
REDU Revista de docencia

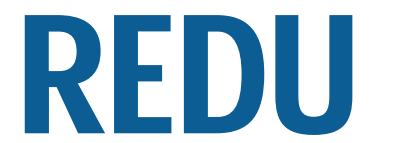

Revista de Docencia Universitaria

Vol $11 \mathrm{~N}^{0} 1$

Enero - Abril, 2013 


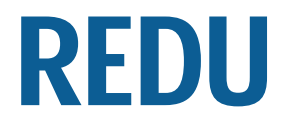

\section{Revista de Docencia Universitaria}

\section{Vol $11 \mathrm{~N}^{0} 1$}

Enero - Abril, 2013

Sede editorial: Facultad de Ciencias de la Educación

Rúa Xosé María Suárez Núñez, s/n. Campus Vida

15782 Santiago de Compostela

Teléfono con extensión: +34 981563100 Ext.13825

Fax: +34 981530494

\section{Edita:}

RED-U(Red Estatal de Docencia Universitaria)

http://www.red-u.org/

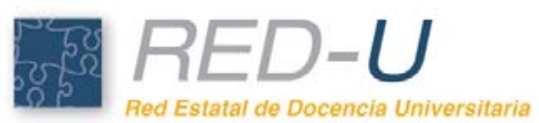

\section{Edición on-line}

http://www.red-u.net/

Fecha de edición: 2013

ISSN: 1887-4592 


\section{EQUIPO EDITORIAL}

\section{Director}

Miguel A. Zabalza Beraza, Profesor catedrático Univ. de Santiago de Compostela (miquel.zabalza@usc.es).

\section{Asesores Editoriales RED-U}

Amparo Fernández March, presidenta de RED-U (ICE - Universidad Politécnica de Valencia)

Araceli Adam Salvatierra, secretaria de RED-U (Universidad Politécnica de Catalunya

\section{Secretarias de Dirección}

Manuela Raposo Rivas, Profesora Titular de la Universidad de Vigo (mraposo@uvigo.es)

Lina Iglesias Forneiro, Profesora Titular de la Universidad de Santiago de Compostela (lina.iglesias@usc.es)

Secretaría técnica: Pablo César Muñoz Carril, profesor de la Universidad de Santiago de Compostela.

\section{Asesores Técnicos:}

D. José Pereira Uzal (jose@arcade.es) Arcade consultores

D. Miguel Zapata (mzapata@um.es ), profesor de la Universidad de Alcalá de Henares.

\section{COMITÉ CIENTFEICO}

Dra. Itzíar Alkorta Idiakez, Universidad del País Vasco, España

Dr. Manuel Area Moreira, Universidad de La Laguna, España

Dr. Manuel Cebrián de la Serna, Universidad de Málaga, España

Dra. María Isabel de Almeida, Universidad de Sao Paulo, Brasil

Dr. Ángel Díaz Barriga, Universidad Nacional Autónoma de México, México

Dr. Roberto Di Napoli, Goldsmiths College, University of London, Reino Unido

Dra. Amparo Fernández March, Universidad Politècnica de Valencia, España

Dra. Isabel Flávia Gonçalves Fernandes Ferreira Vieira, Universidade do Minho, Portugal

Dr. Antoni Font Ribas, Universidad de Barcelona, España

Dr. Franco Frabbonni, Universidad de Bolonia, Italia

Dra. Mercedes González Sanmamed, Universidad de A Coruña, España

Dr. Günter L. Huber, Universidad de Tübingen, Alemania

Dra. María Soledad Ibarra Sáiz, Universidad de Cádiz, España

Dra. Begoña Learreta Ramos, Universidad Europea de Madrid, España

Dra. Carlinda Leite, Universidade do Porto, Portugal

Dr. Clemente Lobato, Universidad País Vasco, España

Dr. Carlos Marcelo García, Universidad de Sevilla, España

Dr. Miquel Martínez Martín, Universidad de Barcelona, España

Dra. Maria Carmen Monreal Gimeno, Universidad Pablo de Olavide, España

Dr. Carlos Moya Ureta, Instituto Latinoamericano de Estudios Sociales, Chile

Dr. Antonio Nóvoa, Universidade de Lisboa, Portugal

Dr. Javier Paricio Royo, Universidad de Zaragoza, España

Dra. Ángeles Parrilla Latas, Universidad de Vigo, España

Dra. Ángeles Sánchez-Elvira Paniagua, Universidad Nacional de Educación a Distancia, España

Dr. Felipe Trillo Alonso, Universidad de Santiago de Compostela, España

Dra. Carmen Vizcarro Guarch, Universidad Autónoma de Madrid, España 



\section{REDU \\ Revista de Docencia Universitaria \\ Vol 11 No1 \\ Enero - Abril 2013}

\section{Sumario}

Editorial

\section{Monográfico}

\section{Academic Writing}

La escritura académica en la universidad

Anna Camps Mundó; Monserrat Castelló Badía.

Perspectives on Academic Writing in European Higher Education: Genres, Practices and Competences Otto Kruse

First year students' construction of an academic identity in English as a foreign language

Angels Oliva Girbau; Marta Milian Gubern.

Enseñar a escribir textos científico-académicos mediante la revisión colaborativa: El trabajo

final de grado en Psicología

Mariona Corcelles Seuba; Maribel Cano Ortíz; Gerardo Bañales Faz; Norma Alicia Vega

Concepciones y prácticas declaradas de profesores terciarios en torno al leer y escribir en las asignaturas

Paula Carlino; Patricia Iglesia; Irene Laxalt .

¿Para qué se lee y se escribe en la universidad colombiana? Caracterización de prácticas de lectura y escritura en 17 universidades

Mauricio Pérez-Abril; Alfonso Rodríguez Manzano.

Contradictions regarding teaching and writing (or writing to learn) in the disciplines: What we have learned in the USA

David Russell . 


\section{Miscelánea}

La controvertida aplicación de las competencias en la formación docente universitaria

Rosario Mérida Serrano

Inteligencia Emocional y Comunicación: la conciencia corporal como recurso

Rosa María Rodríguez Jiménez, Mạ del Mar Caja López, Patricia Gracia Parra, Paloma Julia Velasco Quintana, Ma José Terrón López

El EEES, identidad y competitividad Europea: Principios fundamentales e interpretación de las principales autoridades

Miguel Angel Comas

Experiencias Creativas para el Desarrollo Humano Sostenible: espacios para la formación profesional en Arte en Costa Rica

Enid Sofía Zúñiga Murillo

Retos institucionales de la formación del profesorado universitario

Inma Torra Bitlloch; Ma Dolores Màrquez Cebrián; Teresa Pagès Costas; Pau Solà i Ysuar; Rafael García Campos; Fidel Molina Luque; Angel-Pío González Soto; Albert Sangrà Morer

La enseñanza de competencias para resolver situaciones conflictivas con pacientes agresivos

en enfermería mediante el Método del Caso (MdC)

Marta Arrue Mauleon

Iniciativa curricular para el desarrollo de competencias transversales en Ciencias de la Salud

Enrique Gil Martín

Evaluando el Practicum en Educación Social: Acciones de Mejora ante la puesta en práctica de los nuevos grados

Sonia Morales Calvo

Reflexiones en torno a la lengua francesa en Castilla y León: secciones bilingües y formación de los profesores de disciplinas no lingüísticas (DNL)

Ana.M. Elisabeth Cuervo Vázquez; Rosa M. Alonso Díaz; Montserrat Sabadell González.

La responsabilidad social de las universidades a través de la memoria cinematográfica

María José Chisvert Tarazona.

Entorno virtual de aprendizaje compartido en Educación Superior

Margarita R. Rodríguez Gallego, Antonia López Martínez

Puntos fuertes y débiles en la formación por competencias según los graduados

universitarios españoles

Andrea Conchado Peiró, José Miguel Carot Sierra 


\section{Recensión}

Zabalza, M.A; Zabalza, M.A. (2012). Profesores y profesión docente. Entre el "ser" y el "estar"

Alfonso Cid Sabucedo

Noticias REDU

Eventos relacionados con Docencia Universitaria

455 
En otoño de 2009 aproveché la convalecencia de un accidente para leerme en detalle los discursos fundamentales de la campaña electoral que dio la presidencia de los Estados Unidos a Barak Obama, accesibles en el sitio http://obamaspeeches.com. No solo el éxito perlocutivo del ya famoso lema Yes We Can, sino muchos otros rasgos que a vuelapluma había ido registrando yo a través de la TV norteamericana un año antes me habían ratificado en el convencimiento del poder de las palabras.

La irrupción del Yes we can en la campaña presidencial de Obama se produce en su discurso pronunciado en Nashua el 8 de enero de 2008, en la noche de las primarias demócratas de New Hampshire en las que se impuso su máxima rival Hillary Clinton. Mas lo que a mí -en definitiva, profesor no de Ciencia Política sino de lo que antaño se llamaba "Poética y Retórica" - más me interesaba era revisar el conjunto de las intervenciones fundamentales del futuro presidente.

Siglos antes de Cristo los griegos aprendieron a ordenar el discurso de un modo tal que pudiera lograr el efecto deseado, pues fueron ellos los primeros en ocuparse de analizar las maneras en que los seres humanos se relacionan verbalmente entre sí, elaborando tratados analíticos y expositivos con los que intentaron descubrir las bases reales de la comunicación humana. La suma de todas sus experiencias expresivas y de normas preceptivas da lugar a la Retórica, el arte o la ciencia del rétor u orador público, cuya paternidad se atribuye a Córax que residía en la Siracusa sicliana, correpondiéndole a su discípulo Tisias llevar la buena nueva a la Grecia continental.

Allí fueron los llamados sofistas los que tomaron la Retórica como cosa suya. Mas, a este respecto, la historia de la palabra guarda una considerable contradicción. Etimológicamente, sofista significa "portador de la verdad", pero quizá hoy en día predomine entre nostros una acepción totalmente contraria, la de sofista como aquel -frecuentemente, un político- que se vale de sofismas, es decir, de razones o argumentos aparentes con los que se quiere defender o persuadir lo que es falso.

La Retórica más genuina no es, en este sentido, nada sofista. Muy claro lo deja Aristóteles cuando en la propia introducción a su tratado, al abordar los usos de la Retórica comienza afirmando que ésta es útil para hacer efectivas la verdad y la justicia, amén de para informar a los ciudadanos del común.

Mas volviendo a las raíces, G. B. Kerford, un estudioso de los primeros sofistas griegos, distinguía tres grados o tipos entre ellos: los sabios, como Solón el legislador, que plasman su sapiencia en forma de leyes; los hombres de Estado, que la aplican a los asuntos prácticos como lo hicieron Pericles o Temístocles; y los maestros de sabiduría que hacían valer su habilidad para transmitir los saberes o para enseñar la elocuencia, como Protágoras, Gorgias o Sócrates.

¿Es aquí donde podríamos "enganchar" -si se me permite tamaño vulgarismolas posmodernas disquisiciones acerca de lo que los anglosajones llaman "Academic 
Writing" y nosotros bien podríamos traducir en román paladino como "Escritura en la Universidad"?

En este sentido, Barack Hussein Obama, profesor universitario, senador y Presidente de los Estados Unidos, ofrece un ejemplo sumamente interesante, al margen de cualquier enjuiciamiento objetivo o partidista de su política. Se trata de una figura perfectamente acomodada a una sociedad de características tan particulares como la del Nuevo Mundo norteamericano, la tierra de promisión donde, sobre los principios políticos que precitarían más tarde en la Revolución francesa, se creó una comunidad de aluvión, crisol de diversas procedencias no exclusivamente europeas, abierta a todas las innovaciones promovidas por el espectacular desarrollo científico y tecnológico que desde la llustración llega hasta la actualidad.

Porque es un hecho que esa Tecnópolis (el nombre que el discípulo de Marshall McLuhan Neil Postman -autor en 1995 de The End of Education- le da, con tintes poco amables, a los Estados Unidos de Norteamérica) constituye un campo privilegiado de aplicación y desarrollo de las nuevas tecnologías, pero a la vez, incluso en pleno Siglo XXI, ostenta también rasgos inconfundibles de una vasta comunidad humana en la que, como en las tribus ancestrales, la oralidad y la palabra conservan un poder sustancial.

Ese énfais retórico acompaña la democracia americana desde los padres fundadores, y tuvo una primera gran figura representativa en el propio Abraham Lincoln, que luchó contra el esclavismo proclamando en 1863 la emancipación. Obama lo recordará a la hora de hacer un llamamiento a sus adversarios políticos en su "Victory Speech", y no por azar el que se convertiría en enero de 2009 en el cuadragésimo cuarto presidente de su país comenzó su campaña dos años antes en el Capitolio estatal de Springfield, Illinois, donde Lincoln había pronunciado en 1858 su trascendental discurso "House Divided". No faltan, asi, comentaristas políticos que los relacionan a ambos por su condición común de concienzudos apóstoles del poder de las palabras que Obama invoca al final de la proclamación de su candidatura en el mismo lugar en que ciento cuarenta y nueve años atrás su antecesor había dado el discurso sobre la "casa dividida".

Bien es cierto que en su campaña fue decisiva la incorporación de todos los recursos de internet, en forma de blogs, chats, redes sociales $y$, sobre todo, el visionado en el portal You Tube de alguno de sus discursos más importantes. Pero en el origen de todo ello, como en el Génesis judeocristiano, está la palabra, que es el sustento de esa oralidad comunicativa que caracteriza a los humanos como seres racionales $\mathrm{y}$, por descontado, como animales sociales. A este respecto, Obama no hace sino aprovechar las nuevas posibilidades tecnológicas de lo que se ha dado en denominar la Galaxia Internet, como otro de sus predecesores había hecho lo propio con los recursos de lo que McLuhan denominaba "la constelación de Marconi". Me estoy refiriendo, claro está, a las "fireside chats", la serie de treinta charlas radiadas que Franklin D. Rooselvelt difundió entre 1933 y 1944 y que tanta influencia tuvieron en la comprensión por parte de la ciudadanía de dos trascendentales iniciativas presidenciales: por una parte, la política del New Deal y, posteriormente, la decisión de entrar en la gran guerra que asolaba Europa. 
Desde mis primeras estancias en los Estados Unidos, hace ya treinta años, siempre me sorprendió la versatilidad y amplitud de la moderna Retórica norteamericana, que se manifiesta en todas las facetas de la vida social y muy especialmente a través de los medios de comunicación, entre los cuales la televisión no ha perdido todavía la preeminencia consabida, pero que cada vez más debe compartir espacios y tiempos de audiencia con internet.

En esta cultura de la palabra oral rediviva nació, se formó y se mueve el actual presidente. Nada extraño, pues, que nos venga de USA esta oleada del "Academic Writing". La trayectoria universitaria de Obama, primero en Ciencias políticas en la Universidad de Columbia y luego, hasta el doctorado, en la Facultad de Derecho de Harvard, potenció en él unas condiciones personales que sin duda poseía de forma innata, como también las disfrutó el presidente Ronald Reagan, cuya profesión de actor no dejó de serle de gran ayuda en las lides comunicativas y políticas (no se puede decir lo mismo, por cierto, de George W. Bush junior).

La eficacia retórica del mandatario reelegido en 2012 se manifiesta también en su capacidad de empatizar con los auditorios, gracias a su pertinente actio, la sobria pero suficientemennte enfática actuación con la que acompaña sus alocuciones. Se trata de expresarse conforme a las expectativas de un determinado auditorio, para elaborar lo que James E. Porter y otros teóricos del "Academic Writing" definen como "Discourse Community" ${ }^{1}$, noción esta última que me recuerda vivamente la que uno de mis colegas, el teórico de la Literatura Stanley Fish denomina "Comunidades interpretativas" ${ }^{2}$. Y no se nos debe escapar, asimismo, el buen tino en la selección de los redactores de los discursos del candidato y en la capacidad de transmitirles las ideas fundamentalees, la inventio retórica o contenido del mensaje, a las que ellos luego habrán de poner las palabras justas, la elocutio, y por debajo de ellas la estructura o dispositio más eficaz. Lo mismo que, en definitiva, todo buen profesor e investigador en definitiva ha de hacer con sus libros, monografías, artículos, ensayos, dictámenes, conferencias, informes, $y$, al comienzo de su carrera, con su propia tesis doctoral. Por cierto, precursor latino de este "academic writing" fue en 1977 el gran semiólogo, medievalista y finalmente novelista italiano Umberto Eco, cuando publicó su libro Cómo se hace una tesis, en el que, sin embargo, los aspectos de la elocutio retórica reciben menos atención que los consagrados a la invenio y la dispositio. .

Reconozcamos, desde ya, nuestras carencias a este respecto. Cuando escribo este editorial sin pretensiones (marzo de 2013) es noticia en la prensa española la publicación por parte de la Comunidad de Madrid de los resultados de las oposiciones de 2011 para profesores de primaria. Con tal motivo se ha desencadenado una campaña que algunos califican ya de "estigmatización social" contra la formación dada en la Universidad a los futuros maestros sobre el supuesto de que solo el $13 \%$ de los más de catorce mil candidatos superó una prueba de conocimientos encuadrables en lo que antaño llamábamos "cultura general".

\footnotetext{
${ }^{1}$ Audience and Rhetoric. An Archeological Composition of the Discourse Community, Prentice Hall, Englewood Cliffs, NJ, 1992

${ }^{2}$ Is there a Text in this Class? The Authority of Interpretative Communities, Harvard University Press, Cambridge/London, 1980.
} 
No voy a entrar en el fondo del asunto, que una de las opositoras suspendidas resume en la afirmación de que "salimos de la Universidad sin saber absolutamente nada. Nos deberían enseñar la realidad del aula y los conocimientos básicos, porque por mucho que Piaget fuera muy bueno, yo no voy a contarles quién era a mis alumnos". Pero sí me interesa destacar otra respuesta, esta vez de un paisano lucense, como yo- que apunta en distinta dirección: "Desde mi punto de vista, el principal problema del sistema educativo es que no sabemos hablar en público. Debería primar más la parte oral en la oposición. Lo principal es saber transmitir los conocimientos: no es sólo lo que sabes, sino cómo lo expresas" ${ }^{3 *}$.

No tengo duda de que ahí reside el meollo del "Academic Writing", que no sólo comprende los distintos géneros de la escritura producida en la Universidad -tanto de los profesores como de los alumnos, que deben ser adiestrados desde el principio en esta retórica académica- sino también los que pertenecen al cauce de la oralidad.

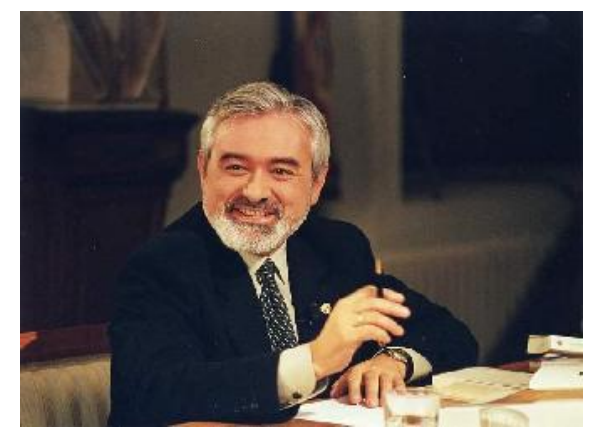

Darío Villanueva

Universidad de Santiago de Compostela (España)

Catedrático de Teoría de la Literatura y Literatura comparada

Exrector de la USC

Secretario de la Real Academia Española

Mail: dario.villanueva@usc.es

\footnotetext{
3 Fuente: Reportaje de Olga. R. Sanmartín, "FUERON A PILLARNOS", El Mundo, viernes 29 de marzo de 2013, página 14.
} 


\title{
REDU
}

Revista de Docencia Universitaria

Vol $11 \mathbf{N}^{01}$

\section{MONOGRÁFICO} Academic Writing

\author{
Coordina:
}

Anna Camps

Universidad Autónoma de Barcelona (España) Montserrat Castelló

Universidad Ramon Llull (España) 



\title{
La escritura académica en la universidad Academic writing at University
}

\author{
Anna Camps Mundó \\ Universidad Autónoma de Barcelona, España \\ Montserrat Castelló Badía \\ Universidad Ramon Llull, España
}

\section{Resumen}

En este texto introductorio del monográfico se definen algunos de los conceptos fundamentales que se relacionan con el constructo de escritura académica. En primer lugar, el concepto de género y su relación con el de sistema de actividad, lo que permite abordar desde un punto de vista dinámico el discurso en los entornos universitarios. A continuación, se discuten las diferentes aportaciones que se han ocupado de la clasificación de los géneros en el ámbito académico universitario lo que permite esbozar una propuesta que sirva de marco integrador para organizar la actividad de escribir y los géneros que se producen en la universidad en esferas discursivas que otorguen sentido y significado a las prácticas de escritura de los estudiantes. En tercer lugar, se ofrecen algunas reflexiones sobre el uso y la enseñanza de los géneros en la docencia universitaria desde la perspectiva apuntada en los apartados anteriores. En la segunda parte del texto se presentan los artículos que forman este monográfico y que ofrecen una panorámica variada y complementaria de las aportaciones actuales en el ámbito de la escritura académica tanto en contextos españoles y latinoamericanos como europeos. A estas aportaciones hay que sumarle la revisión y síntesis de las aproximaciones teóricas, los métodos y los resultados de los movimientos Writing Across Curriculum y Writing In the Disciplines en los EEUU.

Palabras clave: escritura académica; Universidad; Educación Superior; géneros; actividad discursiva.

\begin{abstract}
In this introduction of the Special Issue, some key concepts related to the notion of academic writing are discussed. Firstly, the concept of genre is revised and its relationships with the more broad notion of activity system, which allows for a dynamic approach of discourse and its study in university contexts. Secondly, genre classification proposals in higher education are reviewed and discussed focusing on their contributions and limitations. Based on this review, a new proposal for genre organization is presented, which could act as an integrative framework to organize writing activity and genres in discursive spheres making meaning of the students' writing practices. Thirdly, some recommendations are provided regarding the teaching and learning of writing at university, based on the theoretical perspective developed in previous sections. In the second part of the article, the contributions of the Special Issue are presented. These contributions offer a varied and complementary picture of current
\end{abstract}


trends and perspectives in the field of academic writing in Spanish and Latin-American contexts as well as in Europe. To these contributions it has to be added the review and synthesis of the theoretical discussions, methodological concerns and results of the broadly developed approaches in the USA such as Writing across Curriculum -WAC- and Writing in the Disciplines -WID

Key words: Academic Writing; University; Higher Education; Genres; Discursive activity.

Escribir es una actividad habitual en los entornos universitarios. Una gran parte del conocimiento que se elabora en ellos se plasma y vehicula a través de textos que se leen y producen en una inextricable red de relaciones entre unos y otros, tanto en formatos monológicos como dialógicos. Hablamos de discurso académico para referirnos de una manera muy general a estos usos. El discurso, también el académico, no es simplemente el vehículo por donde transitan los saberes, sino que es instrumento de construcción del conocimiento y de las relaciones que establece con las actividades humanas en que se desarrolla. En palabras de Halliday (1993): "El discurso es la condición esencial del conocimiento, el proceso por el cual la experiencia deviene conocimiento".

El discurso académico adopta formas específicas, adecuadas y adaptadas a las situaciones en que se genera; a estas distintas formas se las denomina géneros discursivos. En la literatura científica sobre la escritura en la universidad este término se ha generalizado. Sin embargo, una lectura atenta de su uso muestra la diversidad conceptual que esconde. Por otro lado, es cada vez más frecuente relacionar este concepto con el de sistema de actividad, lo cual permite abordar desde un punto de vista dinámico el discurso en los entornos universitarios. Con ello se enriquece el mismo concepto de género, pero se hace más compleja su definición.

Sin la pretensión de establecer una definición cerrada de ambos conceptos y de su interrelación, en esta introducción intentaremos delimitarlos en relación con las aportaciones, ya clásicas, de algunos autores que son todavía referentes para su comprensión y de aportaciones posteriores que los han enriquecido. A continuación abordaremos la clasificación de los géneros en el ámbito académico universitario para finalizar con algunas reflexiones sobre el uso y la enseñanza de los géneros en la docencia universitaria.

\section{El concepto de género discursivo}

El concepto de género para referirse a usos diferenciados del lenguaje verbal tiene raíces antiguas, especialmente en la obra de Aristóteles; posteriormente ha sido un término que ha pervivido en los estudios literarios. Sin embargo en la actualidad este concepto ha ampliado su significado para referirse a todos los posibles usos del lenguaje humano considerado éste como una actividad social. Podemos situar esta renovación del concepto de género discursivo en la obra de Mihail Bakhtin. En uno de sus textos fundamentales, El problema de los géneros discursivos, publicado en ruso en 1979, se establecen las características básicas de la noción que de una forma u otra serán la base de las formulaciones posteriores del concepto. La primera idea es que el uso de la lengua está relacionado con la actividad humana y como dicha actividad se 
desarrolla en ámbitos diversos, en diversas "esferas de actividad" dirá Bakhtin, los usos verbales serán también diversos. A pesar de que cada producción oral o escrita es individual, la recurrencia de las situaciones, de las actividades que se llevan a cabo en los distintos ámbitos, da lugar a producciones 'que "reflejan las condiciones específicas y el objeto de cada una de las esferas no sólo por su contenido (temático) y por su estilo verbal, o sea por la selección de los recursos léxicos, fraseológicos y gramaticales de la lengua, sino, ante todo, por su composición o estructuración". Estas condiciones específicas son inseparables, están indisolublemente unidas, y se determinan unas a otras. Por ejemplo, nos es fácil distinguir un artículo de divulgación, de un artículo científico, aunque versen sobre el mismo tema, porque tienen una estructura y unos recursos estilísticos distintos y ello es así porque responden a situaciones diferentes. El primero se sitúa en el ámbito de la comunicación de la ciencia con el conjunto de ciudadanos no especialistas y el segundo en el de la comunicación entre científicos especializados. Así pues, la recurrencia de situaciones da lugar a la recurrencia del conjunto de características verbales de los "enunciados" que se generan en ellas y que llegan a tener cierta estabilidad. Dan lugar a lo que se denomina géneros discursivos.

Una misma persona participa en una gran diversidad de ámbitos ("esferas") de actividad. Los géneros son instrumentos de participación en la actividad social y aprendemos a usar la lengua dentro de las esferas de actividad que han conformado usos verbales específicos. Aprender lengua es aprender a usarla de forma adecuada en cada una de los ámbitos de la actividad humana.

En la obra de Bakhtin se plantea una de las cuestiones importantes en los debates sobre los géneros académicos en el momento actual. Este autor habla de la "relativa estabilidad" de los géneros. Como hemos dicho las producciones verbales en cada ámbito tienden a compartir un conjunto de características que se reiteran, pero esta reiteración es relativa. La actividad humana es dinámica y por lo tanto cambiante. Por ello los géneros serán también cambiantes. Por ejemplo, un artículo científico en nuestros días está muy alejado estilística y estructuralmente de uno escrito en el siglo XIX (Bazerman 1988; 1994). Así explica Bakhtin (1982 [1979]) esta dinamicidad:

\footnotetext{
"La riqueza y diversidad de los géneros discursivos es inmensa, porque las posibilidades de la actividad humana son inagotables y porque en cada esfera de la praxis existe todo un repertorio de géneros discursivos que se diferencia y crece a medida de que se desarrolla y se complica la esfera misma." (p. 248)
}

La estabilidad de los géneros es lo que los convierte en modelos de referencia para la producción verbal y en "horizonte de expectativas" para los receptores (Todorov, 1978). Algunos géneros se institucionalizan y adquieren una gran estabilidad: Pensemos, por ejemplo, en los textos jurídicos, o en las cartas comerciales, y en nuestro ámbito en los informes de laboratorio o en las tesis, al menos durante la última mitad del siglo XX. Esta estabilidad y sobre todo la institucionalización facilitan la participación de los individuos en las comunidades discursivas, por cuanto no se debe negociar en cada caso el tipo de participación requerida; aunque pueden convertirse también en un corsé que impida la verdadera comunicación.

Los conceptos de "esfera de actividad" y de "situación" no son coincidentes. Las esferas de actividad pueden ser consideradas, como decíamos, ámbitos de 
participación en la vida y la actividad humanas. Las situaciones están definidas por el tipo de acción social que se realiza en cierto ámbito de la actividad. Así, el ámbito académico da lugar a géneros discursivos diversos: tesis, informes, reseñas de libros, proyectos, apuntes, etc. Todos ellos responden a tipos de actividades distintas que se llevan a cabo respondiendo a distintas situaciones en una misma esfera de actividad.

Miller (1984), en un imprescindible artículo, define los géneros como acción social y se refiere a ellos como "acciones retóricas tipificadas". Siguiendo a Burke y a Cambbell y Jamieson, precisa que "un género está compuesto por una constelación de formas unidas por una dinámica interna. La dinámica 'funde' características sustantivas, estilísticas y situacionales." ${ }^{\prime i i}$ (p. 152). Es necesario destacar, sin embargo, que las situaciones no vienen definidas sólo ni principalmente por las condiciones materiales de la comunicación sino que son constructos sociales resultado del significado y de la interpretación que los humanos dan a sus actividades. Si la tipificación de una actividad discursiva es útil en la comunicación entra en el stock relativamente estable de nuestros conocimientos y su aplicación puede devenir en rutina o "exigencia".

Al hablar de la "exigencia" que la existencia de los géneros representa para los hablantes o escritores Miller se refiere a que la necesidad de hacerse entender conlleva la necesidad de ajustar el lenguaje a estos usos socialmente tipificados. El término exigencia sugiere determinismo, sin embargo el término alternativo que la autora utiliza, el de "motivo", introduce en el concepto género una idea fundamental lejos de este matiz determinista: los géneros absorben las características de las situaciones recurrentes en que el lenguaje se genera y devienen motivos socialmente compartidos de dichas acciones.

En síntesis, los humanos desarrollamos nuestra actividad, que es en gran parte actividad discursiva, en diferentes ámbitos o esferas de comunicación. En cada una de las esferas se crean situaciones comunicativas recurrentes que dan lugar a formas de uso del lenguaje que denominamos géneros discursivos. Las situaciones y los géneros son construcciones sociales que poseen cierta estabilidad, pero al mismo tiempo son dinámicas y cambiantes. La estabilidad de los géneros conlleva que sean motivos sociales de participación.

Antes de adentrarnos en el entramado de géneros discursivos, entendidos como actividad discursiva que se da en el entorno académico debemos referirnos a ja caracterización de la actividad humana en general para comprender la diversidad de situaciones que se dan en dicho entorno y las interrelaciones entre los diferentes ámbitos comunicativos que se entrecruzan en él. El marco de la teoría de la actividad nos permite avanzar en esta comprensión.

\section{Actividad y sistemas de actividad: la actividad discursiva}

El quehacer humano, la práctica, ha sido abordada desde puntos de vista diversos desde la filosofía, la sociología, la psicología, entre otras ciencias humanas y sociales. Aquí nos basaremos en la aproximación sociohistórica que se desarrolla a partir de las primeras propuestas de Vigotski. Este autor pone de relieve que la actividad específicamente humana se realiza por medio de instrumentos y además se da en 
cooperación con otras personas. Los humanos actúan sobre los objetos a través de instrumentos materiales o simbólicos; uno de ellos y fundamental es el lenguaje. En esta relación mediada el sujeto transforma el objeto y es transformado por él.

Los instrumentos, y el lenguaje por encima de todos, recogen la experiencia de la humanidad y por ello su utilización está marcada por los usos anteriores. Si queremos clavar un clavo utilizaremos un martillo cogiéndolo por el mango y no un destornillador. Si queremos exponer alguna opinión política en un periódico seguramente utilizaremos el lenguaje en forma de artículo de opinión, si nuestra firma es reconocida, o en forma de carta al director, si somos personas anónimas. Es decir acudiremos a instrumentos socialmente elaborados, los géneros discursivos, que nos ofrecen la guía para actuar y que permitirán la comprensión por parte de los lectores.

Leontiev (1972 [1979]) profundizó en el análisis de la actividad humana. Según este autor, la característica básica de la actividad es su orientación al objeto. El proceso de vivir, afirma Leontiev, un sistema de actividades que se suceden una a otra. No es pues una reacción o un agregado de reacciones frente al objeto sino "un sistema con su propia estructura, sus propias transformaciones internas y su propio desarrollo" (p. 11).

Las actividades se basan en motivos que se orientan a finalidades. Los motivos son sociales y los individuos pueden hacerlos suyos. En el marco de las actividades, los individuos o los colectivos llevan a cabo acciones que tienen sus objetivos específicos que toman sentido dentro de ellas. Esto implica que la acción se concibe como forma de participación social que se construye en el marco de la actividad; implica "la asunción por parte de un agente de un objetivo intermedio en el marco de la cooperación social". Supone la separación del objeto de la actividad y su motivo y, por lo tanto implica consciencia. Para alcanzar dichos objetivos se realizan operaciones. Pongamos un ejemplo: Podemos considerar que la actividad humana de comunicar información de hechos acaecidos tiene como uno de sus objetivos mantener la cohesión de un grupo humano. Esta actividad se realiza a través de actos individuales: una persona cuenta a otra algo que ha sucedido. Esta podría ser una forma primera de comunicación de un hecho. Históricamente esta actividad se ha hecho mucho más compleja y mediada por instrumentos cada vez más sofisticados: la prensa escrita, los medios de comunicación audiovisuales, internet. Las acciones y los objetivos específicos de los participantes en este tipo de actividad se han diversificado y podría llegar a ocurrir que alguno de ellos perdiera de vista la finalidad de su acción específica. Por otro lado, las operaciones que implicaba comunicar una noticia oralmente también se han diversificado y multiplicado gracias a las técnicas de comunicación y a su progresiva sofisticación. Los motivos y las finalidades de las actividades cambian con el tiempo. Podríamos pensar que la necesidad de información se asocia en nuestras sociedades al mantenimiento de la democracia, aunque algunos colectivos pueden usarla para todo lo contrario e introducir finalidades espurias como son las de controlar para fines propios a la población. Así, las actividades se transforman y se diversifican. Por ejemplo, las redes sociales se utilizan preferentemente para un tipo de información que los medios más tradicionales no proporcionan.

Han surgido aquí algunos conceptos fundamentales para la comprensión de la actividad humana y también para la actividad discursiva. Destacamos el de sistema de 
actividad y el de motivo relacionado con el de sentido que los participantes otorgan a sus acciones.

El concepto de sistema de actividad fue retomado y modelizado por Engeström y colaboradores (Engeström, Miettinen y Punamäki, 1999). Basándose en el triángulo que representa la relación que Vigotski establece entre sujeto, objeto e instrumento, Engeström lo amplia tomando en consideración la comunidad en que la actividad se lleva a cabo, las reglas sociales que guían la actividad y la división del trabajo que toda actividad implica.. El esquema de la figura 1 representa este modelo:

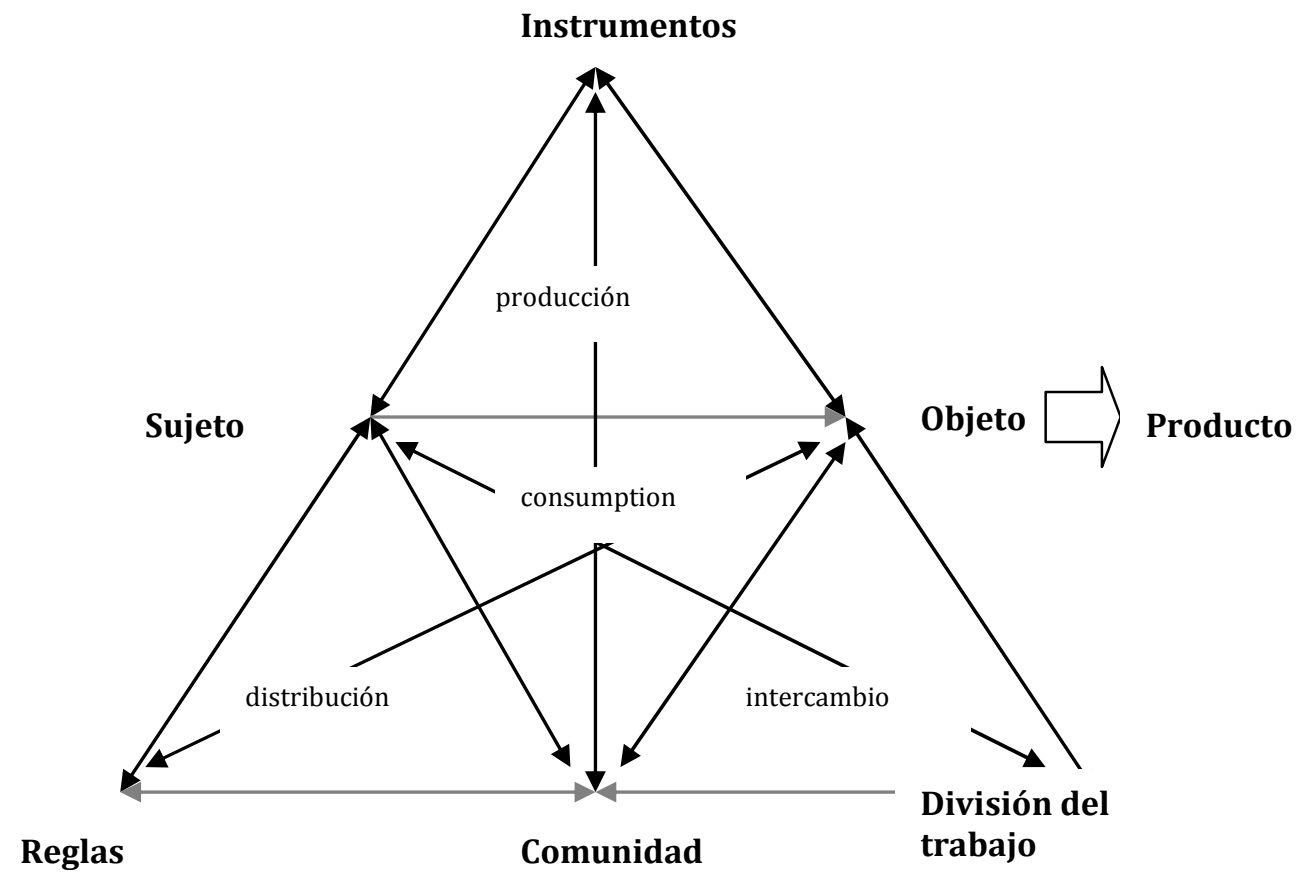

Figura n.1: Modelo del sistema de actividad según Engeström ${ }^{\text {iii }}$

El sujeto es la persona o grupo que actúa. El objeto es el "espacio problema" que orienta la actividad y que se transforma en el resultado o producto de la actividad. Los instrumentos físicos o simbólicos mediatizan la actuación. La comunidad comprende los grupos humanos que comparten el objetivo general y que adoptan unas regulaciones, normas y convenciones, implícitas o explícitas (reglas). Por división del trabajo se entiende tanto la horizontal entre miembros de la comunidad, como la vertical que puede implicar poder o diferencia de estatus.

Los sistemas de actividad en que nos vemos implicados los humanos son muy diversos y la actividad humana se desarrolla en la confluencia no siempre armónica entre sistemas de actividad. Las situaciones de enseñanza de la escritura, por ejemplo, pueden implicar la confluencia como mínimo de dos sistemas de actividad: la comunicativa implicada en la finalidad del texto que se escribe y que se pretende que sea leído por una comunidad que se considera destinataria del texto y la de aprendizaje del género que es objeto de enseñanza. Los sistemas de actividad que confluyen en los ámbitos de la enseñanza y el aprendizaje pueden ser más, como muestran algunas investigaciones: Camps y Uribe (2006), Milian y Camps (2001), 
Milian (1996, 1999) y Russell y Yáñez (2002). También muestran que estas contradicciones pueden ser y son fuente de aprendizaje (Castelló e Iñesta, 2012; Castelló, Iñesta y Corcelles, 2013).

También Leontiev se refería a la diversidad de sistemas de actividad que se entrecruzaban y, siguiendo su distinción entre actividad y acción, afirmaba que una misma acción podía insertarse en sistemas de actividad diversos. Por este motivo, para comprender la actividad de escritura en la universidad no bastará con reconocer el tipo de acciones que los estudiantes llevan a cabo, sino que será necesario profundizar en el conocimiento de los sistemas de actividad que las orientan.

\section{Los géneros en la universidad}

Las clasificaciones de la escritura de los estudiantes universitarios han sido prolijas pero, a pesar de su dispersión, es posible distinguir cuatro grandes perspectivas. En primer lugar, clasificaciones basadas en la teoría que establece tipologías de textos según su función. El trabajo pionero de Kinneavy (1969) es un claro exponente de este enfoque. Este autor desarrolló un modelo según el cual la escritura en la universidad respondía a cuatro grandes finalidades: expresiva, persuasiva, referencial y literaria. En segundo lugar, es preciso mencionar los estudios que establecen clasificaciones empíricas a partir de la información proporcionada por los protagonistas, fundamentalmente profesores pero también estudiantes, acerca de lo que escriben y la finalidad con qué lo hacen (Ganobcsik-Wiliams, 2004; Casanave, y Hubbard, 1992). El tercer enfoque se centra en el análisis de los programas y materiales de las asignaturas y especialmente de las actividades que los profesores proponen a sus estudiantes (Horowitz, 1986; Gillet y Hammond, 2009). Resulta muy difícil integrar de forma coherente los resultados de estos estudios, no sólo porque en ocasiones parten de criterios diferentes a la hora de establecer sus categorías sino también porque bajo denominaciones idénticas a menudo se esconden interpretaciones y significados muy diferentes. Resulta complicado saber por ejemplo qué quiere decir en un determinado contexto escribir un "ensayo" y cuál es la diferencia entre esta actividad y la de escribir un "texto de opinión" o entre un "informe" de una lectura y una "reseña", por citar sólo algunas de las más habituales. Más complicado es todavía averiguar los textos que cada profesor considerará adecuados a la consigna formulada, algo que la mayoría de estudiantes debe inferir a partir de explicaciones y pistas indirectas proporcionadas por el profesor o de los resultados de evaluaciones propias o ajenas. La última perspectiva intenta paliar algunos de estos problemas puesto que establece sus clasificaciones a partir del análisis de los textos producidos por los estudiantes partiendo del análisis de corpus generalmente amplios y representativos de diferentes disciplinas. Algunas propuestas además de establecer diferencias entre los géneros han propuesto la agrupación de los mismos en familias atendiendo a sus características funcionales en un intento de evitar el reduccionismo lingüístico y de trascender las limitaciones disciplinares en clara sintonía con la propuesta de "esferas de actividad" a la que nos hemos referido en el apartado anterior (Gardner y Nesi, 2012). En estos casos, la información de profesores y estudiantes acerca de las finalidades y el significado de los textos que escribían resulta un complemento imprescindible a la hora de establecer su funcionalidad. 
La funcionalidad es ciertamente uno de los criterios necesarios para entender la producción escrita de los estudiantes (y también de los profesores) pero, a nuestro entender, esta funcionalidad resulta muy difícil de captar si no se tienen en cuenta el significado y el sentido que tienen las actividades de escritura en contextos o sistemas de actividad específicos que cuentan con reglas y normas determinadas, con tradiciones y recursos diferenciales en los que determinadas prácticas discursivas son relevantes y más o menos valoradas. Tanto el significado como el sentido de la escritura en el contexto universitario están estrechamente vinculados a la función educativa de la universidad y es esta función la que acaba determinando la funcionalidad de las prácticas discursivas escritas en las que participan los estudiantes $y$, muchas veces también, sus profesores. Partimos de la idea de que la universidad tiene una función formativa y una función investigadora que se interrelacionan. Aquí aparecen ya dos grandes sistemas de actividad que se subdividen en subsistemas relacionados con las diversas disciplinas y ámbitos científicos y profesionales implicados. No siempre es fácil superar las contradicciones que se generan en la confluencia de la docencia y la investigación y tampoco las que se derivan de concepciones disciplinares diferentes que a menudo conviven en un mismo plan de estudios.

\section{¿Cómo organizar los géneros que se enseñan y se aprenden en la universidad?}

Desde nuestra perspectiva las clasificaciones y taxonomías existentes, tanto las que se inspiran en propuestas teóricas como las que se originan empíricamente, a pesar del interés que tienen para comprender las características particulares de los géneros que analizan, no resultan suficientes cuando se trata de pensar en su presencia y organización en las prácticas discursivas que se desarrollan en la universidad desde una perspectiva educativa. Efectivamente, si lo que nos preocupa es la apropiación por parte de los estudiantes de determinados géneros $y$, por extensión de determinadas formas de pensar y de actuar en sus respectivos campos disciplinares, necesitamos un marco integrador que organice la actividad en esferas discursivas que son las que otorgan sentido y significado a las prácticas de escritura de los estudiantes.

Un primer foco de interés y de problematización lo constituye la definición de la propia expresión escritura académica. Una primera respuesta relativamente fácil implicaría considerar que la escritura académica incluye todas las prácticas de escritura que se producen y tienen sentido en el contexto académico. Sin embargo, resulta evidente que existen prácticas de escritura que, o bien se producen en la academia pero aspiran a ser leídas fuera de la misma (recomendaciones, textos divulgativos), o bien tienen finalidades diferentes en los contextos académicos y científico profesionales (tesis, artículos de investigación) ${ }^{\mathrm{iv}}$. Por lo tanto, no todos los textos que se escriben en la universidad comparten finalidades ni responden a las mismas situaciones académicas.

De acuerdo a lo anterior, entendemos que la clasificación, o mejor dicho, la organización de los géneros que se escriben en la universidad debe dar cuenta de las diferentes sistemas de actividad que caracterizan la actividad de las comunidades que integran el contexto universitario: un primer sistema -o esfera- de actividad 
profesional en el que se producen textos de investigación pero también los propios del quehacer profesional, el sistema de actividad de enseñanza y aprendizaje en el que aquello que se escribe y lee está al servicio del aprendizaje, el sistema de actividad social en el que prima el mantenimiento de relaciones y el sistema de actividad académico propiamente dicho que participa de los anteriores pero en el que se producen también textos propios, como por ejemplo las tesis o los proyectos de investigación.

Estos sistemas de actividad difieren en cuanto a las dimensiones que son relevantes a la hora de definir géneros diferentes (Bazerman y Prior, 2004; Russell y Cortes, 2012), y que fundamentalmente tienen que ver con el sentido y significado que otorgan a la escritura, el proceso de composición y las formas en que se elaboran los textos, las características de estos textos en tanto que productos de la actividad y con la audiencia para la que se escribe.

En términos generales, en los sistemas de actividad que caracterizan a las comunidades profesionales el sentido y el significado de la escritura radica en la posibilidad de difundir el conocimiento disciplinar (p.ej. artículos en revistas profesionales) o científico (p.ej. artículos de investigación) y ayudar a resolver problemas habituales del quehacer profesional ( $p$. ej. informes). Los procesos de escritura acostumbran a ser compartidos por varios autores, los productos son altamente especializados, con abundantes términos específicos y mucha información que se da por conocida dado que se espera que la audiencia sean pares con problemas similares.

En los sistemas de actividad vinculados a la docencia y el aprendizaje altamente formalizados como las que caracterizan los contextos universitarios, el sentido y el significado de la escritura acostumbra a estar vinculado a elaborar, construir $-\mathrm{y}$ reflexionar sobre- el conocimiento o a dar cuenta de lo aprendido. Pero además, en múltiples ocasiones a esta función epistémica de la escritura hay que sumarle una función de aprendizaje de las formas comunicativas propias de las comunidades profesionales, lo que convierte a los textos que se producen en metatextos puesto que éstos ya no tienen el sentido que les sería propio sino que se escriben también con la finalidad de que el estudiante aprenda a dominar las claves que los definen (por ejemplo cuando se enseña a redactar un informe de un paciente). En estas situaciones los procesos de composición suelen ser individuales y bastante implícitos y los productos o bien son de uso exclusivo del aprendiz (y autor), por ejemplo los apuntes, o bien reciben valoraciones basadas en criterios muy diversos que generalmente son establecidos por los profesores con más o menos transparencia y claridad en función de los objetivos que se pretendían en cada situación educativa (ortografía, estructura, uso del contenido, nivel de reflexión, etc.). En general, la audiencia se reduce al mismo profesorado o, en ocasiones, al resto de estudiantes, habitualmente del mismo curso, que incluso puede co-evaluar dichos productos.

El sistema de actividad propiamente académico implica la producción de algunos textos cuyo sentido sólo se entiende dentro de la propia academia. La escritura en estos casos está al servicio de determinadas actividades académicas que no tienen correlato en otras esferas de actividad aunque comparten parte de su funcionalidad tanto con la esfera profesional como con la de enseñanza y aprendizaje. Ejemplos emblemáticos son las tesis doctorales, o en la actualidad, los proyectos finales de 
grado. Se trata de actividades que sólo se pueden llevar a cabo en la comunidad académica y que difícilmente la trascienden sin modificaciones sustanciales tanto en sus productos como en los procesos de producción. La finalidad de una tesis es obtener el grado de doctor y para ello precisa de un producto que será evaluado por un tribunal de miembros expertos de la comunidad académica. Incluso en la situación actual en que la mayoría de áreas del saber admiten que la monografía tradicional pueda ser sustituida por un compendio de publicaciones, se requieren algunas modificaciones en el texto final (redactar una introducción y conclusiones que integren las diferentes publicaciones en un todo) y en los procesos (escritura con un director, defensa ante un tribunal) que lo alejan de las características habituales de la publicación de artículos propia de los investigadores ya iniciados que se sitúan en la esfera de actividad profesional. El proceso de redacción de estos textos es también específico puesto que, a pesar de ser compartido con un director y cada vez más con un equipo de investigación, sigue manteniendo el carácter evaluativo individual del candidato. Algo parecido sucede con otros textos propiamente académicos como los exámenes o la presentación de méritos y proyectos. Los productos en estos casos son también especializados con un nivel alto de codificación que varia en función de las características (sociales, culturales y históricas) y normas específicas de cada comunidad académica. Finalmente, la audiencia es la propia academia e incluso la subcomunidad definida por el área de conocimiento o la disciplina.

A los anteriores se le puede añadir un último sistema que caracteriza la actividad social académica que utiliza la escritura con finalidades empáticas y afectivas e incluso organizacionales. Los emails, anuncios, planes de reuniones, entre otros muchos, son ejemplos de los textos propios de esta comunidad cuyo sentido es establecer o mantener las redes sociales específicas de los contextos universitarios. Los procesos que caracterizan la escritura son, en estos casos, variados pero siempre rápidos y guiados por la funcionalidad, algo que también impregna los productos en los que evidentemente prima la comunicación tanto de hechos como de emociones y actitudes. La audiencia es versátil en función de las comunidades pero también puede ser uno mismo cuando se trata de un diario -tanto del que enseña como del que aprende- en el que las voces de la comunidad se integran con la propia.

El hecho de que estos sistemas o esferas de actividad impregnen una gran parte de las actividades discursivas que se producen en los contextos universitarios da cuenta de la variedad y riqueza de las prácticas de escritura pero a la vez dificulta enormemente la organización de los géneros que se producen en dichos contextos. Además, en las actividades de estudiantes y profesores, que además participan de más de un sistema de actividad, se producen múltiples interacciones y relaciones que dan origen a géneros intermedios que responden de manera particular a las exigencias de cada esfera de actividad. La figura 2 da cuenta de estas relaciones y puede servir de marco conceptual integrador para pensar los géneros y sus relaciones en el contexto universitario desde la perspectiva que estamos defendiendo. 


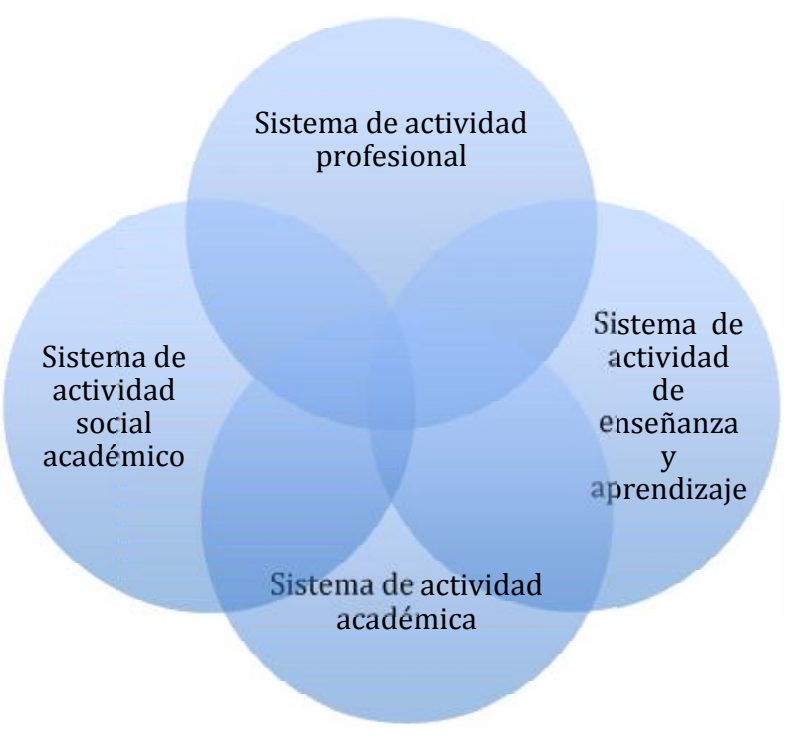

Figura n.2.

Tal como hemos adelantado, la participación tanto de estudiantes como de profesores en más de uno de estos sistemas de actividad genera no pocas tensiones y contradicciones que, si bien, siguiendo los planteamientos de Engeström y Sannino (2011) pueden ser fuente de aprendizaje, también dificultan la apropiación por parte de ambos colectivos de los géneros propios de cada esfera de actividad. Pensemos por ejemplo, en los estudiantes de grado a los que se les pide informes o análisis de casos con la interesante intención de que las actividades a las que se enfrenten sean lo más funcionales, auténticas y significativas posible. En muchas de estas ocasiones, los estudiantes deben "descubrir" cuáles son las claves que rigen la comunicación en el sistema de actividad profesional para producir los textos requeridos de acuerdo a lo que se espera de los mismos en contextos profesionales. Sin embargo, deben hacer esto desde su rol de estudiantes y sin abandonar el sistema de actividad de enseñanza y aprendizaje que les es propio, puesto que el texto va a ser evaluado y en muchas ocasiones va a tener como único destinatario este mismo profesor. Mención especial merece la situación en la que a un estudiante de doctorado se le pide que publique un artículo antes de leer la tesis doctoral, requisito que empieza a ser frecuente en las universidades españolas. Este estudiante cuya actividad principal se sitúa en el sistema de actividad de enseñanza y aprendizaje, debe producir un texto propio de la comunidad profesional, en este caso de investigadores, y consecuentemente debe aprehender las características básicas de este sistema de actividad que le exige cambios en sus acciones y en su identidad. La resolución de estas tensiones pasa por repensar la forma en que se concibe el texto, su rol como escritor, las convenciones o recursos que son necesarios, las reglas de comunicación preestablecidas y su posición en la comunidad, aspectos todos ellos que inciden de forma directa en el texto final que el estudiante es capaz de producir (Castelló, Iñesta y Corcelles, 2013). Algo parecido sucede con el profesor acostumbrado a escribir artículos de investigación que debe enseñar a sus estudiantes qué es y cómo se escribe el proyecto final de grado por citar otro de los retos actuales a los que se enfrentan la mayoría de profesores en la 
actualidad. Las situaciones que ejemplifican contradicciones y tensiones entre sistemas de actividad son múltiples y variadas y resulta conveniente tenerlas en cuenta a la hora de diseñar propuestas de enseñanza y aprendizaje de los géneros propios de las diferentes esferas de actividad que conviven en la universidad si queremos ayudar a los estudiantes a resolverlas de manera efectiva.

En el marco disciplinar universitario se da también la confluencia de diversos sistemas de actividad. Los escritos que se producen en el marco de las disciplinas se insertan en sistemas de actividad diversos; por un lado en el ámbito o esfera de la enseñanza en que los textos que se producen tienen como función bien el mostrar lo que se aprende para que sea evaluado por el profesor: exámenes, resúmenes y síntesis, reseñas, etc., bien servir de instrumento de aprendizaje: ensayos, "papers" de diverso alcance, o bien coadyuvar a los procesos de elaboración de otros textos o del conocimiento que se mostrará de diferentes maneras. Por otro lado los ámbitos profesionales de las diversas disciplinas confluyen constituyendo sistemas de actividad que se entrecruzan con los formativos. En ambos ámbitos se producen textos que se inscriben en géneros diversos, unos altamente institucionalizados, otros más abiertos. Algunos estudios (Flower, 1987; Camps y Uribe, 2008) han mostrado que las finalidades que los alumnos asumen en el marco de estos sistemas en confluencia no son siempre compartidas por todos ellos $y$, por lo tanto el sentido que dan a su actividad escritora puede no ser coincidente. La escritura de un texto puede tener para el alumno solo el sentido de mostrar su conocimiento para aprobar la asignatura; otro alumno, en cambio puede pretender escribir para aprender a insertarse en una comunidad profesional.

\section{La enseñanza de los géneros en la universidad}

La mayoría de los estudios que se han ocupado del análisis de los géneros académicos a los que nos hemos referido en el apartado anterior no se han planteado, al menos de forma explícita, cuáles son los que deberían primarse y por qué en contextos de enseñanza y aprendizaje universitarios. Su foco de atención ha sido la descripción, análisis y categorización de las prácticas existentes pero en contadas ocasiones al análisis le ha acompañado la reflexión respecto al sentido y/o necesidad de la promoción de unos géneros frente a otros.

Ésta sí ha sido la preocupación de movimientos tan importantes en el contexto de los Estados Unidos como los que respectivamente se conocen como "escribir a través del curriculum" (writing across curriculum -WAC-) o "escribir en las disciplinas" (writing in the disciplines -WID-). Dado que ambos son analizados por uno de sus protagonistas de excepción, D. Russell, en este mismo monográfico no es nuestra intención matizar sus aportaciones, si bien buena parte de las afirmaciones siguientes tienen su origen en algunas de las reflexiones y estudios propiciados por estos movimientos, (especialmente el segundo por su mayor conexión con la investigación), y se nutren de algunas de las críticas que han permitido su avance y evolución (Bazerman, 2013).

Desde el punto de vista de la docencia universitaria $y$, a partir de la problematización de las formas tradicionales de enseñanza y aprendizaje, se podrían identificar dos enfoques: a) el que defiende que los géneros discursivos propios de los 
entornos científicos y profesionales deben ser y pueden ser objeto de enseñanza específica dado que se considera que la función del aprendizaje universitario es inserir al aprendiz en la esfera discursiva propia del ámbito en que se desarrollará su vida profesional (Bartholomae, 1986; Bazerman, 1998), y b) el que considera que las situaciones académicas y las profesionales son irreductibles, es decir son ámbitos -0 esferas discursivas- diferentes con fines propios y particulares en cada caso, que, consecuentemente, dan lugar a géneros diversos (Freedman, Adam y Smart, 1994; Russell y Yañez, 2002; Lea y Street, 1998; Robinson-Pant y Street, 2012).

La definición de género que hemos avanzado apunta hacia el segundo punto de vista. Pero adoptarlo de forma extrema sería seguramente simplificador. A continuación formulamos algunas cuestiones que puedan dar lugar a la reflexión y, orientar la investigación sobre los usos del discurso en la enseñanza universitaria:

1. Las finalidades de la actividad docente y/o académica y de la actividad científica y/o profesional son diversas. Así, será diferente el diálogo que tiene como finalidad enseñar del que se produce entre colegas con el fin de construir nuevo conocimiento. En el primer caso, por ejemplo, el dominio de conceptos implicados en un determinado campo del conocimiento no se puede presuponer y habrá que prever cómo pueden acceder a dicho dominio los aprendices. La enseñanza requiere secuenciación y programación, aunque se programe a través de actividades o proyectos.

2. Cualquier aproximación al estudio de las prácticas discursivas en la universidad debería situar el énfasis en la necesidad de que las actividades de lectura y escritura tengan sentido para los estudiantes. El sentido de estas actividades y de las prácticas discursivas viene dado por la situación que las genera y también por la forma en qué los participantes las conciben. Algunos estudiantes son capaces de atribuir a actividades habituales como tomar apuntes un sentido instrumental. Para la mayoría, sin embargo, tienen un sentido puramente de recopilación o repetición de los contenidos (Castelló y Monereo, 2005). Han sido varios los estudios que, sobre todo desde el movimiento británico conocido como academic literacies -ACLITs- se han ocupado de poner de manifiesto las percepciones diferentes -incluso opuestas- que a menudo exhiben profesores y estudiantes frente a las actividades de escritura que enfrentan (Lea y Street, 1998; Lea 2012).

3. Como hemos explicado, los ámbitos discursivos académicos, de enseñanza y aprendizaje y científico/profesional tienen múltiples interrelaciones. Los discursos de uno y otros ámbitos se entrecruzan en prácticas diversas de formación (inicial y permanente), de información (lecturas, conferencias, etc.), de inserción a través de las prácticas (en escuelas, hospitales, empresas, etc.). La participación de los estudiantes en estos ámbitos es una ocasión inmejorable para evidenciar la necesidad de apropiación progresiva de los géneros que son propios. Es preciso recordar que los estudiantes cuando acceden a la universidad son ajenos a las practicas discursivas de estas comunidades a las que se van a incorporar. Sólo conocen algunos contextos de la comunidad de enseñanza y aprendizaje debido a su participación en la escuela que requerirán ser repensados porque la universidad implica formas de actuación y discursos específicos diferentes de los que utilizaron en niveles educativos anteriores.

4. A pesar de la especificidad de los géneros científico/profesionales y los 
géneros educativo/académicos, se puede considerar que hay formas de reflexión sobre la realidad, formas de defender una idea, estilos de argumentación que se siguen en determinados campos del saber que deberían conformar situaciones enseñanza en las que los estudiantes pudiesen participar activa y críticamente, haciendo uso de los géneros propios de estos campos del saber, sin olvidar que el aprendizaje y usos de estos géneros en entornos académicos constituye una actividad específica diferente de la que se espera que lleven a cabo en entornos propiamente profesionales.

Desde el punto de vista metodológico, la enseñanza de los géneros en la universidad puede beneficiarse de algunas de las premisas que hemos venido argumentando como necesarias para la enseñanza de la escritura (Camps, 2003; Castelló, 2008; Castelló, 2009) y que concuerdan con los postulados epistemológicos del movimiento ACLITs. En contextos anglosajones, viene siendo habitual distinguir tres grandes aproximaciones en la enseñanza de la escritura académica (Lea y Stierer, 2000; Robinson-Pant y Street, 2012). La primera denominada técnicas o habilidades de estudio (Study Skills) presume que la alfabetización de los estudiantes tiene que ver con un conjunto de técnicas, reglas y normas para escribir que los estudiantes deben aprender y que pueden ser trasferidas a otros contextos. La segunda aproximación conocida como socialización (Socialitzation) pone el énfasis en las actividades que permiten que los profesores inculquen las bases de una nueva cultura, la académica. Esto implica que los estudiantes deben conocer las características de esta cultura, participar de algunas de sus actividades para socializarse y actuar como miembros de pleno derecho en la misma. La tercera aproximación, la de las alfabetizaciones académicas $^{v}$ (Academic Literacies), entiende la alfabetización como una práctica social y presupone que tanto la escritura como la lectura en el contexto universitario tienen una función epistémica y de construcción de identidad más que relacionarse con el dominio de técnicas o la socialización en una cultura diferente (Ivanic, 1998). Desde esta aproximación no se niega la relevancia de las técnicas o la socialización, más bien se consideran aspectos necesarios pero no suficientes de la alfabetización académica de los estudiantes. Como Lea (2012) sugiere, el foco se traslada a la construcción del sentido y de una identidad como autor. Esto exige situarse en un nivel epistemológico en el que lo cuenta es qué tipo de conocimiento se genera y para qué sirve o en qué esfera de actividad se sitúa.

Desde el punto de vista educativo, este tercer enfoque exige ir más allá de la del conocimiento y dominio de los recursos discursivos propios de cada situación comunicativa y discernir cuándo, cómo y por qué son adecuados dichos recursos en función de los propios objetivos discursivos (Camps y Castelló, 1996). Esto también implica atender a algunas características de los géneros discursivos que a menudo permanecen ocultas a los ojos de los estudiantes y que se relacionan directamente con el sentido de los mismos, como la voz y la posición del autor, la manifestación de su identidad, el nivel de contribución epistemológica que requiere o el diálogo con otros textos y autores. 


\section{Las contribuciones a este monográfico}

Los artículos que contribuyen a este monográfico ofrecen un amplio y diverso panorama sobre la escritura en la universidad. Los dos primeros ofrecen visiones generales de la investigación en Europa y en Estados Unidos, dos áreas con tradiciones docentes e investigadoras diferentes pero que comparten algunos enfoques teóricos que permiten interesantes aproximaciones.

El artículo de David Russell sintetiza algunas investigaciones que ilustran las aproximaciones teóricas, los métodos y los resultados, así como las aplicaciones que han tenido en la enseñanza de la escritura en el marco de las disciplinas en la universidad. La revisión de estos estudios se organiza críticamente a partir de algunas de las contradicciones que el análisis de la actividad escritora y de enseñanza de la escritura plantea: escribir para aprender o aprender a escribir; aprender un género discursivo o aprender contenidos; la relación entre desarrollo y aprendizaje; la transferencia y la transversalidad de la escritura entre el entorno académico y el profesional; la complejidad de la escritura de tesis en que confluyen una gran diversidad de sistemas de actividad y, finalmente, la incidencia de las aproximaciones denominadas críticas que han desembocado en numerosos estudios de corte etnográfico. En su conclusión el autor se refiere a otra contradicción actual de los estudios universitarios en general: la tensión entre equidad social y excelencia en la especialización y sitúa la escritura como un instrumento poderoso para implicar críticamente a los estudiantes en la comunicación con los otros y con el mundo.

El punto de partida del artículo de Otto Kruse lo constituye la mirada a la diversidad de culturas escritas en Europa y la forma en que han influido en la escritura académica. A partir de esta visión general desarrolla tres perspectivas interconectadas a través de las cuales se puede analizar la escritura en la educación superior. La primera de ellas plantea de qué forma los diferentes géneros discursivos tienen funciones diversas para la enseñanza, que hay que conocer si se quieren usar adecuadamente. La segunda perspectiva se centra en las prácticas de enseñanza y en los factores contextuales y organizativos que condicionan la escritura de los estudiantes. Finalmente, la tercera perspectiva aborda el tema de la competencia escrita entendida como la confluencia y coordinación de diversos componentes que habrá que conocer para que la competencia se desarrolle. Este último enfoque es crucial para conectar el conocimiento disciplinar, las habilidades comunicativas, el dominio de los procesos y procedimientos y las demandas lingüísticas y genéricas de los textos que se aprenden a escribir.

El enfoque del tercer artículo de este grupo es algo distinto. Mauricio Pérez y Alfonso Rodríguez, basándose en una amplia investigación de tipo descriptivo caracterizan las prácticas de lectura y escritura en 17 universidades colombianas. La intención del estudio es ofrecer un diagnóstico de la situación con el fin de fundamentar la necesaria renovación de los usos escritos en la educación superior. Los datos en que se basa proceden de una encuesta a estudiantes, de documentos institucionales y programas, de 20 estudios de caso de prácticas destacadas y finalmente de grupos de discusión. El artículo destaca algunos resultados referidos a lo que se lee y se escribe en la universidad (el primer lugar lo ocupan los apuntes de clase y la respuesta a evaluaciones respectivamente); a las finalidades de la lectura, que se 
muestran muy diversas y variadas. El análisis de los cursos que se ofrecen en las universidades da cuenta de que el predominio lo tienen los cursos que tratan la escritura y la lectura como competencia general y no como competencia específica de campos disciplinares; el análisis de las prácticas muestra que las mejores experiencias vividas por los estudiantes se circunscriben en su gran mayoría al ámbito de las disciplinas. Las conclusiones avanzan algunos caminos que se deberían seguir para superar una situación que se considera, por lo menos, problemática.

Un segundo grupo de aportaciones tienen como objeto aspectos diversos implicados en el proceso de enseñar o aprender a escribir en la universidad, como son las concepciones de los profesores, las actitudes y la identidad de los estudiantes como escritores miembros de la comunidad académica, y finalmente la función de la evaluación colaborativa y su incidencia en la mejora de los textos escritos.

El primero de este grupo, firmado por Ángels Oliva y Marta Milian estudia la construcción de la identidad académica a través de la escritura en inglés como lengua extranjera. Las autoras se basan en aportaciones socio-constructivistas, estudios sobre el género, la lingüística sistémico-funcional y la teoría de la actividad para explorar las relaciones entre géneros e identidad de estudiantes de primero de Humanidades que participan en un contexto de enseñanza de la escritura en el que se prima la reflexión sobre la naturaleza y características del sistema de actividad académico.

En el siguiente artículo de este segundo bloque, Paula Carlino, Patricia Iglesia e Irene Laxalt examinan las concepciones sobre lectura y escritura que pueden inferirse de las prácticas declaradas de 544 profesores argentinos. Concretamente, los datos se refieren a las acciones que los encuestados realizan para ayudar a afrontar las dificultades de los alumnos para leer comprensivamente y escribir con claridad. Aunque muchos declaran ocuparse de la lectura y/o escritura en sus materias, predominan quienes manifiestan intervenir sólo al inicio de estas tareas (solicitando trabajos, dando instrucciones, enseñando técnicas) o al final (corrigiendo producciones de los alumnos). Algunos de ellos manifiestan asimismo promover la interacción entre profesor y alumnos y/o entre pares, a través de la discusión sobre lo leído o lo escrito. Las autoras relacionan sus resultados con los marcos teóricos de "escribir a través del curriculum" y "enseñanza dialógica" para inferir las concepciones que podrían corresponder a las prácticas declaradas, además de proponer líneas de actuación en la formación de los futuros profesores.

Por último el trabajo de Mariona Corcelles, Maribel Cano, Gerardo Bañales y Norma Vega, se sitúa en el reto de enseñar a escribir el trabajo final de Grado. La situación educativa diseñada para promover la apropiación de las características de este género específico incluye la revisión colaborativa de tres versiones del texto final, además de otros aspectos que tienen por objetivo ayudar a los estudiantes a construir su identidad como autores. En el artículo se analizan las sugerencias de cambio que ofrecen tanto profesores $(n=8)$ cómo compañeros $(n=35)$ en las situaciones de revisión colaborativa y el impacto de las mismas en el texto final. Los resultados revelan que, si bien los compañeros fueron revisores más activos que los profesores en términos cuantitativos, la naturaleza de sus sugerencias fue de menor calado y tuvo un impacto menor en la calidad del texto. Curiosamente los estudiantes que fueron capaces de introducir cambios relacionados con la presencia del autor en el texto son los que más mejoraron sus textos. 


\section{Referencias bibliográficas}

Bakhtin, M. (1982 [1979]). El problema de los géneros discursivos. En Estética de la creación verbal. Madrid: Siglo XXI, pp. 248-293. (Orig. ruso: éstetika slovesnogo tvorchestva, Izdatel 'stevp "iskusstvo", 1979)

Bartholomae, D. (1986). Inventing The University. Journal of Basic Writing, 5 (1), 4-23.

Bazerman, C. (1994). Systems of genres and the enactment of social intentions. Genre and the new rhetoric, 79-101.

Bazerman, C. (1988). Shaping Written Knowledge. Madison: University of Wisconsin Press.

Bazerman, C., \& Prior, P. A. (2004). What writing does and how it does it: An introduction to analyzing texts and textual practices. Lawrence Erlbaum Associates.

Camps, A. (2003) (Coord.) Secuencias didácticas para aprender a escribir. Barcelona: Graó.

Camps, A. \& Castelló, M. (1996). Las estrategias de enseñanza y aprendizaje de la escritura. En C. Monereo \& I. Solé (coords.): El asesoramiento psicopedagògico: una perspectiva profesional y constructivista. Madrid: Alianza Editorial, pp. 321342.

Camps, A. \& Uribe, P. (2008). La construcción del discurso escrito en un entorno académico: una visión de la dinámica del aprendizaje de los géneros discursivos. En J.L. Barrio (coord.): El proceso de enseñar lenguas. Madrid: La Muralla, pp. 2756.

Casanave, C. \& Hubbard P. (1992). The writing assignments and writing problems of doctoral students: faculty perceptions, pedagogical issues, and needed research. English for Specific Purposes 11; 33-49.

Castello, M. \& Monereo, C. (2005). Students' note-taking as a knowledge construction tool. L1-Educational Studies in Language and Literature, 5 (3), 265-285. . I: 0,322 (NRS); Q: 1 (NRS); Cites SCl: 5

Castelló, M. (2008). Escribir trabajos de investigación con alumnos de grado. Textos. Didàctica de la lengua y de la literatura, 50, 21-29

Castelló, M. (2009). Aprender a escribir textos académicos: ¿copistas, escribas compiladores o escritores?. En J.I. Pozo y Pérez Echeverría, M. P. (Coords.), La Psicología del aprendizaje universitario: de la adquisición de conocimientos a la formación en competencias (pp.120-133). Madrid: Morata.

Castelló, M. Iñesta, A. \& Corcelles, M. (2013). Ph. D. students' transitions between academic and scientific writing identity: learning to write a research article. Research in the Teaching of English, v.47. 442-478.

Castelló, M. \& Iñesta, A. (2012). Texts as Artifacts-in-Activity: Developing Authorial Identity and Academic Voice in Writing Academic Research Papers in M. Castelló \& C. Donahue (Eds.). University writing: Selves and Texts in Academic Societies (pp.179-200). Bingley, UK: Emerald group Publishing Limited. 
Engeström, Y., Miettinen, R. \& Punamäki, R-L. (Eds) (1999), Perspectives on Activity Theory, Cambridge University Press, Cambridge.

Engeström, Y., y Sannino, A. (2011). Discursive manifestations of contradictions in organizational change efforts: A methodological framework. Journal of Organizational Change Management, 24(3), 368-387.

Flower, L. (1987). Interpretative acts: cognition and the construction of discourse. Occasional paper, 1. Berkeley/ Pittsburgh: CSWL. University of California/ Carnegie Mellon University. (trad. cat.: Actes interpretatius: cognició i la construcció del discurs. Articles, 5, 1995, 81-101)

Freedman, A.; Adam, Ch. \& Smart, G. (1994). Wearing suits to class: simulating genres and simulation as genre. Written Communication, 11 (2), 193-226

Ganobcsik-Williams, L. (2004). A Report on the Teaching of Academic Writing in UK Higher Education. Royal Literary Fund.

Gardner, S. y Nesi, H. (2012). A Classification of Genre Families in University Student Writing. Applied Linguistics 26, 1-29; doi:10.1093

Gillett, A. \& Hammond A. (2009). Mapping the maze of assessment: an investigation into Practice. Active Learning in Higher Education, 10; 120-37.

Halliday, M.A.K. (1993). Towards a language-based theory of learning. Linguistics and Education, 5, 93-106

Horowitz, D. (1986). What professors actually require of students: academic tasks for the ESL classroom. TESOL Quarterly 20, 3; 445-62.

Ivanic, R. (1998) Writing and Identity: The Discoursal Construction of Identity in Academic Writing. John Benjamins: Amsterdam

Kinneavy, J. E. (1969). The basic aims of discourse. College Composition and Communication. 20, 5; 297-304.

Lea, M. R. (2012). New Genres in the Academy: Issues of Practice, Meaning Making and Identity. In M. Castelló, C. Donahue (ed.). University Writing: Selves and Texts in Academic Societies (Studies in Writing, Volume 24), Emerald Group Publishing Limited, pp.93-109

Lea, M. R. \& Street, B. V. (1998). Student writing in higher education: An academic literacies approach. Studies in Higher Education, 23 (2), 157-172. doi:10.1080/03075079812331380364

Lea, M. \& Stierer, B. (2000). Student Writing in Higher Education: new contexts Open University Press: Buckingham

Leontiev, A.N. (1979). El problema de la actividad en psicología. En La actividad en psicología, La Habana: Libros para la educación, pp 7-27. (Original ruso: en Voprosy filosofii, 9, 95-108, 1972)

Leontiev, A.N. (1981). Problemas del desarrollo del psiquismo. La Habana (Cuba): Editorial Pueblo y Educación.

Lillis, T. (2001). Student Writing: Access, Regulation, Desire, Routledge: London 
Milian, M. (1996). Contextual factors enhancing cognitive and metacognitive activity during the process of collaborative writing. En G. Rijlaasrsdam et al. (eds.), Effective Teaching and Learning of Writing (pp. 372-386). Amsterdam: Amsterdam University Press.

Milian, M. \& Camps, A. (2001) Interacción de contextos en el proceso de composición escrita en grupo. Lengua, discurso, texto (I Simposio Internacional de Análisis del Discurso), Vol. II. Madrid: Visor. pp. 2767-2780.

Miller, C.R. (1984). Genre as a social action. Quarterly Journal of Speech, 70, 151-167.

Robinson-Pant, A. y Street, B. (2012). Students' and Tutors' Understanding of 'New' Academic Literacy Practices. In Montserrat Castelló, Christiane Donahue (ed.) University Writing: Selves and Texts in Academic Societies (Studies in Writing, Volume 24), Emerald Group Publishing Limited, pp.71-92

Russell, Ch. \& Yáñez, A. (2002) 'Big Picture People Rarely Become Historians': Genre Systems and the Contradictions of General Education. En Ch. Bazerman, \& D.R. Rusell, (eds.): Writing Selves/Writing Societies. Research from Activity Perspectives, Fort Collins, Colorado: The WAC Clearinghouse and Mind, Culture, and Activity.

Todorov, T. (1978). L'origine des genres. En Todorov, T. (ed.): La notion de litterature et autres essais. París: Seuil, pp. 27-46. (Trad. cast.: El origen de los géneros. En M.A. Garrido Gallardo (comp.): Teoría de los géneros literarios. Madrid: Arco/Libros, pp. 31-48).

Vigotsky, L.S. (1977 [1934]). Pensamiento y Lenguaje. Buenos Aires (Argentina): La Pléyade.

\section{Notas}

\footnotetext{
'Bakhtin (1982) define los enunciados como "unidades de la comunicación discursiva" con "unas fronteras bien definidas" determinadas "por el cambio de los sujetos discursivos, es decir, por la alternancia de los hablantes" (p. 260)

ii "a genre is composed of a constellation of recognizable forms bound together by an internal dynamic". The dynamic "fuses" substantive, stylistic, and situational characteristics" (p. 152)

iii University of Helsinki. Center for Activity Theory and Development

http://www.edu.helsinki.fi/activity/pages/chatanddwr/activitysystem . Consulta realizada el 30.01.06

iv Para ampliar el análisis de las fronteras entre géneros y contextos, ver al respecto Russell y Cortés, 2012.

` La expresión no tiene una traducción fácil ni univoca. Hemos optado por la traducción literal del mismo pese a ser conscientes de las connotaciones diferenciales que tanto los términos de "alfabetizaciones" como la de "académicas" tienen en castellano.
} 
Cita del artículo:

Camps, A. y Castelló, M. (2013). La escritura acadèmica en la universidad. REDURevista de Docencia Universitaria. Número Monográfico dedicado a Academic Writing. Vol.11 (1) Enero-Abril. pp. 17-36. Recuperado el (fecha de consulta) en http://www.red-u.net

\section{Acerca de las autoras}

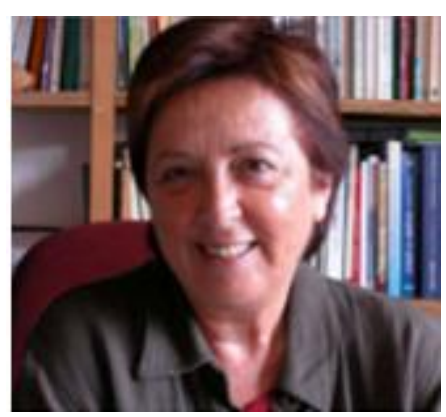

\section{Anna Camps Mundó \\ Universitat Autónoma de Barcelona \\ Departamento de Didáctica de la Lengua \\ Mail: Anna.camps@uab.cat}

Catedrática emérita de Didàctica de la Llengua. Es maestra, licenciada en Pedagogía i en Filología románica y Doctora en Psicología.

Directora del grupo de investigación GREAL, (UAB).

Sus líneas de investigación prioritarias giran en torno de la enseñanza de la lengua escrita y de la reflexión metalingüística y de la enseñanza de la gramática en los niveles de Primaria y Secundaria.

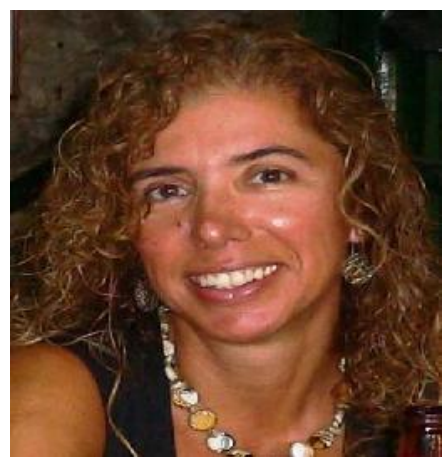

\section{Montserrat Castelló Badia}

\section{Universitat Ramon Llull}

Departamento de Psicologia

Montserratcb@blanquerna.url.edu

Profesora catedrática de la Facultad de Psicología, Ciencias de la Educación y del Deporte Blanquerna. Universidad Ramon Llull.

Directora del grupo de investigación SInte-lest (URL), miembro del seminario SINTE.

Sus líneas de investigación versan sobre la enseñanza de la escritura académica y la identidad, la regulación socialmente compartida de la escrituray la escritura como herramienta epistémica 


\title{
Perspectives on Academic Writing in European Higher Education: Genres, Practices, and Competences
}

\author{
Perspectivas sobre la escritura académica en la educación superior europea: \\ Géneros, prácticas y competencias
}

Otto Kruse

Zurich University of Applied Sciences, Switzerland

\begin{abstract}
Academic writing in European higher education is a little explored field hidden behind fifty major language and fifty national educational systems of which it is part. The article aims at developing a perspective for studying the diversity of writing in Europe and finding a shared language for analyzing student writing. The article recaps baselines theories for the teaching of writing and reports on three important European writing traditions. The main part of the paper is devoted to three foundational conceptions for writing: The role of genres in understanding student writing, the function of writing practices for the organization of student writing and the meaning of writing competences for the teaching of writing.
\end{abstract}

Key words: Academic Writing; Higher Education; Genres; Competences; Writing practices.

\section{Resumen}

La escritura académica en la educación superior europea es un campo poco explorado y oculto bajo cincuenta lenguas y otros tantos sistemas educativos nacionales que la componen. El artículo tiene por finalidad elaborar una perspectiva que nos permita estudiar la diversidad que implica escribir en Europa y desarrollar un lenguaje compartido para analizar la escritura de los estudiantes. El artículo revisa las teorías básicas en la enseñanza de la escritura y se centra en tres importantes tradiciones de escritura europeas. La parte principal del documento está dedicada a tres conceptos fundamentales para la escritura: el papel de los géneros en la comprensión de la escritura de los estudiantes, la función de las prácticas de escritura para organizar lo que escriben los estudiantes y el significado de las competencias comunicativas para la enseñanza de la escritura.

Palabras clave: Escritura académica; Educación Superior; Géneros; Competencias; prácticas de escritura. 


\section{Background and Aims}

In the past decades, writing in European higher education has been affected by several epoch-making influences like internationalization and new language demands, the rise of the new literacies as a result of the computer and internet age, the political changes in European higher education exemplified most clearly by the Bologna process, and, finally, the new philosophies of and technologies for the teaching of writing, many of them imported from the US. We have to recognize that we - the writing teachers and researchers - are part of rapidly changing education systems in which we find many actors like teaching and learning specialists, online learning divisions, disciplinary teachers, pedagogical societies, politicians and parliaments, university associations, Google, Microsoft, Facebook, and Apple. All of them influence how students write and how their teachers make use of writing for learning.

Much of the writing in higher education, on the other hand, is still connected with traditions which have grown as part of the teaching philosophies and writing cultures established at the universities in the last centuries (Chitez \& Kruse 2012). These traditions form a baseline for each country from which new developments set out and from which new writing practices and new forms of teaching are initiated. Knowing these different starting points is necessary for understanding the variety of writing cultures in Europe but is not sufficient for understanding the directions which the new developments point at. A new dynamic has taken hold of all higher education in Europe with similar problems and similar questions for each country. Whether the solutions will be alike is not yet clear but it is obvious that all countries can only gain from an exchange on their writing policies. We find models for good academic writing not only in the Anglo-Saxon world but also in the various European traditions. What unites the teaching of writing in Europe at the moment are the similarities in the transformation processes with their various stresses and challenges.

In this paper, I will first have a short look at the different basic writing cultures which were established in Europe in the past and which, to a certain extent, still form the base for academic writing. The linguistic and cultural diversity of the European continent is both an enriching and a dividing factor when textual norms and directions for student writing are in question. Writing routines as they exist in primary, secondary and higher education are strongly rooted in national cultures but are difficult to compare and difficult to evaluate with respect to their effects on intellectual and literacy development.

The main part of this paper will be devoted to the elaboration of three different perspectives by which student writing in higher education can be analyzed. Perspective one is on genre and analyzes the various textual forms which are used for teaching. It will be shown that each genre has a different function for teaching which we have to understand if we want to use it purposefully. The second perspective is on writing practices and looks at the different contextual and organizational factors which determine student writing. It will be shown that formal aspects of writing assignments are all but trivial for understanding and conceptualizing student writing in educational programs. The third perspective is on competences. If we want to teach writing deliberately and use it effectively for student learning we have to break it down into its various components to understand how it develops. It will be shown that a look at 
writing competences is essential for connecting writing with disciplinary knowledge, communicative abilities, process and procedural skills, genre and language demands.

All three perspectives are interconnected with the main conceptual approaches to academic writing which have led theory building and research in the past decades. Probably the most fundamental approach for modern writing pedagogy is the process approach which, starting in the 1970s (Emig 1971, Flower \& Hayes 1980, 1981, Perl 1994, Nystrand 2006), introduced a completely new way of teaching writing grounded on an understanding of what writers do or think and how writers develop and replaced traditional writing instruction based on the teaching of "good language" or correct textual norms. Main concerns of the process approach are the planning of writing, revision, feedback, and reflection (cf. Pritchard \& Honeycut 2006). Although criticized for not paying enough attention to the social context (Kent 1999), genre (Hyland 2003) and to language (Bizzell 1982, Hyland 2011), this approach still forms the base of all writing instruction.

The study of contextual factors affecting literacy practices has been influenced most sustainably by another influential approach, the Writing-in-the-Disciplines (WID) tradition (Russell 2001). WID approaches were able to demonstrate the impact of disciplinary affiliation on the way writing is valued, used, and taught (Langer and Appelbee 1987, Walvoord and McCarthy 1990, Thaiss and Zawacki, 2006, Poe, Lerner and Craig 2010). All of these studies were able to demonstrate that writing is grounded on the epistemic assumptions of the respective disciplines and is used to introduce students to the various forms of disciplinary thinking and arguing. All of these studies see the disciplinary context as a the most influential source of making students engage in their majors and getting acquainted with the respective genres and rhetorical practices. Writing, from this perspective, is a means of integration into and specialization in their fields of study. Adaptations of this approach to the needs of European universities are frequent (for instance, Deane and O'Neill 2011).

The UK-based Academic Literacies Approach (ACLIT) is a third way of conceptualizing academic writing. ACLIT is somewhat critical about the assumption "that students are merely acculturated unproblematically into the academic culture" through writing as Lea $(2004,741)$ states. Lea wants to see writing studied in a broader context and understands it as a more diverse activity than just being tied to disciplinary discourse and academic writing in its narrow sense. To the ACLIT approach, writing should not be seen as a set of universal skills but rather as an open field also affected by new literacies (Street 2005) and multiple genres (Lea 2004). A critical attitude towards the aspect of power involved in the rituals and mysteries of academic writing is recommended (Lea and Street 1998). The developmental aspect is stressed thus seeing literacy as a continuous way of interacting with written products from many sources.

Rarely mentioned in the context of basic theories of writing, but very influential in most European countries are approaches to writing from the English as a Foreign Language disciplines, may they be called EAP (English for Academic Purposes), ESP (English for Specific Purposes), ESL/ EFL (English as a Second/ Foreign Language) or TESOL (Teachers of English to Speakers of Other Languages). In many universities, L1 writing teachers and L2 English (writing) teachers do similar work although with little 
interaction among each other and little coordination of their work (Kruse 2013, in press). The teaching of writing in English is usually reserved to postgraduate levels and to faculty. Basic literature for this kind of teaching comes from two sources. One is the work of John Swales (1995, 2004, Swales \& Feak 2011) who analyzed the main academic research genres and connected genre teaching with the teaching of English as a foreign language. Swales' works are used in all parts of the world for the teaching of writing in English as L2, but are more and more used in L1 contexts, too. A second source is the work of Ken Hyland who analyzed academic discourse in English with respect to such linguistic variables as metadiscourse, citation practices, hedging or selfreference (Hyland, 1998, 1999, 2000, 2005) and connected this with the teaching of writing, both in L1 and L2 (Hyland, 2002, 2003, 2011). Typical for L2-approaches to writing is their unproblematic attitude towards the teaching language which has been abandoned by the process approaches some 50 years ago. At European universities, problems from this approach arise when English language norms collide with those of the national languages. At many universities, especially in Eastern and Central Europe, the problems seems to be that English writing instruction is the only one available while for the national language writing is hardly ever taught at all (Harbord 2011).

\section{European Traditions}

European academic cultures have their common roots in medieval university structures and teaching practices which were built on Latin as a lingua franca. With the change from Latin to national languages in the 17th and 18th century, new models of academic discourse in the national languages were developed and institutionalized. The shift from oral to written forms of research communication (Ong 1982, Russell 2001, Kruse 2007) led to new forms of discourse based on written text. Students were made to write papers instead of disputing their theses in front of an audience (Kruse 2007). At the beginning of the 19th Century, three essentially different modes of using writing for teaching and of teaching writing emerged in France, England, and Germany. All three had considerable influence on writing in Europe and contributed to it differently. Each of these writing cultures was adopted and transformed by the respective national educational systems and led to unique academic cultures. Different from the US, where writing courses have been introduced as early as 1872 (Russell, 2001), European universities have seen the teaching of writing not as a field in which they should engage. The silent assumption always was that writing follows thinking and that teaching should place more emphasis on developing critical thinking skills than in developing linguistic competence.

The French tradition is closely related to essay writing. There are four different forms which are called commentaire composé, dissertation, étude d'un texte argumentative, and discussion. Donahue $(2002, p, 136)$ explains that these essay forms are used already in the lycée, the last three years of secondary education but still are in use at the first years in higher education, both as exams to a given topic or as a takehome assignments. These essays are characterized, among other things, by easily recognizable external structures, by a "strong reliance on paraphrase without citing", by a statement of "the problem" and "the plan" at the beginning and a thesis 
statement at the end. All of these essays train certain intellectual and linguistic skills by introducing students to scholarly argumentation and by making them refer to ideas and facts from the respective disciplines. Today, the variety of genres in French higher education is much greater (Delcambre \& Reuter 2010, Chitez \& Kruse, 2012) and several research-related genres (term papers, research papers) are equally used. For the French writing culture, however, the training of students in using well-structured, linguistically and argumentative well composed papers, is still a baseline.

Another tradition of essay writing developed in the UK. It is often seen in connection with tutoring systems used in Oxford and Cambridge (for instance, Palfreyman 2008). Tutorials are additional teaching structures which accompany regular courses and independent library work. Similar to the German seminars, they connect writing with oral communication among students or between student(s) and tutor and introduce students to scholarly work. As in France, essays were used for learning and assessment in most education programs, except for some science disciplines. The types of essays are numerous. There are narrative, descriptive, evaluative, personal, argumentative, comparative, critical, expository and otherand other essays. Today, a whole industry seems to offer students support for essay writing or even writes essays for them. Nesi \& Gardner (2012, p. 38) speak of essays not as a single genre but as a "genre family". They consider the social purpose of essays "to demonstrate/ develop the ability to construct a coherent argument and employ critical thinking skills." In their corpus of student texts, essays are only one out of 13 genre families. What makes the genre interesting for understanding writing cultures is its peculiar approach to teaching thinking. It is a variable genre which allows adaptation to many teaching purposes and to many desired student activities.

A third tradition of student writing was developed in the German-speaking countries and is connected with seminar teaching (Kruse 2007, 2012, Pohl 2009, Macgilchrist \& Girgensohn 2011, Chitez \& Kruse 2012). At the beginning, seminars were teaching arrangements offered to small groups of selected students in addition to the regular study programs. Students would work with a teacher on a selected research field or topic. Seminars always included student writing and made students submit papers in regular intervals. Seminar writing practices were originally connected to the introduction of libraries at the institutes which were used to make students study original sources instead of simply recap knowledge from their professor's lectures. In contrast to essays, seminar papers are a longer form of student papers usually devoted to a self-chosen topic. In the 20th century, seminars were offered to all students in undergraduate as well as graduate programs and the writing of seminar papers became identical with academic writing itself. Academic writing in this sense is markedly different from writing in secondary education and demands an "immediate immersion in the discourse of specific knowledge communities" (Foster 2002, p, 213). It gives students, as Foster (2002) continues to explain his observations from German universities, a "wide autonomy in choosing the occasions, time frames, deadlines, physical sites and working rhythms for writing." It seems that a model of student writing has appeared with seminar writing which is different from essay writing. It is more closely connected with research-oriented teaching and the practices of thesis writing than the essay. It does, however, not provide the opportunity to train thinking skills in a more selective and less demanding way as the essay forms would allow. With 
the new structures of the Bologna process, however, writing becomes more regulated in German contexts and essay writing slowly moves into the curricula as an additional genre.

The models for student writing these three countries provided have been transferred to several other European countries. The German kind of seminar writing has spread with Humboldt's university model to most Northern and Eastern European countries while the French model has influenced several of the Romance countries. The export of essay writing in the UK tradition is of more recent origin and seems to follow the teaching of English foreign or academic language.

Since the first writing centers have been opened in Europe some 20 years ago, the need for improved writing instructions is widely recognized by university administrations and educational politics but not very often resources for such institutions are provided. Most changes in the teaching of writing are rather grass-root initiatives from study programs or individual teachers. First-year writing courses, writing-intensive seminars, portfolio courses or provision for thesis writing have become common at many universities. Little of this is communicated within universities, however, and even less to a wider public. Little of this is initiated by specialized writing teachers or writing centers but more by disciplinary teachers who look for new ways of using writing in their classes. Textbooks on student writing are available in almost every country and teachers who would like to improve on their writing pedagogy will find many ideas and instructions there.

Associations on the research or teaching of writing like the Special Interest Group Writing (as part of EARLI, the European Association for Research in Learning and Instruction), the EATAW (European Association for the Teaching of Academic Writing) and the EWCA (European Writing Centre Association) have started to organize discourses across Europe, while national organizations are connecting writing teachers and researchers at national levels in several countries. One of the oldest of such institutions is the yearly Writing Development in Higher Education Conference in England; another example is the bi-annual conference of the Swiss Forum for Academic Writing. New Europe-bound journals like the Journal of Writing or the Journal of Academic Writing have also been successful in initiating exchange on research and teaching. A showcase for research from Europe is the Studies in Writing Series, edited by Gert Rijlaarsdam, which recently published a comprehensive volume on European writing research (Torrance et al. 2012). The influence of US-bound research and teaching organizations has contributed much to develop a theoretical and methodological base for research and teaching (see, for instance, Dean \& O'Neill, 2011, Castelló \& Donahue 2012, Delcambre \& Donahue 2012, Russell \& Cortes, 2012).

\section{Perspectives on the Teaching of Writing}

To develop an European view on academic writing we need some kind of metacommunication on the purposes, practices and textual forms of student writing. As we have seen, student writing in national contexts is often bound to traditional genres which seem to be unique or at least do not seem to equal any genre in the Englishspeaking communities. The "referat", for instance, is a genre used in Ukraine. For 
anyone not socialized in Ukrainian higher education, it is hard to understand what this practice means. From descriptions of Ukrainian colleagues, I know that the referat is connected with literature searches, reading and summarizing so that we might assume that it equals a term paper. Still, it would take a closer examination to find out what exactly students have to do when writing a referat, how much time they are granted for writing it and what their teachers would expect from them.

To analyze and compare student writing across cultures it is important to have concepts at hand by which writing practices can be examined. Three such concepts or perspectives are outlined in the next chapters, one focusing on genre and the functions of writing, the second on context and writing practices, the third on writing competences and skills. These perspectives may not be the only one ones that matter but cover a good part of what should be studied when a writing practice like the referat is at question.

\section{Perspective I: Genres and their Functions for Learning}

When we look at student writing, it makes a difference which kind of texts we make our students write and which kind of thinking, acting, reading the production of the text will demand. It has been said, that genres like the various essay genres are integral part of writing cultures. We may call such genres, which are used for teaching, "educational genres". They are created by certain institutions in a local, national or international context and are often used for long periods of time once they are established and accepted by all stake holders. Nobody in the UK would doubt that the essay is a main teaching genre, as little as anyone in Germany would question the usefulness of the seminar paper. Both, the essay and the seminar paper are flexible genres (or genre families) which may be adapted to the teaching needs of many disciplines and several levels of higher education.

Genres may be seen as results of categorizing texts (or other cultural products) into prototypical classes which fulfill similar functions and share some rhetorical, structural or linguistic features. In its broadest sense, the term "genre", as Hyland $(2003,21)$ says, "refers to abstract, socially recognized ways of using language". When certain kinds of texts are used repeatedly in a context they will stabilize. Those using the genre will learn to discriminate it from other genres and expect it be used in a certain context or for a special purpose. It should be noted that the term "genre" is also used to make distinctions between other cultural products like film, art, or music. For individuals in literate societies, understanding genres is an important part of cultural knowledge and allows individuals a quick orientation in the constant flow of textual (or other cultural) products from many sources and media.

There are three main theories trying to account for the various aspects of genres, as Hyon (1996) suggested, each characterized by a slightly different perspective on their topic. The New Rhetoric approach (Miller 1998) sees genre as "social" or "rhetorical action" which is connected to recurring situational demands or "rhetorical situations". The constructivist impetus of this approach places the emphasis more on their pragmatics than on their linguistic or structural form and sees genres based "in the conventions of discourse that a society establishes as ways of "acting together'" 
(Miller 1998, p. 136). This approach has been most influential in the US-American tradition of teaching of writing.

The Systemic Functional Approach (Halliday 1985) led to an understanding of genre as a "staged, goal-oriented, purposeful activity in which speakers engage as members of our culture" (Martin 1984, p. 25). This approach is less hesitant in describing the linguistic means used in genres and their structure which is mainly given by its different parts. It places special emphasis on the analysis of generic text types such as description, narration, or recounts as basic elements used by all genres (see Martin \& Rose 2008). It is mainly based in Australia (also called "Sydney school") and mainly used in primary/ secondary school and second language teaching. The third line of genre theory is connected with the ESP (English for Special Purposes) approach (Swales 1990, 2004) and sees genre characterized by communicative purposes. It stresses the connection of genres with discourse communities using a certain genre or genre system. The detailed analysis of academic genres like the research article made it an important resource for teaching in all academic fields. While originally developed in the context of teaching English as second or academic language (for instance, Swales \& Feak 2012), today it is largely used in L1 teaching as well.

No matter which definition one would follow, there are a several aspects of genre which all theoreticians would agree with. Genres are closely connected to communities of users. The members of these communities use the genre actively (by writing) or passively (by reading). Genres are integrated in cultural or professional contexts and are used for defined purposes in these contexts. Educational genres as used in primary, secondary, and higher education are firmly integrated in learning arrangements and teaching philosophies. Such genres like, for instance, the essay forms, the term paper or the thesis variations have been used for many generations and tend to be highly conventionalized by descriptions in textbooks, on assignment sheets or university websites. Educational genres show similarities to those genres used by researchers for reporting, discussing or presenting research results (Swales 1995,2004 ) but still remain some features characterizing them as learning genres used to train students in various aspects of disciplinary thinking and professional practice.

With Emig's (1971) distinction of learning-to-write vs. writing-to-learn, the notion of writing as a tool for learning has been established. The cognitive approach to writing, however, focused more on the "how" of the writing process than on what is learned by it. The genre approach for the teaching of writing is a good base to make distinctions between different kinds of writing. As we have seen, the purpose of essays is a different one than that of the seminar paper. Both genres make student do different things, create different kinds of thought and meet other expectations of their teachers. Nesi \& Gardner (2012, p. 22) state this in the following way: "If you ask a student in Sociology, or in Engineering, what is involved in writing assignments in their discipline, they will soon start to explain that there are different types of writing essays, research proposals, reports, projects and more - and that each of these has a different function, each relates differently to research or practical work being done and to reading and lectures in the discipline; and so each is organized differently."

In an analysis of a large corpus of student texts, Nesi et al. (2008) and Nesi \& Gardner (2012) isolated about 100 different genres and categorized them into 13 
"genre families" which they then assigned to five major functions of student writing. These functions are:

Demonstrating knowledge and understanding: To this category belong genre families like "explanations" which make students explain facts or concepts like "code switching", "fast mapping" or the "functioning of the eye." The second genre family Nesi \& Gardner (2008) mention is "exercises" which were often tasks from mathematics or statistics and demanded deductions or applications of formulae to certain problems.

Developing powers of informed and independent reasoning: "Critiques" and "essays" are the two genre families covering this function of writing. Both, analytical and critical evaluations of topics which make students discuss, analyze, critique, review, argue interpret or compare a topic. Both genre families are further subdivided.

Developing research skills: "Literature surveys", "methodology recounts" and "research reports" are the most important genre families of this section. The genres subsumed under these families are useful for preparing, reporting, summarizing and reviewing research and research literature. Long essays and theses are important parts of this category.

Preparing for professional practice: A considerable number of genres are directly related to professional issues like case studies, problem questions, design specifications, and proposals. "Problem questions" are called assignments from Law in which a scenario is developed for which the writer has to develop an analysis of the legal situation. "Design specifications" are genres typical for constructive disciplines like computer sciences and engineering which demand the design of a product or the production of other artifacts like a user's manual. Proposals for business projects are another writing assignment type for this category of genres.

Writing for oneself and others: In this category fell narrative recounts, which are to a large part narrations on historical or personal events. Another group of texts are fictional narratives. The third genre family is called "empathy writing" and contains media-related texts for a non-academic audience to prepare students "for professional engagement outside the academy" (Nesi \& Gardner 1012, p. 216).

The description of these functions is probably the most detailed account of the uses of genres in student writing ever given on the base of an empirical study. It demonstrates that little is won if we continue to argue in a global way about "academic writing" as long as we don't make distinctions about its different forms and uses. A simple formula for "writing to learn" seems not applicable; there are simply too many forms of learning and genres used for learning.

Looking for a common denominator of student writing, we might argue that it is connected with various ways of dealing with knowledge, such as developing, collecting, searching, testing, systematizing, analyzing, criticizing, reflecting, applying or transforming knowledge. All these ways of handling knowledge may be seen as some kind of disciplinary or critical thinking as, for instance, brought forward by Bean (1996). 
It is also worth looking at the various connections of writing with teaching forms like lectures, seminars, projects and practice courses. Writing may also be looked at as a way of academic acculturation (Prior \& Bilbro 2012) allowing students to react to disciplinary knowledge at several levels: From the learning of basic skills and actively (re-) constructing disciplinary knowledge to the acquisition of critical thinking and research skills and from a reflection of and familiarization with professional practice to professional knowledge communication.

\section{Perspective 2: Writing Practices and the Contexts of Student Learning}

A second perspective useful for looking at student writing is devoted to a very practical point of view: How is student writing initiated, organized, structured, and graded? Student writing is, as the last chapter has shown, part of complex learning arrangements in which students write and develop as writers. The organization of writing in such contexts depends on the setup of the study program as well as on the genres chosen. The term "writing practices", in addition, refers to what writers, tutors and teachers actually do with texts and how they arrange the production of texts in the classroom or in the students' overall learning context.

It may be felt that such formal aspects do not have much weight compared to, for instance, the question of what students write or which genres they use. But this is not true. The organization of writing matters and initiating student writing in the wrong way may turn an assignment from a positive learning experience into a useless routine. Not all kinds of writing are helpful for learning (Russell 2001). Too much or too little time granted for writing, too many or too few writing assignments at a time, too much or too little guidance for an assignment may all be detrimental to its expected gain.

For a European perspective on writing, writing practices are an important matter as they allow comparing writing on some unobtrusive and easy-to-catch aspects. How many assignments students get, for instance, or how much time is granted for a paper can be assessed with little reference to language-bound issues like genre or to discipline-specific aspects like the rhetorical qualities of texts. The term "writing practices" does not belong to the most important analytical categories for the study of writing but matters the more when it comes to very practical questions of writing pedagogy. Looking at thesis writing, for instance, shows that the length of theses and the time granted for writing vary greatly. Samac, Prenner and Schweth $(2009,20)$ report from a study in Austria that "...not everywhere but at most universities a Bachelor thesis has to be written. Some require two theses whereas some students may have the choice to write a long or two short ones, in some instances with the specification that one is on basic research, the other practice-oriented. Only in rare cases no Bachelor thesis are required". [translated by the author]

The number of ECTS points (European Credit Transfer System), according to the same authors (p. 21), centres around the average of 10, with extremes of 4 and 20 credit points while its length varies between 25 and 100 pages. Each credit point covers 25-30 hours of work. We can assume that variations are even greater when we look at thesis writing in other European countries. Needless to say, a thesis demanding a workload of 100 hours gives writing a different meaning than one requiring 500 . 
The most important dimensions of writing practices may be subsumed under the headings of instruction, time, and collaboration. These aspects may seem formal but open a window on several deeper features of writing like writer autonomy, author roles, feedback, or supervision. "Instruction", in this sense, covers all information given to students make them understand what is expected from them and any kind of guidance provided to them. "Time" covers all regulations concerning deadlines and temporal arrangements of writing while "collaboration" covers all relationships and interactions that arise from writing, including those to peers, disciplinary of professional communities. The following list of issues covers the most important aspects of writing practices, which should be taken into account when systematically integrating writing in a study program. They are organized along the headings of "instruction", "time", and "collaboration".

\section{Instruction}

In most fields of learning, instruction would be treated separately from practice. In academic writing, however, instruction is integrated in its practice as some kind of provision or guidance while writing. It is a common practice at European universities to initiate student writing for learning and/or assessment without teaching it beforehand. Similar to many complex competences, it may be assumed that writing can be learned only in connection with its practice and not in an abstract, decontextualized way. In most cases, writing instruction is, therefore, part of writing-to-learn arrangements when students receive a writing assignment and start working on it. Instruction may be given before, during, and after the actual writing takes place. The most important kinds of writing instruction seem to be the following:

Initiating writing: The kinds of instruction students receive with their assignment is an important factor for success in writing. Such instructions should refer to the assignment purpose, the task, the process, and the product (Bean 2011). Writing assignments may vary with the level of education. At the entrance level, assignments should be more elaborate and oral instructions should be added to make students understand what they are supposed to do. At higher levels when students are acquainted with the writing culture of their institution and discipline instructions may be restricted to genre, deadline, and size of the paper. A good assignment would make students feel that they are not fulfilling an assignment but write for a larger purpose (Sommers \& Saltz 2004). It should be kept in mind, however, that most assignments need negotiation and that a productive writing culture should allow students to clarify all open questions.

Selection of a topic: Choosing a topic is not only a matter of practicability but also a matter of competence and motivation. Students are generally more motivated for writing when they can write on a topic of their own choice than when working on a default topic. It is, however, an additional effort of teaching to making students understand what the selection of a topic demands. The choice of a topic is closely connected to motivation. Students need to be interested in a topic to keep up motivation while writing an extended paper or a thesis. It is interest in the topic that makes writing a meaningful activity (cf. Hidi \& Boscolo 2006). Strategies for providing 
topics vary greatly. The essay writing tradition set out with prepared topics for all participants but often allows choosing from several alternatives. Strategies from seminar writing might provide overall themes from which students would select one and adapt it to their own interests. Thesis writing is even more inventive in providing topics. In some institutions, students are completely free to choose a topic from all fields of their discipline while it remains a matter of negotiation with a supervisor to delineate the topic. Other institutions may make suggestions for topics and let students choose one of them.

Supervision, tutoring, and feedback: Probably the most effective part of instruction for student writers comes from supervision or tutoring activities connected with feedback. Sommers (2007 quoted from Rogers 2010, p 372) concluded from a study of over 400 undergraduate students from Harvard that feedback matters "when, and only when, students and teachers create a partnership through feedback - a transaction in which teachers engage with their students by treating them as apprentice scholars and evolving writers, offering honest critique paired with instruction." What seems to be needed most by students is a direct and flexible communication on the student's accomplishments with support for the further development of the text.

Evaluation and assessment of writing: Variations in assessing and grading student texts are great and may be connected with different forms of feedback for the writer. It makes a difference whether and to what degree student writers are informed about the criteria their texts will be assessed by or not. Assessment is part of the overall writing culture of an institution and is an important factor in encouraging or hindering students to develop as writers (Harrington 2010).

\section{Time}

The amount of time granted for writing is not only connected to the scope of a paper but also to the kind of independence required in fulfilling a certain assignment. Foster (2006, p. 61) argues that time patterns as indicated by deadlines, free time or time autonomy shape students' work in essential ways and may be characteristic for certain academic cultures. He noticed a markedly different degree of time autonomy in the German university system as compared to the US-American one. Seminar papers, term papers and theses demand more time and need more time autonomy for students as, for instance, essay writing which may restrict time to a couple of days. Still, it would be unwise to see a more generous time frame generally as more favorable than a more restricted one. A five-minute prompt during a lecture may work well as a clue for reflection and a one-week assignment may be perfect for writing a summary of a research article or a book chapter. It is the right mix of different assignments lengths that counts.

Independent work and autonomy: Writing is one of the most important tools to induce independent work in a study program. Writing an extended paper makes students read and produce knowledge instead of receiving it from lectures or text books. Using time well for a writing task has to be learned and time may alternatively be used productively or spent with procrastination. If ample time is granted, there is an obligation for the teachers to provide provision for writers so that they can 
overcome writer's blocks and stay in flow. The more time is granted the better the supervision or the tutoring offered should be.

Dealing with deadlines: By adopting the Bologna changes, European universities moved away from the traditional wide-stretched, liberal study programs and introduced some variation of the three plus two year structure characterized by less time and assessments for every course. This led to a higher number of writing assignments and a greater workload for students through writing. The general policy concerning deadlines has changed very much with these developments. While European universities traditionally had rather open and negotiable deadlines, today the trend moves towards strict deadlines. Local cultures, however, may preserve old traditions or confuse negotiable with strict deadlines.

Author roles: The development of appropriate and variable author roles depends on a frame for student writing allowing enough time for extended papers going beyond the surface level and managing the difficulties connected with entering disciplinary conversations (Russell \& Foster 2002). For student writers, the transition from secondary school to university demands students not only to adapt to new genres and adopt new rhetorical practices but also to find into new roles connected with the organization of extended writing/ research projects.

\section{Collaboration}

Although seemingly a solitary activity, writers, in fact, interact with others in many ways. Much of this interaction takes place on paper (or screen) and connects writers with the ideas, publications and research results of other members of a disciplinary or professional community. Bruffee (1984) called this kind of interaction "collaboration" as distinct from "cooperation" which involves partners actually working together. He views academic discourse as an eternal conversation on a topic which individual scholars join with their own contributions. Any discourse in academic fields shows the signs of collaboration and interaction with others by its citation practices and the ways of referring to others' works, as Hyland (2000) has shown. Understanding and practicing this kind of collaboration is one of the main prerequisites to give texts a quality as "academic". For the learning of writing, the direct forms of cooperation among students, however, are of no less importance. Students need responses to their writing in order to revise their papers and improve their quality (Beach \& Friedrich 2006). Cooperation on writing also allows reflection to raise awareness of writing and increases motivation. There are many ways in which responses can be provided for student writers:

Group work: Student papers can be written individually or cooperatively as group work. There are arguments for both, individual and cooperative writing. Cooperative writing helps students reflect their writing and exchange competences necessary for composition.

Peer feedback: There are many forms in which feedback may be given. There are oral and written forms. Feedback can be given in a formal or informal way and can be supported by feedback templates, scoring grids, or criteria lists. It may be given on 
proposals, drafts or submitted papers. It can be provided by peers, tutors, supervisors or disciplinary teachers (for an overview, see Beach \& Friedrich, 2006).

Student support groups: Cooperation among students can be organized in various ways. Feedback groups can be implemented in seminars or organized among thesis writers. Self-directed support groups as initiated by Girgensohn (2007, Macgilchrist \& Girgensohn 2011) allow students to learn about writing and support each other in their individual writing projects. Such groups may be initiated by a writing center or by the students themselves.

There are large differences in European higher education systems, with respect to all aforementioned aspects, i.e., to which degree students may write cooperatively, in which way peer feedback is organized, how much time autonomy is granted, and how writing is assessed. To a large extent, these practices determine the meaning that writing receives within an institution and the role it assumes for the development of student writers.

\section{Perspective 3: Writing Competences and the Teaching of Writing}

Writing is not a simple skill that is learned for once and then practiced invariantly. This truth stands at the beginning of any deliberate teaching of writing. But if writing is not "simple" as a skill, what is it then? Complex skills are called "competences" or "competencies". Such competences are thought of as clusters of abilities, knowledge, understanding, action skills, experience, and motivation (Klieme et al. 2007, 37). Competences are not only mental units but are rooted in active engagement, experience, and action. Most competences are actively regulated and developed consciously. To understand the connection of the various kinds of sub-competences, a competence model is needed which explains how they interact and relate to performance. Many of the sub-skills of competences run automatically and are unconscious during action.

To understand and teach academic writing it is necessary be aware of the hierarchical structure of writing competence and the many sub-skills necessary to perform writing well. Stating competences positively is an important step for getting away from deficit models of writing which ground the teaching on a lack of linguistic or thinking skills. Writing competences may be seen as clusters of skills which are connected to several areas like disciplinary knowledge, communicative skills and so on. We can teach all these aspects separately, if necessary, to make them routinize them and make them available automatically.

Competences in educational programs develop along the demands of the teaching assignments. The writing competences students develop will depend on how we make them write and how we instruct them. In order to instruct them well, we have to know how the respective competences are structured.

Looking at academic writing as a competence, we have to assume that it is an integrative activity that stands at the interconnection of several domains like disciplinary knowledge, procedural and process skills, communicative and discourserelated abilities, understanding of media for text production and communication, genre and textual knowledge, and, finally language competence. What makes writing 
an integrative activity is its potential to connect these domains. It should, however, be noted that writing also has the function to train student competences in each of these fields. The main domains to which writing competences are connected have been outlined, for instance, by Kruse \& Jakobs (1999), Beaufort (2007), Kruse \& Chitez (2012). They may be described as follows:

Connections to disciplinary knowledge: Academic writing must be connected with disciplinary knowledge otherwise content cannot be generated. Many studies have shown how writing and disciplinary thinking (for instance, Langer \& Appelbee, 1987, Walvoord \& McCarthy, 1990) relate on each other and how disciplinary thinking is shaped by writing. Disciplinary knowledge itself, of course, is not part of writing competence but academic writing cannot be created without it. The reliance on disciplinary knowledge is a prerequisite for writing but also the result of it. Students construct knowledge (or at least they learn to do so) by writing and in the same time acquire disciplinary knowledge by reading and synthesizing it.

Connections to process and procedural skills: Teaching the writing process is probably the most important domain for writing teachers. Writers should learn the steps in which writing proceeds, the recursive nature of writing, the actions of planning, idea generation, and revision. In research writing, writers also have to acquire knowledge about process management and co-ordinate writing with research. Process competence, however, is not only a prerequisite for writing but is also built by writing. It cannot be learned in an abstract way but it can be supported by writing instruction and supervision.

Connections to communication and discourse knowledge: Relating a paper and its content to its audience and situating it in a discursive community demands another set of skills which is connected to an understanding of the respective communities and to the degree in which students feel connected to them (for instance, Bruffee, 1984). Discourse and citation practices (Hyland 2000) as well as the construction of appropriate author roles and of an authorial voice (Nelson \& Castelló, 2012) form the linguistic side of this competence field.

Connections to media use: Media use is a fourth part of writing competence which has become of special importance with the new media and new literacies. The various media used to produce, communicate, discuss, publish and submit text are by no means self-evident for students. The new literacies demand not only new competences, strategies and dispositions to handle the new media but also the participation in a new global community (Leu et al., 2007, Coiro et al., 2008). Due to the same authors, one of the greatest challenges of the new literacies is their constant change and the need of adapting to them. The new literacies are neither a single nor a homogeneous set of competences and they are related to reading as well as to writing and communicating.

Connections to genre knowledge: As has been explained, genre is a main concept to make understand how writers relate textual features to contextual purposes. Genres mediate all writing processes in higher education and connect them to overall functions for thinking, learning, research and professional practice. Genre awareness (Devitt 2009) or genre understanding (Swales 1995, Hyland 2003) are competences 
which students have to develop in order to become members of academic communities.

Connections to linguistic skills: Writing, finally relies in many ways on language competence from basic aspects of spelling, grammar, and syntax to rhetorical means like hedging, metadiscourse, intertextuality, and self-reference (for an overview, see Kruse \& Chitez, 2012). Recently, many linguistic features of academic writing have been explored by corpus studies and the development of student writers with respect to their linguistic skills has been demonstrated, for instance by Pohl (2007) and Steinhoff (2007).

Looking at writing from the perspectives of a competence model shows that writing is not an isolated skill but integrated in an array of neighboring competences. It can evolve only when all of them are addressed and develop at equal pace. Writing competence is not separable from disciplinary knowledge and critical thinking, from understanding writing processes and procedures, from communicative and discursive skills, from media use and new literacy demands and, finally, from genre and language competences. Writing develops, as Rogers (2010, p. 374) summarizes from a review on longitudinal studies in the US "in multidimensional and nonlinear ways" and depends on a large number of factors related to classroom practices, student engagement, teacher behaviors and genres. We need models, therefore, not only for skills development, but also for the development of writers and have to cross-check these developments with overall goals for higher education.

\section{Conclusions}

New orientations for the study and teaching of academic writing in Europe have to be found in the divers field of higher education and the changing field of academic literacy. What leads European universities into a new understanding of academic writing cannot be put in a single model or even ideology but has to be developed in practice. The teaching of is not done by writing teachers alone but by all disciplinary teachers who make use of writing in their classes or supervise student theses.

Development of the teaching of writing in Europe demands both an exchange on traditional genres and an exchange on new writing practices or best-practice models. Bologna has set the frame for the teaching in higher education to which the teaching of writing has to comply. In many ways, Bologna supports the introduction of new teaching models and provides arguments for a deliberate and explicit teaching of all important competences connected with academic writing and their various transition fields to learning and research.

The teaching of writing will continue to stand in the conflicting field between national languages and English. Models to connect the teaching of both are yet to develop. Undergraduate education will continue to be taught in national languages while graduate education will continuously move towards English as language for writing and instruction. The teaching of writing has to be done in both languages and has to be grounded in a conception of multilinguality. 
What unites the teaching of writing in Europe is its future rather than its past. Transformation processes are very similar in most countries and the directions of change seem identical (though not necessarily the solutions). International projects and a transfer of teaching approaches seem a good way of learning from each other. Hopefully, writing in Europe will maintain (part of) its cultural diversity and will gain a shared identity not by adopting a unified model but by understanding similarities on the background of cultural variation.

\section{References}

Banzer, R., Kruse, O. (2011). Schreiben im Bachelor-Studium: Direktiven für Didaktik und Curriculumentwicklung. In: B.Berendt (Ed.), Neues Handbuch Hochschullehre. Ergänzungslieferung G 4.8. Berlin: Raabe Verlag.

Beach, R. \& Friedrich, T. (2006). Response to writing. In: C. A. MacArthur, S. Graham, and J. Fitzgerald (Eds.), Handbook of writing research (p. 222-234). New York: Guilford Press.

Bean, J. (1996). Engaging ideas. The professor's guide to integrating writing, critical thinking, and active learning in the classroom. San Francisco: Jossey-Bass.

Bean, J. (2011). Backward design: Towards an effective model of staff development in writing in the disciplines. In: M. Deane and P. O'Neill (Eds.). Writing in the disciplines (p. 215-236). Houndsmills: Palgrave Macmillan.

Beaufort, A. (2008). College writing and beyond: A new framwork for university writing instruction. Logan, Utah State University Press.

Bizzell, P. (1982). Cognition, convention, and certainty. PRE/TEXT 3(3). (Reprinted in Victor J. Vitanza (Ed.) (1993). PRE/TEXT. The first decade (p. 65-92). Pittsburg, Pa: Pittsburg University Press.)

Bruffee, K. A. (1984). Collaborative Learning: Higher Education, Interdependence, and the Authority of Knowledge. Baltimore: Johns Hopkins University.

Castelló, M. \& Donahue, C. (2012). Introduction. In: Montserrat Castelló \& Christiane Donahue (Eds.). University writing. Selves and texts in academic societies (p. XIIIXXVII). Bingley, UK: Emerald.

Chitez, M. \& Kruse, O. (2012). Writing cultures and genres in higher education. In: M. Castelló \& C. Donahue (Eds.), University writing. Selves and texts in academic societies (p. 151-176). Bingley, UK: Emerald.

Coiro, J., Knobel, M., Lankshear, C. \& Leu, D. J. (2008). Central issues in new literacies and new literacies research. In: J. Coiro, M. Knobel, C. Lankshear \& D.J. Leu (Eds), Handbook of research on new literacies. Mahwah, NJ: Lawrence Erlbaum.

Deane, M. \& O'Neill, P. (2011). Writing in the Disciplines: Beyond Remediality. In: M. Deane \& P. O'Neill (Eds.), Writing in the disciplines (p. 3-13). Houndsmills, UK: Palgrave Macmillan. 
Delcambre, I. \& Donahue, C. (2012). Academic writing activity: Student writing in transition. In: M. Castelló \& C. Donahue (Eds.). University writing. Selves and texts in academic societies (p. 151-175). Bingley, UK: Emerald.

Delcambre, I. \& Reuter, Y. (2010). The French didactics approach to writing, from elementary school to university. In: C. Bazerman, R. Krut, K. Kundsford, S. McLeod, S. Null, P. Rogers \& A. Stansell (Eds.), Traditions of writing research (p. 17-30). New York: Routledge.

Dell'Aversano, C. \& Grilli, A. (2006). La scrittura argumentativa. Dal saggio breve alla tesi di dottorato. Le Monnier Università.

Devitt, A. (2009). Teaching critical genre awareness. In: C. Bazerman, A. Bonini \& D. Figueiredo (Eds.). Genre in a Changing World. Perspectives on Writing (p. 337351). Fort Collins, Colorado: The WAC Clearinghouse and Parlor Press. Available at http://wac.colostate.edu/books/genre/.

Donahue, C. (2002). The lycée-to-university progression in French students' development as writers. In: D. Foster \& D.R. Russell (Eds.). Writing and learning in cross-national perspective. Transitions from secondary to higher education ( $p$. 134-191). Urbana, III: NCTE.

Emig, J. (1971). The composing process of twelfth graders. Urbana, Ill: National Council of Teachers of English.

Flower, L. \& Hayes, J. R. (1980). The dynamics of composing: Making plans and juggling constraints. In: L.W. Gregg \& E.R. Steinberg (Eds.). Cognitive processes in writing (p. 31-50). Hillsdale, NY: Lawrence Erlbaum.

Flower, L. \& Hayes, J. (1981). A cognitive process theory of writing. College Composition and Communication, 32, 365-387.

Foster, D. (2002). Making transitions to university: Student writers in Germany. In: D. Foster \& D.R. Russell (Eds.): Writing and learning in cross-national perspective. Transitions from secondary to higher education (p. 192-241). Urbana, III: NCTE.

Foster, D. (2006). Writing with authority: Students' roles as writers in cross-national perspective. Carbondale: Southern Illinois University Press.

Girgensohn, K. (2007). Neue Wege zur Schlüsselqualifikation Schreiben. Autonome Schreibgruppen an der Hochschule. Wiesbaden: VS Verlag.

Halliday, M.A.K. (1985). An introduction to functional grammar. London: Arnold.

Harbord, J. (2010). Writing in Central and Eastern Europe: Stakeholders and directions in initiating change. Across the Disciplines: A Journal of Language, 7. Available at http://wac.colostate.edu/atd/articles/harbord2010.cfm.

Harrington, K. (2010). The role of assessment in "writing in the Disciplines". In: M. Deane \& P. O'Neill (Eds.). Writing in the disciplines (p. 48-64)). Houndsmills, UK: Palgrave Macmillan.

Hayes, J. R. \& Flower, L. S. (1980). Identifying the organization of writing processes. In L. Greg \& E. Steinberg (Hrsg.). Cognitive Processes in Writing. (p.3-30). Hillsdale: Erlbaum. 
Hidi, S. \& Boscolo, P. (2006). Motivation and writing. In: C. A. MacArthur, S. Graham, and J. Fitzgerald (Eds.). Handbook of writing research (p. 144-158). New York: Guilford Press.

Hyland, K. (1998). Hedging in scientific research articles. Amsterdam: John Benjamins.

Hyland, K. (1999). Text, processes and practices. London: Longman.

Hyland, K. (2000): Disciplinary discourses: Social interaction in academic writing. Harlow: Pearson Education.

Hyland, K. (2002). Teaching and researching writing. Harlow: Pearson Education.

Hyland, K. (2003). Genre-based pedagogies: A social response to process. Journal of Second Language Writing, 12/ 1, 17-29.

Hyland, K. (2005). Metadiscourse. Exploring interaction in writing. London: Continuum.

Hyland, K. (2011). Learning to write: Issues in theory, research and pedagogy. In: R.M. Manchón (Ed.). Learning-to-write and writing-to-learn in an additional language (p. 17-36). Amsterdam: John Benjamins.

Hyon, S. (1996). Genre in three traditions: Implications for ESL. TESOL Quarterly, 30, 693-722.

Kent, T. (Ed.) (1999). Post-process theory. Beyond the writing-process paradigm. Carbondale: Southern Illinois University Press.

Klieme, E., Avenarius, H., Blum, W., Döbrich, P. et al. (2007). Zur Entwicklung nationaler Bildungsstandards. Eine Expertise. Herausgegeben vom Bildungsministerium für Bildung und Forschung (BMFB) Berlin. Berlin: BMBF.

Kruse, O. (2007). The origins of writing in the disciplines: Traditions of seminar writing and the Humboldtian ideal of the research university. Written Communication 23/3, 331-352.

Kruse, O. (2012). Das Seminar: Eine Zwischenbilanz nach 200 Jahren. In: B. Kossek \& C. Zwiauer (Eds.). Universität in Zeiten von Bologna. Zur Theorie und Praxis von Lehr- und Lernkulturen (p. 89-110). Wien: Vienna University Press.

Kruse, O. (2013, in press). Wissenschaftliches Schreiben mehrsprachig unterrichten: Was ist möglich, was ist nötig? OEDaF Nachrichten.

Kruse, O. \& Chitez, M. (2012). Schreibkompetenz im Studium: Komponenten, Modelle und Assessment: In: U. Preusser \& N. Sennewald (Eds.). Literale Kompetenzentwicklung an der Hochschule (p. 57-84). Frankfurt am Main: Peter Lang.

Kruse, O. \& Jakobs, E.-M. (1999). Schreiben lehren an der Hochschule: Ein Überblick. In: O. Kruse, E.M. Jakobs \& G. Ruhmann (Eds.). Schlüsselkompetenz Schreiben. Konzepte, Methoden, Projekte für Schreibberatung und Schreibdidaktik an der Hochschule (p. 19-34). Neuwied: Luchterhand.

Langer, J. A. \& Applebee, A. N. (1987). How Writing Shapes Thinking: A Study of Teaching and Learning. Urbana, III: NCTE. 
Lea, M. \& Street, B. (1998). Student writing in higher education: An academic literacies approach. Studies in Higher Education, 23/2, 157-172.

Lea, M. (2004). Academic literacies: a pedagogy for course design. Studies in Higher Education 29/6, 739-756.

Leu, D. J., Zawilinski, L.J., Castek, M., Banerjee, B., Housand, C., Liu, Y. \& Neal, M.O. (2007). What is new about the new literacies of online reading comprehension? In: L. S. Rush, J. Eakle \& A. Berger (Eds.). Secondary school literacy: What research reveals for classroom practices (p. 37-68). Urbana, IL: National Council of Teachers of English.

Macgilchrist, F. \& Girgensohn, K. (2011). Humboldt meets Bologna. Developments and debates in institutional writing support in Germany. Canadian Journal for the Study of Discourse, 23/1, 1-27.

Martin, J.R. (1984). Language, register and genre. In: F. Christie (Ed.). Children writing: Reader (pp. 21-29). Geelong, Vic: Deakin University Press.

Martin, J.R. \& Rose, D. (2008). Genre relations. Mapping culture. London : Equinox.

Miller, C.R. (1974). Genre as social action. Quarterly Journal of Speech, 70, 151-167. (Reprinted in T.B. Farell (Ed.) (1998), Landmark essays on contemporary rhetoric (p. 123-143). Mahwah, NJ: Lawrence Erlbaum).

Nelson, N. \& Castelló, M. (2012). Academic writing and authorial voice. In: M. Castelló \& C. Donahue (Eds.), University writing. Selves and texts in academic societies ( $\mathrm{p}$. 33-52). Bingley, UK: Emerald.

Nesi, H. \& Gardner, S. (2012). Genres across the disciplines. Student writing in higher education. Cambridge: Cambridge Applied Linguistics.

Nesi, H., Gardner, S., Thompson, P. et al. (2008). An investigation of genres of assessed writing in British higher education: Full research report. ESRC End of Award Report (RES-000-23-0800) Swindon: ESRC.

Nystrand, M. (2006). The social and historical context for writing research. In: C. A. MacArthur, S. Graham, \& J. Fitzgerald (Eds.). Handbook of writing research (p. 11-27). New York: Guilford Press.

Ong, W. J. (1982). Orality and Literacy: The Technologizing of the Word. London: Routledge.

Palfreyman, D. (Ed.) (2008). The Oxford tutorial: "Thanks, you thought me how to think" (2nd. Ed.). Oxford: OxCHEPS.

Perl, S. (Ed.) (1994). Landmark essays on writing process. Davis, CA: Hermagora Press.

Pohl, T. (2007). Studien zur Ontogenese wissenschaftlichen Schreibens. Tübingen: Niemeyer.

Pohl, T. (2009). Die studentische Hausarbeit. Rekonstruktion ihrer ideen- und institutionsgeschichtlichen Entstehung. Heidelberg: Synchron.

Poe, M., Lerner, N. \& Craig, J. (2010). Learning to communicate in science and engineering: Case studies from MIT. Cambridge, MA: MIT Press. 
Prior, P. \& Billbro, R. (2012). Academic enculturation: Developing literate practices and disciplinary identities. In: M. Castelló \& C. Donahue (Eds.). University writing. Selves and texts in academic societies (p. 19-32). Bingley, UK: Emerald.

Pritchard, R. J. \& Honeycott, R. L. (2006). The process approach to writing instruction: Examining its effectiveness. In: C. A. MacArthur, S. Graham \& J. Fitzgerald (Eds.). Handbook of writing research (p. 275-292). New York: Guilford Press.

Rogers, P. (2010). The contribution of North American longitudinal studies of writing in higher education to our understanding of writing development. In: C. Bazerman, R. Krut, K. Lundsford, S. McLeod, S. Null, P. Rogers \& A. Stansell (Eds.). Traditions of writing research (p. 365-377). New York: Routledge.

Russell, D.R. \& Foster, D. (2002). Introduction: Rearticulating articulation. In: D. Foster \& D.R. Russell (Ed.). Writing and learning in cross-national perspective. Transitions from secondary to higher education (p. 1-48). Urbana, III: NCTE.

Russell, D.R. (2001). Where do the naturalistic studies of WAC/WID point? A research review. S. H. Mcleod, E. Miraglia, M. Soven, C. Thaiss (Eds.). WAC for the new millenium: Strategies for continuing writing-across-the-curriculum programs ( $p$. 259-298). Urbana: NCTE.

Russell, D. R. (2002). Writing in the academic disciplines: A curricular history. Carbondale, III.: NCTE (2nd Ed.).

Russell, D. R. \& Cortes, V. (2012). Academic and scientific texts: The same or different communities? In: M. Castelló \& C. Donahue (Eds.). University writing. Selves and texts in academic societies (p. 3-18). Bingley, UK: Emerald.

Samac, K., Prenner, M. \& Schweth, H. (2009). Die Bachelorarbeit an Universität und Fachhochschule. Wien: Facultas/UTB.

Sommers, N. \& Saltz, L (2004). The novice as expert: Writing the freshman year. College Composition and Communication, 56(1), 124-149.

Sommers, N. (2007). Across the drafts. Unpublished manuscript.

Steinhoff, T. (2007). Wissenschaftliche Textkompetenz. Sprachgebrauch und Schreibentwicklung in wissenschaftlichen Texten von Studenten und Experten. Tübingen: Niemeyer.

Street, B.V. (2005). Introduction. New literacy studies and literacies across educational contexts. In: B.V. Street (Ed.). Literacies across educational contexts. Mediating learning and teaching (1-24). Philadolphia: Caslon Publishing.

Swales, J. M. (1990). Genre analysis. English in academic and research settings. Cambridge: Cambridge University Press.

Swales, J. M. (2004). Research genres. Explorations and a pplications. Cambridge: Cambridge University Press.

Swales, J. M. \& Feak, C. B. (2012). Academic writing for graduate students. Essential tasks and skills (3rd. Edition). Ann Arbor: University of Michigan Press. 
Thaiss, C., Myers Zawacki, T. (2006). Engaged writers and dynamic desciplines. Research on the academic writing life. Portsmouth: Boynton/Cook.

Torrance, M.; Alamargot, D.; Castello, M.; Garnier, F., Kruse, O, Mangen, A. Tolchinski, L., van Waes, L. (Eds.) (2012). Learning to Write Effectively: Current Trends in European Research. Bradford: Emerald.

Walvoord, B. E. F. \& McCarthy, L. P. (Eds.) (1990). Thinking and writing in college: A naturalistic study of students in four disciplines. Urbana, IL: National Council of Teachers of English.

Cita del artículo:

Kruse, O. (2013). Perspectives on Academic Writing in European Higher Education: Genres, Practices, and Competences. Revista de Docencia Universitaria. REDU. Número monográfico dedicado a Academic Writing. Vol.11 (1) Enero-Abril. pp. 37-58 Recuperado el (fecha de consulta) en http://www.red-u.net/

\title{
Acerca del autor
}

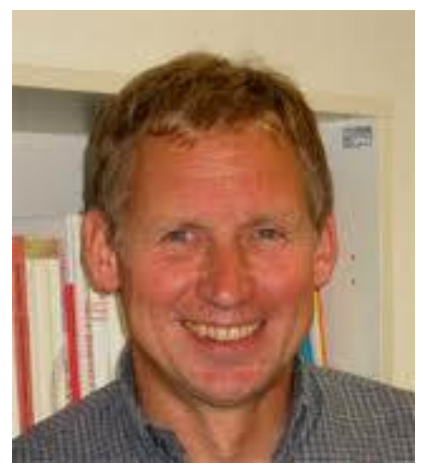

\section{Otto Kruse}

\author{
Zurich University of Applied Sciences (Switzerland) \\ Language Competence Centre \\ Mail: otto.kruse@zhaw.ch
}

Otto Kruse is a trained psychologist. He received his doctoral degree and habilitation from the Technical University of Berlin, then worked in clinical psychology, student counseling and social work before he received his current position as a professor in the field of applied linguistics at the Zurich University of Applied Linguistics. He is specialized in the teaching of writing and has given numerous writing courses in different study programs and in further education. He was and is involved in several international research projects studying what writing in European higher education means to teaching and learning. 


\title{
First year students' construction of an academic identity in English as a foreign language
}

\author{
Construcción de la identidad académica a través del inglés como lengua \\ extranjera en alumnos de primero
}

\author{
Angels Oliva Girbau \\ Universitat Pompeu Fabra, España \\ Marta Milian Gubern \\ Universitat Autònoma de Barcelona, España
}

\begin{abstract}
This paper studies the construction of an academic identity through writing in English as a foreign language, using a theoretical approach based on socio-constructivism, genre theory, systemic-functional linguistics and cultural-historical activity theory. Specifically, we study the relationship between academic genres and identity in first-year Humanities students, and to what extent a reflective social approach to writing instruction can foster students' initiation by providing spaces to analyse and discuss the nature of the academic activity system. The theoretical underpinnings present students' identity in construction as an essential element in the process of acquisition of academic genres, hence key to students' success at university, and students' identities as key elements of the academic activity system, at the same level as the other three - its communication tools, users, and goals. The theoretical framework guided the design of a course on academic English as a foreign language for first-year Humanities students at Universitat Pompeu Fabra. The course materials were used simultaneously as research tools to gather the data for the study, using an emic perspective as a teacher/researcher. The results show a close relationship between students' problems with the acquisition of genres in English and conflicts between their identities and other elements of the academic activity system, particularly their relation to other members and to ideational content, and hence students' inability to make relevant contributions due to problems using academic genres in English.
\end{abstract}

Key words: Academic identity, Cultural-Historical Activity Theory, ACLITS, WAC/WID, activity system, academic genres, discourse communities.

\section{Resumen}

El presente artículo estudia la construcción de la identidad académica a través de la escritura en inglés como lengua extranjera, desde la base teórica del socioconstructivismo, la teoría de género, la lingüística sistémico-funcional y la teoría de actividad histórico-cultural. Concretamente, se analiza la relación entre 
los géneros académicos y la identidad académica en alumnos de primero de Humanidades, y hasta qué punto un planteamiento social reflexivo de la enseñanza de la escritura contribuye a mejorar la integración de los alumnos, proveyéndoles de espacios y herramientas para el análisis del sistema de actividad académico. Conceptualizamos la identidad en construcción de los aprendices como un elemento esencial del proceso de adquisición de los géneros académicos, y por tanto clave para su permanencia en la universidad. Las identidades de los estudiantes son vistas como elementos clave del sistema de actividad académico, al mismo nivel que sus otros componentes - herramientas de comunicación, usuarios y metas. A partir de este marco, se diseñó un curso de inglés para usos académicos para alumnos de primero de Humanidades en la Universidad Pompeu Fabra (Barcelona). Los materiales del curso se usaron como herramientas para recoger datos para un estudio émico, evidenciando la relación entre los problemas de los estudiantes con la adquisición de géneros académicos en inglés y los conflictos que experimentan cuando sus propias identidades entran en contradicción con elementos del sistema, particularmente en relación con otros miembros del sistema y con el contenido del campo, lo que dificulta que los alumnos puedan construir conocimiento a través de los géneros en inglés.

Palabras clave: Identidad académica, Teoría de la Actividad Histórico-Cultural, ACLITS, WAC/WID, sistema de actividad, géneros académicos, comunidades discursivas.

\section{Introduction}

This piece of research stems from the conceptualisation of students' initiation into higher education as a never-ending process of negotiation of meaning and identity between instructors, students, and the genres that mediate the construction of knowledge and the relationships within the academic community. The theoretical framework in which this action research project is embedded borrows from genre theory (Bakhtin, 1986; Bazerman, 1994; Devitt, 1993, 1994, 1996, 2004) and systemic functional linguistics (Halliday, 1978, 1993, 1994) for the conceptualisation of academic genres; cultural-historical activity theory for the relationship between genres, subjects and context (Leontiev, 1978; Engeström, 1995; Russell \& Yáñez, 2003); and from socio-constructivism (Vygotsky, 1978; Bruner, 1985) for our views on learning and the role of genres in higher education.

Interrelations among the cited perspectives are made evident by several scholars (among others, Roth and Lee, 2007; Street, 1999, 2009, Lea \& Street, 2006), pouring on foundational concepts of developmental psychology and cognition, literacy and rhetorical studies, and cultural anthropology. We'll focus successively on these strands taking into account the flow of similarities among them.

\section{Activity Theory and CHAT}

Historically linked to the work of Vygotsky, Activity Theory and Cultural Historical Activity Theory provide tools for understanding human functioning. Both approaches share a view of learning common to other learning theories within the sociocultural family: social contexts shape individuals by inserting them in social activities, and at the same time individuals continually shape and reshape contexts performing their activities. Vygotsky explained the relationship between individuals and learning by means of the semiotic mediation, mainly through the use of language signs interacting with others in the ZPD; Luria and Leontiev incorporated societal, cultural and historical 
dimensions to a theory of human mental functioning, creating what is known as the second-generation activity theory.

An activity system is conceived as a system of human action upon an object in order to achieve a definite goal or outcome; it is, according to Leontiev, a social format organizing and regulating human behavior. Activities differ according to the different goals they pursue; goals experiment continuous changes along the evolving history of humankind. Therefore, activity systems are not static, but dynamic systems which are constantly re-created by micro-level interaction among their components. In fact, along with the evolving societal development, an activity system may unfold into two or more systems by opening its boundaries and losing its self-containment. Entities belonging to an activity system -objects, rules, means of production, outcomes- may be used in other activity systems in a sort of exchange. This situation gives rise to the possibility for contradictions that transcend the individual subject and its relations to other elements in the activity system (Roth \& Lee, 2007).

Using activity systems, we can analyze the way in which specific tools are implemented to mediate the goals and the object (focus or problem) of a community, and how they change over time in relation to the subjects, either individuals or groups who work towards some results, while their participation in different activity systems contributes to build their social identity (Russell \& Yáñez, 2003). These objects or motives are not frozen, but change and adapt through time. The existence of the objects involves the existence of some general, shared goals, which are nonetheless constantly challenged at an individual level.

From a social perspective, participating in an activity system entails one's engagement with the identity and goals of the system. New participants imitate what they perceive to be tools and forms of use, and internalize them if they perceive that they are successful employing them. Drawing from Activity Theory and CulturalHistorical Activity Theory, we envision higher education as a set of interconnected activity systems. Activity theory conceptualizes the context of communication as a network of dynamic systems that are made up of human agents, goals and discourse.

In the academic context, by making explicit to students the mechanics of the community they intend to join through the study of its specific discourse patterns and their own development as members of it, we turn writing instruction into a tool of inclusion that grants first-year students prompt access to their field of knowledge as learners and active participants, challenging the power hierarchy of the academic activity systems. For novice writers, academic identity is defined by their learner status and their perception of themselves within the activity systems of higher education. Their conceptualization as academic writers depends on the relationship they perceive they have or may potentially have in the future to the goals, tools and users of the system.

Participation in the academic community requires students to a) accept the entry rules of the community, b) have their participations sanctioned by the expert members of the communities, and c) actively participate in the exchanges of the community so as to be eligible for acceptance and show adherence to the community. 


\section{Systemic functional linguistics and genre}

According to the patterns of activity systems, participating in academic activities is mainly achieved through the use of language tools to fulfil the goals of the activity, following the rules established and accepted by the academic community. These rules to a certain extent are the result of a stabilisation and institutionalisation of language use in the form of genres. Nonetheless, discursive genres act as bridges between societal norms and individual options, between the social perception of genres as routinized ways of using language because of their recurrence, and the individual perception, both of the goal /object of the activity and of the discursive tools to reach it.

Bakhtin (1979) related the concept of genre to the use of language in the diverse spheres of human activity. He uses several labels to refer to these spheres, concomitant with the activity systems described in CHAT -cultural sphere, sphere of human praxis, sphere of discursive communication, determined sphere of common reality, sphere of human activity and life... As multiple and multifaceted these spheres are, so are the uses of language related to them. "Language participates of life through concrete utterances belonging to it, so as life participates of language through these utterances" (p.252). In a given situation or sphere, a speaker/writer chooses to use a given discursive genre. This election follows the consideration of the sphere of action as well as the consideration of the motives and the object of the activity, and also taking into account all the components of the situation -participants, aspects of the communicative action, etc. It is to say that the speaker's discursive intention, even being individual and subjective, adapts and follows a determined generic form. And this occurs in every communicative situation. Generic forms become as necessary as linguistic forms, and contribute to inter-comprehension in the same way as linguistic forms do. But genres guide our discursive processes because of their relationship with the sphere of action and the object of the activity, while linguistic forms only constitute an objective lexical and grammatical collection of options to be used.

Systemic-Functional Linguistics (SFL) started using textual evidence to trace the functions genres perform, and how to reproduce them from a semiotic perspective. SFL developed a comprehensive conception of context or situation in relation to genre development, which included the notions of field, tenor and mode. These components of context determine the occurrence of concrete registers/genres.

According to Halliday (1993: 26) register/genre is a semantic and a functional concept, defined as "the configuration of semantic resources that the member of a culture typically associates with a situation type. It is the meaning potential that is accessible in a given social context". This potential is realised by means of three functional components (Halliday, 1970) through which a text operates - the textual, interpersonal and ideational components. Halliday argued that genres are not defined by their formal qualities per se, but rather by the way such qualities relate to the contents and functions of discourse in the particular context where it commonly takes place. 
The interpersonal component deals with intertextuality and the relationship between the writer and the readers. This component describes the dialogical aspects of texts, how writers speak to the audience about the text and about their relationship to it and thus establish different degrees of closeness with the community of readers. By using modality and metadiscourse, writers can tell their readers how reliable a proposition is, or their inclination towards an idea or opinion. Since academic writing deals with cognition and its limits, it is important for scholars-to-be to gain skills for engaging in remote discussions with other academic writers while clearly establishing their attitude towards their role, their readers and their statements. Modality determines the writer's relationship with the audience by taking out insurance on categorical statements, limiting such statements to conditions under which they can be regarded as objectively valid or acceptable in argument via the use of modal auxiliaries, adjectives, hedges, and such (Nash, 1990).

The ideational component covers the semantic concepts of their field-knowledge and any use of nonstandardised expressions. This component refers to the text epistemological functions, how it contributes to consolidating field knowledge by mirroring the community's background knowledge.

Finally, the textual component covers the compositional patterns of genres and any related formal features. This component shows how language structure binds together the ideational and interpersonal components (Freeman, 1981; Halliday, 1970). It designates linguistic issues - not in terms of grammatical accuracy, but rather in relation to the community's expectations on the form of a particular genre, the generic conventions and the necessary command of English to implement them lexical items, syntactic structures and compositional patterns.

Within the specific context of an activity system, discourse genres are used as social tools that provide speakers with models of suitable responses to situations they are likely to encounter in their usual sphere of action. Discourse and context are thus bound by a mutually defining relationship, as genres build and are built by recurrent situations, facilitating the increasingly complex communication needs of the members of discourse communities by inserting discourse functions into predictable structures (Bazerman, 1995). Genres cannot exist isolated from their users and their sphere of action, because they would be unrecognisable.

\section{Identity and learning}

People's identities are networked across the activities in which they participate and are on a trajectory over time, and this needs to be taken into account both in providing opportunities for participation in educational settings, and in recognising the outcomes from educational provision... One of the outcomes from any social activity is a reconfiguration of participants' subjectivities, both individually and in relation to one another. Learning to feel differently about yourself, even to have a different sense of who you are is in itself a type of learning. In educational contexts 'learning' is not just an increase in knowledge, understanding and capability, but includes the discoursal reconstruction of identity too. (Ivanic, 2006, p.19-20). 
Along with meanings, the members of the academic activity systems also negotiate their identities within the system through the use of genres. Identity (re)construction is therefore regarded as a key element of any activity system, as it mediates the representation of users and their participations in the system. The subjects of the academic activity system are materialised into it through a series of identities or social representations that are constructed through their interactions, and hence through their use of genres. Identities -in plural-are the tools that users construct to represent themselves. Their perceptions of and contributions to the collective and individual goals of the system affect the way users perceive these identities - their roles, their status, their relationship to it, and so on. These identities, therefore, are not stable, but in a permanent state of fluctuation between different parameters, such as learner/expert or professor/researcher. A single user could then support more than one identity at the same time in order to interact in different activity systems or within the same activity system if he or she had different roles to perform in it.

When applying the concept of activity system to the context of higher education, we reject the view of academia as a single activity system, as this would overlook the multiplicity of voices, literacies, identities, and individual and collective goals presentday university comprehends. Instead, we interpret academia as a network of interrelated, sometimes overlapping and sometimes concentric activity systems, in which users may switch across groups, or participate simultaneously in different ones.

Newcomers acquire genres in different ways -by trial-and-error or by imitation, for example- as they participate in the exchanges of their activity system. As new users acquire genres, they also internalise the object/motive of the activity and the identity of the group. Since the acquisition of genres occurs dialectically, there are tensions and conflicts between the different objects, goals, tools and subjects of the multiple activity systems learners operate in when they attempt to transfer genres across different activity systems (Russell \& Yáñez, 2003). For first-year students, academic identity is defined by their learner status and their perception of themselves within the activity systems of higher education. Students' self-image as academic writers depends on the relationship they perceive they have or may potentially have in the future to the goals, tools and users of the system. For learners initiated into multilingual contexts, the number of genres multiplies, and so do the conflicts between them, since generic knowledge is not transferable across contexts, disciplines or languages. In the academic context, by making explicit to students the mechanics of the community they intend to join through the study of its specific discourse patterns and their own development as members of it, we turn writing instruction into a tool of inclusion that grants first-year students prompt access to their field of knowledge as learners and active participants, challenging the power hierarchy of the academic activity systems:

By gaining a grasp of how entire discursive systems operate through generic turns, we can locate ourselves, our potential speech acts and the criteria our utterances should seek to meet; we can start to understand what we can achieve rhetorically at any moment, and what we cannot, and how. (Bazerman, 1995, p.99).

Within a community of knowledge, authors read each other's works and respond to them unendingly, which results in the fact that all the academic texts of a field are interconnected into a knowledge network. Each text depends on the existence of many other texts, which themselves were written on the basis of former texts. Their 
recurrence and index of referentiality constitute marks of status and recognition in their relation to other members of the discipline, so that they both co-operate and compete simultaneously. In the context of higher education, literacy performs three functions. Firstly, the socio-constructivist approach to education inseparably connects discourse, cognition and learning. Vygotsky (1978) viewed learning as an individual achievement that is mediated by social interaction and constructed when comprehensible meaningful discourse triggers higher mental processes. Secondly, in the current teaching practice writing is the prevailing form of evaluation, as students are required to read and write extensively across a wide range of genres and, perhaps, different languages. The focus of this paper, however, is on another function of writing, academic genres as proof of a subject's belonging to a discipline, as genres and identity are mutually constructed. The identity of academic writers is mutually dependant of their academic literacy, the genres they can employ and their impact. By displaying their knowledge of the forms, concepts and relations assigned to a certain genre, academic subjects reassert their identity as members of the group and contribute to its goals. This is a never-ending process of renegotiation of status, as "the rules of the game constantly change in response to a wide range of intellectual, material, and political forces within and outside the community" (Russell, 1991, p.14). Within the same community of knowledge, users' status are sometimes unstable particularly for new less established members - and may fluctuate between expert and novice, as new genres emerge or change through time (Carlino, 2004).

\section{Integrating research and instruction}

By using qualitative research to analyze a group of first-year Humanities students, we attempt to provide some insights on students' initiation into higher education as a cultural phenomenon, describing students' construction of their academic identity through their writing, as their realisation of academic genres can provide clues as to their perceptions of the knowledge and the system of meanings of the academic system of activity they are about to join. Driven by our focus on the relationship between context and text, we chose to use an ethnographic approach to data gathering because it could provide an in-depth analysis of the context of particular forms of literacy Awareness of this process and its implications can make students feel more comfortable with their own struggle in seeing themselves as part of the academic activity system. Hence, we designed research tools and course materials jointly, so as to obtain as much information as possible in order to analyse students' participation in progress. This could potentially undermine the object of the analysis, as one of the problems for academic literacies researchers who are also practitioners is that being inside the system, one risks taking a subjective view on students' writing. However, the insertion of research into course design had many advantages that compensated this risk.

Firstly, it narrowed down three gaps - the gap between research and instruction, the gap between text and context/users, and the one between expert and novice writers -, contextualizing academic literacies by linking "activities of reading and writing and the social structures in which they are embedded and which they help to 
shape" (Barton and Hamilton 1998:6). Secondly, using compulsory and voluntary coursework rather than only voluntary activities contributed to widen the range of student types participating in the study.

In the case study, we analyse first-year Humanities students' participations in a course on English for academic purposes aimed at mainly native Catalan and Spanish speakers from Universitat Pompeu Fabra (Barcelona)' during the academic year 201011.

\section{Course design: Identity, literacy and inclusion through reflective activities}

In our vision of present-day higher education, the role of writing instructors consists of guiding students as they learn to interact with the components of the system they are attempting to join, increasing students' awareness of the nature of the system and enhancing their process of genre acquisition through opportunities to partake of interactions with other members of the field. Consequently, we abandon deficit models of teaching academic discourse (Lea \& Street, 2006), breaking away from the myths of transience and transparency that blame students for their inability to write within the generic boundaries of academic communities. In practice, this goal demands a reflective social approach to writing instruction that fosters students' successful initiation into university by providing spaces to analyse and discuss the nature of the academic activity system.

In this particular case, we followed the lead of the US movement Writing in the Disciplines (also known as Writing Across the Curriculum), and its British counterpart Academic Literacies for the design of the course materials, as they both appeared in a similar context to the one we currently experienced by Catalan higher education, and they embody the principles we summarised in our theoretical framework. We have used as references the work of Lillis, Street, Lea and Ivanic for Academic Literacies; and Russell's detailed descriptions of the history of Writing Across the Curriculum ${ }^{\mathrm{ii}}$.

\section{Description of course materials}

The materials for the course, doubled up as research tools, were designed following the guiding principles we have described in our theoretical background:

- Literacies are multiple and changing, as they depend on the social practices of different cultural and linguistic contexts.

- Genres, goals, knowledge and identity are all mutually dependant, and they are simultaneously built in the context of the academic activity system through participation.

- The expert members of the academic activity system in charge of instructing novices should be aware of the context-specificity of genres, their mutable nature and their three-fold use for personal meaning-making, as proof of insiderdom, and as a test of belonging and knowledge of the field.

These materials for the seminars consisted of reading and writing activities to guide students' understanding and competence using academic genres, and some 
reflective activities based on Academic Literacies practices that were aimed at 1 ) raising students' awareness of the components and mechanics of the academic activity system, 2) guiding students' analysis of written genres and how they reflect the underlying assumptions and roles of the people who use them, and 3) providing students with opportunities to critically discuss and challenge the academic status quo as new members of $\mathrm{it}^{\mathrm{iii}}$ - the features of genres, their transparency, identity issues, power relations, and so on. A brief description of the course activities is provided below.

\section{Awareness activities}

They start at a very basic level, with a description of the concept of activity system applied to students' context, and explicitly naming and discussing its components, taking into account students' previous background to point out elements that may be more implicit to students' conceptualisation of the academic activity system (such as the readers, the collective goals of the system, the relationship between status and genres, and such). The resources for raising students' awareness comprehend anything between the explicit teaching of what an activity system is and the components that make it up to activities in which students infer the relationships between these components out of a sample text.

\section{Analysis activities}

Students analyse academic genres and the underlying assumptions that determine their functions and features. This analysis starts at textual level, but students are requested to discuss textual features in relation to the context of use, and the power relationships and marks of status associated to them, insisting on how textual features cast an image of text authors and their perceptions of their readers, field, knowledge, and such. Language forms are therefore always connected to the knowledge and particular ways of communication of the field.

\section{Contestation activities}

The goal of such activities is to offer students opportunities for critical reading and analysis, so that they can challenge the prescriptive view on specific genres as permanent, correct or transparent. These activities connect language with what individuals do, exploring the connections between the implicit assumptions on language made by individuals and social institutions, and to what extent "by engaging in an existing practice we are maintaining a particular type of representational resource; by drawing on a particular type of representational resource, we are maintaining a particular type of social practice" (Lillis \& Scott, 2007, p.12).

The categorisation of these kinds of activities is not strict, however, as there are no clear-cut limits between the goals we have set for each type of activity in the sense that activities aimed at, for example, raising awareness, are intrinsically connected to analysis and contestation. In terms of goals, therefore, rather than a hierarchical 
classification, we find a continuum that mirrors students' gradual understanding of the nature of the academic activity system and their subsequent critical attitude towards some of its aspects as full active members of it.

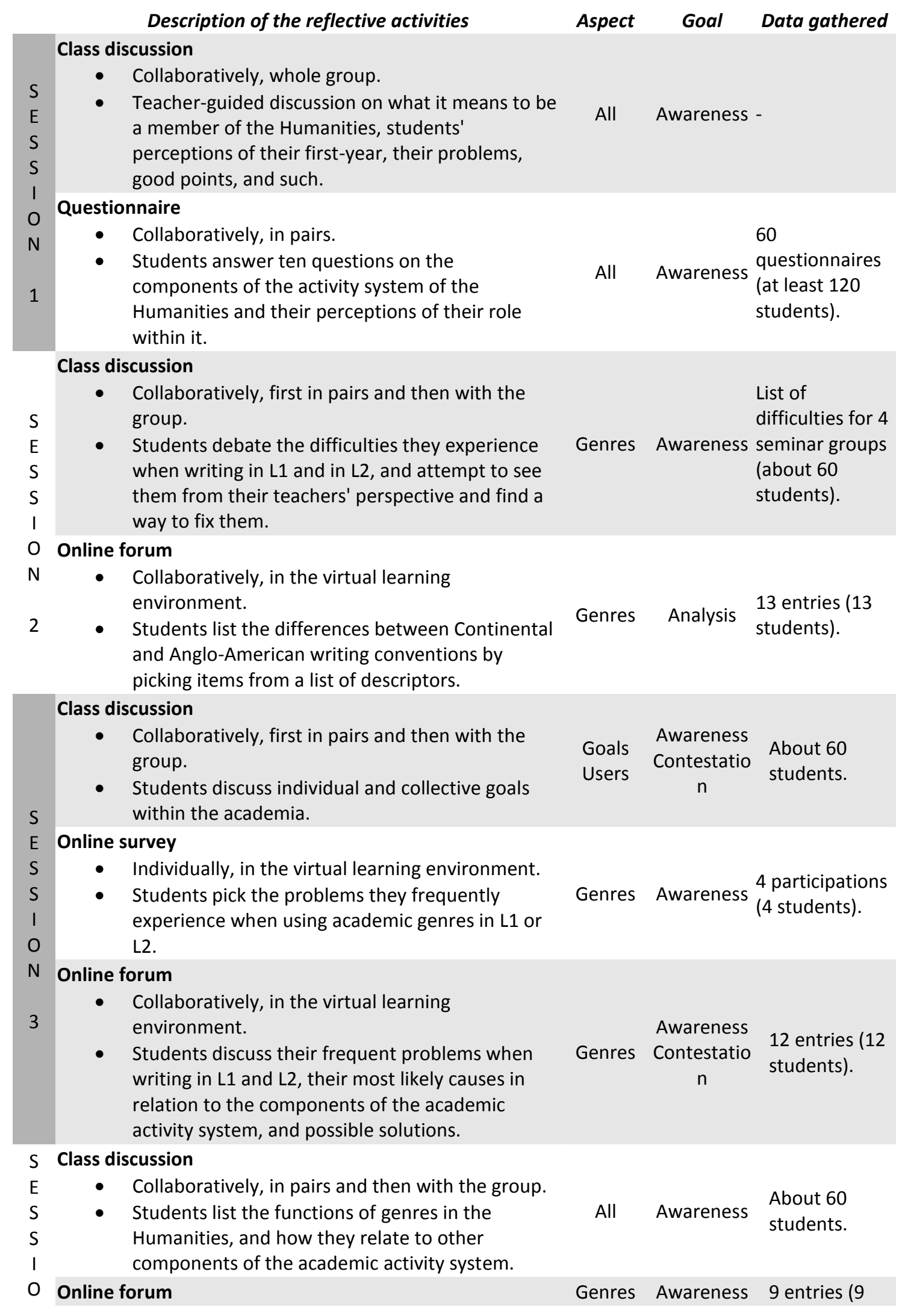




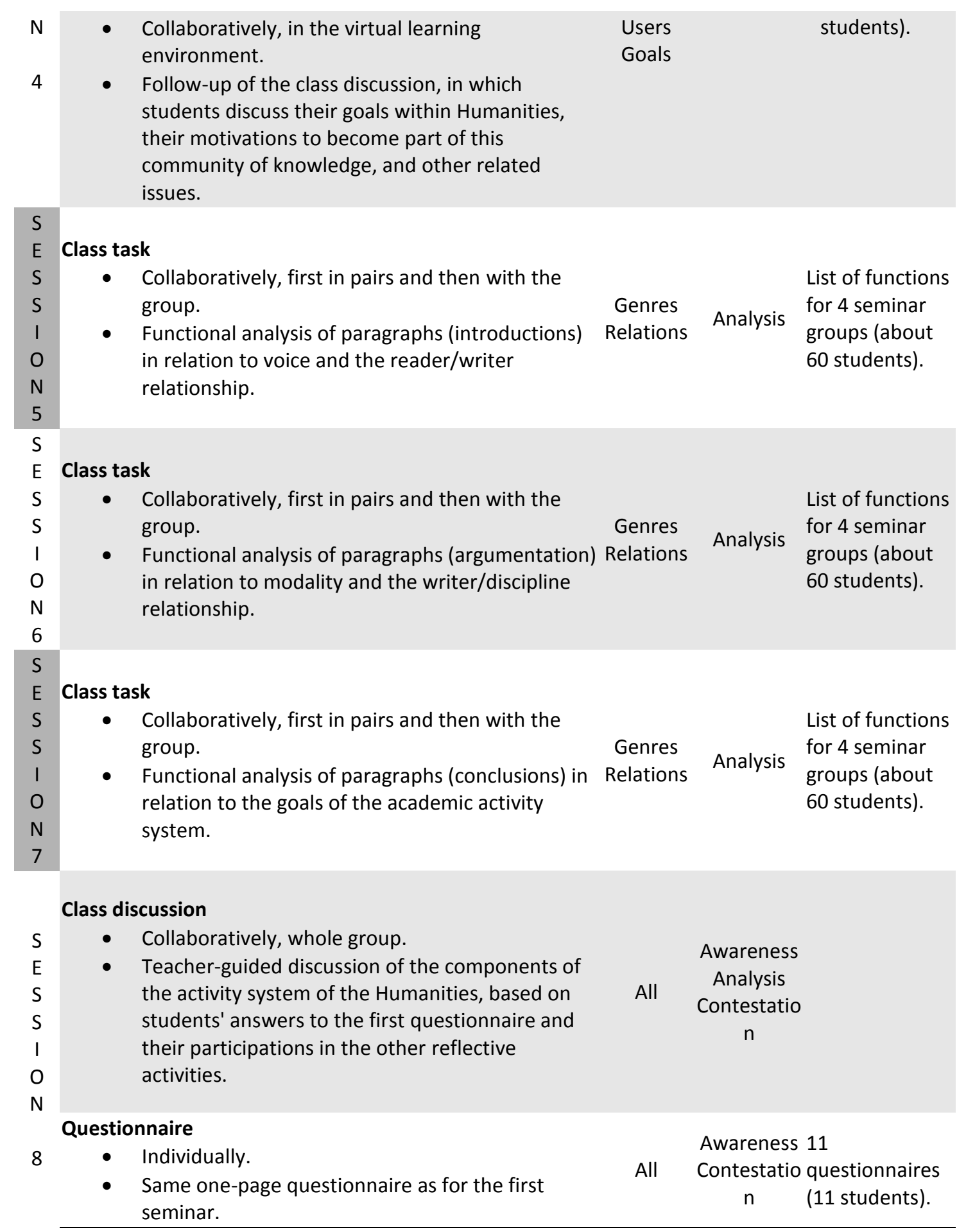

Table n. 1. List of reflective activities/research tools.

Table 1 sums up the reflective activities for each session. The first column contains session numbers, one to eight. The second column contains the list of activities by type: class discussion or task, questionnaires, online forums or surveys; and it provides a brief description of each activity and the interaction patterns it entails (collaborative or individual). The third column lists what components of the academic activity system the reflective activity deals with, either genres, goals, users, relationships, or all of them. The fourth column displays the goals for each activity - 
raising awareness, enabling analysis or promoting contestation, as discussed in the previous paragraphs. The last column lists the data gathered by means of each activity, and hence the number of students who took part in each one.

\section{Example of an activity}

As an illustration of the methods we used during the seminars, we shall briefly describe the reflective activity we used in seminar number five. The goal for this seminar was to engage students into the analysis of the textual marks of voice and writer/reader relationships in academic genres based on the study of introductory paragraphs. At the start of the lesson, the seminar instructor introduces the notion of discourse as social action, using everyday examples. The seminar instructor asks students to list the functions of sentences in introductory paragraphs, based on their own experience. The list is written down on the board, and rephrased collaboratively. Using this list, students analyse in pairs the functions of sentences in a paragraph. After a while, the seminar instructor conducts students' discussion of what function is performed by each sentence, and what resources the author has used to achieve it.

\begin{tabular}{|c|c|}
\hline Focus & One of the functions of a sentence in an introduction is... \\
\hline Ideational component & $\begin{array}{l}\text { - To present the topic and point of view. } \\
\text { - To explain information. } \\
\text { - To contain the main ideas of the text. } \\
\text { - To state your thesis. }\end{array}$ \\
\hline Textual component & $\begin{array}{l}\text { - To state the problem/issue to be discussed. } \\
\text { - To describe text organisation. } \\
\text { - To go from general to specific. } \\
\text { - To be a summarised version of the whole paper. }\end{array}$ \\
\hline Interpersonal component & $\begin{array}{l}\text { - To help the audience understand the text. } \\
\text { - To get the audience interested. } \\
\text { - To give you the main idea of the text and the general topic. } \\
\text { - To be very reader-focused. }\end{array}$ \\
\hline
\end{tabular}

Table n. 2. Students' list of functions as gathered in session 5 .

The combined list of functions for introductory paragraphs students contributed is displayed in table number 2 , in which items are arranged according to Halliday's functional components of discourse. After writing the list on the board, the seminar instructor asked students to connect these items to one or more of the components of academic activity systems, in order to increase their awareness of the connections between genres and all the other components (subjects, identities, goals, results). At the end of the class, the seminar instructor asks students to guess the status of the author of the text, and justify their decision based on the paragraph itself, exploring the textual realisations of status and identity in genres, and challenging some of their assumptions about the role of novices and experts $\underline{3}$. The ultimate goal of these tasks is to enable students to articulate the conceptions of genres, making explicit what was implicit: their views of themselves as members of the Humanities, and how challenged they feel by expert genres and status. 


\section{Research outcomes: Identities in conflict}

The data gathered along the seminars in the course have a rather limited impact due to the large number of students enrolled in the subject who did not attend the seminars, as shown in table 1 when comparing the number of questionnaires obtained in the first and last seminar sessions, sixty and eleven respectively. The reasons for the high rate of absenteeism are mainly connected to the students' level of English. Some students could skip the course by producing a language certificate, so the most language-proficient students disappeared from the course after the third week. In addition, many of the students who had never studied English before or who felt they had a very low level either did not attend at all or dropped out after the first assignments. Consequently, the number of students' contributions is too low to draw general conclusions as to the efficacy of the materials.

In spite of the lack of data coming out of the questionnaires, discussions and participation in the reflective activities we described above, either in class setting or in online forums, offer some insights into the students' evolving awareness of their position in academic activity systems, though conflicts and tensions derived of their English proficiency may have blurred their own vision as new members of the Humanities academic activities.

Through the analysis of students' participation we constructed a picture of students' relationship to the components of the academic activity system as mediated by Anglo-American academic genres (Rienecker \& Stray, 2003). The results show a close relationship between students' problems with the acquisition of academic genres in English and conflicts between their newly constructed identities and other elements of the academic activity system, particularly their relation to the other members of the academic activity system and to its ideational content, and hence students' insecurity to make a relevant contribution due to their problems using academic genres in a foreign language.

Faced with a writing task in English, students struggled between their will to create and their will to communicate, a conflict that challenged their still vulnerable academic persona. Their wish to contribute was still strongly individualistic, rather a personal challenge than a contribution to collective goals. Students felt that they needed to assert the legitimacy of their belonging to the academic activity system, which depended solely on their ability to articulate their contributions in an academic manner and submit them to the approval of an audience superior in status to them. In this respect, academic socialisation overlaps first-year students' entrance into maturity and their reach for new more powerful and independent roles. Because of their acute perceptions of status and the power relations their own novice status entails, students are not interested in constructing knowledge with their peers, who are in the same precarious position as they are and competing for the same posts. At the initiation stage, students do not view the social construction of knowledge within their discipline as a feasible goal due to their problems dealing with materials by other authors, and because at this point reasserting their academic identities is much more important as an individual goal than the collective goals and patterns of interaction established by the community. 
Students' initial attitude towards academic genres in English is rather negative, due to their perception of the level of formality that these genres demand, their objective structure that feels very complicated, uncomfortable" ${ }^{\text {iv }}$. However, explicit teaching and discussion of the nature of the academic activity system made students aware of other levels of difficulties besides their foreign language skills. After the seminar sessions, and as shown in the materials gathered using the reflective activities, students' trouble understanding and producing such genres was no longer purely linguistic, but also determined by cultural differences, problems finding an audience, lack of content and procedural knowledge, issues of status and identity, and their relationship to the other components of the academic activity system.

The students' need to translate and to use a dictionary openly refer to their lack of proficiency in English. Thinking in English is equated here to thinking in a different kind of way, rather than in a different language, a way that students find so challenging that they resort to "cheating" in order to regain control of their voices. The following quote from the forum on students' difficulties writing academic genres illustrates students' ambivalence between their focus on their language difficulties and the hints at other issues (the italics are ours):

It's very difficult to change our way of thinking. Normally when I write an assignment I start writing in English, but then I forget a lot of things that I wanted to write, therefore I first write in Spanish or Catalan and then I translate to English. Well, I know it's wrong, but if I write directly in English, I can't control my ideas. I need to have a plan with what I want to say and then I put on the paper in English. Oh! and always with my dictionary next to me.

When asked to reflect on their problems with academic genres, students realised that they lacked control over contents, form, audience and reception. Even though this lack of control existed in other subjects too, it was exposed even more clearly by their deficiencies writing in English, and because of the different planning and writing processes Anglo-American writing requires from them. In the same forum, another student compared the cognitive processes to the discursive ones. The cognitive processes described in the quote (marked in italics) correspond to students' description of academic genres in their L1. From students' point of view, cognition is a deeply subjective chaotic lonely process, in contrast to the required linear structure and accountability of academic genres:

From my point of view, the main source of the problems we experience when we write an academic paper is the difference between mental structure and discursive one. On one hand, mental structure is not linear: it does not use discursive arguments and it presupposes a lot of things because the subject is the only one who works with it. Besides, it employs many digressions, excursions and associations with memories and other mental issues.

As a consequence, students' still half-constructed academic identity was undermined by their inability to communicate transparently using academic genres in English. In students' view, the foreign language problem was materialised in their frequent grammatical and lexical errors, even though these accounted for $10 \%$ of their grade in written assignments. Thus, students initially argued that they were able to use academic genres in their native tongue, even though when asked explicitly about their problems they listed the same issues for genres in both languages. This contradiction 
may reflect students' reluctance to admit to deeper problems with genres beyond spelling or language accuracy, since admitting to other issues may indicate they are not suitable to join the academic community.

Students' relationship to the genres used in the academic context is extremely complex because genres materialise students' relationship to the components of the academic activity system they are attempting to join. By gaining a deeper understanding of how to use generic tools to interact in the system, students gain more control of the image they project and their relationship to the ideational contents of the Humanities and other members of the system. Students' insecurities with the foreign language and the increasing pressure on new students to solve their writing issues outside the system can only prevent students and faculty from seeing the deepest issues that undermine students' successful entrance into the academic system. Students' self-image, their career and their successful access and interaction in the academic community can be negatively affected by their need to fit apparently contradictory academic identities for different disciplines and languages, and by students' lack of awareness of the role of writing in the construction and development of an academic persona in their first language and in English. By the end of the term, however, some students had a more hopeful view of their chances to participate in the academic community through genres in English. As a token of this attitude change, when asked about what it felt like to be a member of the Humanities in the questionnaire for seminar 8 , one of the students replied: "I feel like a beginner in all the areas, but I have in my hands the opportunity to discover a lot of ideas" .

\section{Conclusions}

The course materials exposed a strong connection between students' problems acquiring academic genres and the conflicts derived from the construction of their academic identity in relation to the components of the academic activity system. Explicit discussion of this process helped students become aware of the elements that make up the academic activity system and their relationship to themselves, improving their perception of the role of academic genres in English by denying their transparency and exposing students' difficulties, thus changing their focus from language to contents, and from the textual features of genres to goals, functions and relationships.

Students' struggle with the acquisition of academic genres should not be regarded from a purely textual point of view, as it is embedded in the conflicts derived from their process of initiation into the academic activity system. Students' construction of their identity as members of the academic field is mutually dependent on their relationship to the novice and expert members of the academy, to its contents and its goals, and to the tools used to interact, mark one's status, initiate new members and negotiate the goals of the academic activity system ${ }^{\mathrm{vi}}$.

By incorporating the findings of research on literacies and instruction, we can abandon deficit models of teaching academic discourse to offer more students the opportunity of contributing to the knowledge community. Learning from research can 
help writing instructors to guide students as they learn to interact with the components of the system they are attempting to join, thus enriching their process of genre acquisition and awareness with the opportunity to partake of its interactions. Initiation becomes a process of negotiation of identity and meaning between instructors, students, and the texts that mediate the construction of knowledge and the relationships in the academic community.

\section{Referencias bibliográficas}

Bakhtin, M. M.; Holquist, M. and Emerson, C. (1986). Speech genres and other late essays. Austin, Texas: University of Texas Press.

Bazerman, C. (1994). Constructing experience. Illinois: Southern Illinois University Press.

Bazerman, C. (1995). Systems of genre and the enactment of social intentions. In A. Freedman and P. Medway (Eds.). Genre and the new rhetoric (pp. 79-101). London: Taylor \& Francis.

Bruner, J. (1985) Vygotsky A Historical And Conceptual Perspective in Wertsch, V. (ed) Culture, communication and cognition: Vygotskyan perspectives. Cambridge: Cambridge University Press.

Carlino, P. (2004). La Distancia que Separa la Evaluación Escrita Frecuente de la Deseable. Revista de Educación de la Universidad de los Andes, vol. 13(1), 8-17.

Devitt, A. (1993). Generalizing about Genre: New Conceptions of an Old Concept. College Composition and Communication, 44, 573-86.

Devitt, A. (1994). Genre and the New Rhetoric. London: Taylor \& Francis.

Devitt, A. (1996). Genre, Genres and the teaching of genre. College Composition and Communication, 47, 605-615.

Devitt, A. (2004). Writing genres. Carbondale, US: Southern Illinois University Press.

Engeström, Y. (1995). Expansive Learning at Work: Toward an Activity Theoretical Reconceptualization. Journal of Education and Work, 14 (1), 133 -156.

Freeman, D. (1981). Essays in modern stylistics. London: Methuen.

Halliday, M.A.K. (1970). Functional Diversity in Language as Seen from a Consideration of Modality and Mood in English. Foundations of Language, 6, 322-361.

Halliday, M.A.K. (1978). Language as social semiotic. London: Edward Arnold.

Halliday, M.A.K. (1993). Towards a Language-Based Theory of Learning. Linguistics and Education, 5, 93-116.

Halliday, M.A.K. (1994). An Introduction To Functional Grammar. London: Longman.

Ivanic, R. (1998). Writing and Identity: The Discoursal Construction of Identity in Academic Writing. Amsterdam: John Benjamins. 
Ivanič, R. (2006). Language, learning and identification. Language, culture and identity in applied linguistics, 7-29.

Lea, M.R. \& Street, B. (2006) The "Academic Literacies" Model: Theory and Applications. Theory Into Prcatice, 45(4), 368-377.

Leontiev, A.N. (1978). Activity, consciousness and personality. Englewood Cliffs: Prentice- Hall.

Lillis, T. and Scott, M. (2007). Defining Academic Literacies Research: Issues of Epistemology, Ideology and Strategy. Journal of Applied Linguistics, 4 (1), 5-32.

Nash, W. (1990). The writing scholar: Studies in academic discourse. London: Sage.

Rienecker, L. \& Stray Jorgensen, P. (2003). The (im)possibilities in teaching university writing in the Anglo-American tradition when dealing with continental student writers, in L.A. Björk, G. Braüer, L. Rienecker and P. Stray (eds.). Teaching academic writing in european higher education. Dordrecht: Kluwer Academic Publishers, p. 101-112.

Roth \& Lee (2007). Vygotsky's Neglected Legacy: Cultural-Historical Activity Theory. Review of Educational Research, 77, 186-232.

Russell, D. (1987). Writing Across the Curriculum and the Communications Movement: Some Lessons from the Past. College Composition and Communication, 38 (2), 184-219.

Russell, D. (1991). Writing in the academic disciplines, 1870-1990: A curricular history. Illinois: Southern Illinois University Press.

Russell, D. and Yáñez, A. (2003). Teoría de la Actividad Histórico-Cultural Vygotskiana y la Teoría del Sistema de Géneros: Una Síntesis sobre la Escritura en la Educación Formal y la Escritura en otras Prácticas Sociales. Entre Lenguas, 8, 67-82.

Russell, D.; Lea, M.; Parker, J.; Street, B. and Donahue, T. (2009). Exploring notions of genre in 'academic literacies' and 'writing across the curriculum': approaches across countries and contexts. In C. Bazerman, A. Bonini and D. Figueiredo (eds.). Genre in a Changing World. Perspectives on Writing. Colorado: WAC Clearinghouse/Parlor Press, pp. 459-491.

Street, B. (1999). Academic literacies. In C. Jones, J. Turner and B. Street (eds) Students Writing in the University: cultural and epistemological issues. Amsterdam: John Benjamins.

Street, B. (2009). Academic Literacies approaches to Genre?. Fifth International Symposium on Textual Genre Suties (VSIGET). Published in RBLA Belo Horizonte, 10 (2), 347-361.

Vygotsky, L.S. (1978). Mind in society: The development of higher psychological processes. Cambridge: Harvard University Press. 


\section{Notes}

' You may read more about Universitat Pompeu Fabra and its language policies at www.upf.edu, available in Catalan, Spanish and English.

ii For further information on these two trends, Russell, Lea, Parker, Street \& Donahue (2009) described and compared them.

iii The course materials combined genres written by expert authors (canonically written or not) and novice authors (mainly previous students of the subject).

iv Both quoted from seminar 1 questionnaire.

${ }^{\vee}$ Quoted from the questionnaire from seminar 8.

${ }^{v i}$ According to Ivanic (2004:20), "the capacity to examine critically their own processes of identification gives people agency and control over the contribution they are making to the circulation discourses, and ultimately to the potential transformation of their social world."

Cita del artículo:

Oliva, A.; Milian, M. (2013). First year students' construction of an academic identity in English as a foreign language. Revista de Docencia Universitaria. REDU. Número monográfico dedicado a Academic Writing. Vol.11 (1) Enero-Abril. pp. 59-77 Recuperado el (fecha de consulta) en http://www.red-u.net/

\section{Acerca de las autoras}

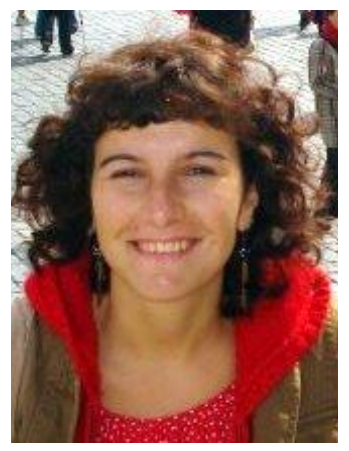

\section{Angels Oliva Girbau}

\section{Universitat Pompeu Fabra}

Facultad de Humanidades y Facultad de Traducción

Mail: angels.oliva@upf.edu

Angels Oliva Girbau es Licenciada en Filología Inglesa por la Universitat Autònoma de Barcelona, tiene un máster de Enseñanza del Inglés como Lengua Extranjera por la Universitat Pompeu Fabra, y es Doctora en Didáctica de la Lengua y la Literatura por la Universitat Autònoma de Barcelona. Trabaja como docente en la UPF desde 2005, donde ha participado en la adaptación al plan Bolonia de asignaturas en lengua inglesa en el grado de Humanidades, en el de Publicidad y Relaciones Públicas y en el de Traducción e Interpretación, y donde tutoriza aprendices de docentes de inglés en las prácticas en institutos de secundaria. Sus líneas de investigación se sitúan en la metodología docente de y en lenguas extranjeras, y en la relación entre identidad, escritura y multilingüismo. Es miembro del grupo GREILI. 


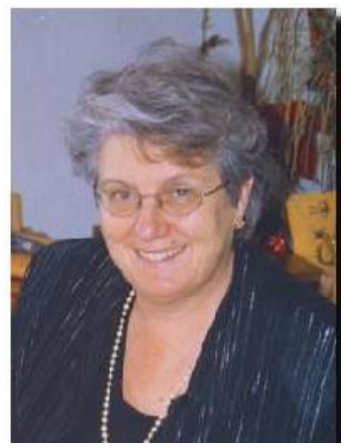

\section{Marta Milian Gubern}

\section{Universitat Autònoma de Barcelona}

Facultad de Educación

Mail: marta.milian@uab.cat

Marta Milian Gubern es Licenciada en Filología (Idiomas Modernos) y en Filología Catalana por la Universidad de Barcelona, y Doctora en Filosofía y Letras por la Universidad Autónoma de Barcelona. Es docente de la Facultad de Ciencias de la Educación de la UAB desde 1980, donde ha participado también como profesora de la enseñanza de la composición escrita, en el programa de Doctorat de Didàctica de la llengua i la Literatura, coordinado por el Departament de Didàctica de la llengua i la literatura. Su trayectoria investigadora se orienta a la enseñanza y aprendizaje de la composición escrita y géneros discursivos, la enseñanza y aprendizaje de la gramática y la actividad/reflexión metalingüística en entornos plurilingües. Es miembro activo del grupo GREAL. 


\title{
Enseñar a escribir textos científico-académicos mediante la revisión colaborativa: El trabajo final de grado en Psicología
}

\author{
Teaching academic writing through collaborative peer review: The undergraduate \\ thesis in Psychology
}

\author{
Mariona Corcelles Seuba \\ Maribel Cano Ortiz \\ Universitat Ramon Llull, España \\ Gerardo Bañales Faz \\ Norma Alicia Vega \\ Universidad Autónoma de Tamaulipas, Méjico
}

\section{Resumen}

En los últimos años, han sido abundantes las propuestas en contextos universitarios diseñadas para mejorar la alfabetización académica de los estudiantes pero pocas se han centrado en la enseñanza de un género tan específico como el artículo de investigación. En el marco del Trabajo Final de Grado de Psicología, el presente estudio analizó una intervención que tuvo por finalidad la mejora de la calidad de la escritura de este género discursivo. La intervención combinó la revisión colaborativa entre pares con la revisión del profesor. Analizamos el tipo y la cantidad de sugerencias propuestas por los compañeros y por el profesor y la aceptación o no de estas sugerencias para conocer su impacto en la calidad final del texto. La muestra se compuso de 35 estudiantes y 8 profesores. Se utilizó un diseño longitudinal expost facto de grupo único con medidas repetidas en el que la variable dependiente fue la calidad del texto y las variables independientes la revisión entre iguales y la del profesor. Los resultados del estudio muestran que esta metodología favoreció la revisión continua del texto lo que repercutió favorablemente en su calidad final. Los compañeros ofrecieron más sugerencias que los profesores y obtuvieron una mayor ratio de aceptación. Sin embargo, estas sugerencias se dirigieron mayoritariamente a la revisión de los aspectos formales mientras que las de los profesores aludieron, en primer lugar, a la coherencia y cohesión del texto, en segundo lugar, a los aspectos formales y por último, a la voz e implicación del lector. Estas últimas sugerencias fueron las que tuvieron una mayor incidencia en la calidad final de los textos.

Palabras clave: enseñanza de la escritura, escritura académica, revisión colaborativa, artículo científico, trabajo final de grado, universidad. 


\begin{abstract}
Last years, numerous proposals have been designed to improve students' academic literacy at the university context but few have focused on teaching a specific genre such as the research article. Within the framework of the undergraduate thesis in Psychology, this study examines an intervention that aimed to improve the writing quality of this type of discourse. Such intervention combined collaborative peer review with the review of the teacher. We analyzed the type and number of suggestions made by peers and by the teacher and its acceptance or rejection to understand its impact on the final text quality. The sample involved 35 students and 8 teachers. A longitudinal design ex-post facto in a single group with repeated measures was used in which the dependent variable was the quality of the text and the independent variables were peer and teacher review. Results of the study showed that this methodology promoted the continuous revision of the text, which affected the quality of the final texts. Peers provided more suggestions than teachers and also had greater acceptance rate. However, these suggestions were mainly directed to the revision of the formal aspects while the teacher's suggestions referred, firstly, to aspects of coherence and cohesion, secondly, to formal aspects and finally, to involvement of the reader and author's position which were aspects that had a greater impact on the final texts quality.
\end{abstract}

Key words: teaching writing; academic writing, research article, undergraduate thesis, University.

\title{
Introducción
}

En las últimas décadas, la importancia de la escritura académica en contextos de aprendizaje universitario ha sido puesta de manifiesto desde diferentes perspectivas de forma reiterada. Por un lado, la investigación ha señalado cómo la escritura contribuye al aprendizaje de diferentes disciplinas y por lo tanto se constituye en una herramienta epistémica (Tÿnajala, Mason y Lonka, 2001); por otro lado, numerosos estudios han demostrado cómo en las diferentes disciplinas se promueven formas particulares de escribir que, en definitiva, están vinculadas a modos específicos de pensar $\mathrm{y}$ de promover conocimiento en las diferentes comunidades académicas (Bazerman, Little, Chavkin, Fouquette, Bethel y Garufis, 2005; Becher, 2001; Berkenkotter y Huckin, 1995; Carlino, 2002; 2003; 2005; Lemke, 1997; Prior, 2006; Russell, 1997; entre otros).

Sin embargo, la investigación también ha puesto reiteradamente de manifiesto que estas formas de pensar y de comunicarse específicas de cada una de las disciplinas no sólo no resultan evidentes para los estudiantes universitarios sino que, además, éstos tienen numerosas y variadas dificultades para concebir y manejar la escritura como herramienta de aprendizaje y de socialización disciplinar (Carlino, 2004; Sabaj, 2009; Solé, Mateos, Miras, Martín, Castells, Cuevas y Gràcia, 2005). En este contexto, la necesidad de promover la alfabetización académica -academic literacy- de los estudiantes universitarios viene siendo argumentada tanto desde perspectivas lingüísticas, como pragmáticas, educativas o psicológicas, entre otras (Carlino, 2005; Castelló, 2008a; Donahue, 2002; Lea y Stierer, 2000; Russell, 1997).

De modo general, desde todas estas perspectivas se comparte la necesidad de que los estudiantes se conviertan en miembros letrados de una determinada comunidad científica, lo que implica conocer y dominar el uso de unas determinadas reglas y convenciones, además de comunicarse y producir conocimiento en el marco de los que dichas convenciones permiten. Aprender una materia tiene que ver con el dominio de los recursos discursivos de cada disciplina y saber usar estos recursos es lo 
que permite que el estudiante gradualmente se erija como miembro de una determinada comunidad (Bazerman, 2004; Bazerman et al., 2005; Sabaj, 2009).

En los últimos años, han sido abundantes las propuestas diseñadas para promover esta alfabetización en diferentes contextos universitarios (Cassany, López y Martí, 2000; Moyano, 2004; Squillari, Bono y Rinaudo, 2003; Vásquez, 2005; entre otros), sin embargo, han sido muy pocos los trabajos que se han centrado en la enseñanza de un género específico como el artículo científico o el informe de investigación (Bazerman, Keranen y Encinas, 2012; Castelló, González y Iñesta, 2010; Castelló y Iñesta, 2012; Castelló, Iñesta y Monereo, 2009; Castelló, Iñesta, Pardo, Liesa, y Martinez-Fernández, 2012; Lonka, 2003). Éste es precisamente el género del que nos ocupamos en este estudio, en el contexto de la escritura del trabajo de final de grado en los estudios de psicología. En el marco de la renovación curricular y metodológica que supone para la universidad española el proceso de incorporación al Espacio Europeo de Educación Superior, la mayoría de los nuevos títulos de grado contemplan la escritura de un texto, comúnmente denominado Trabajo de Final de Grado, cuyas características se asemejan notablemente a las del género académico-científico ya sea en su modalidad de artículo de investigación o de monografía de investigación (Mateo et al., 2009). Probablemente por la especificidad de sus características, que le erigen tanto en vehículo de comunicación especializada como de construcción de saberes, el aprendizaje de este género textual es extremadamente complejo y su enseñanza poco frecuente (Castelló et al., 2012; Sabaj, 2009). La investigación sobre las características que definen a este género discursivo ha puesto de manifiesto que para que se produzca este aprendizaje hay que atender a tres dimensiones definitorias del mismo: a) la estructura y organización de la información, b) la necesidad de dominar los recursos discursivos que permiten a los autores posicionarse en el texto y delimitar su identidad y c) la necesidad de manejar recursos intertextuales que permiten dialogar y discutir con otros autores además de incorporar parte de su discurso y de sus trabajos en el propio.

Además, los estudios que se ocupan de la enseñanza de la escritura han demostrado ampliamente la importancia del conocimiento del proceso de escritura, así como de los procesos de planificación y especialmente de revisión a la hora de escribir textos complejos de mayor calidad. Precisamente, en contextos de educación superior, la revisión colaborativa o entre pares se ha revelado como una de las mejores formas de promover procesos de revisión de alto nivel que, en último término, inciden no sólo en la calidad final de los textos que escriben los estudiantes, sino en la posibilidad de profundizar y adquirir más conocimientos sobre el tema del que se escribe (Castelló et al. 2012; Castelló, González y Iñesta, 2010; Venables y Summit, 2003). Ésta es también la pretensión de nuestro estudio: promover la revisión entre pares como una forma de incidir en la calidad de los textos y en el conocimiento de los estudiantes sobre las características del proyecto de grado.

La investigación sobre escritura ha proporcionado variadas evidencias acerca de algunas de las bondades de la revisión entre iguales en diferentes contextos. En primer lugar, la revisión conjunta conlleva un mayor número de análisis y transformaciones del texto que los que se ponen en marcha en situaciones de revisión individual tanto en estudiantes de primaria como de secundaria o universidad (Allal, 2000; Lindblom- 
Ylänne, Pihlajamäki y Kotkas, 2006). En segundo lugar, tanto en estudiantes de grado como de postgrado o doctorado, la revisión en parejas incrementa la conciencia sobre el proceso de escritura y consiguientemente incide en la regulación del proceso de composición (Lonka, 2003; Castelló, 2008b; Castelló, Gonzalez y Iñesta 2010). En tercer lugar, en todos los contextos la interacción entre iguales facilita el ajuste a la audiencia y, en último término, la consideración de la escritura como una actividad interactiva y dialógica (Berg, 1999; Castelló y Iñesta, 2012; Hui-Tzu, 2006; Nystrand, 1989; Nelson y Schunnn, 2009; Prior, 2006, entre otros).

Sin embargo, no todas las situaciones de revisión entre iguales resultan igualmente útiles cuando se trata de evaluar su impacto en la mejora de la calidad de los textos y los resultados de la investigación no son unánimes al respecto (Cho y Schunn, 2007; Gibbs y Simpson, 2004; Gielen, Peeters, Dochy, Onghena, y Struyven, 2010; Topping, 2010). Así, algunos estudios señalan la necesidad de tener en cuenta la calidad de las ayudas o retroalimentaciones (Liang, 2010), además de la formación previa de los estudiantes revisores (Hui-Tzu, 2006). También los aspectos sociales parecen relevantes a la hora de explicar los resultados negativos de algunos estudios en los que la revisión entre iguales no implicó una mejora de la calidad de los textos. En este sentido, Freedman (1992) pone de manifiesto cómo en algunas ocasiones los alumnos evitan realizar evaluaciones negativas, ya sea porque no quieren asumir el rol de profesores o simplemente por las connotaciones socialmente negativas que este hecho conlleva. También, Yarrow y Topping (2001) muestra que hay que planificar la composición de los grupos o las parejas para que la interacción realmente ayude al aprendizaje.

En conjunto, los resultados contradictorios de la investigación sobre revisión entre iguales parecen estar relacionados con una insuficiente atención al impacto diferencial que pueden tener en los textos variables específicas vinculadas a las características de la revisión que se lleva a cabo (Topping, 2010), de lo que se deduce la necesidad de dedicar mayores esfuerzos al estudio del tipo de comentarios que ofrezcan los compañeros, a su formación como revisores y especialmente al análisis y la categorización de las diferentes tipologías de ayudas que ofrecen los iguales. Esto es lo que nos proponemos en nuestra investigación, cuya finalidad consiste en analizar el impacto de la revisión colaborativa en la escritura del proyecto de grado que los estudiantes de psicología realizan al finalizar sus estudios. Para conseguirlo nos planteamos los siguientes objetivos:

1. Describir y analizar el tipo y la cantidad de sugerencias de cambios propuestas por los compañeros y por los profesores en las diferentes revisiones de los textos.

2. Analizar la aceptación de dichas sugerencias de cambios en las revisiones que los estudiantes hicieron de sus textos para cada tipo de sugerencias y en su totalidad.

3. Valorar si mejora la calidad de los textos realizados por los estudiantes. 


\section{Enseñando a escribir el proyecto de grado: descripción de la intervención}

\section{El contexto de la intervención}

El contexto de intervención se ubica en el denominado espacio de seminario de cuarto curso del grado de Psicología de la Universidad Ramon Llull. El espacio de seminario lo constituyen grupos de 12 a 15 estudiantes y un profesor que se reúnen dos veces por semana en sesiones de 2 horas, con el objetivo de guiar el desarrollo del trabajo de final de Grado, a partir de la implantación de los nuevos planes de estudio ajustados a la normativa del Espacio Europeo de Educación Superior (EEES).

La organización del seminario es de un curso académico con actividades diversas en cada semestre. En el primer semestre, las ayudas se dirigen a la selección de lecturas para el marco teórico, la definición del diseño de la investigación y la planificación de su puesta en práctica. En el segundo semestre, los estudiantes llevan a cabo la recogida y el análisis de datos junto con la escritura del trabajo. Existen dos tipos de proyectos de grado, el de intervención y el de investigación. Los estudiantes escogen el que resulta más adecuado en función de sus preferencias. El primero acostumbra a estar estrechamente vinculado al trabajo realizado en las prácticas finales y consiste en el diseño, descripción y evaluación de una intervención en el ámbito psicológico que el estudiante haya llevado a cabo. El segundo consiste en el diseño y desarrollo de un trabajo de investigación empírico. En ambos casos, el producto final es el de un artículo científico -de investigación o de intervención- cuya escritura resulta altamente problemática para los estudiantes. Estos problemas de escritura básicamente tienen que ver con las dificultades de los estudiantes para establecer una posición clara a lo largo del texto de forma argumentada utilizando de forma ajustada los recursos discursivos y las convenciones propias de los textos científicos en psicología. Por este motivo, diseñamos una intervención para ayudar a los estudiantes a hacer visible su voz en el texto ajustándose a los requisitos de la escritura científica en psicología y, en definitiva, a mejorar la calidad de su escritura académica.

\section{Descripción de la intervención en escritura}

La intervención fue diseñada siguiendo el modelo desarrollado por Castelló (2008a) para la enseñanza de la escritura, inspirado a su vez en varios trabajos previos desarrollados en ocasiones en otros niveles educativos (Camps 2003a; 2003b; Lea y Stierer, 2000). Este modelo atiende a tres momentos del proceso de enseñanzaaprendizaje, estrechamente relacionados, que conllevan actividades diferenciadas y que detallamos a continuación.

\section{Antes de la escritura:}

A lo largo del primer semestre, los estudiantes desarrollaron actividades que implicaban leer para escribir y hablar sobre la escritura (Castelló, 2002). La finalidad de estas actividades era explicar, compartir y discutir las características del género textual (artículo científico en psicología). Para ello, se proporcionó un dossier con 
explicaciones y ejemplos específicos de las características propias de este género, organizado en tres grandes bloques (Castelló, Corcelles, Iñesta, Bañales y Vega, 2011):

a) Los recursos discursivos que sirven al autor de un texto científico para posicionarse y hacer visible su voz -a partir de matizadores, potenciadores, valoraciones o expresiones de autoreferencia- e implicar al lector - utilizando la primera persona del plural, haciendo comentarios o aclaraciones, así como recurriendo al conocimiento compartido, ofreciendo guías o planteando preguntas-. Éstos son los que Hyland, (2002; 2005) considera como los recursos propios de la modalización del discurso científico.

b) La utilización de las citas como herramientas intertextuales y dialógicas diferenciando entre el uso de citas directas, indirectas, integradas o no integradas- (Swales, 1990; Teberosky, 2007).

c) La organización y estructura de la información del artículo científico en distintas secciones -introducción, método, resultados y discusión- (Swales, 1990, 2004).

Para entender el impacto de estas características en los textos y en el lector, se leyeron y analizaron de forma compartida artículos y textos previamente escritos por los estudiantes, buscando en ellos ejemplos del uso de determinados recursos discursivos y discutiendo su función en el texto, es decir, cómo los autores los utilizaban para hacer visible su voz, cómo citaban y cómo estructuraban el artículo.

En base a la discusión y el análisis de estos textos se consensuó una guía para ayudar a los estudiantes a tener presentes los aspectos mencionados al escribir sus textos.

\section{Durante la escritura}

En el segundo semestre, los estudiantes empezaron a escribir sus textos con la ayuda de la guía de escritura para regular su proceso escrito y ajustarse a las características del género. Se planificaron tres entregas del texto escrito: una inicial al finalizar el primer mes de trabajo (en la que se esperaba que estuviera escrito, como mínimo, el apartado de la introducción del artículo), una intermedia al finalizar el segundo mes (con el artículo casi completo, o, al menos, con la introducción, método, resultado y conclusión) y una final al tercer mes de trabajo (con el artículo ya terminado en su versión final). Después de la primera y segunda entrega los estudiantes, organizados en 16 parejas y un trío, tenían que revisar el texto de su compañero, haciendo uso de la revisión por pares como mecanismo de ayuda entre iguales para la regulación de la escritura. Para ello, se explicaron las características del método de revisión por pares, los profesores constituyeron las parejas de revisión a partir de criterios de afinidad temática y rendimiento similar y se dedicó una sesión al inicio del semestre a formar a los estudiantes en la tipología de sugerencias que podían proporcionar a sus compañeros. En el caso del trío, para favorecer la interdependencia entre los estudiantes, se organizó evitando que hubiera una relación recíproca entre el texto que cada alumno revisaba y el revisor del texto del alumno, es decir, el alumno 1 revisó al alumno 2 , el 2 al 3 y el 3 al 1. 
Después de cada entrega, las parejas de estudiantes se ofrecían de manera recíproca sugerencias de cambio respecto al texto escrito a partir de comentarios que inserían en el texto usando el programa informático de revisión Word y que discutían en las sesiones de seminario. Por otra parte, el profesor también realizaba sus comentarios sobre el texto en las dos entregas que además podían complementarse con tutorías individuales.

\section{Después de la escritura}

Una vez recibidas las sugerencias del compañero y del profesor, los estudiantes revisaban sus textos y los modificaban según su criterio y estas revisiones se incorporaban a la siguiente entrega del texto.

\section{Método}

\section{Participantes}

La muestra analizada en este estudio estaba compuesta por 35 estudiantes $(71,4 \%$ mujeres y 28,6\% hombres) agrupados en 8 seminarios con 8 profesores. Los estudiantes se organizaron en 16 parejas y un trío. La selección se realizó entre todos los estudiantes que cursaban seminario de cuarto curso de la Facultad de Psicología $(n=89)$ y los criterios para llevarla a cabo fueron los siguientes: estudiantes que hubieran escogido realizar el proyecto de investigación, que sus profesores aceptaran participar en el proyecto y que además hubiesen asistido a todas las sesiones y cumplido con todas las entregas y revisiones del texto.

\section{Procedimiento de recogida de datos}

Se utilizó un diseño longitudinal ex-post facto de grupo único con medidas repetidas en el que la variable dependiente fue la calidad del texto y las variables independientes la revisión entre iguales y la del profesor.

Se recogieron las versiones electrónicas de los textos elaborados por los alumnos y los comentarios realizados por los compañeros y por el profesor, que los estudiantes colgaban en la plataforma virtual de la Universidad, como parte del proceso de enseñanza-aprendizaje. Esto implica que se analizaron dos versiones de los textos elaborados por los alumnos correspondientes a la primera y última entrega (inicial y final) y los comentarios realizados por los compañeros y profesores en la primera y segunda entrega (no se realizaron comentarios en la tercera ya que representaba el texto final).

\section{Procedimiento de análisis de los datos}

Teniendo en cuenta las características del género del artículo científico (Iñesta, 2009; Swales, 1990, 2004) y la propuesta para su análisis que realizamos en estudios anteriores (Castelló et al., 2011), diseñamos una rúbrica (ver Anexo 1) que constaba de 
varios criterios de evaluación agrupados en las dimensiones que se detallan a continuación:

a) Estructura del género discursivo: Valoramos el grado en que el texto se organizaba en distintas secciones -introducción, método, resultados y discusión /conclusiones - siguiendo la estructura propia de un artículo científico. A continuación, se detallan los criterios de evaluación para cada una de estas secciones:

a.1) Título: Los criterios se referían al grado en que se presentaba un título claro y atractivo que sintetizara el tema o variables principales del estudio dentro de una extensión adecuada (máximo 2 frases).

a.2) Resumen: Evaluamos el grado en que se presentaba de manera clara y concisa una síntesis del contenido del estudio en términos de objetivos, participantes, método, resultado y conclusiones sin utilizar más de 250 palabras.

a.3) Introducción: En este caso, los criterios se referían al grado en que la estructura de la introducción estaba organizada siguiendo los 3 movimientos de Swales, (1990, 2004), para progresar en la argumentación y explicación del contenido siguiendo el género discursivo propio del artículo científico. Dichos movimientos hacen referencia a: 1) "Establecer un territorio": el autor debería mostrar la relevancia del ámbito de investigación haciendo referencia a los autores previos y a las generalizaciones más relevantes sobre el tema objeto de estudio; 2) "Establecer un nicho": el autor debería situar su estudio en el ámbito de investigación presentado anteriormente, revisando los estudios previos e identificando el "vacío" en la investigación previa; 3) Por último, "Ocupar el nicho": el autor debería mostrar claramente cuáles son los propósitos, objetivos, preguntas de investigación y/o hipótesis del estudio para mostrar al lector cómo éste ocupará el vacío en el ámbito de la investigación previa.

a.4) Método: Tuvimos en cuenta el grado en que se describía claramente la muestra, los materiales e instrumentos de recogida de datos y el procedimiento utilizado en el estudio.

a.5) Resultados: Los criterios se referían al grado en que en el texto se presentaban claramente los resultados de acuerdo a las preguntas $u$ objetivos del estudio.

a.6) Discusión y conclusiones: Evaluamos el grado en que se presentaban claramente las implicaciones de los resultados obtenidos con respecto a los objetivos y/o hipótesis del estudio, señalando las semejanzas y diferencias en relación a otros estudios, las limitaciones y los aspectos para futuras investigaciones.

b) Coherencia: Los criterios se referían al grado en que las ideas se organizaban evidenciando un eje organizador de la información.

c) Cohesión: Valoramos el uso de conectores adecuados (no repetitivos) para facilitar el enlace de los párrafos y la progresión lógica de la información.

d) Uso de los recursos discursivos para manifestar el posicionamiento del autor (modalización): Los criterios se referían a la presencia de recursos discursivos como: matizadores y potenciadores, valoraciones de las afirmaciones $y$ 
elementos de autoreferencia que permitieran de manera clara manifestar la voz del autor (Hyland, 2002; 2005).

e) Uso de los recursos discursivos para implicar al lector (modalización): Evaluamos el grado en que se manifestaba la presencia de recursos discursivos para implicar al lector como: el uso de la primera persona del plural, la realización de comentarios, aclaraciones y preguntas, el ofrecimiento de guías al lector y las referencias al conocimiento compartido (Hyland, 2002; 2005).

f)Intertextualidad: Valoramos la utilización de citas indirectas (integradas y no integradas) y directas de manera correcta y equilibrada a lo largo del texto (Swales, 1990; Teberosky, 2007).

g) Aspectos formales: Los criterios se referían al grado en que los textos presentaban problemas a nivel de gramática, ortografía y puntuación.

Cada dimensión se evaluó con una escala Likert del 1 al 5 (valores de menor a mayor puntuación) de acuerdo a los criterios de evaluación especificados anteriormente y en la rúbrica (ver Anexo 1). Estos criterios se revisaron utilizando textos similares a los del estudio y se efectuaron diversos ajustes a los descriptores hasta alcanzar el consenso.

Una vez consensuados los criterios de evaluación de cada dimensión por los investigadores (autores del presente artículo), tres jueces - dos autores del artículo y un estudiante de doctorado implicado en el proyecto y entrenado previamente-, analizaron conjuntamente la calidad del $30 \%$ de los textos totales, llegando a un acuerdo del $97 \%$. Los casos dudosos fueron discutidos hasta llegar a un consenso en la valoración. Posteriormente, se procedió de manera independiente al análisis de la totalidad de los textos restantes.

En cuanto al análisis de la cantidad y tipos de sugerencias de cambio realizados tanto por los compañeros como por los profesores, en primer lugar, se analizaron todos los comentarios $y$, después de discutir el tipo de cambio que promovían se realizaron las categorías. Éstas fueron agrupadas en tres dimensiones consensuadas siguiendo el procedimiento de la grounded theory. Las tres dimensiones establecidas son las siguientes:

a) Comentarios que se refieren a la falta de coherencia y cohesión del texto: En esta dimensión incluimos las categorías que hacían referencia a la coherencia (añadir información relevante o eliminar información irrelevante, estructurar la información siguiendo un orden lógico), y a la cohesión (conectar diferentes frases, evitar repeticiones de palabras, utilizar conectores lógicos, cambiar el orden de la información, elaborar o clarificar frases confusas y clarificar los objetivos).

b) Comentarios que se refieren a la voz e implicación al lector (modalización): En esta dimensión incluimos las categorías que hacían referencia a la utilización de recursos para posicionarse y hacer visible la voz del autor en el texto (justificar el propio punto de vista -con citas y/o argumentos-, utilizar enfatizadores, matizadores o marcadores de actitud), y a las que referían a la 
implicación al lector (metacomentarios de guía al lector, autoreferencia, preguntas al lector o referencias al conocimiento compartido).

c) Comentarios que se refieren a aspectos formales del texto: En esta dimensión incluimos categorías que hacían referencia a la tipografía y al estilo (eliminar un espacio, poner negrita, poner cursiva, tipo de letra, sangría, errores de letras, etc.), a la gramática y sintaxis (concordancia de género y número, orden de las palabras, etc.), a la ortografía y puntuación (normas ortográficas, acentos, comas, puntos, paréntesis, etc.) y, por último, a la revisión formal de citas (incumplimiento de la normativa APA).

Posteriormente, tres jueces - dos autores del presente artículo más un estudiante de doctorado implicado en el proyecto- analizaron de nuevo los comentarios tratando de adscribirlos a las tres dimensiones establecidas. En este caso, la ratio de acuerdo llegó al 98\%. Los pocos casos en los que persistían dudas fueron discutidos por todo el equipo hasta que se obtuvo un acuerdo en la adscripción a una determinada categoría.

\section{Resultados}

Hemos organizado la presentación de los resultados en función de su respuesta a cada uno de los objetivos planteados en el estudio.

En relación con el primer objetivo, en la Tabla n. 1 mostramos una síntesis del tipo y la cantidad de sugerencias de cambios efectuadas por estudiantes y profesores en la primera y segunda revisiones del texto.

\begin{tabular}{|c|c|c|c|c|c|c|}
\hline \multirow{2}{*}{$\begin{array}{c}\text { Tipo de } \\
\text { sugerencia }\end{array}$} & \multicolumn{2}{|c|}{ Revisión 1} & \multicolumn{2}{|c|}{ Revisión 2} & \multicolumn{2}{|c|}{ Total } \\
\hline & Compañero & Profesor & Compañero & Profesor & Compañero & Profesor \\
\hline $\begin{array}{l}\text { Cohesión y } \\
\text { Coherencia }\end{array}$ & $285(29,6 \%)$ & $82(58,2 \%)$ & $203(28,1 \%)$ & $78(63,9 \%)$ & $488(29 \%)$ & $160(60,8 \%)$ \\
\hline $\begin{array}{c}\text { Voz e implicación } \\
\text { al lector }\end{array}$ & $106(11 \%)$ & $15(10,6 \%)$ & $52(7,2 \%)$ & $26(21,3 \%)$ & $158(9,4 \%)$ & $41(15,6 \%)$ \\
\hline $\begin{array}{l}\text { Aspectos } \\
\text { Formales }\end{array}$ & $572(59,4 \%)$ & $44(31,2 \%)$ & $467(64,7 \%)$ & $18(14,8 \%)$ & $1039(61,6 \%)$ & $62(23,6 \%)$ \\
\hline Total & 963 & 141 & 722 & 122 & 1685 & 263 \\
\hline
\end{tabular}

Tabla n. 1. Tipo y cantidad de sugerencias de cambios efectuadas por los compañeros y por los profesores

Como podemos observar, los compañeros sugieren más cambios que los profesores en ambas revisiones y en todas las categorías (cohesión y coherencia; voz e implicación al lector y en los aspectos formales). Tanto en el caso de los compañeros como en el de los profesores, el menor número de sugerencias se refieren a la utilización de recursos para posicionarse y hacer visible la voz del autor en el texto y para implicar al lector, mientras que las sugerencias relativas a cambios formales son las más frecuentes en las revisiones de los compañeros (tanto en la primera como en la segunda revisión). En cambio, en las revisiones de los profesores, se hace más hincapié 
en las cuestiones referidas a la cohesión y coherencia, tanto en la primera como en la segunda revisión.

Esta diferencia en el tipo y cantidad de sugerencias, tal y como se observa en la tabla n. 2, es significativa en ambas revisiones tanto para las revisiones entre compañeros $(t(35)=6.83, p<.001)$ como para las revisiones de los profesores $(t(35)=$ $6.16, p<.001)$.

\begin{tabular}{|c|c|c|c|c|}
\hline \multicolumn{5}{|c|}{ Media de sugerencias de cambios que recibe cada estudiante por parte del compañero ( $n=35$ ) } \\
\hline & Media & Desviación estándar & $t$ & $p$ \\
\hline Revisión 1 & 27.51 & 20.32 & 6.83 & $.000^{* * *}$ \\
\hline Revisión 2 & 20.62 & 16.45 & & \\
\hline \multicolumn{5}{|c|}{ Media de sugerencias de cambios que recibe cada estudiante por parte del profesores ( $n=35$ ) } \\
\hline Revisión 1 & 4.02 & 4.52 & & \\
\hline Revisión 2 & 3.48 & 2.67 & & \\
\hline
\end{tabular}

Fuente: elaboración propia

Tabla n. 2. Significación respecto a la cantidad de sugerencias de cambios realizadas por los compañeros y profesores entre la primera y la segunda versión.

Nota: $* * *$ valores significativos $p<.001$

El análisis cualitativo de la tipología de comentarios y sugerencias de cambios pone de manifiesto que los compañeros son más específicos y concretos en la formulación de sus apreciaciones lo que explicaría la mayor cantidad de sugerencias. Así, en el caso de los comentarios relativos a la necesidad de mejorar la coherencia y cohesión del texto, los estudiantes detectaron principalmente dificultades referidas a la necesidad de ampliar o sintetizar el contenido y a la falta de claridad en frases confusas, tal como ilustran los siguientes ejemplos:

"Podrías poner algún ejemplo de esto, de algún profesional que no lo comparta; y ya que citas el DSM-IV, poner las características diagnósticas basadas en el mismo y complementarlas con la definición que pones a continuación" (S 25)

"Para mi gusto, también podrías comentar otros trabajos que hayan estudiado algo similar (tal y como lo hiciste en la introducción) que corroboren también tus hipótesis"( $\mathrm{S}$ 26)

"Creo que se tendría que arreglar esta frase, me parece que no queda claro a qué conduce" (S 14)

En el caso de los profesores, los comentarios relativos a ésta dimensión fueron menos concretos y muchos de ellos se realizaron en formato de pregunta requiriendo de justificación. Aquellos más frecuentes hicieron referencia a dificultades vinculadas a la elaboración y estructuración lógica de la información, a la utilización de los 
conectores y a la clarificación de objetivos, tal y como se muestra en los siguientes ejemplos:

"Hace falta explicitar más el nicho" (P3)

"El texto está desorganizado, hace falta más orden y estructura. El primer párrafo se podría enlazar con el tercero, algunas ideas se repiten, hace falta sintetizar. Esto sólo se puede hacer definiendo claramente el objetivo y pensando en la estructura en función de este objetivo" (P6)

"¿Por qué pones la definición del TDAH aquí? Queda poco cohesionado con el texto. Pasa lo mismo con "HHSS", mejor realizar una introducción que relacione los dos temas" (P1)

"Hace falta definir el objetivo específico i el vacío o problema al que se quiere responder antes de decidir cómo se organizará la información"

En el caso de los comentarios relativos a la voz e implicación al lector (modalización), si bien los que refieren a manifestar la voz del autor fueron muy escasos tanto en el caso de los estudiantes como en los profesores, los que realizaron los compañeros, en casi todos los casos, se relacionaron con recursos destinados a implicar al lector, tal como ilustran los siguientes ejemplos:

"¿Lo pones en cursiva para vincular al lector con la pregunta de después (eficacia terapia - eficacia supervisiones) o para qué?" (S 15)

"Está bien que hagas pensar/reflexionar un poco al lector sobre el tema con una pregunta." (S 22)

"En este párrafo, para reconducir el tema de la depresión, que es lo que nos interesa, añade algo como: "Volviendo al tema de la... bla, bla, bla" para que así vayas situando al lector. iPiensa-Io!" (S 25)

En cambio, en el caso de los profesores, además de estos comentarios para implicar al lector, se observaron comentarios referidos a las dificultades para mostrar el posicionamiento del autor y para justificar sus ideas mediante citas o argumentos, como podemos ver en los siguientes ejemplos:

"Faltaría una redacción introductoria en el apartado de resultados para guiar al lector." (P2)

"Si añades unos párrafos-guía (por ejemplo: -Hemos organizado en función de...- o -En primer lugar revisaremos... después presentaremos... y finalmente....- será mucho más fácil seguir la voz del autor y entender la estructura del texto." (P4)

"-En opinión del autor...- No, mejor poner -Consideramos...- "(P5)

"Se trata de una escritura aún elaborativa: mucho resumen y poca voz del autor."(P1)

"Podrías argumentar mejor esta idea y poner una cita para dar fuerza a tu posicionamiento." (P3)

Por último, respecto a los comentarios relativos a los aspectos formales, los estudiantes detectaron mayoritariamente errores en la ortografía y la puntuación así como incumplimientos de la normativa APA en relación a la citación, tal y como muestran los siguientes ejemplos:

"Pienso que se tendría que quitar esta coma." (S13) 
"Reemplaza el "sobretodo" por el "más común". Quita el punto y pone coma y la "p" de mayúscula a minúscula." (S29)

"Tendrías que poner la bibliografía en orden alfabético según normativa APA." (S35)

"Al citar a un autor, no hace falta poner el nombre; solo apellido y año "Subirats (2004)." (S30)

En el caso de los profesores, los comentarios más frecuentes respecto a esta categoría, también hicieron referencia a los mismos aspectos: errores en la aplicación de la normativa APA (en la primera versión de los textos), ortográficos y de puntuación:

"Tienes que revisar la normativa APA en las referencias, especialmente la de las revistas. Se pone "Referencias bibliográficas" y no "bibliografía"." (P3)

"Normativa APA: En cursiva ponemos el volumen pero no el número de la revista." (P2)

"Numerar las tablas y citarlas en el texto dentro del apartado de resultados." (P7)

"Vigilar el tema de la ortografía y la puntuación" (P8)

En relación al segundo objetivo, relacionado con el análisis de la aceptación de las sugerencias de cambios, en la Tabla n. 3, podemos observar, en términos generales, los porcentajes de las aceptaciones tanto en la primera revisión como en la segunda revisión entre compañeros y el profesor.

\begin{tabular}{|c|c|c|c|}
\hline \multicolumn{4}{|c|}{ Total de sugerencias recibidas y total de aceptadas Revisión 1} \\
\hline \multicolumn{2}{|c|}{ Compañeros } & \multicolumn{2}{|c|}{ Profesor } \\
\hline Total de recibidas & Total de aceptadas & Total de recibidas & Total de aceptadas \\
\hline 963 & $698(72,48 \%)$ & 141 & $98(69,5 \%)$ \\
\hline \multicolumn{4}{|c|}{ Total de sugerencias recibidas y total de aceptadas Revisión 2} \\
\hline \multicolumn{2}{|c|}{ Compañeros } & \multicolumn{2}{|c|}{ Profesor } \\
\hline Total de recibidas & Total de aceptadas & Total de recibidas & Total de aceptadas \\
\hline 722 & $473(65,5 \%)$ & 122 & $52(42,6 \%)$ \\
\hline \multicolumn{4}{|c|}{ Total de sugerencias recibidas y total de aceptadas entre las dos revisiones } \\
\hline 1685 & $1171(69,4 \%)$ & 263 & 150 (57 \%) \\
\hline
\end{tabular}

Tabla n.3. Número total de sugerencias recibidas y aceptadas de los compañeros y profesores

El porcentaje de aceptación de las sugerencias de los compañeros fue, en todos los casos, más elevado que las del profesor. En referencia a las sugerencias aceptadas del profesor podemos observar una tendencia a una menor aceptación de las sugerencias de cambio en la segunda revisión $(42,6 \%)$ respecto a la primera revisión (69,5\%).

Respecto al impacto de las sugerencias de cambio aceptadas para cada dimensión de análisis, la Tabla n. 4 recoge los porcentajes, medias y desviaciones 
estándar para la primera y segunda revisión de los textos, tanto de los compañeros como del profesor.

\begin{tabular}{|c|c|c|c|c|c|c|}
\hline & \multicolumn{6}{|c|}{ Sugerencias aceptadas Revisión 1} \\
\hline & \multicolumn{3}{|c|}{ Compañeros $(n=698)$} & \multicolumn{3}{|c|}{ Profesores $(n=98)$} \\
\hline & Porcentaje & Media & $D S^{1}$ & Porcentaje & Media & $D S^{1}$ \\
\hline Cohesión y Coherencia & $189(27 \%)$ & 65.62 & 30.36 & $51(52 \%)$ & 54.60 & 38.15 \\
\hline Voz e implicación al lector & $82(11,8 \%)$ & 59.26 & 42.48 & $7(7,2 \%)$ & 42.30 & 49.35 \\
\hline \multirow[t]{4}{*}{ Aspectos Formales } & $427(61,2 \%)$ & 77.38 & 26.01 & $40(40,8 \%)$ & 96.43 & 6.79 \\
\hline & \multicolumn{6}{|c|}{ Sugerencias aceptadas Revisión 2} \\
\hline & \multicolumn{3}{|c|}{ Compañeros $(n=473)$} & \multicolumn{3}{|c|}{ Profesores $(n=52)$} \\
\hline & Porcentajes & Media & $D S^{1}$ & Porcentajes & Media & $D S^{1}$ \\
\hline Cohesión y Coherencia & $113(23,9 \%)$ & 52.97 & 34.75 & $34(65,4 \%)$ & 42.37 & 35.22 \\
\hline Voz e implicación al lector & $27(5,7 \%)$ & 41.02 & 41.86 & $11(21,1 \%)$ & 38.59 & 44.46 \\
\hline Aspectos Formales & $333(70,4 \%)$ & 63.50 & 30.99 & $7(13,5 \%)$ & 32.14 & 46.43 \\
\hline
\end{tabular}

Tabla n. 4. Descripción de la cantidad de sugerencias aceptadas de tutores y compañeros en la primera y Nota: $1=$ Desviación estándar segunda revisión

Así pues, en la primera revisión, del total de sugerencias aceptadas de los compañeros $(n=698)$ se incorporaron con mayor frecuencia las referidas a los aspectos formales (61.2\%), y en menor porcentaje a la coherencia y cohesión (27\%) y a la voz e implicación al lector (5,7\%). En cambio, del total de sugerencias aceptadas por parte de los profesores $(n=98)$, las que se incorporaron con mayor frecuencia fueron las relativas a la cohesión y coherencia (52\%), en segundo lugar, las que referían a los aspectos formales (40.8\%) y por último, a la voz e implicación al lector (7,2\%).

Además, la aceptación del tipo de sugerencias en la segunda revisión fue similar a la primera. En el caso de los compañeros ( $n=473$ ), los aspectos formales fueron las sugerencias que se aceptaron en mayor porcentaje (70.4\%), seguido de los aspectos relacionados con la cohesión y la coherencia (23.9\%), y, en tercer lugar de los relacionados con la voz e implicación al lector (5.7\%). Respecto a las sugerencias aceptadas del profesor $(n=52)$, se incorporaron mayoritariamente los aspectos de cohesión y coherencia (65.4\%) y en menor medida los que tenían que ver con la voz e implicación del lector (21.1\%) y aspectos formales (13.5\%).

Con la finalidad de analizar si los cambios entre la primera y la segunda revisión eran significativos, comparamos las medias de cada una de las dimensiones de análisis ( $t$ test de medidas repetidas). Como se observa en la Tabla $n$. 5 , respecto a la aceptación de las sugerencias de los compañeros, existen diferencias significativas entre la primera revisión y la segunda respecto a la cohesión y coherencia (t (29) = - 
2.096; $p=.045)$. Estas diferencias indican que se incorporaron menos sugerencias en la segunda revisión, dado que la media de aceptaciones en la segunda revisión fue inferior a la de la primera (ver Tabla n. 4). En las otras dimensiones de análisis -voz e implicación al lector y aspectos formales-, no existen diferencias significativas en los cambios entre la primera y segunda versión.

Respecto a los profesores, existen diferencias significativas en los cambios relativos a aspectos formales ( $t(4)=-9.196 ; p=.001$ ). En este caso, la media de aceptación de sugerencias fue inferior en la segunda revisión (ver Tabla n. 4), es decir en la segunda revisión se incorporaron menos sugerencias sobre los aspectos formales. En las otras dimensiones de análisis -cohesión y coherencia y voz e implicación al lector- no existen diferencias significativas.

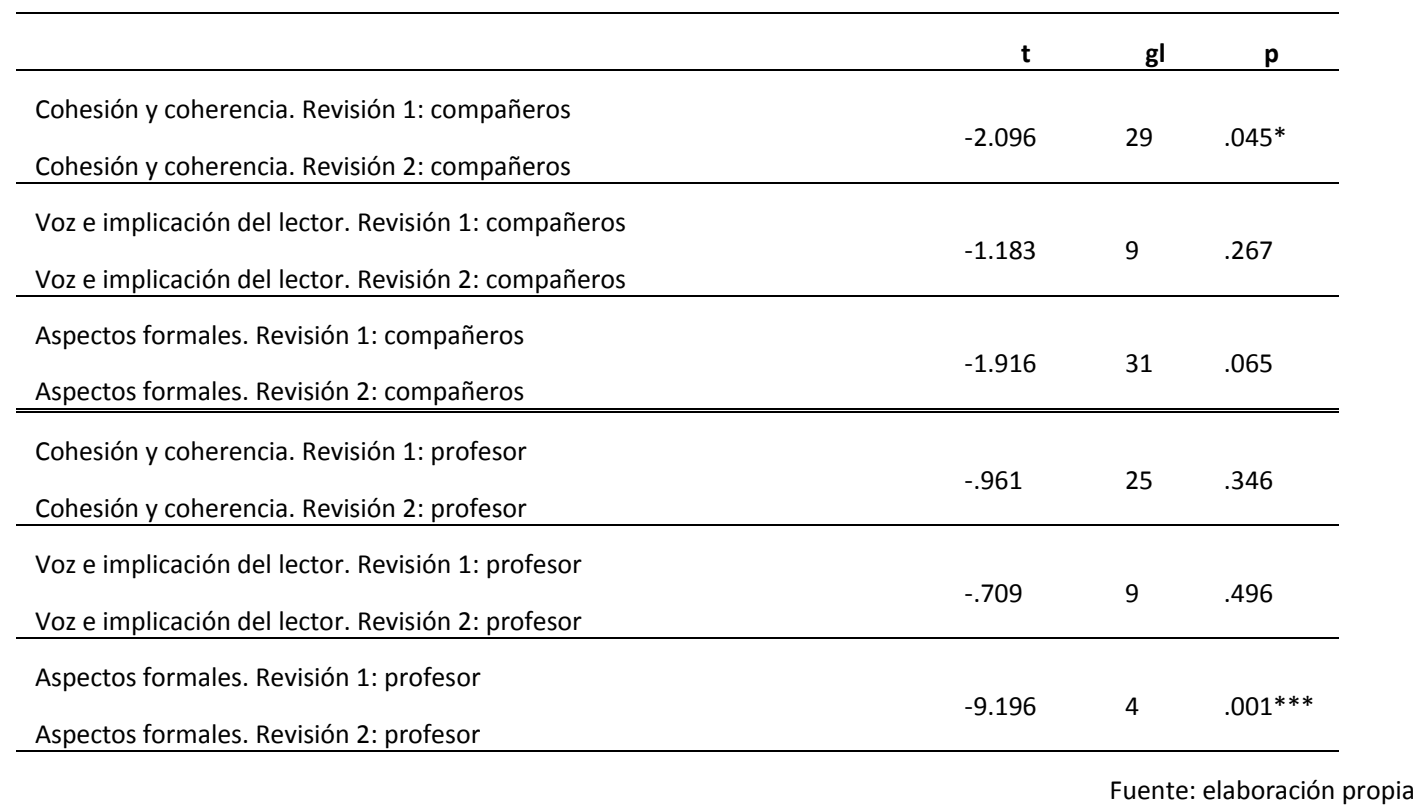

Tabla n. 5. Prueba t de medidas repetidas: cambios aceptados en la revisión 1 y 2 entre compañeros y profesor

Nota: *valores significativos $\mathrm{p}<.05 ; * * *$ valores significativos $\mathrm{p}<.001$

Finalmente, respecto al tercer objetivo del estudio, se agruparon los resultados de las diferentes dimensiones contenidas en la rúbrica (ver anexo 1) dada su alta correlación, obteniendo una medida global de calidad. Los resultados de la calidad global de los textos (con un valor máximo de 5 ) indican que los textos finales fueron significativamente mejores que los iniciales $(t(35)=6.88 ; p<.001)$ (ver Tabla $n .6)$.

\begin{tabular}{|c|c|c|c|c|c|}
\hline \multicolumn{2}{|c|}{ Texto inicial (versión 1) } & \multicolumn{2}{|c|}{ Texto final (versión 2) } & & \\
\hline \multicolumn{2}{|c|}{$\mathrm{N}=35$} & \multicolumn{2}{|c|}{$\mathrm{N}=35$} & \multirow[b]{2}{*}{$\mathbf{t}$} & \multirow[b]{2}{*}{ p } \\
\hline$M$ & DS & $M$ & DS & & \\
\hline 2.46 & $(.86)$ & 3.35 & $(.92)$ & 6.88 & $.000 * * *$ \\
\hline
\end{tabular}

Fuente: elaboración propia

Tabla n. 6. . Prueba de muestras relacionadas respecto a la calidad de los textos en sus versiones iniciales y finales.

Nota: ${ }^{* * *}$ valores significativos $p<.001$ 
El análisis detallado en función de las dimensiones de aceptación de sugerencias de cambio (coherencia y cohesión, voz e implicación del lector y aspectos formales) permite puntualizar estos resultados. La relación entre la aceptación de los cambios en los aspectos formales o los relativos a la cohesión y coherencia y la calidad de los textos no es significativa. En cambio, la aceptación e incorporación de las sugerencias respecto a la voz e implicación del lector correlacionan significativamente con la mejora en la calidad de los textos, tanto en la primera como en la segunda revisión (ver Tabla n. 7). Así pues, los aspectos relacionados con la modelización del discurso han supuesto mejoras significativas en la mejora de la calidad global de los textos finales.

\begin{tabular}{lcccc}
\hline & $\begin{array}{c}\text { Calidad } \\
\text { versión 1 }\end{array}$ & $\begin{array}{c}\text { Calidad versión } \\
1 \text { Voz }\end{array}$ & $\begin{array}{c}\text { Calidad } \\
\text { versión 2 }\end{array}$ & $\begin{array}{c}\text { Calidad versión } \\
\text { 2 Voz }\end{array}$ \\
\hline Calidad del texto en la versión 1 & & $.763^{* * *}$ & .196 & .165 \\
$\begin{array}{l}\text { Calidad del texto en la versión 1: } \\
\text { Voz e implicación del lector } \\
\text { Calidad del texto en la versión 2 }\end{array}$ & & & & .331 \\
\end{tabular}

Fuente: elaboración propia

Tabla n. 7. Correlación de la calidad de los textos entre la versión 1 y la versión 2 en función de las sugerencias de cambio aceptadas respecto a la voz e implicación del lector.

Nota: $* *$ valor significativo $p<.01 ; * * *$ valores significativos $p<.001$.

\section{Discusión y conclusiones}

En el presente estudio hemos analizado una intervención para la mejora de la escritura académica en estudiantes de grado de psicología que combina la revisión colaborativa entre pares con la revisión del profesor a lo largo del proceso de escritura. Los resultados del estudio muestran que la metodología adoptada favoreció la revisión continua del texto incidiendo positivamente en la calidad de los textos finales y reafirma estudios previos que también coinciden en considerar que una mayor revisión del texto incide en la mejora de la calidad final del texto (Castelló et al., 2012; Castelló, González y Iñesta, 2010; Paulus, 1999; Venables y Summit, 2003).

Sin embargo, más allá de constatar esta mejora de los textos finales, nuestro objetivo se cifró en discernir los aspectos de la revisión que efectivamente se vinculan a la mejora de la escritura. Para ello, analizamos las características de las sugerencias ofrecidas tanto por los compañeros como los profesores, así como el grado de aceptación de las mismas por parte de los escritores.

En este sentido, un primer resultado del estudio indica que los compañeros fueron más prolijos y ofrecieron más sugerencias de cambio que los profesores. Este resultado pone de manifiesto que la implicación y el compromiso de los estudiantes en la revisión recíproca de sus textos fue elevada lo que refuerza el argumento de algunos autores (Yarrow y Topping, 2001; Topping, 2005; Topping, 2010) relativo a la importancia de considerar la revisión entre pares como un recurso que enriquece la tarea del profesor y a la vez la de los estudiantes. 
No obstante, a pesar que los compañeros ofrecieron más sugerencias que los profesores, resulta relevante constatar que éstas difirieron significativamente en cuanto a su tipología. Mientras que las sugerencias de los compañeros se centraron en los aspectos formales, las de los profesores fueron preferentemente dirigidas a la mejora de la coherencia y la cohesión del texto.

También fue diferencial la aceptación de dichas sugerencias y su consiguiente impacto en la revisión y mejora de los textos. Los estudiantes aceptaron en mayor proporción las sugerencias de los compañeros cuando hacían referencia fundamentalmente a los aspectos formales del texto. En cambio, los cambios relacionados con los aspectos de coherencia y cohesión del texto, la presencia y voz del autor en el texto o los relacionados con la implicación del lector, de mucho más calado e impacto en la calidad final del texto, estuvieron mayoritariamente vinculados a la aceptación de las sugerencias de los profesores. Si bien es cierto que determinados estudios han enfatizado el mayor ajuste que en determinadas situaciones puede suponer la mediación entre iguales dado que los estudiantes comparten un marco similar de experiencia que les permite ser más directos y utilizar un lenguaje más próximo y más sensible a las dificultades del aprendiz (Good y Brophy, 1987; Vigotsky, 1995; Duran y Monereo, 2012), resulta también evidente que un mayor dominio de los recursos discursivos es relevante cuando se trata de escribir textos complejos como el proyecto final de grado. En este caso, los profesores no sólo disponían de mayor dominio de estos recursos, sino que los estudiantes probablemente les atribuían de forma clara más credibilidad y, consecuentemente, hicieron más caso de sus sugerencias, especialmente en lo relativo a aspectos relevantes.

Como ya hemos comentado, no todas las situaciones de revisión colaborativa resultan igualmente útiles ni beneficiosas. Por este motivo, diversos estudios recomiendan la formación de los estudiantes en calidad de revisores (Hui-Tzu; 2006; Liang, 2010 y Topping, 2010) algo que también tuvimos en cuenta en nuestro estudio, aunque, a la luz de los resultados, la formación realizada no permitió a los estudiantes ir mucho más allá de las cuestiones formales. Es este sentido, consideramos que en la formación inicial se debería incidir más en la reflexión sobre la tipología y la forma de sugerirse los cambios para que ésta fuera realmente un mecanismo de ayuda al proceso metalingüístico de la composición escrita. También es posible que se necesitara más tiempo del que dispusimos para que los estudiantes pudiesen incorporar todos los recursos discursivos a su repertorio de revisiones, pero también es probable que resulte más difícil de conseguir dado que ésta era la primera vez que se enfrentaban a un reto tan complejo como la escritura del proyecto de grado y que, a la vez participaban en una propuesta de revisión colaborativa. Las exigencias de la escritura de textos académico-científicos como el que tenían que elaborar estos estudiantes requiere, además del dominio de recursos discursivos propios, conocer a la comunidad de referencia para poder dialogar con sus autores, desarrollar una identidad como autores diferente de la del estudiante que escribe y entender el texto como un artefacto que evoluciona con la propia actividad de escritura (Prior, 2006). Estos son retos complejos que exigen que los estudiantes se enfrenten y sean capaces de resolver importantes tensiones y contradicciones que aparecen cuando les pedimos que escriban para $-y$ desde- la comunidad profesional o científica de referencia pero 
desde su condición de estudiantes y, por lo tanto, en el marco de la comunidad académica. Los resultados de algunos trabajos recientes muestran que estas contradicciones se resuelven sólo cuando se instaura un proceso continuado de reflexión que permita a los estudiantes tomar consciencia de los mismos y regular su actividad en consecuencia (Castelló y Iñesta, 2012; Castelló, Iñesta y Corcelles, 2013).

Somos conscientes de que el estudio adolece de algunas limitaciones entre las que destacan características de la muestra, que resulta particular por cuanto se inscribe en unos estudios con una larga tradición en la realización de trabajos de grado (antes tesis de licenciatura), lo que propició que los profesores estuvieran altamente sensibilizados por el tema y muy dispuestos a desarrollar una propuesta que les ayudase a enseñar a escribir este tipo de texto. Además, el trabajo se circunscribe a los estudios de psicología, lo que restringe su transferencia dadas las implicaciones disciplinares que tienen estos textos. En este sentido, durante el curso 2012-13 hemos iniciado una intervención, con premisas similares pero con las necesarias adaptaciones, en los estudios de grado de educación infantil y primaria de la misma Universidad que nos puede permitir avanzar en la contextualización a otras comunidades y disciplinas.

Por otra parte, hubiera sido conveniente tener en cuenta el análisis de la pertinencia de las sugerencias de cambio y de la aceptación diferencial de las mismas por parte de los escritores, aspectos que se tomarán en consideración para el análisis de futuras intervenciones. Esto nos permitiría conocer con más detalle el impacto de las sugerencias y revisiones en los textos finales. Finalmente, consideramos que en futuros estudios también sería interesante conocer la interacción que se desarrolla en cada pareja y de la manera en que los estudiantes adoptan los roles de revisor y escritor y los gestionan adecuadamente para comprender cuáles son las actuaciones que inciden tanto positiva como negativamente en la calidad del proceso y el producto final.

Si bien es cierto que en el ámbito internacional -fundamentalmente en los países de tradición anglosajona- la investigación sobre la escritura académica está ampliamente consolidada y su enseñanza en la educación superior está generalizada, la situación en nuestro contexto es claramente diferente. Son escasos los trabajos sobre la enseñanza de la escritura académica y su inclusión en los currículos universitarios y las propuestas existentes son todavía dispersas y poco conocidas. Esperamos con nuestro trabajo pueda contribuir a la necesaria reflexión sobre la alfabetización académica de nuestros estudiantes y de cómo cuándo y por qué llevarla a cabo.

\section{Referencias Bibliográficas}

Allal, L. (2000). Metacognitive regulation of writing in the classroom. En A. Camps \& M. Milian (eds.) Metalinguistic activity in learning to write (pp. 145-166). Amsterdam: Amsterdam University Press.

Bazerman, C. (2004). Speech acts, genres, and activity systems: How texts organize activity and people. En C. Bazerman \& P. Prior (Eds.), What writing does and how 
it does it: An introduction to analyzing texts and textual practices (pp. 309-339). Mahwah, NJ: Lawrence Erlbaum.

Bazerman, C., Little, J., Chavkin, T., Fouquette, D., Bethel, L. \& Garufis, J. (2005). Writing Across the Curriculum. Reference guides to rhetoric and composition, Parlor Press and WAC Clearinghouse. Recuperado de: http://wac.colostate.edu/books/bazerman wac/

Bazerman, C., Keranen, N., \& Encinas, F. (2012). Facilitated immersion at a distance in second language scientific writing. En M. Castelló \& C. Donahue (Eds.), University writing: Selves and texts in academic societies (pp. 235-248). Bingley, UK: Emerald Group Publishing Limited.

Blanch S., Corcelles, M., Duran, D., Dekhinet, R. \& Topping, K. (2012). La escritura y corrección de textos a través de tutoría entre iguales, recíproca y virtual, para la mejora en inglés y español. Revista de Educación. doi:10.4438/1988-592X-RE2012-363-190.

Becher, T. (2001). Tribus y territorios académicos: La indagación intelectual y las culturas de las disciplinas. Barcelona: Gedisa.

Berg, E. C. (1999). The effect of trained peer response on ESL students' revision types and writing quality. Journal of Second Language Writing, 8, 215-241.

Berkenkotter, C. \& Huckin, T. N. (1995). Genre knowledge in disciplinary communication. Mahwah, NJ: Lawrence Erlbaum Associates.

Camps, A. (2003a). Texto, proceso, contexto y actividad discursiva: puntos de vista diversos sobre la actividad de aprender y enseñar a escribir. En J. Ramos (coord.) Enseñar a escribir sin prisas... pero con sentido (pp. 86-103). Sevilla. M.C.E.P.

Camps, A. (2003b). Secuencias didácticas para aprender a escribir. Barcelona. Graó.

Carlino, P. (2002). Enseñar a escribir en la Universidad: cómo lo hacen en EE.UU. y por qué. Unipluriversidad, 2 (2), 57-67.

Carlino, P. (2003). Alfabetización académica: Un cambio necesario, algunas alternativas posibles. Educere Investigación, 6 (20), 409-420.

Carlino, P. (2004). El proceso de escritura académica: Cuatro dificultades de la enseñanza universitaria. Educere Investigación, 8 (26), 321-327.

Carlino, P. (2005). Escribir, leer, y aprender en la universidad. Una introducción a la alfabetización académica. Buenos Aires, Fondo de Cultura Económica.

Cassany, D., López, C., \& Martí, J. (2000). Divulgación del discurso científico: La transformación de redes conceptuales. Hipótesis, modelo y estrategias. Discurso y sociedad, 2 (2), 73-103.

Castelló, M. (2002). De la investigación sobre el proceso de composición a la enseñanza de la escritura. Revista Signos, 35 (51-52), 149-162.

Castelló, M. (2008a). Escribir trabajos de investigación con alumnos de grado. Textos de didáctica de la lengua y la literatura, 50, 21-29. 
Castelló, M. (2008b). From self-regulation to socially regulation of writing: Different voices in graduate students' writing. International Journal of Psychology, 43 (23), 356- 366.

Castelló, M., Corcelles, M., Iñesta, A., Bañales, G \& Vega, N. (2011). La voz del autor en la escritura académica. Una propuesta para su análisis. Revista Signos, 76 (44), 105-117.

Castelló, M., González, L. \& Iñesta, A. (2010). La regulación de la escritura académica en el doctorado: El impacto de la revisión colaborativa en los textos. Revista Española de Pedagogía, 68 (247), 521-537.

Castelló, C., \& Iñesta, A. (2012). Texts as artifacts-in activity: Developing authorial identity and academic voice in writing academic research papers. En M. Castelló \& C. Donahue (Eds.), University writing: Selves and texts in academic societies (pp. 179-200). Bingley, UK: Emerald Group Publishing Limited.

Castelló, M., Iñesta, A. \& Corcelles, M. (2013). Ph. D. Students' Transitions Between Academic and Disciplinary Writing Regulation: Learning to Write a Research Article. Reseaerch in the Teaching of English (aceptado. Publicación prevista: Mayo).

Castelló, M., Iñesta, A., Pardo, M., Liesa, E., \& Martinez-Fernández, R. (2012). Tutoring the end-of-studies dissertation: Helping psychology students find their academic voice. Higher Education, 63 (1), 97-115.

Castelló, M., Iñesta, A., \& Monereo, C. (2009). Towards self-regulated academic writing: An exploratory study with graduate students in a situated learning environment. Electronic Journal of Research in Educational Psychology, 7 (3), 1107-1130.

Cho, K., \& Schunn, C. D. (2007). Scaffolded writing and rewriting in the discipline. Computers and Education, 48, 409-426.

Donahue, C. (2002). Effets de l'écrit sur la construction du sujet textuel à l'université. Spirale, 29, 75-99.

Duran, D. \& Monereo, C. (2012). Entramados. Métodos de aprendizaje cooperativo y colaborativo. Barcelona: Horsori.

Freedman, S. W. (1992). Outside-in and inside-out: Peer response groups in two ninthgrade classes. Research in the Teaching of English, 26 (1), 71-107.

Gibbs, G. \& Simpson, C. (2004). Conditions under which assessment supports students' learning. Learning and Teaching in Higher Education, 1, 3-31.

Gielen, S., Peeters, E., Dochy, F., Onghena, P., \& Struyven, K. (2010). Improving the effectiveness of peer feedback for learning. Learning and Instruction, 20 (4), 304-315.

Good, T., \& Brophy, J. (1987). Looking into classrooms (4th ed). New York: Harper \& Row.

Hui-Tzu, M. (2006). The effects of trained peer review on EFL students' revision types and writing quality. Journal of Second Language Writing, 15 (2), 118-141. 
Hyland, K. (2002). Authority and invisibility: Authorial identity in academic writing. Journal of Pragmatics, 34, 1091-1112.

Hyland, K. (2005). Stance and engagement: A model of interaction in academic discourse. Discourse Studies, 7 (2), 173-192.

Lea, M. R., \& Stierer, B. (2000). Student writing in higher education: New contexts. Buckingham, UK: SRHE \& Open University Press.

Lemke, J. (1997). Aprender a hablar ciencia. Lenguaje, aprendizaje y valores. BCN. Paidos.

Levy, C.M. \& Ransdell, S.E. (1996). The science of writing: Theories, methods, individual differences, and applications. Mahwah, N.J: L. Erlbaum.

Liang, M.Y. (2010). Using synchronous online peer response groups in EFL writing: revision-related discourse. Language Learning \& Technology, 14 (1), 45-64.

Lindblom-Ylänne, S., Pihlajamäki, H., \& Kotkas, T. (2006). Self-, peer- and teacherassessment of student essays. Active Learning in Higher Education, 7, 51-62.

Lonka, K. (2003). Helping doctoral students to finish their thesis. En L. Bjork, G. Brauer, L. Rienecker \& P.S. Jorgensen (Ed.), Teaching Academic Writing in European Higher Education (pp. 113-131). Netherlands: Kluwer Academic Publishers.

Mateo, J. (coord.) (2009). Guia per a l'avaluació de competències en el treball de final de grau en l'àmbit de les ciències socials i jurídiques. Barcelona: Agència per a la Qualitat del Sistema Universitari de Catalunya.

Moyano, E.I. (2004). La escritura académica: una tarea interdisciplinaria a lo largo de la currícula universitaria. Revista Texturas, 4 (4), 109-120.

Nelson, M. \& Schunn, C. (2009). The nature of feedback: How different types of peer feedback affect writing performance. Instructional Science, 37 (4), 375-401.

Nystrand, M. (1989). A social-interactive model of writing, Written Communication, 6 (1), 66-85.

Paulus, T. M. (1999). The Effect of peer and teacher feedback on student writing. Journal of Second Language Writing, 8 (3), 265-289.

Prior, P. (2006). A Sociocultural theory of writing. En MacArthur, C.A., Graham, S. \& Fitzgerald J. (Eds.), Handbook of writing research (pp.54-65). New York : Guilford.

Russell, D. (1997). Rethinking genre in school and society: An activity theory analysis. Written Communication, 14 (4), 504-554.

Sabaj, O. (2009). Descubriendo algunos problemas en la redacción de artículos de investigación científica (AIC) de alumnos de postgrado. Revista Signos, 42 (69), 107-127.

Solé, I.; Mateos, M.; Miras, M.; Martín, E.; Castells, N.; Cuevas, I. \& Gràcia, M. (2005). Lectura, escritura y adquisición de conocimientos en Educación Secundaria y Educación Universitaria. Infancia y Aprendizaje, 28 (3), 329-347. 
Squillari, R., Bono, A. \& Rinaudo, M. (2003). La formación en valores a través de las tareas de escritura trabajando con estudiantes universitarios. Ponencia presentada el Congreso Latinoamericano de Educación Superior en Siglo XXI. Universidad Nacional de San Luis.

Swales, J. (1990). Genre analysis. English in academic and research settings. Cambridge: Cambridge University Press.

Teberosky, A. (2007). El texto académico. En M. Castelló, Escribir y comunicarse en contextos científicos y académicos. Conocimientos y estrategias (pp.17-46). Barcelona: Crítica y fundamentos 15. Graó.

Topping, K. (2005). Trends in peer learning. Educational Psychology, 25(6), 631-645.

Topping, K. (2010). Methodological quandaries in studying process and outcomes in peer assessment. Learning and Instruction, 20 (4), 339-343.

Topping, K. J., Dehkinet, R., Blanch, S., Corcelles, M., \& Duran, D. (2012) Paradoxical effects of feedback in international online reciprocal peer tutoring. Computer Education. doi: 10.1016/j.compedu.2012.10.002

Vásquez, G. (2005). Diseño e implementación del Centro de Escritura Académica de Postgrado 27 (CEAP), Instituto de Lingüística, Facultad de Filosofía y Letras (UBA). Recuperado de: http://www2.udec.cl/catedraunesco/10VASQUEZ.pdf

Venables, A. \& Summit, R. (2003). Enhancing scientific essay writing using peer assessment. Innovations in Education and Teaching International, 40 (3), 281291.

Vygotsky, L. (1995). Pensamiento y lenguaje. Barcelona: Paidós, 1934.

Yarrow, F. \& Topping, K. (2001). Collaborative writing: The effects of metacognitive prompting and structured peer interaction. British Journal of Educational Psychology, 71, 261- 282. 
Anexo 1:

Rúbrica de evaluación de la calidad de los textos

\begin{tabular}{|c|c|c|c|c|c|}
\hline DIMENSIONES & 5 & 4 & 3 & 2 & 1 \\
\hline $\begin{array}{l}\text { Estructura del } \\
\text { género: Titulo }\end{array}$ & $\begin{array}{l}\text { Presenta un título } \\
\text { claro y atractivo que } \\
\text { sintetiza el tema o } \\
\text { variables principales } \\
\text { del estudio dentro } \\
\text { de una extensión } \\
\text { adecuada (máximo } \\
2 \text { frases) }\end{array}$ & $\begin{array}{l}\text { Presenta un título } \\
\text { claro que sintetiza } \\
\text { el tema o variables } \\
\text { principales del } \\
\text { estudio, pero } \\
\text { contiene algún } \\
\text { problema: palabras } \\
\text { redundante, es } \\
\text { demasiado largo... }\end{array}$ & $\begin{array}{l}\text { El título sintetiza el } \\
\text { tema o variables } \\
\text { principales del } \\
\text { estudio, pero es } \\
\text { poco claro }\end{array}$ & $\begin{array}{l}\text { Presenta un título } \\
\text { confuso que carece } \\
\text { de una síntesis del } \\
\text { tema o variables } \\
\text { principales del } \\
\text { estudio, y que } \\
\text { además tiene una } \\
\text { extensión } \\
\text { inadecuada de } \\
\text { palabras y varias } \\
\text { palabras } \\
\text { redundantes }\end{array}$ & No contiene título \\
\hline $\begin{array}{l}\text { Estructura del } \\
\text { género: Resumen }\end{array}$ & $\begin{array}{l}\text { Presenta de manera } \\
\text { clara y concisa una } \\
\text { síntesis del } \\
\text { contenido del } \\
\text { estudio en términos } \\
\text { de objetivos, } \\
\text { participantes, } \\
\text { método, resultado y } \\
\text { conclusiones sin } \\
\text { utilizar más de } 250 \\
\text { palabras. }\end{array}$ & $\begin{array}{l}\text { Presenta de manera } \\
\text { clara y concisa una } \\
\text { síntesis del } \\
\text { contenido del } \\
\text { estudio en términos } \\
\text { de objetivos, } \\
\text { participantes, } \\
\text { método, resultado y } \\
\text { conclusiones, pero } \\
\text { utiliza más de } 250 \\
\text { palabras. }\end{array}$ & $\begin{array}{l}\text { Presenta de manera } \\
\text { clara y concisa la } \\
\text { mayoría de } \\
\text { elementos del } \\
\text { contenido del } \\
\text { estudio, pero } \\
\text { algunos son } \\
\text { confusos o se } \\
\text { omiten. }\end{array}$ & $\begin{array}{l}\text { Presenta de manera } \\
\text { confusa y parcial el } \\
\text { contenido del } \\
\text { estudio. }\end{array}$ & $\begin{array}{l}\text { No contiene } \\
\text { abstract }\end{array}$ \\
\hline $\begin{array}{l}\text { Estructura del } \\
\text { género: } \\
\text { Introducción }\end{array}$ & $\begin{array}{l}\text { Realiza claramente } \\
\text { los } 3 \text { movimientos } \\
\text { en la introducción. }\end{array}$ & $\begin{array}{l}\text { Realiza los } 3 \\
\text { movimientos } \\
\text { aunque uno de ellos } \\
\text { puede presentarse } \\
\text { de manera confusa } \\
\text { o tendría que } \\
\text { ampliarse. }\end{array}$ & $\begin{array}{l}\text { Realiza sólo } 2 \\
\text { movimientos. }\end{array}$ & $\begin{array}{l}\text { Realiza sólo } 1 \\
\text { movimiento. }\end{array}$ & $\begin{array}{l}\text { No realiza ninguno } \\
\text { de los } 3 \\
\text { movimientos. }\end{array}$ \\
\hline $\begin{array}{l}\text { Estructura del } \\
\text { género: Método }\end{array}$ & $\begin{array}{l}\text { Describe de manera } \\
\text { adecuada y clara la } \\
\text { muestra, los } \\
\text { materiales e } \\
\text { instrumentos de } \\
\text { recogida de datos y } \\
\text { el procedimiento } \\
\text { utilizado en el } \\
\text { estudio. }\end{array}$ & $\begin{array}{l}\text { Describe de manera } \\
\text { adecuada y clara la } \\
\text { muestra, los } \\
\text { materiales e } \\
\text { instrumentos de } \\
\text { recogida de datos y } \\
\text { el procedimiento } \\
\text { utilizado en el } \\
\text { estudio, pero } \\
\text { algunos detalles son } \\
\text { mejorables. }\end{array}$ & $\begin{array}{l}\text { Describe } \\
\text { la mayoría de } \\
\text { apartados } \\
\text { metodológicos de } \\
\text { un proyecto, pero } \\
\text { deberían ser } \\
\text { mejorados en } \\
\text { muchos detalles. }\end{array}$ & $\begin{array}{l}\text { Describe } \\
\text { dos apartados } \\
\text { metodológicos del } \\
\text { proyecto que } \\
\text { pueden ser } \\
\text { mejorados en } \\
\text { muchos detalles, } \\
\text { además carece de } \\
\text { más de dos } \\
\text { apartados } \\
\text { esenciales. }\end{array}$ & $\begin{array}{l}\text { El apartado no } \\
\text { existe }\end{array}$ \\
\hline $\begin{array}{l}\text { Estructura del } \\
\text { género: } \\
\text { Resultados }\end{array}$ & $\begin{array}{l}\text { Presenta } \\
\text { claramente los } \\
\text { resultados de } \\
\text { acuerdo a las } \\
\text { preguntas u } \\
\text { objetivos del } \\
\text { estudio. }\end{array}$ & $\begin{array}{l}\text { Presenta } \\
\text { claramente los } \\
\text { resultados de } \\
\text { acuerdo a las } \\
\text { preguntas u } \\
\text { objetivos del } \\
\text { estudio, pero se } \\
\text { observan problemas } \\
\text { (tablas, gráficos..). }\end{array}$ & $\begin{array}{l}\text { Presenta } \\
\text { claramente los } \\
\text { resultados de la } \\
\text { mayoría de } \\
\text { preguntas u } \\
\text { objetivos del } \\
\text { estudio, pero } \\
\text { algunos no se } \\
\text { corresponden o se } \\
\text { explican de manera } \\
\text { inadecuada. }\end{array}$ & $\begin{array}{l}\text { Presenta resultados } \\
\text { confusos, } \\
\text { desordenados o } \\
\text { desvinculados de las } \\
\text { preguntas u } \\
\text { objetivos del } \\
\text { estudio. }\end{array}$ & $\begin{array}{l}\text { No contiene el } \\
\text { apartado. }\end{array}$ \\
\hline
\end{tabular}




\begin{tabular}{|c|c|c|c|c|c|}
\hline $\begin{array}{l}\text { Estructura del } \\
\text { género: Discusión } \\
\text { y Conclusiones }\end{array}$ & $\begin{array}{l}\text { Presenta } \\
\text { claramente las } \\
\text { implicaciones de los } \\
\text { resultados } \\
\text { obtenidos con } \\
\text { respecto a los } \\
\text { objetivos y/o } \\
\text { hipótesis del } \\
\text { estudio, señala } \\
\text { semejanzas y } \\
\text { diferencias en } \\
\text { relación a otros } \\
\text { estudios, las } \\
\text { limitaciones del } \\
\text { estudio y los } \\
\text { aspectos para } \\
\text { futuras } \\
\text { investigaciones }\end{array}$ & $\begin{array}{l}\text { Presenta } \\
\text { claramente las } \\
\text { implicaciones de los } \\
\text { resultados } \\
\text { obtenidos con } \\
\text { respecto a los } \\
\text { objetivos y/o } \\
\text { hipótesis del } \\
\text { estudio, señala } \\
\text { semejanzas y } \\
\text { diferencias en } \\
\text { relación a otros } \\
\text { estudios, las } \\
\text { limitaciones del } \\
\text { estudio y los } \\
\text { aspectos para } \\
\text { futuras } \\
\text { investigaciones, } \\
\text { aunque alguno de } \\
\text { estos aspectos es } \\
\text { mejorable. }\end{array}$ & $\begin{array}{l}\text { Presenta algunas } \\
\text { implicaciones de los } \\
\text { resultados } \\
\text { obtenidos con } \\
\text { respecto a los } \\
\text { objetivos y/o } \\
\text { hipótesis del } \\
\text { estudio, pero no las } \\
\text { contrasta en } \\
\text { relación a otros } \\
\text { estudios, tampoco } \\
\text { señala las } \\
\text { limitaciones del } \\
\text { estudio ni los } \\
\text { aspectos para } \\
\text { futuras } \\
\text { investigaciones }\end{array}$ & $\begin{array}{l}\text { Presenta una } \\
\text { discusión o } \\
\text { conclusión } \\
\text { desvinculada o } \\
\text { confusa en relación } \\
\text { a las implicaciones } \\
\text { de los resultados } \\
\text { obtenidos y los } \\
\text { objetivos y/o } \\
\text { hipótesis del } \\
\text { estudio. }\end{array}$ & $\begin{array}{l}\text { No contiene el } \\
\text { apartado }\end{array}$ \\
\hline Coherencia & $\begin{array}{l}\text { Todas las ideas se } \\
\text { organizan siguiendo } \\
\text { una secuencia } \\
\text { lógica evidenciando } \\
\text { un eje organizador } \\
\text { de la información. }\end{array}$ & $\begin{array}{l}\text { La mayoría de las } \\
\text { ideas se organizan } \\
\text { siguiendo una } \\
\text { secuencia lógica } \\
\text { evidenciando un eje } \\
\text { organizador de la } \\
\text { información. }\end{array}$ & $\begin{array}{l}\text { Algunas de las ideas } \\
\text { se organizan } \\
\text { siguiendo una } \\
\text { secuencia lógica y } \\
\text { es difícil evidenciar } \\
\text { un eje organizador } \\
\text { de la información. }\end{array}$ & $\begin{array}{l}\text { Muy pocas ideas se } \\
\text { organizan siguiendo } \\
\text { una secuencia } \\
\text { lógica y no se } \\
\text { evidencia un eje } \\
\text { organizador de la } \\
\text { información. }\end{array}$ & $\begin{array}{l}\text { Carece de un } \\
\text { desarrollo lógico y } \\
\text { progresivo de las } \\
\text { ideas a lo largo de } \\
\text { todo el texto y no } \\
\text { es visible un eje de } \\
\text { organización de la } \\
\text { información. }\end{array}$ \\
\hline Cohesión & $\begin{array}{l}\text { Contiene variedad } \\
\text { de conectores } \\
\text { adecuados que } \\
\text { facilitan el enlace } \\
\text { entre los párrafos y } \\
\text { la progresión lógica } \\
\text { de la información }\end{array}$ & $\begin{array}{l}\text { Contiene variedad } \\
\text { de conectores } \\
\text { adecuados que } \\
\text { facilitan el enlace } \\
\text { entre los párrafos y } \\
\text { la progresión lógica } \\
\text { de la información, } \\
\text { pero no todos son } \\
\text { adecuados. }\end{array}$ & $\begin{array}{l}\text { Contiene pocos } \\
\text { conectores que se } \\
\text { usan de forma } \\
\text { repetitiva aunque } \\
\text { se, mantiene el } \\
\text { enlace entre los } \\
\text { párrafos que } \\
\text { facilitan la } \\
\text { progresión lógica de } \\
\text { la información. }\end{array}$ & $\begin{array}{l}\text { Contiene muy pocos } \\
\text { conectores que se } \\
\text { usan de forma } \\
\text { inadecuada. }\end{array}$ & No hay conectores. \\
\hline $\begin{array}{l}\text { Recursos } \\
\text { discursivos para } \\
\text { manifestar el } \\
\text { posicionamiento } \\
\text { del autor }\end{array}$ & $\begin{array}{l}\text { Utiliza recursos } \\
\text { discursivos variados } \\
\text { que le permiten } \\
\text { manifestar su } \\
\text { punto de vista de } \\
\text { manera clara }\end{array}$ & $\begin{array}{l}\text { Utiliza recursos } \\
\text { discursivos variados } \\
\text { que le permiten } \\
\text { manifestar su } \\
\text { punto de vista de } \\
\text { manera clara, pero } \\
\text { algunos son } \\
\text { ocasionalmente } \\
\text { inadecuados }\end{array}$ & $\begin{array}{l}\text { Utiliza recursos } \\
\text { discursivos que le } \\
\text { permiten } \\
\text { manifestar su } \\
\text { punto de vista de } \\
\text { manera clara, pero } \\
\text { se utilizan } \\
\text { frecuentemente de } \\
\text { manera repetitiva. }\end{array}$ & $\begin{array}{l}\text { Utiliza recursos } \\
\text { discursivos de } \\
\text { manera escasa y/o } \\
\text { inadecuada que } \\
\text { hacen difícil ver su } \\
\text { punto de vista. }\end{array}$ & $\begin{array}{l}\text { No utiliza recursos } \\
\text { discursivos que le } \\
\text { ayuden a } \\
\text { manifestar su } \\
\text { punto de vista }\end{array}$ \\
\hline $\begin{array}{l}\text { Recursos } \\
\text { discursivos para } \\
\text { implicar al lector }\end{array}$ & $\begin{array}{l}\text { Utiliza recursos } \\
\text { discursivos variados } \\
\text { que le permiten } \\
\text { implicar al lector de } \\
\text { manera clara }\end{array}$ & $\begin{array}{l}\text { Utiliza recursos } \\
\text { discursivos variados } \\
\text { que le permiten } \\
\text { implicar al lector de } \\
\text { manera clara, pero } \\
\text { algunos son } \\
\text { ocasionalmente } \\
\text { inadecuados }\end{array}$ & $\begin{array}{l}\text { Utiliza recursos } \\
\text { discursivos que le } \\
\text { permiten implicar } \\
\text { al lector de manera } \\
\text { clara, pero se } \\
\text { utilizan } \\
\text { frecuentemente de } \\
\text { manera repetitiva }\end{array}$ & $\begin{array}{l}\text { Utiliza recursos } \\
\text { discursivos de } \\
\text { manera escasa y/o } \\
\text { inadecuada que } \\
\text { hacen difícil ver la } \\
\text { implicación del } \\
\text { lector }\end{array}$ & $\begin{array}{l}\text { No utiliza recursos } \\
\text { discursivos que le } \\
\text { ayuden a implicar } \\
\text { al lector }\end{array}$ \\
\hline Intertextualidad & $\begin{array}{l}\text { Utiliza citas } \\
\text { indirectas } \\
\text { integradas y no } \\
\text { integradas de } \\
\text { manera correcta y } \\
\text { equilibrada, } \\
\text { haciendo a su vez }\end{array}$ & $\begin{array}{l}\text { Utiliza citas } \\
\text { indirectas } \\
\text { integradas y no } \\
\text { integradas de } \\
\text { manera correcta, } \\
\text { pero abusa en } \\
\text { varias ocasiones de }\end{array}$ & $\begin{array}{l}\text { Utiliza en su } \\
\text { mayoría citas } \\
\text { directas }\end{array}$ & $\begin{array}{l}\text { Utiliza citas de } \\
\text { manera escasa, } \\
\text { inadecuada o } \\
\text { repetitiva a lo largo } \\
\text { del texto }\end{array}$ & No utiliza citas \\
\hline
\end{tabular}




\begin{tabular}{|l|l|l|l|l|l|}
\hline & $\begin{array}{l}\text { un uso puntual de } \\
\text { citas directas }\end{array}$ & las citas directas & & \\
\hline Aspectos formales & $\begin{array}{l}\text { El texto está exento } \\
\text { de problemas } \\
\text { gramaticales, } \\
\text { ortográficos o de } \\
\text { puntuación. }\end{array}$ & $\begin{array}{l}\text { El texto tiene } \\
\text { algunos problemas } \\
\text { gramaticales, } \\
\text { ortográficos o de } \\
\text { puntuación. }\end{array}$ & $\begin{array}{l}\text { El texto tiene } \\
\text { bastantes } \\
\text { problemas } \\
\text { gramaticales, } \\
\text { ortográficos o de } \\
\text { puntuación. }\end{array}$ & $\begin{array}{l}\text { El texto tiene } \\
\text { muchos problemas } \\
\text { gramaticales, } \\
\text { ortográficos o de } \\
\text { puntuación. }\end{array}$ & $\begin{array}{l}\text { El texto está lleno } \\
\text { de errores } \\
\text { gramaticales, } \\
\text { ortográficos o de } \\
\text { puntuación. }\end{array}$ \\
\hline
\end{tabular}

Cita del artículo:

Corcelles, M.; Cano, M.; Bañales, G.; Vega, N.A. (2013). Enseñar a escribir textos científico- académicos mediante la revisión colaborativa: El trabajo final de grado en Psicología. Revista de Docencia Universitaria. REDU. Número monográfico dedicado a Academic Writing. Vol.11 (1) Enero-Abril. pp. 79-104. Recuperado el (fecha de consulta) en http://www.red-u.net/

\section{Acerca de las autoras y autor}

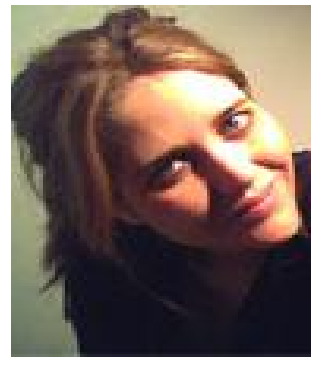

\section{Mariona Corcelles Seuba}

\section{Universitat Ramon Llull}

Departamento de Psicología

Mail: Marionacs@blanquerna.url.edu

Doctora en Psicología de la Educación por la Universidad Ramon Llull y licenciada en Filosofía por la Universidad de Barcelona. Actualmente es profesora ayudante doctora de la Facultad de Psicología, Ciencias de la Educación y del Deporte Blanquerna. Miembro del Seminario de Investigación Interuniversitario en Estrategias de Enseñanza y Aprendizaje (SINTE www.sinte.es ). Sus áreas de investigación son el aprendizaje entre iguales, la escritura epistémica y la didáctica de la filosofía.

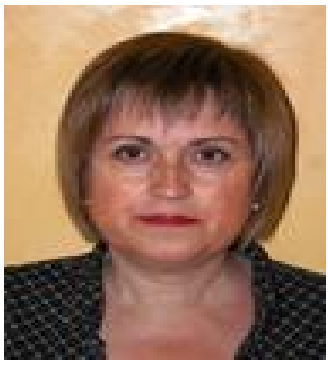

\section{Maribel Cano Ortiz}

\section{Universitat Ramon Llull}

Departamento de Psicologia

Mail: Maribelco@blanquerna.url.edu 
Doctora en Psicología por la Universidad Ramon Llull. Máster interuniversitario en Estrategias de Aprendizaje. Licenciada en Psicología y Logopedia por la Universidad Autónoma de Barcelona. Actualmente es profesora asociada de la Facultad de Psicología, Ciencias de la Educación y del Deporte Blanquerna. Miembro del Seminario de Investigación Interuniversitario en Estrategias de Enseñanza y Aprendizaje (SINTE www.sinte.es). Sus áreas de investigación son la escritura epistémica, los procesos de enseñanza y aprendizaje y la orientación educativa.

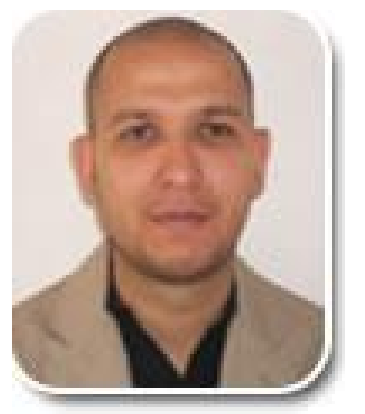

\section{Gerardo Bañales Faz}

Universidad Autónoma de Tamaulipas

Unidad Académica Multidisciplinaria de Ciencias, Educación y Humandidades

Mail: gbanales@uat.edu.mx

Doctor en Psicología de la Educación por la Universidad Ramon Llull y licenciado en Ciencias de la Educación por la Universidad Autónoma de Tamaulipas (UAT). Miembro del Seminario de Investigación Interuniversitario en Estrategias de Enseñanza y Aprendizaje (SINTE www.sinte.es). Actualmente es profesor- investigador de la Universidad de Tamaulipas (UAT). Sus áreas de investigación son los procesos de escritura de textos académicos y la enseñanza de la escritura académica en la universidad.

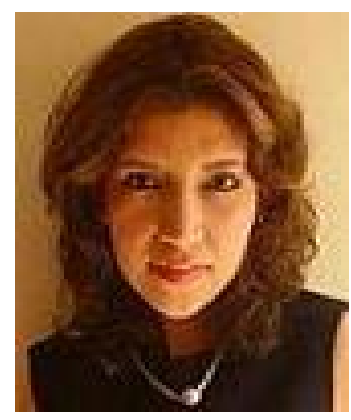

\section{Norma Alicia Vega}

Universidad Autónoma de Tamaulipas

Unidad Académica Multidisciplinaria de Ciencias, Educación y Humandidades

Mail: navegalo@uat.edu.mx

Doctora en Psicología de la Educación por la Universidad Ramon Llull y licenciada en Ciencias de la Educación por la Universidad Autónoma de Tamaulipas (UAT). Miembro del Seminario de Investigación Interuniversitario en Estrategias de Enseñanza y Aprendizaje (SINTE www.sinte.es).

Actualmente es profesora- investigadora de la Universidad de Tamaulipas (UAT). Sus áreas de investigación son la comprensión de múltiples textos y la enseñanza de estrategias de comprensión lectora en contextos escolares. 


\title{
Concepciones y prácticas declaradas de profesores terciarios en torno al leer y escribir en las asignaturas
}

\author{
Tertiary teachers' conceptions and declared practices about reading and writing \\ in their disciplines
}

\author{
Paula Carlino \\ CONICET-Univ. de Buenos Aires, Argentina \\ Patricia Iglesia \\ Univ. de Buenos Aires, Argentina
}

Irene Laxalt

Univ. Nacional del Centro de la Provincia de Buenos Aires, Argentina

\section{Resumen}

Este trabajo examina las prácticas declaradas y las concepciones sobre lectura y escritura que pueden inferirse de ellas, a partir de un estudio entre profesores terciarios argentinos. Para ello, administramos una encuesta a profesores de diversas asignaturas en 50 instituciones, que forman a los futuros docentes secundarios. Obtuvimos 544 respuestas válidas. En este artículo, enfocamos una de las preguntas abiertas que proponía describir las acciones que se realizan para ayudar a afrontar las dificultades de los alumnos para leer comprensivamente y escribir con claridad, reconocidas por la mayoría de los encuestados. Aunque muchos declaran ocuparse de la lectura y/o escritura en sus materias, predominan quienes manifiestan intervenir sólo al inicio de estas tareas (solicitando trabajos, dando instrucciones, enseñando técnicas) o al final (corrigiendo producciones de los alumnos). Una proporción menor de docentes expresan que median los procesos de leer o escribir durante las clases. Algunos de ellos manifiestan asimismo promover la interacción entre profesor y alumnos y/o entre pares, a través de la discusión sobre lo leído o lo escrito. De estas respuestas es posible abstraer dos modalidades con que los profesores incluyen la lectura y/o escritura en sus materias: una "periférica" y otra "entrelazada". De acuerdo con los marcos teóricos de "escribir a través del currículum" y "enseñanza dialógica", interpretamos estos resultados en términos de las concepciones que podrían corresponder a las prácticas declaradas. Discutimos también la incidencia que estas prácticas tendrían sobre la formación de los futuros profesores.

Palabras clave: enseñanza, lectura, escritura, disciplinas, concepciones, profesores 


\begin{abstract}
This paper examines the declared practices and conceptions on reading and writing that can be inferred from a study in Argentina. After passing a survey to teachers of various subjects in 50 institutions that train future high-school teachers, we collected 544 valid responses. In this article, we focus on one of the open questions that describe the actions taken to address the students' difficulties to write and understand what they read, recognized by most respondents. Although many teachers claim to deal with reading and/or writing in their field, most of them tend to intervene at the beginning of the assignments (requiring tasks, giving guidelines, providing techniques) or at the end (correcting students' productions). A smaller proportion of teachers expressed that they support students during these tasks and make time for it in their classes. Some of them also promote interaction between teacher and students or among peers, through discussion on what has been read or written. Two modes of dealing with reading and/or writing within their subjects emerged from these data: the "peripheral" and the "intertwined" teaching. According to the theoretical "writing across the curriculum" and "dialogic teaching" approaches, we examine these results in terms of the conceptions which could correspond to the declared practices. We also discuss the impact that these teaching practices would have on the training of future teachers.
\end{abstract}

Key words: instruction, academic literacy, disciplines, conceptions, teachers

\title{
Introducción'
}

¿Qué lugar ocupan la lectura y la escritura en la enseñanza de las diversas asignaturas en la formación docente? ¿Se las concibe y utiliza como tareas que se ponen en juego sólo al momento de la evaluación de los contenidos impartidos en ellas o también se promueve su empleo como instrumentos de aprendizaje? ¿Se las considera herramientas aún en construcción, por lo cual se las orienta y acompaña al interior de cada materia, o bien se supone que los alumnos no requieren ayudas pedagógicas para leer y escribir porque han aprendido a hacerlo con anterioridad? ¿Se contemplan así en la enseñanza de cada asignatura los procedimientos de estudio específicos o se los exige sin más?

Con estos interrogantes de partida, investigamos cómo se piensan las relaciones entre lectura, escritura, enseñanza y aprendizaje disciplinar, en los institutos que forman a profesores secundarios a nivel nacional ${ }^{2}$. En Argentina, los profesores que se preparan para enseñar en las escuelas secundarias (con alumnos de entre 12 y 18 años) reciben generalmente su formación de cuatro años en instituciones terciarias no universitarias, los llamados "Institutos de Formación Docente" (IFD). Desde hace un lustro, los IFD son objeto de políticas de mejora, dependientes del Ministerio de Educación de la Nación. En este contexto, el presente estudio apuntó a brindar información útil para diseñar acciones de desarrollo profesional docente y eventualmente para contribuir a cambios curriculares $y / 0$ institucionales en la formación de grado.

De acuerdo con marcos teóricos convergentes, que abrevan en las corrientes de escribir a través del currículum, la socio-retórica, la enseñanza dialógica, el constructivismo, el socio-constructivismo, y la teoría de las situaciones didácticas, la indagación relevó si la lectura y la escritura se incluyen en la enseñanza al servicio del aprendizaje de los contenidos de las diversas asignaturas, y cómo se lo hace. 
El propósito fue averiguar en qué medida existen docentes $\mathrm{y} / \mathrm{o}$ acciones institucionales que procuran integrar el trabajo sobre la comprensión y producción de textos en la formación de los futuros profesores secundarios, trascendiendo el uso del escribir como canal para que los alumnos comuniquen lo que han estudiado por su cuenta al momento de ser evaluados y del leer como extracción de un significado inmanente al texto. En tanto prácticas de estudio, la lectura y la escritura podrían estar presentes de diversas maneras en todas las materias. Por ello, se encuestó a profesores de las distintas carreras y asignaturas que se imparten. En particular, la investigación procuró determinar de qué modos los profesores de institutos de formación docente manifiestan orientar el análisis y elaboración de textos que requieren de sus alumnos durante el cursado de sus materias. Asimismo, analizó qué concepciones podrían subyacer a estos modos. Para conocer el lugar que otorgan a las actividades de leer y escribir para aprender sus asignaturas, y a cómo intervienen en ellas, la encuesta incluyó preguntas relativas a la enseñanza y al aprendizaje, y no sólo a la lectura y escritura.

En este trabajo, pasamos revista a los marcos teóricos desde los que se realizó el estudio, describimos el método utilizado y analizamos uno de los campos abiertos de la encuesta. Nos centramos así en las respuestas al ítem que pedía describir las acciones que realizan los profesores para ayudar a afrontar las dificultades de los alumnos "para leer comprensivamente y escribir con claridad", declaradas por el $90 \%$ de los encuestados.

Detallamos a continuación las opciones teóricas del equipo de investigación, el GICEOLEM $^{3}$, en lo que respecta al objeto de enseñanza de las asignaturas, el aprendizaje, la noción de alumno, y de enseñanza. No todos los conceptos que desarrollamos en lo que sigue son retomados en la interpretación de los datos analizados en el presente artículo dado que algunos se utilizaron para el análisis de otras preguntas de la encuesta. Sin embargo, hemos querido incluirlos en este trabajo para mostrar el entramado de conceptos con los que pensamos el estudio y las cuestiones indagadas.

\section{Opciones teóricas desde las que se construyó la encuesta y se analizaron los datos}

Las opciones teóricas en las que se funda este estudio atañen a cómo el equipo de investigación entiende cada componente del sistema didáctico (Chevallard, 1997): los objetos de conocimiento propios de las asignaturas en el nivel superior, la enseñanza del profesor, y el aprendizaje del alumno. De acuerdo con Camps (2003), diversas disciplinas son necesarias para pensar la enseñanza de un saber, aunque ninguna de éstas pueda "aplicarse" en el aula (ni en la investigación) sino que han de pensarse como referencias teóricas para entender los problemas que surgen, aunque estos problemas han de definirse desde la propia enseñanza (y la investigación). Con esta intención, explicitamos nuestras concepciones sobre la enseñanza y aprendizaje de dos tipos de objetos de conocimiento: el saber conceptual de las asignaturas (sus sistemas de conceptos) y el saber hacer correspondiente a las prácticas de lectura y escritura esperadas dentro de ellas (quehacer de lector y escritor en cada materia). Asimismo, caracterizamos nuestra noción de alumno en la educación superior. 


\section{El objeto de enseñanza: los saberes disciplinares y las prácticas de lectura y escritura involucradas}

En el equipo de investigación que desarrollamos este estudio entendemos que los saberes que han de ser enseñados en las distintas carreras de la formación docente incluyen, además del conocimiento conceptual proveniente de cada disciplina, las prácticas de lectura y escritura que necesitan cumplimentar los alumnos para cursar las materias y las que precisarán ejercer los futuros profesores en cada área de estudio. Siguiendo a Bogel y Hjorthoj (1984, p. 12), asumimos que "una disciplina es un espacio discursivo y retórico, tanto como conceptual". Por ello, su aprendizaje involucra adquirir su sistema de conceptos y métodos, junto con usos del lenguaje específicos. Estos usos, de naturaleza práctica y situada, sólo pueden aprenderse en el contexto dentro del cual ocurren y a través de la ayuda de quienes tienen experiencia en ellos (véase, por ejemplo, Gottschalk y Hjorthoj, 2004; Mitchell, 2010; Purser, Skillen, Deane, Donohue y Peake, 2008).

A diferencia de lo que supone el sentido común, cuestionamos que la lectura y la escritura sean habilidades generales que puedan aprenderse de una vez y para siempre para luego aplicarse a cualquier texto y situación (cf. Rose, 1985; Russell, 2002). Si bien existen algunos saberes sobre lo escrito que son transferibles (ortografía, gramática), los aspectos más sustantivos no son universales. Así, las cuestiones dependientes del contenido y propósito de escritura (desarrollo conceptual, adecuación a la situación comunicativa, modos de argumentar considerados válidos, jerarquización de las nociones, etc.) adquieren particularidades en cada ámbito, y son inherentes a determinadas comunidades de práctica. Las formas de lo escrito relativas a estos aspectos -denominados profundos o de primer orden, porque hacen referencia al significado- no son aceptadas por igual en todas las disciplinas. Los modos de lectura y escritura requeridos en cada asignatura no resultan de la mera prolongación de procedimientos elementales adquiridos en forma general antes de ingresar a la educación superior. Las investigaciones muestran, en cambio, que las maneras de leer y escribir apropiadas para un determinado ámbito precisan ser reconstruidas para poder servir en otro, y que esto ocurre a través de un largo aprendizaje no exento de obstáculos, que deben ser enfrentados.

Así, la noción sobre el escribir en la cual se basa el presente estudio se contrapone a la habitual concepción "autónoma" de la escritura, generalmente implícita, pero advertida y combatida por uno de los exponentes del movimiento "escribir a través del currículum": la creencia de que se trata de "una simple habilidad universalmente aplicable, en gran parte no relacionada con el 'contenido' [...], una técnica separada e independiente" de lo que se escribe (Russell, 1990: 55). Junto con éste y otros impulsores del movimiento "escribir a través del currículum", adherimos a la noción de "escritura como una actividad retórica compleja involucrada [embedded] en las diversas prácticas de las comunidades de discurso académico" y "condicionada por ésta[s]" (Russell, 2002: 9 y 12).

Es necesario entonces resaltar el contraste entre dos visiones de la escritura porque de ellas depende que se la considere un saber ya adquirido, que los profesores pueden suponer en sus alumnos, o bien un objeto de enseñanza del cual han de 
ocuparse en sus asignaturas. En el primer caso, se concibe a la escritura como un canal de comunicación que sirve para transmitir un pensamiento elaborado con anterioridad, una forma extrínseca que se aplicaría a un contenido pre-existente. En contraposición, la postura alternativa entiende el escribir como una actividad social, lingüística y cognitiva que permite repensar aquello sobre lo que se escribe, y dar origen a ideas que no existían antes de escribir. Según tratamos al final de este apartado, la escritura es un instrumento de elaboración, y no sólo un medio de transmisión de lo ya sabido. Considerar que la escritura es una actividad cognoscitiva, que incide en la comprensión de los contenidos disciplinares que los docentes deben ayudar a aprender, ha llevado a que en este estudio se incluyeran preguntas relativas a qué hacen los profesores de cualquier materia para promover y orientar la producción escrita, ya que hacerlo contribuye a que los alumnos se apropien de los conceptos de sus asignaturas. Considerar a la escritura una práctica social situada, tal como desarrollamos más adelante, advierte que sólo ciertas situaciones de escritura promueven este uso epistémico.

En igual sentido, es preciso tener presentes las concepciones contrapuestas sobre la lectura. Es común suponer que leer es una destreza separable del contenido. En palabras de una profesora: "Si [los alumnos] tienen lectura comprensiva, no deberían tener problemas con el texto" (en Carlino, 2005: 85). De este modo, se considera que la lectura -en tanto competencia independiente del material que se leepuede ser aprendida y enseñada de una vez y para siempre. Entender a la lectura como habilidad general, aprendida previamente, lleva a pensar que los alumnos puedan "aplicarla" por su cuenta para leer los textos de cada asignatura. Esta concepción suele ir de la mano de una idea extractiva del leer, en la cual se supone que el significado es inmanente al texto y que basta sólo con decodificarlo para acceder a aquél. Desde esta perspectiva, no es preciso que el profesor intermedie la lectura porque se da por supuesto que de un mismo texto sólo emerge una única comprensión posible. La concepción contraria, que fundamenta este estudio, se basa en los modelos interactivos sobre el proceso de lectura. En ellos, se considera que el significado de lo leído depende no sólo de lo que aporta el texto sino también de lo que aporta el lector, es decir, de sus conocimientos previos y de su propósito de lectura (Haswell et al., 1999; Lerner, 1985; Smith, 1988). Así, las categorías conceptuales con las que un alumno se acerca a un texto le ayudan a percatarse de ciertas ideas y oscurecen $u$ ocultan otras interpretaciones potenciales. También, vuelven importantes unas y secundarias otras. Sólo la intervención del profesor -conocedor de los marcos conceptuales de su disciplina- puede ayudar a los alumnos a saber qué buscar en los textos para hallar lo que resulta relevante para su materia (cf. Carlino, 2003, 2005). Desde esta concepción interactiva y constructiva de la lectura, el docente debería ayudar a confrontar las diversas interpretaciones poniéndolas en relación con el conocimiento disciplinar. En virtud de ello es que la encuesta diseñada indagó acerca de si los profesores intervienen en las actividades de lectura que proponen a sus alumnos y cómo lo hacen.

Así pues, el enfoque al que adhiere este estudio subraya que en cada dominio del conocimiento se lee y se escribe según modos particulares. La reiteración de estos modos de actuar frente a situaciones similares es lo que configura los géneros de los discursos académicos y disciplinares. En contraste con la idea extendida de que un 
género es un tipo de texto, las investigaciones socio-retóricas cuestionan que los géneros discursivos consistan en conjuntos de rasgos textuales. Por el contrario, entienden a los géneros discursivos como acciones retóricas típicas motivadas por situaciones afines (Miller, 1984). Los géneros incluyen propiedades de cierto tipo de textos pero las exceden, porque -en tanto prácticas sociales - involucran habituales formas usar el lenguaje, concepciones compartidas, adopción de valores, posicionamientos epistemológicos y relaciones entre lectores y escritores, que ponen en juego cuestiones de poder. (Artemeva, 2008; Bazerman, 1988; Freedman y Medway, 1994; Miller, 1984; Russell, Lea, Parker, Street y Donahue, 2009).

Si se sigue esta concepción, los modos en que se lee y escribe en las disciplinas (y no sólo los tipos de texto) han de formar parte del saber a enseñar en las asignaturas, en tanto le cabe a cada docente -con apoyo de la institución educativa- la responsabilidad de ayudar a los alumnos a ejercer las prácticas de interpretación y producción de textos necesarias para participar en su materia ${ }^{4}$.

De igual manera, entendemos que las prácticas de estudio características de un determinado campo del saber deben enseñarse en las asignaturas, es decir, trabajarse y realimentarse. Las formas de estudiar dependen sutilmente de lo que ha de estudiarse: no se lee, estudia y prepara un examen de la misma manera en matemática que en historia, en biología, en psicología o en literatura. Por tanto, para aprender a hacerlo, los alumnos necesitan orientación de los profesores de esas asignaturas. Igualmente, para tomar buenos apuntes en clase, resumir un texto de una materia, o elegir qué información de internet es confiable, de calidad y relevante, se requiere la ayuda de quien conoce el tema para saber qué datos jerarquizar, qué conceptos seleccionar y retener de las clases, de los libros o de internet. Este quehacer letrado no podría ser impartido en forma general por un especialista (por ejemplo, un profesor de Letras, de Educación o de Psicología), quien difícilmente sabría distinguir lo importante de lo secundario en dominios de conocimiento alejados de su área disciplinar (Biggs en Chalmers y Fuller, 1996; Torres, 2009).

Ahora bien, estos usos de la lectura y la escritura vinculados con la actividad de estudiar han sido descritos como "estrategias de aprendizaje" (Castelló, 2000), como procedimientos constitutivos de la actividad intelectual que ha de llevar a cabo el alumno para convertir en conocimiento propio aquello que le es enseñado. En este sentido, las investigaciones señalan que ciertas tareas de lectura y escritura ponen en marcha procesos cognitivos que inciden en cómo los alumnos elaboran y se apropian de la información recibida. En ausencia de esta acción cognitiva, no ocurre un verdadero aprendizaje, sino que el estudiante apenas logra asir una información superficial, que no perdura más allá del momento de la evaluación. El aprendizaje duradero y productivo se alcanza sólo en la medida en que el alumno relaciona la información nueva con sus conocimientos preexistentes. Lo que interesa aquí es destacar que determinados usos de la lectura y la escritura conllevan una actividad cognoscitiva que favorece esta puesta en relación. De acuerdo con las condiciones en que se realicen, las prácticas de estudio pueden o no resultar herramientas epistémicas, es decir, instrumentos semióticos que cambian las condiciones de trabajo de la mente humana e inciden en la construcción del propio conocimiento (cf. Bazerman, 2009). Las investigaciones sobre "leer y escribir para aprender" (Langer, 1986) y sobre la potencial función epistémica de la escritura (Scardamalia y Bereiter, 
1985) subrayan que no cualquier tarea de producción o comprensión de textos impulsa la elaboración del conocimiento, por lo cual diferentes propuestas de aula que incluyen leer o escribir generan variable actividad cognitiva en quien las lleva a cabo, y afectan de forma diferente los aprendizajes conceptuales que logra realizar a partir de ellas (Bangert-Drowns, Hurley, y Wilkinson, 2004; Langer y Applebee, 1987). En este sentido, Carter, Miller y Penrose (1998) señalan que escribir debe ser reconocido en las clases como un proceso intelectual, un "método" para pensar y establecer relaciones, lo cual contrasta con la consideración usual de la escritura como producto textual. Teniendo en cuenta lo anterior, el presente estudio apuntó a recabar datos sobre las funciones que los profesores de IFD atribuyen a la escritura y lectura en relación con el aprendizaje de las materias que imparten, y relevó los usos que suscitan de ellas en sus materias. Se indagó pues en qué medida las condiciones en que se plantea leer y escribir en las asignaturas permiten a los alumnos emplear las tareas propuestas como instrumentos de aprendizaje.

\section{El aprendizaje}

La concepción de aprendizaje en la que se basa esta investigación conjuga el constructivismo piagetiano, el socioconstructivismo vigostkyano-wertschiano y las corrientes de "aprendizaje situado", en oposición a la idea de aprendizaje como recepción pasiva y como tarea solitaria y descontextualizada. Estas corrientes muestran que para lograr aprender un sistema de conceptos, es decir, para volver propio un saber conceptual que inicialmente es ajeno, el alumno debe operar sobre la información que le llega, debe re-elaborarla, procesarla, digerirla, en fin, transformarla, para volverla compatible con el conocimiento que ya tiene. En este proceso, los conocimientos preexistentes del aprendiz se ven modificados, recíprocamente, por el nuevo saber que incorpora.

Ahora bien, esta doble acción transformadora -denominada por Piaget asimilación y acomodación- no ocurre en un alumno aislado e independiente de sus pares y de su docente. Por el contrario, la acción cognitiva del alumno está impulsada, generalmente, por el trabajo compartido y la confrontación de ideas dentro de un grupo, con integrantes que disponen de distintos conocimientos.

Por su parte, para entender cómo se aprenden las prácticas de estudio vinculadas con cada disciplina resulta útil recurrir a las nociones de comunidad de práctica, de participación y de aprendizaje guiados. Estas nociones, puestas en relación con el concepto de género (Artemeva, 2008), permiten conceptualizar el desafío que enfrentan los estudiantes del nivel superior al intentar estudiar las materias que cursan: la lectura y escritura requeridas para participar en las diversas asignaturas y carreras implican para ellos adentrarse en comunidades de prácticas especializadas, de las que permanecerán excluidos a menos que sus miembros (los profesores) estén dispuestos a compartir sus saberes (sus modos de hacer para entender lo que leen, sus procedimientos para escribir sobre sus temas).

Si se tienen en cuenta estas nociones, es preciso reconocer que aprender conceptos y aprender prácticas lleva tiempo, más tiempo que el que usualmente se cree. En ninguno de los casos alcanza con exponerse al saber -o al saber hacer- sino que se requiere transformar, poner en relación, jerarquizar, confrontar, participar, 
devenir, intentar, reajustar, persistir. Una enseñanza acorde a este principio debe prever estos tiempos y esta actividad del alumno.

La concepción de aprendizaje resumida en este apartado se refiere, así, a cómo se adquieren los conocimientos conceptuales de cada asignatura, por un lado, y los quehaceres prácticos vinculados con sus formas de leer y escribir, por el otro. Estas nociones han orientado la elaboración de la encuesta, que apunta a conocer en qué medida y de qué forma los docentes promueven la participación de los alumnos en clase, su acción cognitiva sobre los contenidos que imparten y el trabajo compartido de interpretación y producción de textos, necesario para cursar las materias.

\section{El alumno de la educación superior}

En consonancia con las ideas sobre aprendizaje desarrolladas arriba, es preciso explicitar cómo concebimos al alumno de la educación superior. Creemos necesario hacerlo para distinguir nuestro enfoque de la habitual suposición de que, por ser adultos, los estudiantes universitarios o terciarios deberían ser autónomos (Diment y Carlino, 2006; Estienne y Carlino, 2004). Este presupuesto se funda en una falacia: equiparar edad biológica con adquisición de los saberes necesarios para desempeñarse en forma autónoma en las prácticas de estudio propias del nivel superior. Desde esta usual suposición, los alumnos -por ser adultos- han de saber autorregularse en estas prácticas. Sin embargo, el problema de esta equivalencia estriba en que la maduración cronológica no garantiza haber aprendido a ejercer las prácticas de estudio correspondientes. Como hemos señalado, esas prácticas sólo se aprenden en situación de participar sostenidamente en ellas con guía de quienes están familiarizados. Si los alumnos no han ejercido ciertas prácticas de escritura y estudio distintivas de la educación superior, entonces no las habrán aprendido y difícilmente podrán ser autónomos en ellas. Siguiendo a Chanock (2001), reconocemos que la autonomía es un resultado de la participación cultural y no del crecimiento biológico. La autonomía no es un estadio madurativo que concierne a todo quehacer del adulto. Por el contrario, un adulto es autónomo en aquello sobre lo que tiene experiencia y no lo es respecto de las prácticas culturales de las que ha carecido. De este modo, los estudiantes de IFD sólo pueden ser autónomos en aquellas prácticas que hayan aprendido a ejercer en su experiencia previa. $Y$ serán dependientes en aquéllas que aún les quedan por aprender. Es probable que las formas de estudiar en la educación superior y los modos de leer y escribir disciplinares sean poco familiares para ellos. En este caso, precisarán de la heterorregulación de sus docentes más experimentados para que, poco a poco, logren aprender a participar en ellas y puedan prescindir de su guía y retroalimentación (Wertsch, 1978).

\section{La enseñanza}

La noción de enseñanza que supone este estudio atañe a todas las decisiones relativas a cómo el docente y la institución procuran favorecer el aprendizaje de los alumnos. Enseñar abarca, pero excede, la exposición del profesor. Incluye asegurar ciertas condiciones, crear determinados dispositivos y prever particulares intervenciones de los profesores para potenciar la adquisición de conocimiento por parte de los estudiantes. Tal como mencionamos en el apartado sobre El objeto de enseñanza, este 
conocimiento consiste tanto en el sistema de conceptos de una disciplina como en sus prácticas de uso del lenguaje. Enseñar por tanto no es sólo exponer conceptos sino ayudar a los alumnos a participar en esas prácticas al tiempo que el docente ofrece condiciones para que esta participación guiada sea posible.

Para pensar con qué criterios un profesor organiza e interviene en sus clases, resulta instructivo apelar a la Teoría de las Situaciones Didácticas, formulada inicialmente por Brousseau para la enseñanza de la matemática. Se trata de un modelo de las interacciones didácticas, que propone pensar la enseñanza como un proceso centrado en la producción de conocimientos en el entorno escolar. El modelo contempla dos tipos de interacciones necesarias para que los alumnos construyan conocimiento en forma guiada: "a) la interacción del alumno con una problemática que ofrece resistencias y retroacciones, que operan sobre los conocimientos [...] puestos en juego (sujeto-medio) y b) la interacción del docente con el alumno a propósito de la interacción del alumno con la problemática [...] (medio)" (Sadovsky, 2005: 19). Se postula la necesidad de crear un "medio", pensado y sostenido con una intencionalidad didáctica, para que ambos tipos de interacciones tengan lugar.

Según este modelo, para planificar un proceso de enseñanza es necesario prever situaciones que contemplen los dos tipos de interacciones. Por una parte, es preciso que el profesor organice en clase un medio en torno a una situación problemática para que los alumnos puedan interactuar con él. La necesidad de un medio está dada por varias razones. En primer lugar, para favorecer la interacción del alumno con una problemática que lo desafía. Asimismo, porque la relación didáctica va a extinguirse en el futuro, y en ese momento el alumno deberá hacer frente a situaciones problemáticas, desprovistas de intenciones didácticas. Finalmente, porque asumir, entender e implicarse en un problema genuino permite que la situación cobre sentido para el alumno, más allá de cumplir con la tarea escolar para obedecer al docente.

Por otra parte, en las clases, el alumno ha de interactuar con quienes representan el saber cultural. De este modo, tampoco puede faltar la interacción con el docente. Una visión de la enseñanza que se centre exclusivamente en los procesos de producción de conocimientos de los alumnos en interacción autónoma con un medio, sin las retroacciones de sus pares, ni la mediación de quienes representan el saber cultural (los docentes), estaría desconociendo el carácter social y cultural de la construcción de conocimientos escolares. Una de las funciones del docente es hacer que las respuestas a problemas particulares se inserten en un sistema organizado de conocimientos, que permite abordar cuestiones que van mucho más allá del contexto que las hizo observables, proceso que no se da de manera automática. El docente, por ser representante del saber, aceptará algunas producciones pero no otras que le resulten distantes del conocimiento que quiere instituir.

En este modelo teórico, las interacciones del tipo sujeto-medio, independientemente de la mediación del docente, se describen a través del concepto de situación adidáctica. En ella, el alumno pone en juego sus propios conocimientos pero también los modifica, rechaza o genera otros nuevos a partir de las interpretaciones que hace de los resultados de sus acciones con el medio (retroacciones del medio). 
Las interacciones alumno-docente se describen a través del concepto de contrato didáctico que, entre otras cuestiones, explica las regulaciones que realiza el profesor, teniendo como referencia tanto la clase como la disciplina o conjunto organizado de saberes: "El docente, por ser representante del saber [...] tolerará -aunque provisoriamente- algunas producciones pero no lo hará con otras que pueden parecerle muy alejadas de aquello que quiere instituir" (Sadovsky, 2005: 22). ${ }^{5}$

Como complemento de lo anterior, la concepción de enseñanza que manejamos considera que al profesor y a la institución educativa les caben otras responsabilidades coadyuvantes: contribuir al incremento de la confianza de los alumnos en sí mismos como aprendices, favorecer sus ganas de sostener el esfuerzo y perseverancia necesarios, y su entusiasmo por aprender individual y colectivamente. Estas cuestiones, vinculadas con la construcción de la autoimagen de los estudiantes y su percepción de autoeficacia (Bandura, 1987), a su vez se relacionan tanto con las interacciones que promueve el docente en las situaciones de enseñanza, como con la calidad de participación guiada que se les ofrece, tal como se desarrolla a continuación.

De acuerdo con las oportunidades de interacción que se promueven en una clase, las formas en que un docente organiza e interviene en las situaciones didácticas que propone pueden ser pensadas en un continuum que va del monólogo a un diálogo con multiplicidad de voces. En el primer polo, el profesor expone la mayor parte del tiempo y los alumnos escuchan predominantemente. En el segundo, los alumnos son alentados a participar y sus aportes son escuchados y retomados de algún modo por el docente, para ayudar a reformularlos, para matizarlos, o para legitimarlos. Aquí el intercambio se potencia por las diversas relaciones que se establecen entre el habla, la lectura y la escritura (se habla para escribir y leer, para planificar un texto o para abordar otro; se escribe para intervenir oralmente en clase y para elaborar y discutir lo leído) (Cartolari y Carlino, 2011). En la enseñanza dialógica, la participación oral y escrita se interpenetran, lo mismo que el aporte de cada alumno y del profesor. Las interacciones entre pares enriquecen el diálogo entre alumnos y docentes (Dysthe, 1996; Wells, 1987). Si se vinculan estas nociones con las del párrafo previo, puede notarse que a mayor dialogicidad de las clases, mayor probabilidad de que los estudiantes se sientan tenidos en cuenta, valorados e incluidos $y$, por tanto, de que asuman con menor temor el riesgo de exponerse al participar. Esto, a su vez, llevaría a potenciar su interés, compromiso y tesón, en un entorno en el cual confían que les proveerá, a través del diálogo, las ayudas pedagógicas necesarias para aprender. Asimismo, si se relaciona la perspectiva de la enseñanza dialógica con la teoría de las situaciones didácticas, puede notarse que convergen en destacar la necesidad de participación activa de los alumnos y en reformular el rol del profesor como bisagra entre el saber disciplinar y el conocimiento producido por el estudiante, en vez de concebir al docente como transmisor directo del conocimiento.

En sentido convergente, para pensar cómo los docentes pueden ayudar a los alumnos a aprender los modos de leer y escribir propios de cada asignatura, este estudio abreva en las corrientes que impulsan ocuparse de estas prácticas desde todos los espacios curriculares. Nos referimos al movimiento escribir a través del currículum (WAC), vinculado con "escribir para aprender", y a escribir en las disciplinas (WID), relacionado con "aprender a escribir" (Bazerman, Little, Bethel, Chavkin, Fouquette y 
Garufis, 2005; Russell, 2002; Russell et al., 2009). Desde estos enfoques, se promueve incluir, orientar, y retroalimentar la lectura y la escritura dentro de cada materia, tanto por su potencial epistémico (porque leer y escribir pueden favorecer el aprendizaje de los contenidos a través de la participación activa de los alumnos), como por tratarse de usos del lenguaje con especificidades disciplinares, que no se aprenden sino situadamente (Artemeva, 2008; Freedman, 1994). Una idea afín desarrollada por Lerner (2001) es que las instituciones educativas y los docentes tienen la responsabilidad de enseñar los saberes prácticos implicados en los diversos modos de lectura y de escritura necesarios para participar en los diferentes ámbitos del mundo letrado. Esto incluye hacerse cargo de trabajar en clase los procesos de búsqueda y elaboración de información, de interpretación y producción de textos requeridos para aprender las asignaturas. La autora los denomina "quehaceres de lector y escritor" para subrayar que se trata de "contenidos" prácticos que como tales han de incluirse en la enseñanza. En coincidencia con estos enfoques, Carlino (2013), explicita dos objetivos de la alfabetización académica "que, si bien relacionados, conviene distinguir: enseñar a participar en los géneros propios de un campo del saber y enseñar las prácticas de estudio adecuadas para aprender en él".

Como desarrollamos más arriba, aprender a participar en una típica práctica de lenguaje en respuesta a situaciones retóricas similares, es decir, aprender un género discursivo, implica adquirir conocimientos que incluyen pero transcienden el conocimiento de los textos. Los alumnos han de desarrollar determinadas identidades (que involucran saberes sobre textos, quehaceres de lector y escritor, concepciones, valores y relaciones sociales) para lograr participar en estas prácticas de lectura y escritura. Según Prior y Bilbro (2011), el aprendizaje de los géneros disciplinares debe ser conceptualizado como parte de un proceso de enculturación académica. Esta idea también está presente en la metáfora del alumno como ingresante a una nueva cultura, con los desafíos de integración que esto supone y la necesidad de que los profesores den la bienvenida al recién llegado (Carlino, 2003, 2005). El proceso consiste en la incorporación paulatina de los estudiantes a las actividades de determinados grupos sociales en las que se utiliza el lenguaje (oral y escrito) de cierta manera, incorporación posibilitada por la mediación de los más experimentados.

Recíprocamente, entendemos que enseñar estas prácticas equivale al conjunto de decisiones e intervenciones del profesor que facilitan que los alumnos progresivamente ejerzan estas actividades, lo cual no se logra explicando verbalmente un conocimiento teórico o haciendo ejercitar porciones de ese saber fuera de contexto. La enseñanza fragmentada y no situada de ese complejo saber-hacer lo disuelve, lo desvirtúa, lo transmite carente de sentido, y por tanto, ha sido objetado como una transposición didáctica ilícita (Chevallard, 1997; Lerner, 2001), que convierte el saber práctico que originalmente se pretende enseñar en una serie de conocimientos declarativos cuya suma no da por resultado el aprendizaje que se pretendía lograr. $\mathrm{Ni}$ aprender a reconocer clases de textos, ni ejercitar el uso de conectores fuera de una tarea de escritura relevante para quien escribe, ni distinguir el texto del paratexto o hacer ejercicios de coherencia y cohesión sin un propósito de lectura o escritura que lo justifique, ayudan a aprender a participar en las prácticas que se pretende enseñar. En todo caso, pueden ayudar a tomar conciencia (conceptualmente) de ciertos aspectos de la lengua pero eso no equivale a aprender a 
hacer uso de este conocimiento en las situaciones en que podría resultar relevante (Carlino, 2013). Para ello, es preciso que los diseños curriculares y los profesores prevean tiempo didáctico para que los alumnos ejerzan las prácticas de lectura, escritura y estudio específicas de cada asignatura, con acompañamiento y enseñanza situada del docente para que puedan hacerlo.

La concepción de enseñanza en la que se fundamenta este estudio también presta atención al tiempo de clase que es preciso destinar para favorecer el aprendizaje de los estudiantes. Sabiendo que "el tiempo de asimilación del alumno (...) es un tiempo prolongado y sostenido en simultaneidad, al igual que el tiempo de producción del científico o del escritor, y francamente opuesto al tiempo escolar, caracterizado por la fragmentación, la disociación y la sucesión" (Castedo, 2011), es preciso que los docentes prevean formas de organizar las actividades de enseñanza para sostener el trabajo en profundidad sobre ciertos contenidos centrales de las asignaturas que imparten.

Por último, reconocemos que los procesos de enseñanza se dan dentro de un sistema didáctico enmarcado en contextos institucionales, que los condicionan. En este sentido, lo que manifiestan los docentes en la encuesta no debería ser entendido en términos de concepciones o prácticas idiosincrásicas, es decir, meramente individuales, sino como indicador de modos de hacer colectivamente esperados y de algún modo funcionales para la institución.

Teniendo en cuenta esta caracterización de las nociones que el estudio explora, la encuesta incluyó preguntas sobre cómo los profesores organizan la enseñanza para propiciar la participación efectiva de los alumnos, si se ocupan de acompañar las tareas de interpretación y elaboración de textos que requieren en sus materias y cómo lo hacen. También indagó sobre el grado de apoyo que los docentes reciben de su institución para llevar adelante esta agenda.

\section{Método}

La información se relevó a través de un cuestionario con 32 preguntas cerradas y abiertas, administrado mediante un formulario en línea. Considerando como universo de estudio a los docentes de distintas asignaturas que forman a los futuros profesores de nivel secundario, trabajamos con una muestra probabilística y estratificada, de alcance nacional, constituida por 50 instituciones de formación docente. Respondieron la encuesta en forma válida 544 profesores.

Además del tratamiento estadístico de los datos, realizamos un análisis cualitativo de los campos abiertos del cuestionario. Éste consistió en la lectura y relectura cuidadosa de todas las respuestas a cada pregunta abierta y en la posterior clasificación de las mismas en categorías, progresivamente refinadas. Se atendió a que esta categorización fuera exhaustiva, en el sentido de clasificar todas las respuestas de todas las unidades informantes, y que las categorías fueran excluyentes entre sí, por lo cual cada segmento de análisis de una respuesta se adjudicó a una y sólo una categoría. Las escasas respuestas que contenían información que podía corresponder a dos categorías (por ejemplo, si describían dos tipos de intervención del docente, en vez de un tipo) fueron segmentadas y esos segmentos fueron los categorizados. La 
paulatina construcción de las categorías, entonces, se realizó a partir de los datos mismos, enfocados según los marcos teóricos de los investigadores, puestos en relación con la propiedad o atributo a la que aludía la pregunta abierta. Ninguna categoría fue definida a priori sino que todas surgieron interactivamente de la lectura reiterada de los datos y los marcos teóricos del equipo de investigación. Para garantizar la confiabilidad de la categorización, se realizó la tarea entre las tres autoras, que debieron llegar a acuerdos en todos los casos. Una vez establecido el sistema de categorías y asignada cada respuesta a una categoría, se cuantificó el número de respuestas por categorías.

Así, los datos analizados en este artículo provienen de nuestra lectura reiterada de las respuestas a un campo abierto de la encuesta, en el que los encuestados describieron lo que hacen con relación a las dificultades de los alumnos para leer y escribir en sus materias. Intentando abstraer los rasgos distintivos de estas descripciones, obtuvimos más de una docena de códigos que fueron discutidos y reformulados progresivamente. Una vez establecidos los acuerdos interjueces, se procedió a un reagrupamiento de estos códigos en categorías más abarcativas que, a la vez de representar los rasgos empíricos comunes a los códigos absorbidos, fueran relevantes desde un punto de vista teórico según las opciones conceptuales del estudio.

\section{Resultados}

El ítem que analizamos en este trabajo aparece en el cuadro 1 y consta de 3 preguntas cerradas y una abierta.

Algunos alumnos de institutos de formación docente tienen dificultades para leer comprensivamente y escribir con claridad.

20 a) ¿Percibe usted este problema en la carrera donde enseña?

1. Sí

2. No

20 b) ¿Ha hablado con otros docentes de la institución sobre este problema?

1. Sí

2. No

20 c) ¿Se hace algo al respecto? (puede marcar más de una opción)

1. Sí, yo por mi cuenta

2. Sí, con otros colegas

3. Sí, a nivel institucional

4. No

20 d) Por favor, describa qué se hace 
Cuadro n.1: Secuencia de preguntas del cuestionario asociadas al ítem que analizamos en este artículo

Los profesores encuestados acuerdan en un 90,2\% percibir dificultades de los alumnos "para leer comprensivamente y escribir con claridad" y 93,1\% declara que "ha hablado con otros docentes de la institución sobre este problema". El análisis de la pregunta sobre si "se hace algo al respecto", en la que se podía marcar más de una opción, revela que 200 profesores (40,6\%) manifiestan trabajar lectura y/o escritura en su materia ("por mi cuenta"), $182(29,8 \%)$ refieren que se lo hace "a nivel institucional", $146(37,1 \%)$ "con otros colegas" y $47(9,6 \%)$ indican que "no se hace nada al respecto".

En lo que sigue, nos centramos en el análisis de lo que los docentes dicen hacer por su cuenta y dejamos para otra publicación los resultados correspondientes a quienes expresaron que se hace algo a nivel institucional o con colegas. Ahora bien, es preciso anticipar que, aunque el campo abierto del ítem pide describir qué se hace para afrontar las dificultades de los alumnos, las descripciones que ofrecen los profesores encuestados revelan algo más que una indicación de cómo enfrentan el problema. Resultan un medio indirecto de conocer si la lectura y la escritura están presentes en las aulas y cómo lo están. También permiten entender qué significa para los encuestados ocuparse de la lectura y la escritura en la enseñanza de sus asignaturas.

\section{Qué hacen los docentes por su cuenta}

De los 200 profesores que señalan que hacen algo por su cuenta, 153 especifican lo que realizan en su asignatura (el resto deja el campo abierto sin completar). De ellos, $43 \%$ manifiesta ocuparse de la lectura, $34 \%$ de la escritura y $22 \%$ de ambas. Un examen cuidadoso de lo que estos profesores describen que realizan en sus materias para encarar las dificultades que tienen los alumnos para "leer comprensivamente y escribir con claridad" permite distinguir dos modos básicos de intervención, que hemos denominado "Trabajo en los extremos" y "Trabajo durante" (véase cuadro 2).

Antes de detallar el tipo de respuestas englobadas en estas dos grandes categorías, conviene destacar que ambas se distinguen por cómo y cuándo interviene el profesor en las tareas de lectura y escritura que propone. En el primer modo, predominante, el docente interviene marginalmente en la lectura y escritura que realizan sus alumnos (al inicio o al final) en tanto que, en el segundo, el profesor interviene durante el transcurso de realización de estas actividades y les dedica tiempo de clase. Ambos modos también se diferencian por las concepciones que es posible inferir de las respuestas, acerca de las relaciones entre leer, escribir, aprender y enseñar en las asignaturas. Al final del trabajo caracterizamos estos modos de incluir la lectura y la escritura en las materias como "periféricos" y "entrelazados", respectivamente. 


\begin{tabular}{|c|c|c|c|c|}
\hline Categoría & Subcategoría & Ejemplo de respuesta & $\begin{array}{c}\% \\
\text { (Parcial) }\end{array}$ & $\begin{array}{c}\% \\
\text { (Total) }\end{array}$ \\
\hline \multirow{4}{*}{$\begin{array}{l}\text { Trabajo } \\
\text { en los } \\
\text { extremos }\end{array}$} & pide trabajo & $\begin{array}{l}\text { "Estimulo la lectura, pido trabajos en los que } \\
\text { exijo buena redacción posterior a la } \\
\text { comprensión del tema." (Formulario 1459, } \\
\text { Biología) }\end{array}$ & $\begin{array}{l}14 \% \\
(22)\end{array}$ & \multirow{4}{*}{$\begin{array}{c}47 \% \\
(N=71)\end{array}$} \\
\hline & $\begin{array}{l}\text { corrige } \\
\text { producto }\end{array}$ & $\begin{array}{l}\text { "Insisto en que los alumnos se expresen tanto } \\
\text { en forma oral y escrita en clase, para poder } \\
\text { observar y corregir los errores que surgen." } \\
(345, \text { Matemática) }\end{array}$ & $\begin{array}{l}14 \% \\
(21)\end{array}$ & \\
\hline & da técnicas & $\begin{array}{l}\text { "Posibilitar la adquisición de técnicas de lectura } \\
\text { y comprensión" (1517, Psicología) }\end{array}$ & $\begin{array}{l}10 \% \\
(15) \\
\end{array}$ & \\
\hline & da pautas & $\begin{array}{l}\text { "Guías de orientación para la lectura de textos } \\
\text { académicos." (1287, Lengua) }\end{array}$ & $\begin{array}{c}9 \% \\
(13)\end{array}$ & \\
\hline \multirow[t]{2}{*}{$\begin{array}{l}\text { Trabajo } \\
\text { durante }\end{array}$} & $\begin{array}{l}\text { trabaja en } \\
\text { clase }\end{array}$ & $\begin{array}{l}\text { "Dentro de la bibliografía selecciono algunos } \\
\text { apartados para ser leídos y trabajados, } \\
\text { explicados en clase. Doy consignas de trabajo } \\
\text { que promuevan la comprensión lectora" (269, } \\
\text { Educación) } \\
\text { "Ocupo mucho tiempo en la comprensión de la } \\
\text { situación problema y poco en la solución } \\
\text { analítica (aplicar formulación). Esto lo } \\
\text { propongo de la siguiente manera: dado un } \\
\text { enunciado discutimos su contenido. Dado el } \\
\text { esquema de una situación problema deben } \\
\text { escribir el enunciado." (289, Física) }\end{array}$ & $\begin{array}{l}30 \% \\
(46)\end{array}$ & \multirow[t]{2}{*}{$\begin{array}{c} \\
\\
37 \% \\
(N=57)\end{array}$} \\
\hline & $\begin{array}{l}\text { trabaja en } \\
\text { clase - proceso } \\
\text { interactivo }\end{array}$ & $\begin{array}{l}\text { "Redactan consignas y se las pasan a otro } \\
\text { compañero para saber si es clara o si realmente } \\
\text { responden a lo pedido. Se trabaja con } \\
\text { redacciones, comentando tanto la corrección en } \\
\text { los conceptos como en la forma de expresarlos, } \\
\text { y también con exposiciones orales, ya que no } \\
\text { sólo tienen dificultades para escribir con } \\
\text { claridad, sino también para expresarse } \\
\text { correctamente en forma oral." (168, Geografía) }\end{array}$ & $\begin{array}{l}7 \% \\
(11)\end{array}$ & \\
\hline Otros & & $\begin{array}{l}\text { "Ofrezco un tiempo para el asesoramiento } \\
\text { sobre los temas cuya lectura no se } \\
\text { comprende."(583, Educación) }\end{array}$ & & $\begin{array}{c}7 \% \\
(N=11)\end{array}$ \\
\hline Impreciso & & $\begin{array}{l}\text { "Se desarrollan tareas específicas para mejorar } \\
\text { la situación." }(29, \text { Historia })\end{array}$ & & $\begin{array}{c}9 \% \\
(N=14)\end{array}$ \\
\hline Totales & & & & $\begin{array}{c}100 \% \\
(N=153)\end{array}$ \\
\hline
\end{tabular}

Fuente: Elaboración propia

Cuadro n.2: Qué declaran los docentes que hacen por su cuenta acerca de la lectura y la escritura en sus materias

\section{Trabajo "en los extremos"}

El primer modo de acción docente, al que denominamos "Trabajo en los extremos", abarca al $47 \%$ de las respuestas de los encuestados que describen qué hacen por su cuenta. Se caracteriza porque el docente suele intervenir al inicio de los procesos de lectura o escritura (requiriendo trabajos, dando pautas, enseñando técnicas) y/o al final (corrigiendo las producciones de los estudiantes). Es decir, el profesor solicita o 
pauta una tarea, explica una estrategia o técnica de estudio, o bien corrige lo hecho por sus estudiantes, pero no interviene en el transcurso entre ambos extremos sino que los alumnos realizan la tarea por su cuenta.

Así, las respuestas categorizadas como "trabajo en los extremos" revelan un docente que contempla la lectura y la escritura en su materia pero no prevé que su intervención sea necesaria más allá de los "márgenes exteriores" (al inicio o al final) de la actividad que propone. Lo que ocurre "en medio", es decir, durante las tareas de lectura o escritura, es responsabilidad de los alumnos. Los docentes encuestados cuyas respuestas se agrupan en esta modalidad probablemente suponen que su intervención al comienzo o fin es suficiente para regular el desempeño de los estudiantes. En cambio, parecen no concebir que los alumnos puedan necesitar orientaciones o realimentaciones durante el proceso. Desglosamos a continuación las subcategorías que integran esta modalidad, a través del análisis de algunos ejemplos. En el cuadro no 2 se especifican los porcentajes de cada una.

\section{Pide trabajo}

Esta subcategoría agrupa las respuestas en las que el docente declara requerir a sus alumnos tareas de lectura y/o escritura. En las siguientes respuestas, hemos destacado los verbos que utiliza, para resaltar que se trata más de una exigencia que de un trabajo guiado por el profesor:

"A partir de lo planteado en clase, a nivel teórico, se solicita a los alumnos la presentación de producciones escritas donde fundamenten ideas del autor, comparen ideas de distintos autores, consulten bibliografía y escriban textos argumentativos sobre diferentes ideas o conceptos, analicen fuentes históricas a partir de enunciados personales." (191, Historia)

"Doy trabajos para que realicen en pequeños grupos donde tienen que interpretar la bibliografía dada y hacer un resumen no textual, sino en el que se note una comprensión de lo leído." (515, Biología)

"Les doy trabajos para desarrollar diferentes temas a fin de que vayan desarrollando sus capacidades tanto en forma escrita como oral, por ejemplo, presentando sus trabajos al resto del grupo." (337, Psicología)

Este último ejemplo hace visible la idea, aparentemente común en estas respuestas, de que asignar esta clase de tareas contribuye a que los estudiantes desarrollen sus capacidades; no aparece en cambio mención a otro tipo de mediación del profesor, que podría incidir en este "desarrollo". En síntesis, la subcategoría "Pide trabajo" muestra a un docente que propone a sus alumnos tareas de lectura y/o escritura pero que, tal vez en virtud del supuesto de que se trata de habilidades generales ya aprendidas en la escolaridad previa, no se ocupa de acompañar (guiar, realimentar) la consecución de estas tareas. Interviene, pues, en el inicio de la tarea al solicitarla.

\section{Da técnicas}

Esta subcategoría reúne las respuestas que expresan enseñar "técnicas de estudio", como un contenido separado de los contenidos disciplinares, antes y/o en forma 
independiente de que los alumnos se enfrenten al desafío de leer o escribir un texto para la asignatura. Se enseña un saber general con la expectativa de que luego, por su cuenta, los alumnos puedan "aplicarlo" a situaciones particulares. En los ejemplos siguientes, los verbos destacados en negrita revelan que el profesor explica la técnica. En cambio, no se hace mención a que ayude a implementarla o trabaje a partir de las dificultades que pudieran surgir durante su empleo, cuando los estudiantes leen o escriben en una situación específica de la asignatura:

"Explico las estrategias de comprensión de texto." (114, Historia)

"Facilito técnicas de estudio." (1444, Historia)

"Doy técnicas de estudio y de análisis y comprensión de texto. Sugiero tener un diccionario a disposición." (226, Antropología)

"Aplico técnicas de lectura comprensiva" (251, Historia)

"Proponer actividades que favorezcan las macro habilidades de leer, escuchar, hablar y escribir para comprender y producir textos orales y escritos". (306, Matemática)

Estas respuestas serían una muestra de que la lectura y la escritura son concebidas como habilidades, es decir, destrezas generalizables, "no relacionadas de modo específico con cada disciplina", que podrían aprenderse "fuera de una matriz disciplinaria" (Russell, 1990: 53) y utilizarse luego en toda situación. En cambio, las investigaciones citadas en nuestro marco teórico conceptualizan la lectura y la escritura como prácticas sociales imbuidas de los propósitos, de los valores y de los conocimientos de quienes participan en ellas, y señalan que sólo se aprenden situadamente (Artemeva, 2008; Russell y otros, 2009).

En resumen, las respuestas categorizadas como "da técnicas" evidencian que el docente interviene en el "extremo inicial" de la tarea, al enseñar la técnica. La puesta en práctica del conocimiento que podría aprenderse en esta enseñanza queda a cargo de los alumnos, como si les resultara evidente saber cómo implementarlo y como si estudiar consistiera en "aplicar" las técnicas, cual formas vacías que pueden emplearse para cualquier texto y situación.

Según Chalmers y Fuller (1996), este tipo de enseñanza ha sido probada como inefectiva ya que sólo se ocupa de transmitir un saber declarativo pero omite enseñar el necesario saber procedimental para que los estudiantes aprendan cómo usarlo recontextualizadamente en las diferentes circunstancias en que precisarían hacerlo. Del mismo modo, para Lerner (2001), de poco sirve que el docente comunique verbalmente este saber práctico. Al contrario, los caminos para desbrozar los textos, para ir comprendiéndolos y para estudiarlos deberían enseñarse como conocimientos en acción, a través de prácticas guiadas que permitan construir los saberes implicados en estas actividades conjuntas. Al tratarse de quehaceres del lector, es imposible aprenderlos como conocimientos declarativos ya que luego no habrá que decirlos sino ejercerlos.

\section{Da pautas}

En aquellas respuestas agrupadas como "da pautas", el docente encuestado manifiesta ofrecer orientaciones, a modo de instrucción inicial, para que los estudiantes realicen el trabajo solicitado. Explica qué espera del alumno en la tarea encomendada, brinda 
lineamientos, aconseja lo que se debería hacer. La diferencia entre esta subcategoría con la previa es que las indicaciones que se proveen al pautar una tarea son específicas para esa tarea y no generales como en las respuestas agrupadas en "da técnicas". La similitud entre ambas subcategorías es que en las dos el docente interviene con su orientación antes de que los estudiantes enfrenten la tarea:

"Cada vez que se propone una actividad de escritura, se ofrecen instrucciones y modelos de resolución para que sirvan como referencia."(936, Lengua)

"Lo que hago es: Recomendar la lectura en general; el releído y corrección de los trabajos que presentan, que lean o hagan leer a un familiar o amigo los trabajos realizados antes de entregarlos." (523, Química)

"Propongo claves para la lectura comprensiva, claves para poner en contexto los términos académicos propios del texto." (1232, Educación)

"Fundamentalmente intento describir con la mayor precisión lo que se espera del alumno." (1284, Biología)

Igual que en las subcategorías previas, en estas respuestas el docente describe sus acciones "al inicio" y no manifiesta acompañar a sus alumnos en las dificultades que puedan encontrar al emplear las pautas para la tarea dada. Así, las tres subcategorías hasta ahora tratadas consisten en intervenciones del profesor antes de que los alumnos lean o escriban. La siguiente subcategoría, por el contrario, reúne las intervenciones que se dan en el "extremo" opuesto, es decir, al final de estos procesos.

\section{Corrige producto}

Las respuestas agrupadas bajo la denominación "corrige producto" expresan situaciones en las que el docente interviene para señalar errores después de que los alumnos han leído o escrito. Estas respuestas consideran los productos por sobre los procesos porque, si bien algunas mencionan que los errores indicados pueden remediarse, la revisión aparece desintegrada del proceso de lectura o escritura y queda fuera del control del productor. Quien señala los errores es alguien distinto de quien los produce y debe subsanarlos; la revisión no se asume como tarea conjunta. La reconsideración de lo escrito o leído se realiza sólo si hay que enmendar faltas. No está contemplada como una labor epistémica que ayude a aprender la asignatura. En los siguientes ejemplos, nuestra negrita destaca que, desde el punto de vista de estos docentes, el trabajo sobre la producción o comprensión de textos se emprende para erradicar falencias del alumno y no como un modo de ayudarle a elaborar la información recibida o a desarrollar sus ideas. La corrección del docente opera sobre el producto más que sobre el productor:

"Remarco en los trabajos prácticos o parciales escritos cada error de ortografía o inconveniente en la redacción, y solicito lo revean, rehagan o reformulen, según cada caso." (456, Biología)

"Corrección de producciones escritas y fundamentación de la calificación para remediar falencias. [...]" (582, Lengua)

"Los trabajos prácticos corregidos pueden volverse a entregar luego de hacer todas las correcciones indicadas por la docente [...]" (272, Geografía)

"Corregir los escritos y dárselos para que los reescriban" (170, Matemática) 
"Se trata de realizar en clase actividades que se supervisan a fin de corregir errores de comprensión." (32, Psicología)

Como puede notarse en el cuadro previo, las dificultades de los alumnos para escribir o para entender lo que leen son expresadas en términos de errores o faltas eludibles y no como indicador de los desafíos inherentes a la tarea. Desvinculados del aprendizaje, los errores no se piensan como oportunidad para discutir y trabajar sobre ellos, o para confrontar interpretaciones, sino que impulsan la necesidad de remediarlos inmediatamente.

En síntesis, las cuatro subcategorías que constituyen el modo "trabajo en los extremos" se refieren a respuestas en las que los docentes encuestados manifiestan ocuparse de la lectura y/o la escritura en sus materias sólo en la periferia de estas actividades. Tal vez por falta de tiempo y por priorizar otros contenidos, o por concebirlas como habilidades ya aprendidas y no como prácticas situadas desafiantes, o en virtud de pensar que los alumnos deberían saber desempeñarse por sí solos, o debido a carecer de formación sobre cómo hacerlo, estos profesores no intervienen durante los procesos para "enseñar en acción" los quehaceres de leer y escribir para estudiar sus asignaturas. Como consecuencia de ello, la lectura y la escritura resultan actividades extrínsecas al aprendizaje de sus materias y difícilmente funcionen para sus alumnos como instrumentos de elaboración cognoscitiva.

\section{Trabajo "durante"}

Al segundo modo de trabajar con la lectura y/o la escritura lo denominamos "trabajo durante". Esta modalidad se diferencia de la anterior porque los encuestados describen ocuparse de los procesos de comprensión y/o producción escrita en sus clases, destinando tiempo didáctico a ello e interviniendo durante la tarea que proponen a fin de ayudar a los estudiantes para que puedan realizarla. A diferencia del modo "en los extremos", en la modalidad "durante", las tareas de lectura y/o escritura aparecen entrelazadas con el aprendizaje de los contenidos conceptuales de las asignaturas, es decir, los profesores se ocupan de la lectura y la escritura como un medio para que sus alumnos trabajen los temas propios de sus materias. Así, la producción y/o interpretación de textos tienden a aparecer integradas a la enseñanza y al aprendizaje de cada asignatura. Al destinar tiempo de clase a tareas vinculadas con leer y/o escribir, el docente tiene ocasión de observar cómo resuelven sus alumnos estas propuestas y qué dificultades presentan durante el proceso y no sólo en los productos. Estas respuestas representan al $37 \%$ de quienes expresaron que hacen algo por su cuenta y describieron qué hacen, y se distribuyen en dos subcategorías: "trabaja en clase" y "trabaja en clase-proceso interactivo". Cabe mencionar que estas subcategorías no se contraponen entre sí sino que las respuestas incluidas en "trabaja en clase-proceso interactivo" reúnen los rasgos de las respuestas categorizadas como "trabaja en clase" y, además, cumplen con algunos rasgos adicionales. En el cuadro no 2 se detallan los porcentajes relativos y a continuación se proveen ejemplos ${ }^{6}$.

\section{Trabaja en clase}

La mayoría de respuestas de la modalidad "trabajo durante" corresponden a la subcategoría "trabaja en clase". En los ejemplos siguientes, la negrita resalta que la 
lectura y/o la escritura se incluyen en las clases para ayudar a entender los contenidos propios de la materia. El uso de la primera persona plural también expresa una mayor implicación del profesor durante las tareas que propone a sus alumnos:

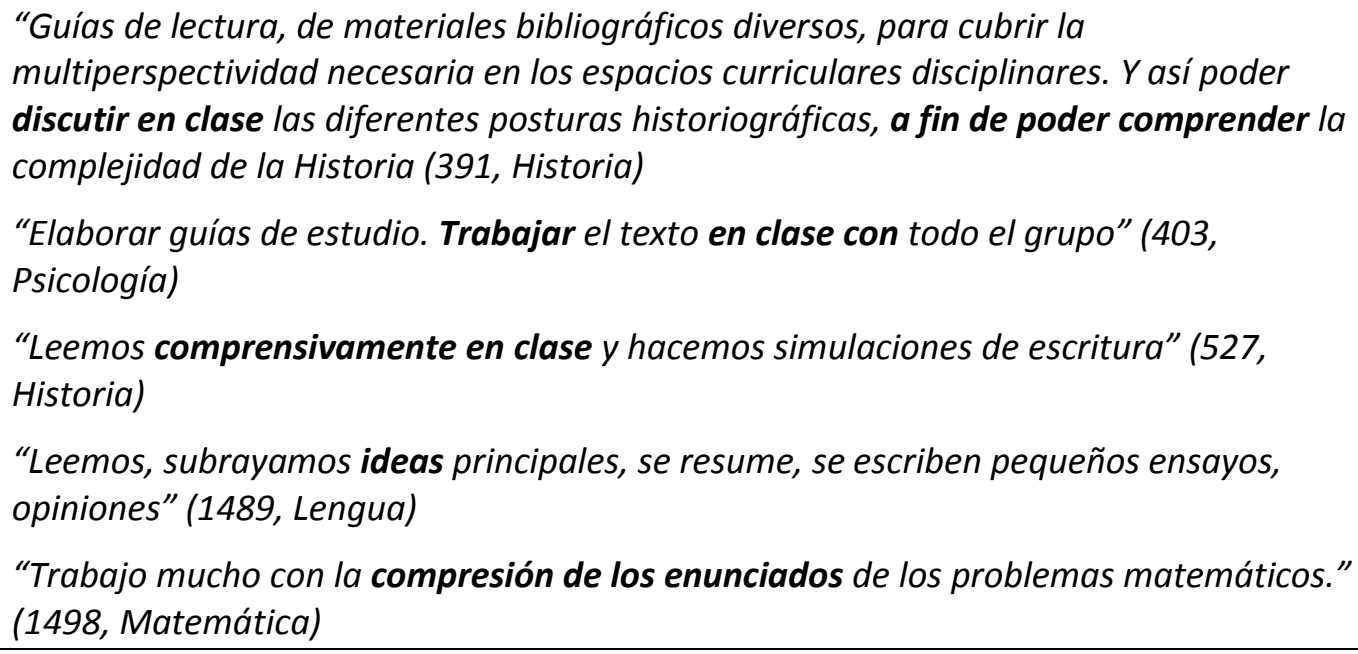

Trabaja en clase - proceso interactivo

Esta subcategoría agrupa las respuestas que comparten los rasgos de la subcategoría anterior ("trabaja en clase") pero en las que, además, los profesores indican explícitamente promover la interacción entre alumnos y docente. Es decir, la enseñanza se encara con un enfoque dialógico, contemplando un ida y vuelta recursivo entre lo que hacen los alumnos y lo que el profesor aporta.

Cuando se trata de tareas de lectura, los docentes declaran destinar tiempo de sus clases a leer y releer en forma conjunta, deteniendo la lectura para trabajar sobre la interpretación de algunos textos, tal como los siguientes ejemplos muestran:

\footnotetext{
"Leemos en conjunto, explicamos párrafos, explicamos con aportes de los alumnos..." (1196, Educación)

"Leemos en voz alta en el aula, tratamos de analizar cada término, verbalizan lo que entendieron, intercambian ideas explicándose, ayudándose entre ellos hasta llegar a lo correcto." (1174, Matemática)
}

Así, la lectura conjunta en clase se vuelve más analítica. Esta modalidad de lectura, en algunos casos, es acompañada por otras acciones docentes que colaboran en la comprensión de lo leído, como por ejemplo, la contextualización de los textos y de los autores que se leen, y el establecimiento de propósitos de lectura.

Del mismo modo, cuando se trata de tareas de producción escrita, los profesores destinan tiempo de clase a realizar revisiones y devoluciones de los trabajos escritos para realimentar las producciones. En algunos casos, también favorecen la interacción entre pares a través de la discusión sobre lo leído o por medio de comentarios sobre lo escrito:

"Redactan consignas y se las pasan a otro compañero para saber si es clara o si realmente responden a lo pedido. Se trabaja con redacciones, comentando tanto la corrección en los conceptos como en la forma de expresarlos" (168, Geografía) 
"En mi materia propongo un TP [trabajo práctico] de exposición de lecturas oral en el primer cuatrimestre y un TP escrito, con investigación bibliográfica y restricción de espacio. Suele tener varias reescrituras, con asesoría de compañeros y docente" $(408$, Psicología)

El trabajo en clase de este modo asegura que leer y escribir sean prácticas enseñadas y no sólo evaluadas:

"Me dedico con cada alumno en particular a hacer tareas de estado de avance en comprensión de textos y en reformulación de escritos. A veces leemos en clase, a veces escribimos en clase. Me preocupa realizar estas actividades, durante la cursada y antes de los primeros parciales para garantizar cierto éxito en las evaluaciones de la mayoría de los alumnos." (538, Biología)

Se nota en las respuestas previas un entrecruzamiento de interacciones: entre alumnos y docente, entre alumno y alumno, entre texto escrito y pensamiento, entre oralidad y escritura. De acuerdo con nuestros marcos teóricos, es esta multiplicidad de voces lo que promovería la elaboración y apropiación del conocimiento (Dysthe, 1996; Wells, 1990). Estas interacciones recursivas permiten, también, que los contenidos se aborden en forma menos instantánea y más sostenida, gracias a los tiempos que demanda detenerse en la interpretación y en la revisión conjunta de los textos. A los fines del aprendizaje, la lentificación del ritmo en que se avanza con nuevos temas, sumado al trabajo dialógico con ellos, ayudaría a que la información novedosa pudiera ponerse en relación con los conocimientos que ya tienen los alumnos. También favorecería que los estudiantes vinculasen unos temas con otros y los integrasen con lo que piensan, lo cual a su vez evitaría que fueran retenidos como datos inconexos e improductivos, sólo utilizados en el momento del examen.

Esta labor didáctica, que convierte a las aulas en un ámbito para la elaboración colectiva del conocimiento y no sólo para su transmisión, plantea desafíos curriculares que no desconocen los docentes encuestados, en cuanto al tiempo de las clases, la cantidad de contenidos y la carga de trabajo que demanda del profesor:

"También la corrección, la devolución, la re-escritura, el volver a corregir y devolver y así sucesivamente... pero lleva tiempos que no tenemos... Nuestras cargas horarias reconocidas por el sistema son los dos módulos frente a la totalidad del grupo." (339, Educación)

En síntesis, las respuestas categorizadas como "trabaja en clase - proceso interactivo" expresan, según nuestros marcos teóricos, las formas de organización de la enseñanza y de intervención docente que más ayudan a aprender porque implican sostener el trabajo sobre los contenidos por más tiempo y porque favorecen el establecimiento de relaciones entre ideas a través de entrelazar la lectura y escritura con la enseñanza de los conceptos disciplinares. 
Concepciones subyacentes a la enseñanza periférica ("en los extremos") y a la enseñanza entrelazada ("durante") de la lectura y escritura en las materias

A partir de las respuestas sobre lo que manifiestan los docentes acerca de lo que hacen en sus materias respecto del leer y escribir de sus alumnos, es posible inferir ciertas concepciones que estarían implícitas en esas prácticas declaradas. Entendemos las concepciones como ideas que "lleva[n] a formas particulares de acción social, [...] decisiones, [...] elecciones y omisiones" (Ivanic, 2004, p. 124).

Así, de la modalidad de inclusión periférica de la lectura y/o escritura ("Trabajo en los extremos"), en la cual leer y escribir reciben un tratamiento marginal (al inicio y/o al final de la tarea), podemos colegir que la producción e interpretación de textos son escasamente consideradas un objeto de enseñanza al interior de cada materia. La concepción subyacente a ello las entendería como habilidades independientes del aprendizaje de una disciplina. Es posible adjudicar a estas respuestas gran afinidad con la creencia cuestionada por Russell (1990): el supuesto de que escribir (y leer) son habilidades universalmente aplicables, no relacionadas con los contenidos, técnicas independientes de lo que se escribe y lee.

Esta enseñanza periférica también podría ser el producto de que el profesor no otorga al leer y escribir suficiente importancia respecto del aprendizaje de su asignatura y por ello no le dedica tiempo de clase. En este sentido, lectura y escritura serían pensadas como tareas de extracción o transcripción de un conocimiento ya dado (en el texto o en el alumno), cuya apropiación por parte de los estudiantes corre por carriles ajenos al leer y escribir. Leer sería sacar el significado que un texto contiene; escribir sería comunicar lo que quien escribe ha pensado/aprendido previamente. Asimismo, estos profesores encuestados considerarían que estas destrezas ya fueron aprendidas en la escolaridad previa y que por tanto son aplicables en cualquier situación posterior. Los alumnos, por haber llegado al nivel terciario, deberían ser autónomos y autorregular sus procesos de lectura y escritura. Ello justificaría el hecho de considerar que no es necesario acompañar con enseñanza las tareas de producción e interpretación de textos, sino sólo pautarlas y/o corregirlas.

Las nociones anteriores se asemejan a las que surgen de una investigación con entrevistas a profesores universitarios de diversas asignaturas (Carlino, 2010; véase asimismo, Carlino, 2008). Estas concepciones también se alínean con las que autores de otros países han puesto de manifiesto (Carter, Miller y Penrose, 1998; Chanock, 2001; Creme \& Lea, 1998; Lea y Street, 1998; Purser, 2012; Russell, 1990). Asimismo, resulta interesante comprobar que las respuestas que corresponden a la modalidad que denominamos "periférica" se aproximan a pensar la escritura y lectura como derivados secundarios de las situaciones de evaluación, lo cual ha sido constatado con frecuencia en ámbitos diversos y distantes del argentino, entre ellos, China:

"Los profesores de Lengua ponen énfasis en escribir porque la escritura es considerada una de las habilidades del lenguaje [...] pero no conceptualizan realmente la escritura como herramienta para enriquecer el aprendizaje y el pensamiento crítico [...]. Profesores en otras disciplinas emplean las tareas de escritura como instrumentos de evaluación y las consideran 'subproductos' de sus materias, no como 'escribir para aprender'” (Wu, 2012: 144). 
En contraposición, los profesores encuestados que proveen respuestas del tipo "Trabajo durante" podrían estar guiados por la idea de que leer y escribir son prácticas inherentes al aprendizaje de los contenidos disciplinares. En este sentido, dedicarían tiempo de clase al trabajo con la lectura y escritura porque éstas serían pensadas como actividades de elaboración (comprensión, clarificación, puesta en relación, integración, discusión, reformulación, etc.) del conocimiento implicado, es decir, actividades con poder epistémico. En consonancia con Carter, Miller y Penrose (1998), esta modalidad "entrelazada" daría cuenta de una concepción del escribir y leer como procesos intelectuales, instrumentos para pensar y establecer relaciones, lo cual contrasta con la consideración de la escritura como mero producto textual, y de la lectura como simple input o entrada de información.

Además, a estas respuestas que declaran que el profesor interviene durante las clases mediando la relación entre los alumnos y los textos dados para leer o para escribir podría adjudicárseles una concepción sobre el aprendizaje de la lectura y escritura como no acabado en ningún nivel educativo, aprendizaje que ha de realizarse al leer y escribir textos concretos en situaciones definidas y auténticas: "aprender a escribir como una realización social e intelectual en continuo desarrollo, ligada al aprendizaje disciplinar" (Russell, 2002: 9). En forma recíproca, las respuestas categorizadas como "trabajo durante" indican que los profesores implícitamente asumen la necesidad de enseñar a leer y escribir de modo entramado con la enseñanza de los conceptos disciplinares, para que sus materias sean aprendidas. Finalmente, estas respuestas darían cuenta de una visión de estudiante adulto que sigue necesitando la heterorregulación del profesor para producir e interpretar textos en cada asignatura.

\section{Conclusión}

En este trabajo, analizamos parte de los datos recogidos en un estudio a nivel nacional entre profesores de diversas disciplinas y carreras de las instituciones que forman a los futuros docentes secundarios argentinos. Partimos de detallar las opciones teóricas con las que nuestro equipo de investigación encaró la investigación, con el propósito de mostrar la complejidad de los referentes necesarios para comprender la enseñanza y el aprendizaje del leer y escribir en las asignaturas. Nos centramos en el análisis de uno de los campos abiertos del instrumento, que solicitaba describir qué se hace con relación a la lectura y escritura de los alumnos. De las respuestas examinadas, inferimos las concepciones sobre lectura, escritura, su aprendizaje y enseñanza, que podrían corresponder a las prácticas declaradas por los encuestados.

Casi la totalidad de quienes respondieron la encuesta perciben que los estudiantes tienen dificultades para leer y escribir en sus materias, y más de un tercio declara ocuparse de ello en su asignatura. Ahora bien, este panorama pudo ser precisado a partir del análisis cualitativo de las respuestas abiertas con respecto a qué hacen frente al problema constatado.

De los docentes que se ocupan del asunto en forma individual, predominan los que declaran intervenir periféricamente, al inicio o al final de la lectura y/o escritura que requieren, por medio de tareas que solicitan, pautan, corrigen o enseñan técnicas generales para ser luego aplicadas, pero no refieren acciones que acompañen la 
actividad lectora o escritora de los alumnos en torno a los contenidos disciplinares. Se trata por tanto de una inclusión marginal o poco integrada de la lectura y/o escritura en sus materias.

En contraste, un porcentaje menor describe ocuparse de la lectura y/o escritura durante sus clases entramando estas tareas con la enseñanza de su disciplina; son minoritarias las respuestas en las que también se expresa que se lo hace interactivamente entre lo que los docentes aportan y lo que hacen los alumnos. Estas intervenciones entretejen las orientaciones para leer y escribir con el trabajo sobre los contenidos propios de cada materia y por ello parte de la labor en clase es volver sobre, retomar, esos contenidos a través de la lectura y/o la escritura.

Es decir, la mayor proporción de las acciones que se realizan tienden a abordar la lectura y/o la escritura como un agregado en la periferia de la enseñanza. Son menos frecuentes las acciones que integran el leer y escribir para aprender los contenidos disciplinares $y$, al hacerlo, siguen enseñando estos contenidos por medio de la producción o discusión de textos, en vez de avanzar permanentemente con nueva información.

Si retomamos el interrogante al que en forma indirecta este ítem de la encuesta pretende aportar (¿de qué maneras la lectura y la escritura están presentes en las aulas, qué hacen los docentes con relación a ellas, qué significa para los encuestados ocuparse de la lectura y la escritura en la enseñanza de sus materias?), es posible afirmar que muchos encuestados, incluso más de los previstos según nuestra hipótesis inicial, se ocupan de la lectura y la escritura. Empero, "ocuparse" adquiere dos significados contrastantes. En buena parte de las respuestas, hace referencia a que los docentes realizan algo respecto de la lectura y la escritura que resulta de añadir a lo que enseñan ciertas acciones situadas en los márgenes de esa enseñanza. En cambio, un grupo menor de profesores se ocupan del leer y escribir en el transcurrir de sus materias y acompañan la lectura y la escritura como un modo de ayudar a elaborar los contenidos disciplinares. "Ocuparse" significa aquí destinar tiempo de clase e intervenir durante los procesos de interpretación y producción escrita. Así, uno de los significados de ocuparse corresponde a agregar un contenido (la lectura y/o la escritura); el otro equivale a entretejer ese contenido con el trabajo sobre los temas de las asignaturas.

Estos resultados son, a nuestro juicio, potencialmente útiles para pensar la formación docente y las políticas educativas del sector. Indican que es insuficiente incentivar la idea de que es necesario ocuparse de la lectura y la escritura transversalmente en la educación superior. Importa asimismo clarificarse que no da lo mismo el cómo, el modo en que es posible hacerlo.

Según los marcos teóricos enunciados al comienzo del artículo, lo que resulta más provechoso para el aprendizaje es entramar o tejer el trabajo sostenido de lectura y escritura con la enseñanza de cada materia al servicio del aprendizaje de sus propios contenidos, en vez de añadir o coser un contenido ajeno en el contorno de las asignaturas.

Ilustran este entramado las respuestas de los docentes que ven valioso discutir con los alumnos sus interpretaciones sobre los textos, aunque resulten alejadas de la que el profesor considera adecuada. Estos profesores, que hacen lugar al trabajo 
conjunto sobre la interpretación de lo leído, probablemente conciben que comprender los textos de su asignatura requiere algo más que su explicación. Por eso, están dispuestos a dedicar tiempo de clase al aporte de cada uno, a apreciar qué han entendido los alumnos para volver a explicar en función de ello o incluso porque valoran la confrontación entre pares como un medio de promover la transformación de sus ideas de partida. El trabajo que realizan sobre la interpretación de la bibliografía resulta una ayuda pedagógica imprescindible para que sus alumnos entiendan los conceptos de su materia y aprendan a leer sus textos.

Esta forma de ocuparse también ha quedado ejemplificada en los docentes que no devienen meros correctores de escritos, sino que orientan en el transcurso de la producción (y no sólo al final) para que los textos mejoren en los aspectos profundos o de primer orden (coherencia, pertinencia de los contenidos conceptuales, adecuación), además de en los denominados superficiales o de segundo orden de consideración (ortografía, gramática). Intervenir durante el proceso apunta, también, a que mejore el escritor y no sólo el escrito, y lleva a promover un uso epistémico de la escritura en vez de un uso comunicativo únicamente. De este modo, la escritura no se emplea aquí sólo como instrumento de evaluación y la intervención del docente no se queda en los bordes de la materia. Los profesores que dicen ocuparse de la lectura y la escritura según este modo entramado lo expresan en términos de hacerse cargo de enseñar su disciplina, sin convertirse, por ello y por un rato, en docentes de Lengua.

Añadir o coser un contenido foráneo en las materias no es igual, de cara al aprendizaje de los conceptos disciplinares, a entrelazarlo o tejerlo con ellos. Por tanto, las políticas educativas interesadas en mejorar la formación docente tienen ante sí un desafío de mayor envergadura que promover que las instituciones y los profesores se ocupen de la lectura y la escritura a lo largo y ancho de la formación. Resulta necesario que los modos de ocuparse también sean objeto de reflexión para que incluir la comprensión y producción escrita en las asignaturas contribuya al aprendizaje de los estudiantes.

Para concluir, quisiéramos hacer notar que las concepciones que inferimos de ambas modalidades de acción docente pueden considerarse parte constitutiva de ellas si se entiende, con Gee, el conglomerado de práctica y representación como "discurso". Estas concepciones interactúan con las prácticas logrando naturalizar lo que no es sino una alternativa entre otras. Según este autor, las ideas generan condiciones para que ciertas realidades sean concebidas como posibles o imposibles, a través de favorecer o volver impensables determinadas acciones:

Las ideas no sólo reflejan la "realidad" sino que parcialmente ayudan a crearla o constituirla [...] Las teorías fundan creencias, las creencias llevan a acciones y las acciones crean mundos (realidades) sociales (Gee, 1990: 8 y 23 ).

Por ello, porque prácticas y concepciones son pensadas aquí como caras de una misma hoja, cualquier rediseño curricular o acción formativa que se emprenda deberá, a nuestro juicio, ayudar a revisar a la vez unas y otras. 


\section{Referencias bibliográficas}

Artemeva, N. (2008). Toward a Unified Social Theory of Genre Learning. Journal of Business and Technical Communication, 22(2), 160-185.

Bandura, W. (1987). "Mecanismos autorreguladores" y "Autoeficacia”. En Pensamiento y acción. Fundamentos sociales. Barcelona: Ed. Martínez Roca. Edición original en inglés de 1986.

Bangert-Drowns, R., Hurley, M., \& Wilkinson, B. (2004). The effects of school-based writing-to-learn interventions on academic achievement: A meta-analysis. Review of Educational Research, 74(1), 29-58.

Bazerman, C. (1988). Shaping Written Knowledge. The Genre and Activity of the Experimental Article in Science. Madison (Wisc.): University of Wisconsin Press.

Bazerman, C., Little, J., Bethel, L., Chavkin, T., Fouquette, D. y Garufis, J. (2005). Reference Guide to Writing across the Curriculum. West Lafayette, Indiana: Parlor Press.

Bazerman, Ch. (2009). Genre and cognitive development: Beyond writing to learn. En Ch. Bazerman, A. Bonini y D. Figueiredo (2009). Genre in a Changing World. Perspectives on Writing (pp. 38-49). Fort Collins, Colorado: The WAC Clearinghouse and Parlor Press.

Bogel, F. Y Hjortshoj, K. (1984). Composition Theory and the Curriculum. En F. Bogel y K. Gottschalk (Eds.), Teaching Prose. A Guide for Writing Instructors (1-19). Nueva York: Norton.

Camps, A. (2003). Miradas diversas a la enseñanza y el aprendizaje de la composición escrita. Lectura y Vida, 24(4), 2-11.

Carlino, P. (2003). Leer textos científicos y académicos en la educación superior: Obstáculos y bienvenidas a una cultura nueva. Uni-Pluri/Versidad, 3(2), Universidad de Antioquia, Medellín, Colombia, 17-23. http://aprendeenlinea.udea.edu.co/revistas/index.php/unip/article/viewArticle/ $\underline{12289}$ (último acceso 25/02/13)

Carlino, P. (2005). Escribir, leer, y aprender en la universidad. Una introducción a la alfabetización académica. Buenos Aires: Fondo de Cultura Económica.

Carlino, P. (2008). Concepciones y formas de enseñar escritura académica: un estudio contrastivo. Signo y Seña, 16, pp. 71-117. Disponible en: https://sites.google.com/site/jornadasgiceolem/universidad (último acceso 25/02/13)

Carlino, P. (2010). Reading and writing in the Social Sciences in Argentine universities. En Charles Bazerman, Robert Krut, Suzie Null, Paul Rogers, Amanda Stansell (Eds.), Traditions of Writing Research. Oxford: Routledge / Taylor \& Francis, 2010 (pp. 283-296). https://sites.google.com/site/jornadasgiceolem/universidad (último acceso 25/02/13)

Carlino, P. (2013, en prensa). Alfabetización académica diez años después. Revista $\begin{array}{lllll}\text { Mexicana de Investigación } & \mathrm{n}^{\circ} & 57 .\end{array}$ http://www.comie.org.mx/v1/revista/portal.php 
Carter, M., Miller, C. \& Penrose, A. (1998). Effective Composition Instruction: What Does the Research Show? Center for Communication in Science, Technology and Management, Publication Series, № 3, April, North Carolina State University. http://www4.ncsu.edu/ crmiller/Etcetera/Effective Comp Instr.pdf (último acceso 25/02/13)

Cartolari, M. y Carlino, P. (2011). Leer y tomar apuntes para aprender en la formación docente: un estudio exploratorio", Revista Magis 4(7), 67-86. http://revistas.javeriana.edu.co/index.php/MAGIS/index (último acceso 25/02/13)

Castedo, M. (2011). Lenguaje y pedagogía para una educación inicial inclusiva "en" y "de" las diversas culturas escritas. X Congreso Nacional de Lectura. Fundalectura. Bogotá. Colombia. 4 de mayo de 2011.

Castelló, M. (2000). El conocimiento que tienen los alumnos sobre la escritura. En J. I. Pozo y C. Monereo (coord.), El aprendizaje estratégico (197-217). Madrid, Aula XXI Santillana.

Chalmers, D. y Fuller, R. (1996). Teaching for Learning at University. London: Kogan Page.

Chanock, K. (2001). From Mystery to Mastery, Actas de la Conferencia Australiana sobre Lenguaje y Habilidades Académicas "Changing Identities", Universidad de Wollongong, New South Wales, 29-30 de noviembre de 2001.

Chevallard, Y. (1997). La transposición didáctica. Del saber sabio al saber enseñado. Buenos Aires: Aique. Edición original en francés de 1991.

Creme, Ph. y Lea, M. (1998). Student writing: challenging the myths. Proceedings of the 5th Annual Writing Development in Higher Education Conference. Centre for Applied Language Studies, University of Reading, April 1-2, 1998. http://www.rdg.ac.uk/AcaDepts/cl/CALS/wdhe98/cremelea.html (último acceso 25/02/13)

Lea, M. y Street, B. (1998). Student Writing in Higher Education: An Academic Literacies Approach. Studies in Higher Education, 23(2), 157-172.

Diment, E. y Carlino, P. (2006). Perspectivas de alumnos y docentes sobre la escritura en los primeros años de universidad. Un estudio exploratorio, Memorias de las XIII Jornadas de Investigación en Psicología y Segundo encuentro de investigadores en Psicología del Mercosur "Paradigmas, Métodos y Técnicas", Facultad de Psicología de la Universidad de Buenos Aires, 10-12 de agosto de 2006, Tomo l, pp. 202-204. https://sites.google.com/site/jornadasgiceolem/universidad (último acceso 25/02/13)

Dysthe, O. (1996). The Multivoiced Classroom: Interactions of Writing and Classroom Discourse. Written Communication, 13(3), 385-425.

Estienne, V. y Carlino, P. (2004). Leer en la universidad: enseñar y aprender una cultura nueva. Uni-Pluri/Versidad, 4(3), Universidad de Antioquia, Medellín, Colombia, pp.

9-17.

Disponible

en: 
https://sites.google.com/site/jornadasgiceolem/universidad (último acceso 25/02/13)

Freedman, A. (1994). 'Do as I Say': The Relationship between Teaching and learning New Genres. En Genre and the new Rhetoric. London: Taylor and Francis.

Freedman, A. y Medway, P. (1994). Genre and the new Rhetoric. London: Taylor and Francis.

Gee, J. (1990). Social Linguistics and Literacies: Ideology in Discourses. London: The Falmer Press.

Gottschalk, K. y Hjortshoj, K. (2004). The Elements of Teaching Writing. A Ressource for Instructors in All Disciplines. Boston: Bedford/St. Martin's.

Haswell, R., Briggs, T., Fay, J., Gillen, N., Harrill, R., Shupala, A. Y Trevino, S. (1999). Context and Rhetorical Reading Strategies". Written Communication, 16(1), 3-27.

Ivanic, R. (2004). Discourses of writing and learning to write. Language and Education, $18(3), 220-244$.

Langer, J. (1986). Learning through writing: Study skills in the content areas. Journal of Reading, 29(5), 400-406.

Langer, J. y Applebee, A. (1987). How writing shapes thinking: A study of teaching and learning. Urbana, Illinois, National Council of Teachers of English.

Lerner, D. (1985). La relatividad de la enseñanza y la relatividad de la comprensión: Un enfoque psicogenético. Lectura y Vida. Revista Latinoamericana de Lectura, 6(4), 10-13.

Lerner, D. (2001). Leer y escribir en la escuela: lo real, lo posible y lo necesario. México: Fondo de Cultura Económica.

Miller, C. (1984). Genre as Social Action. Quarterly Journal of Speech, 70, 151-167.

Mitchell, S. (2010). Now you don't see it; now you do: Writing made visible in the university. Arts and Humanities in Higher Education, 9(2), 133-148.

Prior, P. y Bilbro, R. (2011). Academic Enculturation: Developing Literate Practices and Disciplinary Identities. En Montserrat Castelló y Christiane Donahue (ed.), University Writing: Selves and Texts in Academic Societies (Studies in Writing, Volume 24), Londres: Emerald Group Publishing Limited, pp.19-31.

Purser, E. (2012). Teaching Academic Writing at the University of Wollongong. En Christopher Thaiss, Gerd Bräuer, Paula Carlino, Lisa Ganobcsik-Williams y Aparna Sinha (Eds.), Writing Programs Worldwide: Profiles of Academic Writing in Many Places (pp. 55-68). Anderson, South Carolina: Parlor Press and Fort Collins, Colorado: The WAC Clearinghouse. 2012. http://wac.colostate.edu/books/wpww/

Purser, E., Skillen, J., Deane, M., Donohue, J., y Peake, K. (2008). Developing Academic Literacy in Context. Zeitschrift Schreiben. Disponible en: http://www.zeitschriftschreiben.eu/Beitraege/purser Academic Literacy.pdf

Rose, M. (1985). The language of exclusion. Writing instruction at the university. College English, 47(4), 341-359. 
Russell, D. (1990). Writing Across the Curriculum in Historical Perspective: Toward a Social Interpretation. College English, 52, 52-73.

Russell, D. (2002). Writing in the Academic Disciplines. A Curricular History. Carbondale and Edwardsville: Southern Illinois University Press.

Russell, D., Lea, M., Parker, J., Street, B. y Donahue, T. (2009). Exploring Notions of Genre in "Academic Literacies" and "Writing Across the Curriculum": Approaches Across Countries and Contexts. En Ch. Bazerman, A. Bonini, y D. Figueiredo (Eds.), Genre in a Changing World. Perspectives on Writing (pp. 401-429). Fort Collins, Colorado: The WAC Clearinghouse and Parlor Press.

Sadovky, P. (2005). La Teoría de las Situaciones Didácticas: un marco para pensar y actuar la enseñanza de la matemática. En Humberto Alagia, Ana Bressan y Patricia Sadovsky (2005), Reflexiones teóricas para la Educación Matemática. Buenos Aires: Libros del Zorzal.

Scardamalia y Bereiter, (1985). Development of dialectical processes in composition. En D. Olson, N. Torrance y A. Hildyard (Eds.), Literacy, Language and Learning. Cambridge: C.U.P.

Smith, F. (1988). Understanding Reading. Hillsdale, N.J.: Lawrence Erlbaum Associates.

Torres, M. (2009). Entrevista sobre Enseñar a estudiar. Las prácticas del lenguaje en contextos de estudio. Revista 12(ntes), 2(1), 1-4.

Wells, G. (1987). Apprenticeship in literacy. Interchange, 18(1/2), 109-123.

Wells, G. (1990). Creating the Conditions to Encourage Literate Thinking. Educational Leadership, 47(6), 13.

Wertsch, J. (1978). Adult-child interaction and the roots of metacognition. Quarterly Newsletter of the Institute for Comparative Human Development, 2(1), 15-18.

WU, D. (2012). Xi'An International Studies University. En Christopher Thaiss, Gerd Bräuer, Paula Carlino, Lisa Ganobcsik-Williams y Aparna Sinha (Eds.), Writing Programs Worldwide: Profiles of Academic Writing in Many Places (pp. 139-146). Anderson, South Carolina: Parlor Press and Fort Collins, Colorado: The WAC Clearinghouse. 2012. http://wac.colostate.edu/books/wpww/

\section{Notas}

${ }^{1}$ El presente trabajo se realizó en el marco del PICT 2010-893 con financiación de la ANPCyT de Argentina. Parte de los resultados fueron presentados en las Jornadas Nacionales Cátedra UNESCO de Lectura y Escritura "Lectura, escritura y aprendizaje disciplinar". Facultad de Ciencias Humanas, Universidad Nacional de Río Cuarto, 9-10 de septiembre de 2010.

${ }^{2}$ La investigación se realizó durante 2009-2010 con apoyo del Instituto Nacional de Formación Docente (INFD), dependiente del Ministerio de Educación de la Nación. Además de las autoras, integraron el equipo de investigación Leandro Bottinelli, Manuela Cartolari y Marta Marucco.

${ }^{3}$ GICEOLEM: Grupo para la Inclusión y Calidad Educativas a través de Ocuparnos de la Lectura y Escritura en todas las Materias. https://sites.google.com/site/giceolem2010/

4 Utilizamos el término disciplina para referirnos a una ciencia o campo de investigación. Usamos asignatura o materia para designar el espacio curricular dentro del sistema educativo que tiene por objeto enseñar una disciplina. La diferencia principal entre una y otras estriba en su propósito: 
producir conocimiento o acercar ese conocimiento a una población no especializada como los alumnos.

${ }^{5}$ Aunque el análisis de la pregunta de la encuesta analizada en este trabajo no retoma estos conceptos, otros ítems del instrumento informan en qué medida los profesores encuestados consideran las interacciones alumno-medio y docente-alumno al pensar las situaciones de enseñanza que dicen realizar.

6 El $16 \%$ de las respuestas restantes corresponden a "otros" (7\%) o son imprecisas o confusas y no permiten comprender qué hacen los docentes en sus clases (9\%).

Cita del artículo:

Carlino, P.; Iglesia, P.y Laxalt, I. (2013). Concepciones y prácticas declaradas de profesores terciarios en torno al leer y escribir en las asignaturas. Revista de Docencia Universitaria. REDU. Número monográfico dedicado a Academic Writing. Vol.11 (1) Enero-Abril. pp. 105-135. Recuperado el (fecha de consulta) en http://www.redu.net/

\section{Acerca de las autoras}

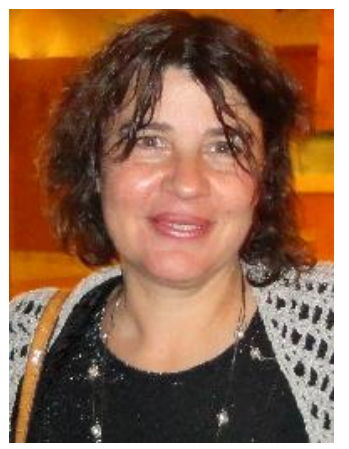

\section{Paula Carlino}

Universidad de Buenos Aires

Consejo Nacional de Investigaciones Científicas y Técnicas (CONICET)

Mail: paulacarlino@yahoo.com

Doctora en Psicología, Investigadora del CONICET en el Instituto de Lingüística de la Universidad de Buenos Aires. Dirige el GICEOLEM, equipo pluridisciplinar que investiga las relaciones entre enseñar, aprender, leer y escribir en diversas disciplinas del nivel secundario, superior y de posgrado. Entre sus libros, se destacan: Leer y escribir con sentido. Una experiencia constructivista en educación infantil y primaria (coordinado con D. Santana, Madrid: Visor, 1996); Escribir, leer y aprender en la universidad. Una introducción a la alfabetización académica (2005), distinguido como "Mejor Libro de Educación"; Lectura y escritura, un asunto de todos (coordinado con S. Martínez, 2009), declarado "de Interés educativo" por el Senado de la Nación Argentina; Writing Programs Worldwide: Profiles of Academic Writing in Many Places (co-editado con C. Thaiss y otros, 2012). Véase: https://sites.google.com/site/giceolem2010/ 


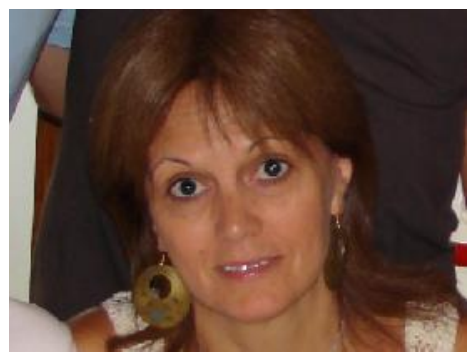

\section{Patricia Iglesia}

\section{Universidad de Buenos Aires}

Departamento de Biología

Mail: psmiglesia@gmail.com

Licenciada y Profesora en Ciencias Biológicas egresada de la Facultad de Ciencias Exactas y Naturales de la Universidad de Buenos Aires. Especialista en Docencia Universitaria de la misma universidad. Realizó estudios de posgrado en procesos de lectura y escritura, actualmente se encuentra elaborando la tesis correspondiente a la Maestría en Escritura y Alfabetización de la UNLP. Es Jefa de Trabajos Prácticos en la asignatura Biología del Ciclo Básico Común (UBA) y Profesora del Instituto de Formación Docente 174) de provincia de Buenos Aires. Forma parte del GICEOLEM. En los últimos años ha participado de diferentes espacios y publicado artículos que abordan el leer y escribir para aprender en asignaturas de ciencias naturales, en universidades e instituciones de formación docente. Entre ellos, es coautora de Writing to Learn Biology in the Framework of a Didactic-Curricular Change in the First Year Program at an Argentine University, accessible en http://wac.colostate.edu/books/wpww/chapter3.pdf

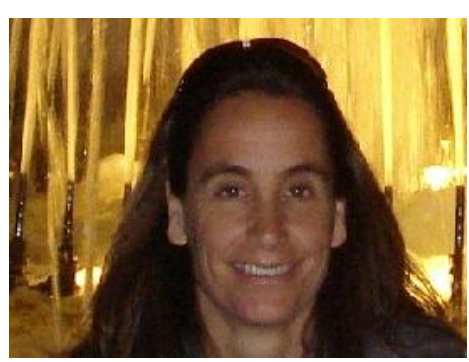

\section{Irene Laxalt}

Universidad Nacional del Centro de la Provincia de Buenos Aires

Facultad de Ciencias Humanas

Mail: irenelaxalt@gmail.com

Profesora en Ciencias de la Educación (Facultad de Ciencias Humanas, Universidad Nacional del Centro de la Provincia de Buenos Aires). Realizó estudios de posgrado en Educación y en Alfabetización y escritura. Actualmente se encuentra en etapa de revisión de tesis correspondiente a la Maestría en Educación (UNCPBA) y elaborando el Trabajo de campo para obtener el título de Especialista en Alfabetización y escritura (UNLP). Es Jefa de Trabajos Prácticos en asignaturas pertenecientes al Departamento Epistemológico- Metodológico. Ha participado de varios equipos técnicos que dan formación continua a maestros de escuelas primarias. Forma parte del GICEOLEM, equipo de investigación que analiza las relaciones entre la enseñanza, el aprendizaje, la lectura y la escritura en diversas disciplinas coordinado por Paula Carlino. 


\title{
¿Para qué se lee y se escribe en la universidad colombiana? Caracterización de prácticas de lectura y escritura en 17 universidades
}

\author{
What is read and written in Colombian university? Characterization of literacy \\ practices in 17 universities
}

\author{
Mauricio Pérez Abril \\ Pontificia Universidad Javeriana, Colombia \\ Alfonso Rodriguez Manzano \\ Universidad del Atlántico - Universidad del Norte, Colombia
}

\section{Resumen}

El propósito de la investigación ¿Para qué se lee y se escribe en la universidad colombiana? Un aporte a la consolidación de la cultura académica del país fue caracterizar las prácticas de lectura y escritura dominantes en la universidad colombiana. Se trató de un estudio descriptivo e interpretativo basado en datos de naturaleza cuantitativa y cualitativa. Se estudiaron 17 universidades. Se analizaron cinco fuentes: a) encuesta a estudiantes; b) programas de los cursos que las universidades ofrecen como apoyo para los procesos de lectura y escritura; c) documentos de política institucional; d) grupos de discusión de estudiantes, docentes e investigadores; y e) 17 estudios de casos de prácticas destacadas. Los resultados generales señalan que las universidades sí se ocupan de fortalecer los procesos de lectura y escritura académica, principalmente desde la oferta de cursos de lectura y escritura como competencias genéricas, mas no como prácticas epistémicas y disciplinares específicas. Los apuntes de clases son el texto más leído y escrito en la mayoría de las carreras, lo cual indica que para los estudiantes esa particular forma de apropiación del saber que la universidad distribuye es una prioridad, más que su uso en prácticas académicas y campos de aplicación. En el análisis de prácticas destacadas se encuentra que para estos docentes, leer y escribir son más efectos y condiciones de una postura sobre el conocimiento, que fines en sí mismos. En este artículo se presenta un resumen de la investigación más una selección y discusión de algunos resultados claves.

Palabras clave: Alfabetización académica, lectura, escritura, didáctica universitaria, cultura académica.

\begin{abstract}
The purpose of the research What is read and written in Colombian university? A contribution to the consolidation of the academic culture of the country was to characterize the literacy practices prevailing in Colombian university. It was a descriptive and interpretive study based on quantitative and qualitative data. We studied 17 universities. Five sources were analyzed: a) survey to students; b) courses that
\end{abstract}


universities offer to support reading and writing processes, c) institutional policy documents, d) focus groups of students, teachers and researchers; e) 17 case studies of outstanding practices. The overall results indicate that universities do dedicate to strengthening the processes of academic reading and writing, mainly offering literacy courses as generic skills, but not as specific disciplinary epistemic practices. The class notes are the most read and written text in most careers, indicating that for students that particular form of appropriation of knowledge which distributes college is a priority, rather than its use in academic and practical fields application. The analysis of outstanding practices shows that for these teachers, reading and writing are more effects and conditions of a position on knowledge than ends in themselves. This article is a summary of the research and a selection and discussion of some key results.

Key words: Academic literacy, reading, writing, university teaching, academic culture.

\section{Contexto}

La importancia de la lectura y la escritura en el desarrollo de un país y en la vida social y ciudadana no tiene discusión. De un lado, un país con débiles niveles de lectura y escritura difícilmente puede construir condiciones para una vida democrática sólida. De otro lado, la productividad científica y la pertenencia a la vida académica se vinculan de modo directo con las capacidades de lectura y escritura. De algún modo, formarse como ingeniero, como biólogo o como filósofo consiste básicamente en aprender a leer, escribir y pensar como tales. Los bajos índices de producción académica y científica de países como el nuestro pueden explicarse, al menos en parte, desde las características de las prácticas de la lectura y la escritura que se promueven en nuestras universidades.

Esta investigación ${ }^{1 .}$ se ocupó de caracterizar las prácticas de lectura y escritura universitarias desde una postura que toma distancia de la perspectiva dominante en los estudios realizados en el país. La tradición investigativa colombiana se ha centrado en estudiar variables relacionadas con las capacidades, dificultades y déficits de los estudiantes como ámbitos de interpretación (Henao \& Castro, 2001). Hay algunos estudios que se ocupan de asuntos curriculares y del análisis de las condiciones de contexto, la procedencia social y familiar. Los enfoques dominantes de estos estudios provienen fundamentalmente del campo de las ciencias cognitivas, de teorías psicolingüísticas y en algunos casos de la sociología. Son realmente pocos los estudios en los que el interés se haya centrado en el análisis de las prácticas pedagógicas que circulan en las aulas universitarias. Es decir, son muy ausentes las investigaciones realizadas desde el campo de la pedagogía y la didáctica universitaria. Los 54 investigadores, de las 17 universidades participantes que conformamos el equipo de esta investigación, coincidimos en optar por una perspectiva que se ocupara del análisis de elementos de orden pedagógico y didáctico, especialmente de la enseñanza como objeto de análisis (Camps, 2004), con el fin de ampliar la perspectiva investigativa nacional.

En este marco, el problema que se abordó en esta investigación fue el siguiente: la universidad promueve ciertos modos de leer y escribir, ciertas prácticas de lectura y escritura, en atención a un tipo de cultura académica que intenta favorecer. Detrás de dichas prácticas es posible leer la idea de cultura académica que circula en nuestro 
país. Esas prácticas, además de estar determinadas por las capacidades lectoras y escritoras de los estudiantes, por sus características socioculturales y sus trayectorias académicas, están marcadas de modo fuerte por las prácticas académicas de lectura y escritura que se propician en la formación universitaria y por los dispositivos didácticos y pedagógicos que configura y promueve.

Nos preguntamos entonces: ¿Cuáles son las características y las condiciones de posibilidad de las prácticas académicas de lectura y escritura en la universidad colombiana? ¿Cuáles de estas prácticas son valoradas positivamente por los estudiantes? ¿Cómo se explica la presencia de esas prácticas en algunos contextos universitarios? Estos interrogantes incluyen, en relación con las prácticas de enseñanza, otros como los siguientes: ¿Qué se pide leer al estudiante? ¿Para qué se pide leer y escribir? ¿Qué se hace con lo que se lee y se escribe? ¿Cuáles son los mecanismos de legitimación, valoración y evaluación de los productos de lectura y escritura en la universidad? ¿Qué clase de apoyos reciben los estudiantes antes, durante y después de la lectura y escritura de textos?

\section{Antecedentes y referentes}

Un referente clave de esta investigación es el concepto de alfabetización académica, pues constituye una de las prioridades actuales de la formación de los profesionales en las diversas disciplinas y universidades del mundo. Paula Carlino (2005), entre otros, ha documentado e investigado dicha problemática, especialmente en la cultura de lengua inglesa, y ha reconocido diversas modalidades a través de las cuales se aborda este asunto al interior de las universidades. Desde esos estudios es claro que en la actualidad a la universidad le corresponde trabajar sistemáticamente la lectura, la escritura y la oralidad "a través del curriculum" (Carlino 2005), es decir, al interior de las diversas asignaturas y espacios académicos. Esto implica un trabajo situado en los campos disciplinares particulares.

De otro lado, en este estudio asumimos que leer, escribir, hablar son prácticas que en la universidad cumplen una función epistémica (Olson, 1998), es decir, están ligadas a las formas de conocer y de aprender en un campo disciplinar específico, lo que implica además del dominio de una estructura de conceptos de la disciplina, la participación en una comunidad académica, unas prácticas particulares (unos modos de investigar, de publicar, de validar el conocimiento) y el dominio de unos géneros discursivos, también específicos.

La revisión de estudios desarrollados en Colombia sobre lectura y escritura académica en el ámbito universitario de los últimos 10 años señalan que el tema ha sido realmente tratado, a juzgar por el volumen y variedad de trabajos. En ellos se pueden reconocer varias líneas de investigación dominantes; una preferencia en cuanto al nivel educativo privilegiado y una serie de calificativos reiterados para denominar el desempeño encontrado en los estudiantes. De ellos también se derivan vacíos o necesidades de los que precisamente la investigación que aquí presentamos se ha ocupado. 
En el más reciente de ellos, Uribe-Álvarez y Camargo-Martínez (2011), a partir del análisis de unos cuarenta trabajos entre los que se cuentan bases de datos, memorias en páginas web y CD-ROM de congresos, coloquios y encuentros, señalan varias de las tendencias que serán confirmadas en los últimos trabajos consultados. La primera de ellas es el énfasis en los estudiantes como objeto de análisis, $77 \%$, sobre todo en los primeros y segundos semestres; mientras que para el caso de los docentes y las prácticas es de apenas el $23 \%$. Así mismo, se concluye que las perspectivas teóricas que predominan en los informes de investigación se refieren de forma mayoritaria al enfoque lingüístico textual y al análisis del discurso con un porcentaje del 60\%; le siguen el enfoque sociocultural con el $10 \%$ y el cognitivo con el $8 \%$. El constructivista y el semántico comunicativo cuentan apenas con el $5 \%$ y el $3 \%$ respectivamente. Finalmente, se señala que las problemáticas que abordan los investigadores se centran en tópicos tales como el diagnóstico sobre los procesos lectores y escritores (32\%), las dificultades que presentan los estudiantes para comprender y producir textos académicos (también 32\%); le siguen la preocupación por el uso apropiado de estrategias cognitivas y metacognitivas (15\%), el desarrollo de competencias $(10 \%)$ y, los aspectos actitudinales (8\%) y de motivación (3\%).

Asimismo, en un trabajo de revisión de 132 textos entre ponencias, artículos y libros denominado "El estado de la investigación en la enseñanza de la lectura y la escritura en la universidad colombiana y latinoamericana", Murillo (2009) señala que las líneas de trabajo más representativas son: la enseñanza de la lectura y la escritura a docentes, futuros docentes o docentes en ejercicio; la lectura y la escritura en disciplinas distintas de las Ciencias Humanas; las políticas institucionales; y la reflexión acerca del concepto de didáctica. La línea de trabajo acerca de la formación docente, según esta investigadora, está caracterizada por la preocupación por el diseño y evaluación de estrategias metodológicas que buscan la efectividad en los procesos de enseñanza de la lectura y la escritura; las formas metodológicas más reseñadas son los proyectos de aula y el seminario taller; se evidencia asimismo que los trabajos de investigación o las experiencias investigativas se realizan principalmente con los estudiantes de los primeros semestres para diagnosticar el estado de la lectura y la escritura con el que ingresan a la universidad. Según este estudio, los trabajos sobre la lectura y la escritura en las disciplinas consisten generalmente en propuestas de intervención pedagógica que buscan el mejoramiento de los procesos de enseñanza y aprendizaje de la lectura. El modo de organización discursivo que más se trabaja es la argumentación; los tipos de texto más recurrentes son el resumen, el ensayo y reseña; generalmente desde la lingüística textual, la semiótica discursiva y la psicología cognitiva. Los trabajos referidos a las políticas institucionales, a su vez, remiten a la implementación de una orientación remedial, el estudio de los contenidos no vistos en el bachillerato y el estímulo del placer de leer.

Un tercer estudio revisado señala que la mayor parte de esas investigaciones sentencian "las dificultades que presentan los estudiantes del ámbito universitario para comprender y producir textos académicos", y "las debilidades detectadas en buen número de diagnósticos que han precedido las propuestas de cambio y que siguen haciendo parte de las preocupaciones de las universidades". Este informe también señala la escasez de investigaciones sobre los factores pedagógicos y didácticos y la ausencia de trabajos acerca del papel del conocimiento previo, el uso de 
claves de recuperación o el papel de los esquemas en el desarrollo de competencias lectoras y escritoras (Camargo, Z., Caro, M., Castrillón, C. \& Uribe, G., 2008).

Un cuarto trabajo acerca la investigación de la lectura y la lectura durante los últimos 20 años (Ulloa, 2008), denominado "Lectura, escritura y conocimiento en la educación superior", coincide con el anterior en que esos estudios se han realizado desde la lingüística textual, la semiótica discursiva y la psicología cognitiva; se trabaja más la lectura que la escritura, sobre todo desde la noción de competencia; y muy poco la lectura y la escritura vistas como prácticas sociales, como expresiones de la cultura escrita, como instituciones sociales.

Esta constancia de la investigación de la lectura y la escritura en Colombia y su enfoque desde las disciplinas mencionadas, es también señalada por la profesora Murillo (2009) como un rasgo que diferencia la investigación colombiana de la realizada en otros países aledaños, como los del Cono Sur. En los últimos 30 años afirma en "El estado de la investigación en la enseñanza de la lectura y la escritura en la universidad colombiana y latinoamericana"-, los estudios han experimentado una tendencia hacia la contextualización, enfocando inicialmente los procesos cognitivos redaccionales para luego centrarse en las prácticas sociales e institucionales en donde éstos tienen lugar.

En Argentina, como en otros países de la Región -según esta investigadora-, el objeto de análisis se ha ampliado: de la descripción de las dificultades de los alumnos se estaría pasando a la indagación de las relaciones de ese aprendizaje con las cátedras o disciplinas universitarias.

En este mismo sentido, según la revisión de Giovanna Carvajal (2008), las dos tendencias investigativas que se observan son la lectura en sus procesos pedagógicos desde las tres disciplinas mencionadas y los trabajos hechos a partir del movimiento "Escribir a través del Currículo" (Carlino, 2004), ambas en general se orientan a diagnosticar los llamados problemas de lectura y escritura en el estudiante universitario colombiano. Carmen Arias (2008), en esta misma línea, reitera la constancia, para el caso de la capital del país, de las investigaciones acerca de las competencias comunicativas, la inclinación por los colegios de primaria y secundaria y el predominio de las metodologías cualitativas.

A pesar de la relevancia de estos trabajos, resulta evidente la ausencia de estudios desde la perspectiva pedagógica y didáctica, así como aquellos relacionados con las características de las prácticas de lectura y escritura que circulan en la universidad colombiana.

A continuación se expone una síntesis de la metodología empleada, con el fin de brindar un marco para comprender la selección de resultados que se presenta más adelante. Cabe aclarar que por la magnitud de la investigación, en este artículo se ha optado por elegir, poner en relación y discutir los principales resultados de las distintas fuentes. 


\section{Método}

En la investigación participaron 17 grupos de investigación del mismo número de universidades. Las 17 universidades eran de diferentes regiones del país, con diversidad de características en cuanto a tamaño (número de estudiantes y número de programas), ubicación geográfica y naturaleza (pública o privada). El estudio se realizó desde un enfoque mixto, con un diseño de carácter descriptivo e interpretativo que articula datos de tipo cualitativo y cuantitativo. Con el propósito de caracterizar las prácticas de lectura y escritura en las universidades, se decidió trabajar sobre cinco fuentes complementarias de información: a) una encuesta a estudiantes; b) los programas (syllabus) de los cursos que las universidades ofrecen como apoyo para los procesos de lectura y escritura; c) documentos de política institucional sobre lectura y escritura académica; d) grupos de discusión de estudiantes, docentes e investigadores; y e) 17 estudios de casos de prácticas destacadas de docentes: uno por universidad, excepto en una de ellas. A continuación reseñamos brevemente cómo se realizó la recogida y análisis de los datos para cada una de las fuentes. La selección de resultados que se presenta en este texto corresponde principalmente a las tres primeras fuentes, pero consideramos clave para el lector tener una panorámica general de la estructura de toda la investigación. Por esta razón exponemos la opción metodológica correspondiente a cada fuente.

\section{La encuesta a estudiantes}

La encuesta se diseñó a partir de la revisión de instrumentos e investigaciones nacionales e internacionales previas. Un referente internacional clave estuvo constituido por los estudios basados en encuestas, publicados por Bernard Lahire (2004). El instrumento se validó por juicio de expertos, se discutió en cada uno de los 17 grupos de investigación y se realizaron cinco pilotajes con estudiantes. El instrumento aplicado quedó conformado por 22 preguntas organizadas en cuatro secciones así:

- la primera recogió los datos que identifican al estudiante;

- la segunda contuvo preguntas de opción múltiple que indagaron, de acuerdo con la propia experiencia de la universidad donde estudian, sobre la presencia de la lectura y la escritura en actividades académicas, los tipos de documentos leídos y escritos, los idiomas y los propósitos de la lectura y la escritura en dichas actividades académicas;

- la tercera consistió en tres preguntas abiertas en las que se pidió el nombre del profesor y de la actividad académica que recuerdan como una experiencia significativa relacionada con la lectura y la escritura;

- la cuarta contuvo preguntas para conocer la presencia y las características de la práctica de lectura y escritura señalada por ellos como la más significativa, en una asignatura, cursada el semestre anterior. Se indagó aquí acerca de las actividades de lectura, los propósitos, los tipos de documentos leídos y las actividades que se gestionaron con ellos, así como el tipo de documento que 
escribió y la clase de apoyos a la escritura recibidos, lo que incluye la eventual revisión y la evaluación.

La muestra de estudiantes de cada universidad, para la aplicación de la encuesta, se construyó en atención al tamaño (número de estudiantes) de cada una, y en proporción al número de programas que se ofrecían, de tal modo que para cada universidad se pudiera contar con representatividad para cada una las agrupaciones de programas UNESCO ${ }^{2}$. Los estudiantes encuestados debían haber cursado más del $50 \%$ de las asignaturas de su programa durante el segundo semestre del 2008, pues nos interesaba que se hubiera recorrido un trayecto significativo del plan de estudios. Una vez definido el número de estudiantes por cada agrupación de programas, se estableció aleatoriamente a quienes debía ser aplicado el instrumento. Se trató de un muestreo aleatorio estratificado. Finalmente, la muestra quedó constituida por 3715 estudiantes de las 17 universidades colombianas de diferentes regiones del país, y es representativa a tres niveles: para el total de las universidades, para cada una de éstas y por agrupaciones de programas, según las áreas UNESCO, tanto a nivel general como por universidad. Los resultados que se presentan en este texto corresponden a datos generales agrupados, de todas las universidades y procesados desde la estadística descriptiva ${ }^{3}$. El margen de error fue del $5 \%$. El grado de confiabilidad de la encuesta, según el estadístico Alpha de Cronbach fue de 0.90 .

\section{Documentos de política institucional de lectura y escritura y análisis de programas (syllabus) de cursos de lectura y escritura}

Se identificaron los documentos oficiales en los que se plantean líneas de política institucional, criterios o disposiciones asociadas con las prácticas de lectura y escritura en la universidad. Igualmente se recopilaron los programas de los cursos que se ofrecen, en los que de manera explícita se hace referencia a la lectura y escritura en las 17 universidades. Los cursos podían ser ofrecidos desde el interior de las carreras o desde las facultades o departamentos de lingüística, de comunicación o lenguaje. Los programas fueron identificados en una búsqueda exhaustiva en los catálogos en línea y a través de cartas dirigidas a los decanos y directores de carrera y departamento. Se identificaron 415 programas (syllabus) diferentes de cursos de lectura y escritura ofertados por las universidades participantes. Se tomaron los años 2008 y 2009 como rango de tiempo en que se debían haber dictado los cursos. Es importante aclarar que realmente en las 17 universidades se atienden más de 415 grupos de estudiantes, pues un mismo programa se dicta para más de un grupo en cada semestre. Pero para la investigación interesaba estudiar los 415 programas de diferente nominación.

Respecto a los documentos de política institucional, es importante señalar que en muy pocas universidades se identificaron documentos en los que directamente se plantearan directrices al respecto. En algunos casos, los documentos no eran conocidos por el grupo de docentes ni, incluso, por los directivos. Es decir, no cumplían realmente una función regulativa. Por ello esta fuente permitió evidenciar la ausencia de una política institucional sobre lectura y escritura, al menos a nivel de los documentos. Si bien la fuente no cumplió la expectativa inicial planteada en el proyecto, se analizó el contenido de los pocos textos encontrados. 
Para el caso de estas dos fuentes documentales, los textos se procesaron desde una perspectiva inductiva para construir un sistema de categorías y descriptores, apoyados en la técnica de análisis del contenido (Bardin, 1986). Se conformaron subequipos de investigadores para proponer las categorías y descriptores, a partir de la lectura de una muestra de textos. Una vez se contó con una depuración de las categorías y descriptores, a partir de las deliberaciones de los sub-equipos de investigación conformados en el proyecto, se definió un instrumento, con su correspondiente glosario y manual de aplicación, que permitiera una descripción unificada de la heterogeneidad de los documentos al interior de cada universidad. La información derivada del procesamiento se consolidó en una base de datos y se procesó desde la estadística descriptiva. De este modo, se identificaron las tendencias, enfoques, propósitos, estrategias didácticas, resultados, tipos textuales abordados, etcétera, evidenciadas en los cursos que se ocupan de la lectura y la escritura en las diferentes universidades. Se utilizaron procedimientos y herramientas que buscaron garantizar criterios estandarizados en la recolección de información, procesamiento y unificación de la información como la base de datos Access, instrumentos y sus correspondientes los manuales y glosarios.

\section{Estudios de casos de prácticas destacadas}

Además de describir las prácticas existentes en las universidades, desde las fuentes señaladas, nos interesaba construir saberes derivados de experiencias destacadas, bajo el supuesto de que es posible tomar la práctica como fuente de saber, y de que existe una epistemología de la práctica que puede tomarse como fuente de conocimiento (Schön, 1992). Así, en este proyecto decidimos estudiar experiencias docentes que se destacaran por ser espacios en los que leer y escribir fueran prácticas vividas por los estudiantes como experiencias formativas importantes, para desde su análisis cualitativo en profundidad, reconocer los rasgos que las caracterizan.

Para seleccionar las experiencias definimos una matriz de criterios relacionados con la trayectoria de las mismas en el tiempo, la solidez conceptual, el nivel de reflexión adelantada por los docentes que la lideraban, el reconocimiento por parte de los pares como experiencia destacada y la existencia de elaboraciones escritas sobre ella; pero el criterio central consistió en que la experiencia fuera identificada por los estudiantes como una práctica destacada. Es importante señalar que el concepto de "destacada" en esta investigación es más un efecto del estudio, que una noción previa, pues precisamente el interés de la investigación consistía en identificar cuáles eran algunos rasgos que caracterizaban las prácticas que en las universidades son reconocidas como destacadas.

Metodológicamente procedimos de la siguiente manera: a) realizamos una relectura del estado de arte y de los conceptos claves de la investigación, con el fin de discutir los criterios de selección de las experiencias y los tópicos de análisis; b) decidimos como opción metodológica "los estudios de casos" debido, entre otras razones, a que permite la comprensión del fenómeno social estudiado desde diversidad de fuentes y desde las condiciones concretas de su existencia, igualmente porque permite, desde la interacción directa con los contextos, comprender su complejidad (Stake, 1999; Flyvbjerg, 2005; Yin, 2004) y, c) acordamos una estructura 
de tópicos comunes, mínimos, así como una guía para el análisis, con el fin de abordarlos en el estudio de cada uno de los casos.

Los criterios que tuvimos en cuenta para seleccionar definitivamente los casos estudiados fueron:

- Garantizar que la experiencia contaba con una trayectoria verificable, esto significa que se pudiera evidenciar que no se trataba de una experiencia coyuntural, que se hubiese implementado, por ejemplo, en una sola ocasión. Para verificar este criterio se debió acudir a otras fuentes.

- Que fuera reconocida por sus pares (docentes) como una práctica destacada.

- Que contara con materiales que permitieran su sistematización: documentación, registros audiovisuales, fotográficos, textuales, testimonios, etcétera.

- Que contara con escritos de reflexión, ponencias, etcétera, es decir, publicaciones referidas a la práctica.

- Que contara con una apuesta conceptualmente consistente.

- Que, si se trataba de una asignatura, se estuviera llevando a cabo preferiblemente con estudiantes que ya hubiesen cursado la mitad del programa (de quinta matrícula en adelante).

Una vez acordada la perspectiva metodológica para los estudios de casos, se definió una estructura mínima de categorías generales para el análisis y unos parámetros unificados para la elaboración de los informes correspondientes a cada universidad. Posteriormente se realizó un meta-análisis de los 17 casos, a partir de los informes, para acceder a unas conclusiones sobre los rasgos que caracterizan las prácticas como destacadas. Para postular los rasgos se realizó un análisis inductivo al interior de cada categoría.

\section{Grupos de discusión}

Como investigadores, en el transcurso de la investigación decidimos incluirnos como una fuente más de información, en la medida que la mayoría contábamos con trayectoria en el campo. La decisión fue optar por los grupos de discusión, desde el enfoque de Jesús Ibáñez (1979), como alternativa metodológica; esta decisión se tomó luego de análisis colectivos y discusiones sobre diversas perspectivas cualitativas. Como postura epistemológica para esta fuente, acogimos los planteamientos de Irene Vasilachis (2007), quien establece distinciones entre una epistemología del sujeto cognoscente y una epistemología del sujeto conocido, que en la tradición de la investigación suelen permanecer aisladas. En esta perspectiva, debe entenderse el sujeto conocido no sólo como proveedor de datos útiles, sino produciendo conocimiento en interacción con el sujeto cognoscente (Vasilachis, 2007).

Metodológicamente, decidimos trabajar con grupos de discusión, para incluirnos como equipo de investigadores (sujetos a la vez cognoscentes y conocidos) y como fuente clave de la investigación. Cabe señalar que las discusiones en el orden conceptual de la perspectiva de Ibáñez enriquecieron mucho la investigación y a la vez se convirtieron en un espacio valioso de formación conjunta. Igualmente las experimentaciones en el orden de lo metodológico nos dieron pistas claves para 
avanzar en los diseños de los grupos de discusión de estudiantes y docentes al interior de las universidades.

De manera muy esquemática podríamos decir que un Grupo de Discusión (GD) es un mecanismo de producción de discursos organizados, estructurados por un investigador que explora un fenómeno social específico. Desde esta postura, el investigador asume una función-sujeto: “él es el lugar en donde la información se transduce en significación (y en sentido). En las perspectivas empirista y formalista el investigador es una ecuación personal, un rozamiento, un ruido que hay que reducir: la información y el sentido no se comunican" (Ibáñez, 1979, p.34). La organización de la puesta en escena del GD obedece de modo directo y explícito a los intereses del investigador, quien configura la situación de intercambio verbal y está totalmente implicado. Así, el GD opera como una "máquina" de producción de discursos en atención a un plan trazado por el investigador.

En esta perspectiva, el investigador -en tanto sujeto que cuenta con un recorrido en un campo particular, pues ha investigado, reflexionado, problematizado, teorizado sobre el fenómeno que se está estudiando- se considera autorizado, legítimo (PérezAbril, 2009) para proponer una ruta de análisis, una hipótesis que guíe su exploración. Él mismo es la medida de su propia investigación.

Luego de definir las pautas teóricas y metodológicas comunes, los grupos de discusión que efectuamos fueron los siguientes:

- GD entre los investigadores del estudio. En diciembre de 2009 llevamos a cabo cinco grupos de discusión en los que los participamos todos los investigadores de los equipos de las 17 universidades. Dichos grupos de discusión fueron transcritos y analizados para ser retomados en las interpretaciones generales de resultados.

- GD con Docentes y Estudiantes de las universidades (uno para cada grupo poblacional). Cómo el propósito principal del análisis de esta fuente consistía en la visualización e interpretación de las prácticas en función de los campos de formación disciplinar, determinamos la importancia de que asistiera un estudiante o docente por cada área UNESCO, por lo que los grupos estuvieron conformados entre 8 y 10 sujetos. Como antes señalamos, para llevar a cabo estos grupos de discusión escribimos un documento explicativo que se constituyó en la pauta para efectuarlos, generando así condiciones de realización similares en las universidades y que en todos los casos se planteara la misma situación de apertura.

Los diálogos de todos los GD fueron transcritos por un único equipo de sociólogos expertos en análisis de datos cualitativos; los GD de docentes y estudiantes se enviaron a cada una de las universidades para su interpretación, en tanto que los desarrollados entre investigadores fueron analizados por un equipo interinstitucional, que se encargó de observar las distintas posturas, las semejanzas, las diferencias y las tensiones, en relación con los hallazgos de la investigación y las lecturas que los investigadores realizaron de éstos. 
Estas fueron las cuatro entradas metodológicas correspondientes a las cinco fuentes. Veamos a continuación una selección de los principales resultados de las diferentes fuentes de la investigación, las relaciones entre ellos y una discusión.

\section{Resultados}

En este artículo optamos por elegir algunos resultados relevantes de las diferentes fuentes, que ilustran las problemáticas claves tratadas en la investigación.

\section{Textos más leídos y razones por las que se leen}

Uno de los ítems centrales de la encuesta indagó por los materiales que más leen los estudiantes para responder a sus compromisos académicos. Los apuntes de clase son el texto al que más acuden. Recordemos que los encuestados eran estudiantes que habían cursado más del $50 \%$ de las asignaturas de la carrera. Para responder este ítem de la encuesta se les pidió tomar como referencia las asignaturas cursadas el semestre inmediatamente anterior.

\begin{tabular}{|l|c|}
\hline & \% \\
\hline Apuntes de clase propios & 83,68 \\
\hline Materiales elaborados por profesor & 79,78 \\
\hline Paginas web, blogs & 76,82 \\
\hline Libros o capítulos propios carrera & 72,79 \\
\hline Libros de consulta & 59,88 \\
\hline Resúmenes de libros o artículos & 53,99 \\
\hline Artículos científicos & 40,45 \\
\hline Documentos periodísticos & 39,23 \\
\hline Informes de investigación & 35,74 \\
\hline Apuntes de clase de otro compañero & 33,27 \\
\hline Literatura (novela cuentos) & 26,57 \\
\hline Otro & 1,94 \\
\hline
\end{tabular}

Tabla n. 1. Textos más leídos por los estudiantes

Como se observa en la tabla 1 , el texto que los estudiantes afirmaron que más leen es los apuntes de clase propios $(83,68 \%)$ y los materiales elaborados por el profesor $(79,78 \%)$; la lectura de páginas web y blogs también ocuparon un lugar importante (76,82\%).

En los grupos de discusión (GD) de estudiantes se indagó precisamente sobre lo que los estudiantes pensaban respecto a que los apuntes de clase fuese el texto más leído y más escrito (el género dominante). Frente al tema hubo afirmaciones, réplicas, adhesiones. En las relecturas y reflexiones derivadas de los análisis de las 
transcripciones, se hallaron elementos claves relacionados con una posibilidad interpretativa de las virtudes de los apuntes de clase, las razones de su existencia y sus sentidos.

El hecho de que los apuntes de clase tuviesen ese protagonismo como tipo de texto más leído y escrito en la universidad se cuestionó, por parte de los estudiantes, desde la perspectiva del papel que deberían tener las fuentes primarias en el ingreso al campo disciplinar; desde la ausencia de textos en los que el análisis, la crítica y la toma de posición frente al saber tuviesen un papel central y por su orientación a la evaluación. Pero el análisis de los GD igualmente fue revelando elementos de valoración positiva del rol del docente como académico experto, como miembro de la comunidad disciplinar, que guía la aproximación inicial a un campo de saber, labor compleja para un estudiante que está recién ingresando a la cultura académica. Igualmente se fue abriendo una línea de reflexión relacionada con la función epistémica de la escritura al tomar apuntes en clase: los apuntes se revelaron, desde este análisis, como un espacio de condensación, de comprensión de un saber difícilmente asible para los estudiantes, como muestra el ejemplo siguiente.

\footnotetext{
"siempre... siempre se necesita como profundizar más y eso es algo que uno no va a encontrar ágilmente en los libros, sino en personas que ya han estudiado más, personas que...mmm ... se han tomado la molestia como de... de poner de...sí, de poner para uno, en palabras y en textos específicos sobre la carrera de uno, esos conocimientos organizados ...... a los que uno pueda acceder y en los que tiene que profundizar luego, porque pues lo que los profesores dicen, exponen, generalmente es algo sobre lo que ellos leyeron mucho, no te están transmitiendo todo... entonces nos muestran como también muchos hilos..." (TGDE) ${ }^{4}$.
}

Este es el tipo de problemáticas que enfrenta un estudiante que ingresa al pregrado, a juzgar por la información analizada en los GD. Según sus palabras, el estudiante al llegar a la universidad se encuentra con dos situaciones: debe (le piden) leer mucho en las asignaturas y el tiempo no alcanza. De otro lado, no cuenta con las suficientes herramientas académicas, específicas, para abordar aquellos textos propios de la disciplina. De hecho, los tipos textuales especializados que deben enfrentarse en la cotidianidad académica son poco abordados como objetos de estudio en los cursos de los primeros semestres de la carrera, mucho menos en la educación básica. Al respecto los estudiantes afirmaron: "La universidad supone que uno llega sabiendo leer artículos científicos en ingles, artículos de revisión, informes de laboratorio, historias clínicas...", "no se nos enseña a leer esos textos, es como si uno debiera saberlo, pero ni el colegio ni la universidad lo enseña" (TGDE).

Esta situación explica, al menos en parte, por qué eligieron como alternativa central de ingreso a la disciplina la voz del docente capturada en los apuntes de clase, y esto se puede vincular, por supuesto, con una economía cognitiva. Por esta razón, asistir a clase resulta un asunto "casi que de supervivencia" (GDE), afirmaron los estudiantes. Se asiste para tomar apuntes, que son la versión procesada por el estudiante, de otra versión: la del docente. Pero en fin, esa es la opción "más cómoda" (GDE), afirmaron. El estudiante asiste. Toma apuntes. Los complementa con los de otros compañeros. Por eso, dijeron, "tomamos fotocopias de los apuntes de otro" (GDE). Podríamos afirmar que los apuntes de clase adquieren un valor simbólico de cambio (Bourdieu, 1985), en el mercado lingüístico, en este caso, del aula. 
Esa situación pareciera imponer restricciones frente a expectativas y necesidades académicas latentes que los estudiantes señalaron, y que se evidenciaron en dos tipos de cuestionamientos planteados en los GD: a) "necesitamos ir más allá de lo básico" (GDE), afirmaron, para profundizar en esos saberes que se están comprendiendo. Señalaron que sabían que se debe ir a las fuentes directas, a los artículos e investigaciones, incluso en la lengua original. Pero, además de la restricción de tiempo, señalaron que b) no contaban con las herramientas académicas requeridas, pues en el colegio, afirmaron, "no se aprende a leer y escribir artículos científicos, informes de laboratorio" (GDE) y otros tipos de textos. Pero, agregaron, "la universidad tampoco lo enseña" (GDE), supone que la competencia lectora y escritora general es suficiente.

\section{¿Para qué se lee en la universidad?}

Otro de los ítems de la encuesta indagó sobre los propósitos de la lectura en la universidad. En la tabla 2, los resultados muestran una variedad importante.

\begin{tabular}{|l|c|}
\hline & \% \\
\hline Realizar una exposición & 86,39 \\
\hline Responder a una evaluación escrita & 85,99 \\
\hline Responder a una evaluación oral & 69,51 \\
\hline Participar en discusiones grupales & 59,51 \\
\hline Diseñar un proyecto & 49,31 \\
\hline Elaborar un escrito académico & 45,91 \\
\hline Trabajar en el marco de un proyecto & 38,91 \\
\hline Asistir a eventos académicos & 30,12 \\
\hline Escribir artículos o ponencias & 25,84 \\
\hline Realizar una relatoría & 24,82 \\
\hline
\end{tabular}

Tabla n. 2. Propósitos de la lectura

Las opciones que obtuvieron las mayores frecuencias están relacionadas directamente con la evaluación, hecho que complementa los análisis planteados en relación con las razones de lectura de apuntes de clase y materiales elaborados por el docente. Al parecer, los apuntes cumplen doble función: apoyan el ingreso al campo disciplinar y son la base para preparar las evaluaciones. Los porcentajes más altos fueron: realizar una exposición (86,39\%), responder una evaluación escrita (85,99\%), responder una evaluación oral $(69,51 \%)$ y participar en discusiones grupales $(59,51 \%)$.

Los resultados relacionados con los textos que escriben los estudiantes fueron muy similares a los hallados en el campo de la lectura y parecen corroborar las tendencias expuestas. Veamos. 


\section{¿Qué es lo que más se escribe?}

En cuanto a los textos que se escriben, los resultados de la encuesta mostraron las siguientes tendencias centrales.

\begin{tabular}{|l|c|}
\hline & $\%$ \\
\hline Apuntes de clase & 91,50 \\
\hline Resúmenes & 82,90 \\
\hline Ensayos & 78,03 \\
\hline Informes & 71,69 \\
\hline Reseñas & 30,90 \\
\hline Memorias, protocolos, actas, diarios & 22,94 \\
\hline Relatorías & 19,71 \\
\hline Comentarios o aportes para foros & 15,11 \\
\hline Artículos científicos & 11,81 \\
\hline Documentos periodísticos & 10,73 \\
\hline Comentarios o aportes discusión en línea & 9,46 \\
\hline Crear blogs & 7,88 \\
\hline Textos literarios & 7,37 \\
\hline Otro & 2,20 \\
\hline
\end{tabular}

Fuente: elaboración propia

Tabla n.3. Textos que más se escriben

Los apuntes de clase (91,50\%), resúmenes (82,90\%), ensayos $(78,03 \%)$ e informes $(71,69 \%)$ fueron los que se identificaron como los que más se escriben. $\mathrm{Si}$ bien en esta tendencia aparecieron los ensayos, informes y resúmenes como textos que se escriben frecuentemente, al preguntar por las razones por las que esos textos se escriben, tal como se observa en la tabla 4, los resultados indican que se trata dominantemente de escribir para la evaluación. Es decir, la escritura de ensayos, resúmenes e informes estarían cumpliendo una función evaluativa similar al examen. Al parecer no hay diferencia entre escribir un ensayo y realizar un examen, en cuanto a su función.

\begin{tabular}{|l|c|}
\hline & $\%$ \\
\hline Responder a una evaluación escrita & 80,18 \\
\hline Responder a una evaluación escrita & 80,18 \\
\hline Presentar informes & 77,66 \\
\hline Diseñar un proyecto & 48,06 \\
\hline Elaborar una relatoría & 23,92 \\
\hline Redactar ponencias & 16,56 \\
\hline Presentar informes & 77,66 \\
\hline Responder a una evaluación escrita & 80,18 \\
\hline Elaborar escritos para una exposición & 83,68 \\
\hline Elaborar notas personales & 66,87 \\
\hline Escribir artículos para ser publicados & 11,84 \\
\hline Otros & 3,63 \\
\hline
\end{tabular}

Tabla n. 4. Razones de escritura de los textos 
Vale la pena señalar que si bien la lectura de documentos académicos se realiza predominantemente en español, el $60 \%$ de los estudiantes leyó al menos un texto completo en inglés el semestre anterior a la aplicación de la encuesta, lo que estaría mostrando un consumo importante de literatura en segunda lengua. De otro lado, aunque las prácticas de lectura orientadas funciones distintas a la evaluación y al trabajo en las asignaturas fueron escasas, algunos datos (tabla 5) muestran que comienzan a emerger lecturas orientadas a la participación en dinámicas propias de las comunidades disciplinares como la participación en eventos académicos $(37,46 \%)$, los grupos de estudio $(46,73 \%)$ o la pertenencia a grupos y semilleros de investigación $(20,17 \%)$. Por lo anterior, podemos considerar que comienzan a ser tomadas en cuenta algunas dinámicas propias de la cultura académica.

\begin{tabular}{|l|c|}
\hline & $\%$ \\
\hline Asignatura & 88,78 \\
\hline Grupo de estudio & 46,73 \\
\hline Evento académico & 37,46 \\
\hline Semillero (o grupo) de investigación & 20,17 \\
\hline Curso extra curricular & 19,98 \\
\hline Otra & 13,50 \\
\hline Concurso & 7,80 \\
\hline Club de la lectura & 5,43 \\
\hline \multicolumn{2}{|c|}{ Fuente: elaboración propia }
\end{tabular}

Tabla n.5. Actividades académicas para las que se lee

\section{Características de los cursos de lectura y escritura que ofrecen las universidades}

Para el análisis documental de esta fuente se identificaron los cursos que se ofrecían en las 17 universidades para apoyar los procesos de lectura y escritura. La búsqueda fue exhaustiva, pues se quería contar con una cifra que diera cuenta de la tendencia en la oferta curricular al respecto. Se identificaron 415 programas (syllabus) de cursos de lectura y escritura ofertados por las universidades participantes, entre los años 2008 y 2009. Esta cifra evidenció que las universidades sí se ocupan de los procesos de lectura y escritura, al menos que sí lo hacen desde la oferta curricular al respecto. Más de la mitad de los cursos (52\%) pertenecía al ciclo de fundamentación y el $81 \%$ fue de carácter obligatorio. El $83,7 \%$ se ofrecían desde las facultades o departamentos de lingüística, de comunicación o lenguaje. La estrategia metodológica principal empleada fue el taller $(65 \%)$. El ensayo $(47,7 \%)$, el resumen $(34,5 \%)$ y la reseña $(21,9 \%)$ fueron los tipos de textos más trabajados. 


\begin{tabular}{|l|c|}
\hline & \% \\
\hline Escritura (como competencia general) & 85,5 \\
\hline Lectura (como competencia general) & 75,4 \\
\hline Conocimientos sobre el lenguaje y los textos & 45,3 \\
\hline Oralidad (como competencia general) & 31,6 \\
\hline Lectura / escritura en relación con saberes disciplinares & 22,9 \\
\hline
\end{tabular}

Fuente: elaboración propia

Tabla n.6. Objeto de trabajo de los cursos

De otro lado, como se observa en la tabla 6, la mayoría de cursos se ocupan de la lectura y la escritura como competencias generales, es decir, no vinculados de modo directo con los campos disciplinares. Uno de los resultados clave se relaciona con el bajo porcentaje de cursos de lectura y escritura vinculados directamente a las disciplinas (22,9\%). Esta cifra da cuenta de una tendencia, en las universidades estudiadas, a concebir la lectura y la escritura como competencias generales y no como especificidades de los campos disciplinares.

Pero, vale la pena señalar que los estudiantes participantes en los grupos de discusión valoraron esos cursos de lectura y escritura como espacios importantes para su desarrollo integral, pues allí, dicen ellos, "se lee sobre temas de actualidad, de contexto y se aprende a escribir textos importantes para la vida." (GDE), así no sean del ámbito disciplinar particular. De hecho valoraron los espacios para leer literatura, aprender sobre arte, etcétera, ya que brindan un marco para "situar las profesiones" (GDE), dicen los estudiantes. Por tanto, los cursos "genéricos" de lectura y escritura resultaron valorados, pero con una función que no es la epistémica - disciplinar.

\section{Espacios curriculares en los que los estudiantes viven las mejores experiencias de lectura y escritura}

Con el fin de identificar los espacios curriculares en los que los estudiantes vivieron las experiencias destacadas de lectura y escritura, incluimos en la encuesta una pregunta abierta: "Escriba el nombre de la asignatura (s) o de la actividad (es) en la que ha vivido la mejor experiencia de lectura y escritura en la universidad".

Los 3715 estudiantes encuestados referenciaron 3720 espacios académicos en los que vivieron sus mejores experiencias al respecto. Esos registros fueron categorizados inductivamente para obtener estos resultados:

\begin{tabular}{|l|c|}
\hline & $\%$ \\
\hline Asignaturas de la disciplina & 71,8 \\
\hline No referencia asignatura & 10,67 \\
\hline Lectura y escritura como competencia general & 9,19 \\
\hline Asignaturas de la disciplina vinculadas a investigación & 6,05 \\
\hline Espacios alternos & 0,38 \\
\hline Ética humanismo ciudadanía & 0,73 \\
\hline Lectura y escritura en la disciplina & 0,70 \\
\hline Otro & 0,48 \\
\hline
\end{tabular}

Tabla n.7. Asignaturas o actividades en las que se viven las mejores experiencias de lectura y escritura 
Como se observa en la tabla 7 , la gran mayoría $(71,8 \%)$ de los espacios académicos en los que los estudiantes dijeron haber vivido experiencias destacadas de lectura y escritura son asignatura de las disciplinas específicas (neurofisiología, etnohistoria, etología, pensamiento lógico 1, etcétera). Los cursos generales en los que se enseña a leer y escribir, como competencia general, aparecieron muy poco referenciados como significativos, solo un 9,19\%. Estos datos pueden interpretarse desde diversos ángulos, pero es evidente que las prácticas de escritura y lectura académicas que los estudiantes señalan como destacadas están vinculadas de manera fuerte con las disciplinas.

Por su parte, los análisis de los grupos de discusión de los investigadores de las 17 universidades, como los de los estudiantes y profesores que se hicieron en cada universidad, permitieron hacer explícitas las condiciones en las que llegan los estudiantes a la universidad y lo que ésta hace al respecto. En los GD de los investigadores se señaló que la educación básica forma a los estudiantes, en el mejor de los casos, en maneras genéricas de leer y escribir, es decir, no ligadas a la especificidad de las disciplinas y, por tanto, desde esta formación no se puede esperar que al llegar a la universidad logren reconocer los elementos particulares de esos textos especializados, en función de los campos disciplinares con los que allí se los pone en relación.

\section{Algunos rasgos de las prácticas destacadas}

Como se señaló, otra fuente de la investigación fueron los estudios de casos. En la investigación se identificaron casos de prácticas destacadas en las 17 universidades, desde los parámetros definidos. En cada universidad se estudió, mínimo un caso, excepto en una universidad, pues los estudiantes no postularon ninguna experiencia como destacada. Por esta razón se estudiaron dos casos de una universidad. Los 17 casos estudiados corresponden a espacios académicos propios del campo disciplinar (por ejemplo: teorías del estado, prácticas de escritura en el componente de psicoanálisis II, medio ambiente e industria, programa de alfabetización académica del programa académico de fonoaudiología, etcétera), hecho que coincide con la naturaleza de las asignaturas nombradas por los estudiantes como aquellas en las que vivieron experiencias destacadas al respecto.

La construcción del balance general de los estudios de casos y de los hallazgos logrados en un análisis por categorías, y subcategorías, permitió llegar a las siguientes consideraciones que revelan el carácter destacado de las experiencias referenciadas, que, desde la perspectiva de la investigación, se pueden considerar rasgos que caracterizan las prácticas destacadas de enseñanza de la lectura y la escritura en la universidad colombiana. Algunos de los rasgos son:

- Se evidencia un propósito explícito de los docentes destacados de incidir en los procesos de formación de los estudiantes, en un sentido amplio, tanto en el campo disciplinar al que pertenecen como en su dimensión ciudadana. En esos procesos formativos leer y escribir son más bien condiciones o efectos de una postura de los docentes sobre el conocimiento y sobre su función social, política, más que fines en sí mismos. 
- Es evidente una función epistémica de la lectura y la escritura al interior de los espacios académicos estudiados, hecho que contrasta con los resultados generales derivados de la encuesta y los análisis de los cursos. Los docentes destacados proponen a sus estudiantes abordar los textos especializados como objetos de lectura y escritura con el propósito de comprenderlos, describirlos, explicarlos, pero ese no es el fin último, se trata de ir más allá: para generar nuevas producciones, publicar, o para participar en dinámicas académicas propias de las comunidades disciplinares. El trabajo sobre la lectura y la escritura, en estos espacios, es tomado más como un proceso complejo que como producto. Se evidencian acciones de planeación, documentación, revisión, lecturas de pares y discusión, que permiten que la actividad de leer y escribir "se convierta en un instrumento para la reorganización del saber y para la creación de nuevas perspectivas e ideas sobre un fenómeno" (Castelló, 2005, p. 3).

- La experiencia personal que vive el docente con la lectura y escritura, sus trayectorias y su manera de vivir en el campo disciplinar, en esas prácticas específicas, marcan de manera clara los modos en que configuran sus propuestas de trabajo con los estudiantes. Su tradición lectora, la pasión por el saber y la relación permanente que tienen con la escritura inciden de modo implícito en las maneras como configuran su acción de formación. Podemos afirmar, dese el análisis de estos casos, que el tipo de relación que el docente tiene con su campo de saber se refleja en la acción docente, y esa particular manera de relacionarse con el saber se transmite indefectiblemente a los estudiantes, y no siempre de modo explícito.

- Existe una tensión entre la planeación y la flexibilidad. En estos docentes es clara la manera sistemática como piensan, planean y configuran su acción didáctica: los tiempos están claramente pensados, los propósitos, las acciones, los productos académicos, las características de los textos que se piden leer y escribir, las prácticas a las que se desea que sus estudiantes se vinculen, etcétera. Pero, a la vez, esas prácticas se flexibilizan en su puesta en escena, es decir, estamos frente a un esquema flexible que es posible gracias a la existencia de una previa planeación.

- Existe una proximidad del docente hacia los estudiantes de forma permanente. Los estudiantes no están solos en sus búsquedas conceptuales, teóricas e interrogativas, hay un docente que los acompaña desde la confrontación, revisión, discusión y cuestionamiento en los procesos que debe adelantar para la construcción de los saberes que se proponen. Este acompañamiento se complementa con una alta exigencia académica, que opera a modo de reto cognitivo para que los estudiantes se pongan a la altura de las condiciones.

- Finalmente, otro rasgo, ligado al anterior, se relaciona con el hecho de que los docentes de estas prácticas están permanentemente reflexionando, cuestionando, modificando sus prácticas como resultado de la revisión crítica sobre sus propias acciones. Podría afirmarse que estos docentes desarrollan su práctica con un alto componente de revisión y reflexión crítica sobre sí mismos, que en varios casos se vincula con el desarrollo de sistematizaciones sobre sus 
propias prácticas, la escritura y publicación de textos analíticos, la participación en eventos en los que se socializa y discute la labor docente.

\section{Conclusiones y discusión}

En primer lugar, es importante señalar que si bien no fue posible identificar unas directrices explícitas de políticas institucionales al respecto, las universidades colombianas sí están preocupadas por el tema de la lectura y la escritura académicas. A juzgar por los resultados, el esfuerzo de la universidad al ofrecer un buen número de cursos orientados a fortalecer los procesos de lectura y escritura -a los que asisten simultáneamente estudiantes de diferentes programas- y el hecho de que éstos sean obligatorios, lo demuestra. Como se señaló, estos cursos no resuelven los problemas de lectura y escritura específicos de las disciplinas, porque se basan en una concepción de lectura y escritura como competencias generales, no como especificidades disciplinares, y sabemos que la lectura y la escritura deben pensarse como especificidades de los campos disciplinares (Olson, 1998).

Pero también es cierto que gran parte de los estudiantes que ingresan a la universidad colombiana llegan con debilidades básicas en lectura y escritura, y sin las herramientas necesarias para ingresar a la vida académica de la educación superior, según lo revelan los resultados de las evaluaciones de egreso de la educación básica, que se realizan cada año (ICFES, 2010). De algún modo, estos cursos intentan enfrentar esta situación. $O$ en otras palabras, los cursos pensados como competencias generales, dadas las condiciones de la educación básica colombiana, se requieren, especialmente en los primeros semestres, pero no son suficientes, pues no aportan elementos suficientes para fortalecer los procesos de lectura y escritura en su función epistémica.

El trabajo que habría que fortalecer al interior de las disciplinas -una vez los estudiantes ya han ingresado a la vida académica, al haber recorrido un buena parte de su plan de estudios- supone una dinámica concreta en la que hay que realizar acciones sistemáticas sobre esas prácticas de lectura y escritura propias de los campos de conocimiento específicos. En este aspecto, a juzgar por los resultados de este estudio, es en el que no es claro el trabajo que se realiza en las universidades de nuestro país. Aquí queda planteado un reto concreto de política institucional.

Lo anterior nos permite afirmar que la disyuntiva relacionada con si la lectura y la escritura en el contexto universitario deben ser pensadas como competencias genéricas o específicas, es una falsa dicotomía. La oferta de cursos generales, a los que asisten estudiantes de diferentes programas, cumple una doble función en los primeros semestres: a) sirve de puente entre la educación básica y la universidad y b) apoya el ingreso a la vida académica especializada y a las dinámicas de comunidades disciplinares.

En este sentido, la investigación permite señalar la necesidad de realizar acciones sistemáticas al interior de las asignaturas y demás espacios disciplinares, para fortalecer la escritura y la lectura con función epistémica. Esto puede contribuir a generar condiciones para un fortalecimiento de las capacidades académicas, que a su vez son la base para un incremento de la productividad científica en el país. Es importante, igualmente, repensar la forma como estamos vinculando a nuestros 
estudiantes a las comunidades de práctica y a las dinámicas de producción, circulación y validación del conocimiento. Acciones como promover en los estudiantes su participación en procesos de investigación, la asistencia activa a eventos, congresos, los incentivos académicos para promover la escritura de ponencias, etcétera, son prioritarios para la formación de un futuro profesional.

En segundo lugar, el hecho que los apuntes de clase fuese el texto más leído y más escrito por los estudiantes, y que la lectura de fuentes primarias como artículos de investigación o libros teóricos fuese bajo obliga a repensar las prácticas de enseñanza que la universidad promueve. Acudir al registro de las notas de clase, en la proporción que muestran los resultados de este estudio, y que esa práctica esté asociada a la evaluación, indican que la fuente central de conocimiento es la voz del docente, y que las demás fuentes poco son empleadas en el pregrado. Es claro que la toma de apuntes por sí sola, vinculada a la evaluación, no es indicador de un trabajo intelectual con el saber. Como señala Paula Carlino (2005), las prácticas evaluativas tienen gran impacto en lo que aprenden los alumnos, pues no se trata de un componente más del proceso didáctico. En los modos de evaluar se configura lo que se espera que el estudiante haga con el conocimiento en construcción. Cabe preguntarnos si lo que ocurre en el acto evaluativo es una mera devolución de información, una rendición de cuentas, o si realmente hay una orientación hacia la apropiación y uso consistentes del conocimiento.

Si bien este resultado asigna al docente universitario un papel y responsabilidad relevantes en el acompañamiento de los estudiantes, para el ingreso a las disciplinas, hay una especie de dependencia excesiva del docente. Por supuesto que esa una función central del profesor de relevar, ordenar, presentar una estructura de conceptos, para reducir la complejidad del campo conceptual a su cargo, que exige elegir del amplio espectro de conocimientos aquellos conceptos, teorías, posturas que permiten dibujar el mapa básico del campo disciplinar, con el fin de garantizar a sus estudiantes una primera aproximación al mismo. Pero a eso no se puede reducir el acto de enseñar. Si bien el estudiante asume el discurso del docente como la voz del experto por excelencia (Cartolari \& Carlino, 2011), se requiere ir a las fuentes primarias de la disciplina, desbordar los límites del aula para acceder a las dinámicas de producción, circulación del conocimiento en las comunidades especializadas.

De otro lado, como se observó en los resultados, además de los apuntes de clase, las páginas web y los blogs presentaron una frecuencia alta de lectura entre los estudiantes. Esta situación es explicable desde las condiciones del contexto actual de circulación y acceso a la información. Si bien es necesario reflexionar sobre la necesidad de incorporar las tecnologías en los procesos educativos, es clave preguntarnos por los alcances y limitaciones de la web como fuente legítima de conocimientos.

Frente a este panorama, el estudio de las prácticas destacadas indica que la relación que tiene el docente con su campo de conocimiento, así como su participación activa en la comunidad disciplinar, en los procesos de investigación y circulación del conocimiento, son elementos que determinan su forma de hacer docencia. Un docente que está vinculado a la comunidad de práctica, que está investigando, publicando, configura prácticas en las que logra producir retos importantes en sus estudiantes y de algún modo, contribuir a la generación de un 
deseo de saber. Cabe señalar que en las experiencias de los docentes destacados, leer y escribir, como prácticas epistémicas, son más efectos y condiciones de una postura sobre el conocimiento, que fines en sí mismas. Hace falta más investigación en profundidad sobre experiencias destacadas, en nuestro país, desde una epistemología de la práctica (Schön, 1992), para construir saberes sobre las prácticas de enseñanza.

Finalmente, consideramos que las tendencias halladas en esta investigación explican, al menos en parte, los bajos índices de productividad científica del país. Los estudios recientes sobre el tema en América Latina y el Caribe (Santa \& Herrero, 2010) indican que en 2007, Colombia generó el 0,1 \% de la productividad científica mundial y el 3,3\% de Latinoamérica y el Caribe. Por supuesto que estas cifras también se explican desde la débil tradición en producción científica de nuestro país, la baja inversión en investigación en educación y el no contar con políticas claras y decididas al respecto. Esta preocupación por la productividad científica, se vincula de modo directo con la idea de desarrollo de un país.

\section{Referencias bibliográficas}

Arias Castilla, C. (2008). Estado del arte en didácticas para la formación de las competencias comunicativas en las facultades de educación de Bogotá. Horizonte pedagógico, vol. 9, año 1, n¹, 2007; y vol. 10, n¹, 2008.

Bardin, L. (1986). Análisis de Contenido. Madrid: Akal.

Camps, A. (2004). Objeto, modalidades y ámbitos de la investigación en didáctica. Lenguaje, 32, 7-27.

Carlino, P. (2005). Escribir, leer y aprender en la universidad. Una introducción a la alfabetización académica. Buenos Aires: Fondo de Cultura Económica.

Carlino, P. (2004). Escribir a través del currículum: tres modelos para hacerlo en la universidad. Lectura y Vida. 25(1), 16-27.

Carvajal B., G. (2008). Prácticas de lectura y escritura, tecnologías de la información y procesos de producción y apropiación de conocimientos en el ámbito universitario, en: $2^{\circ}$ Encuentro Nacional y $1^{\circ}$ Internacional sobre lectura $y$ escritura en educación superior, Memorias, Bogotá, 18-19 de septiembre de 2008.

Bourdieu, P. (1985). ¿Qué significa hablar? Economía de los intercambios lingüísticos. Madrid: Akal.

Cartolari, M. \& Carlino, P. (2011). Leer y tomar apuntes para aprender en la formación docente: un estudio exploratorio. Magis. Revista Internacional de Investigación en Educación, 4 (7), 67-86.

Castelló, M. (2005). La escritura epistémica: Concepciones y estrategias de regulación en estudiantes de doctorado. Ponencia. IV Jornada de Desarrollo Humano y Comunicación Alcalá de Henares. Madrid.

Flyvbjerg, B. (2005). Cinco equívocos sobre la investigación basada en estudios de caso. Estudios Sociológicos. 23 (2), 561-590. 
Camargo, Z., Caro, M., Castrillón, C. \& Uribe, G. (2008). Estado del arte de las concepciones sobre prácticas de lectura y escritura en la universidad colombiana (Informe: insumo proyecto de investigación). Recuperado de: http://www.colombiaaprende.edu.co/html/mediateca/1607/articles248749 Estado del arte lectura escritura en la U.pdf

Henao, M \& Castro, J. (2000). Estados del arte de la Investigación en Educación y Pedagogía en Colombia 1989 - 1999. Bogotá: ICFES.

Ibáñez, J. (1979). Más allá de la sociología. El grupo de discusión: Técnica y crítica. Madrid: Siglo XXI.

ICFES (2010). Saber 5․ Y 9o. Resultados Nacionales. Resumen ejecutivo. Bogotá. ICFES. Recuperado de: http://www.icfes.gov.co/resultados/saber-pro-resultadosindividuales/resultados-agregados-saber-pro/11-inicio/pruebas-saber

Lahire, B. (comp) (2004). Sociología de la lectura, Barcelona: Gedisa.

Murillo Fernández, M. (2009) "El estado de la investigación en la enseñanza de la lectura y la escritura en la universidad colombiana y latinoamericana", en: REDLEES, Tercer Encuentro Nacional y $2^{\circ}$ Internacional de Lectura y escritura en educación superior. Memorias, Cali, 8-9, octubre, 2009 [CD-ROM].

Olson, D. (1998). El mundo sobre el papel. El impacto de la escritura y la lectura en la estructura del conocimiento. Barcelona: Gedisa.

Pérez-Abril, M (2009). A propósito de la legitimidad en la investigación cualitativa. magis, Revista Internacional de Investigación en Educación, 2, 235-240.

Santa, S. \& Herrero, V. (2010) Producción científica de América Latina y el Caribe: una aproximación a través de los datos de Scopus (1996-2007). Revista Interamericana de Bibliotecología, 33 (2), 379-400.

Schön, D. (1992). La formación de profesionales reflexivos. Hacia un nuevo diseño de la enseñanza y el aprendizaje en las profesiones. Barcelona: Paidós.

Schön, D. (1998). El Profesional Reflexivo: Como Piensan los Profesionales Cuando Actúan. Barcelona: Paidós.

Stake, R. (1999). Investigación con estudio de casos. Madrid: Morata.

Ulloa S. A. (2008). Lectura, escritura y conocimiento en la educación superior, en: REDLEES, 3er Encuentro Nacional y $2^{\circ}$ Internacional de Lectura y escritura en educación superior. Memorias, Bogotá, ,18-19 de septiembre de 2008 [CD-ROM].

Uribe-Álvarez, G. \& Camargo-Martínez, Z. (2011). Prácticas de lectura y escritura académicas en la universidad colombiana. Magis. Revista Internacional de Investigación en Educación, 3 (6), 317-341.

Vasilachis de Gialdino, I. (2007). El aporte de la epistemología del sujeto conocido al estudio cualitativo de las situaciones de pobreza, de la identidad y de las representaciones sociales [99 párrafos]. Forum Qualitative Sozialforschung / Forum: Qualitative Social Research, 8(3), Art. 6.

Yin, R. (2004). Case Study Methods. Cosmos Corporation. Complementary Methods for Research in Education. Washington DC: American Educational Research Association. 


\section{Notas}

1. El proyecto se tituló: ¿Para qué se lee y se escribe en la universidad colombiana? Un aporte a la consolidación de la cultura académica del país, desarrollado entre 2009 y 2011. Código: PREO0439015708. Dirigido por Gloria Rincón (Universidad del Valle) y Mauricio Pérez Abril (Pontificia Universidad Javeriana). Financiada por COLCIENCIAS más 17 universidades: Universidad de la Amazonia, Universidad de Antioquia, Universidad del Atlántico, Universidad Autónoma de Occidente, Unidad Central del Valle, Universidad de Córdoba, Universidad de Caldas, Universidad Católica de Pereira, Universidad del Cauca, Universidad de Ibagué, Pontificia Universidad Javeriana de Bogotá, Pontificia Universidad Javeriana de Cali, Fundación Universitaria Monserrate, Universidad del Pacífico, Universidad del Quindío, Universidad Pedagógica Nacional y Universidad del Valle. En el proyecto participaron 54 investigadores de 17 grupos de investigación, uno de cada universidad. El proyecto contó con la asesoría e interlocución de Anna Camps (Universidad Autónoma de Barcelona), Paula Carlino (CONICET y Universidad de Buenos Aires)y Luiz Percival Leme Britto (Universidad do Oeste do Pará).

2. Debido a que la organización académico-administrativa de las universidades en Colombia es muy diversa, se acogió la clasificación de programas de pregrado propuesta por UNESCO que se compone de las siguientes áreas: Educación; Humanidades y Artes; Ciencias sociales, Educación comercial y Derecho; Ciencias; Ingeniería, Industria y Construcción, Salud y Servicios sociales; y Recreación.

3. También se realizaron análisis factoriales, pero en este texto no se presentan.

4. TGDE: Transcripción de los grupos de discusión de estudiantes.

Cita del artículo:

Pérez-Abril, M., Rodríguez, A. (2013). ¿Para qué se lee y se escribe en la universidad colombiana? Caracterización de prácticas de lectura y escritura en 17 universidades. Revista de Docencia Universitaria. REDU. Número monográfico dedicado a Academic Writing. Vol.11 (1) Enero-Abril. pp. 137-160. Recuperado el (fecha de consulta) en http://www.red-u.net/ 


\section{Acerca de los autores}

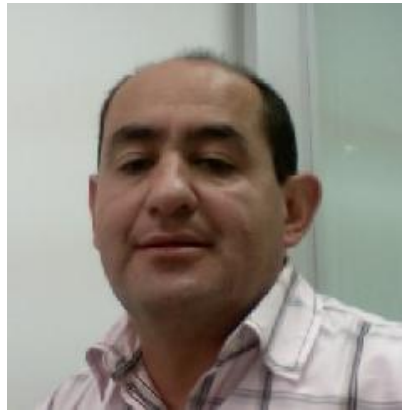

\section{Mauricio Pérez Abril}

Pontificia Universidad Javeriana. Bogotá - Colombia

Mail: mauricioperezabril@gmail.com

Profesor e investigador de la Facultad de Educación. Formador de formadores. Sus campos de investigación son la cultura académica, la didáctica de la lengua y las políticas públicas de lectura y escritura. Director del Grupo de investigación: Pedagogías de la Lectura y la Escritura. Es candidato a doctor en educación de la Universidad Pedagógica Nacional de Colombia. Miembro de la Red Colombiana para la Transformación Docente en Lenguaje.

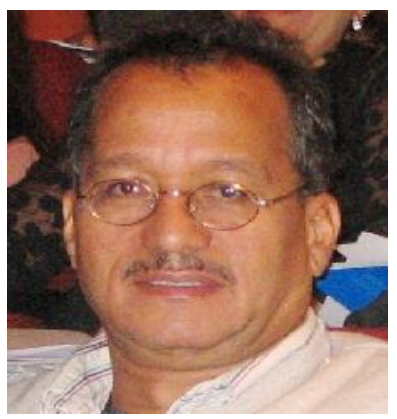

\section{Alfonso Rodríguez Manzano \\ Universidad del Atlántico - Universidad del Norte. \\ Barranquilla - Colombia.}

Mail: alfrodriguezm@yahoo.es

Docente - investigador de la Facultad de Ciencias Humanas de la Universidad del Atlántico y del Instituto de estudios en educación de la universidad del Norte. Es miembro de los grupos de investigación GILKARí y Lenguaje y Educación. Es magíster en letras de la Universidad de Paris III y magíster en filosofía de la Universidad del Valle. Sus campos de investigación son las relaciones entre literatura, educación y cultura. 


\title{
Contradictions regarding teaching and writing (or writing to learn) in the disciplines: What we have learned in the USA
}

\author{
Contradicciones acerca de cómo promover la escritura epistémica en las \\ disciplinas: lo que hemos aprendido en EEUU
}

David R. Russell

lowa State University, USA

\section{Resumen}

En este artículo se describe la tradición de la investigación anglófona de América del Norte acerca del rol de la escritura en la enseñanza y el aprendizaje en la educación superior. La tradición de investigación está asociada con un movimiento de reforma de la educación de más de cuarenta años, denominado "escritura a través del currículo" (Writing Across the Curriculum; WAC) o "escritura en las disciplinas" (Writing in the Disciplines; WID). El movimiento anima a los profesores de las diferentes disciplinas a interesarse por mejorar la escritura de sus estudiantes y su formación disciplinar a través de la escritura. La investigación desarrollada bajo los movimientos WAC y WID utiliza métodos propios de la investigación educativa ( $y$, en menor medida, de la lingüística aplicada) para comprender los roles que juega la escritura en el trabajo y la formación disciplinar, a menudo en relación con la escritura en otras instituciones (empresas, gobierno, etc.). Situar en primer plano la escritura en los movimientos WAC y WID ha revelado seis contradicciones estructurales e institucionales en la educación superior de los EEUU: 1) la escritura como herramienta transversal frente a la escritura especializada, 2) la concepción del género como un contenedor de contenido -dualismo forma/contenido- versus su concepción como acción social, 3) la escritura como medio para evaluar el aprendizaje de contenidos frente a la escritura como una herramienta de desarrollo intelectual, profesional o personal, 4) la escritura para conseguir la meta social de la escolarización (epistémica) versus escribir para contribuir a la meta social del trabajo (pragmática) 5) las tesis de máster o doctorado como el último obstáculo educativo versus su consideración como la primera actuación profesional, 6) y la escritura (y su enseñanza) para la reproducción social y/o disciplinar versus la escritura (y su enseñanza) para el cambio social y/o disciplinar.

Palabras clave: Escritura en las disciplinas, la escritura para aprender, las contradicciones, la escritura a través del currículo, género 


\begin{abstract}
This article describes a tradition of Anglophone North American higher education (HE) research concerning the role of writing in learning and development. The research tradition is associated with a forty-year-old education reform movement called Writing Across the Curriculum (WAC) or Writing in the Disciplines (WID). The movement encourages teachers in different disciplines to become interested in their students' writing and to improve their writing and their disciplinary education (formation) through writing. The research on WAC/WID uses methods familiar in educational research (and to a lesser extent applied linguistics) to understand the roles writing plays in disciplinary work and disciplinary formation, often in relation to writing in other institutions (business, government, etc.). The foregrounding of writing in WAC/WID has reveled six structural, institutional contradictions in US HE: 1) writing as transversal versus writing as specialized; 2) genre conceived as a container of content--a form/content dualism--versus genre conceived as social action; 3 ) writing as a means of assessing learning of content versus writing as a tool of intellectual / professional / personal development; 4) writing for a social motive of schooling (epistemic) versus a social motive of work (pragmatic); 5) the masters or doctoral thesis as a last educational hurdle versus a first professional performance; 6) and (teaching) writing for social/disciplinary reproduction versus (teaching) writing for social/disciplinary change.
\end{abstract}

Key words: Writing in the disciplines, writing to learn, contradictions, writing across the curriculum, genre

\title{
Introduction
}

This article describes a tradition of Anglophone North American higher education (HE) research concerning the role of writing in learning and development. It is not a formal literature review but rather an introduction to this 30 year-old tradition (for reviews see Russell 1997; Russell 2002; Russell 2009). Several research studies are briefly summarized to illustrate the theory, the methods, selected results, and interventions developed for and resulting from the research. The fundamental theoretical frame for this research tradition derives from several sources familiar to European researchers in didactics and, to a lesser extent, applied linguistics, although neither applied linguistics nor European didactics have much influenced this North American tradition, which owes more to an indigenous tradition called Rhetoric and Composition (Donahue 2004). The major influences are Vygogsky's genetic theory of development (1978, 1986) and a theory of genre very much influenced by Bakhtin (1981) and Julia Kriseva's (1980) notions of intertextuality. But this framework is built upon American pragmatism (Dewey) social interactionism (Mead), and a U.S. interpretation of Schutz's (1989) phenomenological sociology, the "social construction of reality" (Berger and Luckmann 2011).

The methods are those familiar in social sciences research in education (survey, ethnographic observation and interviewing, quasi-experimental comparison-group studies, etc.), often combined with linguistic and rhetorical analysis of texts. But the results of this research and the interventions described require some understanding of the differences between U.S. and European educational contexts.

Over the last 30 years, research on writing in North American HE has often exposed deep contradictions among the conflicting societal demands and interests in 
HE. By contradictions I mean deep and historical tensions within and between organizations, instutions or social structures, not simply conflicts or problems (though these often grow out of contraductions). The concept has its socilogical origins in marxist anlaysis of the contradiction between use value and exchange value in capitalist societies. Medical institutions thus face a contradiction between the patient as a person in need of help and the patient as a source of profit (e.g., cost savings) (Leont'ev 1981). The concept is specifically developed in many versions of cultural historical activity theory, not limited to it. Attention to writing exposes the contradictions between the formation of students specifically (professionally) versus generally (for citizenship); between research (and reproduction) versus professional practice (service); between the interests of teaching versus research. All of these generate conflicts and problems that students and teachers experience in using writing for teaching, learning, and researching in higher education.

I begin with a central intervention and the central contradiction around it, a forty-year-old higher education reform movement called Writng Across the Curriculum (WAC). (For an overview see Russell 2002; Bazerman, Bethel, Chavkin, Fouquette \& Garufis 2005).

\section{Cognition: Writing to learn, learning to write, and the WAC movement}

A first contradiction is one embedded in the very deepest conceptions of writing: writing viewed as an autonomous transcription of speech versus writing viewed as an integral part of the intellectual activity of a discipline: in other words, writing as transversal versus writing as specialized. A corollary to this contradiction is that between writing as a means of examining (and therefore of selecting students) and writing as a means of teaching and learning.

This contradiction is played out in historical and institutional terms specific to the US. Contrary to most other nations, almost all American HE institutions offer a general skills writing course, called "first year composition," required of almost all students since the emergence of the modern university on the model of von Humboldt in the 1870 s at Harvard. But first-year composition was founded and in many ways continues on the assumption that writing is transversal and constitutes a set of discrete and easily transferrable skills, which should have been learned earlier, in secondary or elementary school. However, there is little evidence that the skills acquired in the course are transferred to other courses in other disciplines (Wardle 2007). The course has nevertheless persisted for 140 years, for reasons I have discussed elsewhere (Russell 2002).

In the 1970s, student writing became a major issue in higher education, following the social upheavals of the 1960s. Numerous American universities began to accept students from working class and racial minority backgrounds - what is called "open enrollment." Many universities responded by requiring remedial supplementary courses in writing for students they considered not sufficiently prepared. Many other universities, however, responded to the influx of new students from divers backgrounds by encouraging teachers in different disciplines to become interested in 
their students' writing. These universities called on experts in composition to aid teachers in the disciplines to use writing more effectively. Thus the Writing Across the Curriculum (WAC) movement was formed (Russell 2002), as a response to the limitations of the notion of writing as transversal and writing skill as easily transferrable.

In the US today, $50 \%$ of institutions of HE in a recent national survey reported offering some program to improve student writing across the curriculum (Thaiss \& Porter 2010). Some 65\% of Ph.D. granting universities reported such a program. In general, WAC programs do not replace first-year composition courses but rather are intended to compliment them. WAC programs frequently offer workshops where experts in composition teach teachers in other disciplines techniques to help them improve their students' learning of the discipline through writing. Many institutions also offer courses in each program of study (major) designated as "writing intensive," where the students write more often, where they receive more attention from their teachers, and where the number of students is generally lower (Bazerman, et al. 2005). A few universities have each department construct a plan for improving their students' writing in the program of study, from first year to last, as we shall see.

The central theoretical concept of WAC-from its Vygotsian origins in the work of James Britton and his colleagues in the UK (1975) - is that students not only learn to write but also write to learn. This concept provides a deeper understanding of writing than the transmission (sender-receiver model.) Writing is a tool for learning instead of merely a tool for assessing learning. Writing is conceived as a means of engaging students with the problems and methods of a discipline as well as a means of sorting students.

Recent large-scale survey research (NSSE, 2008) of more than 23,000 students in 82 U.S. universities found that students who reported doing more extensive writing with certain qualities in their courses was highly correlated with positive dimensions of student engagement and learning. The report concluded:

"when institutions provided students with extensive, intellectually challenging writing activities, the students engaged in more deep learning activities such as analysis, synthesis, integration of ideas from various sources, and grappled more with course ideas both in and out of the classroom. In turn, students whose faculty assigned projects with these same characteristics reported greater personal, social, practical, and academic learning and development" (pp. 20-21)

However, the specific cognitive effects of writing and their mechanisms are by no means well understood. In what is still the most comprehensive review of research on writing to learn, Klein (1999) found that there is no evidence of a general effect of writing on learning, such that the amount of writing is correlated with gains in learning. Rather, as with the NSSE study, certain uses of writing have shown significant positive effects in a range of studies. Klein suggests that the most promising research is in the area of genre, and it is around genre that most WAC research and theory has centered, but with a reconceived conception of genre, which brings us to a second contradiction. 


\section{Form/content dualism versus genre as social action}

In traditional conceptions of writing, there is a separation between the form and the content. University teachers often profess themselves able to teach the content but not the form of writing. Often writing experts are expected to teach proper form, which thought to be transversally available for the writing of any content. This is particularly felt in US HE, where first-year composition exists. Yet university teachers are clearly able to write in the ways expected of their disciplines and writing experts are not able to write successfully in any and all disciplines (at least not without immersion in the activity of the field through long practice).

The WAC movement approached this contradiction through the research on writing in the disciplines and professions: WID, as this research tradition is called. Methodologically, this research came out of Dell Hymes's linguistic ethnography (1974). The seminal study, "Strangers in Strange Lands" (1987), by McCarthy (a student of Hymes), showed how a first-year university student struggled to understand the very different writing expectations of three of his courses, with consequences for his identity work as a student. Yet the professors were largely unaware of the differences in their expectations and the struggles of their students. This study also signaled a tradition of studying teachers and students who are involved in WAC programs.

Theoretically, this research was propelled, from the early 1990s, by taking the concept of genre from language study and reformulating it with a concept from phenomenological sociology, Alfred Schutz's (1989) typifcation.

The link between form and content is genre, but genre conceived not as textual forms subject to taxonomic classification, but "genre as social action." This approach is called North American Genre theory (to distinguish it from Language for Special Purposes or Systemic Functional Linguistics theories) (Coe \& Freedman 1998). It is also often called New Rhetoric (to emphasize its focus on the communicative force of writing). In this perspective, a genre is, in Carolyn Miller's famous phrase, "typified rhetorical actions based in recurrent situations" that form and shape expectations and responses $(1994$, p. 31; 1984). Genre is not seen as similar formal features or as packeted speech (Wertsch, 1994), but as typified actions that over time have been routinized, "stabilized-for-now" (in Schryer's phrase, 1993) in ways that have proven useful in some recurring situation - that is, in some context recognized (interpreted) as similar, as typical, by participants. In time, these interactions come to be taken for granted. Genres become not simply forms of words, but forms of life, as Bazerman said, following Wittgenstein (1994). A genre is a sort of path in the forest of our communal life, begin by someone and followed by others who need a route to a destination.

This phenomenology of genre is compatible with Vygotsky's view of mediated action (Russell 2010). Put simply, a genre is the ongoing use of certain material tools (marks, in the case of written genres) in certain ways that people recognize as having worked once and might work again, a typified, tool-mediated response to conditions recognized by participants to be recurring. This perspective on genre presupposes pragmatics, discourse in context. But it also embraces philosophical pragmatism (Dewey and Mead), communication as mediating symbolic interaction. 
In the case of writing in higher education, the social action, the activity, is teaching and learning-disciplinary learning, primarily. And the genres have developed to support learning (Russell 1997). For example, the experimental report in natural sciences is more than a collection of linguistic elements shard by diverse documents. The genre is rather a typified response to a recurring action: that is, to communicate the results of an experiment to other researchers who are occupied with this specific problem (Bazerman 1988). It is not, finally, the form but the function that makes the difference between one genre and another. The lab report done by undergraduate students in a laboratory course in chemistry, for example, shares-often exactly-the linguistic forms of a professional research article. But its function is different - to teach beginners, not to inform experts. In this concept of genre, genre as social action, a genre tells us not only what one can say, but also what motives one can have, within some system of social activity.

The theory of genre as social action is a concept that is less transversal and much more specific and heterogeneous. The concept of genre as social action links the genre to specific contexts and practices, activities and acts. Note that it is impossible to determine the genre with linguistic analysis alone. One must interrogate the context by sociological or social psychological methods (interviews, observations, etc.), although linguistic analysis is also important and often necessary. One cannot read off motives, contexts, from texts alone (Coe \& Freedman 1998).

The concept of genre as social action is similar in important was to much continental European theorizing of genre-not surprisingly, perhaps, given similar roots in continental sociology, Bakhtin, and Vygotsky. In particular French and Swiss research in the didactics of writing has looked beyond analysis of linguistic features and individual skills to view genres in terms of their role in social systems/networks and institutions, such as disciplines. (For a comparison of North American and French views of genre in research on writing, see Donahue 2009.)

Research and interventions based on the theory of genre as social action use writing to simultaneously initiate students into the practices of writing AND thinking common in a field. For example, a team of researchers at North Carolina Sate developed an online tutorial designed to support students in the process of writing laboratory reports in chemistry, a genre that is frequent is natural sciences and engineering, but often reduced to a formulaic "recipe" without intellectual interest or engagement on the part of students. The online tutorial, Labwrite, is designed to teach students not only "the how" of writing the genre (the linguistic and rhetorical conventions) but also "the why" of writing it: the motives and social roles expected, its function in the scientific practice of science (Carter, Ferzli \& Wiebe 2004, 2007).

Labwrite guides the students through the process of understanding and representing (textually, mathematically and graphically) a laboratory experiment. Instruction on writing a laboratory report begins even before the students enter the laboratory, continues in the laboratory, and ends after they leave. The goal of instruction is not to improve their writing but to improve their learning: to teach scientific concepts and scientific methods using writing as a means. Because the students write to learn, they are evaluated on their learning, and not on their writing per se. The genre is a tool for learning science in the context of specialized formation. And the students learn the genre "as a matter of course," in acting in the course 
context from beginning to end, not apart from it. In order to develop this tutorial, the researchers used the results of research on expert scientists who write experimental reports -25 years of WID research, historical, linguistic, and ethnographic on scientific writing in situ. (For another approach, the Science Writing Heuristic, see Hand 2007)

A double blind comparison study of the students using using Labwrite, versus students using the usual paper guide, showed that the students using LabWrite 1) better understood the concepts taught in the chemistry course 2) better understood the scientific method taught, and 3) had a more positive attitude toward laboratory work than students who did not use Labwrite. It should be noted that the research did not try to determine if the students wrote better but rather if they learned better (Carter, Ferzli, \& Weibe 2004). Carter (2007) theorized out of this work that there are certain "apprentice genres" that are characteristic of formation in each field, genres which may provide a key to not only learning but also development in a field.

\section{Development and learning}

A third contradiction is between learning and development. Much research, such as the study above, has attempted to measure the effects of writing on learning, but learning conceived in terms of discrete, short-term gains of the type usually measured by conventional educational tests. In these terms, writing often showed no effect or even negative effects (Klein 1999). However, writing's possible effects on longer-term intellectual development are more difficult to measure but potentially more important to educators. Bazerman $(2007,2009)$ has elaborated Vygotsky's genetic theory of development in relation to genre. Piaget argued that one must reach a certain level of cognitive development before one can learn in certain ways. Vyotsky turned that on its head. In Vygotsky's theory learning precedes development (1986). Bazerman suggests that genres-particularly written genres--"provide highly differentiated, scaffolded communicative spaces in which we learn the cognitive practices of specialized domains" (2007, 2009).

In Vygotsky's theory, tool-mediated semiotic activity transforms cognition by internalizing external cultural tools and processes. As one internalizes through learning, a cognitive reorganization can take place that Vygotsky (1997) calls development. Accumulated learning may lead to qualitative change: reformulated functional cognitive systems or development. In Vygotsky's famous example, children at about two years old imitate the speech of others in a babbling that Piaget dismissed as "egocentric" speech awaiting a developmental shift. Vygotsky theorized that this "egocentric speech" is learning that becomes, in time, internalized to produce a qualitatively different use of the learning, as a tool of self-regulation, around age three. Children learn to talk to themselves out loud, and eventually this talk is internalized as a new stage of cognitive development: conscious thought including planning, self-regulation, and so on.

Genres, then, Bazerman suggests, may provide zones of proximal development as Vygotsky terms them. External cultural tools (e.g., genres) that become internalized have the potential for refiguring prior engagements with the material and social worlds. Bazerman argues that genres are important to this qualitative shift from 
accumulated learning to cognitive refiguration or development. External tools, such as genres that students internalize, have the potential to refigure engagement with the world. One sees the world differently. One sees prior learning differently.

Bazerman's example is grammar (2007, 2009). Students memorize discrete grammar rules, learn the parts of speech, and manipulate diagrams of sentences, perhaps. But for most people all of that is just learning, often quickly forgotten or, if remembered, not integrated into regular activity. But for many people-such as language teachers - that accumulated learning comes to transform the way one thinks about communication, at some point. Experts in language think in terms of this developed and, for them, developmental understanding of grammar. They use it to change the way they write, to evaluate the way people speak and write in different ways, to manipulate language in new ways, to generate new problems, and so on. Students of language begin in other words to have a disciplined understanding that it allows them to see the world in new ways, through the lens of grammar. They become, in effect, specialized. Bazerman (2009) describes this process as "punctuated periods of marked development where the learning become integrated with other existing or parallel developed functional systems to create new functional systems" (p. 290)

A recent study (Bazerman, Ewing, Simon, \& Piang, under review) provides evidence for Bazerman's theory from a case study of students in a two-year teacher education study program leading to licensure. The students' written work and their comments about it were coded to index the qualitative development of disciplinary understanding that students expressed in and about the genres assigned in the program. Findings "provide strong evidence that genres and structured parts of genres can direct the kind of thoughts expressed by students." By assigning particular genres of professional education, in a context that motivates and supports students, the teachers lead students to produce "particular kinds of expressed thoughts in addressing the intellectual challenges of the genre. Thus genres can provide opportunities to practice and learn particular kinds of thinking." There was also evidence that development was uneven, with "time lags in development and punctuated moments of reorganizations, with the reorganization not being stabilized until coherent representations of the relations of thought are developed."

Further research is ongoing in this line (Russell \& Harms 2009), despite the difficulties of such longitudinal study. A more pressing issue for writing in $\mathrm{HE}$, one firmly linked to development, is that of transfer across domains. And here contradictions are manifold, embedded in the very problem addressed.

\section{Transfer and the transversal}

Concepts of development and genre as social action have also informed research on the transition from writing in HE to writing in professional work-fundamentally different activity systems nevertheless linked through what Spinuzzi (2004) has called "genre ecologies." (One example is the intertextual linkage we noted between the student's lab report and a scientist's experimental article.) This transition from "school to work" is crucial to understanding the contradiction in HE between traditional values 
of liberal education and new demands on $\mathrm{HE}$ from employers, government employability agendas, and so on.

The most extensive study of the transition was conducted by a group of Canadian researchers (Dias et al. 1999; Freedman, Adam \& Smart 1994). A central but uncomfortable finding is that there is little 'transfer' between learning in the genres of professional education in HE and learning the genres of professional work. These researchers found that students attributed their learning to schooling (writing for the teacher, for a mark) even when teachers attempted to simulate the workplace: by assigning workplace genres, having professionals in the field attend student presentations, etc. That is, students recognize and create texts as belonging to the activity and genre ecology of schooling, though the texts teachers assign for reading or writing may have been drawn from or intended for a workplace ecology of genres. This, the researchers argue, is because the social motive of schooling (epistemic) is fundamentally different than that of work (pragmatic). And the genres of school and work-however similar in form they may be-perform different social action.

This fundamental contradiction in social motives generates other contradictions. Writing in schooling is primarily individual, done for assessment typically, and leads to a mark. Incorporating other students' work is often considered cheating. Writing in professional environments is primarily collective, collaborative, and leads to a product or service. In schooling, then, there is little "document cycling"-feedback and revision loops common in professional workplaces (Goswami \& Odell 1985).

An obvious intervention, then, is to immerse students in a target genre ecology beyond the classroom, in a workplace, as with internships or service learning. However, this is expensive and difficult to control. Based on North American genre studies on communication in professional organizations, a group of researchers have been constructing multi-media simulations of fictional organizations, represented by fictional Internet and intranet sites, to create an activity system that mediates between schooling and work (Fisher 2006, 2007; Russell \& Fisher 2009) (see Figure 1). Students role-play as they collaboratively engage in workplace-like activities in the fictional online learning environment, using the sorts of tools and genres typical in workplaces in a particular sector (databases, files of documents, meeting minutes, videoed meetings, synchronous and asynchronous communication, etc.). 


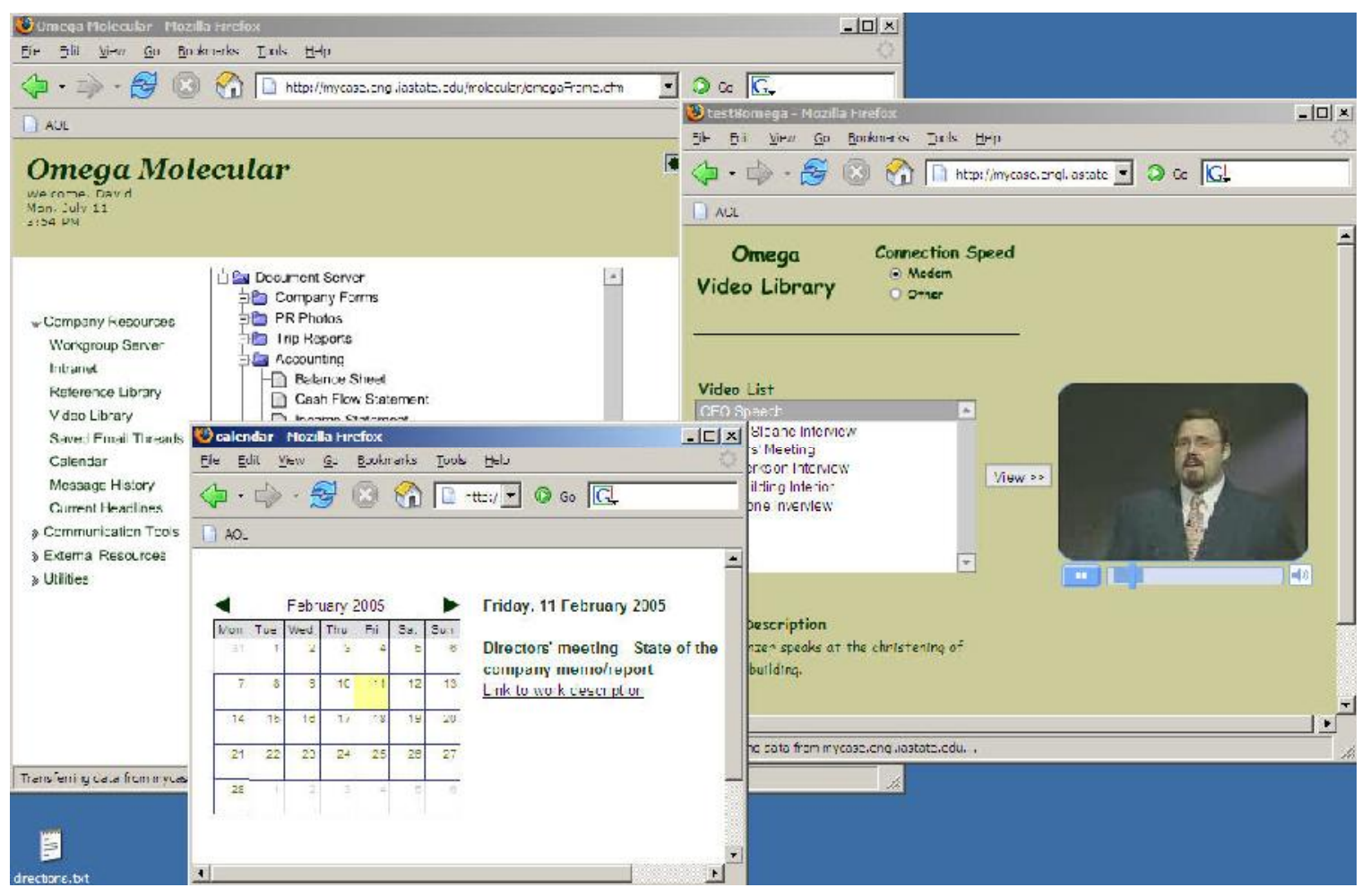

Figure n. 1. Online multi-media simulation of a fictional workplace

Students play the role of consultants (or interns) in the fictional organization. The "consultants" produce texts in a range of genres (written, oral, visual and electronic) that arise in the fictional activity (and genre) system of the company. The students' texts ("work deliverables") are submitted to characters in the simulation, such as the CEO in a fictional biotechnology startup company, pictured here in a video (see Figure 1). And the characters reply to the students-as-consultants through a closed email system (though it is actually-as the students are told-the teacher who is replying, in character, using a special role-sensitive email system). There is also a document server with a universe of documents from various departments of the organization. And in the interactions of fictional characters and students-as-consultants, that document universe is brought into circulation through the genre ecology. There students must act on deadlines, face ethical dilemmas "seeded" into the simulation, and deal with "emergencies," such as an anti-GMO demonstration outside the company headquarters. In other words, the elements of time and space, although fictional, are added to the fictional case, to create a learning environment where students experience genre as social action in workplaces.

Research into students' learning in these environments suggests that students are much more likely to attribute their learning in the online simulation environment to contexts of professional work than to contexts of schooling, as compared to their attributions of other parts of their courses that use more traditional learning environments (e.g., Blackboard ${ }^{\mathrm{TM}}$ and face-to-face instruction) (Fisher, 2006). These attributions seem to be shaped by the changes in classroom rules, division of labor, and community that the simulation affords, and by the contradictions between the genre ecologies of schooling and workplace (mediated by the simulation as teaching tool). For example, in the engineering and business simulations, students draw freely 
from each other's work as it is posted to a shared file space, and from previous students' work published in the simulation (students add to the simulation over time). This literacy practice is, as noted above, atypical in schooling but highly typical in the workplace, where people often draw from a common pool of documents and where documents cycle through multiple readers in the division of labor. The goal of the online multi-media simulations is to exploit the contradictions between the two activity systems in order to produce reflective practice and reflective practitioners (Schön 1987, 1999).

\section{Contradictions of graduate education: Lamination of activity and multi-genre in the thesis}

A contradiction similar to the one noted above between the institutional demands of $\mathrm{HE}$ and the professional demands of employment exists also in the genres of the Masters and Ph.D. thesis (US dissertation). These genres have become a major issue with the exponential growth of post-graduate education-and a major object of North American genre research for 25 years. From the first ethnographic studies of Ph.D. students (Berkenkotter, Huckin \& Ackerman, 1988), US research has found that doctoral students write the genre of the thesis with great difficulty, and often at the price of profound conflicts of identity, long periods of inactivity, often, and other costs, human and financial. Caught between the activity systems of the institution and its requirements, on one hand, and the discipline and its practices on the other, newcomers to the genre and activity of the thesis/dissertation bring their own sociocultural history and take an active role in learning to write it. Disciplinary enculturation is less a slow absorption or unconscious, passive assimilation and more a conscious, often chaotic battle, though often hidden from the view of advisor and departments.

Paul Prior's longitudinal studies (1998), have found that students at this level engage in a process of "gentrification," reclassifying texts, attributing similarities, as they learn-and sometimes reject-powerful disciplinary and institutional practices. Each student participates in multiple networks of activity simultaneously-university, department, discipline, committees, job market, family. And each must negotiate this "laminated" activity in writing this most important document of their career (See also Blakeslee, 1997; Casanave, 2002).

An ethnographic study of 11 students using activity theory (Lundell \& Beach, 2003) isolated two central contradictions of graduate education revealed in their processes of thesis writing:

- Students must write in the format and style required by the rules of the department and university, but the formats and styles do not easily translate into presentation or publications for the job market.

- They must conform to the practices of the thesis director, who is often uninformed about the department/university rules, and who does not give them the rules. 
Paré and Starke Meyerling's study (2009) of 60 Ph.D. students and their thesis directors found that the thesis is not only a double genre-a last exercise at the university, a final test, but also, entirely or partially, the first significant contribution to the disciplinary conversations. The thesis is also a multi-genre, which responds to multiple exigencies, functions in multiple systems of activity, and is addressed to multiple readers. Not only does the thesis contain a number of distinct embedded genres, each difficult to master (e.g., literature review, essay, experimental report), but it also responds to different social actions in many contexts, including the dyad of the director and student, the thesis committee, the department, the university, the disciplinary community, hiring committees, and the broader social structures of research in the field. Thus, the thesis is perhaps the most complex genre written in the university (whether by students, teachers, or researchers) in its multiplicity of intellectual, rhetorical and social demands.

In the last five years, interventions to support thesis writers have proliferated in the North America. Even prestigious universities such as Yale have what is called a graduate writing center, modeled on undergraduate writing centers. Here students can find individual help, group support, workshops, translation support, and so on.

\section{Contradictions of criticality}

In the 1990s the WAC movement received a few criticisms that it did not have a sufficiently radical stance: specifically, that the movement was not critical of disciplinary writing practices (Mahala 1991; LeCourt 1996; Malinowitz 1998; Horner 2000). These critics accused WAC/WID of being complicit with the patriarchal and hegemonic power of disciplines and thus excluding students from radical critique of disciplinary practices and power. Critics also maintained WAC/WID at that time conceived of disciplinary discourses as single, simple constructs rather than multivoiced and contested practices, a criticism that was often valid. Both of these criticisms point to a fundamental contradiction in US higher education. It is at bottom a very conservative institution, charged with reproduction of knowledge and practices. Yet it is also often expected to be an institution for fostering change, and thus deeply political, even-or perhaps especially-in its written discourse, though this political aspect is generally hidden.

In response in part to such criticisms, a number of fine-grained ethnographic studies of students wrestling with the political dimensions of disciplinary discourse were conducted. David Seitz (2004), for example, studied students who were in a cultural studies course in which the teacher encouraged them to be critical, in the radical political sense mentioned above. Seitz followed a group of students in the course, including during the term after they left it, to their working class lives in Chicago. Seitz found that the students themselves offering a powerful critique of the critical teacher. Similarly, Lucille McCarthy and a teacher-researcher from philosophy she collaborates with, Steve Fishman, did a series book-length ethnographic studies of university students focusing on race and class, which very much complicate notions of the critical. Thaiss \& Zwacki (2006) studied the writing practices of professors in several disciplines at a US research university in comparison to the ways they teach 
writing to students. They found that in research writing professors navigate multiple boundaries, even disciplinary ones, stretch genre expectations, and generally deal with complexity. While some of the professors invited students to engage with ambiguity and variation in writing expectations, most relied on teaching a normative version of their discipline's genres or of more generalized academic genres.

Those answering the radical critics of WAC/WID emphasize the potential for change in teachers, departmental curricula, and disciplines through writing experts working with all the above to expose practices long unexamined and imagine new uses for writing--"subversion from within." (Maimion \& McLeod 2000; Bazerman 1992). Change in disciplines then comes from the inside as more voices with more diverse views enter into the discussion.

Case study and longitudinal studies of professors suggest the possibilities and limits of such an approach, given the contradictions between expectations for teaching and research in US universities (Walvoord 1997; Walvoord \& McCarthy 1990). Deep change requires long-term commitments to collaboration with teachers in the disciplines and institutional structures to support that. Interventions at the level of disciplinary organizations occurred most notably in Engineering, whose national accrediting agency, $A B E T$, made communication one of its six major criteria for certifying university departments of engineering as compliant with standards. This spawned collaboration and research over the last decade under the rubric of EngiComm (Leydens, \& Schneider 2009). More recently, a discipline-level multiuniversity intervention and research project in Computer Science, funded by the National Science Foundation, attempts to construct and test a four-year framework for developing students' communication-primarily written-in that field (Carter, Vouk, Gannod, Burge, Anderson \& Hoffman 2011). At the university level, there are ongoing projects to conduct in every department a survey and iterative reform of curricula and pedagogy. The University of Minnessota (2013) Writing Enhanced Curriculum, for example, has a team of WAC experts work with individual departments to survey their needs, change curriculum, develop interventions, and assess them in a three-year iterative cycle. (See also Graves, Hyland \& Samuels 2010; Toronto, NCSU.). However, these are rare.

\section{Conclusion}

In summary, over the last four decades, the foregrounding of writing in US HE has reveled six contradictions. 1) North American WAC/WiD efforts with university teachers exposed a contradiction between writing viewed as an autonomous transcription of speech or thought and writing viewed as an integral part of the intellectual activity of a discipline: in other words, writing as transversal versus writing as specialized. Closely related is 2) a contradiction between genre conceived as a container of content--a form/content dualism--versus genre conceived as social action-uniting form and content in activity. Thus is highlighted a tension between writing taught in situ by teachers in the disciplines through the activity of the disciplines and writing taught in separate, add-on ways, formally separated from instruction in the discipline. 3) Attention to writing similarly indexes a contradiction in 
HE between discrete learning of content versus intellectual / professional / personal development. The slogan "students should write to learn" masks the potentially deeper function of writing in discipline-specific genres, which may provide access to socially and economically important know-how and roles. 4) Writing may provide a means of accessing that know-how and those roles, but there is a fundamental contradiction between the social motive of schooling (epistemic) and the social motive of work (pragmatic), which makes it very difficult to learn write in the ways disciplinary and professional do within HE. 5) This contradiction between formal schooling and professional work is played out in writing (and directing) the genre of the thesis, although again, the role of writing in the transition is often hidden.

Finally 6) attention to the relationship between writing and disciplinary work has bared a fundamental contradiction in US HE. HE is bottom a very conservative institution, whose goal is reproduction of knowledge and social practices. Yet the modern university is also a driver of change, in various and contested directions. These tensions-deeply political in the broadest sense of the term-are played out in the written discourse of disciplines, whose conventions and values are dynamic and contested in the very structure of their discourse, though this political aspect is generally submerged in their routine work.

For professionals writing routine genres-genres they know well-writing does not need to be a focus of attention. It seems uninteresting, invisible, something learned long ago. In routine genres there seems to be no "writing," only "writing it up," the last and least interesting step. This invisibility is fine-useful-until it breaks down under the pressure of new challenges, and thus writing is bumped up into conscious attention, with the attendant emotions of anxiety. This is a normal part of learning to write a new genre in a new activity system (a scientist having to write a press release, for example (Smart, 2000)).

Yet for students, the specialized genres of the disciplines of higher education are for the most part new challenges, and bring anxiety. Indeed, the genres often go by the same names as those the students wrote in secondary school (essay, report), and thus bring attendant confusion and unlearning necessary to sort out the differences in the kinds and disciplines of thinking/writing (Graves et al., 2010; Donahue, 2008). So students must consciously wrestle with writing. Students must struggle to acquire on their own the genre know-how that is for their professors so routinized, operationalized in the activity and "content" of the field, as to be invisible to them. Yet this is the very know-how on which not only students' grades but also their success beyond the university depend, in large part (as employer surveys so often point out (e.g., Bowers \& Metcalf, 2008)). This is the fundamental and unchanging challenge of teaching and learning (with) with writing.

Yet in recent years, with the new challenges of higher education, what is true for individuals has become true for the HE sector writ large. The old ways of teaching and learning (with) writing no longer work smoothly. Student writing development has thus become an object of conscious attention, a "problem" in $\mathrm{HE}$, because it reveals fundamental contradictions in contemporary HE. Higher education sits between two contradictory pressures, what Burton Clark (1989) has called disciplinary excellence versus social equity. On one end, the intake end of social equity, far more students (and far more diverse students) come streaming into higher education-bringing in a 
far greater diversity of linguistic resources, not only international students but also students from different cultural and class backgrounds. Many of these new students do not know the ways of reading and writing that seem to be second nature to traditional students. Moreover, the patterns of participation have changed, with many students are in HE part time, balancing schedules and, more importantly, spending a large part of their time away from the academic environment and its traditional discourses.

On the other end, disciplinary excellence, HE is also changing. Excellence used to mean reproducing an intellectual (and in many ways a social) elite, to carry on what was then the relatively stable work of the disciplines and professions. But changes in both knowledge and work mean that post-industrial societies and economies need more "knowledge workers," and this knowledge work depends on written communication in and between specialisms. HE is essential to the emplyability agendas of modern societies, and this means HE must use and teach more sophisticated and diverse genres of written commuication. Students are leaving higher education to enter far more specialised and yet far more interdisciplinary workplaces. As the pace and complexity of global communication increases, the division of intellectual labor increases, and with it the importance of writing. Students will have to have greater linguistic and rhetorical flexibility to effectively enter and eventually transform professions and institutions.

Between these two contradictory pressures, a changing notion of social equity on one hand and a changing notion of disciplinary excellence on the other, sits student writing in higher education. Because it is no longer possible for HE to simply skim the cream and pour the rest out, it is no longer possible to ignore issues of teaching and learning, and no longer possible to leave the problem of writing development solely in the hands of individual students, unaided by academic staff.

Attention to writing, then, is not a distraction from teaching content, but a means of teaching it more effectively, because content is seen not as a thing to be put into students' minds but resources for engaging with, communicating with, others and the world-most powerfully, usually, through writing. In this sense, writing is no longer a remedial subject, another course or courses to be taken outside a curriculum (the knee-jerk reaction of modern HE to problems is to create another course). Writing is rather a shared responsibility, a means of teaching and learning and critical thinking for both students and researchers. And as the tradition of research I have touched upon here suggests, writing is also an interesting object of research and tool of pedagogical experiment and reform in its own right.

\section{References}

Bakhtin, M. (1981). The dialogic imagination. Austin: University of Texas Press.

Bazerman, C., Simon, K., Ewing, P. and Pieng, P. (under review). Domain-Specific Cognitive Development through Writing Tasks in a Teacher Education Program

Bazerman, C. (1988). Shaping Written Knowledge. Madison: University of Wisconsin Press. 
Bazerman, C. (1992). From cultural criticism to disciplinary participation: Living with powerful words. Writing, teaching, and learning in the disciplines, 61-68.

Bazerman, C. (1994). Systems of genres and the enactment of social intentions. Genre and the new rhetoric, 79-101.

Bazerman, C. (2009). Genre and cognitive development: Beyond writing to learn. Pratiques 143/144, 127-138.

Bazerman, C. (2009). Genre and Cognitive Development: Beyond Writing to Learn. In Genre in a Changing World, Perspectives on writing (pp. 279-294). Fort Collins, Colo.: WAC Clearinghouse; West Lafayette, Ind: Parlor Press. Retrieved from a http://wac.colostate.edu/books/genre/

Bazerman, C. \& Russell, D. (2003). Writing Selves/Writing Societies: Research from Activity Perspectives, Perspectives on Writing. Fort Collins, CO: WAC Clearinghouse. http://wac.colostate.edu/books/selves societies

Bazerman, C., \& Prior, P. A. (2004). What writing does and how it does it: An introduction to analyzing texts and textual practices. Lawrence Erlbaum Associates.

Bazerman, C., Joseph, L., Bethel, L., Chavkin, T., Fouquette, D., \& Garufis, J. (2005). Reference Guide to Writing across the Curriculum. West Lafayette, IN: Parlor Press and The WAC Clearinghouse http://wac.colostate.edu/books/bazerman wac/.

Beaufort, A. (1999). Writing in the real world: Making the transition from school to work. Teachers College Pr.

Beaufort, A. (2006). Writing in the professions. Research on composition: Multiple perspectives on two decades of change, 217-242.

Berger, P. L., \& Luckmann, T. (2011). The social construction of reality: A treatise in the sociology of knowledge. Open Road.

Berkenkotter, C., Huckin, T. N., \& Ackerman, J. (1988). Conventions, conversations, and the writer: Case study of a student in a rhetoric $\mathrm{Ph}$. D. program. Research in the Teaching of English, 9-44.

Blakeslee, A. M. (1997). Activity, context, interaction, and authority: Learning to write scientific papers in situ. Journal of Business and Technical Communication, 11, 125-169.

Bowers, M. Y., \& Metcalf, M. A. (2008). What employers want and what students need: Integrating business communication into undergraduate and graduate business courses. Proceedings of the 2008 Association for Business Communication Annual Convention (pp. 1-13).

Britton, J. N. (1975). The Development of Writing Abilities (11-18). London: Macmillan Education.

Britton, J. N. (1975). The Development of Writing Abilities (11-18). London: Macmillan Education. 
Carter, M. (2007). Ways of knowing, doing, and writing in the disciplines. College Composition and Communication, 58(3), 385.

Carter, M., Ferzli, M., \& Wiebe, E. (2004). Teaching genre to English first-language adults: A study of the laboratory report. Research in the Teaching of English, 395-419.

Carter, M., Ferzli, M., \& Wiebe, E. N. (2007). Writing to Learn by Learning to Write in the Disciplines. Journal of Business and Technical Communication, 21(3), 278.

Carter, M., Vouk, M., Gannod, G. C., Burge, J. E., Anderson, P. V., \& Hoffman, M. E. (2011). Communication genres: Integrating communication into the software engineering curriculum. Software Engineering Education and Training (CSEE\&T), 2011 24th IEEE-CS Conference on (pp. 21-30).

Casanave, C. P. (2002). Writing games: Multicultural case studies of academic literacy practices in higher education. Lawrence Erlbaum.

Christie, F., \& Martin, J. R. (2005). Genre and institutions: Social processes in the workplace and school. Continuum Intl Pub Group.

Clark, B. R. (1989). The Academic Life. Educational Researcher, 18(5), 4.

Coe, R. M., \& Freedman, A. (1998). Genre theory: Australian and North American approaches. Theorizing composition: A critical sourcebook of theory and scholarship in contemporary composition studies, 136-147.

De Vise, D. (2011, September 13). U.S. falls in global ranking of young adults who finish college. Washington Post. Washington, D.C.

Dias, P., Freedman, A., Medway, P., \& Paré, A. (1999). Worlds Apart: Acting and Writing in Academic and Workplace Contexts. Mahwah, NJ: Lawrence Erlbaum Associates.

Donahue, T. (2009). Genre and disciplinary work in French didactics research. In Genre in a Changing World (pp. 424-441). Fort Collins, Colo.: WAC Clearinghouse / Parlor Press. Retrieved from http://wac.colostate.edu/books/genre/

Fisher, D. (2007). CMS-based simulations in the writing classroom: Evoking genre through game play. Computers and Composition, 24(2), 179-197.

Fisher, D. D. (2006). Remediating the professional classroom: The new rhetoric of teaching and learning. lowa State University.

Freedman, A., Adam, C., \& Smart, G. (1994). Wearing suits to class: simulating genres and simulations as genre. Written Communication, 11, 193-226.

Goswami, D., \& Odell, L. (1985). Writing in Nonacademic Settings. Guilford Press.

Graves, R., Hyland, T., \& Samuels, B. M. (2010). Undergraduate Writing Assignments: An Analysis of Syllabi at One Canadian College. Written Communication, 27(3), 293.

Hand, B. M. (2007). Science inquiry, argument and language: A case for the Science Writing Heuristic. Sense Pubns. 
Horner, B. (2000) Terms of Work for Composition: A Materialist Critique, Albany: State University of New York Press.

Hymes, D. H. (1974). Foundations in sociolinguistics; an ethnographic approach. Philadelphia,: University of Pennsylvania Press.

Klein, P. D. (1999). Reopening inquiry into cognitive processes in writing-to-learn. Educational Psychology Review, 11, 203-270.

Kristeva, J. (1980). Desire in language: A semiotic approach to literature and art. Columbia University Press.

LeCourt, D. (1996) “WAC as Critical Pedagogy: A Third Stage?” JAC, 16: 389-405.

Leont'ev, A. N. (1981). The problem of activity in psychgology. In J. V. Wertsch (Ed.), The concept of activity in soviet psychology (pp. 40-71). Armonk, NY: M.E. Sharpe.

Leydens, J. A. \& Schneider, J. (2009). Innovations in composition programs that educate engineers: Drivers, opportunities, and challenges. Journal of Engineering Education 98(3), 255-271.

Lundell, D. B., \& Beach, R. (2003). Dissertation Writers' Negotiations with Competing Activity Systems. Writing Selves, Writing Societies: Research from Activity Perspectives. Eds. Charles Bazerman and David R. Russell. Fort Collins, CO: WAC Clearinghouse.

Mahala, D. (1991) "Writing Utopias: Writing Across the Curriculum and the Promise of Reform," College English, 53, 773-89.

Malinowitz, H. (1998) "A Feminist Critique of Writing in the Disciplines," in S. Jarratt and L. Worsham (eds) Feminism and Composition Studies: In Other Words (pp. 291-312), New York: Modern Language Association of America.

McCarthy, L. P. (1987). A stranger in strange lands: A college student writing across the curriculum. Research in the Teaching of English, 233-265.

McLeod, S., \& Maimon, E. (2000). Clearing the air: WAC myths and realities. College English, 573-583.

Miller, C. (1984). Genre as social action. Quarterly Journal of Speech, 70, 151-167.

Miller, C. R. (1994). Rhetorical community: The cultural basis of genre. Genre and the new rhetoric, 67-78.

NSSE (National Survey of Student Engagement). (2008). Promoting Engagement for All Students: The Imperative to Look Within. Bloomington, IN: National Survey of Student Engagement. http://nsse.iub.edu/NSSE 2008 Results

Paré, A., D. Starke-Meyerrin, \& McAlpine, L. (2009). The Dissertation as Multi-Genre: Many Readers, Many Readings. Genre in a Changing World, Perspectives on Writing (pp. 179-195). West Lafayette, IN: Parlor Press and The WAC

Prior, P. A. (1998). Writing/disciplinarity: A sociohistoric account of literate activity in the academy. Mawah, N.J.: Lawrence Erlbaum. 
Russell, D. (1997). Rethinking genre in school and society. Written Communication, 14(4), 504-554.

Russell, D. (2001). Where Do the Naturalistic Studies of WAC/WID Point? A Research Review. In WAC for the New Millennium: Strategies for Continuing WritingAcross-the-Curriculum Programs. Urbana, IL: NCTE.

Russell, D. R. (2002). Writing in the academic disciplines]: a curricular history. Carbondale: Southern Illinois University Press.

Russell, D. R. (2007). Rethinking the articulation between business and technical communication and writing in the disciplines: Useful avenues for teaching and research. Journal of Business and Technical Communication, 21(3), 248.

Russell, D. R. (2009). Texts in contexts: Theorizing learning by looking at genre and activity. Rethinking Contexts for Learning and Teaching: Communities, Activites and Networks (pp. 17-30). Taylor \& Francis US.

Russell, D. R. (2010). Writing in multiple contexts: Vygotskian CHAT meets the phenomenology of genre. Traditions of writing research, 353-364.

Russell, D.R., \& Fisher, D. (2009). Online, multimedia case studies for professional education: Revisioning concepts of genre recognition. In J. Giltrow \& D. Stein (Eds.), Genres in the Internet: Issues in the theory of genre, Pragmatics \& Beyond New Series (pp. 163-191). Amsterdam?? Philadelphia: John Benjamins Publishing Company.

Russell, D.R., \& Yañez, A. (2003). "Big Picture People Rarely Become Historians": Genre Systems and the Contradictions of General Education,. Writing Selves/Writing Societies: Research from Activity Perspectives. Fort Collins, CO: The WAC Clearinghouse. Retrieved from http://wac.colostate.edu/books/selves societies/

Schön, D. A. (1987). Educating the reflective practitioner. Jossey-Bass San Francisco. Retrieved from http://www.thecommonwealthpractice.com/reflectivepractitionerreview.pdf

Schön, D. A. (1999). The reflective practitioner (Vol. 1). Basic books. Retrieved from http://sopper.dk/speciale/arkiv/book49.pdf

Schryer, C. F. (1994). The lab vs. the clinic: Sites of competing genres. In A. Freedman \& P. Medway (Eds), Genre and the new rhetoric. (105-124).

Schutz, A. (1989). The Structures of the Life-World: Alfred Schutz and Thomas Luckmann. Trans, by Richard Zaner and Tristham Englehardt. Evanston: Northwestern University Press.

Scott, M. \& Lillis, T. (2008) 'Defining academic literacies research: Issues of epistemology, ideology and strategy'. Journal of Applied Linguistics, 4, pp. 5-32.

Seitz, D. (2004). Who can afford critical consciousness?: practicing a pedagogy of humility. Hampton Pr.

Slevin, J. (2001) Introducing English: Essays in the Intellectual Work of Composition. Pittsburgh: University of Pittsburgh Press. 
Smart, G. (2000). Reinventing expertise: Experienced writers in the workplace encounter a new genre. Transitions: Writing in academic and workplace settings, 223-252.

Spinuzzi, C. (2003). Tracing Genres through Organizations: A Sociocultural Approach to Information Design. Cambridge, MA: The MIT Press.

Thaiss, C., \& Porter, T. (2010). The state of WAC/WID in 2010: Methods and results of the US survey of the international WAC/WID mapping project. College Composition and Communication, 61(3), 534-570.

Thaiss, C., \& Zawacki, T. M. (2006). Engaged writers dynamic disciplines. Engaged writers dynamic disciplines. New York: Heineman

University of Minnesota - WEC Home Page. (2013). Retrieved February 15, 2013, from http://wec.umn.edu/index.html

Vygotsky, L. (1978). Mind in society. Cambridge, MA: Harvard UP.

Vygotsky, L. (1986). Thought and Language. Cambridge, MA: MIT Press.

Walvoord, B. E. F., \& National Council of Teachers of English. (1997). In the long run?: a study of faculty in three writing-across-the-curriculum programs. Urbana, III.: National Council of Teachers of English.

Walvoord, B. E., \& McCarthy, L. P. (1990). Thinking and Writing in College: A Naturalistic Study of Students in Four Disciplines (p. 281). Urbana, Illinois: NCTE.

Wardle, E. (2007). Understanding Transfer from FYC: Preliminary Results of a Longitudinal Study. WPA: Writing Program Administration, 31(1-2), 65-85.

Wertsch, J. V., \& Wertsch, J. V. (1991). Voices of the mind: Sociocultural approach to mediated action. Harvard University Press.

Cita del artículo:

Russell, D.R. (2013). Contradictions regarding teaching and writing (or writing to learn) in the disciplines: What we have learned in the USA. Revista de Docencia Universitaria. REDU. Número monográfico dedicado a Academic Writing. Vol.11 (1) Enero-Abril. pp.161-181. Recuperado el (fecha de consulta) en http://www.red-u.net/ 


\title{
Acerca del autor
}

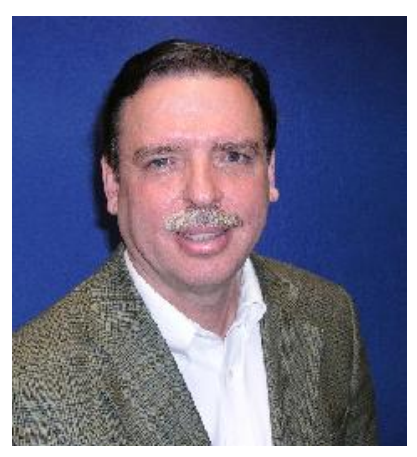

\section{David R. Russell}

\author{
lowa State University \\ Department of English \\ Mail: drrussel@iastate.edu
}

David R. Russell is professor of English at lowa State University, where he teaches in the programs in Rhetoric and Professional Communication and Applied Linguistics and Technology. His research interests are in writing in the disciplines, international writing instruction, and, recently online multi-media case studies. His book, Writing in the Academic Disciplines: A Curricular History, examines the history of United States writing instruction since 1870 . He has published more than 50 articles on writing in the disciplines and professions, drawing mainly on cultural historical activity theory and rhetorical genre theory. 


\section{REDU}

Revista de Docencia Universitaria

Vol 11 №1 



\title{
La controvertida aplicación de las competencias en la formación docente universitaria.
}

\author{
The Controversial Application of Competences in Teacher Training at University
}

\author{
Rosario Mérida Serrano \\ Universidad de Córdoba, España
}

\section{Resumen}

Se presenta una clarificación conceptual del término competencias, explicitando nuestra posición personal e indagando en diferentes corrientes teóricas que aportan diferentes visiones de este constructo. Su polisemia y ambigüedad genera diferentes concepciones y, como consecuencia, ampara diversas prácticas educativas en el ámbito universitario. Una caracterización exhaustiva de este concepto permite identificar como rasgos relevantes su carácter holístico, integral, contextual, axiológico, transferible y evolutivo.

Se muestra la potencialidad de las competencias para instaurarse como marcos de referencia del nuevo modelo docente universitario propuesto en el Espacio Europeo de Educación Superior. De este modo, las competencias aparecen como amplias finalidades que enmarcan los propósitos y el perfil formativo y profesional de las titulaciones, al tiempo que facilitan la selección de unos contenidos entendidos como soportes culturales que facilitan la adquisición de un conocimiento relevante, profundo, interdisciplinar, flexible y creativo. Las competencias permiten diseñar situaciones de aprendizaje y elegir principios metodológicos que surgen de la implicación del alumnado en procesos de investigación para adquirir nuevos saberes. Del mismo modo, la evaluación se ve afectada por la inclusión de las competencias en el currículum, necesitando aplicar una concepción formativa que se concreta en el uso de diversos instrumentos para valorar el proceso y no solo los resultados académicos.

Finalmente analizamos algunas circunstancias que han dificultado la aplicación real de las competencias en los escenarios de educación superior, proponiendo la red RIECU como ejemplo de buena práctica para el desarrollo de competencias profesionales en los futuros docentes de Educación Infantil.

Palabras clave: Competencias docentes, competencias profesionales, innovación educativa, formación del profesorado.

\section{Abstract}

This paper presents a conceptual clarification of the term 'competences', outlining the authors' personal stance and examining different theoretical currents which offer differing views of this construct. Its 
polysemy and ambiguity generate different conceptions and, consequently, encompass different educational practices within university education. An exhaustive characterisation of this concept helps to identify its significant features: its holistic, comprehensive, contextual, axiological, transferable and evolutionary nature.

This paper shows the potential of competences to offer frameworks of reference for the new model of university teaching proposed in the European Higher Education Area. Hence, competences appear as broad ends in themselves which frame the purposes and formative and professional profile of university course programmes, whilst at the same time facilitating the selection of contents, understood as cultural media, which in turn facilitate the acquisition of relevant, in-depth, interdisciplinary, flexible and creative knowledge. Competences allow us to design learning situations and to choose methodological principles which emerge from involving students in research processes in order to acquire new knowledge. Similarly, evaluation is affected by the inclusion of competences in the curriculum, requiring the application of an approach to training which is materialised through the use of different instruments to evaluate the processes and not just academic results.

Finally, the paper analyses certain circumstances which have hindered the real application of competences in scenarios of higher education, proposing the RIECU network as an example of good practices for the development of professional competences in prospective Pre-Primary school teachers.

Key words: Teaching competences, professional competences, education innovation, teacher training.

\section{Introducción}

Leíamos recientemente en las páginas de esta misma revista las interesantes reflexiones o catarsis, como él las Ilamó, de Miguel Ángel Zabalza (2011) en relación a lo que ha supuesto el popularmente llamado proceso de Bolonia, o Espacio Europeo de Educación Superior (en adelante, EEES), si se quiere denominar de forma más académica. He tenido la oportunidad de implicarme en este proceso desde la doble perspectiva de profesora y gestora, participando en una serie de actuaciones que han abarcado desde la experimentación de los créditos ECTS (European Credits Transfer System) hasta el diseño de los Planes de Estudio y la implantación de los nuevos Grados.

Las directrices propuestas en la Declaración de Bolonia (1999) dibujan un escenario docente prometedor para la Educación Superior. Comienza a hablarse de la importancia del aprendizaje centrado en el alumnado, de la necesidad de incrementar la dimensión práctica de los estudios académicos, de la renovación de las metodologías (De Miguel, 2005 y 2006 e Informe de la Comisión para la Docencia de las Universidades Andaluzas coordinado por Sola y Pérez, 2006), de los cambios en la concepción de la evaluación universitaria (Yorke, 2003 y Woolf, 2004) y de la modularidad de los contenidos (Zabala, 1999, Tobón, 2006 y Bautista-Cerro, 2007).

Diversos estudios (Delors, 1996, Bricall, 2000, Informe DeSeco, 2003, González y Wagenaar, 2003 y MEC, 2006), tanto a nivel nacional como internacional (Hirsch and Weber, 1999 y Weber and Duderstadt, 2010), muestran la oportunidad de poner en marcha una transformación profunda en la formación universitaria que permita consolidar la identidad europea, que facilite la movilidad y reconocimiento de los estudiantes universitarios, que potencie las redes de conocimiento e investigación y que contribuya a transferir los aprendizajes teóricos a entornos profesionales cada vez más flexibles, cambiantes y complejos (Van Damme, 2001, Kivinen and Nurmi, 2003, Rué, 2004 y 2007 y Dolado, 2010). 
Es en este escenario de reformas y promesas de profundas transformaciones educativas en las instituciones de Educación Superior donde se enmarca la incorporación del discurso de las competencias en el contexto educativo universitario. Las reformas de otras etapas del sistema educativo español, desde Educación Infantil a Educación Secundaria, incluyen también las competencias como finalidades para el desarrollo de las capacidades del alumnado, proponiendo una forma de trabajar en clase activa, globalizada, integral y centrada en la resolución de tareas.

En este clima de cambio, en la primera década del siglo actual los anhelos reformistas en el ámbito educativo prometían, para algunos docentes universitarios, la oportunidad de practicar metodologías en la universidad que fueran valoradas y reconocidas desde un marco normativo que, por primera vez, focalizaba su atención en la docencia y respaldaba las prácticas innovadoras que se venían desarrollando en las aulas por profesorado voluntarista y comprometido con la mejora docente. La recién iniciada evaluación de la labor docente del profesorado universitario y las diversas reivindicaciones para incrementar su importancia en los procesos de acreditación y promoción profesional, nos sitúan en un momento donde se habla, se propone y se desea revalorizar la función docente y educativa de la universidad.

Es en este contexto donde se inicia una prometedora línea de innovación docente (Mérida, 2007a, 2007b, Mérida y Dueñas, 2008, Mérida, Gil y Gallego, 2008, Mérida, Angulo, Gil y Mañas, 2008, Mérida, González y Olivares, 2011, Mérida et al., 2011, Gómez, Mérida y González, 2011 y Mérida, González y Olivares, 2012), centrada en el desarrollo de competencias profesionales en el alumnado de la titulación de Magisterio de Educación Infantil. Se pretende incorporar modificaciones que afecten a los pilares básicos del currículum universitario (Rodríguez, 2008 y López Ruiz, 2011) a partir de la conexión del escenario académico y los contextos profesionales o escuelas infantiles.

Con esta finalidad se ha ido construyendo en el transcurso del tiempo la Red de Infantil CEP-Escuela-Universidad (RIECU) que integra a personas que pertenecen a la formación inicial docente -alumnado y profesorado de la Facultad de Ciencias de la Educación- y personas que pertenecen a la formación continua docente -maestras y asesoras del Centro de Profesorado de la etapa Infantil- El interés compartido de todos los agentes de la red es el diseño, aplicación y valoración del método de Proyectos de Trabajo (en adelante, PT). Esta red está consolidándose como una comunidad de práctica, la cual según Wenger (2001: 100) tiene como características básicas 'el compromiso mutuo, la configuración de una acción colectiva y el uso de un repertorio compartido'. En la segunda parte de este trabajo se narra la experiencia de innovación desarrollada en el presente curso académico 2011/12.

\section{Justificación. Marco de referencia}

\section{¿Qué significa competencia? La controvertida polisemia del término}

Una de las innovaciones que sustentan el cambio en la docencia universitaria impulsado por Bolonia es el enfoque por competencias. Pero, antes de comenzar a valorar las potencialidades de este constructo en el terreno educativo, es necesario 
tener en cuenta, como se ha indicado en otros trabajos (Mérida, 2009 y Pérez et al., 2009) qué entendemos por competencias para, en consonancia con las propuestas de algunos autores (Gimeno, 2008), evitar conceptuarlas como destrezas y habilidades, lo cual supondría una vuelta atrás, acercándonos a concepciones curriculares tecnocráticas y desfasadas.

Es este uno de los peligros que se ha de tener en cuenta al aplicar el concepto de competencia educativa al ámbito universitario. Igualmente, hemos de tener presentes los datos de estudios europeos (Eurydice 2011 y 2012) que nos informan sobre las desigualdades sociales, la escasa atención a la diversidad, los niveles de fracaso y abandono de los sistemas educativos... Son indicadores preocupantes para la equidad y cohesión social que debieran ser tenidos en cuenta al aplicar las competencias.

Como contrapartida, en este trabajo se suscribe una concepción de competencia identificada con la capacidad de las personas para responder satisfactoriamente a las demandas de un contexto real, poniendo en marcha comportamientos holísticos y globales que incluyen la dimensión cognitiva, los procedimientos, las actitudes, los valores y las emociones.

Esta concepción de competencia ayuda a entender el conocimiento universitario como la adquisición de recursos personales que permiten comprender y dar respuesta a los problemas y situaciones de la vida real. No se trata de adquirir conocimientos teóricos descontextualizados y difícilmente transferibles a contextos vitales y profesionales, sino de usar herramientas transdisciplinares que faciliten el entendimiento de la realidad compleja y cambiante en la que nos situamos.

Desde esta concepción, y siguiendo las directrices que se propone en los Descriptores de Dublín (2004), las competencias en la universidad han de poseer las siguientes características: carácter holístico e integrado, carácter situado y contextual, carácter axiológico y emocional, carácter transferible y carácter evolutivo.

Sintetizando, los rasgos básicos de las competencias según las palabras de Pérez et al. (2009: 18): “ (...) constituyen un 'saber hacer' complejo y adaptativo, esto es, un saber que se aplica no de forma mecánica sino reflexiva, es susceptible de adecuarse a una diversidad de contextos y tiene un carácter integrador, abarcando conocimientos, habilidades, emociones, valores y actitudes".

\section{Estado actual de la cuestión de las competencias en el ámbito universitario.}

En este momento, en cualquiera de las etapas que integran nuestro sistema educativo, se puede apreciar la apuesta decidida por un modelo curricular basado en competencias. Prueba de ello son las normativas que regulan las enseñanzas desde la etapa infantil hasta la universitaria, así como el modelo de formación permanente del profesorado.

Un proceso similar ocurre en el sistema universitario, en donde a partir de la Declaración de Bolonia (1999) fueron sucediéndose diversas reuniones y Declaraciones de los Ministros de Educación europeos para consensuar la agenda y acciones conducentes a la armonización del sistema universitario europeo. En este contexto, y durante la última década del siglo pasado, se producen diversos estudios sobre las competencias y la educación superior, tanto a nivel internacional como nacional 
(Perrenoud, 2001, González, y Wagenaar, 2003, Informe DeSeco, 2003, Rychen y Salganik, 2003, De Miguel, 2006, Mérida, 2006, Sola y Pérez, 2006, Garagorri, 2007, Zabala y Arnau, 2007 y Pérez et al., 2009, entre otros). En estos años, se estudia la naturaleza, definición y componentes de las competencias, se establecen complejas clasificaciones atendiendo a diversos criterios -transversales o genéricas, específicas o profesionales, sistémicas, intrapersonales, interpersonales...-, se abordan las repercusiones de las competencias en las decisiones metodológicas y modalidades de enseñanza, se comienzan a experimentar diferentes modelos e instrumentos de evaluación, se indagan las competencias más valoradas por los agentes del sistema laboral y productivo, se exploran formas de inclusión de las competencias en los nuevos Planes de Estudio... En definitiva, podemos afirmar que nos encontramos en una fase de indagación teórica, de exploración de referencias prácticas, de aplicación de innovaciones docentes que tienen como eje central el enfoque de competencias... Se está produciendo la construcción de un discurso dominante centrado en las virtualidades de las competencias para el diseño y desarrollo del currículum universitario.

Hemos de tener en cuenta que los estudios y prácticas aludidas en el párrafo anterior son realizadas, en la mayoría de los casos, por profesorado voluntario, que se caracteriza por un fuerte compromiso con la mejora y la innovación educativa. Suelen poseer una trayectoria docente consolidada que les permite abordar experiencias novedosas e implicarse colectiva y profundamente en ellas.

Sin embargo, es a partir del RD 1393/2007, cuando se oficializa el proceso de inclusión de las competencias como requisito obligatorio de los nuevos Planes de Estudio que se elaboraron para adaptar las enseñanzas universitarias españolas a la nueva estructura de los estudios universitarios europeos -grado, máster y doctoradoEn él se recogen las competencias genéricas o transversales establecidas en los Descriptores de Dublín (2004) y se añaden una serie de competencias específicas o profesionales en el caso de las profesiones con atribuciones profesionales reguladas por el Estado.

La publicación de documentos oficiales, que incluían las competencias profesionales en diversas ramas de conocimiento, pusieron de manifiesto que el concepto de competencia utilizado oficialmente dista bastante del que hemos suscrito en este trabajo. En muchos ámbitos se incluyeron, bajo el nombre de competencias, objetivos muy concretos que hacían referencia al dominio de destrezas o habilidades. De este modo se elaboraron listados inconmensurables que debían ser distribuidos entre las diferentes asignaturas, materias y módulos. El profesorado universitario se vio obligado a repartir las competencias, debiendo asumir un número poco razonable para su trabajo y evaluación.

Por tanto, en nuestra opinión, la incorporación de las competencias en muchos planes de estudio no ha supuesto una incidencia generalizada de cambio en las prácticas docentes universitarias. Más bien ha respondido a demandas tecnocráticas y oficialistas que el profesorado, en general, ha vivido como una imposición normativa más que como una oportunidad de innovación. Como señalan Weber y Duderstadt (2010) las competencias que los estudiantes han de desarrollar son lo suficientemente complejas como para requerir una 'comunidad' coherente que apunte, siquiera desde 
una ética de mínimos, hacia direcciones 'creíbles' para ella misma. Lo contrario es la 'excepcionalidad' de algunos profesores en un modelo donde impera el desajuste entre lo que se dice y lo que se hace.

Actualmente, como apuntan Tierno, Iranzo y Barrios (en prensa) existe un movimiento crítico que señala las competencias como un modelo curricular que responde a un movimiento utilitarista y de mercantilización. Las críticas se centran en torno a un doble debate: competencias frente a contenidos $y$ enfoque por competencias frente a otros planteamientos práctico-reflexivos (De Ketele, 2006, Zabalza, 2006, Gimeno Sacristán, 2008; Villa, 2008; Carabaña, 2011; De la Orden, 2011). Junto a esta corriente crítica existen múltiples casos de aplicación y buenas prácticas en el trabajo por competencias, pero experimentados más desde iniciativas individuales que desde compromisos organizativos e institucionales (González Maura, 2006, Hirtt, 2010 y Guzmán y Marín, 2011).

\section{Del dicho al hecho... hay un buen trecho. Del prometedor discurso de las competencias a su implantación}

Si bien hemos esbozado un prometedor e ilusionante escenario de transformación del modelo docente universitario de la mano del trabajo por competencias, no es menos cierto que del discurso teórico profesado al discurso practicado hay una distancia enorme.

Los principios explicitados sustentaban un prometedor proceso de cambio en los contextos universitarios, sin embargo diferentes situaciones han truncado las expectativas iniciales que, al menos desde nuestro punto de vista, eran elevadas. Veamos algunas de las situaciones que han contribuido a que no se haya producido una verdadera transformación en las prácticas docentes universitarias:

(1) Se trata de una reforma que ha seguido un proceso jerarquizado, comenzando desde arriba e implicando, sobre todo, a los gestores y responsables de las políticas universitarias (Tonucci, 2010). El modo de hacer las reformas en otras etapas del sistema educativo nos indica que es muy difícil que un proceso de cambio sea exitoso si no se implica y surge desde la base del sistema (Fullan, 1992, Hargreaves, 1996, Bolívar, 2000, Lieberman y Miller, 2003, Knight, 2005, Fernández Cruz, 2006 y Marcelo, 2011). Es decir, el análisis de otros procesos reformistas nos muestra que cualquier cambio en las prácticas educativas se apoyan en la convicción, motivación y compromiso del profesorado, como agentes imprescindibles que lideran las transformaciones deseadas. Este principio no se ha respetado en el caso del EEES puesto que el profesorado universitario, el cual en su mayoría adolece de una formación didáctica básica, se ha socializado en una cultura profesional que premia y valora en exceso la función investigadora frente a las labores docentes. Su inmersión en una institución jerárquica, que potencia la promoción profesional prioritariamente por los logros conseguidos en el ámbito investigador le enseña, a través del silencioso pero eficaz currículum oculto, que si aspira a tener un buen status y consideración profesional ha de dirigir sus esfuerzos hacia las tareas investigadoras.

(2) El discurso reformista introducido por el EEES, y concretamente el trabajo por competencias, ha estado tintado de un matiz ideológico desde sus inicios. Un sector importante de la comunidad universitaria ha percibido las competencias como un 
retroceso, una vuelta a modelos tecnocráticos ya superados (Torres, 2006, Gimeno, 2008, y Angulo 2010). El discurso de las competencias al surgir del ámbito empresarial y de la cualificación profesional, se ha identificado, en más ocasiones de las deseables, con una mercantilización de los procesos educativos aportando una visión demasiado utilitarista de la formación superior. De hecho, todos conocemos las resistencias y oposición frontal que ha tenido el proceso de Bolonia por parte de determinados colectivos que han percibido el cambio como una privatización de la educación pública y una sumisión de la academia a las leyes del mercado y del tejido productivo.

(3) Se ha deteriorado el deseo de alcanzar una verdadera ciudadanía europea. Se pretendía configurar un sistema universitario competitivo frente a los países de Asia y EEUU, al tiempo que favorecer la movilidad de estudiantes y profesorado, ampliar las fronteras de empleabilidad y hacer más transparentes y reconocibles las titulaciones expedidas por los diferentes estados. Sin embargo, este estado de opinión favorable al proyecto europeo ha decrecido en los últimos años. La crisis económica, las presiones de otros países comunitarios, las dificultades de empleo, los flujos migratorios y la difícil situación por la que atraviesa el sueño europeo genera actualmente un ambiente hostil y escéptico que desanima las ilusiones reformistas y cuestiona los beneficios reales de una educación superior armonizada a nivel europeo.

(4) La burocratización en el cambio de los planes de estudio universitarios ha incidido negativamente en el trabajo por competencias. La regulación de unos requerimientos básicos para incluirse en las memorias de las titulaciones oficiales que debían ser verificadas por diferentes agencias de acreditación nacionales o autonómicas, ha producido diversa normativa legal en la que se han definido por real decreto las competencias de diversas profesiones, prioritariamente las que tienen atribuciones profesionales. Los grupos de expertos que han participado en la definición de estas competencias en las diversas titulaciones no han tenido demasiado claro qué son las competencias y cuál es su función para la mejora del aprendizaje.

(5) La transformación de las culturas profesionales necesita tiempo. En efecto, una reforma de la envergadura propuesta requiere apoyarse en procesos de transformación cultural, los cuales son lentos, inestables y dilemáticos. La influencia esperada del profesorado innovador sobre los menos implicados no ha sido muy efectiva, puesto que los segundos no veían con buenos ojos los tiempos de dedicación que requería el nuevo formato docente. Comienzan a resurgir en el ámbito universitario discursos ya usados en otras etapas educativas que apelan a la sobreprotección, infantilización y pérdida de conocimientos que genera el nuevo modelo. Sin demasiadas evidencias comienza a crearse un estado de opinión resistente al cambio, que cuestiona los beneficios del modelo pedagógico propuesto y aboga por una vuelta a los métodos más tradicionales.

(6) Este estado de opinión adverso se ve reforzado por las restricciones económicas y materiales que se derivan de la crisis económica. Cualquier propuesta de cambio a coste cero es rechazada por el colectivo docente, el cual tenía altas expectativas respecto a la reducción del tamaño de los grupos, y a las posibilidades de tutorización y seguimiento más personalizado que ello podría generar. El nuevo modelo educativo propuesto implica una dedicación horaria mayor y exige unos formatos metodológicos plurales que requieren más implicación docente. 
Para no ofrecer una visión excesivamente pesimista, también deseamos aludir al profesorado que tras involucrarse en un proceso de cambio y modificar su dinámica de aula ya no puede dar marcha atrás, al reconocer que ha experimentado procesos creativos, ricos y estimulantes, tanto para su desarrollo profesional como para la mejora del aprendizaje de su alumnado, a los que no está dispuesto a renunciar.

\section{Las competencias como marco conceptual útil para el diseño de experiencias de innovación docente.}

Como señala López Ruiz (2011) parece claro que la introducción del enfoque basado en competencias en la Educación Superior no afecta únicamente a la reformulación de los planes de estudio, sino que además implica una reconstrucción de los programas de cada módulo o asignatura integrante y una profunda redefinición del proceso de enseñanza-aprendizaje convencional en las instituciones universitarias.

A continuación intentamos vislumbrar cuáles son las repercusiones del enfoque por competencias en los diversos elementos del currículum universitario.

\section{Las competencias como marcos de referencia para la identificación de finalidades y la selección de los contenidos}

En el contexto español las competencias universitarias han sido definidas y establecidas por las administraciones centrales y autonómicas a través de diferente normativa según se tratara, o no, de profesiones reguladas o con competencias profesionales específicas. Este marco normativo establece por primera vez las competencias como grandes metas formativas que han de haber alcanzado los estudiantes universitarios que hayan acabado sus estudios de grado, máster y doctorado.

Se han definido diferentes tipos de competencias: genéricas o transversales, dedicadas a la formación común que ha de poseer cualquier estudiante universitario; y profesionales o específicas, referidas a la cualificación profesional que deben alcanzar los estudiantes de una determinada titulación. Se trata de complementar el necesario bagaje cultural de cualquier estudiante con el dominio de las herramientas y requisitos básicos necesarios para ejercer una determinada profesión.

Las competencias, por tanto, deben actuar como referencias formativas globales que orientan y direccionan el aprendizaje de las diferentes asignaturas y materias en las que se articula el plan de estudios. Suponen una estrategia para clarificar el punto de llegada de nuestro viaje formativo, instaurándose como 'hojas de ruta' para alcanzar el perfil definido en la titulación.

Por otra parte, uno de los pilares básicos de la innovación propuesta por el EEES es la reflexión sobre el tipo de conocimiento que deseamos que el alumnado adquiera. Tomando en consideración los avances tecnológicos, digitales, culturales y sociales acontecidos en la sociedad de la información (Gutiérrez y Tyner, 2012), como contexto que envuelve a las instituciones universitarias, podemos pensar que la mera repetición memorística de contenidos no resulta útil para capacitar a la futura ciudadanía a 
intervenir, y si es posible transformar, contextos inciertos, flexibles, globales y complejos. Es por ello, que el tipo de conocimiento que se ha de construir en las instituciones de educación superior ha de estar caracterizado por ser:

- Un conocimiento profundo, que responda a los aspectos más relevantes de la materia, lo cual requiere una compleja labor de síntesis de los programas de modo que permitamos que el alumnado analice, comprenda, establezca relaciones, construya transferencias con situaciones prácticas y, en definitiva, desarrolle capacidades mentales de orden superior. Debemos dirigir nuestros esfuerzos a conseguir un tipo de conocimiento que tenga valor de uso y no sólo de cambio (Winter, 2003).

- Un conocimiento interdisciplinar que permita a los estudiantes analizar y comprender cualquier realidad desde una mirada complementaria, en la que intervienen diferentes enfoques disciplinarios, en la que las materias son herramientas de análisis necesarias pero insuficientes por sí mismas, que necesitan apoyarse en otras para alcanzar una comprensión global del fenómeno. Habría que empezar a avanzar, como propone Lasnier (2000), hacia programas más integrados y menos sobrecargados que permitan abordar situaciones globales más acordes con la naturaleza holística de las competencias.

- Un conocimiento como construcción social. A menudo en el ámbito universitario el profesorado se obsesiona con 'dar todo el programa', lo cual conlleva una aproximación superficial, mecánica y reproductiva del aprendizaje.

- Un conocimiento útil y relevante. El alumnado ha de estar motivado para implicarse en sus aprendizajes y poder alcanzar un conocimiento significativo. Una motivación intrínseca, centrada en la pasión por conocer, en la satisfacción por saber, no se alcanza mediante programas cuyos contenidos hacen alusión prioritariamente a conceptos teóricos descontextualizados, totalmente ajenos a su futuro desempeño profesional.

- Un conocimiento flexible y emergente. La selección de contenidos y el diseño de los programas, generalmente, se realiza de forma previa a conocer las características, intereses y motivaciones de los destinatarios. El desarrollo del currículum en el aula ha de responder a principios de apertura, flexibilidad y adaptación a los elementos emergentes que van apareciendo al interactuar los protagonistas del hecho educativo.

- Un conocimiento actual y original. Además de la función de transmisión del saber acumulado por las generaciones anteriores, en la universidad se ha de promover un conocimiento interesado por los desafíos y retos sin resolver que nos presenta nuestra sociedad actual. La creatividad, la indagación y la exploración de situaciones novedosas son ejercicios en los que han de adquirir práctica nuestros estudiantes.

De todo lo expuesto podemos concluir que conceptuamos las competencias como marcos de referencia que orientan la práctica educativa y que nos ayudan a seleccionar los contenidos que vamos a trabajar. 


\section{Las competencias como marcos de referencia para las decisiones metodológicas y el diseño de situaciones de aprendizaje}

Siguiendo las pautas que nos ofrece Pérez et al. (2009), identificamos los siguientes principios de intervención educativa: (1) Aprendizaje basado en la investigación; (2) Autenticidad; (3) Participación; (4) Reforzar la autoestima y ofrecer confianza; (5) Primar la comunicación; (6) Fomentar el uso de las TIC; (7) Reflexión, autonomía y responsabilidad; y (8) Aprendizaje cooperativo.

\section{Aprendizaje basado en la investigación}

Las competencias también tienen incidencia en los criterios metodológicos que suscribimos, así como en las actividades que ponemos en marcha en las aulas. $\mathrm{Si}$ tenemos en consideración las conclusiones de los informes internacionales elaborados sobre las reformas de las instituciones de educación superior -en Francia, el Informe de Jacques Attali (1997), en Reino Unido el Informe Dearing (1997) y en Estados Unidos el Informe Boyer (2003)- podemos afirmar que, actualmente, una de las debilidades más relevantes de los sistemas universitarios es el poco uso que realizan de la indagación como medio para aprender, abusando de técnicas basadas en la simple transmisión de conocimiento. Adoptar la perspectiva del aprendizaje basado en la investigación supone incentivar el papel activo del alumnado como constructor de sus aprendizajes y favorecer su implicación en un proceso de exploración, indagación y análisis permanente, para alcanzar unos niveles de autonomía intelectual que le permitan autoformarse y seguir 'aprendiendo a aprender'.

\section{Autenticidad}

Este principio hace alusión a recorrer caminos de aprendizaje partiendo de las experiencias cotidianas de la vida para seguir avanzando en procesos de abstracción y teorización creciente. Tradicionalmente en los contextos universitarios se ha empleado el método deductivo, partiendo de la lógica epistemológica disciplinar como forma de plantear secuencial y linealmente el conocimiento deseado. La costumbre academicista nos ha abocado a prescindir de las vivencias y partir desde el principio con formalizaciones abstractas que, seguramente, tienen sentido para el conocimiento del experto, pero que producen desconexión y rechazo en la mente del aprendiz.

\section{Participación}

Desde una perspectiva sistémica del aprendizaje (Brofenbrenner, 1979) la calidad de los contextos donde se desarrolla el hecho educativo repercute directamente tanto en los procesos como en los resultados obtenidos. Es por ello que la docencia universitaria ha de incorporar la implicación del alumnado en todas las fases del proceso de enseñanza-aprendizaje, tomando en consideración y consultándoles las diferentes decisiones que se van adoptando. Corresponsabilizar al alumnado en su proceso formativo significa ofrecerle la oportunidad no solo de responder pasivamente a los requerimientos del docente, sino facilitar su análisis y toma de decisiones en 
aspectos relevantes del currículum, acostumbrándose de este modo a implicarse activamente en procesos democráticos de elección, y aprendiendo a compartir la responsabilidad que se deriva de intervenir en situaciones complejas.

\section{Reforzar la autoestima y ofrecer confianza}

El clima de aula entendido como el ambiente psicosocial creado a partir de la comunicación, actitudes, comportamientos y valores que ponen en marcha los protagonistas del hecho educativo -docentes y discentes- en un contexto físico determinado, es el responsable del incremento u obstaculización del aprendizaje alcanzado. La literatura pedagógica (Doyle, 1981) nos indica las repercusiones que tiene la calidad de los contextos psicosociales en el desarrollo de un ambiente de seguridad y confianza que propicie el aprendizaje. Es evidente, desde la perspectiva de la enseñanza como proceso relacional (Álvarez y Del Río, 1985), que la calidad de los intercambios personales está mediada por el nicho ecológico en el que tiene lugar. Especialmente significativas son las expectativas que el docente tiene respecto a las posibilidades de aprendizaje de su alumnado. Incluir factores valorativos y emocionales en el marco de las competencias supone incorporar un factor tradicionalmente excluido de la formación universitaria, que facilita el abordaje de las emociones, los sentimientos, las actitudes y los valores como parte fundamental de los procesos de aprendizaje. Educar con co-razón, significa ser permeable a la abundante literatura científica (Bar-On y Parker, 2001 y Toro, 2005) relacionada con la inteligencia emocional que demuestra el carácter indivisible de las personas y el sustrato emocional presente en cualquier actividad humana. No podemos dar la espalda al sentido común y a las múltiples evidencias que demuestran que el desarrollo intelectual y cognitivo se apoya en una base afectivo-emocional equilibrada, en el que la autoestima y valoración del aprendiz de sus propias posibilidades de aprendizaje juegan un papel muy relevante para apropiarse de nuevos conocimientos.

\section{Primar la comunicación}

En el apartado anterior nos hemos referido a las situaciones educativas como procesos relacionales y afectivo-emocionales, ahora nos centramos en la enseñanza como proceso comunicativo. Concebir los procesos de enseñanza-aprendizaje como actos comunicativos de carácter multidireccional -profesorado-estudiantes, estudiantesestudiantes- supone asumir que el intercambio comunicativo es la piedra angular sobre la que se construyen los conocimientos.

\section{Fomentar el uso de las TIC}

El desarrollo tecnológico actual nos ofrece un conocimiento en red que resulta muy atractivo para nuestros estudiantes. Trabajar por competencias significa abordar el conocimiento desde la búsqueda y selección de la información, hasta su análisis y comprensión. Las TIC nos ofrecen posibilidades ilimitadas que no debemos desaprovechar en los entornos educativos universitarios. No obstante, debemos emplear los entornos digitales como herramientas de alfabetización y construcción de 
conocimientos incorporando un uso crítico y personal de las mismas al servicio de un modelo pedagógico activo.

\section{Reflexión, autonomía y responsabilidad}

El aprendizaje universitario ha de favorecer los procesos de autonomía y autorregulación del aprendizaje. Hemos de superar modelos reproductivos y apostar decididamente por situaciones donde el alumnado tenga la posibilidad de tomar conciencia de sus propias fortalezas y debilidades en cada ámbito del saber y del hacer. La metacognición y el uso consciente de las estrategias de aprendizaje más ajustadas a sus estilos cognitivos les permitirá aprovechar sus potencialidades y acercarse al saber desde un posicionamiento único e intransferible.

\section{Aprendizaje cooperativo}

El aprendizaje cooperativo se ha mostrado como una herramienta de apropiación del saber muy potente, al tiempo que aparece como una competencia profesional muy deseada en los entornos laborales actuales. El trabajo en equipo favorece el debate, la crítica compartida y la negociación sobre situaciones dilemáticas, facilitando la aplicación de conceptos teóricos en el análisis y posicionamiento ante diversas situaciones reales. En este sentido las comunidades de práctica o de indagación (Wells, 2002) nos ofrecen una organización del aula donde el aprendizaje dialógico, entendido como un proceso interactivo, democrático y simétrico que se sustenta en el debate y en la argumentación discursiva, se constituye como estrategia privilegiada de apropiación del saber. Por tanto, crear comunidades de aprendices en las aulas universitarias propicia la adquisición de competencias donde la colaboración, convivencia e iniciativa son claves para cultivar una cultura de aprendizaje compartido basado en la investigación y confrontación de ideas y puntos de vista personales.

\section{Las competencias como marcos de referencia para la evaluación}

La evaluación de las competencias es uno de los 'talones de Aquiles' de la actual reforma propiciada por el EEES. Modificar la concepción de evaluación que tradicionalmente se ha practicado en los entornos universitarios, así como las acciones de acreditación que de ella se derivan, supone uno de los elementos más complejos que debe afrontar la reforma de las instituciones de educación superior. Sin embargo, es difícil que una innovación curricular sea efectiva si no va acompañada de innovaciones en el modo de concebir la evaluación (Dochy, Segers y Dierick, 2002 y Watts y García-Carbonell, 2006).

Dochy, Segers y Dierick (2002) consideran que el reto más importante de nuestras universidades es pasar de una cultura del examen a una cultura de la evaluación, entendiendo por esta última una evaluación formativa, dirigida a mejorar el proceso de aprendizaje del alumnado y a favorecer su implicación en los procesos evaluativos. Bonsón y Benito (2005) consideran que una forma de entender este proceso de cambio puede ser la de pasar de una evaluación del aprendizaje a una 
evaluación para el aprendizaje. En este sentido, Carless, Joughin y Mok (2006) y Boud y Falchikov (2007) prefieren utilizar el concepto evaluación orientada al aprendizaje.

Si hemos suscrito en los epígrafes previos que la calidad de los aprendizajes está influenciada por el diseño de los ambientes de enseñanza donde estos tienen lugar, estaremos de acuerdo en que la verdadera evaluación, para ser educativa, ha de estar dirigida a todos los elementos que intervienen en los procesos de enseñanzaaprendizaje: profesorado, alumnado, recursos, calidad de las situaciones educativas, selección de contenidos y estrategias de evaluación. Esta mirada global nos permitirá avanzar desde una posición focalizada en los resultados o rendimientos académicos de los estudiantes hacia una evaluación que valora la pertinencia y ajuste de los diferentes elementos del currículum para la adquisición de competencias por parte de los estudiantes.

Además de una mirada global, la evaluación educativa ha de ser formativa, es decir ha de ayudar a los estudiantes a conocer cuáles son los verdaderos propósitos de su aprendizaje, ofreciéndoles unos criterios claros y transparentes, conocidos y negociados desde el inicio del proceso de enseñanza-aprendizaje. Como apunta López Ruiz (2011), la evaluación universitaria, al trabajar por competencias, ha de experimentar un giro copernicano, incluyendo junto a la evaluación del docente, la autoevaluación y la coevaluación o evaluación entre iguales. Como bien es conocido, lo que no se evalúa se devalúa, y los fines de la tarea educativa constituyen para los aprendices sus referencias básicas a alcanzar, y los elementos más importantes para articular sus esfuerzos y organizar sus tareas.

Además, la evaluación de competencias no solo se dirige al ámbito cognitivo, como ya señalamos previamente, las competencias incluyen el ámbito del saber hacer y la dimensión afectivo-emocional y actitudinal. No se puede afirmar que se está trabajando por competencias cuando se deja fuera la valoración de los procesos actitudinales. Aunque se trata de contenidos actitudinales que plantean más complejidad a la hora de valorarlos, tanto por su carácter innovador, como por su naturaleza intangible y poco concreta, se ha de hacer un esfuerzo por integrarlos dentro de la evaluación de competencias (Tejedor, 1998, Nieto, 2000, Trillo, 2005, Zabalza, 2006 e Ibarra y Rodríguez, 2010). Especialmente útiles para este propósito son instrumentos que incluyan evidencias como pueden ser los portafolios, los diarios, los registros de observación, las narraciones y todas aquéllas técnicas más cualitativas que, si bien ofrecen menos economía de tiempo y esfuerzo que los exámenes tradicionales, nos muestran una información mucho más profunda e integral del avance de nuestros estudiantes.

A continuación narramos una experiencia de innovación docente que, con el objetivo de desarrollar competencias profesionales en los futuros maestros y maestras de Educación Infantil, ha supuesto una transformación del modelo docente desarrollado. 


\section{Trabajando las competencias profesionales de los futuros docentes de Educación Infantil a través de RIECU (Red de Infantil Escuela-CEP- Universidad)}

La experiencia de innovación que presentamos intenta incorporar los cambios que hemos ido describiendo en la primera parte de este trabajo. Se hace un esfuerzo para que exista coherencia entre los supuestos teóricos suscritos y las acciones que, a nivel de docencia real, se ponen en práctica. El propósito perseguido es transferir el conocimiento académico a los escenarios educativos infantiles, centrándonos en el desarrollo de competencias profesionales del alumnado universitario. Siguiendo las recomendaciones propuestas por López Ruiz (2011: 299) se trata de alcanzar un enfoque de competencias caracterizado por los rasgos que se describen en el siguiente cuadro:

\section{MODELO TRADICIONAL ENFOQUE DE COMPETENCIAS}

Individualismo docente Equipos docentes

Planes fragmentados: materias disciplinares Planes integrados: módulos interdisciplinares

Programas organizados por temas Programas estructurados en núcleos problemáticos

Lección magistral

Métodos docentes innovadores

Manual único y documentos

Fuentes de información y recursos didácticos complementarios

Profesor transmisor

Profesor facilitador

Alumnado pasivo y receptivo

Alumnado activo y constructivo

Calificación final: examen

Evaluación holística: evidencias

Aula aislada

Diversos espacios y ambientes

Institución académica cerrada

Comunidad de aprendizaje

Fuente: López Ruiz (2011)

Cuadro n.I. Líneas de avance desde el modelo tradicional al enfoque de competencias

El deseo de desarrollar competencias profesionales en el alumnado del Grado de Educación Infantil nos impulsó a solicitar un proyecto de Innovación Educativa ${ }^{1}$ que, centrado en el aprendizaje del método de Proyectos de Trabajo, nos permitiera

\footnotetext{
${ }^{1}$ Este proyecto ha sido financiado por el Vicerrectorado de Calidad e Innovación Docente de la Universidad de Córdoba en la convocatoria de Proyectos de Mejora de la Calidad Docente 2010/11. Es una continuidad de las acciones de innovación desarrolladas desde el curso académico 2004/05 y ha contribuido a consolidar la creación de una comunidad de práctica en la que colaboran las escuelas infantiles, el Centro de formación del Profesorado y la Universidad.
} 
colaborar con maestras en ejercicio expertas en esta metodología para crear una comunidad de práctica.

Es una continuidad de las acciones de innovación desarrolladas desde el curso académico 2004/05 que ha contribuido a consolidar la creación de una comunidad de práctica en la que colaboran agentes formativos responsables de la formación inicial alumnado y profesorado de la Facultad de Ciencias de la Educación- con agentes formativos responsables de la formación continua -asesoras del Centro de Profesorado (en adelante, CEP), maestras de infantil en ejercicio y niños y niñas de esta etapa- En total han participado más de seiscientas personas en un proyecto que, centrado en la metodología de PT en las aulas infantiles, ha intentado crear sinergias entre todos los participantes y elevar el desarrollo profesional de las maestras de infantil, las asesoras y el profesorado universitario, al tiempo que se incrementa el nivel de aprendizaje y la adquisición de competencias por parte de los y las estudiantes universitarias y el alumnado de la etapa infantil de tres a seis años que ha colaborado en la experiencia.

Nos proponemos mejorar la capacitación didáctica de nuestro alumnado para que, en sus futuras intervenciones docentes, tengan la posibilidad de aplicar la metodología de PT en las aulas infantiles. Se trata de una tarea compleja, al ser una metodología centrada en un modelo socioconstructivista de aprendizaje, que se asienta sobre principios educativos como la actividad, el conflicto sociocognitivo, la exploración, la investigación y la cooperación en el aula. Dichos principios, para ser trabajados posteriormente en las escuelas infantiles han de ser vivenciados como estudiantes de la facultad porque, siguiendo el principio de ejemplaridad, debemos ofrecerles modelos docentes que puedan transferir a contextos prácticos (Zabalza, 2003), y no sólo indicar unos principios teóricos incoherentes con sus prácticas en el aula universitaria. Es decir, nos enfrentamos al doble reto de conocer, comprender y aplicar una metodología activa en las aulas universitarias, con el fin de capacitar a los estudiantes universitarios para que posteriormente la apliquen en las aulas infantiles.

Esta experiencia de innovación, tal y como hemos señalado en la fundamentación teórica, nos ha permitido trabajar las competencias modificando cada uno de los elementos del currículum universitario:

- Incidiendo en la selección de contenidos, los cuales presentan un enfoque globalizado centrado en problemas surgidos de los intereses de los niños y niñas de infantil.

- Afectando a la configuración de equipos docentes integrados por profesionales de distintos niveles educativos -maestras, asesoras y profesoras-

- Repercutiendo en la elección de una metodología activa, basada en la indagación de los niños y niñas y en la reflexión-acción de los estudiantes universitarios bajo la supervisión y tutela de las maestras y profesoras universitarias.

- Utilizando diferente documentación, recursos y materiales más allá del libro de texto. 
- Desarrollando situaciones de enseñanza-aprendizaje en diferentes espacios y ambientes: las aulas infantiles, las aulas universitarias, el entorno y la comunidad.

- Poniendo en práctica una evaluación holística, formativa y global, centrada en la recogida y análisis de evidencias -a través de la estrategia de documentación educativa, la cual ha sido incluida en un portafolios-

- Consolidando una comunidad de práctica en la que todos los agentes implicados han participado en el diseño, desarrollo y evaluación de un PT en las aulas infantiles.

\section{Objetivos de la experiencia de innovación docente}

- Consolidar estrategias de participación conjunta entre las escuelas, el CEP y la universidad.

- Establecer redes de colaboración entre la formación inicial y permanente del profesorado de Educación Infantil.

- Colaborar con el alumnado universitario para facilitar la aplicación de los PT en contextos educativos reales.

- Favorecer el aprendizaje y enriquecimiento mutuo entre el profesorado y el alumnado universitario.

- Asesorar al alumnado universitario en el desarrollo de actividades lúdicas, creativas y relevantes para desarrollar competencias en la infancia.

- Evaluar las competencias profesionales del alumnado universitario puestas de manifiesto durante la aplicación del PT.

\section{Fases de la experiencia de innovación docente}

Al participar personas pertenecientes a diferentes instituciones, ha sido necesario articular nuestro trabajo en varias fases:

(1) Planificación y diseño de la Red. Hemos mantenido diversas reuniones y entrevistas las asesoras del CEP y el profesorado universitario para tomar decisiones en relación al cronograma de trabajo, para seleccionar los centros y las maestras con las que nuestro alumnado universitario desarrollaría los PT, para consensuar los materiales y orientaciones de los PT ofrecidos a las maestras y a los estudiantes universitarios y para establecer los roles y funciones que cada agente formativo debía asumir.

(2) Desarrollo de los Proyectos de Trabajo en los colegios de Educación Infantil. Hemos tenido una reunión con todos los agentes, celebrada en el Salón de Actos de la Facultad de Ciencias de la Educación, a la que han acudido setenta estudiantes de Magisterio, cinco profesores y profesoras universitarias, veintisiete maestras de Educación Infantil y una asesora del CEP, en la que se ha adscrito el alumnado de Magisterio al centro y a la maestra correspondiente y se ha presentado el cronograma de trabajo. En esta fase el alumnado universitario ha sido tutorizado por maestrastutoras especialistas en esta nueva metodología, con carácter previo a acudir a los 
diversos colegios para aplicar sus respectivos PT -el alumnado universitario ha permanecido durante tres días en los colegios desarrollando sus proyectos-.

(3) Evaluación de la experiencia de innovación. Hemos aplicado diversos instrumentos de evaluación elaborados 'ad hoc' para recoger información de todos los colectivos implicados -estudiantes, maestras, niños y niñas de infantil, asesoras y profesorado universitario- Organizamos un grupo focal con las maestras de infantil, las asesoras y el profesorado universitario para evaluar la experiencia, incidiendo en el análisis de debilidades, fortalezas y sugerencias de mejora para el curso siguiente.

\section{Tejiendo la red. Red de Infantil Escuela-CEP-Universidad en acción}

En esta fase se produce el encuentro de todos los agentes implicados: asesoras de infantil, maestras, profesorado y alumnado universitario. Ya ha concluido el curso de formación sobre PT organizado por el CEP y se han seleccionado a las veintisiete maestras participantes. Por su parte, el alumnado universitario ha recibido formación sobre los PT en las clases de Didáctica General de la facultad y se han distribuido por parejas y tríos para ser adjudicados, por sorteo, a las maestras elegidas. Es la etapa en la que los PT diseñados por el alumnado de Magisterio se aplican en las aulas infantiles. El proceso se articuló del siguiente modo:

a) Presentación de los agentes de RIECU y elección del tema del PT. Se realiza en el Salón de Actos de la Facultad de Ciencias de la Educación y asisten una asesora de infantil, las veintisiete maestras seleccionadas, cinco docentes universitarios y los setenta estudiantes de Magisterio matriculados en la asignatura de Didáctica General. Se realiza una presentación oficial de la Red de Infantil Escuela-CEP-Universidad y la asignación del alumnado de Magisterio a la maestra correspondiente, utilizando el azar como procedimiento de adjudicación. En esta sesión, el alumnado universitario junto con su maestra, consensuan y eligen la temática del PT, siguiendo las sugerencias de la maestra como portadora de los intereses de los niños y niñas preescolares.

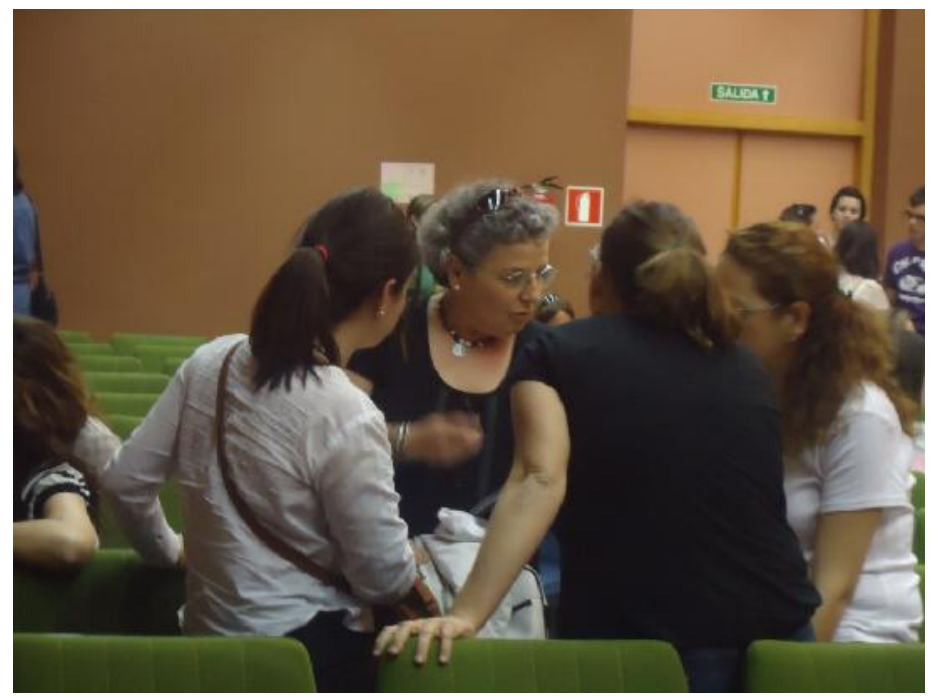

Figura .1. Grupo de estudiantes y maestra negocian el tema del PT 
b) Recogida de ideas previas sobre los PT en los colegios. El alumnado de Magisterio acude a los colegios de Infantil para desarrollar tres actividades, que suponen el inicio de los PT: (1) Asamblea con el alumnado para recoger sus ideas previas; (2) Realización de un mapa conceptual sobre lo que el alumnado sabe del tema del PT, para su posterior comparación con otro mapa conceptual empleado como evaluación final; (3) Elaboración de una carta a las familias de los niños y niñas de Infantil para solicitar la colaboración de las familias y el suministro de materiales.

c) Recogida de información y materiales aportados por las familias. El alumnado, fuera del horario escolar, tiene un encuentro con su maestra tutora del colegio para recoger la información aportada por las familias, con la intención de analizarla e ir proponiendo actividades en consonancia con los materiales recogidos.

d) Tutorización de la planificación de los PT. El alumnado de Magisterio, dividido en cuatro grupos de dieciocho o diecinueve personas, es tutorizado por las cuatro maestras expertas en PT que, al mismo tiempo, han sido las ponentes del curso de formación permanente sobre PT realizado en el CEP. Los estudiantes universitarios les presentan su propuesta de PT, que han elaborado con el apoyo del profesorado universitario en las clases de Didáctica General, teniendo en cuenta las ideas previas de los niños y niñas de infantil, así como la información y recursos suministrados por las familias.

e) Supervisión y últimos ajustes de los PT. Cada grupo de estudiantes universitarios (dos o tres miembros) se reúnen con su respectiva maestra tutora, fuera del horario lectivo, para mostrar su propuesta de PT, con el fin de obtener su visto bueno e introducir las últimas modificaciones antes de ponerlo en práctica en el aula con los niños y niñas.

f) Asistencia a los colegios de infantil durante tres días consecutivos para desarrollar los PT. Los estudiantes, organizados en pequeños grupos de dos o tres miembros, acuden a la clase de su maestra-tutora y desarrollan el PT planificado. El primer y segundo día lo dedican a desarrollar actividades para modificar sus ideas previas y ampliar conocimientos y experiencias sobre el tema tratado, y el último día, aplican la evaluación debiendo realizar, al menos: (1) Mapa conceptual final para verificar los conocimientos adquiridos; (2) Organización en un rincón de los materiales aportados por todas las personas implicadas; y (3) Elaboración de un cuaderno/dossier del alumnado para entregarlo a sus familias en el que se recogen las evidencias de los trabajos realizados por los niños y niñas. 


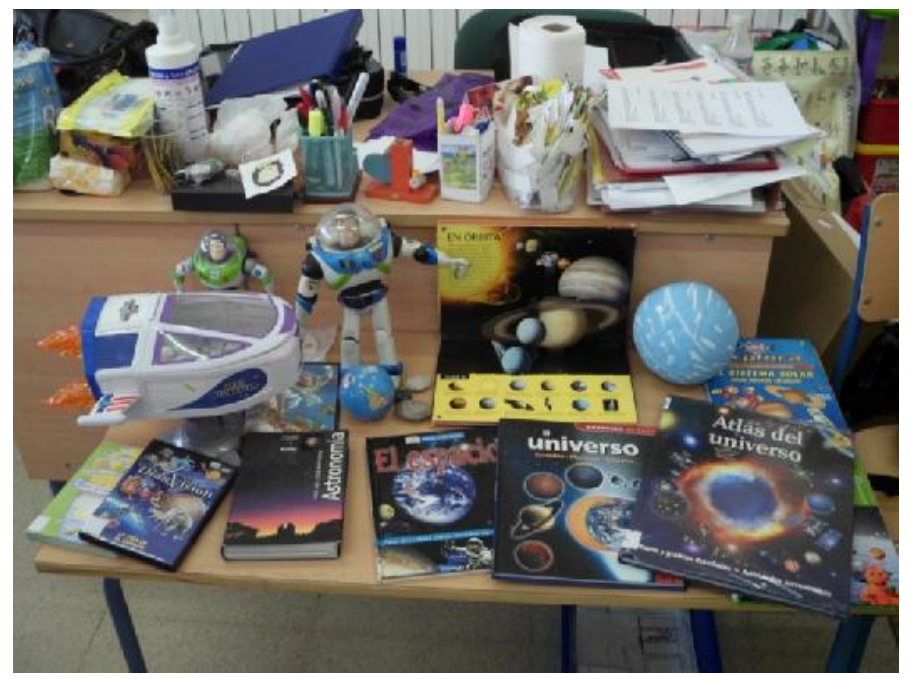

Figura n.2. Rincón del PT 'El Universo'

\section{Evaluando RIECU}

Los instrumentos de evaluación de RIECU no solo se han implementado en esta última fase. Se ha realizado la evaluación prioritariamente a través de tres herramientas: (1) Escalas Likert ${ }^{2}$ elaboradas 'ad hoc', las cuales han sido pilotadas previamente y ajustadas a las exigencias estadísticas para garantizar su fiabilidad y validez; (2) Entrevistas semiestructuradas; (3) Grupo focal en el que participan las dos asesoras de infantil, las veintiocho maestras y tres profesoras universitarias.

Las escalas Likert han sido cumplimentadas por los estudiantes universitarios, antes y después de participar en la experiencia, para verificar si se ha producido mejora en el dominio de sus competencias profesionales. Las maestras han evaluado, también a través de una escala Likert, la calidad de las competencias mostradas por el alumnado universitario en su actuación en el aula al desarrollar los PT. Se han correlacionado las escalas respondidas por el alumnado universitario y las maestras para analizar si existe coherencia en la evaluación de ambos agentes.

Por su parte, las maestras también han respondido una escala Likert para evaluar los aprendizajes que ellas han obtenido al participar en esta experiencia. Al tratarse de una red, la intención es que todas las personas que participan obtengan beneficios para su aprendizaje.

Otra mirada ha sido la proporcionada por las asesoras y el profesorado universitario, las cuales han mostrado su opinión de esta experiencia de innovación a través de entrevistas semiestructuradas, focalizando su interés en los complejos mecanismos de coordinación que conlleva este trabajo colaborativo.

Por último, la valoración de la experiencia de innovación se ha realizado de forma conjunta entre el profesorado universitario, las maestras y las asesoras desde

\footnotetext{
2 Son escalas psicométricas utilizadas en encuestas de investigación, principalmente en Ciencias Sociales. Al responder a una pregunta de un cuestionario elaborado con la técnica de Likert se especifica el nivel de acuerdo o desacuerdo con una declaración (elemento, ítem o reactivo o pregunta). Siempre hacen referencia a un procedimiento de escalamiento en el que el sujeto asigna una valoración a un conjunto específico de categorías o cuanficador es lingüí scos o numér i cos de freauenci a (siempre, a veces, nunca, etc,) o de cantidad (todo, algo, nada, etc.). (Cañadas y Sánchez, 1998).
} 
una mirada más cualitativa a través del grupo focal, lo cual nos ha permitido analizar las fortalezas, debilidades y sugerencias de mejora de este proyecto.

\section{Discusión y conclusiones}

Tras realizar un proceso de confrontación y triangulación de los datos obtenidos en los instrumentos descritos en la fase de evaluación (Escalas Likert de alumnado y maestras y contenido del grupo focal) se han obtenido las siguientes conclusiones, las cuales, por claridad expositiva, se van a organizar por colectivos:

a) Todos los agentes implicados:

La implicación en una comunidad de práctica requiere un proceso de colaboración lento y un esfuerzo añadido por parte de los profesionales implicados. La pertenencia a diferentes instituciones genera discursos, prácticas y pautas de actuación marcadas por las necesidades que plantean los diferentes contextos. Las lógicas que se imponen en cada escenario educativo, pese a tener un elemento común, que es el trabajo por competencias a través de los PT, no siempre son convergentes. Para armonizar actuaciones conjuntas y procesos de innovación, que integren la escuela y la universidad, es necesario crear una dinámica relacional y comunicativa asentada en un proceso de aprendizaje dialógico, simétrico y multidireccional. Es necesario participar en una comunicación horizontal y empática, donde la actitud de escucha y la asertividad presidan las interacciones. Este diálogo y negociación de roles es imprescindible para acometer una actividad de cooperación que supere las tradicionales relaciones entre las escuelas y la universidad, donde las primeras acogen al alumnado de prácticas y tratan de aplicar las pautas elaboradas unilateralmente por la academia. Si las maestras de infantil participan en el diseño de la innovación aportando ideas, sugerencias y propuestas, su actitud no es de meras receptoras, por el contrario, asumen un papel activo en toda la experiencia. En definitiva, se trata de ir construyendo lentamente procesos de transformación cultural que requieren tiempo y nuevos modos de interacción, tal como indicamos en el marco teórico de este trabajo.

Es necesario trabajar en un foco de interés compartido, deseado por todas las agencias formativas que intervienen. En este caso, los PT es una metodología en la que estaban interesadas desde la formación continua antes de iniciar la comunidad de práctica. Su trayectoria no ha sido forzada para adaptarla a los requerimientos o líneas de investigación de las profesoras universitarias. Por tanto, se ha respetado uno de los puntos de partida o premisas necesarias para ir consolidando una comunidad de práctica, según propone Wenger (2001).

b) Estudiantes universitarios:

Es necesario incentivar la participación del alumnado universitario en la experiencia de innovación, puesto que su nivel de trabajo e implicación, al tener que desarrollar un PT, normalmente, es superior al resto de compañeros que cursan la asignatura de forma tradicional. La documentación necesaria, su participación en contextos de enseñanza reales, la preparación de recursos y materiales, su indagación teórica y el aprendizaje del método de PT, necesitan un esfuerzo sobreañadido que debe ser valorado. 
El alumnado de Magisterio muestra una percepción elevada respecto al dominio de competencias profesionales conseguido al aplicar el método de PT en las aulas infantiles. Sus aprendizajes más relevantes los atribuyen al contacto con maestras de excelencia que actúan como modelos profesionales. La interacción con los niños y niñas, el trato con las familias, la motivación ante los aprendizajes y el respeto a la individualidad y diversidad de cada aprendiz son las competencias más destacadas por el alumnado universitario. Igualmente, el alumnado universitario indica que adquiere competencias al actuar en un escenario educativo real, donde tienen que actuar con los recursos disponibles y donde, según su opinión, tienen que responder a múltiples y simultáneas demandas de los niños y niñas que son imprevisibles, y por tanto, imposible de predecir. La opinión expresada por los estudiantes universitarios que participan en RIECU es consistente con la valoración manifestada por otro alumnado de Educación Superior respecto a las bondades de los contextos escolares como entornos privilegiados para la adquisición de competencias profesionales docentes (Zabalza, 2006 y Mérida, González y Olivares, 2011, Mérida et al., 2011, Gómez, M érida y González, 2011 y Mérida, González y Olivares, 2012).

c) Maestras:

Por su parte, las maestras de Infantil expresan que en algunos casos han percibido al alumnado de Magisterio un poco desorientado y desbordado por su actuación en la práctica. Han necesitado clarificar 'sobre el terreno' y negociar los roles que asumían cada una de ellas - la maestra y la alumna de Magisterio-, puesto que, en ocasiones, actuaban como meras observadoras y otras modificaban demasiado la dinámica y rutinas establecidas en el aula. Entre las virtualidades que ofrece el alumnado de Magisterio, las maestras subrayan la aportación de recursos - sobre todo virtuales-, su creatividad, energía y entusiasmo. En cuanto a las debilidades más relevantes expresan su poca capacidad de escucha a los saberes infantiles, su actitud excesivamente directiva y la dificultad para atender los diferentes ritmos de los niños y niñas.

d) Asesoras de Infantil:

Las asesoras de Infantil del Centro de Profesorado manifiestan su valoración positiva de la experiencia e indican su deseo de continuar consolidando la red. Consideran que es necesario intensificar los procesos de comunicación y trabajo conjunto entre las maestras y las profesoras universitarias, profundizando en la negociación de documentos comunes que faciliten un trabajo coordinado y coherente en las aulas infantiles y universitarias. También indican su deseo de perfeccionar los criterios y el sistema de selección de las maestras más idóneas para participar en la experiencia.

e) Profesorado universitario:

Las profesoras universitarias expresan su valoración de la experiencia como positiva, tanto por sus repercusiones en el alumnado universitario, como para ellas como profesionales que, a medida que transcurre el tiempo, pierden contacto con la docencia real. Supone un puente que las aproxima a situaciones de enseñanzaaprendizaje concretas y les posibilita la interacción con profesionales de excelencia de esta etapa. Consideran que amplían su formación y les supone un reto para imbricar la teoría y la práctica, o mejor, para analizar situaciones de la práctica desde los fundamentos teóricos disponibles. El enriquecimiento profesional, percibido por las docentes universitarias que participan en esta experiencia de innovación, coincide con 
los beneficios sugeridos en la literatura científica centrados en la relación teoríapráctica (Wells, 2002, Zabala y Arnau, 2007 y De la Orden, 2011).

Finalmente, la debilidad más señalada por todos los colectivos implicados es el escaso margen temporal disponible para el desarrollo de un PT (tres días), lo cual genera una actitud de precipitación, en la que difícilmente se puede observar, aprender y reflexionar sobre el proceso vivido. Proponen, como sugerencia para el curso próximo, la vinculación de esta experiencia de innovación al Practicum I, cuya duración de un mes pudiera permitir adquirir competencias profesionales de forma más reflexiva y sosegada.

\section{Referencias bibliográficas}

Álvarez, A. y Del Río, P. (1985). La influencia del entorno en la educación y la aportación de los modelos ecológicos. Infancia y Aprendizaje, 29, 3-32.

Angulo, F. (2010). La educación y el currículum en el espacio europeo: Internacionalizar o globalizar? En J. Gimeno, Saberes e incertidumbres sobre el currículum. M adrid: Morata, 476-497.

AAVV (2004). "Dublin" descriptors for the Bachelor's, Master's and Doctoral awards. A report from a Joint Quality Initiative informal group, celebrated 23 march. Recuperado el 23 de febrero de 2012 en: http://institucional.us.es/eees/formacion/Descriptores_Dublin_Ensenanza.pdf

Bar-ON, R. y Parker, J. (2001). The Handbook of Emotional Intelligence. Theory, developmental and application at home, school, and in the workplace. San Francisco: Jossey-Bass.

Bautista-Cerro, M. J. (2007). El largo camino de las competencias. Diseño de perfiles y programas. Acción Pedagógica, 16, enero-diciembre, 6-12.

Bolívar, A. (2000). Los centros educativos como organizaciones que aprenden. Promesa y realidades. Madrid: La M uralla.

Bonson, M. y Benito, A. (2005). Evaluación y Aprendizaje. En A. Benito y A. Cruz (Eds.). Nuevas claves para la docencia universitaria en el Espacio Europeo de Educación Superior (87-100). M adrid: Narcea.

Boud, D. y Falchikov, N. (2007). Rethinking Assessment in Higher Education. Learning for the long term. Londres: Routledge.

Brofenbrenner, U. (1979). The Ecology of Human Development: Experiments by Nature and Design. Cambridge, M A: Harvard University Press.

Cañadas, I. y Sánchez, A. (1998). Categorías de respuesta en escalas tipo Likert. Psicothema, 10 (3), 623-631.

Carabaña, J. (2011). Competencias y universidad, o un desajuste por mutua ignorancia. Bordón, 63 (1), 15-31.

Carless, D., Joughin, G. y Mok, M. M. C. (2006). Learning-oriented assessment: principles and practice. Assessment \& Evaluation in Higher Education, 31 (4), 
395-398.

De Ketele, J. M. (2006). Caminhos para a Avaliaçao de Competencias. Revista Portuguesa de Pedagogia, 40 (3), 135-147.

De La Orden, A. (2011). Educación y Competencias. Bordón, 63 (1), 47-61.

De M iguel, M. (2005). Cambio de paradigma metodológico en la Educación Superior, Cuadernos de Integración Europea, 2, 16-27.

De Miguel, M. (coord.) (2006). Metodologías de enseñanza y aprendizaje para el desarrollo de competencias. Orientaciones para el profesorado universitario ante el Espacio Europeo de Educación Superior. Madrid: Alianza Editorial.

Declaración de Bolonia (1999). Comunicado de la Conferencia de Ministros Europeos responsables de la Educación Superior. Recuperado el 11 de junio de 2008 en http://www.eees.es/pdf/Bolonia ES.pdf

Delors, J. (1996). La educación encierra un tesoro. Informe a la UNESCO de la Comisión Internacional sobre la Educación para el s. XXI. Recuperado el 14 de enero de 2012 en http:// www.unesco.org/delors/delors s.pdf.

Dochy, F., Segers, M. y Dierick,S. (2002). Nuevas vías de aprendizaje y enseñanza y sus consecuencias: una era de evaluación. Red-U, 2 (2), 13-30.

Dolado, J.J. (2010). Disfunciones en el sistema universitario español: diagnóstico y propuestas de reforma. En D. Peña (editor). Propuestas para la Reforma de la Universidad Española. Madrid: Fundación Alternativas.

Doyle, W. (1981). Research on classroom context. Journal of Teacher Education, 32 (6), 2-7.

Eurydice (2002). Las competencias clave. Un concepto en expansión dentro de la Educación General Obligatoria. Ministerio de Educación, Cultura y Deporte. Secretaría General de Educación y Formación Profesional. Recuperado el 25 de junio de 2012 en:

http:// eacea.ec.europa.eu/education/eurydice/key data en.php

Eurydice (2011). Modernisation of Higher Education in Europe: Funding and the social dimensión. Brussels: Education, Audiovisual and Culture Executive Agency (EACEA P9 Eurydice). Recuperado el 25 de junio de 2012 en: http://eacea.ec.europa.eu/education/eurydice/key data en.php

Eurydice (2012). Cifras clave de la Educación 2012. Evolución de los sistemas educativos europeos durante la última década. Brussels: Education, Audiovisual and Culture Executive Agency (EACEA P9 Eurydice). Recuperado el 25 de junio de 2012 en:

http://es.scribd.com/doc/486889/competencias-clave-Eurydice\#outer page 4

Fernández Cruz, M. (2006). Desarrollo profesional docente. Granada: Grupo Editorial Universitario.

Fullan, M. (1992). Successful school improvement. Buckingham: Open University Press.

Garagorri, X. (2007). Currículo basado en competencias: aproximación al estado de la cuestión. Innovación Educativa, 161, 47-55. 
Gimeno, J. (2008). Educar por competencias ¿Qué hay de nuevo? Madrid: M orata.

González Maura, V. (2006). La formación de competencias profesionales en la Universidad. Reflexiones y experiencias desde una perspectiva educativa. XXI Revista de Educación, 8, 175-187.

González, J. y Wagenaar, R. (2003). Tuning educational Structures in Europe. Final Report. Phase one. Bilbao: Servicio de Publicaciones de la Universidad de Deusto. Recuperado el 18 de junio de 2010 en http:// www.relint.deusto.es/TuningProject/background.asp

Gutiérrez, A. y Tyner, K. (2012). Educación para los medios, alfabetización mediática y competencia digital. Comunicar, 38, pp. 31-39.

Guzmán, I. y Marín, R. (2011). La competencia y las competencias docentes: reflexiones sobre el concepto y la evaluación. REIFOP, 14 (1), 151-163. Recuperado el 28 de junio de 2012 en: http//www.aufop.com

Hargreaves, A. (1996). Profesorado, cultura y modernidad: cambian los tiempos, cambia el profesorado. Madrid: Morata.

Hirsch, W.Z. and Weber, L. E. (1999). Challenges Facing Higher Education at the Millennium. París and Oxford: American Council on Education/ oryx Press and IAU Press/Pergamon.

Hirtt, N. (2010). La educación en la era de las competencias. REIFOP, 13 (2), 108-114. Recuperado el 28 de junio de 2012 en: http// www.aufop.com

Ibarra, M. S.; Rodriguez-Gómez, G. (2010) Aproximación al discurso dominante sobre la evaluación del aprendizaje en la universidad. Revista de Educación, 351, 385-407.

Informe Bricall (2000). Informe universidad 2000. Conferencia de Rectores de las Universidades Españolas (CRUE). Biblioteca digital de la Organización de Estados Iberoamericanos para la Educación, la Ciencia y la Cultura. Recuperado el 2 de febrero de 2011 en: http://www.oei.es/ oeivirt/ bricall.htm.

Informe DeSeco (2003). OCDE-CERI, Definition and selection of competencies. Theoretical and conceptual foundations: strategy paper on key competencies. Recuperado el 23 de mayo de 2010 en http://mt.educarchile.cl/M T/iibrunner/archives/libros/Competencias/OECD2005.pdf.

Kivinen, O. and Nurmi, J. (2003): Unifying Higher Education for Different Kinds of Europeans. Higher Education and Work: a comparison of ten countries. Comparative Education, 39(1), pp. 83-103.

Knigth, P. T. (2005). El profesorado de Educación Superior. Formación para la excelencia. Madrid: Narcea.

Lasnier, F. (2000). Réussir la formation par compétences. M ontreal: Guérin.

Lieberman, A. y Miller, L. (Eds.) (2003). La indagación como fase de la formación del profesorado y la mejora de la educación. Barcelona: Octaedro.

López, J. I. (2011). Un giro copernicano en la enseñanza universitaria: formación por competencias. Revista de Educación, 356, 279-301. 
Marcelo, C. (Coord.) (2011). Evaluación del desarrollo profesional docente. La Coruña: Davinci.

MEC (2006). Propuestas para la renovación de los métodos en la Universidad. Madrid: Secretaría de Estado.

Mérida, R. (2007a). El prácticum y la formación en competencias del maestro de Educación Infantil en España. Revista Iberoamericana de Educación, 42 (7), 663-686.

Mérida, R. (2007b). Hacia la convergencia europea: los Proyectos de Trabajo en la docencia universitaria. Electronic Journal of Research in Educational Psychology, 5 (3), 13, 825-852. Recuperado el 19 de julio de 2012 en http://www.investigacion-psicopedagogica.org/revista/new/ultimonumero.php

Mérida, R. y Encinas, C. (2008). Un puente entre la escuela y la universidad. Cuadernos de Pedagogía, 380, 24-26.

M érida, R., Angulo Romero, J. y Gil del Pino, C. (2008). Applying the ECTS

Mérida, R., Angulo, J., Gil, C. y Mañas, J. (2008). El crédito europeo: experimentación en el título de maestro de Educación Infantil. I Jornadas sobre Experiencias Piloto de Implantación del Crédito Europeo en las Universidades Andaluzas, (783-789). Cádiz: Servicio de Publicaciones de la UCA.

M érida, R., Barranco, B., Criado, E., Fernández, N., López, R. M. y Pérez, I. (2011). Aprender investigando en la escuela y en la universidad. Una investigaciónacción a través de los Proyectos de Trabajo. Obstáculos y dificultades para la investigación escolar. Investigación en la Escuela, 73, 65-76.

Mérida, R., Gil, C. y Gallego, R. (2008). El prácticum y las competencias específicas en la titulación de Magisterio de Educación Infantil. En M. Agudo, Experiencias Piloto de Implantación del Crédito Europeo en la Universidad de Córdoba (121134). Córdoba: Facultad de Derecho y CC Económicas y Empresariales de la UCO.

Mérida, R.; González, E. y Olivares, M.A. (2011). Adquisición de competencias profesionales del alumnado de Magisterio de Educación Infantil a través de una red de colaboración escuela-universidad. Revista de Investigación en Educación, 9 (2), 184-199.

Mérida, R.; González, E. y Olivares, M.A. (2012). Redes de aprendizaje escuelas infantiles-universidad. Evaluación de una experiencia de innovación. Cultura y Educación: revista de teoría, investigación y práctica, 24 (1), 95-110.

Mérida, R. (2006). Nueva percepción de la identidad profesional del docente universitario ante la convergencia europea. Revista Electrónica de Investigación Educativa, 8 (1). Recuperado el 22 de junio de 2012 en: http://redie.uabc.mx/vol8nol/contenido-merida.html

M érida, R. (2009). La adquisición de competencias en M agisterio de Educación Infantil: tendiendo puentes entre el contexto académico y los escenarios laborales. Bordón, 61 (2), 93-107. 
Nieto, S. (2000). El discurso del profesorado universitario sobre la evaluación del aprendizaje de los alumnos como estrategia de innovación y cambio profesional: exposición y análisis de una experiencia. Revista de Educación, 322, 305-324.

Pérez, A., Soto, E., Sola, F., M. y Serván, Ma J. (2009). Orientar el desarrollo de competencias y enseñar cómo aprender. La tarea del docente. Guía para la adaptación al Espacio Europeo de Educación Superior, 4. Córdoba: Consejería de Innovación, Ciencia y Empresa de la Junta de Andalucía y Vicerrectorado de EEES y Estudios de Grado.

Perrenoud, P. (2001). The key to social fields: competencies of an autonomous actor. En D. S. Rychen y L.H. Salganik (Eds).Defining and Selecting Key Competencies. Gottingen: Hogrefe y Huber, pp.121-150.

Rodríguez, R. (2008). Un modelo basado en las competencias: Hacia un nuevo paradigm en la enseñanza universitaria. Contextos Educativos, 11, 131-147.

Rué, J. (2004). Conceptuar el aprendizaje y la docencia en la Universidad mediante los ECTS. Revista Interuniversitaria de Formación de Profesorado, 18, pp. 179-195.

Rué, J. (2007). Enseñar en la Universidad. El EEES como reto para la Educación Superior. Madrid: Narcea.

Rychen, S.R. y Salganik, L.H. (2003). A holistic model of competence. En D.S. Rychen y L.H.Salganik (Eds). Key Competencies for a successful life and a well-functioning society. Gottingen: Hogrefe y Huber, 41-62.

Sola, M. y Pérez, A. (coord.) (2006). Informe de la Comisión de Innovación para la Docencia de las Universidades Andaluzas. Recuperado el 13 de abril de 2010 en http://www.uhu.es/convergencia_europea/documentos/metodologia/INFORME -CIDUA-PDF.pdf

System to the Childhood Education Teaching Degree in Andalusia. European Journal of Education. Research, Development a Policy, 43 (4) 527-543.

Tejedor, F. J. (Dir.) (1998). Las estrategias utilizadas por los profesores universitarios para la evaluación del aprendizaje de los alumnos. Madrid: CIDE-Informe.

Tierno, J.M., Iranzo, P. y Barrios, Ch. (en prensa). El compromiso organizativo e institucional para diseñar y evaluar competencias en la universidad. Revista de Educación, 361.

Tobón, S. (2006). Formación basada en competencias. Pensamiento complejo, diseño curricular y didáctica. Bogotá: Ecoe.

Tonucci, F. (2010). Apuntes sobre el manifiesto. Educar(nos), 49, 9-11.

Toro, J. M. (2005). Educar con co-razón. Bilbao: Desclée de Brouwer.

Torres, J. (2006). La desmotivación del profesorado. Madrid: M orata.

Trillo Alonso, F. (2005). La evaluación de los estudiantes universitarios. El caso de la Universidad de Santiago de Compostela. Santiago de Compostela: Universidad.

Van Damme, D. (2001): Higher Education in the age of globalisation: The need for a new regulatory framework for recognition, quality assurance and acreditation. 
Introductory Paper for the UNESCO Expert Meeting. Paris, 10-11 September 2001.

Villa, A. (2008). La excelencia docente. Revista de Educación, número extraordinario, $177-212$.

Watts, F. y García-Carbonell, A. (2006). La evaluación compartida: investigación multidisciplinar. Valencia: Servicio de Publicaciones de la Universidad Politécnica de Valencia.

Weber, L. E. and Duderstadt, J.J. (2010). University Research for Innovation. London: Económica Ltd.

Wells, G. (2002). Indagación dialógica. Hacia una teoría y una práctica socioculturales de la educación. Paidós: Barcelona.

Winter, R. (2003). Contextualising the Patchwork Text: Addressing problems of coursework assessement in Higher Education. En R. Winter, J. Parker y P. Ovens, The Patchwork Text: A Radical Re-assessment of Coursework Assignments Innovations in Education and Teaching International Special Issue, vol. 40 (2), 112-122.

Woolf, H. (2004). Assessement criteria: reflections on current practices. Assessement and Evaluation in Higher Education, 29 (4), 479-493.

Yañiz, C. y Villardon, L. (2006). Planificar desde competencias para promover el aprendizaje. Bilbao: Universidad de Deusto.

Yorke, M. (2003). Formative assessement in higher education: Moves towards theory and the enhancement of pedagogic practice. Higher Education, 45 (4), 477-501.

Zabala, A. (1999). Enfoque globalizador y pensamiento complejo. Barcelona: Graó.

Zabala, A. y Arnau, L. (2007). Cómo aprender y enseñar competencias. Barcelona: Graó.

Zabalza M.A. (2011). M etodología docente. Revista de Docencia Universitaria. REDU. Monográfico: El espacio europeo de educación superior. Hacia dónde va la Universidad Europea?. 9 (3), 75-98. Recuperado el 13 de febrero de 2012 en http://redaberta.usc.es/redu

Zabalza, M. A. (2003). Diseño curricular en la universidad. Competencias del docente universitario. Madrid: Narcea.

Zabalza, M.A. (2006). Competencias docentes del profesorado universitario. Madrid: Narcea.

Cita del artículo:

Mérida Serrano, R. (2013). La controvertida aplicación de las competencias en la formación docente universitaria. Revista de Docencia Universitaria. REDU. Vol.11 (1) Enero-Abril. pp. 185-212. Recuperado el (fecha de consulta) en http://www.redu.net/ 


\section{Acerca de la autora}

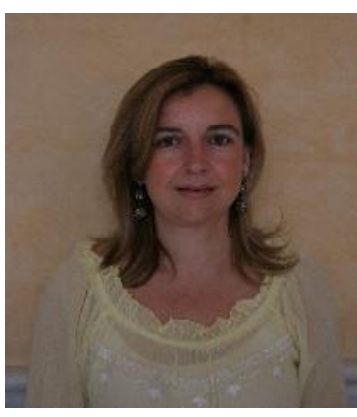

\section{Rosario Mérida Serrano}

Universidad de Córdoba

Departamento de Educación

Mail: rmerida@uco.es

Es maestra de Educación Infantil y doctora en Psicopedagogía, actualmente es profesora de la titulación de Grado de Magisterio de Educación Infantil e imparte docencia en el Máster de Educación Inclusiva y Educación Secundaria. Sus principales líneas de investigación son: la formación docente inicial y continua del profesorado de infantil, la investigación cualitativa y la convivencia escolar. 


\title{
Inteligencia Emocional y Comunicación: la conciencia corporal como recurso
}

\author{
Emotional Intelligence and Communication: body awareness as a resource
}

\author{
Rosa María Rodríguez Jiménez \\ $M^{a}$ del Mar Caja López \\ Patricia Gracia Parra \\ Paloma Julia Velasco Quintana \\ $M^{a}$ José Terrón López
}

Universidad Europea de Madrid, España

\section{Resumen}

Los cambios producidos en los procesos de enseñanza- aprendizaje requieren de los docentes formación competencial que motive a los alumnos para que sean capaces de desarrollarse como personas y realizar aprendizajes significativos a lo largo de toda su vida. En línea con este objetivo es necesaria una formación específica del profesorado que promueva el desarrollo de las competencias de tipo emocional. En el contexto educativo, una de las claves del éxito (la consecución de un proceso de enseñanza-aprendizaje efectivo y de calidad) se encuentra en el modo en el cual se establecen las dinámicas interpersonales. Un buen docente es aquel que no sólo "sabe", sino que "es". La presencia del docente en el aula, su cercanía al alumno, su capacidad para gestionar sus emociones, para comunicarse de modo eficaz y empatizar con él, son todos ellos aspectos que inciden sin lugar a dudas en el clima de trabajo, en el grado de motivación del alumno, en los resultados de aprendizaje, y claro está, en su desarrollo personal. Cuando hablamos de aspectos comunicativos, aquellos de tipo no verbal cobran una gran importancia. Los docentes apenas reciben formación de tipo experiencial sobre aspectos relacionados con la presencia del cuerpo y su expresión. El conocimiento enactivo a través de un programa de concienciación corporal y comunicación no -verbal posibilita el desarrollo de competencias intra e interpersonales. Se presentan en este trabajo los resultados preliminares de un programa de formación realizado en este ámbito con profesores universitarios de distintas áreas de conocimiento.

Palabras clave: Competencias docentes, trabajo corporal, inteligencia emocional, conocimiento enactivo, comunicación no-verbal, concienciación corporal

\section{Abstract}

Nowadays, the changes in the teaching-learning processes need competence trained teachers so as to motivate students in their personal growth leading to significant life- long learning. In order to achieve 
this objective, specific teacher training that recognizes the importance of developing emotional competences becomes necessary. In an educational context, one of the keys to success (securing an effective quality teaching-learning process) is found on the way interpersonal dynamics are established. A proficient teacher is the one that not only has a good competence in declarative knowledge but also in skills or know how to and existential knowledge (savoir-être).. The presence of the teacher in the classroom, his proximity to the student, his ability to manage emotions, to communicate effectively and to feel empathy, are all aspects that undoubtedly affect the work atmosphere at the level of student motivation towards improving learning outcomes, and ultimately, in his personal development. When we talk about the aspects of communication, non-verbal ones acquire a great importance. Teachers hardly receive non significative experiential training on aspects related to body presence and its expression in the classroom (somatic aspects). Body knowledge through a program of body awareness and nonverbal communication enables the development of intra and interpersonal skills, aiming to cover this training gap. This paper presents the preliminary results of a training program conducted in this area with teachers from diverse subject areas in the context of higher education.

Key words: Teacher competences, body work, emotional intelligence, enactive learning, non-verbal communication, body awareness.

\section{Introducción. Competencias docentes}

El nuevo paradigma educativo en educación superior (EEES) supone, como ya sabemos establecer los objetivos de aprendizaje de nuestros alumnos en términos de adquisición de competencias, tanto específicas de las materias como genéricas o transversales. Son ya muchos los autores que están estudiando la mejor manera de integrar el desarrollo competencial en los nuevos grados y la manera de desarrollar y evaluar este desarrollo en el alumnado. También somos conscientes de que este nuevo paradigma educativo supone un gran cambio en la manera de enseñar (y de aprender). El hecho de que el estudiante se sitúe en el centro, hace que los procesos de enseñanza-aprendizaje se transformen para adecuarse a las nuevas exigencias. Pero no son sólo los estudiantes los que cambian la forma de aprender, los docentes debemos también propiciar nuevos entornos que favorezcan el aprendizaje, diseñar nuevas actividades formativas que integren las competencias, motivar a los alumnos en esta nueva estructura, etc. pues ese aprendizaje dependerá de cómo planteemos nuestro trabajo docente. Tal y como señala Cano (2008) en estos tiempos cambiantes, resulta importante que el docente desarrolle determinadas competencias pues a través de su trabajo conseguirá un mejor desarrollo en sus estudiantes.

Debemos pues precisar qué competencias deben tener los docentes para conseguir que el cambio sea un éxito. Es decir, definir las nuevas competencias que deben poseer los profesores de ahora y que ya no son las que se precisaban hace unos años. Se trataría, en palabras de Zabalza (2009), de buscar un marco de referencia en el que ir vislumbrando la docencia universitaria y la formación específica necesaria para desarrollarla adecuadamente. En esta línea, existen diversas propuestas de cuáles son las competencias docentes en diversas etapas educativas $y$, en particular, en el ámbito universitario que nos preocupa Cano (2008), Valcárcel (2003), y Zabalza y Zabalza (2011a) coinciden en varios aspectos. En particular, hablan de que las cualidades personales de los docentes juegan un papel fundamental, por lo que es necesario incluir en el desarrollo competencial de los docentes las habilidades sociales y afectivas. Otros estudios (Sutton y Wheatley, 2003) nos indican que para lograr unos procesos de enseñanza-aprendizaje efectivos y de calidad se requiere que los docentes 
desarrollen la competencia emocional pues a través de su propio bienestar personal conseguirán un mejor desarrollo socio-emocional de sus alumnos.

Si queremos docentes competentes, debemos reforzarles aportándoles la formación requerida para el ejercicio de su profesión. Es por tanto crucial una formación integral que contemple el desarrollo de competencias emocionales y que ponga de manifiesto la importancia del aspecto humano en el aula ("Ios profesores enseñan tanto por lo que saben como por lo que son"); la complicidad y empatía entre profesor y alumno, favorecen la comunicación y por tanto el proceso de enseñanza/aprendizaje. Como cita Zabalza (2011b):

La enseñanza es un ejercicio "cuerpo a cuerpo" con nuestros estudiantes. Se trata de un proceso mediado por las características personales de ambos, las suyas como estudiantes y las nuestras como profesores o profesoras.

$Y$ cuanto más vulnerables son esas personas con las que trabajamos, más importancia adquiere nuestra condición de persona y la forma en que nuestras características personales influyen en la forma llevar a cabo nuestro trabajo (p.48).

En este proyecto abordamos el espacio donde este "cuerpo a cuerpo" toma lugar; ganando conciencia corporal sobre uno mismo, somos capaces de identificar nuestros patrones perceptivos, cognitivos y expresivos, llegando a detectar de manera más precisa los de los demás. El proceso de comunicación se clarifica con el descubrimiento de nuevas posibilidades de habitar el cuerpo. Este proyecto se centra en este trabajo de apertura de la conciencia corporal como premisa hacia una comunicación intra e inter personal más atenta y sensible. En esta sensibilidad, reside para nosotras la clave de una modulación empática de las características humanas de profesores y alumnos en el aula, en pro de una enseñanza eficiente y satisfactoria.

En este artículo presentamos los resultados preliminares de este proyecto de desarrollo de competencias intra e interpersonales en docentes universitarios mediante la realización de un programa de concienciación corporal y comunicación noverbal.

\section{Competencias emocionales y Corporalidad}

Cuando se analiza el número de publicaciones aparecidas en relación a la profesión docente, cabe destacar la poca representatividad que presentan los aspectos emocionales en la misma. En este sentido, Sutton y Wheatley (2003) justificaban la poca investigación en este ámbito en el sentido de que los trabajos sobre aspectos cognitivos del profesorado son relativamente recientes (entorno a los años 70) y aquellos centrados en las emociones comenzaron en los años 80 pero no se empezaron a aplicar al ámbito educativo hasta los 90.

El estudio de las emociones presenta una perspectiva multifactorial que según los mismos autores se puede categorizar en: a) valoración, relacionada con diferencias culturales; b) experiencia subjetiva; c) cambios fisiológicos; d) expresión emocional y e) tendencias de acción. Lo que parece evidente, y así avalan los estudios realizados (Sutton y Wheatley, 2003), es que la complejidad de las emociones determina el modo en que los profesores se manifiestan en su labor docente. Tanto las emociones positivas (gozo, excitación, amor, deseo de cuidar al otro, etc.), como las negativas 
(enfado, frustración, tristeza, ansiedad, etc.) afectan sin lugar a dudas al trabajo que el docente realiza en un aula.

La influencia de las emociones se produce por una parte a nivel cognitivo, puesto que afectan a la atención, a la memoria, al pensamiento y a la capacidad de categorización y de resolución de problemas (Niedenthal y Kitayama, 1994). Así, los profesores que experimentan emociones más positivas pueden generar mayor número de ideas y estrategias para la enseñanza. Esto les puede llevar a obtener mejor sus objetivos lo que a su vez influye en emociones positivas convirtiéndose en un bucle. Por otra parte, las emociones afectan a la motivación del profesor; en este sentido existen evidencias experimentales que señalan que inducir estados de buen humor en los docentes aumenta la auto-eficacia (Bandura, Self-Efficacy: The Exercise of Control, 1997).

Asimismo los estudiantes son conscientes de las emociones de los profesores (Frenzel, Goetz, Lüdtke, Pekrun, y Sutton, 2009; Hargreaves, 2000) y se ven influenciados por ellas. De hecho, existen estudios en niveles universitarios (Emmer E. , 1994) sobre el valor que los estudiantes dan a que los profesores se ocupen de ellos. Existen muchas maneras en las cuales se comunican las emociones tanto de modo voluntario (consciente) como involuntario (o inconsciente). Aquí aparecen sin lugar a dudas los aspectos no verbales como son la utilización del espacio, los movimientos realizados y las cualidades de los mismos, la gestualidad facial, el tono de voz, su intensidad, el foco visual, etc. (Darwin, 1984; Prozesky, Communication and Effective Teaching, 2000).

Es conocido que los procesos de aprendizaje son más fructíferos cuando el ambiente del aula resulta agradable, cuando se estimulan y propician emociones positivas (Erk, y otros, 2003) y cuando se establecen correctos canales de comunicación entre el elemento docente y discente. Las investigaciones de Hamann y Mao (2002) demuestran, por ejemplo que las miradas agradables y las palabras amables activan nuestro sistema cortical de gratificación, lo que se traduce en mayores niveles de motivación e implicación con una determinada actividad.

Así, se hace necesario insistir en procesos de regulación emocional, que permitan al docente gestionar con mayor eficacia los aspectos emocionales (Sutton R. , 2004). Estos procesos se enmarcan en el concepto de inteligencia emocional. Con posterioridad al concepto de inteligencias múltiples (Guilford y Hoepfner, 1971) introducido por Gardner (1995) en 1990 , Salovey y colaboradores (1995) acuñan el término 'Inteligencia Emocional' haciendo referencia a la integración de la inteligencia intrapersonal y la interpersonal y, describiéndola como la "forma de inteligencia social que implica la capacidad de supervisar a uno mismo y a otros, sus sentimientos y emociones, para diferenciar entre ellos, utilizando esta información para conducir a la vez, el pensamiento y la acción" (Salovey, Mayer, Goldman, Turvey, y Palfai, 1995, p. 4). Goleman (1996) desarrolla el concepto de "Inteligencia Emocional" (IE) ligando cada subgrupo (intrapersonal e interpersonal) con habilidades prácticas emocionales concretas, o competencias generales, como se las conoce en el entorno académico. Así, distingue entre competencias intrapersonales entre las cuales se encuentra la autoconciencia, la autorregulación y la automotivación, de las competencias interpersonales entre las que tenemos la empatía y las habilidades sociales. 
Por tanto, la Inteligencia Emocional (IE) se entiende como la capacidad de conciencia y control de las emociones propias y el manejo adecuado de las mismas en un contexto social. Los resultados obtenidos con el TMMS, Trait Meta-Mood Scale (Salovey, Mayer, Goldman, Turvey, y Palfai, 1995), demuestran que los subfactores que componen la IE, tienen efectos diferenciales en la salud de las personas. Las personas con dificultades para regularse a nivel emocional presentan mayor número de síntomas físicos; sintomatología depresiva y ansiedad, mayor tendencia al pensamiento negativo o disminución en su funcionamiento físico y social (Extremera y Fernández-Berrocal, 2002; Fernández-Berrocal, Ramos, y Extremera, 2001; Salovey, Mayer, Goldman, Turvey, y Palfai, 1995). Por el contrario, una elevada habilidad para tomar conciencia y controlar nuestras emociones, incide directamente en la reducción del malestar subjetivo y las emociones negativas. Así, las personas con una alta capacidad para clarificar y regular sus propias emociones presentarán menor sintomatología ansiosa y depresiva, más satisfacción vital y en definitiva mejor salud mental y física (Domenech Delgado, 1995; Fernández-Berrocal, Ramos, y Extremera, 2001; Extremera y Fernández-Berrocal, 2006). El desarrollo de competencias como la autoconciencia, la confianza en uno mismo, la empatía y la gestión de las emociones e impulsos perturbadores, mejoran la conducta de las personas (docentes, en nuestro caso) y su rendimiento en entornos educativos (Mearns y Cain, 2003).

Por tanto, la mejora de la inteligencia emocional de los docentes tendrá resultados en el nivel de bienestar y en el grado de percepción de salud de los mismos, y por tanto, incidirá de manera positiva en su actuación en el aula. Esta mejora de la IE se puede abordar desde distintos enfoques, siendo el del trabajo corporal y la comunicación no- verbal uno de los más innovadores.

El estudio del lenguaje no verbal ha adquirido gran relevancia como tema de investigación en el ámbito de la psicología. De Paulo (1992) destacó como principales características su naturaleza irrepetible, su conexión con las emociones, su accesibilidad al observador, su velocidad y su carácter único.

Es conocido que en un mensaje un 75 a un 90 por ciento del impacto, se transmite de forma no verbal (Galloway, Nonverbal: Authentic or Artificial, 1977) por lo que tiene sentido elegir este canal como un instrumento para mejorar el nivel competencial del docente. El contenido verbal del mensaje que quiere transmitir un docente pierde importancia respecto a otros aspectos como son el tono de voz, la expresión facial, la postura, los gestos, etc., pudiendo tanto reforzarlo como modificarlo e incluso llevarlo a contradicciones El lenguaje no verbal puede transmitir a los alumnos coherencia o no en los mensajes lanzados por el profesor así como predisponer emocionalmente a los mismos para el resto del tiempo de clase.

Ambady y Rosenthal (1993) señalaron que el estudio de pequeñas muestras de lenguaje no verbal de los profesores en las enseñanzas medias y universitarias, permitía predecir los índices de satisfacción que dichos profesores recibían por parte de sus estudiantes en las encuestas de fin de curso. Estos resultados han sido corroborados por Babab, Avni-Babad y Rosenthal (2004) en un estudio más amplio llevado a cabo en el ámbito universitario, y en el que el lenguaje no verbal de los profesores era evaluado en diferentes situaciones (en el primer minuto de la primera clase, dando clase en general, dialogando con los estudiantes y hablando acerca del 
curso en su despacho). Entre las situaciones estudiadas, se observó que la valoración de pequeñas muestras del lenguaje no verbal del profesor al impartir clase tenía buena correlación con los resultados de los ítems de las encuestas que hacían referencia al estilo expresivo del profesor (humor, entusiasmo, claridad y apreciación general). Para estos autores, los buenos docentes presentan un perfil no verbal intenso y rico en detalles. Son expresivos con sus rostros, manos, cuerpo y voz y permanecen más tiempo de pie que sentados, aprovechando en su movimiento todo el espacio del aula. Muestran una fuerte orientación hacia la audiencia. Hacen continuos cambios de todos estos factores para captar el interés de los estudiantes. No obstante, a pesar de esta gran actividad, están relajados y evitan demostrar comportamientos negativos.

El desarrollo de competencias tanto de tipo profesional como personal a través del movimiento y el lenguaje no verbal no es algo nuevo (Boyatzis, Mckee, y Johnston, 2008; Camerino I Foguet, Castañer I Balcells, y Buscá, 1999; Coates, 2002; Erk, y otros, 2003; Galloway, 1977; Guilford y Hoepfner, 1971; Hamann y Mao, 2002; Rizzolatti y Sinigaglia, 2006). En empresas líderes a nivel mundial desde hace tiempo se vienen incorporando programas de coaching que integran niveles no sólo intelectuales sino también físicos y emocionales para promover entornos de trabajo saludables al tiempo que eficientes y productivos. Los programas son diseñados por terapeutas y educadores especializados en Análisis del Movimiento Laban, Terapias corporales como la Danza Movimiento Terapia y técnicas de Educación Somática del Movimiento (SME) como Body Mind Centering, la técnica Alexander o Feldenkrais entre otras.

Los procesos de grupo que integran aprendizaje intelectual, artístico e intuitivo son recursos educativos y terapéuticos que basan su efectividad en el fenómeno de la enacción; se aprende a través de la acción consciente (Fischman, 2005; Varela F. , 1988). Un uso más consciente del cuerpo y su movimiento favorecen un aprendizaje más significativo, mayor control sobre la vivencia y por tanto sobre la emoción.

La comunidad docente carece en su mayoría de una formación específica en conciencia corporal que les permita utilizar sus habilidades comunicativas de manera más eficiente en los ámbitos antes mencionados. Hoy en día, las actividades formativas para el profesorado en materia de competencias (Benito, Bonsón, y Icarán, 2005; De Miguel Díaz, 2006; García Olalla y Poblete Ruiz, 2007; Blanco Fernández, 2009; Rué Domingo, 2008; Poblete Ruiz y Villa Sánchez, 2007; Terrón, 2007; Zabalza M. Á., 2003; García García, Terrón López, y Blanco Archilla, 2010; Velasco Quintana, y otros, 2011) se llevan a cabo en España sin incluir un trabajo vivencial que contemple la importancia de la conciencia corporal en el proceso comunicativo a niveles inter e intra personales. Se detecta pues, una falta de reconocimiento de la importancia de integrar el aprendizaje enactivo de competencias en la formación del profesorado y como consecuencia un vacío importante en la formación integral de los docentes con implicaciones directas sobre el grado de satisfacción con sus habilidades comunicativas a nivel inter personal; profesor/ profesor y profesor/alumno. No es así en países como Estados Unidos donde estudios como los de Bat-Sheva (1994) mostraron hace ya tiempo la importancia y la aplicación de experiencias en movimiento y conciencia corporal desde la Danza Movimiento Terapia en los procesos de enseñanzaaprendizaje.

Asimismo estudios más recientes demuestran que programas de trabajo corporal específicos son especialmente adecuados para la mejora de la empatía en entornos 
laborales, repercutiendo por tanto en la mejora de las habilidades relacionales, la inteligencia emocional, el bienestar psicológico y la satisfacción vital (Fischman, 2005). Estas mejoras son clave en el desarrollo de competencias profesionales como el liderazgo, la resolución de conflictos y las habilidades comunicativas.

Las últimas investigaciones en neurociencia y el desarrollo de un conjunto de disciplinas bajo el nombre de Ciencias Somáticas del Movimiento (ISMETA) avalan, especialmente desde los años 70, la importancia que un correcto desarrollo motor y trabajo corporal tienen en el funcionamiento de distintas funciones cerebrales (Ramachandran, 2008; Rizzolatti y Sinigaglia, 2006). Se ha comprobado que cambios en el repertorio de conductas motoras, producen cambios en los niveles cognitivos o niveles mentales (Payne, Dance Movement Therapy: Theory and practice, 1992). Las cualidades de cualquiera de nuestros movimientos son manifestaciones de cómo la mente se expresa a través del cuerpo en ése momento. Los cambios en cualidades del movimiento indican que la mente ha cambiado de enfoque en el cuerpo. Del mismo modo, cuando dirigimos la mente o atención a distintas partes de nuestro cuerpo e iniciamos el movimiento desde esas zonas, cambiamos la cualidad de nuestro movimiento. Así encontramos que el movimiento puede ser un modo de observar la expresión de la mente a través del cuerpo, y también puede ser un modo de producir cambios.

Igualmente, está ampliamente demostrada la conexión entre las emociones y el comportamiento motor; es lo que algunos autores denominan "comportamiento motor emocional» (Capello, 2008). El movimiento es considerado así un proceso dinámico en el que a través del cuerpo se pueden expresar emociones, percepciones, motivaciones e interacciones interpersonales (Lapierre y Aucoutourier, 1983). Algunos investigadores como Capello (2008) se basan en estas premisas para desarrollar competencias generales en personas adultas a través de programas de movimiento creativo.

La incorporación de elementos no verbales y de movimiento creativo en el proceso de enseñanza - aprendizaje ha demostrado ser una herramienta potente para la adquisición de competencias generales como se puede constatar en investigaciones previas (Coates, 2002; Bat-Sheva, 1994; Rodríguez Jiménez, Terrón López, y Gracia Parra, 2010; Rodríguez Jiménez, 2010). De hecho, autores como Alles (2005) incorporan en las publicaciones sobre adquisición y evaluación de competencias enfocadas al desarrollo profesional, métodos distintos a los tradicionalmente utilizados como son aquellos relacionados con el trabajo corporal.

A partir de todo lo anterior se justifica el proyecto descrito en este trabajo. Es evidente el interés que suscita tratar de mejorar las competencias intra e interpersonales de los profesores (en definitiva, inteligencia emocional) y una de las formas de implementarlo sería mediante un programa que ponga el énfasis en la comunicación no verbal y en el aprendizaje experiencial (Bat-Sheva, A concept of "body knowledge" and an evolving model of "movement experience": Implications and application for curriculum and teacher education, 1994).

Un profesor consciente de sus patrones de conducta no verbal, podrá mejorar su comunicación obteniendo mayor seguridad y control sobre el impacto de la misma en su entorno de trabajo, tanto fuera como dentro del aula. Aunque se han realizado 
algunas acciones (casi todas en ámbito no universitarios) encaminadas a esta mejora no se ha valorado como han evolucionado los profesores que participaron en dichos programas (Camerino I Foguet, Castañer I Balcells, y Buscá, 1999; Álvarez de Arcaya Ajuria, 2004). De hecho, en este trabajo se pretende precisamente recoger la percepción sobre los profesores implicados en el proceso a través de los diarios reflexivos realizados durante la realización de la formación, utilizando metodología cualitativa tal y como se explica a continuación.

\section{Metodología}

La metodología planteada para la realización del proyecto ha sido la de tipo Investigación - Acción, en adelante IA, en concreto el modelo de Kemmis (Kemmis y Mctaggart, 1988). Esta metodología está reconocida como la investigación más potente realizada entre el profesorado gracias a su proceso transformador desde la reflexión. A través de ella se generan comunidades de aprendizaje por parte de equipos de profesores, lo que incide en la creación de una cultura institucional orientada al cambio, a la reflexión, a la mejora profesional del docente, que incide en la calidad de la enseñanza. Este planteamiento metodológico genera una mejora a partir de una transformación desde la reflexión conjunta del profesorado (Sandín Esteban M. P., 2003; Latorre Beltrán, 2003; Elliot, 2000). La IA está orientada a mejorar la formación del profesorado, a reflexionar sobre la propia práctica docente, a generar comunidades de aprendizaje, y dinamizar procesos de reflexión y cambio. Supone una investigación aplicada, en contextos reales, con incidencia directa en la intervención educativa. Este diseño de investigación que genera descripciones densas de todo lo que acontece y de los procesos de reflexión y cambio del profesorado serán perfectamente transferibles a otros contextos educativos que quieran vivenciar experiencias de formación en este sentido. Los procesos de cambio y mejora continua del profesorado sólo son patentes y tienen impacto si se viven desde dentro, si el docente percibe la necesidad de mejora y está dispuesto a generar procesos de cambio.

Así esta metodología es relevante en cuanto a que:

- Integra la acción de formación en conciencia corporal del propio profesorado en su mismo ámbito de práctica docente.

- El foco es la mejora de la práctica docente incidiendo sobre la mejora en sus habilidades de comunicación a nivel intra e inter personal.

- El profesorado puede aplicar los conocimientos adquiridos de manera inmediata en su práctica y esto repercutirá sobre el diseño y mejora de las herramientas formativas que se utilicen.

- El profesorado participa de manera activa en el proceso siendo testigo y artífice del impacto de su aprendizaje.

- El profesor se implica en el proceso utilizando sistemas de reflexión y evaluación basados en sus experiencias y percepciones de cambios en su manejo del entorno laboral.

Según esta metodología las fases de trabajo que se han llevado a cabo en el proyecto son las siguientes: 


\section{Fase I. Planificación de la acción}

Así pues la hipótesis de acción es ¿cómo podríamos mejorar el grado de inteligencia emocional de nuestros docentes?; ¿cómo podemos mejorar sus competencias intra e interpersonales? Consideramos que una respuesta factible es a través de programa de trabajo corporal y comunicación no-verbal.

La planificación de la acción se llevó a cabo a través de las siguientes fases:

\section{Formación del grupo participante.}

Se invitó al profesorado interesado a participar en el programa de trabajo corporal. La comunicación se realizó mediante directores de departamento y herramientas de comunicación on-line. En esta etapa se explicó al profesorado los requisitos y las implicaciones de su participación. En la reunión de presentación del programa estaban el grupo investigador y el grupo participante.

El grupo participante se constituyó a través de profesores que de manera voluntaria manifestaron su deseo de participar en el programa, por lo que eran docentes con una predisposición a investigar y reflexionar sobre aspectos comunicativos mediante herramientas basadas en lo corporal. Los participantes eran inicialmente 22 docentes de distintas áreas de conocimiento, pertenecientes a las distintas Facultades de la Universidad a la que pertenecen los autores: Escuela Politécnica, Artes y Comunicación, Ciencias Sociales, Ciencias de la Salud, Ciencias Biomédicas y Actividad Física y del Deporte. Algunos de ellos habían participado ya en años anteriores en programas de formación del profesorado sobre conciencia corporal y comunicación no-verbal, dirigidos por la responsable del proyecto. En esas convocatorias habían manifestado su interés por profundizar en esta línea de conocimiento, lo cual fue uno de los factores que impulsó la puesta en marcha del programa.

\section{Diseño programa}

Diseñar un programa de trabajo corporal específico, orientado a la mejora de las competencias interpersonales de los docentes. En esta etapa se estableció también el calendario de las sesiones de movimiento así como de las reuniones de trabajo de los participantes en la investigación.

\section{Diseño de la recogida de información}

En la fase de diseño también hay que contemplar y decidir qué información se recogerá y cómo y en qué momentos. Se utilizaron técnicas de recogida de información diversas, basadas fundamentalmente en la observación participante:

- Diarios de observación del personal investigador que estuvo presente en las sesiones de formación de los docentes.

- Diarios reflexivos por parte de los propios docentes al finalizar cada sesión de trabajo corporal y al final de cada semana de trabajo entre una sesión y la siguiente respondiendo de manera concreta a dos preguntas: ¿qué he descubierto en la sesión de trabajo? y ¿cómo me ha ayudado esto durante la semana de trabajo entre una sesión y la siguiente?.

- Recogida de información durante las reuniones mediante actas de reunión.

- Entrevistas semi- estructuradas al grupo participante. 
- Grabación y análisis de los comentarios derivados de cada sesión práctica así como en las reuniones de todos los participantes. Se garantizó a los participantes la confidencialidad del material (evidencias) recogido que será utilizado sólo con fines de investigación.

Una vez diseñado el plan de acción se acometió con el grupo de participantes la fase de acción más observación.

\section{Fase 2. La acción más observación}

Las tareas de acción y de observación, en este proyecto, se realizaron en paralelo, por lo que se ha optado por ponerlas en una única fase unificada.

La acción se llevó a cabo tal y como se había diseñado a través de las siguientes fases:

\section{Desarrollo}

Implementar el programa de trabajo corporal y movimiento con el grupo participante trabajando sobre competencias vinculadas a la inteligencia emocional. El programa consistió en la realización de 1 sesión semanal de 2 horas de duración, durante un total de 13 semanas.

Durante el desarrollo del programa, y en paralelo al mismo, se mantuvieron 3 reuniones conjuntas entre el equipo investigador y el equipo participante para analizar los avances realizados en el proceso.

\section{Evaluación}

Consiste en el análisis del impacto de este programa en el entorno profesional y la realización de un informe final.

\section{Fase 3. La reflexión}

La reflexión se llevó a cabo en las siguientes fases:

\section{Recopilación de la información}

Las investigadoras recogieron las notas tomadas en los diarios reflexivos de los participantes buscando evidencias de:

- Comentarios relacionados con aprendizajes explícitos en cuanto a conciencia corporal adquirida.

- Relevancia de estos conocimientos en el plano intra-personal; ¿me ayudan a conocerme mejor?, ¿He adquirido una mayor conciencia de mi cuerpo?

- Relevancia de estos conocimientos en el plano intra e inter-personal; ¿me ayudan a relacionarme de manera más consciente en mi ámbito laboral?

- Otras consecuencias del trabajo; aparición de otras reflexiones en relación al impacto del trabajo tanto a nivel intra como inter personal, con connotaciones negativas o positivas para el profesor. 


\section{Reducción y representación}

Se seleccionaron aquellos comentarios y/o unidades de significado que eran independientes y recurrentes, de modo que fueran representativos de las reflexiones del profesorado, mediante un proceso inductivo de categorización.

\section{Validación}

Observación de saturación en comentarios relacionados a las siguientes temáticas:

- Evidencia de adquisición de mayor conciencia corporal.

- Evidencia de mejora en la confianza y la autoestima.

- Evidencia del impacto de los anteriores en sus habilidades y roles.

- Evidencia de una mayor conciencia de su práctica en relación a sus habilidades inter e intra comunicativas.

- Evidencia de cambio en sus valores y creencias en relación al cuerpo y su potencial expresivo.

- Evidencia de cambios en su cosmovisión, es decir, en la percepción del docente en cuanto a la relación entre la institución, la educación, el cuerpo y la comunicación.

\section{Interpretación}

Suficiente saturación en los apartados anteriores así como otros que pudieran aparecer, así como las notas del equipo investigador, servirían para interpretar el impacto de la acción.

\section{Conclusiones}

La acción se modifica en base a los resultados recogidos.

Del análisis de resultados se revisarán las acciones acometidas, esto es, de la reflexión conjunta del equipo investigador, se obtendrán resultados de cuyo análisis obtendremos una mejora de las acciones a acometer.

\section{Programa de trabajo corporal}

\section{Fundamentos}

El programa de trabajo corporal ha sido diseñado desde un amplio prisma de técnicas y enfoques que toman como base la vivencia del cuerpo en primera persona. Las dos áreas de conocimiento de las que se nutre el proyecto son la Danza Movimiento Terapia (DMT) y la Educación Somática del Movimiento (Body Mind Centering, 2001). Estas dos prácticas comparten un eje fundamental sobre el que se vertebra el proyecto; el aprendizaje enactivo (Varela F. , 1988).

El abordaje de la práctica del movimiento desde la perspectiva del conocimiento enactivo implica reconocer el vínculo inseparable entre conocimiento y movimiento; el conocimiento surge inevitablemente de la acción en el mundo y la acción no es otra cosa sino movimiento. Los participantes son en última instancia, los que actúan desde su experiencia operando con el entorno a través del movimiento. Es desde la vivencia, 
observación y modulación de sus capacidades individuales de introspección e interacción, que surge el conocimiento y este se transforma en el verdadero contenido de las sesiones.

La Danza Movimiento Terapia propone un enfoque holista de la vida y el comportamiento humanos. A la hora de trabajar en el ámbito educativo, facilita un encuadre vivencial a través del cual los profesores, como participantes reflexivos, pueden explorar, observar y evaluar con seguridad, su propia experiencia y práctica docente.

El Movimiento Somático como práctica, facilita un proceso de cuestionamiento en el lugar del cuerpo y su movimiento. La experiencia humana puede ser desde este enfoque, observada, experimentada, compartida e investigada, fomentando el ejercicio de la presencia consciente y desde ahí, la reorganización y cambios de nuestros patrones conductuales. En este proyecto se ha trabajado con el enfoque somático que propone la escuela Body Mind Centering, fundada en 1973 por Bonnie Bainbridge Cohen.

El proyecto se desarrolla a lo largo de 13 sesiones en las que se pretende involucrar a los profesores participantes en un proceso de autodescubrimiento, desarrollo de la conciencia corporal y exploración de los vínculos entre experiencia corporal y conocimiento práctico personal, desembocando en un conocimiento más profundo de sus habilidades comunicativas en el contexto educativo y contribuyendo a su crecimiento y desarrollo profesionales.

El conocimiento corporal surge de la relación de los participantes con su vivencia del movimiento. El trabajo va cobrando sentido conforme los participantes toman conciencia, a través de sus propias reflexiones, de la relación complementaria entre la experiencia verbal y la no verbal; el concepto último, es el sentido encarnado de la experiencia.

La DMT y el Movimiento Somático, transmitidos desde esta metodología de conocimiento enactivo, procuran facilitar un proceso de aprendizaje en el que los profesores puedan armonizar los aspectos afectivos y cognitivos de su práctica docente. Se promueve así, la adquisición de conocimiento práctico, útil para la vida profesional.

\section{El aprendizaje enactivo. Abordaje enactivo.}

El término enacción proviene del verbo inglés to enact. Este neologismo significa "llevar a cabo", "poner en ejecución", "representar" o "actuar" (Varela F. , 1988). Desde el campo de la neurobiología, Maturana y Varela (1985), proponen con este término, plantear una nueva perspectiva del conocimiento. Varela, Thompson y Rosch (1997) indican que bajo dicha perspectiva la cognición no es sólo la representación de un mundo pre-dado por una mente pre-dada sino que tiene en consideración la variedad de acciones que un ser realiza en el mundo. El mundo emerge de cómo actuamos, esto es, cómo nos movemos, cómo nos tocamos, cómo respiramos y cómo comemos. Todo ello es lo que implica el término "en-acción". La acción significa producir "algo" por medio de una manipulación concreta. 
Este movernos en el mundo o "enactuar" va forjando la historia de lo que nos será posible más adelante. A través de una espiral de "enacciones" y mientras el sistema esté "activo" o "vivo", vamos creando los "mundos" que conocemos y habitamos (Varela F. , 1988). En lugar de habitar en un mundo pre-establecido, predefinido, prefabricado, el autor sugiere que vamos haciendo surgir nuestro mundo "desde un trasfondo... lo relevante es aquello que nuestro sentido común juzga como tal, siempre dentro de un contexto" (Varela F. , 1988, p. 89).

\section{La Danza Movimiento Terapia (DMT)}

La DMT es una especialidad de la psicoterapia que utiliza el cuerpo y sus expresiones no verbales como un proceso para promover la integración emocional, cognitiva y física del individuo (Stanton-Jones, 1992). Pertenece al grupo de las artes creativas en psicoterapia como el arte terapia, la musicoterapia y la dramaterapia. Según la Asociación Americana de Danza Movimiento Terapia (fundada en 1966 y responsable de establecer los estándares de calidad para la profesión de Danza Movimiento Terapia, oficialmente reconocida en Estados Unidos), se define como "el uso psicoterapéutico del movimiento para promover la integración emocional, cognitiva, física y social del individuo".

La DMT se basa en el principio de que existe una conexión entre la personalidad y el modo en que una persona se mueve; y que cambios en el patrón de movimiento pueden producir cambios en la psyche promoviendo una mayor salud emocional y física (Flaum Cruz y Florence Berrol, 2004). Como apunta Fischman (2005):

Se entiende aqui que la DMT es una modalidad o un abordaje enactivo que tiene como trasfondo una teoría del conocimiento que implica que el cuerpo conoce a través de su acción y crea los mundos en que vive, a la vez que es afectado y transformado por su contexto (p.31).

Este abordaje inminentemente enactivo permite un aprendizaje que emana de la relación consciente con la realidad corporal de cada individuo; es por tanto no imitativo, adaptativo a las distintas predisposiciones, habilidades y experiencias de los participantes.

La DMT como técnica corporal, se nutre de conocimientos sobre dinámica de grupos, desarrollo evolutivo, teorías psicológicas y psicoterapéuticas, análisis de movimiento, en concreto Laban Movement Analysis (Laban, 1987), y técnicas de danza como la Improvisación Contact, el Release, etc.

\section{Educación del Movimiento Somático: Body Mind Centering}

El término Educación del Movimiento Somático (Somatic Movement Education, SME) aglutina a un conjunto de disciplinas que se preocupan por crear condiciones de aprendizaje para ampliar la conciencia sobre el cuerpo y sus procesos físicos y subjetivos. Entre ellas cabe citar disciplinas como el Body Mind Centering ${ }^{\circledR}$, Método Feldenkrais ${ }^{\circledR}$, Alexander Technique y Rolf Technique. Este enfoque educativo y terapéutico, se ha desarrollado durante el último siglo a través de éstas y otras 
escuelas que comparten un proceso de continuo cuestionamiento, en torno al misterio de cómo la conciencia habita el cuerpo (ISMETA).

La educación del Movimiento Somático se ocupa de la experiencia consciente del cuerpo; de lo que le pertenece, experimenta y regula desde su propia existencia. Thomas Hanna (1976), el primero en acuñar el término, lo define como el estudio del ser desde la perspectiva de la experiencia vivida, incluyendo las dimensiones de cuerpo, psique y espíritu. El Movimiento Somático, pues, describe movimiento que es sentido y realizado desde la propia percepción del cuerpo, en lugar de desde el estímulo de una fuente externa.

Body-Mind Centering ${ }^{\circledR}\left(\mathrm{BMC}^{\circledR}\right)$ propone un enfoque integral y encarnado del movimiento, el cuerpo y la conciencia. Es un estudio vivencial basado en la fisicalización y aplicación de principios anatómicos, fisiológicos, psicofísicos y del desarrollo. Este enfoque utiliza diversos recursos para asistir el proceso de somatización; el movimiento consciente, el contacto, la voz y el sonido y la mente (Body Mind Centering, 2001). El propósito del estudio en BMC, es reconocer la diferenciación de cada una de las estructuras corporales y su expresión en movimiento; piel, sistema óseo, muscular, órganos, etc., para facilitar una mayor conciencia e integración de las mismas.

El estudio del Body-Mind Centering ${ }^{\circledR}$ es un proceso creativo en el cual la integración del material se lleva a cabo en un contexto de auto-descubrimiento y apertura. Cada persona es el estudiante, el profesor y la materia de estudio, el objetivo subyacente del trabajo es descubrir la facilidad con la que se llevan a cabo los procesos de transformación.

\section{Objetivos de trabajo}

Para la consecución del desarrollo de competencias a través de un abordaje enactivo y somático, los siguientes objetivos se presentan como indispensables:

- Asegurar la creación de un clima de trabajo en el que el participante se sienta seguro y cómodo para explorar su corporalidad, con autonomía e independencia y como parte del grupo, participando en la emergencia de un soporte grupal.

- Facilitar estructuras de trabajo donde la experiencia corporal pueda ser verbalizada, compartida y dotada de significado.

- Desarrollar una continuidad en la temática de las sesiones, asegurando que los contenidos respondan a un equilibrio entre las necesidades y expectativas del propio grupo y las metas que se le plantean abordar en forma de contenidos didácticos de trabajo.

\section{Estructura del programa de movimiento}

\section{Estructura general}

La estructura general del programa de movimiento se ha dividido en tres áreas de trabajo según las competencias que se pretenden desarrollar en cada una de ellas. 
El programa de movimiento se dividió en 13 sesiones de las cuales, 10 se dedicaron propiamente al trabajo corporal, manteniendo una estructura similar entre ellas y 3 se dedicaron al 'seguimiento e integración' de los contenidos visitados en las anteriores. Se organizaron 3 niveles de trabajo consecutivos en cuanto al desarrollo de competencias en el siguiente orden:

- Nivel 1: área intra-personal. Se dedicaron 3 sesiones de trabajo al desarrollo de competencias propias del área intrapersonal: autoconfianza, autoestima y autocontrol. En esta fase de trabajo, estas competencias consolidan la base del trabajo que a posteriori se llevará a cabo, estableciendo los parámetros sobre los que los participantes podrán continuar modulando su capacidad para hacerse cargo del proceso de aprendizaje.

- Nivel 2: área inter-personal. Sin dejar de fomentar el desarrollo de las competencias del nivel anterior, se dedicaron de nuevo 3 sesiones al trabajo al desarrollo de las habilidades comunicativas, la empatía y la toma de decisiones. En este nivel, los participantes han tomado confianza con el sistema de trabajo y son capaces de verbalizar la emergencia de conocimiento desde la experiencia individual y grupal, con mayor confianza. Asimismo, la confianza en el proceso les permite modular su participación en las sesiones de acuerdo a sus necesidades y expectativas, ejerciendo una toma de decisiones basada en la escucha de lo inter-personal y que en última instancia contribuye al desarrollo de una interrelación más consciente y empática.

- Nivel 3: integración. En esta última fase se dedicaron 4 sesiones al desarrollo de habilidades de observación y análisis del movimiento con el objetivo de facilitar herramientas para una observación objetiva de los procesos intra e inter personales. Se utiliza el Sistema de Análisis del Movimiento Laban (LMA) para profundizar en la auto-observación de patrones de movimiento y reflexionar sobre las aplicaciones al entorno laboral. Los niveles 1 y 2 sirven de base para garantizar la interiorización de este recurso así como para facilitar la comunicación efectiva y la escucha activa a la hora de compartir con el grupo, el conocimiento que se desprende de su aplicación en la práctica.

Las tres sesiones de 'seguimiento e integración' se llevan a cabo al principio del programa (sesión 1), al final del Nivel 2 (sesión 8) y al finalizar el Nivel 3 para concluir con el programa (sesión 13). En ellas se pone especial énfasis en la verbalización de los procesos de observación de las dinámicas intra e inter personales, se realizan dinámicas que ayuden a integrar e interiorizar los contenidos visitados en cada nivel y en el caso de la primera y la última, se estimula que los participantes expresen sus expectativas y grado de satisfacción respectivamente.

Es importante señalar, que los niveles de trabajo planteados y la distribución de las competencias a lo largo de cada uno de ellos, no se ha dado a conocer a los participantes. Se espera con este procedimiento, no condicionar la emergencia de conocimiento en base a la experiencia vivida durante el programa. Las reflexiones de los participantes constituyen por tanto, la evidencia de que el trabajo ha sido efectivo o no, en las áreas competenciales propuestas, posibilitando la identificación de resultados no previstos y la adecuación y re-estructuración del programa según los mismos. 


\section{Estructura de las sesiones}

Las sesiones de trabajo han mantenido una estructura similar con el objetivo de familiarizar a los participantes con una rutina de trabajo y fomentar el equilibrio entre el trabajo individual y grupal y entre comunicación verbal y no verbal:

- Calentamiento. En esta fase se trabaja con la preparación del cuerpo-mente para la sesión fomentando la toma de contacto con las propias percepciones corporales (propiocepción). Normalmente esta fase será guiada por la facilitadora y tendrá como objetivos secuenciales: la apertura de la escucha corporal y los sentidos, la concentración de la mente en el espacio y momentos actuales, la movilización progresiva y consciente del cuerpo y la identificación de temáticas que más tarde se desarrollarán con detalle en la sesión.

- Desarrollo. Una vez se establece un clima adecuado de trabajo, se formulan dinámicas en las que se invita a experimentar y profundizar, de manera vivencial, sobre aspectos concretos de la corporalidad. En parejas, grupos o de manera individual, el participante tiene la oportunidad de sumergirse en una experiencia de contacto consigo mismo y con el otro, a través de la comunicación no verbal. Las dinámicas que se proponen en esta fase y como venimos citando, provienen del BMC y la DMT y pueden llevarse a cabo con énfasis en el contacto, el uso del movimiento expresivo, la visualización, el juego, la imitación o 'mirroring', la observación del movimiento propio o ajeno, como vías hacia una mayor concienciación del continuo percepción/sensación/emoción/cognición/expresión. Dependiendo del contenido de la sesión, se puede estimular la inhibición del lenguaje verbal hasta el final de la sesión, o por el contrario facilitarse momentos para el intercambio de impresiones y reflexiones verbales.

- Reflexión individual. Al final de la parte experiencial, se dedican 15 minutos para que los participantes escriban en su diario reflexivo lo que consideren relevante en relación al trabajo realizado. Estas notas se recogen en cada sesión y se transcriben. Este tiempo de trabajo individual sirve como transición hacia la siguiente fase de verbalización en el 'círculo de cierre' y es un momento importante en el que a menudo surge esa conexión entre los aspectos no verbales de la experiencia somática y aquellos verbales de la experiencia cognitiva.

- Círculo de cierre. En esta fase final, los participantes y facilitadoras se sientan en torno a un círculo y se dejan 15 minutos para que cada uno comparta verbalmente lo que desee. Este es un momento importante del proceso en el que el significado individual del trabajo se comparte y contribuye a la creación de un significado y valor grupal. Es en esta parte de la sesión donde los docentes cuestionan la utilidad y aplicabilidad de la experiencia vivida en la sesión a su ámbito laboral. Es aquí que la experiencia de vida de cada uno de los participantes contribuye a dotar de significado la experiencia presente. El conocimiento emerge de la diversidad encarnada en la corporalidad y al compartirse, permite la constatación de esta pluralidad. Al verbalizar sobre nuestra realidad somática, enactuamos sobre nuestro entorno, dejando entrever la posibilidad de habilitar conexiones psicofísicas antes desconocidas. Las reflexiones son grabadas en audio y transcritas. 
Las facilitadoras de las sesiones de movimiento también realizan su propio diario reflexivo al finalizar cada sesión.

La información que se extrae de esta fase y de la anterior, contribuye a estructurar y moldear las propuestas y contenidos de las sesiones posteriores.

\section{Resultados}

\section{Descripción del grupo}

El grupo participante estaba constituido inicialmente por 22 personas de las cuales, transcurridas ya ocho sesiones de las trece planificadas en el momento de escribir este artículo, continúan 20. Estos profesores muestran un elevado grado de satisfacción con las sesiones de trabajo corporal lo que se evidencia en el compromiso con la actividad, con prácticamente un $90 \%$ de asistencia, y en la expectación que generan muchas de las dinámicas ("estoy muy contenta y ansiosa de lo que nos espera las siguientes clases" [14/02/2012_M1]).

A continuación se detallan algunos de los resultados preliminares, una vez analizada la información recogida por los investigadores. Estos resultados se presentan en dos partes. En primer lugar se muestran los resultados obtenidos en el cuestionario inicial del programa; y en segundo, los resultados preliminares de las reflexiones vertidas por los participantes en las sesiones del programa.

\section{Reflexiones iniciales}

Con el fin de conseguir una "foto inicial" del estado del grupo participante en el curso de formación, en la primera sesión se realizó un cuestionario inicial compuesto por 6 preguntas abiertas en las que se solicitaba una reflexión sobre el estado actual del docente respecto a su conciencia de los aspectos comunicativos no verbales. Estas mismas preguntas serán, de nuevo preguntadas al finalizar las sesiones, con el fin de medir si han aparecido variaciones en las repuestas.

Queremos resaltar en este artículo los resultados referentes a una de las preguntas del citado cuestionario que solicita que los participantes traten de recordar un profesor que conozca y admire y enumeren qué cualidades hacían de esa persona un docente especial. El objetivo de esta pregunta no era otro que tratar de vislumbrar las características que, según los profesores que iban a participar en el curso, debía tener un buen docente.

Tras transcribir las respuestas y analizarlas, aparecen los siguientes códigos preliminares, agrupados en categorías:

\begin{tabular}{|l|c|}
\hline Códigos preliminares & Categorías \\
\hline $\begin{array}{l}\text { Aprendizaje continuo } \\
\text { Experiencia }\end{array}$ & $\begin{array}{c}\text { Lo que sabe el profesor } \\
\text { (Experto en la materia o } \\
\text { Dominio de la materia }\end{array}$ \\
\hline Habilidades comunicativas (verbal y no verbal)
\end{tabular}




\begin{tabular}{|l|r|} 
Innovación & (Competencias didácticas. \\
Participativo & Habilidades como docente) \\
Motivación & \\
Organizado & \\
Justo/noble/Firme & \\
Compromiso & \\
Coherente & \\
Reflexivo & \\
Flexible & \\
\hline $\begin{array}{l}\text { Emociones } \\
\text { Cercanía }\end{array}$ & \\
Paciente & Lo que es el profesor. \\
Asequible & (Características de índole \\
Sincero & personal) \\
Sentido del humor/ alegre & \\
Habilidades sociales & \\
Entusiasmo & \\
\hline
\end{tabular}

Fuente: Elaboración propia

Tabla n.1. Fase inicial de categorización organizada a partir de unidades de significado de primer nivel y códigos de segundo nivel.

La categoría nombrada como Lo que sabe el profesor (Experto en la materia o disciplina) engloba unidades de significado referentes al dominio del profesor de la materia que impartía, su experiencia en el campo de impartición (como investigador, por ejemplo) y la capacidad de aprendizaje continuo. Los profesores destacan, como era esperable, como cualidades del buen docente aquel que conoce bien su materia (y la disfruta), que investiga en ella y que actualiza sus conocimientos.

La categoría nombrada como Lo que hace el profesor (Competencias didácticas. Habilidades como docente) engloba todos aquellos códigos que podemos encuadrar dentro de las habilidades de un profesor relacionadas con aspectos pedagógicos. En este sentido los docentes señalan las habilidades comunicativas orales ("comunica con claridad", "capacidad de comunicar con sencillez conceptos complicados y hacerse entender") como cualidad del buen docente, pero también las no verbales. Un docente capaz de motivar a los alumnos, de introducir elementos innovadores en el aula, firme y justo, comprometido con su profesión o coherente en sus decisiones.

Y una tercera categoría nombrada como Lo que es el profesor (Características de índole personal), en la que los profesores señalan otras características relacionadas con el ser del profesor. En ellas aparecen cualidades como: ser cercano, asequible a alumnos y compañeros, sincero, con buen humor, con control de sus emociones, entusiasta, con "don de gentes"...

Estos resultados obtenidos coinciden con otras experiencias similares recogidas en la literatura pedagógica (Zabalza Beraza y Zabalza Cerdeiriña, 2011b). En ellos, al preguntar por el mejor profesor que recuerdan, todos los participantes señalan con mayor frecuencia otros aspectos no relacionados directamente con el dominio de la 
materia impartida o su trayectoria investigadora, sino con habilidades pedagógicas y otras características personales.

Resulta claro que, aunque los conocimientos específicos o técnicos son fundamentales (el "saber" del docente), no es suficiente con ser un experto en lo que se imparte, es también necesario desarrollar otras cualidades que permitan facilitar el aprendizaje de nuestros estudiantes (lo que "hace") y que impregnen el aula de nuestro "ser", pues nuestra forma de vivir la docencia repercute en la forma de hacer nuestro trabajo.

\section{Resultados preliminares}

A partir de los comentarios vertidos por los profesores en los diarios reflexivos realizados en cada sesión, se identifican cuatro grandes líneas, que describen los pensamientos, impresiones, inquietudes y observaciones de los docentes en torno al desarrollo de competencias intrapersonales, de las interpersonales, a una mayor percepción del entorno y a la posible aplicabilidad de lo desarrollado en el aula.

\section{Desarrollo de Competencias intrapersonales}

En este epígrafe se recogen reflexiones acerca de cómo se sienten los profesores en el transcurso de las dinámicas y sobre cómo influye la sesión en sus estados de ánimo y niveles de energía.

A partir de sus comentarios, se observa que las sesiones producen una relajación en los docentes, que inician con un alto grado de tensión arrastrado de sus quehaceres diarios y logran marcharse con mayor sensación de tranquilidad, bienestar y optimismo,

"como siempre he llegado tensa y acelerada a la sesión y termino con una paz interior y una tranquilidad impresionante" [13/3/2012-11].

Los docentes perciben su participación en estas sesiones como un beneficio personal, "realmente me lo tomo como un tiempo de regalo para mi" [14/02/2012-2].

En ellas, reconocen la conexión entro lo físico y lo emocional. Esta conexión se refleja en comentarios como:

"durante la sesión he ido estableciendo conexiones entre lo físico y lo emocional, hasta el punto de poderme relajar emocionalmente al relajarme físicamente" [14/02/2012-5]

"siento que conecto mi cuerpo y mi mente llegando a una sintonía que me permite avanzar y terminar el día disfrutándolo más" [13/3/2012-11].

En particular, uno de los momentos de las sesiones mejor valorados por los docentes participantes en el programa es el calentamiento inicial, entendido como una herramienta de relajación muy útil que permite desconectar del exterior y que predispone cuerpo y mente para el trabajo de las dinámicas. En este sentido encontramos los siguientes comentarios:

"me encanta la primera parte en la que creo nos hacemos más conscientes de nosotros mismos, de nuestro cuerpo, nos desconectamos de nuestros quehaceres y problemas y 
también entramos en una fase de más relajación, que nos prepara emocionalmente para el resto de la actividad" [21/02/12-M1]

"fantástica relajación inicial, se fomenta la liberación de pensamientos y la conciencia del propio cuerpo preparando la mente para los ejercicios posteriores" [28/02/12-11].

También se observa, a partir de sus comentarios en las reflexiones diarias, cómo los profesores incorporan paulatinamente nuevo vocabulario específico en la descripción de sus estados físicos, "he observado diferencia entre los diferentes estados de tonicidad. La verdad es que en mi caso, algunos factores me han llevado más al terreno de la hiperlaxitud" [13/03/2012-4].

El auto-conocimiento se desarrolla a través de ejercicios tipo mirroring como aquellos en los que "imitan el andar" del compañero. Este tipo de dinámicas se señalan especialmente útiles para este objetivo, "el de hoy es el día que más he aprendido... sobre mí mismo" [13/03/2012_M-3].

\section{Percepción del entorno}

En este epígrafe se analizan las reflexiones vertidas acerca de los ejercicios que se han llevado a cabo dirigidos a incrementar la conciencia de percepción del entorno.

Por un lado, los docentes participantes han comprobado cómo los diferentes estados de tonicidad afectan a la percepción del entorno.

"la sesión de hoy me ha mostrado cómo el cuerpo entero es un órgano perceptivo y cómo soy más o menos sensible a las percepciones dependiendo del estado físico previo. Hay estados de mayor tensión y alerta y estados más relajados. En los estados más tensos se pierden matices perceptivos. En los estados más relajados se perciben más matices" [28/02/2012_7].

También se incrementa la percepción del lugar que ocupa el propio cuerpo, "este curso me está ayudando muchísimo a controlar mi espacio vital" [21/02/2012_M1]. La dinámica titulada "elijo mi espacio" sorprende a los profesores al encontrar placer al ser consciente del lugar que se ocupa, "sin duda el ejercicio que más me ha gustado y con el que más cómodo me he sentido ha sido el último que hemos realizado, localizando nuestro propio sitio en el espacio, en silencio. Además de encontrar mi propio sitio y de por ejemplo dejar que mi cuerpo abrazara la columna del fondo cuando mi cabeza estaba diciendo que no lo hiciera, me ha parecido un ejercicio lleno de belleza corporal" [14/02/2012_M6].

\section{Desarrollo de Competencias interpersonales: El cuerpo como vehículo de expresión.}

En este epígrafe se recogen aquellas reflexiones vertidas por los docentes que hacen referencia al desarrollo de aquellas habilidades o competencias relacionadas con las relaciones con los demás y la integración en el grupo.

Por un lado, se observa una mayor conciencia de la capacidad del cuerpo como vehículo de expresión: "nunca me había planteado que se pudiera hablar con el cuerpo" [14/02/2012_4], "la posibilidad de conocer a otros compañeros simplemente a través del movimiento y el juego me resulta agradable" [21/02/2012-11]. 
En particular, se comprueba como los diferentes estados de tonicidad influyen en la capacidad de comunicación. Por ejemplo los docentes los relacionan de la siguiente manera: "extremos hipotónico / hipertónico = incomunicación; actitud neutra = disfrute, cordialidad, amabilidad" [27/03/2012-2].

Otro hecho destacable que se ha producido en el programa es la mayor comodidad ante el contacto físico que experimentan los docentes después de varias sesiones

"el contacto físico, tocarse, que está tan reprimido en el trato habitual entre personas, va siendo cada vez aquí más natural, menos forzado. Creo que la mayoría nos vamos sintiendo cómodos. Yo estoy a gusto y me parece muy rica la espontaneidad, la improvisación que va apareciendo" [21/02/2012_11].

\section{Aplicabilidad a la docencia}

En este apartado se analizan las reflexiones que hacen los profesores sobre cómo lo vivenciado puede beneficiar su labor docente.

Si bien al inicio del programa se evidencia escepticismo a este respecto ("la verdad es que todavía no soy consciente de cómo aplicaremos lo que hacemos aquí al entorno docente" [14/02/2012_MO]), a medida que el curso ha avanzado, este tipo de comentarios son menos frecuentes y surgen reflexiones concretas sobre su posible aplicabilidad.

Por un lado se establecen analogías entre lo que sucede en las dinámicas y lo que ocurre en el aula. En este sentido, un docente declaraba que "tengo que decir que esas mismas sensaciones aparecen muchas veces también en algunas clases en las que por, ejemplo, toda mi atención está dirigida a un solo alumno, o a algún gesto en concreto de malestar de algún alumno. En ese caso, aparece esa sensación de rigidez muscular y me despisto fácilmente y hace que quiera acabar la clase cuanto antes mejor" [21/02/2012_M6]. Otro hablaba en estos términos sobre su manera de andar y lo que esa forma transmite a los alumnos: "en la medida de lo posible, me planteo corregir mi propia manera de andar. Si ando como si fuese a entrar en una batalla, necesariamente transmitiré inquietud o incluso lejanía a los demás (incluyendo a mis alumnos, claro)" [13/03/2012_M3].

Por otro lado, se identifican otros recursos que pueden ser aplicados directamente al trabajo en el aula, "Ia adaptación activa al grupo, es decir, el grupo influye en mi pero yo influyo en el grupo, puede ser una herramienta útil en la gestión de un aula. Aprender a integrarte en un grupo influyendo a la vez en él sin fricciones puede facilitar la gestión de un grupo de alumnos [21/02/2012_8].

Otra idea la encontramos en estas palabras: "aprender a captar la situación en que se encuentra el alumno a través de su lenguaje, nos permitirá gestionar mejor la clase" [27/03/2012-_4].

En particular, la sesión dedicada a cómo los estados de ánimo (o la conducta) influyen en la comunicación $y$, en particular, la simulación realizada sobre cómo cambiar la actitud negativa de un alumno manejando nuestro propio estado, es destacada muy especialmente por los profesores como la más útil e interesante desde el punto de vista de la aplicabilidad a labor docente,

"ha sido una sesión de gran utilidad para mi docencia" [27/03/2012_M7] 
"me ha parecido una clase muy útil para nuestro día a día y para nuestra labor docente" [27/03/2012_8].

\section{Conclusiones}

Los profesores deben adaptarse rápidamente a todos los cambios que trae consigo el nuevo paradigma educativo de la educación superior en el que estamos inmersos y necesitan de nuevas herramientas que faciliten su labor. Éstas deben proceder de una formación específica que se adapte a sus necesidades, y les prepare para promover aprendizajes significativos y efectivos en sus alumnos. En concreto, se hace necesario ir un paso más allá en la formación específica de cada área de conocimiento y/o pedagógica, incorporando aspectos de carácter emocional que promuevan relaciones interpersonales satisfactorias y útiles para mejorar los procesos de enseñanzaaprendizaje. Pero además consideramos que esta formación debe ser fundamentalmente de tipo vivencial pues la incorporación de procesos de conocimiento enactivo aumenta el grado de autoconocimiento del docente y le hace ser más consciente de sus capacidades expresivas y comunicativas.

El proyecto de desarrollo de competencias intra e interpersonales en docentes universitarios a través de un programa de trabajo corporal y comunicación no verbal que aquí presentamos, aún sin haberse finalizado, muestra resultados preliminares muy prometedores.

Los docentes manifiestan un elevado grado de satisfacción con la iniciativa y se muestran comprometidos con la misma. Prácticamente desde la segunda sesión comienzan a conectar la vivencia a nivel somático y de interrelación con los compañeros, con aspectos relacionados al trabajo en el aula. Las sesiones se perciben como un beneficio tanto a nivel personal como profesional, y los docentes reconocen la conexión entre lo físico y lo emocional e incorporan nuevo vocabulario sobre aspectos corporales. Se comprueba cómo los estados de tonicidad afectan a la percepción del entorno y a la capacidad de comunicación, llevando esto al caso concreto del trabajo en el aula, y a las dificultades que surgen en ocasiones a nivel comunicativo. Los participantes empiezan a ser más conscientes de la información noverbal que se mueve en el aula y en las relaciones con los compañeros, e incorporan recursos que pueden aplicar a la gestión de los grupos en la docencia.

Sin duda alguna, las reflexiones conjuntas por parte de los participantes se dan en un clima de confianza que permite a cada cual enriquecerse personalmente al reconocer los propios límites y ponerlos en común con los demás. A partir de este programa se ha creado una pequeña comunidad de aprendizaje que desea mejorar no sólo en su "saber" sino en su "ser" y esto es lo mejor que, como docentes, podemos ofrecer a nuestro alumnado.

Este programa es fácilmente transferible a cualquier grupo de docentes perteneciente a otros niveles educativos, ya que incide en la competencia emocional docente, que debe ser desarrollada por todos aquellos que quieran favorecer el aprendizaje, independientemente de la edad.

Como líneas de futuro, las autoras de este trabajo esperan seguir reforzando estos resultados preliminares hasta la finalización del proyecto. Igualmente, tienen 
interés en seguir profundizando en esta línea, ofreciendo nuevas posibilidades de formación para docentes que quieran mejorar en su nivel competencial.

\section{Referencias bibliográficas}

Alles, M. A. (2005). Gestión por competencias. El diccionario. Buenos Aires: Granica.

Álvarez de Arcaya Ajuria, H. (2004). Influencias de la comunicación no verbal en los estilos de enseñanza y en los estilos de aprendizaje. Revista de Educación(334), 21-32.

Ambady, N., y Rosenthal, R. (1993). Half a minute: predicting teacher evaluations from thins slices of behavior and physical attractiveness. Journal of Personality and Social Psychology, 64(3), 431-441.

Babad, E., Avni-Babad, D., y Rosenthal, R. (2004). Prediction of Students' Evaluations from Brief Instances of Professors' Nonverbal Behavior in Defined Instructional Situations. Social Psychology of Education, 7(1), 3-33.

Bandura, A. (1997). Self-Efficacy: The Exercise of Control. New York: W. H. Freeman.

Bat-Sheva, K. (1994). A concept of "body knowledge" and an evolving model of "movement experience": Implications and application for curriculum and teacher education. American Journal of Dance Therapy, 16(1), 21-48.

Benito, Á., Bonsón, M., y Icarán, E. (2005). Metodologías activas. En Á. Benito, y A. Cruz, Nuevas claves para la docencia universitaria en el Espacio Europeo de Educación Superior (págs. 21-64). Madrid: Narcea Universitaria.

Blanco Fernández, A. (2009). Desarrollo y evaluación de competencias en educación superior. Madrid: Narcea Universitaria.

Body Mind Centering. (2001). The School for Body-Mind Centering. An embodied approach to movement, body and consciousness. Recuperado el 5 de Abril de 2012, de http://www.bodymindcentering.com/

Boyatzis, R., Mckee, A., y Johnston, F. (2008). Líder emocional. Barcelona: Ediciones Deusto.

Camerino I Foguet, O., Castañer I Balcells, M., y Buscá, F. (1999). Mejora de la comunicación verbal y no-verbal en las aulas universitarias. Revista Electrónica de Formación del Profesorado, 2(1).

Cano García, M. E. (2008). La evaluación por competencias en la educación superior. Profesorado. Revista de curriculum y formación de profesorado, 13(3), 1-16.

Capello, P. (2008). En H. Wengrower, y S. Chaiklin, La vida es danza. Arte y Ciencia de la Danza Movimiento Terapia. Barcelona: Gedisa.

Coates, R. D. (2002). I Don't Sing, I Don't Dance, and I Don't Play Basketball! Is Sociology Declining in Significance, or Has it Just Returned to Business as Usual? Critical Sociology, 28(1), $255-279$.

Darwin, C. (1984). La expresión de las emociones en los animales y el hombre. Madrid: Alianza. 
De Miguel Díaz, M. (2006). Metodologías de enseñanza y aprendizaje para el desarrollo de competencias. Orientaciones para el profesorado universitario ante el espacio europeo de educación superior. Madrid: Alianza Editorial.

De Paulo, B. M. (1992). Non-verbal behavior and self-presentation. Phychological Bulletin, 111, 203-243.

Domenech Delgado, B. (1995). Introducción al síndrome "burnout" en profesores y maestros y su abordaje terapéutico. Psicología Educativa, 1(1), 63-78.

Elliot, J. (2000). La investigación-acción en educación. Madrid: Morata.

Emmer, E. (1994). Teacher emotions and classroom management. Annual Meeting of the American Educational Research. New Orleans.

Erk, S., Kiefer, M., Grothe, J., Wunderlich, A. P., Spitzer, M., y Walter, H. (February de 2003). Emotional context modulates subsequent memory effect. Neurolmage, $18(2), 439-447$.

Extremera, N., y Fernández-Berrocal, P. (2002). Relation of perceived emotional intelligence and health-related quality of life of middle aged women. Psychological report, 91, 47-59.

Extremera, N., y Fernández-Berrocal, P. (2006). Emotional intelligence as predictor of mental, social, and physical health in university students. The Spanish Journal of Psychology, 9(1), 45-51.

Fernández-Berrocal, P., Ramos, N., y Extremera, N. (2001). Inteligencia Emocional. Supresión crónica de pensamientos y ajuste psicológico. Boletín de Psicología, 70, 79-95.

Fischman, D. (2005). La mejora de la capacidad empática en profesionales de la salud y la educación a través de talleres de Danza Movimiento Terapia. Recuperado el 8 de marzo de 2012, de SCribd: Tesis doctoral: http://es.scribd.com/doc/52363713/tesis-doctorado-dra-diana-fischman

Flaum Cruz, R., y Florence Berrol, C. (2004). Dance Movement Therapists in Action. A Working Guide of Research Options. Springfield, Illinois: Charles C Thomas Publisher.

Frenzel, A. C., Goetz, T., Lüdtke, O., Pekrun, R., y Sutton, R. E. (2009). Emotional transmission in the classroom: Exploring the relationship between teacher and student enjoyment. Journal of Educational Psychology, 101(3), 705-716.

Galloway, C. M. (June de 1977). Nonverbal: Authentic or Artificial. Theory into Practice, $16(3), 129-133$.

García García, M. J., Terrón López, M. J., y Blanco Archilla, Y. (Diciembre de 2010). Desarrollo de Recursos Docentes para la Evaluación de Competencias Genéricas. (AENUI, Ed.) ReVisión, 3(3), 17-37.

García Olalla, A., y Poblete Ruiz, M. (2007). Desarrollo de competencias y créditos transferibles: experiencia multidisciplinar en el contexto universitario. Bilbao: Ediciones Mensajero, S.A.U.

Gardner, H. (1995). Inteligencias múltiples. La teoría en la práctica. Barcelona: Paidós. 
Goleman, D. (1996). La inteligencia emocional. Barcelona: Kairós.

Guilford, J. P., y Hoepfner, R. (1971). Analysis of Intelligence. New York: McGraw-Hill.

Hamann, S., y Mao, H. (2002). Positive and negative emotional verbal stimuli elicit activity in the left amygdala. Neuroreport, 13(1), 15-19.

Hanna, E. C. (1976). What is somatics? Recuperado el 7 de septiembre de 2012, de Somatics Educational Resources: www.somaticsed.com/whatls.html

Hargreaves, A. (2000). Mixed emotions: teachers' perceptions of their interactions with students. Teaching and Teacher Education, 16(8), 811-826.

ISMETA. (s.f.). International Somatic Movement Education y Therapy Association. Recuperado el 10 de abril de 2012, de http://www.ismeta.org

Kemmis, S., y Mctaggart, R. (1988). Cómo planificar la investigación-acción. Barcelona: Laertes.

Laban, R. (1987). El dominio del movimiento. Madrid: Fundamentos.

Lapierre, A., y Aucoutourier, B. (1983). La simbología del movimiento. Barcelona: Científico-Médica.

Latorre Beltrán, A. (2003). La investigación-acción: conocer y cambiar la práctica educativa. Barcelona: Graó.

Maturana, H., y Varela, F. (1985). El árbol del conocimiento: Las bases biológicas del entendimiento humano. Santiago de Chile: Editorial Universitaria.

Mearns, J., y Cain, J. E. (2003). Relationships between Teachers' Occupational Stress and Their Burnout and Distress: Roles of Coping and Negative Mood Regulation Expectancies. Anxiety, Stress y Coping: An International Journal, 16(1), 71-82.

Niedenthal, P. M., y Kitayama, S. (1994). The heart's eye: Emotional influences in perception and attention. San Diego, California: Academic Press.

Payne, H. (1992). Dance Movement Therapy: Theory and practice. London: Jessica Kingsley Publishers.

Poblete Ruiz, M., y Villa Sánchez, A. (2007). Aprendizaje basado en competencias: una propuesta para la evaluación de las competencias genéricas. Bilbao: Ediciones Mensajero, S.A.U.

Prozesky, D. R. (2000). Communication and Effective Teaching. Journal of Community Eye Health, 13(35), 44-45.

Ramachandran, V. S. (2008). Los laberintos del cerebro. Barcelona: La liebre de marzo.

Rizzolatti, G., y Sinigaglia, C. (2006). Las neuronas espejo. Los mecanismos de la empatía emocional. Barcelona: Paidós.

Rodríguez Jiménez, R. M. (2010). Uso de la creatividad para el desarrollo de competencias: una propuesta a través del movimiento y la danza creativa. $\mathrm{VI}$ Congreso Internacional Docencia Universitaria e Innovación, (págs. 44-62). Barcelona. 
Rodríguez Jiménez, R. M., Terrón López, M. J., y Gracia Parra, P. (2010). Recursos creativos corporales y uso de la comunicación no-verbal para el desarrollo y evaluación de competencias genéricas en alumnos de grado. Revista de Docencia Universitaria, 8 (1), 142-157.

Rué Domingo, J. (2008). Formar en competencias en la universidad: entre la relevancia y la banalidad. Revista de Docencia Universitaria. Número monográfico I. Formación centrada en competencias. Recuperado el 30 de mayo de 2011 de: http://revistas.um.es/redu/article/view/10631

Salovey, P., Mayer, J. D., Goldman, S. L., Turvey, C., y Palfai, T. (1995). Emotional attention, clarity, and repair: Exploring emotional intelligence using the Trait Meta-Mood Scale. En J. Pennebaker, Emotion, disclosure, and health (págs. 125154). Washington, D.C.: American Psychological Association.

Sandín Esteban, M. P. (2003). La investigación cualitativa en educación. Fundamentos y tradiciones. Madrid: McGraw Hill.

Stanton-Jones, K. (1992). An Introduction to Dance Movement Therapy in Psychiatry. London: Routledge.

Sutton, R. (2004). Emotional regulation goals and strategies of teachers. Social Psychology of Education, 7 (4), 379-398.

Sutton, R. E., y Wheatley, K. F. (2003). Teachers' Emotions and Teaching: A Review of the Literature and Directions for Future Research. Educational Psychology Review, 15 (4), 327-358.

Terrón López, M., Blanco Archilla, Y., Berenguer Ciscar, F., y Learreta Ramos, B. (2007). La coordinación del profesorado como necesidad en la construcción del EEES: Una experiencia en investigación-acción. 150 Congreso Universitario de Innovación Educativa en las Enseñanzas Técnicas. Escuela Universitaria Politécnica de Valladolid.

Valcárcel Cases, M. (Noviembre de 2003). La Preparación del Profesorado Universitario Español para la Convergencia Europea. Recuperado el 13 de Abril de 2012, de Informe final del programa de Estudios y Análisis: http://campus.usal.es/webusal/Novedades/noticias/bolonia/informe final.pdf

Varela, F. (1988). Conocer: Las Ciencias Cognitivas, tendencias y perspectivas. Barcelona: Gedisa Editorial.

Varela, F. J., Thompson, E., y Rosch, E. (1997). De cuerpo presente. Barcelona: Gedisa.

Velasco Quintana, P. J., Terrón López, M. J., Rodríguez Jiménez, R. M., Blanco Archilla, Y., Blanco Fernández, A., Fernández Santander, A., Sáez Pizarro, B. (2011). Identificación y análisis de las herramientas de evaluación por competencias en los grados de la UEM. Actas de las VIII Jornadas Internacionales de Innovación Universitaria. Villaviciosa de Odón: Universidad Europea de Madrid.

Zabalza Beraza, M. Á., y Zabalza Cerdeiriña, M. A. (2011a). Profesores y profesión docente. Entre el "ser" y el "estar". Madrid: Narcea. 
Zabalza Beraza, M. Á., y Zabalza Cerdeiriña, M. A. (2011b). Los profesores y profesoras como personas. En M. Á. Zabalza Beraza, y M. A. Zabalza Cerdeiriña, Profesores y profesión docente. Entre el "ser" y el "estar" (pág. 48). Madrid: Narcea.

Zabalza, M. Á. (2003). Competencias docentes del Profesorado universitario. Madrid: Narcea.

Zabalza, M. Á. (2009). Ser profesor universitario hoy. La cuestión universitaria, 5, 6981.

Cita del artículo:

Rodríguez Jiménez, R.M.; Caja López, M.M.; Gracia Parra, P.; Velasco Quintana, P.J.; Terrón López, M.J. (2013). Inteligencia Emocional y Comunicación: la conciencia corporal como recurso. Revista de Docencia Universitaria. REDU. Vol.11 (1) EneroAbril. pp. 213-241. Recuperado el (fecha de consulta) en http://www.red-u.net/

\section{Acerca de las autoras}

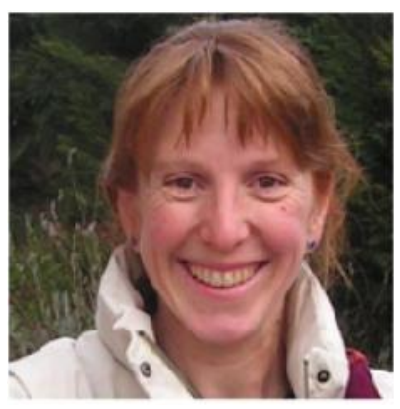

\section{Rosa $M^{a}$ Rodríguez Jiménez}

\section{Universidad Europea de Madrid}

Departamento de Ciencias

Mail: rosamaria.rodriguez@uem.es

Doctora en Ciencias Físicas por la UCM. Diplomada en Educación Especial por la UPS. Bailarina profesional y Máster en Danza Movimiento Terapia por la UAB. Profesora Titular de Física Aplicada en la Universidad Europea de Madrid de la cual es docente desde 1998 de Física, Biomecánica Aplicada a la Danza, DMT y Movement Skills. Miembro titular de la Asociación Española de Danza Movimiento Terapia. Desde 2001 hasta 2009, Directora de la Oficina de Voluntariado y Cooperación en la UEM. Autora de diversas ponencias y publicaciones sobre danza y movimiento creativo, innovación docente, educación y formación en valores en el ámbito universitario, y participante en congresos nacionales e internacionales sobre temas educativos y cooperación al desarrollo. Psicoterapeuta corporal con experiencia en personas con discapacidad, tercera edad, trastornos de la alimentación y colectivos en riesgo de exclusión social. 


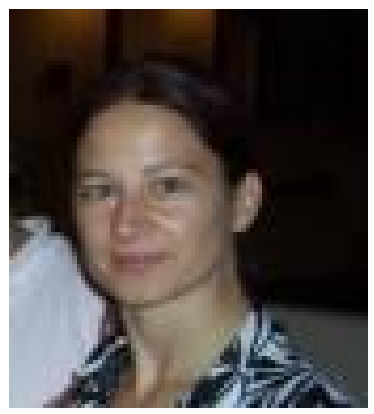

\section{María del Mar Caja López}

Universidad Europea de Madrid

Departamento de Ciencias. Escuela Politécnica.

Mail: mariadelmar.caja@uem.es

Doctora en Ciencias Químicas por la UCLM, desarrolla su labor investigadora en el Instituto de Fermentaciones Industriales (CSIC) y en la Julius-Maximilians UniversitätWürzburg (Alemania), siendo coautora de diversos artículos publicados en revistas del $\mathrm{SCl}$. Desde septiembre de 2009, es profesora adjunta del departamento de Ciencias de la Universidad Europea de Madrid (UEM) e imparte clases de química en los grados de Farmacia, Biotecnología e Ingeniería. Posee estudios no reglados de danza y ha recibido en la UEM diversos cursos de formación relacionados con la innovación docente en general y con la mejora de la actividad docente a través de la comunicación - no verbal en particular.

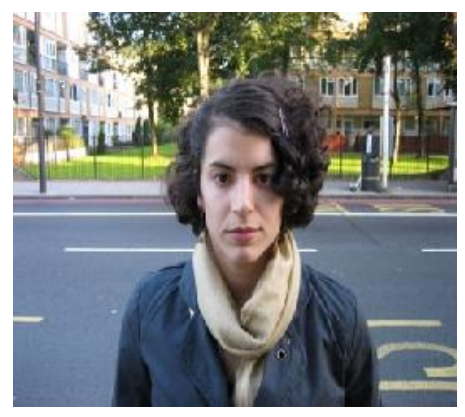

\section{Patricia Gracia Parra}

\section{Universidad Europea de Madrid}

Departamento Fundamentos de la Motricidad y el Entrenamiento Deportivo.

Mail: Patricia.gracia@uem.es

Licenciada en Danza Contemporánea en el London Contemporary Dance School donde además cursa un Máster en Performance y otro en pedagogía de la Danza Contemporánea. Máster en Danza Movimiento Terapia (DMT) en la UAB. Durante siete años de residencia permanente en Inglaterra trabaja y colabora como pedagoga y coterapeuta especialista en pedagogía terapéutica del Movimiento y la Danza en instituciones dedicadas al trabajo educativo-terapéutico con poblaciones infantiles, adolescentes y jóvenes en riesgo de exclusión. En la actualidad compagina su labor como profesora de técnica y didáctica de la Danza Contemporánea en la UEM (Madrid), bailarina, y psicoterapeuta en DMT, manteniendo su investigación y formación en el área de la Educación en Movimiento Somático (SME). 


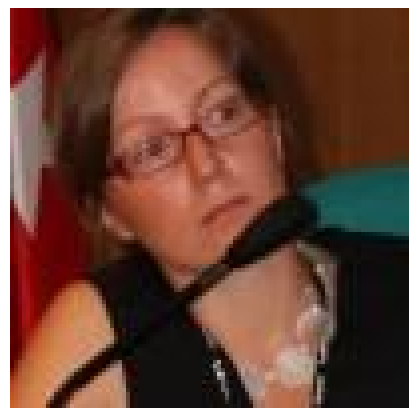

\section{Paloma J. Velasco Quintana}

\section{Universidad Europea de Madrid}

Departamento de Ciencias. Escuela Politécnica.

Mail: pjulia.velasco@uem.es

Doctora en Educación y Licenciada en Ciencias Matemáticas. Actualmente es profesora del departamento de Ciencias de la Escuela Politécnica en la Universidad Europea de Madrid. Con más de 10 años dedicada a la docencia universitaria, su actual línea de investigación está relacionada con el desarrollo de programas de tutoría entre iguales y la aplicación de nuevas metodologías de enseñanza-aprendizaje. Ha participado en varios proyectos de investigación relacionados con dichas líneas. Algunos de los resultados obtenidos se han recogido en diversos trabajos de investigación (comunicaciones en congresos, artículos en revistas, capítulos de libro, etc.).

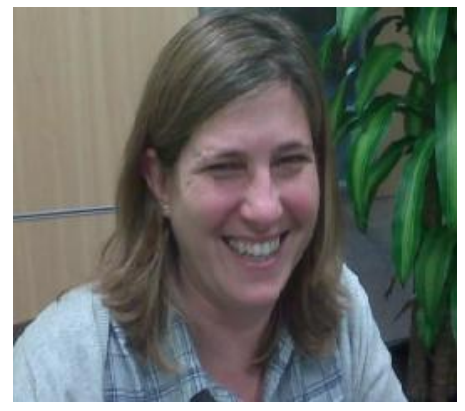

\section{María José Terrón López}

Universidad Europea de Madrid

Departamento de Electromecánica y Materiales

Mail: $\underline{m}$ jose.terron@uem.es

Profesora de la Escuela Politécnica de la Universidad Europea de Madrid (U.E.M.), donde entre otros cargos ha sido directora de departamento y de titulación y responsable del programa de formación del Profesorado. Licenciada en C.C. Físicas, especialidad Electrónica, en la Universidad Complutense de Madrid se doctoró en la Universidad Politécnica de Madrid en el programa de Energía Solar Fotovoltaica. Ha participado en proyectos de investigación nacionales e internacionales en diversas áreas. Su actual línea de investigación está relacionada con la aplicación de nuevas metodologías de enseñanza-aprendizaje y el desarrollo de competencias en los estudiantes, temas en los que tiene diversas publicaciones. Ha participado como experto en conferencias y talleres para la formación del profesorado universitario en diversas universidades españolas. 


\title{
EI EEES, identidad y competitividad Europea: Principios fundamentales e interpretación de las principales autoridades.
}

\author{
The EHEA, European identity and competitiveness: Fundamental principles and \\ interpretation of the principal authorities.
}

\author{
Miquel Àngel Comas
}

Universitat Internacional de Catalunya, España

\section{Resumen}

El proceso de construcción del Espacio Europeo de Educación Superior (EEES) no ha estado exento de controversia y de diferentes niveles de interpretación en su creación, fundamentación y desarrollo.

El objetivo de este artículo es hacer un cierto retrato de la situación e ir más allá del estudio a nivel teórico, en base a los documentos oficiales que han ido acompañando y complementando las diferentes reuniones europeas de Ministros responsables de la educación superior. A partir de entrevistas realizadas en profundidad, en formato semiestructuradas, hemos encontrado interesante captar las opiniones, expectativas y percepciones de actores responsables y activos del proceso de construcción del EEES: Guy Haug, Domenico Lenarduzzi, Marius Rubiralta, Eric Froment, Josep M. Bricall y Jorge Fernández Díaz. Todos ellos, con relevancia política y universitaria, estando implicados desde diferentes instituciones: Conferencia Europea de Rectores, European University Association, Dirección General de Educación de la Comisión Europea y diferentes Ministerios de Educación Superior. Sus contribuciones, han aportado conocimiento para contrastar y ofrecer una mejor y mayor comprensión del despliegue del EEES.

El estudio concluye, partiendo de la triangulación de las aportaciones de los entrevistados y la revisión de documentos oficiales en diferentes ámbitos, que la Universidad (conjuntamente con la investigación - Espacio Europeo de Investigación y la formación permanente - Espacio Europeo de Formación Permanente), es el eje del desarrollo de la sociedad y sello distintivo de Europa, pero necesita adaptarse a los nuevos tiempos y vincularse al concepto de competitividad y eficiencia, y ofrecer una mayor y mejor cooperación y proximidad para favorecer la movilidad y el reconocimiento mutuo. Así mismo, se establece que la creación del EEES responde a una lucha de poder entre el derecho de autonomía de las universidades, el derecho legislativo de los gobiernos estatales y las recomendaciones de la Comisión Europea. Sin embargo, todos coinciden en plantear una reflexión de autocrítica respecto al esfuerzo de información y difusión para evitar posibles interpretaciones erróneas o malentendidos y posibilitar mayores y mejores vías de participación.

Palabras clave: Educación Superior, Universidad, formación permanente, EEES, EEFP, EEI, investigación, Bolonia. 


\section{Abstract}

The process of building the European Higher Education Area (EHEA) has not been without controversy and different levels of interpretation in its creation, foundation and development.

The aim of this paper is to portray the situation and go beyond the theoretical study, based on official documents which have accompanied and complemented meetings of European ministers responsible for higher education. From these deep interviews, in semi-structured format, we found interesting to capture the views, expectations and perceptions of responsible and active actors in the process of building the EHEA: Guy Haug, Domenico Lenarduzzi, Marius Rubiralta, Eric Froment, Josep M. Bricall and Jorge Fernández Díaz. All of them with political and university relevance have been implicated from different institutions: European Conference of Rectors, European University Association, Department of Education of the European Commission and various Ministries of Higher Education. Their contributions have provided knowledge to contrast and offer a better understanding of EHEA display.

Based on the triangulation of the contributions of the interviewees and the review of official documents and statements the study concludes that the university (together with the research European Research Area and lifelong learning - European Lifelong Learning Area) is the fundamental hinge for society development and European seal. But it needs to adapt to changing times and to be linked to competitiveness and efficiency. It also needs to provide more and better cooperation and proximity to encourage mobility and mutual recognition. Besides, the creation of EHEA corresponds to a power struggle between the University autonomy rights, the legislative right of state governments and the European Commission's recommendations. However, all of them agree to raise a self-critical reflection as regards the diffusion of information so as to avoid possible misinterpretation or misunderstanding and enable more and better ways of participation.

Key words: Higher education, University, lifelong learning, EHEA, ERA, ELLLP, Research, Bologna.

\section{Europa y el nuevo reto educativo.}

La educación, la formación y la investigación son ejes prioritarios en las políticas estratégicas de la Unión Europea y responden a una necesidad de crear un sello propio y común, con una clara visión de futuro, de compromiso y de calidad, haciendo que se convierta en un gran reto y oportunidad pedagógica.

Desde el origen del EEES y la Estrategia de Lisboa (COM 2000), se establece la necesidad y el reto de complementar y consolidar el Espacio Europeo de Formación Permanente (COM 2001) con el Espacio Europeo de Investigación (COM 2003). En la Europa del conocimiento (COM 2003) el papel de las universidades pasa a ser prioritario y estratégico y se define en dos fechas 2010 y 2020. La formación permanente y la investigación se conciben sobre la base de la libre circulación de ciudadanos europeos y de la posibilidad de ofrecer mejores oportunidades formativas y laborales, ambos en relación con los principios de movilidad y reconocimiento.

La plena adecuación a la sociedad del conocimiento exige unos mecanismos transparentes y unos instrumentos eficaces que puedan permitir la mejora de la calidad, competitividad, convergencia y reconocimiento de niveles académicos y títulos universitarios, como respuesta a la adaptabilidad del mercado laboral, cada vez más globalizado al adelanto tecnológico y a la plena integración y cohesión social europea. Nos encontramos inmersos en un momento de gran relevancia, trascendencia e interés, donde son muchos los agentes e instituciones que trabajan a 
contrarreloj para saber, conocer y formarse en esta nueva manera de hacer y buscar su máxima aplicación coherente con el entorno más cercano y comunitario. Las nuevas perspectivas en el ámbito de la enseñanza superior, se están dibujando y construyendo $\mathrm{y}$, somos nosotros mismos quienes las estamos buscando y adecuando en la medida de lo posible a nuestro contexto y a nuestro futuro.

La nueva meta educativa que supone el Espacio Europeo de Educación Superior (EEES) se sustenta en unos principios y intereses, con una clara definición europeísta de unificación, convergencia y movilidad, con el objetivo de intentar alcanzar un rasgo distintivo y de calidad en la enseñanza superior, tal y como nos lo especifica la Comisión Europea (COM 2003):

"La Unión Europea (UE) necesita un entorno universitario saneado y emergente. Europa necesita de sus universidades para optimizar los procesos que sustentan la sociedad del conocimiento y lograr el objetivo fijado en el Consejo Europeo de Lisboa de convertirse en la economía más competitiva y dinámica del mundo basada en el conocimiento, capaz de sostener el crecimiento económico y crear una mayor cohesión social".

Este hecho, supone consolidar la idea de que el capital humano y la sociedad del conocimiento, deben convertirse en un gran motor económico para que Europa sea más competitiva a nivel mundial, afirmación ya concretada anteriormente por la propia Comisión Europea (COM 2001):

"La sociedad del conocimiento, en paralelo con otras amplias tendencias socioeconómicas como la globalización, los cambios en la estructura familiar, los cambios demográficos y el impacto de las tecnologías de la sociedad de la información, plantea a la UE ya sus ciudadanos tantos beneficios potenciales como retos. Los ciudadanos tienen nuevas y enormes oportunidades de comunicación, desplazamiento y empleo. Para poder aprovechar estas oportunidades y participar activamente en la sociedad, es fundamental adquirir continuamente conocimiento y aptitudes. Asimismo, las condiciones competitivas ventajosas dependen cada vez más de la inversión en capital humano. Por ello, los conocimientos y las aptitudes son un poderoso motor del crecimiento económico. Teniendo en cuenta la incertidumbre del clima económico actual, la inversión en las personas se convierte en el más importante. En términos económicos, la inserción, empleo y la adaptabilidad de los ciudadanos son vitales para que Europa mantenga su compromiso de convertirse en la sociedad del conocimiento más competitiva y dinámica del mundo".

La idea de profundizar, consolidar y promocionar una sociedad del conocimiento partiendo de un sistema educativo superior europeo, toma un nuevo impulso de gran relevancia y trascendencia, en la nueva era del conocimiento, de la información y de la movilidad laboral, según recoge la AQU y la UNESCO (2000) "una economía cada vez más basada en el conocimiento, no es extraño que mire la universidad como un activo estratégico y decisivo". Claramente nos encontramos ante una revalorización del hecho educativo como fuente de progreso social y económico, coincidiendo con Fernández (2006) "la educación y la formación han pasado a ser puntos claves como elementos decisivos de la competitividad, tanto individual como de las empresas y los países y, además, por sus claras repercusiones en el nivel de empleo". 
La fecha de inicio de este nuevo periodo en la Enseñanza Superior Europea (cronograma 1$)^{1}$ fue establecida para el año 2010. Pero previamente hay que fundamentar los orígenes y hacer una revisión del camino. En este sentido la Magna Charta Universitatum (MCU), que nos orienta hacia las cuestiones esenciales referentes a la vocación de la Universidad para el siglo XXI, junto con la Convención de Lisboa, donde se recoge el reconocimiento de las calificaciones relativas a la Educación Superior en la región europea, son los precedentes del proceso que verdaderamente se inicia con la Declaración de la Sorbona (1998). En esta, se impulsa un proyecto de armonización de los sistemas de educación llamado EEES y finalmente se firman los acuerdos para constituirse en la Declaración de Bolonia (1999), que se convierte en el punto de partida, con la meta de alcanzar el proceso antes de 2010. En estos momentos no hallamos en una segunda fase de consolidación del EEES "Bolonia 2020", establecida en la reunión ministerial de Lovaina de 2009. Dicha etapa es complementaria con el proceso "Horizonte 2020" de la Comisión Europea para impulsar la investigación, la innovación y la competitividad.

Nos encontramos en un momento histórico de cambio donde hay que pensar $\forall$ mucho hacia nuestro nuevo presente universitario o de Enseñanza Superior. El futuro de la humanidad depende en gran medida del desarrollo cultural, científico y técnico donde la Universidad desarrolla un papel clave. Como en todo cambio siempre hay elementos a favor y otros en contra y / o resistentes al cambio, y el mundo universitario no es una excepción. Sin embargo, no debemos olvidar que las sociedades y las culturas evolucionan, progresan y se expansionan día tras día y, quizás fruto también de la aceleración propia de nuestros tiempos, tal y como nos dice Michea (2002): "la noción de "sociedades inmóviles" es pues un mito o una fantasía". Así, coincidiendo también con González y Wagenaar (2003) apuntan que: "una cultura no es una jaula, es un conjunto de códigos a partir de los cuales damos sentido a nuestras experiencias: es por naturaleza mutable, no sólo en sus contenidos sino en las sus reglas de funcionamiento", indicando que el ser humano por su propia naturaleza es activo e interacciona con su entorno, definiendo sus tejidos sociales y comunitarios. Pudiera ser cierto que fruto de los nuevos tiempos, donde se premia la inmediatez, falte la pausa, el reposo y la reflexión para asimilar tantos cambios.

Siguiendo el nuevo camino del marco universitario europeo, la comunidad universitaria, a pesar de los pertinentes cambios propios de un proceso de construcción y de crecimiento busca un consenso colectivo dirigido al beneficio común. Esta reflexión y redefinición constante de uno mismo, está inmersa hoy en día en un lucha continua de identidad; tal como dice Olivas (2007) "la ciudad puede ser una cárcel o la plataforma de liberación y desarrollo creativo del ser humano ", en occidente a pesar de las grandes posibilidades creativas que nos ofrece el mundo actual, reside en un periodo de incertidumbre, es la llamada crisis de valores y de principios individuales y colectivos. Esta definición de uno mismo es necesaria, tal y como dice Morin (2000) "todo desarrollo verdaderamente humano significa desarrollo conjunto de las autonomías individuales, de las participaciones comunitarias y del

\footnotetext{
${ }^{1}$ El conjunto de cronogramas y tablas del presente artículo son de elaboración propia y parten de la Tesis Europea: Comas, MA (2011) L'Espai Europeu d'Ensenyament Superior: origen, fonaments i construcció. Tesis Europea - Universitat Internacional de Catalunya. DART-Europe E-theses Portal:

http://www.dart-europe.eu/full.php?id=456642
} 
sentimiento de pertenencia de la especie humana". Verdaderamente hay una lucha constante hacia el nuevo cambio en el sistema universitario y la universidad no es un órgano aislado, como miembro activo de nuestra sociedad, tal y como dice la AQU y la UNESCO (2000) "es evidente que en los últimos veinte años las universidades no han quedado al margen de la gran oleada de cambios que ha producido en el sistema económico, tecnológico y social en todo el mundo" pero no debemos perder de vista las grandes ventajas y la gran oportunidad que se nos presenta donde el trabajar y crecer en el mismo entorno, por lo tanto el trabajo colaborativo y empático nos puede favorecer y significar un rasgo distintivo de calidad, satisfacción y éxito. "Nuestra sociedad confía hoy más que nunca en las sus Universidades para afrontar nuevos retos, los derivados de la sociedad del conocimiento en el horizonte del presente siglo" (LOU 2001).

Cronograma 1: El Espacio Europeo de Educación Superior

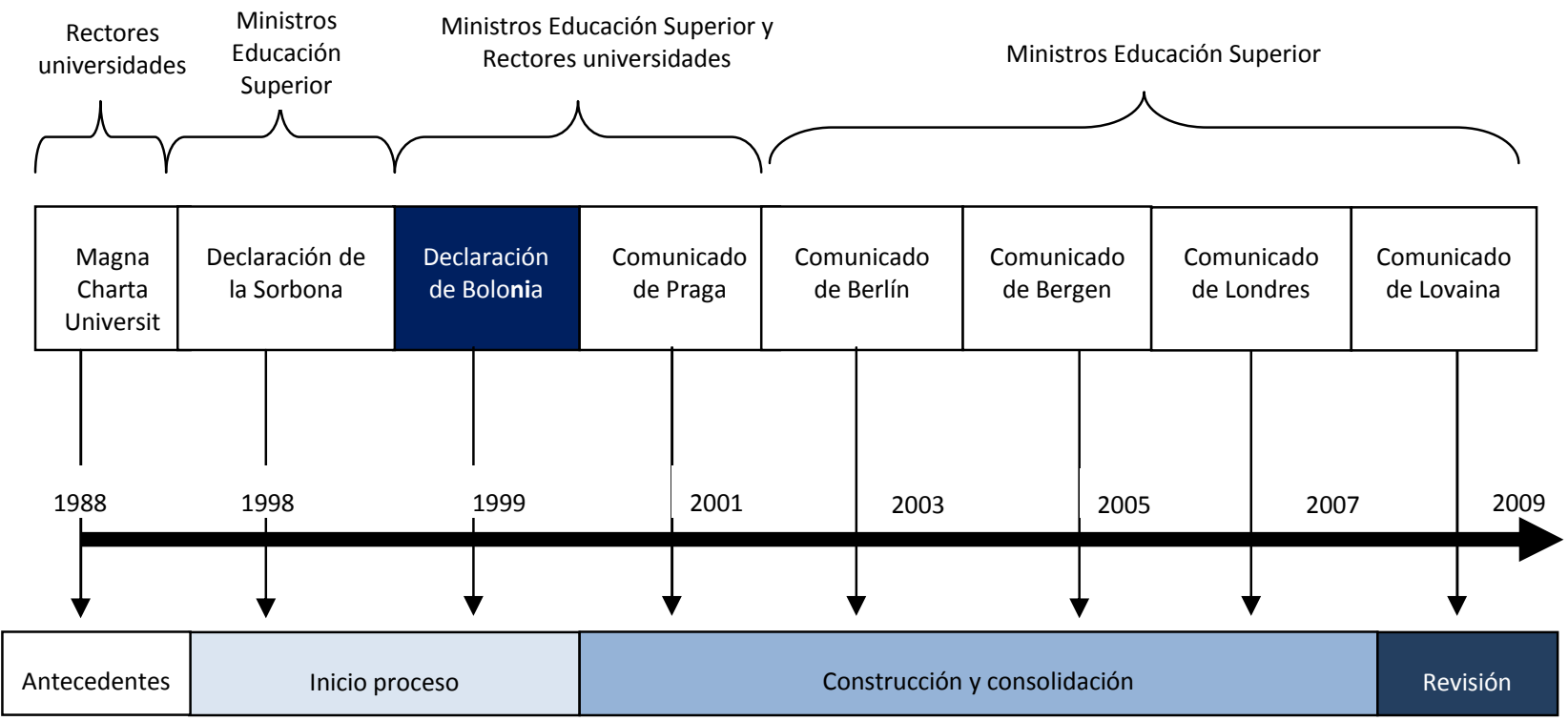

\section{Principios fundamentales del EEES: movilidad y reconocimiento formativo.}

A fin de analizar los diferentes comunicados de los ministros responsables de educación superior que han acompañado el proceso de construcción del EEES, debemos, en primer lugar, establecer el diseño y la colocación de los poderes legislativos de la UE y los Estados miembros en materia de educación. La referencia a la ley es esencial en este caso, como se indica en esta cita de Juristes pour l'Europe (2005) "la definición de las competencias respectivas de la Unión y los Estados miembros permite entender mejor quién hace qué (artículos I-11 a l-18)".

El análisis se basará en una pauta objetiva diferenciando tres categorías de competencias:

a) Exclusivas de la UE.

b) Compartidas, entre la UE y sus Estados miembros. 
c) Subsidiaria, la UE interviene en ausencia del Estado Miembro.

Esta categorización permite una mejor comprensión del grado de responsabilidad legislativa entre Estados y la Unión Europea. En nuestro ámbito, la educación, no es una competencia exclusiva de la UE, ni compartida, sinó subsidiaria. Según la versión consolidada del Tratado de Funcionamiento de la Unión Europea (2008) "la Unión dispondrá de competencia para llevar a cabo acciones para apoyar, coordinar o complementar la acción de los Estados miembros sin por ello sustituir la competencia en estas áreas". La educación se ve a sí misma como un apoyo a los Estados miembros sobre la política educativa, como establece Michel (2003: 138) "en la educación, la acción comunitaria debe respetar plenamente la responsabilidad de los Estados miembros sobre el contenido de la enseñanza y la organización del sistema educativo".

Es fundamental tener en cuenta que las instituciones europeas no tienen capacidad legislativa en materia de educación. Su papel es meramente consultivo y coordinador, como muestra Cussó (2008):

"Esto contrasta con otras áreas, la UE no tiene competencias directas en el campo de la educación. Sin embargo, basándose en una interpretación amplia del concepto de formación profesional, la Comisión Europea ha ido entrando en el campo de la educación. Con el Tratado de Maastricht (1992) que la competencia de la UE en materia de educación fue reconocida oficialmente y, al mismo tiempo, guiados por el principio de subsidiariedad. La Comisión no puede, por ahora, continuar con la orientación en esta área. No obstante, puede confiar en las recomendaciones e incentivos".

Por lo tanto debemos interpretar la competencia en la educación como una competencia nacional, sostenida, apoyada y coordinada por la intervención complementa la UE, según lo indicado por Michel (2003) "la educación fue uno de los primeros sectores para promover el desarrollo de la ley. De hecho, el desarrollo de una política común en materia de educación no se encuentra entre la meta establecida para las Comunidades Europeas". La idea es compartida por Priollaud y Siritzky (2008) "en todos los casos, en virtud del principio de subsidiariedad, las competencias son las de los Estados miembros, las instituciones de la UE están implicadas en apoyo de las políticas nacionales". Pero en todos los casos, el Derecho comunitario sigue siendo aplicable. El principio de no discriminación constante obliga a los Estados miembros.

En esta perspectiva la Comisión Europea se define, según Charlier (2003), como "un catalizador para facilitar la acción conjunta y la cooperación en el principio de subsidiariedad, teniendo en cuenta la diversidad de los sistemas". Estos son los principios de convergencia y reconocimiento de que los registrados en el EEES de la UE el proceso, como dice Haug (2010) "la Comisión ha tenido un papel muy inteligente para apoyar el proceso de convertirse en realidad... la Comisión dice claramente que no va a armonizar el sistema educativo". La construcción de la UE se basa en el respeto a la diversidad y las diferentes realidades de cada país, de la voluntad común de construir juntos y de forma cooperativa. 


\section{Movilidad y reconocimiento formativo}

La educación adquiere una importancia cada vez más como un motor de crecimiento de Europa. Principalmente desde el Tratado de Maastricht, donde se convierte en una de las prioridades de la comunidad, según lo indicado por André (2006) "en noviembre de 1993, después de la entrada en vigor del Tratado de la Unión Europea (Tratado de Maastricht), la educación comenzó a ser parte de las comunidades políticas". La educación se convierte en un eje clave en la mejora de los ciudadanos europeos, como dijo Lenarduzzi (2010) "el proyecto educativo de Europa se basa en los principios de convergencia, cooperación, reconocimiento y movilidad, para fortalecer y permitir más oportunidades a los ciudadanos europeos". Y en el campo de la Educación Superior, debido a la competitividad y la globalización, Europa ha perdido su presencia global y necesita redefinirse a sí misma; como dice Haug (2010) "Bolonia tiene un interés especial para los Estados en aumentar el atractivo del sistema de Educación Superior. Todas las iniciativas para la excelencia, el reconocimiento, el talento... existen para eso". Y de acuerdo con Froment (2010), "frente a un mundo global que se está convirtiendo en uno más abierto que antes, aparece la necesidad de pedir el EEES en un contexto global... tenemos que estar presentes, visibles, legibles, en la competitividad global".

La educación y la formación son factores clave de la economía y desarrollo europeo. Por ello los estados trabajan en la aplicación de las medidas legales adecuadas para facilitar el éxito del EEES. El discurso del presente artículo es la consolidación de la estructura legal del reconocimiento formativo y de la movilidad, que fundamenta la construcción del EEES. Como declaró Haug (2010), "la declaración de la Sorbona y de Bolonia no son documentos académicos, son documentos políticos de la educación superior". Dentro de la Declaración de Bolonia (1999), hay principios jurídicos tales como la movilidad (ver cronograma 2) y reconocimiento formativo (cronograma 3):

Cronograma 2: principios jurídicos de la movilidad

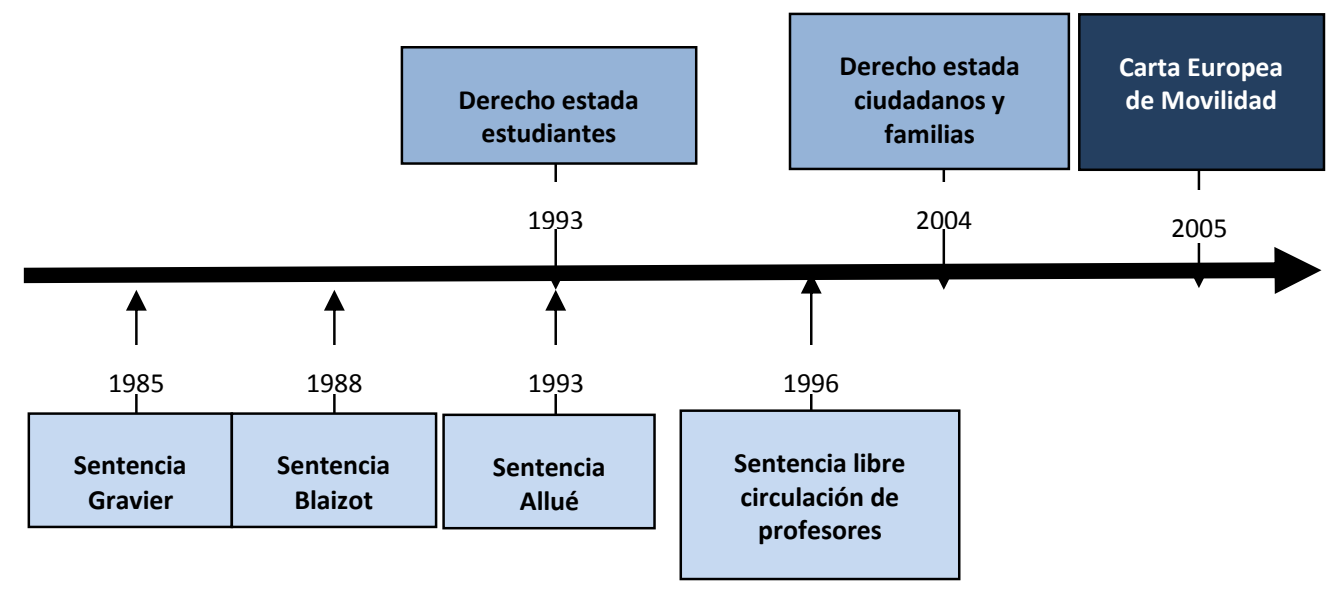

Parlamento Europeo

Derecho de Estadas

Sentencias CJCE 
El caso de Gravier tiene una importancia decisiva y va a servir de precedente en el derecho al reconocimiento del estudiante. Según lo declarado por la Comisión Europea (2006) "en 1985, el Tribunal de Justicia de las Comunidades Europeas su decisión tendrá consecuencias importantes en la cooperación comunitaria en materia de educación y sus desarrollos futuros. Esta es la Sentencia Gravier adoptada el 13 de febrero 1985". La situación que enfrenta la estudiante francesa Françoise Gravier es simple: quería estudiar las técnicas de dibujos animados en Bélgica en una institución de Educación Superior no universitaria, donde por lo visto se imponen cuotas diferentes a las de los estudiantes nacionales. Por lo tanto, como indica la Comisión Europea (2006):

"En nombre del principio de no discriminación, pidió una exención que se le negó y luego interpuso una demanda en la que se apoderó de la Corte. Se estableció que todo estudiante debe tener acceso a cursos de formación profesional en otros Estados miembros, en las mismas condiciones que los nacionales, y que no puede haber discriminación por razón de la nacionalidad en virtud del artículo 7 del Tratado".

Por último, el Tribunal de Justicia en el caso Gravier (1985), se pronuncia sobre cuestiones sobre el tema:

"Cualquier forma de educación que se prepare para una cualificación para una profesión, oficio o empleo, o que proporcione la capacidad de actuar como una profesión, oficio o empleo es la formación profesional, independientemente de su edad el logro educativo de los alumnos o estudiantes, e incluso si el plan de estudios incluye un elemento de la educación general".

La sentencia Gravier representa el punto de partida de la movilidad de los programas europeos de educación: Erasmus, Comett... y por lo tanto contribuyó a la consolidación de un Principios Fundamentales de la UE; según Hernu (2005: 38-39), "el principio de igualdad democrática". Pero antes de estos programas, la Corte emitió un fallo importante: Blaizot, donde la Corte estableció vínculos entre la educación superior y la formación profesional en virtud del artículo 128 del Tratado de Roma.

Otro elemento jurídico importante en la movilidad es el derecho de residencia de los estudiantes (1993), "la presente Directiva tiene por objeto garantizar el acceso a la formación profesional para los nacionales de un Estado miembro organizar el marco en el que ejercen su derecho de residencia". El derecho de residencia para la formación con el tiempo se extenderá a cualquier ciudadano de la UE. Esta ley fue codificada por la Directiva del derecho de libre circulación y residencia de ciudadanos de la UE y sus familiares (2004).

Finalmente, uno de los otros objetivos de la Declaración de Bolonia (1999) ha sido facilitar "la promoción de la movilidad de profesores, investigadores y personal administrativo, el reconocimiento y valoración de los períodos de investigación, la educación y la formación en un contexto europeo, sin menoscabo de sus derechos legales". De aquí parte la sentencia del Tribunal de Justicia contra Luxemburgo 1996 de la Comisión, el reconocimiento de la libre circulación de los maestros, es un caso derivado de una sentencia anterior, la sentencia Allué (1989), que es un principio de prohibición de las diferencias de trato en función de la nacionalidad de los profesores términos de contrato de trabajo y seguridad social. 
Las sentencias de Gravier, Blaizot y los derechos de reconocimiento formativo y de residencia de los estudiantes y profesores fortalecen la libertad de movilidad y, al mismo tiempo, han permitido la construcción de espacios de reconocimiento y la convergencia en la formación. Además, han permitido sentar la base de ejecución de los programas europeos Erasmus, Comenius, Petra y Lingua.

Por último, el Parlamento Europeo, en el origen de la Carta Europea de Calidad para la Movilidad (2005):

"En la educación y la formación, el principio según el cual, para garantizar la calidad global de la movilidad, es conveniente garantizar la libre circulación de la Unión y la aplicación de la medida en los principios establecidos en la Carta y las recomendaciones pertinentes a todos los tipos de movilidad para el desarrollo del aprendizaje profesional: la educación o la formación, el aprendizaje formal o informal, incluyendo los proyectos de trabajo y voluntarios, cortos o largos períodos de la movilidad, la escuela, la educación superior o las medidas de formación profesional en educación y formación durante toda la vida".

La movilidad de los ciudadanos de la UE y la propuesta de eliminar todos los obstáculos a la movilidad entre los Estados miembros reforzará su competitividad, en línea con las disposiciones de la Estrategia de Lisboa.

Es importante y apropiado tratar el debate sobre el término "armonización". Este término ha causado los primeros debates y diferencias entre los Estados y la Comisión con respecto a la extensión de los poderes legislativos en la educación. Esta situación fue causada por la redacción de la Declaración de la Sorbona, o como se especifica Haug (2010):

"La Declaración de la Sorbona, es excelente con las intenciones, una Europa más competitiva, una Europa más coherente y fácil de leer. Sin embargo, hay graves errores en el vocabulario, por ejemplo, la armonización europea no es posible. El Tratado de la UE dice claramente que no va a armonizar el sistema de educación".

Debido a este problema de interpretación, la Declaración de Bolonia ayudó a superar este obstáculo, como lo demuestra Charlier y Croche (2003) "la declaración de Bolonia es, por tanto carece de cualquier mención a una "armonización", interpretándolo como un signo de abandono de la soberanía nacional sobre la Educación Superior, en ello también incide Cussó (2006) "la Declaración de Bolonia, partiendo del proyecto de la Sorbona, abandonaf el término la armonización en favor de "compatibilidad". El debate es el reconocimiento de los títulos, como afirma Haug (2010) "no es necesario anular los títulos nacionales, uno debe encontrar la compatibilidad entre ellos. No se busca la uniformidad $\theta$ para sustituir las cualificaciones nacionales". Con esto en mente, empezamos a buscar la terminología de la mejor manera de definir el marco de trabajo colaborativo en el que los Estados necesitan para construir y consolidar el proceso de construcción del EEES, como lo indica el análisis de Reversat-Pujol (2007) "el uso del término" armonización "en las fuentes del Derecho comunitario fue revelado por muchos de los autores que han puesto de relieve la sustitución de otros términos tales como" coordinación " $y$ " reconciliación". 
El siguiente paso es el reconocimiento de la formación (cronograma 3). Los ECTS (European Credit Transfer System y acumulación de créditos) son esenciales como se detalla en los objetivos de la Declaración de Bolonia (1999);

"Establecimiento de un sistema de créditos - como el sistema de ETCS - como medio de promover la movilidad estudiantil lo más ampliamente posible. Los créditos también podrían obtenerse fuera del sistema de Educación Superior, incluida la educación durante toda la vida".

También la experiencia de los ECTS se consolidará por el programa Erasmus (1987) y por el proyecto Tuning (2000), como ha planteado Haug (2010) en estos dos programas:

"Erasmus tiene un gran impacto, para entrenar, trabajar juntos, a pesar de todas las diferencias entre las universidades para cumplir la movilidad de estudiantes. Todo cambia, el sistema de notas, el sistema de grados, la organización de la enseñanza, exámenes, laboratorio de aprendizaje... era Erasmus... Tuning ha llevado a las universidades a revisar su plan de estudios para hacerlos compatibles con Bolonia. Hágalo de forma inteligente para repensar su plan de estudios, haciendo coincidir las necesidades y demandas de los estudiantes. Para que los estudios sean útiles para la sociedad y el mercado laboral. Esa es la idea de la afinación".

Hay un fuerte vínculo entre estos dos programas y ECTS, tal como se describe en la guía del proyecto Tuning propia a la Comisión Europea (2007):

"Optimización del sistema europeo de sistema de acumulación y transferencia (ECTS, por sus siglas en Inglés) basado en la carga de trabajo de los estudiantes. De acuerdo, Tuning, ECTS no sólo es un sistema diseñado para que los estudiantes puedan moverse más fácilmente a través de Europa, a través de la acumulación y la transferencia de fondos, sino también un sistema que puede simplificar el diseño y desarrollo de programas, especialmente en una relación con la coordinación y la racionalización de las solicitudes de estudiantes a través de la existencia de unidades frente a sus competidores. En otras palabras, el ECTS nos permite planificar y utilizar el tiempo para que los estudiantes para alcanzar la excelencia con los objetivos del proceso educativo, en lugar de considerar un tiempo limitado, como profesores y estudiantes de tiempo ilimitado. De acuerdo con la perspectiva de Tuning, los créditos sólo podrán concederse cuando los resultados del aprendizaje se han cumplido".

Cronograma 3: principios fundamentales del reconocimiento formativo.

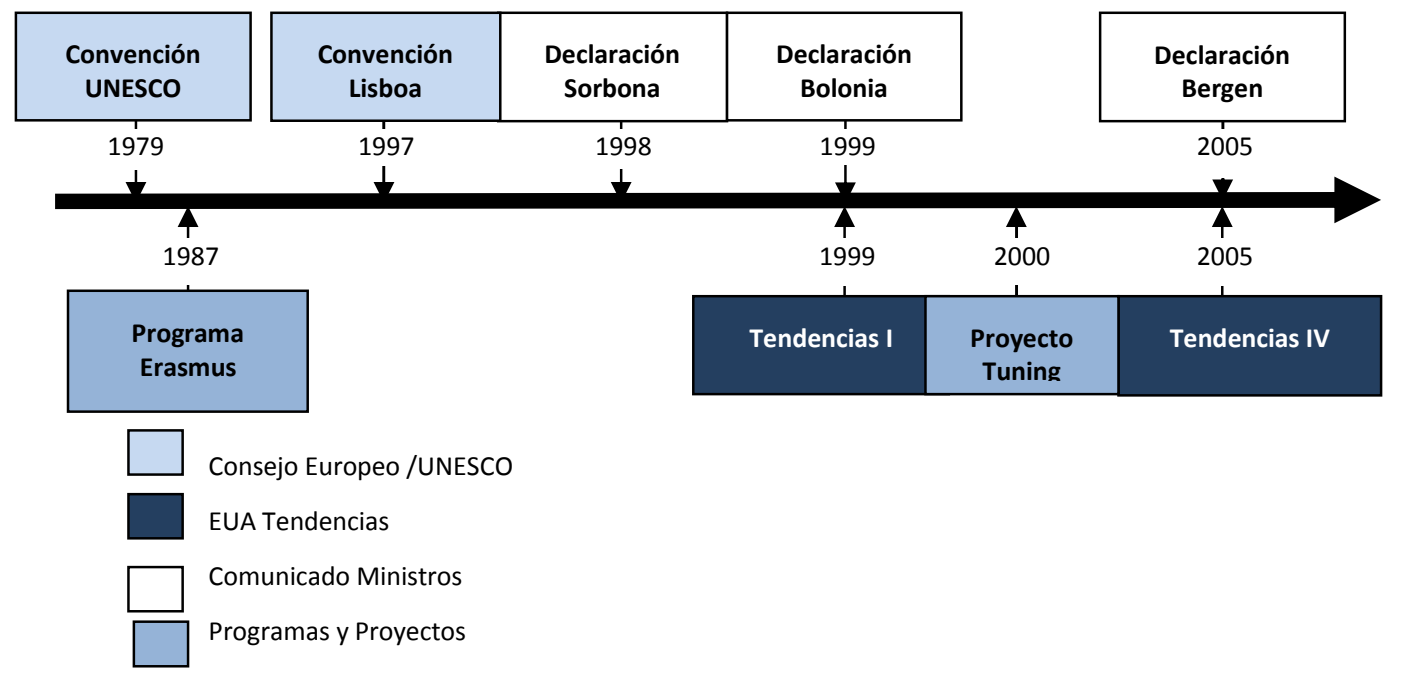


En la Declaración de la Sorbona (1998) era más bien la presencia de una propuesta de "fusión" de estructuras progresivas y grados. Ya en el Convenio de Lisboa (1997) como indica la Declaración de la Sorbona (1998):

"Un convenio sobre la convalidación de las titulaciones universitarias en Europa fue firmado en Lisboa el año pasado. La Convención establece una serie de condiciones básicas, al tiempo que reconoce que los países, a su vez, podrían participar en más proyectos constructivos. Basándose en estos resultados, podemos usarlas para ir más allá. Ya existe un terreno común tanto para el reconocimiento mutuo de diplomas de educación superior con fines profesionales, a través de las directivas de la Unión Europea".

El Convenio de Lisboa (1997) es, por lo tanto, una clave importante, tal como se muestra:

"Reconociendo que el derecho a la educación es un derecho humano y que la educación superior, que desempeña un papel destacado en la adquisición y el avance del conocimiento, constituye un excepcionalmente rico patrimonio cultural y científico, para los individuos y para la sociedad ... Considerando que el reconocimiento de estudios, certificados, diplomas y títulos obtenidos en otro país de la región europea constituye una medida importante para promover la movilidad académica entre las partes ... convencidos de que reconocimiento equitativo de cualificaciones es un elemento clave del derecho a la educación y la responsabilidad de la sociedad".

El Convenio de Lisboa, entre el Consejo de Europa y la UNESCO (1997) estableció la estructura de reconocimiento mutuo con el Convenio sobre Reconocimiento de Cualificaciones relativas a la Educación Superior en la región de Europa. Lisboa es una base de trabajo, seguida de diversas declaraciones y declaraciones de los Ministros de Educación Superior, que forman parte de esta lógica. Que se describen los objetivos de la Declaración de Bolonia (1999).

La Declaración de Bolonia implica un cambio esencial en favor de la creación del EEES, según lo declarado por Crochet (2004):

"Sin embargo, creo que cada eje principal del proceso de Bolonia ofrece un verdadero avance en nuestra educación superior. El eje principal primero es la adopción generalizada de créditos ECTS, lo que es la pieza central del nuevo sistema... Los resultados de graduación de la acumulación de competencias certificadas a través de créditos. Este sistema permite la orientación progresista y alienta la formación durante toda la vida, el reconocimiento, la movilidad y puentes entre los diferentes sistemas de estudio. El segundo es la adopción de un conjunto en dos grados: el grado de licenciatura o seguir el capitán. El primer ciclo se utiliza para ofrecer una formación completa, que confieren un empleo legal. Los estudiantes pueden continuar sus estudios o buscar empleo al final de primer grado, volver a la universidad o la formación a distancia. Bajo ciertas condiciones, el estudiante cuyas elecciones han madurado puede cambiar de dirección entre los dos ciclos. Finalmente, el sistema de dos tiempos sin duda dará lugar a una nueva movilidad entre los dos grados".

Pero fue durante la reunión ministerial de Bergen (2005) donde este plan finalmente consolida la reconciliación y el reconocimiento de los títulos y estructuras;

"En nuestra conferencia en Berlín, se encargó al grupo de seguimiento tres prioridades: el sistema de medida, la garantía de calidad y reconocimiento de títulos y períodos de estudio. Teniendo en cuenta el equilibrio relativo, nos encontramos con que se han logrado progresos realizados en estas tres áreas prioritarias. Será importante conocer los 
avances reales en todos los países participantes. Por lo tanto, reconocemos la necesidad de compartir más de nuestro know-how para desarrollar habilidades tanto en términos de instituciones y gobiernos".

Hay que añadir otro documento creado por la European University Association, lo que consolida la aproximación propuesta de las estructuras y grados: Tendencias IV (2005) "lo que refleja los temas tratados en el informe de progreso del gobierno anterior, es decir tres prioridades que figuran en el Comunicado de Berlín: los problemas de la estructura de grados, de calidad y reconocimiento".

\section{Interpretación de las principales autoridades}

A pesar de la dificultad de poder precisar un número inicial de informantes para entrevistar, coincidiendo con Taylor y Bogdan (1996) "ni el número ni el tipo de informantes se especifica de antemano... es difícil determinar a cuántas personas se Debes entrevistar en un estudio cualitativo". La lista de posibles candidatos podía ser muy elevada, por lo tanto era necesario, tal como indica Camps (2010):

"Empezar con una cierta previsión aunque sea necesario la disposición a redefinir, durante el proceso, el tipo y el número de personas a entrevistar. Este último aspecto aparece naturalmente al final de las entrevistas, cuando se aprecia la falta de aportaciones nuevas que faciliten la comprensión de los temas planteados".

En este sentido el criterio de ir ampliando hasta seis los entrevistados no está motivado por la falta de información, sino por la posibilidad de poder contrastar y consolidar sus aportaciones, mediante el cruce con los textos oficiales (sobre los hechos) y las aportaciones de los entrevistados, como personalidades activas y responsables del proceso del EEES.

La selección de entrevistados (tabla 1), validada por expertos, consta de autoridades representantes de las tres instituciones principales. En cuanto a la Comisión Europea, el Dr. Domenico Lenarduzzi, el representante principal de la CE en la firma de la Declaración de Bolonia; en cuanto a la Conferencia de Rectores Europeos-European University Association, el Dr. Josep M. Bricall y el Dr. Eric Froment, y ambos adquirieron el cargo de la Presidencia de la CRE y la EUA en los primeros pasos del EEES y el Dr. Bricall además fue miembro del Comité de Redacción de la Magna Charta Universitatum. Y los secretarios de Estado de Universidades, el Sr. Jorge Fernández Díaz y el Dr. Màrius Rubiralta, el primero fue el signatario por España de la Declaración de Bolonia y el segundo, principal cargo de universidades a fecha de 2010. Lo que nos permite tener una visión de inicio y fin de la actuación de España en el proceso. Además, la entrevista al Dr. Guy Haug, que ha participado en diferentes fases desde las diferentes instituciones mencionadas y fue uno de los impulsores del programa Erasmus, nos ofrece una visión más global.

Las entrevistas en profundidad responden a un interés cualitativo para favorecer la realización de un marco de comprensión hacia el estado de la cuestión del proceso de creación, constitución y consolidación del EEES. Dichas entrevistas darán pie a poder deducir las consideraciones más relevantes para construir las conclusiones finales. Para ello se estructuró un guión de entrevista en profundidad semiestructurado (tabla 2). 
1. Dr. Josep M. Bricall

Presidente de la Conferencia de Rectores Europeos (1994-1998) y miembro del Comité de Redacción de la Magna Charta Universitatum, firmada en 1988 en Bolonia.

2. Sr. Jorge Fernández Díaz

Secretario de Estado de Universidades, Investigación y Desarrollo (1999-2000) y signatario en representación de España de la Declaración Bolonia en 1999.

3. Dr. Domenico Lenarduzzi

Presidente de la Dirección General de Educación de la Comisión Europa (1981-2001) e impulsor del programa Erasmus.

4. Dr. Guy Haug

Experto asesor de la Comisión Europea en la enseñanza superior, asesor del grupo de trabajo de la Declaración de la Sorbona, previo a la Declaración de Bolonia, impulsor del programa Erasmus, consejero de la EUA, la OCDE y la Unesco, y redactor del informe Trends I y II.

5. Dr. Eric Froment

Tesorero de la CRE (1994-1998), jefe ejecutivo de la Conferencia Nacional Francesa de Rectores (1998-2001), presidente de la EUA (2001-2005) y editor de la EUA Bologna Handbook.

6. Dr. Màrius Rubiralta

Secretario de Estado de Universidades del Ministerio de Educación del Gobierno de España (de 2008 a 2011) y miembro del observatorio del MCU (2009).

Tabla n. 1: Entrevistados

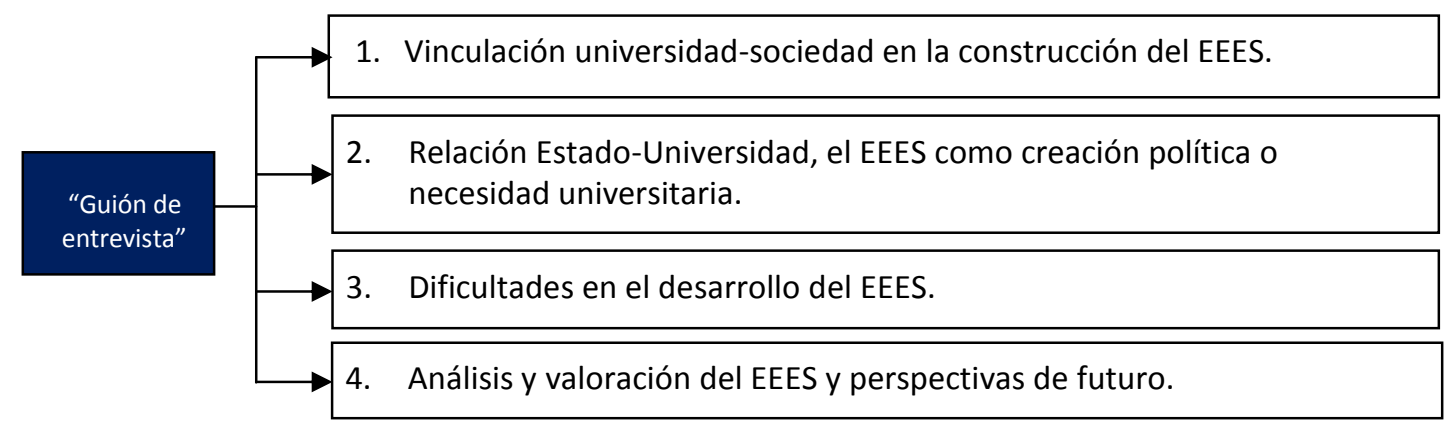

Tabla n. 2: Guión entrevistas en profundidad semiestructuradas

Según los diferentes entrevistados ${ }^{2}$, la Universidad es un elemento clave de la formación, el conocimiento y el progreso social, al tiempo que se convierte en eje del desarrollo de la sociedad y sello distintivo de Europa. Pero necesita adaptarse a los nuevos tiempos y vincularse al concepto de competitividad y eficiencia, y ofrecer más cooperación y cercanía, para favorecer la movilidad y el reconocimiento mutuo. Así Eric Froment considera que:

"Il y a deux rôles. Un rôle présent, qui se joue autour de la connaissance, de la création de la connaissance, la diffusion de la connaissance, la formation permanent et l'articulation avec la société,... c'est à dire qui contribue à la formation, à l'ouverture

\footnotetext{
${ }^{2}$ Las citaciones literales de las entrevistas están en el idioma original, respetando las afirmaciones de los propios entrevistados.
} 
d'esprit, à la connaissance. Et un deuxième rôle qui n'est pas actuellement présente c'est la formation du citoyen européen, le rôle de l'université dans un nouvel espace politique. Il y a une responsabilité des universités dans la formation d'un esprit européen"

La adaptación de la Universidad debe partir de una mirada interna para actualizar sus funciones y objetivos, la relación oferta-demanda, así lo estable Domenico Lenarduzzi:

"L'université était un peu renfermée sur elle-même tout comme le secteur éducatif en général, contrairement à la formation professionnelle, qui par nécessité avait besoin d'une certaine ouverture. L'éducation a perdu à un certain moment le contact avec la société parce qu'elle n'en était plus partie intégrante, elle en était isolée. En effet, elle a commencé à être répétitive et il y avait une grande différence entre l'éducation et le monde socioéconomique. L'éducation a besoin de s'ouvrir au monde et de s'adapter à la réalité. Dès lors, la CE a commencé à parler de la nécessité d'entrer dans la société de la Connaissance. Le rôle de l'université est fondamental pour la société, son problème était de préparer les étudiants à leur intégration dans la vie active. Elle avait par conséquent peur du monde productif. II y a un important apprentissage théorique mais aucune expérience du monde réel. Il y a aussi une absence de "l'esprit de stage ", d'où la difficulté d'accès à l'emploi".

Para ellos la creación del EEES responde a una lucha de poder entre el derecho de autonomía de las universidades y el derecho legislativo de los gobiernos estatales. Sin embargo, todos consideran que la enseñanza superior es fundamental para el progreso, pero que necesita del liderazgo e implicación de los gobiernos para legislar el marco de actuación establecido en los objetivos de Bolonia. Siguiendo las indicaciones de Guy Haug:

"C'est peut être une nécessite politique en face d'un monde qui s'internationalise qui devient un monde plus ouvert qu'avant. C'est le besoin de poser l'EEES dans le contexte mondial, et lui donner une dimension européenne, selon Allegre il faut qu'on soit présent, ont a besoin d'être visible, lisible, sur la compétition mondiale".

Y compartiendo idea de liderazgo político, Jorge Fernández Díaz afirma que: "es evidente que quien tiene que liderar el proceso son los gobiernos, son los políticos porque los estados seguirán siendo muy celosos de ceder estas competencias".

La mayoría coinciden también en considerar que el desarrollo del EEES en España no ha sido el más óptimo, ya que empezó muy tarde. Además, también han coincidido en determinar las dificultades que supone el establecimiento del sistema de grados (cuatro años) y posgrado (un año) por parte de España. Esto es visto como una dificultad en la consecución de los objetivos de la movilidad y el reconocimiento, ya que hay muchos otros países miembros del EEES que han optado por el modelo de grados (tres años) y posgrado (dos años). Afirmación consolidada por Marius Rubiralta:

"El problema de España, no han sido las fases teóricas (Sorbona, Bolonia y las otras) sino su aplicación. La fecha de inicio real en España fue en 2007. Por lo tanto del 07 al 10 pasa todo... en España hasta 2006/07 nadie oyó hablar del EEES, no teníamos ni idea. No sólo estuvimos dormidos mucho tiempo sino que tampoco estábamos preparando el cambio de la estructura universitaria".

De todos ellos hay un reconocimiento hacia el gran beneficio que supone el EEES pero así mismo también coinciden en que es una etapa de un proceso en desarrollo. Aunque no se han alcanzado todos los objetivos propuestos, hay que seguir la línea 
iniciadas por el EEES de cooperación, movilidad y reconocimiento, y consolidar futuras propuestas de comunitarias, competitivas y de difusión. El EEES ha sido un gran logro que ahora necesita su tiempo de reconocimiento. El EEES es fruto de un gran esfuerzo colectivo en beneficio de una mejor identidad, competitividad, movilidad y proyección Europea. Como cita Josep Maria Bricall "En este nuevo espacio europeo qué sentido tiene que cada país tenga su sistema. La investigación y la enseñanza son universales".

\section{Conclusiones}

La creación del EEES no es una acción diferenciada e inconexa de la realidad de la UE, sino que debe interpretarse como un rasgo distintivo de acción comunitaria en materia competencial educativa. Se ha generado el espacio y entorno de movilidad y reconocimiento válido para ofrecer más oportunidades al ciudadano europeo, a la vez de ser una herramienta estratégica. No obstante sería conveniente hacer una reflexión final para no perder el hilo conductor originario y optimizar esta oportunidad pedagógica que a la vez se convierte en un gran avance académico, profesional y político en materias prioritarias de la UE.

El nuevo hito educativo que se origina alrededor del EEES (incluyendo el EEI y el EEFP) se sustenta dentro de unos principios, intenciones, directrices e intereses con una clara definición europeísta de unificación, convergencia y movilidad con el objetivo de intentar alcanzar un rasgo distintivo y de calidad en la enseñanza superior de la UE y donde han intervenido e intervienen tan representantes políticos, académicos y estudiantes, haciendo posible la consecución de los objetivos marcados de la Declaración de Bolonia y, como muy bien afirmó Bricall (2008), "España no puede permitirse quedarse al margen del nuevo tren europeo ni perder esta oportunidad pedagógica".

La UE, en el marco de sus funciones básicas, debe promover el progreso y el bienestar social de los ciudadanos. La plena adecuación a la sociedad del conocimiento exige unos mecanismos transparentes y unos instrumentos eficaces que puedan permitir la mejora de la calidad y la competitividad, la convergencia y reconocimiento de los conocimientos, los niveles académicos y los títulos universitarios como respuesta a un nuevo mercado laboral cada vez más globalizado, el avance tecnológico y la plena integración social europea. Nuestro deber es hacer que la Educación, la Formación y la Investigación, sean un medio imprescindible para promover la cohesión social, la ciudadanía activa, la realización personal y profesional, la adaptabilidad y el empleo.

\section{Referencias bibliográficas}

Arrêt de la Cour du 13 février 1985. - Françoise Gravier contre Ville de Liège. Recuperado el 7 de febrero de 2011, de http://eurlex.europa.eu/LexUriServ/LexUriServ.do?uri=CELEX:61983J0293:FR:PDF

Arrêt de la Cour du 2 février 1988. - Vincent Blaizot contre Université de Liège et autres. Recuperado el 14 de noviembre de 2010, de http://eurlex.europa.eu/LexUriServ/LexUriServ.do?uri=CELEX:61986J0024:FR:PDF 
Arrêt de la Cour du 2 août 1993. - Pilar Allué et Carmel Mary Coonan et autres contre Università degli studi di Venezia et Università degli studi di Parma. Recuperado el 7 de febrero de 2011, de http://eurlex.europa.eu/LexUriServ/LexUriServ.do?uri=CELEX:61991J0259:FR:PDF

Arrêt de la Cour du 2 juillet 1996. - Commission des Communautés européennes contre Grand-Duché de Luxembourg. Recuperado el 14 de noviembre de 2010, de http://eur-lex.europa.eu/LexUriServ/LexUriServ.do?uri=CELEX:61993J0473:FR:PDF

AQU (2003). Marc general per a la integració europea. Barcelona. Comercial Gràfica Anmar, SL. Recuperado el 14 de noviembre de 2010, de http://www.aqu.cat/doc/doc 20197380 1.pdf

Bricall, J. M. (2000). Informe Universidad 2000. Conferencia de Rectores de las Universidades Españolas. Recuperado el 14 de noviembre de 2010, de http://www.oei.es/oeivirt/bricall.htm

Camps, J. (2010). Socialització de gènere a l'aula. Elements per a la comprensió dels condicionants de l'escolarització mixta i diferenciada des de la psicologia social. Tesi doctoral. Universitat Internacional de Catalunya. Recuperado el 10 de marzo de 2011 http://www.tesisenxarxa.net/TESIS UIC/AVAILABLE/TDX-0208111110112//Tesi Jaume Camps.pdf

Charlier, J-E.; Croché, S. (2003). Le processus de Bologne; ses acteurs et leurs complices. Dossier Education et Sociétés. L'influence des organisations Internationales sur les politiques d'éducation. Bèlgica. Recuperado el 10 de marzo de 2011, de http://www.cairn.info/revue-education-et-societes-2003-2page-13.htm

Comas, MA (2011) L'Espai Europeu d'Ensenyament Superior: origen, fonaments i construcción. Tesis Europea - Universitat Internacional de Catalunya. Recuperado el 7 de febrero de 2011, de DART-Europe E-theses: http://www.dart-europe.eu/full.php?id=456642

Comisión Europea (2003). Tuning Educational Structures in Europe. Programa Socrates. Universidad de Deusto i Universidad de Groningen. Recuperado el 7 de febrero de 2011, de http://www.relint.deusto.es/TUNINGProject/spanish/doc fase1/Tuning\%20Educ ational.pdf

Commission Européenne (2006). Histoire de la coopération européenne dans le domaine de l'éducation et de la formation. Office Publications officielles des Communautés Européennes. Luxemburg. Recuperado el 14 de noviembre de 2010, de

http://ec.europa.eu/dgs/education culture/publ/pdf/education/history fr.pdf

Commission Européenne (2007). Introduction à Tuning pour une convergence des structures éducatives en Europe, une contribution des Universités au Processus de Bologne. Recuperado el 10 de marzo de 2011, de

http://tuning.unideusto.org/tuningeu/images/stories/template/General Brochu re French version final.pdf 
Commission Européenne (2009). Guide utilisation ECTS. Direction Générale d'Éducation. Brusselles. Recuperado el 7 de febrero de 2011, de http://ec.europa.eu/education/lifelong-learning-policy/doc/ects/guide fr.pdf

Communiqué de Bergen (2005). L'EEES - Réaliser les objectifs. Recuperado el 10 de marzo de 2011, de http://www.bologna-bergen2005.no/Docs/France/050520 Bergen Communique-Fr.pdf

Communiqué de la Conference des Ministres Chargues de l'Enseignement Superieur (2003). Communiqué de Berlín. Recuperado el 14 de noviembre de 2010, de http://www.bologna-bergen2005.no/Docs/France/030919 Berlin CommuniqueFr.pdf

Communiqué de la Conference des Ministres Chargues de l'Enseignement Superieur (2009). Communiqué de Louvain-la-Neuve. Recuperado el 6 de abril de 2011, de http://www.ond.vlaanderen.be/hogeronderwijs/bologna/links/language/2009 L ouvain Louvain-la Neuve Communiqu\%C3\%A9 FR.pdf

Comunicado de la Comisión de Comunidades Europeas (2000). Hacia un espacio europeo de investigación. Brussel-les. COM (2000) 6 final. Recuperado el 10 de marzo de 2011, de

http://eur-lex.europa.eu/LexUriServ/LexUriServ.do?uri=COM:2000:0006:FIN:ES:PDF

Comunicado de la Comisión de Comunidades Europeas (2001). Hacer realidad un espacio europeo del aprendizaje permanente. Brussel-les. COM (2001) 678 final. Recuperado el 10 de marzo de 2011, de http://eurlex.europa.eu/LexUriServ/LexUriServ.do?uri=COM:2001:0678:FIN:ES:PDF

Comunicado de la Comisión al Consejo, al Parlamento Europeo, al Comité Económico y Social y al Comité de las Regiones (2000). Realización del "Espacio europeo de la investigación": orientaciones para las acciones de la Unión en el ámbito de la investigación (2002-2006). COM (2000) 612 final. Recuperado el 14 de noviembre de 2010, de

http://eur-lex.europa.eu/LexUriServ/LexUriServ.do?uri=COM:2000:0612:FIN:ES:PDF

Comunicado de la Comisión de Comunidades Europeas (2003). El papel de las universidades en la Europa del conocimiento. Brussel·les. COM (2003) 58 final. Recuperado el 10 de marzo de 2011, de http://eur-lex.europa.eu/LexUriServ/LexUriServ.do?uri=COM:2003:0058:FIN:ES:PDF

Comunicado de la Comisión de Comunidades Europeas (2003). El Espacio Europeo de Investigación: un nuevo impulso. Reforzar, reorientar, abrir nuevas perspectivas. Brussel·les. COM (2002) 565 final. Recuperado el 10 de marzo de 2011, de http://eur-lex.europa.eu/LexUriServ/LexUriServ.do?uri=COM:2002:0565:FIN:ES:PDF

Conséil de l'Europe et UNESCO (1997). Convention sur la reconnaissance des qualifications relatives à l'enseignement supérieur dans la région européenne. Lisbonne. Recuperado el 14 de noviembre de 2010, de http://unesdoc.unesco.org/images/0011/001112/111238mb.pdf\#page=37

Conséil de I'Union Européenne (2004). Éducation and Formation. L'urgence des reformes pour réussir la stratégie de Lisbonne. 14358/03 EDUC 168 - COM (2003) 685 final. Recuperado el 14 de noviembre de 2010, de 


\section{http://register.consilium.europa.eu/pdf/fr/04/st06/st06905.fr04.pdf}

Consejo Europeo de Lisboa (2000). Conclusiones de la Presidencia. Recuperado el 7 de febrero de 2011, de http://www.maec.es/es/MenuPpal/Ministerio/EscuelaDiplomatica/Cursossobrer elacionesinternacionales/Documents/WEB\%20-\%20ABELLAN\%202\%20\%20conclusiones\%20CE\%20\%20Lisboa\%202000.pdf

Crochet, M. (2004). Le processus de Bologne. L'aboutissement d'un long cheminement. SER-SA Études. Tom 401. Recuperado el 10 de marzo de 2011, de http://www.cairn.info/article.php?ID REVUE=ETU\&ID NUMPUBLIE=ETU 015\&I D ARTICLE=ETU $015 \quad 0461$

Cussó, R. (2006). La Commission Européenne et l'Enseignement Supérieur : une réforme au-delà de Bologne. Cahiers de la recherche sur l'éducation et les savoirs, núm. 5.

Déclaration de Bologne (1999). Recuperado el 7 de febrero de 2011, de http://www.bologna-bergen2005.no/Docs/France/990719 Bologna Declaration-Fr.pdf

Déclaration de la Sorbonne (1998). Recuperado el 7 de febrero de 2011, de http://www.bologna-bergen2005.no/Docs/France/980525 Sorbonne Declaration-Fr.pdf

EUA (2005). Tendances IV. Etat de la mise en œuvre des reformes de Bologne par les universités européennes. Recuperado el 14 de noviembre de 2010, de http://www.eua.be/eua/jsp/en/upload/TrendsIV FINAL FR.1128074006210.pdf

Fernández, J.E. (2006). Los grandes cambios y la sociedad del conocimiento. La política económica en tiempos de incertidumbre. Netbiblo.

González, J.; Wagenaar, R. (2003). Tuning Educational Structures in Europe. Informe Final. Fase Uno. Universidad de Deusto. Recuperado el 10 de marzo de 2011, de http://www.relint.deusto.es/TUNINGProject/spanish/doc fase1/Tuning\%20Educ ational.pdf

Haug, G.; Kirstein, J. (1999). Tendances I. Tendances des structures d'apprentissage dans l'enseignement supérieur. Conferència Rectors Europeus i Comissió Europea. Recuperado el 18 de marzo de 2011, de http://www.aic.lv/bolona/Bologna/Reports/Trends/trend I.pdf

Haug, G.; Kirstein, J. (1999). Wat it do es say, what it doesn't. Conferència Rectors Europeus i Comissió Europea. Recuperado el 18 de marzo de 2011, de http://www.aic.lv/bolona/Bologna/Reports/Trends/trend I.pdf

Haug, G.; Tauch, C. (2001). Tendances II. Towards the European higher education area: survey of main reforms from Bologna to Prague. Recuperado el 7 de febrero de 2011 de http://www.eua.be/eua/isp/en/upload/OFFDOC BP trend II.1068715483262.pdf

Informe Eurydice (2005). Organización de la Estructura de la Educación Superior en Europa. Dirección General de Educación y Cultura. Comissió Europea. Recuperado el 7 de octubre de 2010, de

http://www.educacion.es/cide/espanol/eurydice/publicaciones/eury2005fshee/ Eury2005fshee-ES.pdf 
Informe Eurydice (2007). Focus sur les estructures de l'enseignement supérieur en Europe. Direction Générale de l'Éducation et de la Culture. Commission Européenne. Recuperado el 7 de octubre de 2010, de

http://eacea.ec.europa.eu/education/eurydice/documents/thematic reports/08 6ES.pdf

Informe Trends III (2003). EUA. Recuperado el 7 de octubre de 2010, de http://www.eees.es/pdf/TrendsIII ES.pdf

Informe Trends IV (2005). EUA. Recuperado el 7 de octubre de 2010, de http://www.eees.es/pdf/TrendsIV ES.pdf

Informe Trends V (2007).EUA. Recuperado el 7 de octubre de 2010, de http://www.gencat.cat/diue/doc/doc 33209799 1.pdf

Journal officiel de I'Union européenne L 394/5. Recommandation du Parlement Européen et du Conseil du 18 décembre 2006. Relative à la mobilité transnationale dans la Communauté à des fins d'éducation et de formation: Charte européenne de qualité pour la mobilité. (2006/961/CE). Recuperado el 29 de noviembre de 2010, de http://eur-lex.europa.eu/LexUriServ/LexUriServ.do?uri=OJ:L:2006:394:0005:0009:FR:PDF

Journal officiel $n^{\circ} \mathrm{L} 158$ du (2004). Directive 2004/38/CE du Parlement européen et du Conseil du 29 avril 2004 relative au droit des citoyens de l'Union et des membres de leurs familles de circuler et de séjourner librement sur le territoire des États membres. Recuperado el 11 de noviembre de 2010, de http://eur-lex.europa.eu/LexUriServ/LexUriServ.do?uri=OJ:L:2004:158:0077:0123:FR:PDF

Journal officiel n L 317 (1993). DIRECTIVE 93/96/CEE DU CONSEIL du 29 octobre 1993 relative au droit de séjour des étudiants. Recuperado el 11 de noviembre de 2010, de http://eur-lex.europa.eu/LexUriServ/LexUriServ.do?uri=COM:1993:0209:FIN:FR:PDF

Juristes pour l'Europe (2005). Quelques bonnes raisons de dire Oui à la Constitution. Recuperado el 10 de marzo de 2011, de http://tamarindo.blogs.com/fredmetey fr/Europereferendum/Juristes\%20pour \%20Europe\%20Appel.pdf

Ley Orgánica de Universidades 6/2001, 21 de desembre. Recuperado el 10 de marzo de 2011, de http://www.boe.es/boe/dias/2001/12/24/pdfs/A49400-49425.pdf

Magna Charta Universitatum (1988). Recuperado el 10 de marzo de 2011, de http://www.magna-charta.org/library/userfiles/file/mc spanish.pdf

Michéa, J. C. (2002). La escuela de la ignorancia. Editorial Acuarela libros. Madrid.

Michel, V. (2003). Recherches sur les compétences de la Communauté. Ed. L'Harmattan. Collection Logiques Juridiques. París.

Morin, E. (2000). Els 7 coneixements necessaris per a l'educació del futur. UNESCO. París. Recuperado el 10 de marzo de 2011, de http://www.unescocat.org/fitxer/516/setconeixements.pdf 
Olives, J. (2007). La ciudad cautiva, vindica los lazos entre urbanismo y filosofía. Diario $A b c$. Entrevista realitzada per Doria, S. al Dr. Josep Olives. Recuperado el 10 de marzo de 2011, de http://www.abc.es/hemeroteca/historico-04-012007/abc/Cultura/la-ciudad-cautiva-de-olives-vindica-los-lazos-entre-urbanismoy-filosofia 153743029720.html

Parlement Européen et Conseil (2005). Charte européenne de qualité pour la mobilité. Recuperado el 7 de febrero de 2011, de http://www.europarl.europa.eu/meetdocs/2004 2009/documents/com/com co $\underline{\mathrm{m}(2005) 0450 / \mathrm{com} \operatorname{com}(2005) 0450 \text { fr.pdf }}$

Parlamento Europeo, el Consejo y la Comisión (2000). Carta de los Derechos Fundamentales de la Unión Europea. Diario Oficial de las Comunidades Europeas, C 364/0. Recuperado el 7 de febrero de 2011, de http://www.europarl.europa.eu/charter/pdf/text es.pdf

Parlamento Europeo (1995). Resolución sobre la comunicación de la Comisión sobre el reconocimiento de diplomas con fines académicos y profesionales (COM(94)0596 - C4-0123/95. Recuperado el 7 de febrero de 2011, de http://eur-lex.europa.eu/LexUriServ/LexUriServ.do?uri=CELEX:51995IP0261:ES:HTML

Taylor, S. J.; Bogdan, R. (1996). Introducción a los métodos cualitativos de investigación. Editorial Paidós. Barcelona.

Tratado de Lisboa (2007). Diario Oficial de las Comunidades Europeas, núm. C 306/01, de 17 de desembre de 2007. Recuperado el 11 de marzo de 2011, de http://eurlex.europa.eu/JOHtml.do?uri=OJ:C:2007:306:SOM:ES:HTML

Cita del artículo:

Comas, M.A. (2013). El EEES, identidad y competitividad Europea: Principios fundamentales e interpretación de las principales autoridades. Revista de Docencia Universitaria. REDU. Vol.11 (1) Enero-Abril. pp. 243-263 Recuperado el (fecha de consulta) en http://www.red-u.net/ 


\section{Acerca del Autor}

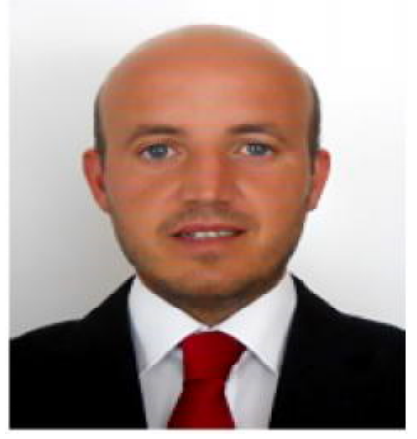

\section{Miquel Àngel Comas}

\section{Universitat Internacional de Catalunya}

Facultad de Educación

Mail: macomas@cir.uic.es

Doctor Europeo por la Universitat Internacional de Catalunya y l'Université Picardie Jules Verne, Licenciado en Pedagogía y Diplomado en Magisterio. Profesor y investigador la Facultad de Educación de la UIC y del GROIE (Grupo de Investigación en Organización de Instituciones educativas). Profesor del Máster Universitario en Recerca en Ciències Humanes, Socials i Jurídiques de la UIC. Líneas de investigación orientadas a la dimensión europea de la Educación Superior, la Formación Permanente y la Investigación. Así como la dirección, gestión y organización de instituciones educativas. Colaborador del Bologna Expert Team, del Bologna Follow-Up Group, del movimiento de participación y reflexión educativa Edu21, y de Capacitas; persona y discapacidad. 


\title{
Experiencias Creativas para el Desarrollo Humano Sostenible: espacios para la formación profesional en Arte en Costa Rica
}

\author{
Creative Experiences from Sustainable Human Development: spaces for \\ vocational training in Art in Costa Rica
}

\author{
Enid Sofia Lúñiga Murillo \\ Universidad Nacional, Costa Rica
}

\section{Resumen}

Este ensayo tiene como objetivo fundamental dimensionar el impacto socioeducativo de la UNARED: Conexiones para la Creatividad en la formación de profesionales en Arte y docentes en Artes, concretamente en las Prácticas Profesionales Supervisadas y las Prácticas Docentes del estudiantado del Centro de Investigación, Docencia y Extensión Artística -CIDEA-, de la Universidad Nacional -UNA-. Para lo cual, se considera imprescindible, en primera instancia, establecer las bases desde las cuales se fundamentan los procesos de la investigación interdisciplinaria en Artes y que el CIDEA lo ha definido como un objetivo estratégico para el quinquenio 2010-2014. Esta RED de trabajo interdisciplinario ha sido constituida desde una visión de Desarrollo Humano Sostenible, la Complejidad aplicada a la Educación, con una metodología basada en la Teoría del Caos, diseñada específicamente para este proceso y con una visión Humanista de la Educación, tal y como lo plantea el Modelo Pedagógico de la Universidad Nacional de Costa Rica. Su fundamento metodológico es la Interdisciplinariedad, donde, estudiantes y docentes en Arte y Educación en Arte han construido y llevado a cabo procesos interdisciplinarios con especialistas en Salud Mental del Sistema Hospitalario Costarricense, adscrito a la Caja Costarricense del Seguro Social -CCSS- con el fin de analizar la viabilidad del trabajo conjunto entre el Arte y la Salud Mental de Costa Rica y mejorar con ello la calidad de vida de la población costarricense.

Palabras Clave: Experiencias Creativas, Desarrollo Humano Sostenible, Pensamiento Complejo, Teoría del Caos, Formación Universitaria de Profesionales en Artes y Enseñanza de las Artes.

\begin{abstract}
The main objective of this paper is to measure the socio-educational impact of the UNARED: Creative Connections for the training of professionals in Art and Art teachers, specifically in the Supervised Professional Practice and Teaching Practices of students of the Centro de Investigación, Docencia y Extensión Artística -CIDEA- of the National University of Costa Rica. For this purpose, it is considered essential first, to establish the basis from which the interdisciplinary research processes in Arts are founded. CIDEA has defined this as a strategic objective for the period 2010-2014. This RED (WEB) of interdisciplinary work has been established from a vision of sustainable human development, the
\end{abstract}


complexity assigned to Education, with a methodology based on Chaos Theory, designed specifically for this process with a humanistic vision of education, such as it is suggested by the Pedagogical Model of the National University of Costa Rica. Its foundation is the Interdisciplinary methodology, where students and teachers in Art and Art Education have built and implemented interdisciplinary processes together with mental health specialists of the National Hospital System, assigned to the Caja Costarricense del Seguro Social -CCSS- (Savings bank of the Welfare State), in order to analyze the feasibility of joint work between the Arts and Mental Health of Costa Rica and thus to improve the citizens' quality of life.

Key words: Creative Experiences, Sustainable Human Development, Complex Thinking, Chaos Theory, University training of Art Professionals and Arts Teaching.

\section{Introducción}

\section{Proyecto UNAREDES: Conexiones para la Creatividad}

El proyecto UNAREDES: Conexiones para la Creatividad, presentada por el CIDEA ante el Consejo Académico -CONSACA- de la Universidad Nacional -UNA- en abril del 2010 y aprobada en mayo de ese mismo año; es una iniciativa estratégica de gestión universitaria del CIDEA, para el fomento del trabajo en red en el campo de la formación profesional de Artistas y Docentes en Arte.

El proyecto plantea como objetivo general (Zúñiga E., 2010,p.6) el "constituir espacios creativos para el Desarrollo Humano Sostenible a través de la UNARED: Conexiones para Creatividad" y como primer objetivo específico el "generar protocolos en arte para la sistematización de experiencias creativas a través de la UNARED: Conexiones para Creatividad"; para luego "sistematizar experiencias creativas por medio de las tecnologías de la comunicación y la información a través de la UNARED: Conexiones para Creatividad" y por último "constituir UNARED virtual para la interacción de conocimientos de experiencias creativas por medio de las tecnologías de la comunicación y el conocimiento por medio de la página web UNARED: Conexiones para Creatividad", en proceso de diseño por estudiantes de cuarto nivel, en el año 2011, de la Escuela de Arte y Comunicación Visual, como parte de sus actividades académicas.

Dicha iniciativa permite constituir un amplio escenario de trabajo que se adapte a las necesidades de las Unidades Académicas del CIDEA y en espacios no tradicionales para la concreción del arte o la educación artística; fortaleciendo, así, la calidad y la pertinencia de la formación profesional de Artistas y Docentes en Arte que se forman en el CIDEA de la UNA.

La metodología diseñada para esta UNARED se basa en el trabajo en red y desde un enfoque de investigación sistémica, en donde las Escuelas de Arte del CIDEA, a través de la consolidación de sus propios espacios creativos, generan protocolos de sistematización de experiencias por medio de las alianzas estratégicas intersectoriales $y$, en concordancia con el pensamiento complejo, se genera en un proceso de trabajo recursivo - artístico y de educación artística en siete estadios de experiencias creativas que serán abordados según la necesidad del fenómeno social desarrollado, todo esto basado en las Siete Leyes del Caos de Briggs y Peat (1999, citados por Zúñiga E., 2010, p. 10) 


\section{Desarrollo Humano Sostenible}

La UNARED: Conexiones para la Creatividad se ha concretado con la articulación de un trabajo conjunto con la Dirección Nacional de Salud Mental de la Caja Costarricense del Seguro Social, con el fin de desarrollar experiencias creativas para la autoexpresión humana y con ello mejorar la calidad de vida de la población costarricense y contribuir a un Desarrollo Humano Sostenible -DHS-.

El concepto de DHS constituido como eje transversal del proyecto UNARED: Conexiones para la Creatividad es considerado como un "proceso de interconexión con la esencia humana dentro de un planeta con recursos finitos y casi agotados" (Zúñiga E., 2010, p. 3).

La anterior conceptualización parte de cinco principios fundamentales del concepto del "Otro Desarrollo" aplicado para los países del Tercer Mundo y que resume Ralph Carmen (2004, p. 43; citando a Nerfin 1975, p.10) como aquel desarrollo:

- Orientado a las necesidades humanas: (...) tanto materiales como no materiales (...) Asegurar la humanización de todos los seres humanos, mediante la satisfacción de sus necesidades de expresión, creatividad, igualdad y convivialidad $\mathrm{y}$ de entender $\mathrm{y}$ hacerse cargo de sus propios destinos.

- Endógeno: (...) brota del corazón de cada sociedad, la cual soberanamente define sus valores y visión a futuro.

- Autosuficiente: (...) cada sociedad se apoya primariamente en su propia fuerza y sus propios recursos, en términos de las energías de sus miembros y sus entornos naturales y culturales.

- Ecológicamente sano: (utiliza racionalmente los recursos de la biosfera, con plena conciencia del potencial de los ecosistemas locales (...) los límites globales (...) impuestos a las generaciones presentes y futuras.

- Basado en transformaciones estructurales: en las relaciones sociales, en las actividades económicas y en su distribución espacial (...) en las estructuras de poder, para así lograr las condiciones de autogestión y participación en la toma de decisiones, por parte de todos los que se ven afectados.

En la formación profesional de Artistas y Docentes en Arte para el desarrollo de experiencias creativas en el marco del proyecto UNARED: Conexiones para la Creatividad, estos cinco principios fundamentales se aplican de la siguiente manera:

- Necesidades Humanas: el Arte y la Docencia en Arte se basan en la comunicación desde un lenguaje estético mediado por distintos mecanismos de expresión, por tanto, desde Conexiones para la Creatividad, las y los participantes del CIDEA -estudiantes y docentes investigadores (as)- reconocen las necesidades de distintos grupos humanos para la autoexpresión desde un lenguaje creativo que permita armonizar su desarrollo mental, social y cultural mediante el desarrollo de estrategias mediadoras de las distintas disciplinas artísticas presentes en la UNA. 
- Endógeno: Las experiencias creativas desarrolladas por las y los participantes en el proyecto Conexiones para la Creatividad son el resultado de un diagnóstico interdisciplinario de las poblaciones con las que se trabaja, por tanto, cada proceso será distinto, en cuanto a su forma y fines, y cada cual es definido por los grupos humanos vinculados al proyecto.

- Autosuficiente y Ecológicamente Sano: La gestión universitaria, desde la UNARED: Conexiones para la Creatividad, garantiza la articulación de procesos intersectoriales que aprovechen al máximo los recursos de las instituciones y organizaciones con las que se participa de manera inter $y$ transdiciplinariamente; minimizando, en la medida de lo posible, el impacto ecológico en su desarrollo.

- Transformaciones estructurales: La UNARED: Conexiones para la Creatividad pretende generar cambios en los enfoques y metodologías de formación profesional de Artistas y Docentes en Arte propiciando en el estudiantado experiencias inter y transdiciplinares, intersectoriales, sistémicas y complejas que, a largo plazo generen cambios en la forma de creación artística y docencia en arte en Costa Rica que estén en estrecha relación con la realidad de la población costarricense, sus necesidades y desarrollo.

Por lo anterior, el concepto de DHS es una arista imprescindible para la comprensión del fundamento epistemológico y metodológico del proyecto UNARED: Conexiones para la Creatividad. Porque se parte de una visión integral del ser humano, sus múltiples realidades y la integralidad de este desde el arte, con experiencias creativas para el mejoramiento de la calidad de vida.

\section{Expresión desde las Artes y Educación en Artes: mecanismos de Autoexpresión Humana}

Al concepto de DHS se le debe sumar el fundamento epistemológico de la Expresión desde las Artes y la Educación en Artes ${ }^{1}$ como mecanismos de la Autoexpresión Humana, todas áreas de impacto del proyecto UNARED: Conexiones para la Creatividad, que fundamentan el mejoramiento de la calidad de vida de la sociedad costarricense.

En primer lugar, la Expresión Artística, facilitada por profesionales en el campo del Arte en el CIDEA, permite el desarrollo de conocimiento y construcción de lenguajes estéticos que propician la creatividad humana pues las manifestaciones artísticas "nos muestran y descubren, principalmente, quiénes y cómo somos los seres humanos, tanto individual como colectivamente: de lo que nos habla el arte, sólo nos habla el arte" (Marín R., 2003, p.6)

Por tanto, la Expresión Artística se vuelve una fuente inagotable para la autoexpresión humana que, mediada por el o la especialista en arte, se convierte en un mecanismo de vivencia, único en cada ser humano, para expresarse, reconocerse y ser reconocido (a) como persona en la sociedad y legitimando su potencial creativo y transformador. 
En segundo lugar, se encuentra la Educación en Artes que, desarrollada por las y los profesionales de la enseñanza en artes, permite la apropiación y el andamiaje neuronal de las y los educandos para la autoexpresión desde el lenguaje estético del Arte en sus diferentes disciplinas; es un proceso sistemático de creación y comunicación humana tal y como lo plantea Francisco Gutiérrez (1975, p.114; citado por Céspedes E., 2003, p.4):

Se da educación en el momento mismo en que se logra generar en el sujeto-agente educativo- acciones creadoras que dinamizan el proceso dándole historicidad (...) se constituye sujeto por medio de una expresión creadora que trasciende. La expresión creadora cristaliza en hechos la realización personal.

En este sentido, el proyecto UNARED: Conexiones para la Creatividad, ha adaptado la descripción del campo de conocimiento propio de la Educación Artística planteada por Marín (2003) para la descripción del campo de la Educación en Artes desde una mirada general e integradora de las Artes, de la siguiente manera:

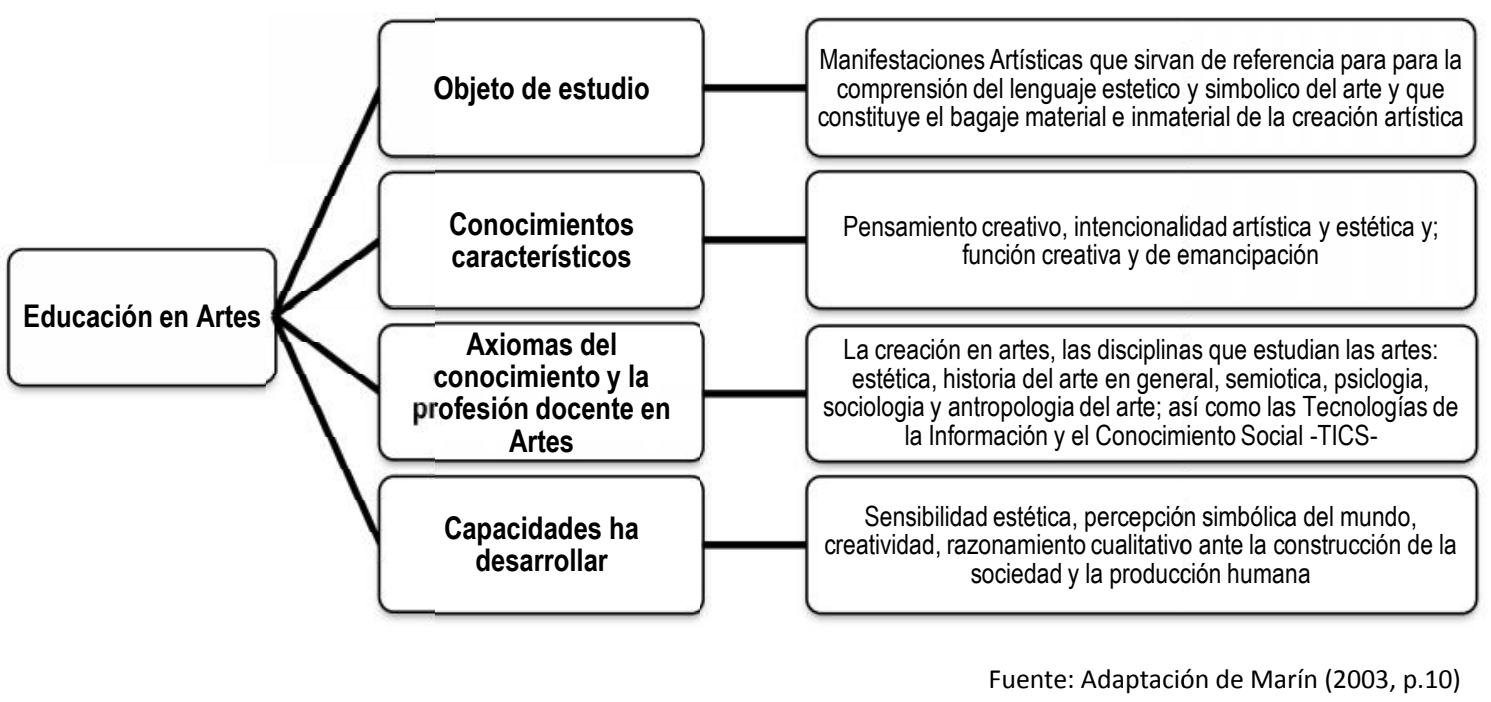

Figura n.1. Esquema del campo integral de la Educación en Artes

De manera integral, se establece que la formación profesional para la Expresión Artística y la Educación en Artes, presentan ejes comunes de desarrollo, como lo son el estudio del arte, sus manifestaciones y su acervo cultural que generan en las sociedades; por tanto, desde la UNARED: Conexiones para la Creatividad se prioriza una metodología que atiendan estos ejes comunes de manera integral e inclusiva en la formación profesional y en el vínculo de la UNA con la Sociedad Costarricense.

\section{Lo Interdisciplinario}

Para el proyecto UNARED: Conexiones para la Creatividad una de las bases fundamentales de cada experiencia creativa, es la necesidad de generar en el estudiantado y en el equipo docente capacidades de pensamiento relacional y construcción de habilidades para unir e integrar situaciones y aspectos que la 
investigación y práctica artística y la docencia especializada en arte separan de otros conocimientos.

En el CIDEA entendemos que un campo interdisciplinario es "un campo de estudio que cruza los límites tradicionales entre varias disciplinas académicas o entre varias escuelas de pensamiento, por el surgimiento de nuevas necesidades" (Zúñiga E., 2011, p.6); en consecuencia, para la UNARED: Conexiones para la Creatividad, es importante que, cada experiencia creativa, construya un lenguaje común entre las disciplinas artísticas con otras áreas del saber que permitan, en docentes y estudiantes, desarrollar habilidades y destrezas para el trabajo interdisciplinar.

EI proyecto UNARED: Conexiones para la Creatividad pretende contribuir a la formación integral de las y los profesionales en arte y de enseñanza de las artes a través de metodologías de investigación y prácticas que se fundamente epistemológicamente en la conexión entre las disciplinas artísticas -primer nivel de interdisciplinariedad e interdependencia- y entre estas con las demás áreas del conocimiento -segundo nivel de interdisciplinariedad e interdependencia- a fin de que sus actividades no se produzcan en forma aislada, dispersa o fraccionada; de manera que se produzcan nuevos conocimientos que sean pertinentes y en concordancia con la realidad costarricense. Entre las características de la Interdisciplinariedad, definidas por el CIDEA (Zúñiga E., 2011, p. 6) y que a las personas participes integran en la UNARED: Conexiones para la Creatividad, se encuentran:

- El requerimiento de que el conocimiento del objeto de estudio se aborde de forma integral, desde varias perspectivas: de manera que las experiencias creativas se constituyan desde las diferentes áreas del conocimiento artístico o científico (social o positivista)

- Elaboración de nuevos enfoques metodológicos, idóneos para la solución de los problemas que se dan en la práctica artística o docente en artes: de tal manera que las experiencias creativas sean en sí mismas únicas, ya que sus metodologías interdisciplinarias sean la respuesta a las necesidades de autoexpresión de las comunidades con las que se trabaja.

- Promueve la complementariedad de diferentes enfoques ante el objeto de estudio: así los conocimientos, habilidades, actitudes, aptitudes y destrezas desarrolladas en las y los participantes del CIDEA que se generan en las experiencias creativas son complementarias con las áreas del saber con las que se trabajan en el desarrollo de las mismas.

- Se la considera una filosofía y marco metodológico que busca sistemáticamente la integración de las teorías, métodos, instrumentos: por tanto las y los participantes del CIDEA practican procesos de investigaciónacción que sistemáticamente y de formal interdisciplinar e interdependiente analizan sus propias prácticas para su diseño, adecuación y evaluación.

A la luz de este enfoque de gestión universitaria, investigación y práctica artística y docente en artes se puede establecer que la UNARED: Conexiones para la Creatividad, más que ser un proyecto aislado del CIDEA plantea acciones integrales para el desarrollo de competencias para un nuevo (a) profesional en artes y docente en artes, desde la misma práctica docente y estudiantil. 


\section{Paradigma Educativo de la formación profesional de Artistas y Docentes en Arte del CIDEA}

Para establecer el impacto en los procesos de formación de profesionales del CIDEA, se requiere establecer cuáles son las bases paradigmáticas que fundamentan los cambios de la UNARED: Conexiones para la Creatividad. Se toma como referente epistemológico el Paradigma Humanista que es punto de partida del Modelo Pedagógico de la Universidad Nacional (Costa Rica).

\section{Paradigma Humanista para la formación profesional de Artistas y Docentes en Arte del CIDEA}

La educación en la UNA se caracteriza por ser epistemológicamente basada en el paradigma humanista de la educación, por tanto, aunque el proceso de investigación y practica de la UNARED: Conexiones para la Creatividad se aborda desde el pensamiento emergente o complejo, el fin último es concretar una educación humanista para la formación de profesionales en Arte y Docentes en Arte.

Es de utilidad, para los efectos de este apartado, contestar la pregunta ¿cuál es el proceso que concreta este paradigma humanista de la educación? Se inicia con enunciar las características fundamentales de humanismo aplicado a la educación y su relación con los procesos llevados a cabo en la UNARED: Conexiones para la Creatividad.

En primer lugar, el pensamiento humanista aplicado a la educación parte de que el objeto de estudio debe ser abordado desde "las necesidades de los individuos como la base de las decisiones educativas" (Hernández G., 1998, p.106) que en la UNARED: Conexiones para la Creatividad es un requerimiento fundamental para el desarrollo de destrezas, habilidades, aptitudes y conocimientos interdisciplinarios en las y los estudiantes e investigadores (as) del CIDEA, es decir, en la UNARED: Conexiones para la Creatividad se trabaja en la construcción conjunta de espacios creativos según las necesidades de autoexpresión de la población por medio de prácticas artísticas y docentes en arte y otras áreas de las ciencias (sociales y duras).

Además, la UNARED: Conexiones para la Creatividad se basa en la concepción de que todas las personas tienen el derecho a la autoexpresión y reafirmación de su humanidad, por tanto se concreta una segunda máxima del paradigma humanista que "concede al conocimiento personal tanto valor como al conocimiento público" (Hernández G., 1998, p.106). Así, el conocimiento personal se concreta en la producción de procesos de autoexpresión que son distintos en cada población abordada y lo interno en cada persona que conforma el grupo; el conocimiento público, en el caso concreto de las experiencias creativas está conformado por las diferentes áreas del saber artístico y del saber científico (social o duro) que, de manera interdisciplinaria, construyen en el proceso de facilitación de expresión y autoexpresión.

El paradigma humanista promueve un aprendizaje significativo que "involucra a la persona como totalidad (procesos afectivos y cognitivos) y se desarrolla en forma experiencial (...) participativo y en un ambiente de respeto, compresión y apoyo" 
(Hernández G., 1998, p.111) por tanto, en la UNARED: Conexiones para la Creatividad esta máxima es de vital importancia, pues las y los estudiantes e investigadores (as) del CIDEA que participan del proceso generan procesos cognitivos y participan, a partir de su propia experiencia, en la construcción de nuevos conocimientos, en el marco del respeto hacia otros y otras profesionales y hacia la dignidad de las personas participes de las experiencias creativas.

De esta manera, en la UNARED: Conexiones para la Creatividad, el DHS está mediado por la autoexpresión humana -individual y colectiva- posibilitada, de manera interdisciplinar, en las sesiones de experiencias creativas; con el fin de concretar la formación desde el paradigma humanista en el que su fundamenta el proceso educativo de la UNA. Lo anterior se puede representar de la siguiente manera:

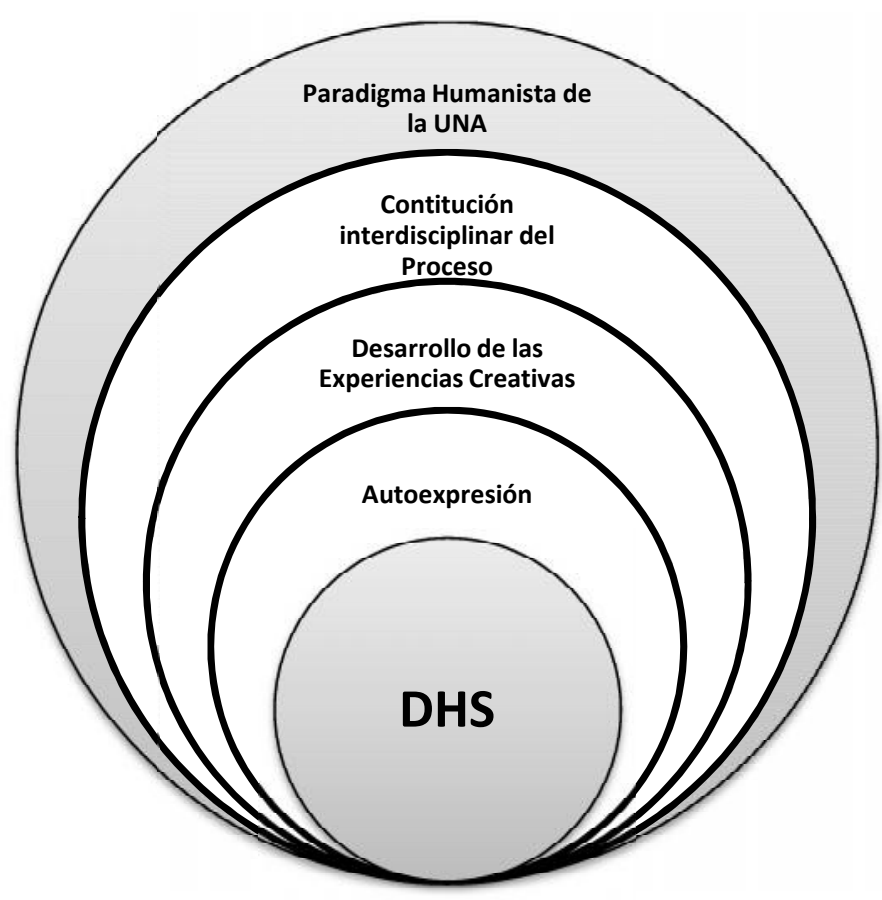

Fuente: Elaboración propia, 2011

Figura n.2. Acople de las acciones y objetivos de UNARED: Conexiones para la Creatividad para concretar la formación desde el paradigma humanista en el cual se fundamenta la UNA

Como se aprecia el proceso de concreción del paradigma humanista, base fundamental de cualquier proceso en la Universidad Nacional, en la UNARED: Conexiones para la Creatividad tiene sus cimientos en la visión de la formación de profesionales en el campo de las Artes y de la Docencia en Artes desde el Desarrollo Humano Sostenible, desde la autonomía, la criticidad, la reflexión y en concordancia con el pensamiento emergente y con ello, dar coherencia y pertinencia a esta UNARED. 


\section{Aportes para un currículo Integral en la formación profesional de Artistas y Docentes en Arte en el CIDEA}

En este proceso de justificación es importante que la UNARED: Conexiones para la Creatividad, establezca las bases en que fundamenta los aportes que impactan en la formación profesional en el CIDEA a partir de un currículo integral a nivel universitario.

\section{Enfoque educativo}

El enfoque educativo del cual parte la construcción de cada una de las experiencias creativas que desarrollan las y los estudiantes e investigadores (as) del CIDEA es desde un Proceso Dialéctico que se basa según Bolaños (2010, pp.92-94) en:

1. Acción socialmente productiva de la educación (...) lo esencial en currículo es la praxis como medio de relación entre el sujeto y la realidad.

2. Sus objetivos se orientan al desarrollo del pensamiento crítico - reflexivo que permita al alumno en un proceso de reflexión - acción lograr incorporarse en el proceso de transformación social (...) el alumnado plantea los objetivos (...) surgen en el proceso de acción - reflexión.

3. El educando es activo y participativo dentro la practica socio-educativa.

4. El docente participa como elemento interactuante con el alumno en el proceso de reflexión-acción. Se caracteriza por su papel crítico y activo dentro de la práctica socio-educativa.

5. El contenido emerge de la reflexión - acción y enfatiza los aportes de la cultura cotidiana.

6. La metodología se centra en procesos sistémicos de acción - reflexión (...) de manera participativa.

7. El contexto socio-cultural es un elemento fundamental puesto que los procesos de acción-reflexión se sustentan en la compresión y transformación de ese contexto. Revaloriza la cultura cotidiana como elemento fundamental para el currículo.

8. Los recursos educativos esenciales para el desarrollo de la practica socioeducativa lo constituyen el medio socio-cultural y los actores sociales.

9. La evaluación se concibe como un proceso constante y participativo en el que se propicia la evaluación formativa, la auto y mutua evaluación.

Lo anterior se puede contextualizar en la UNARED: Conexiones para la Creatividad de la siguiente manera: 


\begin{tabular}{|c|c|c|c|c|c|c|c|c|}
\hline Praxis & $\begin{array}{l}\text { Objetivos de } \\
\text { aprendizaje }\end{array}$ & Estudiantado & $\begin{array}{l}\text { Docentes - } \\
\text { Facilitadores }\end{array}$ & $\begin{array}{c}\text { Contenidos } \\
\text { socioeducativos } \\
\text { de las } \\
\text { experiencias }\end{array}$ & Metodologías & Contexto Social & $\begin{array}{l}\text { Recursos } \\
\text { facilitadores }\end{array}$ & Evaluación \\
\hline $\begin{array}{l}\text { Las y los } \\
\text { estudiantes e } \\
\text { investigadores } \\
\text { (as) del CIDEA, } \\
\text { sobre la propia } \\
\text { práctica de las } \\
\text { experiencias } \\
\text { creativas, } \\
\text { generan nuevos } \\
\text { conocimientos } \\
\text { según el } \\
\text { contexto en } \\
\text { donde trabajen } \\
\text { interdisciplinari } \\
\text { amente }\end{array}$ & $\begin{array}{l}\text { Las y los } \\
\text { estudiantes e } \\
\text { investigadores } \\
\text { (as) del CIDEA } \\
\text { construyen, a } \\
\text { partir de un } \\
\text { diágnostico } \\
\text { interdisciplinar, } \\
\text { los objetivos } \\
\text { de aprendizaje } \\
\text { que el contexto } \\
\text { les demanda }\end{array}$ & $\begin{array}{l}\text { Las y los } \\
\text { estudiantes e } \\
\text { investigadores (as) } \\
\text { del CIDEA son } \\
\text { protagonistas de su } \\
\text { propio proceso de } \\
\text { acción - reflexión y } \\
\text { su condición de } \\
\text { participantes } \\
\text { activos en el } \\
\text { desarrollo de las } \\
\text { experiencias } \\
\text { creativas es vital } \\
\text { importancia para la } \\
\text { asimilación de } \\
\text { nuevos saberes } \\
\text { inter y } \\
\text { transdisciplinares }\end{array}$ & $\begin{array}{l}\text { Las y los } \\
\text { estudiantes e } \\
\text { investigadores } \\
\text { (as) del CIDEA, } \\
\text { se convierten } \\
\text { en facilitadores } \\
\text { de } \\
\text { conocimiento, } \\
\text { con } \\
\text { pensamiento } \\
\text { flexible y } \\
\text { relacional, } \\
\text { capaces de } \\
\text { facilitar } \\
\text { espacios para el } \\
\text { trabajo } \\
\text { conjunto con } \\
\text { especialistas de } \\
\text { otras áreas del } \\
\text { conocimiento }\end{array}$ & $\begin{array}{l}\text { Las y los } \\
\text { estudiantes e } \\
\text { investigadores } \\
\text { (as) en conjunto } \\
\text { con sus docentes } \\
\text { establecen, a } \\
\text { partir del } \\
\text { diagnóstico } \\
\text { interdisciplinario } \\
\text { de la población } \\
\text { con la que se } \\
\text { trabaja sus } \\
\text { características y } \\
\text { sus necesidades } \\
\text { de autoexpresión }\end{array}$ & $\begin{array}{l}\text { Ya que el abordaje } \\
\text { de las experiencias } \\
\text { creativas es } \\
\text { interdisciplinario, } \\
\text { la característica } \\
\text { metodológica } \\
\text { fundamental es el } \\
\text { proceso sistémico } \\
\text { e } \\
\text { interdependiente } \\
\text { derivado de la } \\
\text { acción - reflexión }\end{array}$ & $\begin{array}{l}\text { Las y los } \\
\text { estudiantes e } \\
\text { investigadores } \\
\text { (as) y docentes } \\
\text { del CIDEA } \\
\text { parten de un } \\
\text { contexto socio- } \\
\text { cultural } \\
\text { concreto, con } \\
\text { necesidades } \\
\text { particulares y } \\
\text { abordajes } \\
\text { únicos, lo que } \\
\text { permite }\end{array}$ & $\begin{array}{l}\text { Las y los } \\
\text { participantes del } \\
\text { CIDEA, a partir de } \\
\text { la propia práctica, } \\
\text { producen los } \\
\text { recursos } \\
\text { didácticos } \\
\text { necesarios para la } \\
\text { mediación de } \\
\text { conocimientos } \\
\text { para la } \\
\text { autoexpresión } \\
\text { humana desde un } \\
\text { lenguaje artístico } \\
\text { como una } \\
\text { herramienta } \\
\text { fundamental para } \\
\text { los objetivos de la } \\
\text { experiencia } \\
\text { creativa }\end{array}$ & $\begin{array}{l}\text { La evaluación del } \\
\text { proceso es, en su } \\
\text { totalidad, } \\
\text { formativa, las y los } \\
\text { estudiantes e } \\
\text { investigadores (as) } \\
\text { del CIDEA van } \\
\text { desarrollando } \\
\text { habilidades, } \\
\text { destrezas, } \\
\text { aptitudes y } \\
\text { conocimientos en } \\
\text { la propia práctica } \\
\text { interdisciplinaria } \\
\text { que son evaluadas } \\
\text { después de cada } \\
\text { sesión en conjunto } \\
\text { con las y los } \\
\text { prefesionales de } \\
\text { otras disciplinas } \\
\text { con los que se } \\
\text { trabaja }\end{array}$ \\
\hline
\end{tabular}

Fuente: Adaptación de Bolaños (2010, pp. 92-94)

Tabla n.1. Concreción del enfoque del Proceso Dialéctico en la UNARED: Conexiones para la Creatividad 
Como se aprecia en la Tabla n.1, el desarrollo de las experiencias creativas se centra en las interacciones entre todas las personas partícipes del proceso que es más un "recorrido creativo (...) lo que interesa es la dinamicidad y riqueza de su movimiento que se va generando en el camino, progresivamente, y a la lux de ciertos mojones previsibles para cada transición desde el currículo, pero no limitantes" (Flórez R., 2005, p.279).

Por tanto, el enfoque del Proceso Dialéctico ocurre sistémicamente, en todas las fases de construcción curricular, en donde las experiencias creativas son catalizadores de transformación desde una comunicación horizontal entre todos (as) sus participantes que son parte de una sociedad en permanente cambio, lo que le da pertinencia a este nuevo espacio académico universitario desarrollado desde el Decanato del CIDEA.

\section{Procesos desarrollados por las y los estudiantes e investigadores (as) del CIDEA en la UNARED: Conexiones para la Creatividad}

Las y los estudiantes e investigadores (as) del CIDEA, con la facilitación de conocimiento que desarrollan sus docentes y derivado de un diagnóstico interdisciplinario, planifican las experiencias creativas desde modelos integradores.

Lo anterior, se concreta en la articulación de procesos metodológicos que relacionan sus disciplinas artísticas y docentes artísticas conjuntamente con las disciplinas de las otras ciencias que convergen e interactúan en la experiencia creativa a desarrollar y concretamente se va a describir la metodología estructurada por las y los participantes del CIDEA y de los Hospitales San Vicente de Paul y el Chacón Paut, durante el segundo semestre del 2011, a modo de plan piloto, avalado por las Direcciones Medicas de ambos centros hospitalarios y de la Dirección de Salud Mental de la Caja Costarricense y de Seguro Social.

En este sentido, la articulación entre la salud y el arte, ocurre en la práctica de las y los estudiantes e investigadores (as) del CIDEA quienes, de manera interdisciplinar, propician la transformación de la realidad mediante la integración del conocimiento interdisciplinar a la "realidad histórica y cultural" (Bolaños G., 2010, p.99) que abordan con cada experiencia creativa.

\section{Principios Fundamentales}

En este sentido, las y los estudiantes e investigadores (as) e investigadores (as) partícipes del proceso de la UNARED: Conexiones para la Creatividad, en esta primera etapa, han desarrollado el siguiente proceso de planificación socioeducativa, de manera integral e interdisciplinariamente, que según Bolaños (2010, pp. 110-112) son principios fundamentales para la construcción de un currículo pertinente: 
- Integralidad: las actividades planificadas pertenecen a un concepto integral de mejoramiento de la calidad de vida; las doce sesiones de trabajo se diseñaron en cuatro niveles, desde las necesidades de la población atendida, el abordaje médico y psicológico-psiquiátrico, las posibilidades de abordaje de la danza y las posibilidades de abordaje de la música. Dicho proceso ha sido concebido en tres fases que, sistemáticamente, las y los estudiantes e investigadores (as) e investigadoras de la UNA y la dirección médica de cada hospital aceptaron implementar, consiste en sesiones previas de diseño, ejecución interdisciplinaria, evaluación del proceso y estructuración de siguiente sesión después de cada día de trabajo.

- Proceso participativo: las y los estudiantes e investigadores (as) partícipes de la UNARED: Conexiones para la Creatividad diseñan, ejecutan y evalúan los procesos socioeducativos implementados mediante la participación constante de otras y otros especialistas de área de las artes, la medicina y con las y los participantes propiamente.

- Proceso permanente: la UNARED: Conexiones para la Creatividad, por naturaleza es un proyecto basado en la reflexión-acción y la recursividad; lo que permitió que las y los estudiantes e investigadores (as) partícipes hayan desarrollado competencias para la reflexión y evaluación constante en su propia practica como facilitadores de procesos socioeducativos con enfoque psicosocial, de manera interdisciplinar y transdisciplinarmente.

- Flexibilidad: las y los estudiantes e investigadores (as) e investigadores (as) del CIDEA han desarrollado flexibilidad de pensamiento y aplicación de metodologías, es decir, han generado procesos metacognitivos que son visibles en la atención a las poblaciones con las que trabajan en las sesiones en los hospitales y directamente en las y los participantes, para atender las necesidades específicas individuales y grupales que se presenten.

\section{Características pedagógicas - curriculares esenciales}

Este modelo integrador parte de dos características pedagógicas - curriculares esenciales para la planificación de experiencias socioeducativas con enfoque de atención psicosocial:

\section{Principio de continuidad}

Para Bolaños (2010, p.113) el principio de continuidad responde "a la necesidad de llenar las expectativas y posibilidades que va presentando el individuo, como producto del proceso de desarrollo humano", en el caso del trabajo desarrollado por la UNARED: Conexiones para la Creatividad, las y los estudiantes e investigadores (as) e investigadores (as) del CIDEA, han aplicado este principio de continuidad desde dos puntos de partida, uno las características de las poblaciones atendidas y los objetivos psicosociales que 
abordan las y los especialistas de la salud mental de los hospitales en los que se ha trabajado.

Después, se toma en cuenta el desarrollo de las propias experiencias y los cambios suscitados en las y los participantes; concretando en acciones socioeducativas, el pensamiento interdisciplinario, relacional, flexible y reflexivo necesario en las y los profesionales en Arte y Docencia en Artes del nuevo milenio.

\section{Coherencia horizontal}

El principio de coherencia horizontal está plasmado, para el caso concreto de la UNARED: Conexiones para la Creatividad, en la integración de las posibilidades que brindan las disciplinas artísticas a las necesidades médicas y de salud mental de las poblaciones que se atienden, a través de un diseño basado en un tema, objetivos psicosociales (en donde las y los especialistas en salud mental y médicos participan de manera activa), metodologías, actividades (que se resumen como experiencias creativas a desarrollar), recursos, tiempo, responsables y evaluación del proceso de la sesión (en donde las y los estudiantes e investigadores e investigadoras del CIDEA son los profesionales que marcan las pautas del proceso).

\section{Planeamiento interdisciplinario del proceso}

Como se ha planteado en los apartados anteriores, la interdisciplinariedad es un elemento fundamental del diagnóstico, diseño, ejecución y evaluación de las experiencias creativas de la UNARED: Conexiones para la Creatividad, por tanto, las y los estudiantes e investigadores (as) del CIDEA, en su propia práctica artística y práctica docente han desarrollado una metodología de trabajo que parte de las características de las poblaciones atendidas por las y los especialistas en medicina y salud mental de los hospitales con los que se trabaja, en este sentido, las sesiones se estructuran de la siguiente manera:

- Tema: El tema se define desde el diseño general de las experiencias, según las necesidades que, desde la salud mental y física, deben ser atendidos, se parte de la experiencia y práctica médica de las y los especialistas del área y se analiza su secuencia y horizontalidad de manera interdisciplinaria sumando la experticia y dominio disciplinar de las y los estudiantes e investigadores (as) del CIDEA, tanto en Artes como en Docencia en Artes.

- Objetivos: Responden al proceso definido por las y los especialistas del área médica y del área de salud mental de los centros hospitalarios, además de las y los especialistas de las disciplinas artísticas que participan del proceso. Es importante señalar que los objetivos varían según la población y el hospital donde se trabaja; sumado a esto, también se debe tomar en cuenta los ejes de calidad de vida que se 
desarrollaran y el análisis interdisciplinar que se desprenda de las características de la población atendida.

- Experiencias creativas a desarrollar: En el caso concreto de las experiencias creativas desarrolladas, durante el segundo semestre del 2011, las y los estudiantes e investigadores (as) del CIDEA, han diseñado sesiones de trabajo, mediante protocolos de trabajo, que parten de su propio dominio disciplinar y experticia en el campo de las artes. La estrategia de acción es totalmente recursiva, entre lo inter y transdiciplinar, es decir, se parte del análisis interdisciplinar de las poblaciones, se elaboran estrategias entre los equipos de trabajo, se ejecuta la sesión, de manera transdiciplinar desde las artes y la docencia en artes, y se evalúa interdisciplinarmente el proceso de la misma con los y las especialistas de los centros hospitalarios y se regresa al proceso de diseño transdiciplinar, en el campo de las artes y la docencia en artes, de la siguiente sesión.

- Evaluación de la sesión y observaciones para próxima sesión: La evaluación desarrollada en el proceso de trabajo de las experiencias creativas de la UNARED: Conexiones para la Creatividad, parten del principio de integración y de una visión de proceso inter y transdisciplinar para el mejoramiento de la calidad de vida, por tanto, se evalúa tres procesos, simultáneamente, a saber:

-El proceso de desarrollo psicosocial a través de las experiencias creativas de cada participante al finalizar cada sesión y que permite elaborar, interdisciplinariamente, las observaciones necesarias para el diseño transdisciplinar de la siguiente sesión.

-El desarrollo integral de las y los participantes, en su contexto social y familiar, durante el proceso de trabajo de las experiencias creativas.

-Las habilidades, destrezas y conocimientos, clasificados por competencias que, las y los estudiantes e investigadores (as) del CIDEA, desarrollan duran el diagnóstico, diseño, ejecución y evaluación de las sesiones en cada experiencia creativa desarrollada.

De esta manera, el planteamiento interdisciplinario de la UNARED Conexiones para la Creatividad esta sustentada en los principios del DHS al basarse en las necesidades humanas al reconocer las necesidades de distintos grupos humanos para la autoexpresión desde un lenguaje creativo que permita armonizar su desarrollo mental, social y cultural por parte de las y los estudiantes y docentes de la UNA; endógeno pues las experiencias creativas son el resultado de un diagnóstico interdisciplinario de las poblaciones con las que se trabaja; autosuficiente y ecológicamente sano pues se busca una articulación de procesos intersectoriales que aprovechen al máximo los recursos de las instituciones y organizaciones con las que se participa y que a su vez generen transformaciones estructurales al plantear cambios en los enfoques y metodologías de formación profesional de Artistas y Docentes en Arte. 
Adicionalmente, se busca producir nuevos conocimientos, pertinentes a la realidad de la sociedad costarricense tomando en cuenta los principios de la Interdisciplinariedad de la UNARED Conexiones para la Creatividad, en donde el abordaje del objeto de estudio se de forma integral, en donde las y los estudiantes y docentes del CIDEA elaboran nuevos enfoques metodológicos desde la práctica artística o docente, exigiendo de todas y todos los involucrados de la UNA y de la CCSS una complementariedad de enfoques disciplinares (artes y ciencias -duras y sociales-) en la práctica de procesos creativos y con ello buscar nuevos marcos metodológicos desde la integración de las teorías, métodos, instrumentos.

Por lo anterior, en los espacios creativos desarrollados durante el año 2011 se plateó el siguiente protocolo de trabajo:

\begin{tabular}{|c|c|c|c|c|c|}
\hline Sesión № & & Lugar: & & & \\
\hline Tema & Objetivo & $\begin{array}{l}\text { Experiencias creativas } \\
\text { a desarrollar } \\
\text { transdisciplinariamente }\end{array}$ & Recursos & Tiempo & Responsables \\
\hline \multicolumn{2}{|c|}{ Evaluación de la sesión: } & & & & \\
\hline \multicolumn{2}{|c|}{$\begin{array}{l}\text { Observaciones para próxima } \\
\text { sesión: }\end{array}$} & & & & \\
\hline
\end{tabular}

Fuente: Adaptación de Jiménez, Fidalgo, Zárate y Zárate (2011).

Tabla n.2. Protocolo para el diseño de sesiones de la UNARED: Conexiones para la Creatividad

Este protocolo, resultado del trabajo conjunto de estudiantes/académicos (as) del CIDEA y de especialistas en Salud Mental de la CCSS, permite evidenciar una Praxis académica desde un enfoque Dialéctico, pues cada sesión esta construida interdisciplinariamente procurando registrar la propuesta de sesión, la retroalimentación de la población a la hora de su ejecución y en su etapa de Evaluación; además asegura un análisis integral de todas las personas especialistas en artes y ciencias en su apartado de Observaciones, para ser incluidas en la configuración de la siguiente sesión.

Como se aprecia, la planificación, ejecución y evaluación de cada una de las experiencias creativas de la UNARED: Conexiones para la Creatividad, se desarrollan, sistemáticamente, desde la inter y transdisciplinariedad, tomando en cuenta el contexto y necesidades de las poblaciones atendidas, lo que permite el desarrollo de habilidades, destrezas y conocimientos, de un nuevo perfil de él y la profesional en Arte y de Docencia en Arte, que se forma en el CIDEA; a través del diseño de metodologías propias en materia de gestión universitaria, académica, investigativa y práctica artística y docente en artes. 


\section{Consideraciones Finales para la construcción de competencias en la formación integral de profesionales en Arte y Educación en Arte a partir de las prácticas profesionales y las practicas docentes en la UNARED: Conexiones para la Creatividad}

Esta UNARED tiene impacto en diferentes niveles del ámbito universitario desde una gestión universitaria planteada para trabajar desde la interdependencia, la interconexión de saberes y el desarrollo de actividades en espacios intersectoriales.

Posee, además, metodologías de investigación que impactan en los currículos de las Unidades Académicas del CIDEA, concretamente en los cursos de Práctica Artística y Práctica Docente en artes se puede establecer que la UNARED: Conexiones para la Creatividad, más que ser un proyecto aislado del CIDEA plantea acciones integrales para el desarrollo de competencias para un nuevo (a) profesional en artes y docente en artes, desde la misma práctica docente y estudiantil.

Como se aprecia, para concretar el paradigma humanista, base fundamental de cualquier proceso en la Universidad Nacional y en especial en la UNARED: Conexiones para la Creatividad, tiene sus cimientos en la visión de la formación de profesionales en el campo de las Artes y de la Docencia en Artes desde el Desarrollo Humano Sostenible, desde la autonomía, la criticidad, la reflexión y en concordancia con el pensamiento emergente y con ello, dar coherencia y pertinencia a esta UNARED.

Permitiendo con ello, que las y los estudiantes que participan de la investigación, diseño, ejecución y evaluación de experiencias creativas en espacios hospitalarios, sean parte de un proceso académico universitario concebido desde la visión y misión de la Universidad Nacional y en sinergia con el pensamiento emergente.

Una investigación en Artes planteada desde el Proceso Dialéctico da como resultado que de manera sistémica todas las fases de la construcción de UNARED: Conexiones para la Creatividad para el desarrollo de experiencias creativas en espacios hospitalarios sean catalizadores de transformación desde una comunicación horizontal entre todos (as) sus participantes que son parte de una sociedad en permanente cambio, lo que le da congruencia a este nuevo espacio académico universitario desarrollado desde el Decanato del CIDEA.

Como se aprecia, la planificación, ejecución y evaluación de cada una de las experiencias creativas de la UNARED: Conexiones para la Creatividad, se desarrollan sistemáticamente desde la inter y transdisciplinariedad, tomando en cuenta el contexto y necesidades de las poblaciones atendidas, lo que permite el desarrollo de habilidades, destrezas y conocimientos, de un nuevo perfil de él y la profesional en Arte y de Docencia en Arte, que se forma en el CIDEA; a través del diseño de metodologías propias en materia de gestión universitaria, académica, investigativa y práctica artística y docente en artes.

Actualmente, la UNARED: Conexiones para la Creatividad está sistematizando las experiencias creativas desarrolladas en Costa Rica, específicamente en el Hospital San Vicente de Paul, en la provincia de Heredia, y en el Hospital Psiquiátrico Chacón Paut, en la 
provincia de Cartago, con el fin de evidenciar las competencias generadas en las y los estudiantes e investigadoras del CIDEA que han participado en el plan piloto; estas competencias se clasifican desde el modelo del Instituto Internacional de Planeamiento de la Educación de la UNESCO, con sus "Estrategias de Educación y de Formación para los grupos desfavorecidos: ¿Cómo Articular competencias básicas y técnicas en la capacitación de jóvenes?" de Pedro Milos (2003, pp.17-42):

- La Auto reflexión de la propia práctica profesional en Arte y Docencia en Artes: la formación universitaria de Profesionales en Arte y de Docentes en Arte debe partir de dos principios fundamentales que se concretan en la UNARED Conexiones para la Creatividad; en primer lugar, la responsabilidad de la Universidad Nacional de formar profesionales, en todos los campos del saber, capaces de incidir en la realidad de la sociedad costarricense para potenciar su desarrollo con equidad e inclusión; en segundo lugar, la responsabilidad del CIDEA de gestar espacios para la nueva generación de conocimiento en Arte y de Docencia en Arte, al estar constituido como un Centro de Investigación especializado garante de procesos formativos de calidad y pertinentes a la realidad nacional.

- La Expresión y Comunicación: cada participante del CIDEA, a través del desarrollo de un proceso formativo humanista, dialéctico e interdisciplinario, potencia sus capacidades expresivas y comunicativas, al estar en contacto con otros grupos de profesionales que están inmersos en la realidad nacional y con las poblaciones directamente afectadas, es decir, constituir procesos formativos universitarios que permita a estudiantes y académicos (as) interrelacionarse y retroalimentarse, de manera asertiva y proactiva, con la sociedad costarricense.

- El Pensamiento Crítico y Reflexivo: como base para la innovación y la creatividad, pues la criticidad y la reflexión permiten evaluar diferentes perspectivas sobre un objeto de estudio, clarificar procesos de trabajo académico conjunto e interdisciplinario a través del estudio profundo de situaciones reales con grupos de expertos, que sobre información existente de las poblaciones con las que se trabaja, dando como resultado la generación de propuestas pertinentes y eficaces que, creativamente, innovan en el campo de la formación de profesionales en Arte y Docentes en Arte al transferir conocimientos de las disciplinas artísticas a procesos inter y transdiciplinares al abordar las poblaciones hospitalarias en conjunto con profesionales del área de la salud.

- El Aprendizaje Autónomo: que se especifica al dimensionar a las y los estudiantes universitarios como actores de su propio proceso de aprendizaje, en donde, como sujetos histórico - sociales se desarrollan académicamente en contextos reales de aprendizaje y con ello procuran una formación integral que parte de su participación como grupo de Investigadores (teoría) y Promotores (práctica) del desarrollo social y cultural de la sociedad costarricense.

- El Trabajo Colaborativo: que facilita el trabajo basado en la diversidad de capacidades, enfoques, disciplinas y logro de metas del grupo de trabajo; de 
manera que se facilite el sentido de responsabilidad, investigación, confianza, comunicación asertiva, procesos de evaluación y autoevaluación, aprovechando al máximo las fortalezas y oportunidades individuales y grupales, que en el marco de un proceso formativo universitario, se transforma en una metodología ideal para la generación creativa de nuevos conocimientos, puestos al beneficio de la sociedad, en general y de las poblaciones hospitalarias, en particular.

- La Responsabilidad Social: desarrollada a través de una sistemática revisión de los procesos desarrollados, en conjunto con profesionales de las ciencias sociales y exactas, que le permite a cada estudiante y académico (a) del CIDEA construir una serie de mecanismos de interacción con la sociedad desde una visión ética y con una intencionalidad transformadora.

Como se observa todo el proceso de gestión académica y administrativa de la UNARED: Conexiones para la Creatividad que se plantea desde el Decanato del CIDEA, es integral e inclusivo para el lograr espacios para la formación de un (a) nuevo (a) profesional en Artes y de Docencia en Artes, con competencias para el trabajo inter y transdisciplinar en nuevos espacios de inserción y vinculación con la sociedad costarricense.

Por todo lo anterior, las experiencias creativas contribuirán al Desarrollo Humano Sostenible para la integración de lo cognitivo y lo sensible, en nuevos espacios y a partir de nuevas competencias a través de un currículo universitario que transformará la formación universitaria de profesionales en Arte y Docentes en Arte.

\section{Referencias Bibliográficas}

Bolaños, G. (2010). Introducción al Currículo. Costa Rica: Editorial de la Universidad Estatal a Distancia, EUNED.

Carmen, R. (2004). Desarrollo Autónomo: Humanización del paisaje: una incursión en el pensamiento y la práctica radical. Costa Rica: Editorial de la Universidad Nacional, EUNA.

Céspedes, E. (2003). Principios y Técnicas Recreativas para la Expresión Artística del Niño. Costa Rica: Editorial de la Universidad Estatal a Distancia, EUNED.

Flórez, R. (2005). Pedagogía del Conocimiento. Colombia: McGraw-Hill Interamericana S.A. Hernández, G. (1998). Paradigmas en Psicología de la Educación. México: Editorial Paidós Mexicana, S.A.

Jiménez, P.; Fidalgo, E.; Zárate, O.; y Zárate, S. (2011). Protocolo para el diseño de sesiones de la UNARED: Conexiones para la Creatividad en el Hospital San Vicente de Paul. Documento sin publicar. Heredia: Decanato del CIDEA, Universidad Nacional.

Marín, R. (Coord.). (2003). Didáctica de la Educación Artística. España: Editorial Pearson Educación, S.A. 
Milos, P. (2003). Estrategias de Educación y de Formación para los grupos desfavorecidos: ¿Cómo Articular competencias básicas y técnicas en la capacitación de jóvenes?. Francia: Instituto Internacional de Planeamiento de la Educación de la UNESCO.

Zúñiga, E. (2010). Conexiones para la Creatividad. Perfil de proyecto para el fondo UNARED de CONSACA, documento sin editar. Costa Rica: Decanato del Centro de Investigación, Docencia y Extensión Artística, UNA.

Zúñiga, E. (2011). Iniciativas Interdisciplinarias. Perfil de reestructuración del Programa Fondo Interdisciplinario para la Vicerrectoría Académica, documento sin editar. Costa Rica: Decanato del Centro de Investigación, Docencia y Extensión Artística, UNA.

\section{Notas}

\footnotetext{
${ }^{1}$ Se utiliza el término Educación en Artes, pues Educación Artística hace referencia únicamente a las artes visuales, y el proyecto UNARED: Conexiones para la Creatividad está concebido desde una visión integral de la formación profesional de artistas y docentes en arte, que incluyen las demás disciplinas artísticas que se desarrollan en le CIDEA (Artes Visuales, Danza, Música y Teatro). Se recomienda la lectura del primer capítulo de Marín (2003, p.9) que plantea que "el territorio y contenidos propios de la Educación Artística son las Artes Visuales" la cual es conceptualizada ontológica, epistemológica, metodológica e técnicamente por el Coordinador del libro "Didáctica en la Educación Artística".
}

Cita de Artículo:

Zúñiga Murillo, E (2013). Experiencias Creativas para el Desarrollo Humano Sostenible: espacios para la formación profesional en Arte en Costa Rica. Revista de Docencia Universitaria. REDU. Vol.11 (1) Enero-Abril. pp. 265-284 Recuperado el (fecha de consulta) en http://www.red-u.net/ 


\section{Acerca de la autora}

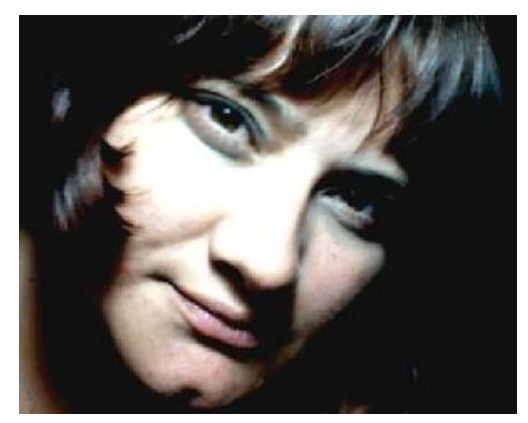

\section{Enid Sofía Zúñiga Murillo}

Universidad Nacional, Costa Rica

Centro de Investigación, Docencia y Extensión Artística CIDEA-

Mail: enidsofia@gmail.com

Máster en Gestión de la Educación con Énfasis en Liderazgo, Universidad Nacional. Licenciada en Administración de Programas de Educación No Formal, Universidad de Costa Rica. Bachiller en Artes Dramáticas, Universidad De Costa Rica. Técnico Medio en Artes con Énfasis en Teatro. Conservatorio de Castella. Actriz profesional desde 1994. Gestora de Proyectos Culturales desde 2000. Investigadora del Centro de Investigación, Docencia y Extensión Artística -CIDEA-, Universidad Nacional desde 2005. Investigadora y Académica en la Administración de la Educación No Formal, Facultad de Educación, Universidad de Costa Rica desde 2007. Sus principales líneas de investigación son la Interdisciplinariedad en la formación profesional de artistas y educadores sociales. 


\section{Retos institucionales de la formación del profesorado universitario}

Institutional Challenges of university teacher training

Imma Torra Bitlloch

Universitat Politècnica de Catalunya, España

María Dolors Màrquez Cebrián

Universitat Autònoma de Barcelona, España

Teresa Pagès Costas

Universitat de Barcelona, España

Pau Solà i Ysuar

Universitat Pompeu Fabra, España

Rafael García Campos

Universitat de Girona, España

Fidel Molina Luque

Universitat de Lleida, España

Àngel-Pío González Soto

Universitat Rovira i Virgili, España

Albert Sangrà Morer

Universitat Oberta de Catalunya, España

\section{Resumen}

El Grupo Interuniversitario de Formación Docente (GIFD) está formado por las unidades y centros de formación (ICE-UB, IDES-UAB, ICE-UPC, CQUID-UPF, ICE-UdG, ICE-UdL, ICE-URV y eLC-UOC) de las ocho universidades públicas catalanas. Estas unidades actualmente se encuentran en un proceso de reformulación de las actividades y planes de formación que están ofreciendo al profesorado universitario para facilitar el desarrollo de su actividad docente. El replanteamiento de sus actuaciones nace de un interés común vinculado a la calidad de la formación docente del profesorado universitario, coherente con los planteamientos promulgados por el Espacio Europeo de Educación Superior (EEES). Este interés y preocupación conduce al desarrollo de un estudio de identificación de las competencias docentes que debe poseer el profesorado universitario para abordar con éxito el desarrollo de las nuevas titulaciones de grado acordes con el EEES. Además este trabajo recoge un cuestionamiento y reflexión en relación a las principales características que deberían tener las actividades y programas de formación dirigidas al profesorado para facilitar su tarea docente; apelando a la labor que, tanto las unidades o centros de formación, las instituciones universitarias a las que pertenecen y los organismos 
vinculados a la acción docente, deberían replantearse para dar respuesta a las nuevas exigencias docentes requeridas al profesorado. El objetivo del trabajo es provocar la reflexión y generar el debate sobre el camino que debe seguir la formación docente del profesorado universitario, como elemento estratégico de la institución, para contribuir a alcanzar los objetivos de calidad a los que las universidades se han comprometido en el proceso de verificación de los nuevos títulos.

Palabras clave: formación, universidad, competencias, profesorado, reconocimiento.

\begin{abstract}
The units or training centers (ICE IDES CQUID, ELC) of the eight public universities in Catalonia, constituted as Interuniversity Group on Teacher Education (GIFD), are currently undergoing a reformulation process concerning the training activities and plans being offered to university teachers, in order to facilitate the development of their teaching activity. The rethinking of their actions is born from a common interest linking the quality of university teachers' training with coherent approaches in the European Higher Education Area (EHEA). This interest and concern has led the group to conduct a research study with the objective of establishing a set of standard teaching skills for the faculty staff to master, with the purpose of successfully undertaking the development of the new degree courses in line with the directives of the European Higher Education Area. This paper contains a thorough examination and analysis regarding the main features that activities and training programs aimed at teachers should include, in order to facilitate the performance of the teaching activity itself, by focusing at the work of training units or training centers, at the academic institutions where they belong, and at the related agencies, and by requesting them to update their educational tasks in order to meet the new requirements that the role of teachers demand. The aim of this work is to stimulate thought and to generate a debate on the direction that university teachers' training must follow, not only as a strategic element of the institution, but also contributing to achieve the quality objectives to which universities have committed themselves in the process of validating the new degrees.
\end{abstract}

Key words: training, university, competences, teachers, teaching recognition.

\title{
Introducción
}

En este artículo se analizan las condiciones en las que se desarrolla la profesión docente en la universidad: sus características, puntos fuertes y puntos débiles, etc. También se plantean cuáles son las acciones que las instituciones universitarias pueden promover para conseguir una mejora continua en la docencia, teniendo en consideración que, algunas de ellas, pueden ser impulsadas de forma individual por cada universidad, mientras que otras pueden requerir la coordinación entre las universidades, o incluso el soporte institucional de la administración responsable de la educación superior.

El análisis de la situación actual arranca de un estudio elaborado por las unidades o centros de formación de las ocho universidades públicas catalanas (GIFD), que ha sido subvencionado parcialmente por el Ministerio de Educación y Ciencia dentro de la convocatoria de Estudios y Análisis del año 2011 (EA2010-0099), y por la Agència de Gestió d'ajuts Universitaris i de Recerca (AGAUR), de la Generalitat de Catalunya $(2010 \mathrm{MQD00049})^{1}$. En este trabajo, teniendo en cuenta que la adaptación al Espacio Europeo de Educación Superior (EEES) está provocando cambios importantes en la tarea docente del profesorado, se plantea que una de las primeras cuestiones que las universidades deben hacerse es cómo motivar al profesorado para que éste aborde el 
cambio cultural que representa la nueva situación. Para ello es vital que la institución defina de forma muy clara qué es lo que espera del profesor, tanto desde un punto de vista individual cómo cuando éste actúa como miembro de un equipo docente responsable de la formación de los titulados universitarios.

\section{El papel de la institución}

Los expertos coinciden en destacar la importancia que adquiere la implicación de la institución en los planes de formación que se diseñen para el profesorado. La tarea docente del profesorado consiste en poner el conocimiento disciplinar a disposición de los estudiantes, con la finalidad que éstos adquieran el perfil profesional que la universidad ha definido para ellos en la nueva propuesta de títulos universitarios. Sin embargo, el proceso formativo de los estudiantes no sólo es responsabilidad de cada profesor individual, sino también de las acciones que el conjunto de profesores emprenda como equipo docente y éstas deben desarrollarse con criterios de calidad y mejora continua, con el fin de conseguir unos resultados de los que la institución debe rendir cuenta.

La nueva definición del perfil profesional de los titulados, basada ahora en las competencias profesionales que estos deben adquirir, modifica la tarea docente del profesorado tanto en su actividad individual en el aula con los estudiantes, como en el seno del equipo docente. Así, se producen cambios en el planteamiento del proceso de enseñanza-aprendizaje y, por tanto deberán replantearse los contenidos y los modelos de formación que se proponen al profesorado; además adquiere una gran importancia la coordinación horizontal y vertical entre los miembros del equipo docente de una misma titulación. El profesorado debe considerarse como una pieza más de la arquitectura que la universidad pone en juego para conseguir una enseñanza de calidad.

La formación del profesor debe garantizar su autonomía en el seno del equipo docente, pero de manera que sea concordante con el perfil de formación establecido por la universidad y adecuada al contexto. En ningún caso el profesorado debe percibir que la necesidad de formación es fruto de una deficiencia propia y una tarea a abordar en solitario, sino que esta obedece al reto que, motivado por el nuevo contexto educativo europeo, ha asumido el conjunto de la institución.

La voluntariedad de la formación, sea inicial o permanente, así como el planteamiento de una formación intrínsecamente relacionada con la actividad del profesorado en la propia institución, son características que distinguen la formación universitaria de la que se realiza para titulados que desempeñan cargos intermedios fuera del ámbito universitario.

La motivación puede conseguirse facilitando la formación en el seno de un grupo de profesores que persigan unos mismos objetivos. En algunos casos puede resultar útil que el grupo de trabajo sea disciplinar, es decir, formado por profesores pertenecientes a una misma disciplina, área de conocimiento, departamento, para que la acción sea aplicable de forma inmediata a un grupo acotado de estudiantes. En otros casos, puede ser más aconsejable el trabajo interdisciplinar entre distintos 
departamentos, facultades o incluso universidades para poder mostrar una gran variedad de contextos donde se desarrolla la acción docente.

Si la institución persigue un cambio cultural real en la universidad, debe dirigir las acciones formativas tanto al profesorado de nuevo ingreso como al profesorado consolidado en la institución y, además, establecer algún sistema de evaluación y/o reconocimiento que no se reduzca únicamente a la antigüedad en la universidad.

\section{Características y modelos de la formación del profesorado universitario}

Cuando se plantea el diseño de la formación del colectivo docente universitario debe tenerse en cuenta que los profesores acceden a la universidad sin que sea requisito tener una formación previa que les capacite para la tarea docente que se les va a encargar. Esta característica diferencia al profesorado universitario de otros colectivos profesionales a los que se les exige una formación y preparación predeterminada para abordar la responsabilidad laboral que van a desarrollar.

Conscientes de esta situación, un significativo número de unidades o centros de formación de las universidades españolas ofrecen de forma diferenciada una formación inicial para el profesorado de nuevo ingreso y una formación continua para el resto de profesorado, toda ella de carácter voluntario.

Estos niveles de formación, que ofrecen la mayoría de universidades españolas, concuerdan con la propuesta de Valcárcel (2003) quien amplía hasta cuatro los niveles de formación, establecidos según el momento de desarrollo profesional en que se encuentra el profesorado universitario:

- Formación previa: dirigida básicamente a personal con posibilidades de iniciar la carrera universitaria.

- Formación inicial: ofertada a los profesores noveles, normalmente con escasa experiencia docente y con una notoria precariedad laboral.

- Formación continua: dirigida a profesores con experiencia y adaptada a las necesidades concretas del propio docente, de su universidad, departamento, etc.

- Formación especializada en enseñanza disciplinar: orientada a aquel profesorado universitario con una larga trayectoria profesional, que por su propia inquietud y por la necesidad de las instituciones donde labora, desee dedicarse al diseño y aplicación de planes de estudios, la innovación, la mejora de la calidad, suponiendo este proceso una formación de formadores.

Cuando un profesor asiste a los cursos que le ofrece su universidad está recibiendo la formación de forma simultánea al ejercicio de la profesión, lo que puede ser aprovechado para que él mismo constate las mejoras que esta formación aporta en su práctica docente. La inmediatez en los resultados puede traducirse en un estímulo para seguir progresando en su formación. En el caso de la formación inicial, se constata que a menudo, el profesorado que realiza esta formación es después el más interesado en participar en la formación continua a lo largo de su carrera profesional. 
Aunque las unidades de formación son muy conscientes que las responsabilidades del profesor universitario en el desarrollo de su profesión abarcan el ámbito docente, el investigador y, en muchos casos, el desempeño de la gestión, la formación que se ofrece al profesorado dentro de la universidad va dirigida mayoritariamente a procedimientos y herramientas que le ayuden en el desarrollo de su tarea docente y, en menor medida, a su tarea investigadora o de gestión. Además, se parte de la suposición que el profesorado posee ya un buen conocimiento de los contenidos y las habilidades propias de la disciplina que debe desarrollar con los estudiantes y se puede decir que casi en ningún caso las unidades o centros de formación presentan oferta de formación disciplinar.

\section{El perfil del profesorado formador}

Hasta este momento, en la mayoría de universidades la formación del profesorado recae en gran parte en profesores de la propia institución. El hecho que un formador pueda ser compañero próximo del profesor que se está formando, puede facilitar el establecimiento de posteriores contactos entre ambos y la creación de nuevas redes de trabajo. Este modelo, largamente utilizado hasta ahora, facilita la transmisión de los valores de la universidad y de su modelo docente. Sin embargo, requiere de la planificación de una formación de los formadores puesto que, solo así, la universidad podrá asegurar que las acciones formativas se realizan con una unidad de criterio.

En algunos casos, la participación como formador del profesorado universitario, de personal externo a la universidad, incluso procedente del ámbito no universitario, puede resultar útil. En este caso se facilita el conocimiento de nuevos contextos y situaciones que pueden ayudar a ampliar el horizonte de la actividad docente.

La unidad o centro de formación de cada universidad deberá valorar en cada caso las ventajas e inconvenientes de cada modelo, y combinar ambas opciones según los contenidos a tratar en cada caso y los objetivos perseguidos.

\section{Características de la formación docente del profesorado de las universidades catalanas}

Desde el Grupo Interuniversitario de Formación Docente (GIFD) se ha realizado un estudio sobre las acciones formativas que, en estos momentos, se están ofreciendo para la formación de su profesorado. Los datos obtenidos han permitido analizar las coincidencias que se producen tanto en lo que se refiere a las modalidades formativas, como a los programas y contenidos que proponen.

Un punto de especial interés era averiguar si la oferta formativa incluía la formación en las competencias docentes identificadas por el grupo de trabajo (competencia interpersonal, metodológica, comunicativa, planificación y gestión de la docencia, trabajo en equipo e innovación. GIFD, 2011), ya sea de forma explícita o bien si su tratamiento se incluye de forma implícita dentro de un contexto de formación más amplio.

Con el objetivo de poder recoger el estado actual de las diferentes unidades de formación del profesorado de las universidades públicas catalanas respecto a las 
acciones que se están realizando, se diseñó y validó una encuesta en línea. Las preguntas se agruparon en dos bloques: en el primero se recogieron los datos de la universidad y de la unidad encargada de la formación del profesorado universitario; en el segundo bloque se agruparon las preguntas que hacían referencia a la información sobre la oferta formativa actual. Por último se dejó un espacio en el cual se pudieran reflejar comentarios generales o sugerencias.

\section{Resultados y análisis de la encuesta}

Los datos que presentamos se refieren a las ocho universidades públicas catalanas consultadas y reflejan la situación actual de las unidades de formación del profesorado universitario público en Cataluña. Hay que destacar que todas las universidades han facilitado la información solicitada a través de sus unidades de formación del profesorado (GIFD, 2011).

1) Sobre la pregunta de si la oferta de formación en docencia se enmarca dentro de un programa establecido y organizado:

El análisis de los resultados nos muestra que todas las universidades disponen de programas de formación, ya sea de formación inicial o de formación continua, dirigidos, respectivamente, a profesores noveles o a profesorado que llevan ya un tiempo ejerciendo la docencia en la universidad. En su oferta las universidades también incorporan actividades de formación concebidas para atender la demanda planteada por el propio profesorado universitario lo que se considera oferta a medida de las necesidades de cada centro.

Estos datos son coincidentes con los obtenidos en un estudio preliminar con 30 universidades españolas, en las que se obtenían resultados parecidos: el 100\% de unidades de formación consultadas ofrecen un programa de formación permanente, mientras que el $86,7 \%$ ofrece un programa de formación específico para noveles.

Todas las unidades y centros de formación consultadas aseguran que la formación en docencia se enmarca dentro de un programa establecido que engloba tanto la formación inicial como la permanente, aunque en diferentes niveles de estructuración. Menos de un $25 \%$ dispone de un programa claramente definido para la formación inicial, en cambio en la formación a demanda es donde se aprecia un mayor porcentaje de actividades formativas estructuradas en programas (entre el $51 \%$ y el $75 \%)$.

Queda aparte la formación a demanda solicitada desde los centros o facultades, que se adapta más a las exigencias y necesidades propias del colectivo de profesores y profesoras de cada centro o facultad, que a una programación organizada desde las unidades de formación y con una finalidad concreta.

2) Sobre la pregunta de qué porcentaje representa la formación en competencias sobre el total de la formación docente, no incluida en cursos específicos:

De acuerdo a las respuestas obtenidas, la formación por competencias representa un porcentaje importante sobre el total de la oferta formativa, incorporándolo en mayor o menor grado en algunos o todos sus programas formativos: 
- De las 8 unidades encuestadas, 3 unidades manifiestan que ésta representa entre el $76-100 \%$ de la formación inicial.

- Respecto a la formación permanente, 5 unidades han respondido que la formación por competencias representa entre el 51-75\% del total.

- En relación a la formación a demanda 4 unidades (50\% de la muestra) consideran que la formación por competencias es equivalente al $26-50 \%$ del total.

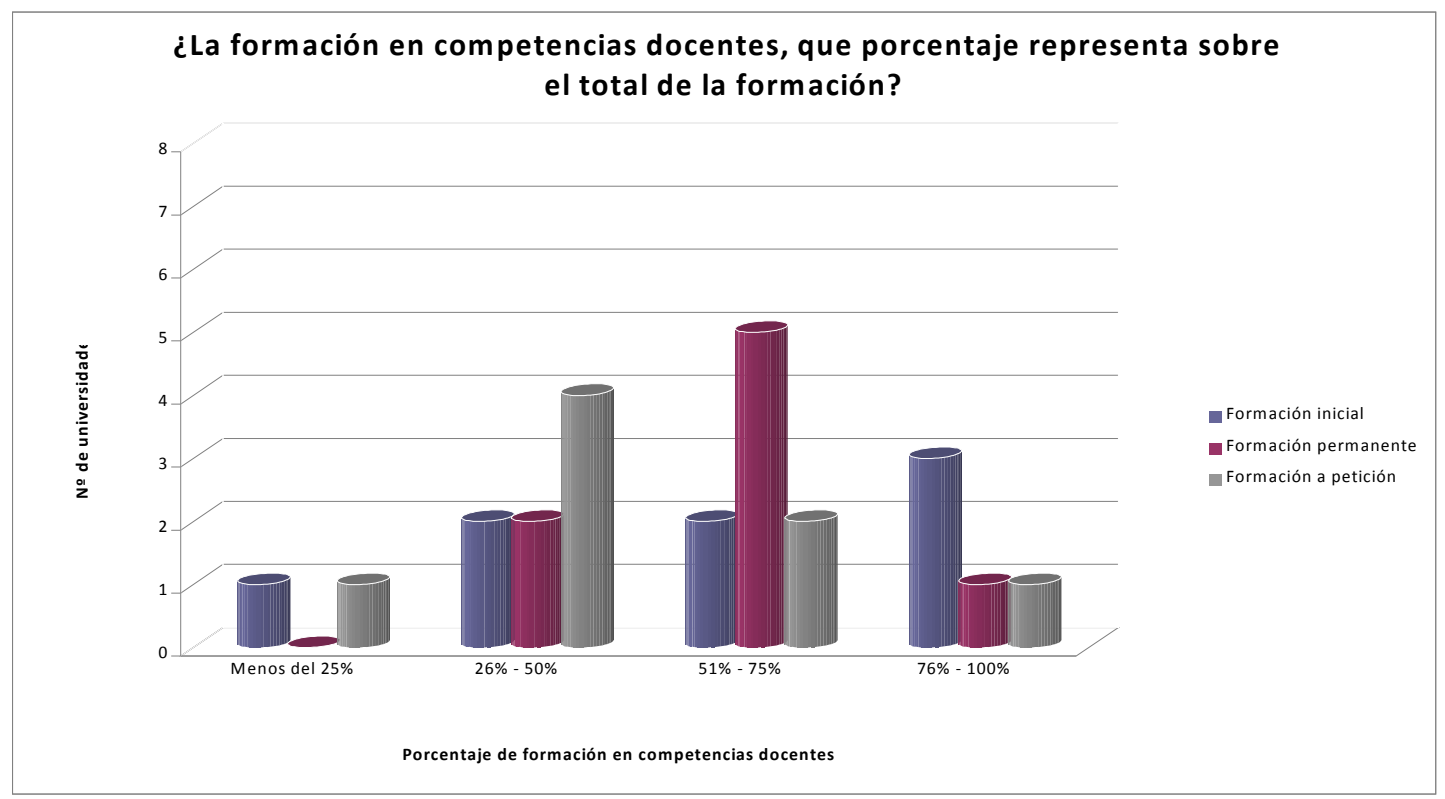

De acuerdo con los resultados obtenidos podemos afirmar que, aunque no se haga de manera explícita en la oferta de las distintas unidades, la formación en competencias adquiere mayor importancia en la primera etapa de la formación del profesorado y a medida que éstos adquieren experiencia la formación se orienta hacia aspectos más específicos e instrumentales.

3) En relación a la oferta formativa de cursos específicos orientados al desarrollo de competencias docentes:

En nuestra encuesta queríamos distinguir entre formación docente no centrada en competencias (pero en la que, de alguna manera, se pudiera estar incidiendo en su adquisición) y formación docente específicamente centrada en el desarrollo de las distintas competencias docentes.

Así, en primer lugar, se preguntaba por un lado qué competencias docentes se trabajan en los cursos de formación, de manera no específica y, por otro lado, sobre el porcentaje que esta formación representaba en relación al total de cursos en cada nivel ofrecidos desde las distintas unidades de formación.

- El análisis de los actuales programas y actividades de formación que tienen las universidades, muestra que todas incluyen en mayor o menor medida la formación en competencias docentes aunque algunas, como el trabajo en 
equipo o la planificación y gestión de la docencia, tienen una presencia muy baja.

- Respecto a la formación inicial la mayoría de unidades trabajan la competencia metodológica, la comunicativa y la de planificación y gestión de la docencia. Las que se encuentran más dispersas son las referentes a la competencia interpersonal y de innovación.

- En la formación continua también se trabajan más la competencia metodológica, la comunicativa y la de planificación y gestión de la docencia incorporándose la interpersonal. Respecto a la formación a demanda las que se hacen más presentes son la metodológica y la que menos la comunicativa.

En segundo lugar, se preguntaba si se ofrecían cursos específicos orientados al desarrollo de las competencias docentes. En caso afirmativo debía señalarse cuáles eran las competencias que se trabajaban.

La oferta de formación orientada específicamente a la adquisición y desarrollo de competencias docentes varía considerablemente entre las distintas universidades.

- Así, en relación a la formación inicial un 33,3\% de las respuestas apuntan a que representa entre el $26-50 \%$ sobre el total de formación, cuando otro 33,3\% afirma que se trata de entre el $51-75 \%$ sobre el total.

- En la formación permanente la opinión está dividida, un 33,3\% afirman que la formación en competencias representa menos del $25 \%$ sobre el total y otro $33,3 \%$ afirman que representa entre el $51-75 \%$ sobre el total.

- Respecto a la oferta de formación a demanda, destacamos que un $42 \%$ de las respuestas afirman que la formación competencial representa menos del $25 \%$ del total.

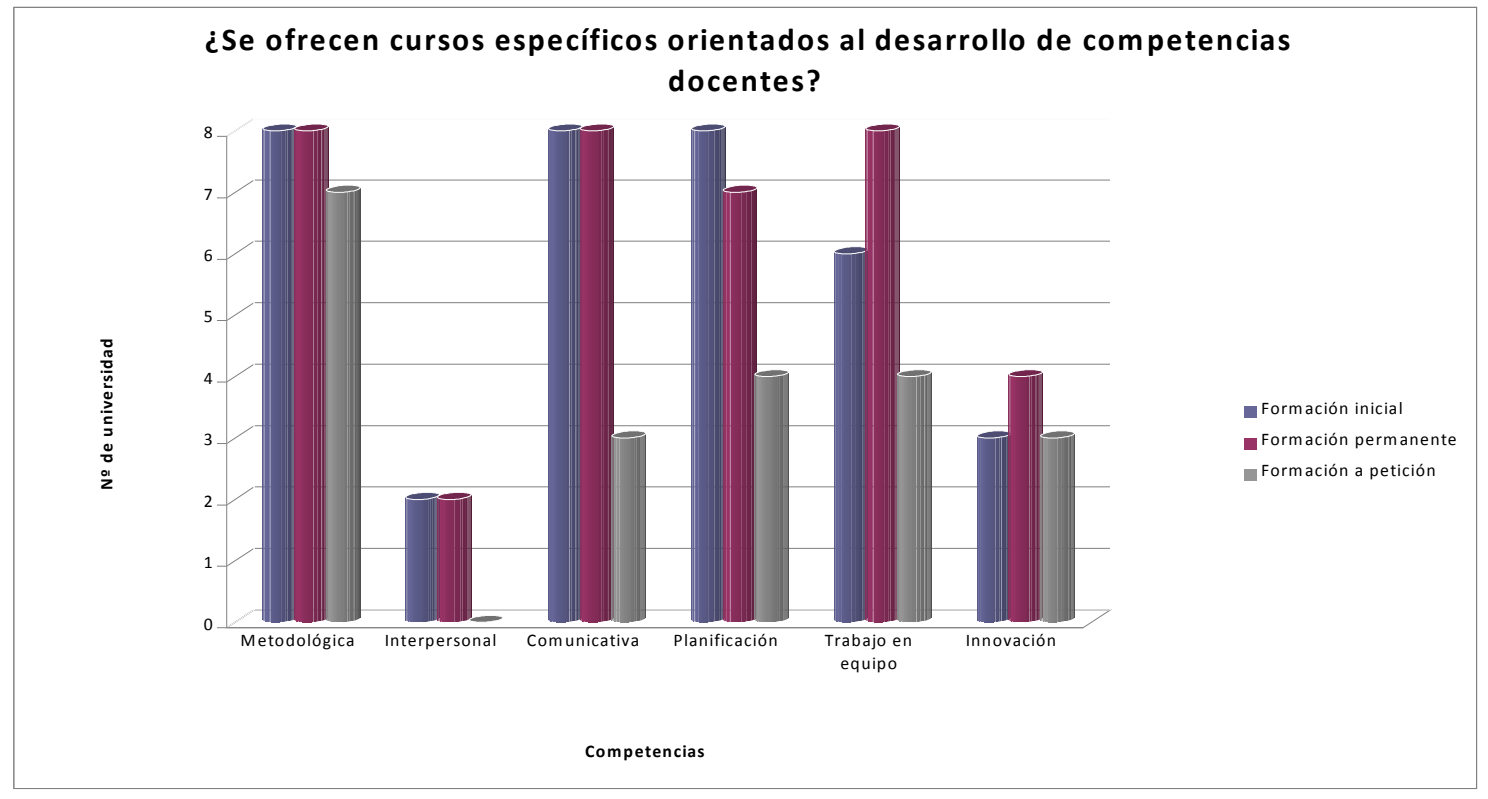

Las competencias más desarrolladas de manera específica en la formación inicial son: 
- Metodológica y comunicativa, en un $100 \%$ de las unidades y trabajo en equipo en un $75 \%$.

- Destaca la competencia de planificación que tiene un resultado nulo para este nivel de formación, seguida de la de Innovación en un 37,5\% y la Interpersonal, en sólo el $25 \%$ de las unidades.

Respecto a la formación permanente, las competencias que han recibido mayor atención son de nuevo:

- La metodológica, comunicativa y planificación y gestión de la docencia (en un $100 \%$ las dos primeras y un $87,5 \%$ la segunda). Siendo nula la de trabajo en equipo.

En la formación a demanda las menos requeridas son:

- La interpersonal (resultado nulo) seguida de la comunicativa y la de innovación con tan sólo un $37,5 \%$ de las unidades. Para este nivel de formación la más trabajada es la Metodológica en un 87,5 \%.

4) Sobre metodologías utilizadas para la formación docente en cada nivel formativo:

- En la formación inicial se utiliza más la metodología de los cursos y talleres $(62,5 \%)$. Seguido de jornadas y asesoramiento (50\%) y seminarios $(25 \%)$.

- En la formación continua o permanente se combinan casi todas las metodologías propuestas [cursos, jornadas, talleres (75\%), seminarios $(62,5 \%)]$, siendo la menos utilizada el asesoramiento en sólo un $25 \%$.

- Sin embargo en la formación a medida predomina el asesoramiento en la que el $62,5 \%$ de las unidades afirman utilizarla. El resto se reparte entre cursos y seminarios $(50 \%)$, talleres $(37,5 \%)$ y jornadas $(25 \%)$.

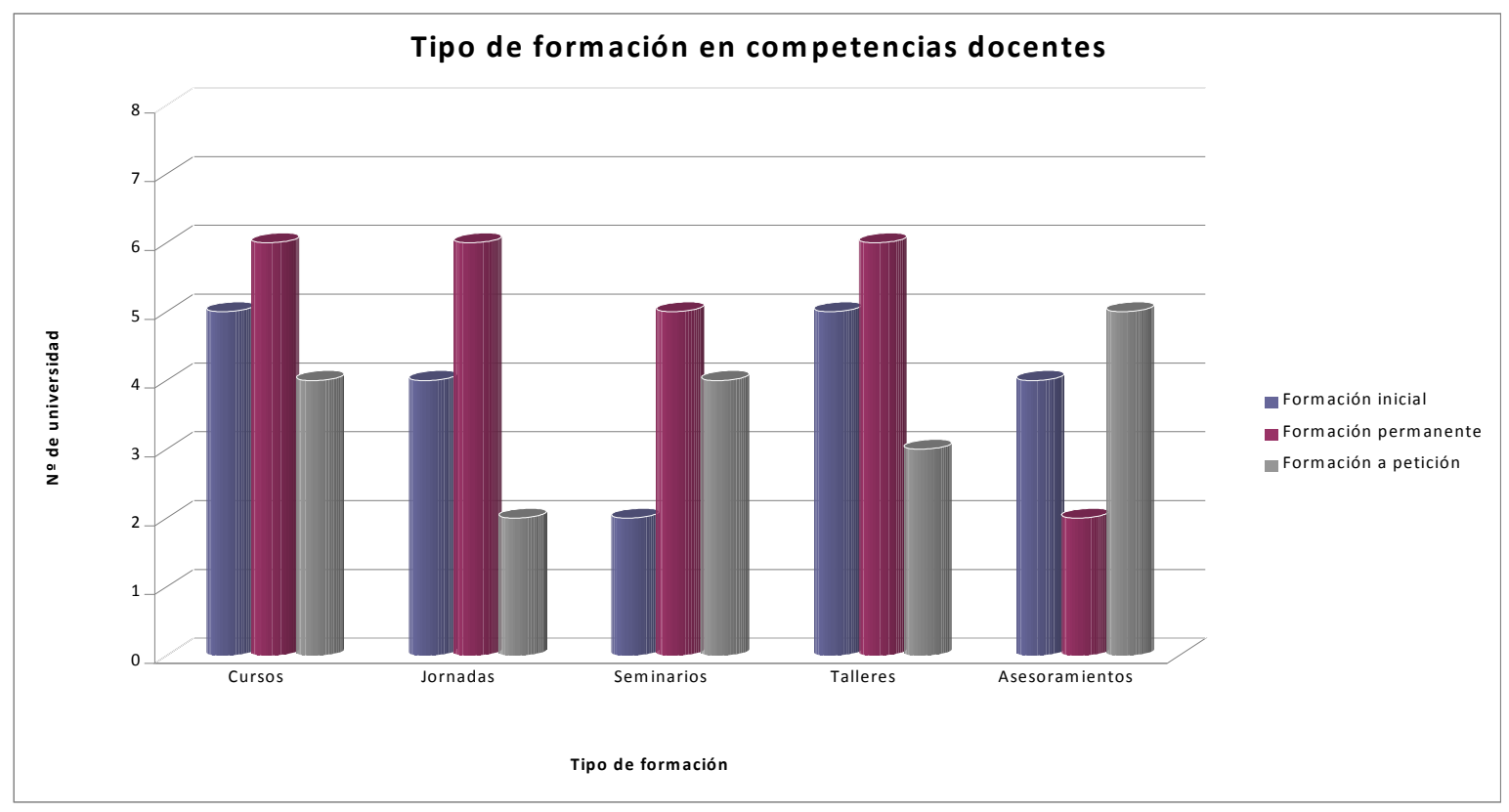


Finalmente la encuesta plantea dos preguntas relativas a la evaluación de la formación en competencias docentes.

Sobre si se realiza un diagnóstico o análisis del nivel competencial del profesorado,

- Los resultados indican que solo 2 unidades hacen un diagnóstico previo a la formación inicial, y solo 1 unidad lo hace respecto a la formación permanente

- 3 unidades lo llevan a cabo durante la formación inicial y 1 unidad en todo tipo de formación.

- 3 unidades evalúan después de la formación inicial y 2 unidades lo hacen después de todo tipo de formación.

Sobre si se hace algún tipo de evaluación o seguimiento del impacto o de la transferencia de la formación realizada

- 3 unidades realizan un seguimiento del impacto de la formación en el nivel de la formación inicial.

- No hay ninguna unidad que haga un seguimiento posterior en los niveles de la formación permanente y a demanda.

\section{Hacia una formación basada en competencias docentes}

Puesto que el perfil de los titulados se tiene que definir a partir de las competencias que deben adquirir, es lógico pensar que el profesorado deba demostrar que es capaz de trabajar en las mismas condiciones. Así, la institución espera que el profesorado sepa repensar su propia disciplina ya que además de poseer los conocimientos y habilidades propias de su área de conocimiento, debe mostrar habilidades y actitudes más genéricas que ayuden a los estudiantes a aprender mejor y a desarrollar el perfil profesional esperado. Se trata más de un cambio cultural que de un cambio de contenidos disciplinares, por lo que debe hacerse más hincapié en las estrategias de relación y trabajo con los estudiantes que en los contenidos a tratar.

En el estudio realizado por el GIFD (GIFD, 2011):, se constató que el profesorado universitario de las ocho universidades públicas catalanas muestra un consenso muy amplio en la definición de las seis competencias que definen la acción docente así como en su priorización. Las competencias por orden de importancias son las siguientes:

1. Competencia comunicativa

2. Competencia metodológica

3. Competencia interpersonal

4. Competencia de planificación y gestión de la docencia

5. Competencia de trabajo en equipo

6. Competencia de innovación 
La pregunta que surgió a partir de esta propuesta fue, ¿cómo se debía plantear la formación del profesorado en las universidades para facilitar que su perfil docente se fuera adecuando progresivamente a las exigencias que cada institución les plantea?.

Los expertos consultados en el estudio llevado a cabo durante el proyecto (GIFD, 2011) coincidieron en considerar que las competencias seleccionadas para trabajar con el profesorado debían ser pocas y presentadas de forma graduada para facilitar un progreso continuo en la actividad docente. Las competencias docentes no son un fin en sí mismo sino una manera de llevar a la práctica los conocimientos que el profesorado desarrolla en su disciplina. La formación en competencias no se puede plantear solo mediante un discurso teórico sino que la propia definición de competencia obliga a ofrecer una formación práctica, contextualizada en la propia área de conocimiento, que se desarrolla a partir de pequeños proyectos de mejora de la docencia. El profesorado, igual que el alumnado, ha de progresar en el dominio de las competencias inherentes a su profesión al ponerlas en práctica durante su actividad cotidiana, percibiendo cómo estas le permiten abordar con éxito los problemas asociados a su actividad docente.

La efectividad de una determinada estrategia de aprendizaje, desarrollada por parte de un profesor individual o por un equipo docente, se pone de manifiesto cuando los estudiantes la perciben como una forma de aprender mejor. Por ello es muy importante que el profesorado establezca sistemas para recoger información sobre cómo el alumnado percibe su actuación para poder plantear propuestas de mejora. La valoración que los estudiantes realizan sobre su propio proceso de aprendizaje, así como los resultados obtenidos por ellos en determinados momentos de su formación, pueden resultar útiles para evaluar el progreso del profesorado y ayudar a reorientar el proceso de formación si se considera oportuno.

De acuerdo con el modelo competencial definido, la formación del profesorado universitario debe presentar estrategias y actividades relacionadas con su actuación en el aula y con su responsabilidad como miembro de un equipo docente. Así, algunas de las características que se espera de la formación se pueden resumir en (GIFD, 2011):

- Ofrecer apoyo mutuo entre profesores, ya sea a través de la figura del profesor mentor o planteando formación colectiva al equipo docente que trabaja en una determinada titulación universitaria.

- Provocar la reflexión del profesorado sobre la propia práctica docente, analizando la idoneidad de su plan de trabajo, su actividad en el aula,...

- Plantear la formación en términos de investigación-acción, provocando que el profesorado trabaje en red con sus compañeros o con otros grupos docentes con los que muestre afinidad.

- Poner en crisis las propias actuaciones a medida que se progresa en el conocimiento teórico sobre los distintos aspectos de la docencia. La construcción de nuevo conocimiento sobre distintas metodologías docentes, estrategias de evaluación, criterios de calidad, etc. ayudarán a visualizar nuevas mejoras de la actividad docente susceptibles de ser abordadas. 


\section{Modelos y antecedentes europeos}

Los resultados de algunos estudios realizados en la década de los 90 por el Banco Mundial o por la UNESCO ya reconocían la importancia del papel del profesorado universitario a partir de la definición de las actitudes y aptitudes necesarias para desarrollar la tarea docente (Valcárcel, 2003). Sin embargo, la falta de modelos institucionalizados para el desarrollo profesional del profesorado universitario, ha dificultado el cambio de concepto por parte del colectivo y se mantiene viva la idea de que para enseñar en la universidad basta con saber la materia. Este concepto, junto con el hecho de que el prestigio profesional viene dado por la labor investigadora y no por la actividad docente, ha llevado a que el profesorado no se sienta implicado en la construcción y desarrollo de su perfil docente.

Desde los años 70 muchas universidades extranjeras mostraron su preocupación por el desarrollo profesional de su profesorado en relación con su actividad docente, de manera que en algunos países la formación del profesorado forma parte intrínseca de los planteamientos estratégicos de la universidad como es el caso de Australia, Canadá, Estados Unidos, Noruega, Reino Unido, Países Bajos y otros países escandinavos (Valcárcel, 2003).

Conscientes de que se puede aprender de los modelos desarrollados en otros países, analizamos las características de algunos modelos europeos que nos pueden resultar próximos.

En el caso del Reino Unido, la formación del profesorado es obligatoria tanto para los profesores noveles como para los permanentes. Ya a principios de los años 90 se inició un proyecto nacional para la formación y calificación de los profesores universitarios, así como de los programas de formación elaborados por las universidades para este fin. Este proyecto fue desarrollado por la Staff \& Educational Development Association (SEDA) ${ }^{2}$ quien estableció unos valores que debería tener el profesorado para su acreditación:

1. Comprensión sobre la forma de aprender

2. Erudición, profesionalidad y práctica ética

3. Trabajar para el desarrollo de unidades de aprendizaje

4. Trabajar de forma efectiva con la diversidad y promover la inclusión

5. Reflexión continua sobre la práctica profesional

6. Desarrollo de las personas y los procesos

En el año 2003, se creó la agencia oficial Higher Education Academy ${ }^{3}$ para promover la educación superior y que incorporó el esquema inicial de la SEDA. La agencia tiene como objetivo principal promover la educación superior y proporcionar consejo para mejorar la experiencia de los estudiantes, apoyar y avanzar en el desarrollo curricular y pedagógico y facilitar el desarrollo profesional de los docentes acreditando estándares de formación inicial y continua.

En este caso, es una agencia oficial quien establece criterios de calidad para la educación superior lo que le permite a su vez apoyar experiencias de mejora que incluyen también la formación del profesorado. 
Otro ejemplo europeo a considerar es el caso holandés donde, el posicionamiento nacional respecto a la necesidad de potenciar la calidad de la docencia, establecer unos estándares profesionales para la docencia universitaria y eliminar barreras en la movilidad de los académicos entre las universidades holandesas, surge de un acuerdo entre las universidades en el seno del Consejo de Rectores de la Asociación de Universidades de los Países Bajos (USNU).

Cada universidad ha tomado en consideración su propia idiosincrasia en lo que se refiere al desarrollo de su personal en temas docentes, pero su actuación es concordante con una reglamentación más amplia definida en el Marco Nacional de Calificaciones en la Educación Superior en los Países Bajos (BKO) ${ }^{4}$. La calificación de la docencia universitaria es el resultado de la evaluación formal de las competencias docentes que además, son reconocidas por el resto de universidades holandesas.

La calificación para la docencia se realiza a partir de la práctica profesional que corresponde a los estándares de la docencia universitaria aceptada mayoritariamente (descriptores de Dublín (JQI, 2004)). Se valora la actuación docente, el diseño de las titulaciones y de las asignaturas, la evaluación de las titulaciones, la orientación y el acompañamiento de los estudiantes. También se considera que el profesorado debe ser lo suficientemente cualificado para garantizar los objetivos de la titulación en lo que se refiere a los contenidos, a la didáctica y a la organización de la misma.

El profesorado es evaluado con criterios conocidos con antelación que abarcan todos los aspectos de su actuación e incluyen la reflexión sobre la propia práctica docente. La evaluación la realiza una junta constituida específicamente para ello y con garantías de confidencialidad, credibilidad y conformidad.

Para conseguir estos objetivos, la universidad facilita el desarrollo profesional del profesorado universitario y los cursos de formación que se ofrecen están orientados a poner en práctica los conocimientos sobre temas educativos.

En este caso se trata de un modelo surgido del acuerdo entre universidades. Cada universidad puede mantener su singularidad mientras que los acuerdos establecidos por el conjunto garantizan una evaluación objetiva y el reconocimiento mutuo de los resultados.

\section{Recomendaciones para la formación del profesorado universitario. Futuras actuaciones}

De la experiencia llevada a cabo y el conocimiento adquirido por el GIFD (2011), consideramos como un elemento fundamental que el profesorado disponga de marcos de aprendizaje. La universidad es un ecosistema de aprendizaje y tiene una responsabilidad institucional en ese sentido ${ }^{5} \mathrm{y}$, cuando hablamos de qué formación debería ofrecer a su profesorado, debería clarificarse primero qué proyecto de calidad docente institucional se desea implementar.

Como principales recomendaciones, extraídas del trabajo del GIFD, para la formación docente del profesorado universitario, se destaca la necesidad de establecer un perfil profesional y definir qué proyectos de calidad docente se pretende alcanzar pues, sin estas cuestiones definidas, no es posible dibujar el perfil 
competencial y por tanto, el perfil profesional del profesorado universitario. Esto implica establecer el nivel de dominio competencial para diseñar la formación (0 -nivel principiante-, 1, 2, 3 y 4 -nivel experto-), teniendo en cuenta que el nivel de experto ofrecería la posibilidad de poder formar a otros profesores y profesoras. Así mismo, debería organizarse la formación como un continuo que permita partir de un mínimo establecido para avanzar hasta la excelencia. A modo de ejemplo, se mencionan como mínimos: saber elaborar una guía docente, conocer y saber aplicar metodologías básicas docentes y saber realizar una tutoría, etc. Con ello, la formación facilitaría la adquisición y el perfeccionamiento progresivo de otras competencias en el desarrollo del ejercicio profesional.

Para poder revisar las estrategias didácticas es fundamental saber interpretar lo que los alumnos perciben de la docencia. Es necesario buscar la coherencia de lo que hacen los profesores respecto de lo que hacen los estudiantes, en caso contrario no podemos esperar buenos resultados. El modelo a seguir en la formación del docente universitario puede adoptar diferentes enfoques en función de aquello que se quiera conseguir. Esta formación puede ser impartida desde el grupo disciplinar (misma área de conocimiento, facultad, departamento, que fortalece a la vez al grupo, consolida, agrupa sinergias) o bien desde el grupo interdisciplinar (diferentes profesores y profesoras, diferentes facultades, universidades, potenciando la riqueza de los diferentes contextos y miradas sobre un tema en concreto).

Es muy importante el papel de las unidades de formación, pero es necesaria la implicación de toda la universidad (responsabilidad institucional), ya que la formación empieza en la selección del grupo de trabajo en el que se integre el profesorado que accede a la función docente.

La formación tiene que estar diseñada a partir de un modelo con un objetivo claro y ajustado al contexto, este modelo debe guardar estrecha relación con el perfil del profesorado que se pretende y éste debe poder disponer del programa formativo desde el inicio. Los destinatarios serían aquellos candidatos que han accedido y se incorporan en la organización. En este sentido se apunta a la formación en docencia universitaria dentro de la formación de doctorandos, asociados, colaboradores, etc.

Podemos identificar tres elementos fundamentales que deberían respetarse en la formación del profesorado universitario (GIFD, 2011):

- La autonomía del propio individuo como profesor/a.

- El perfil de la formación en la universidad.

- El contexto universitario.

En relación a la formación en competencias, se plantea que un modelo puede ser el denominado Modelo 3P de enseñanza: Presagio, Proceso, Producto (Biggs y Collins, 1989). Este modelo se basa en los siguientes aspectos:

- Presagio: Características del profesorado (en base a la concepción de la enseñanza y a los métodos de enseñanza) y contexto institucional (objetivos/competencias, sistema social/clima, institucional, currículumevaluación). 
- Proceso: Enfoques de enseñanza (incluyendo no sólo el tipo de actividades sino también la práctica).

- Producto: Enfoque, resultados y calidad del aprendizaje.

Se entiende que la formación tiene que producir satisfacción al profesorado y que ésta debe serle de ayuda para la resolución de problemas en su práctica diaria. Por ello, en ningún caso debería plantearse la formación como normativa o amenaza que pudiese generar reacciones defensivas y de rechazo.

En la formación del profesorado universitario hay que utilizar metodologías que ayuden y faciliten el aprendizaje a través de la acción, la reflexión de la propia praxis, la autocrítica formativa y, que a su vez promuevan el interés continuo de los docentes por el aprendizaje de sus estudiantes. Es especialmente importante un feedback posterior de los resultados, una vez los profesores y profesoras se han formado, para así poder analizar si la formación ha dado algún resultado o ha incidido de manera positiva en el modelo inicial planteado.

Es necesario pensar también en otras posibilidades de formación como por ejemplo la formación ad-hoc, o formación en el mismo contexto docente. Por ello se plantea extender la opción, ya iniciada en algunas universidades, de ofrecer una formación in-situ, en los propios centros, facultades y departamentos, y el establecer una formación colectiva/colaborativa interuniversitaria, sin prescindir de la formación que se realice en las propias unidades de formación. Se plantea el siguiente interrogante: ¿cómo se puede ayudar al profesorado con incompetencia inconsciente a convertirla en competencia consciente? Antes esta cuestión, se considera que se deberán diseñar revulsivos para desequilibrar la incompetencia inconsciente y así hacerla consciente, ya que es importante el hecho de poder detectar los propios déficits y tener activado un sistema de alertas para poder subsanarlos a través de una formación apropiada.

Finalmente, y aunque no menos importante, la formación debería considerar la necesidad de que el profesorado tenga la habilidad necesaria como para transmitir mensajes positivos a los estudiantes.

\section{Reconocimiento de la formación docente}

Desde la implantación de la LRU (1983) se ha producido en España un aumento progresivo del interés por el desarrollo profesional del profesorado universitario. Sería adecuado que el profesorado reciba algún reconocimiento por la formación realizada puesto que no debemos olvidar que la formación en el ámbito de la docencia es voluntaria. El reconocimiento es necesario porque la formación es una de las estrategias más potentes de las que dispone la institución para garantizar que se apliquen con éxito las propuestas de nuevos títulos, pero también, para evitar la competencia constante que la investigación representa para la docencia. Muchos profesores piensan que el tiempo que están invirtiendo para formarse en temas docentes no les aporta ningún reconocimiento, y que durante este tiempo podrían estar realizando algún trabajo o elaborando alguna publicación que, de buen seguro, será reconocida y valorada en su currículum investigador. 
La normativa actual establece la obligatoriedad de la evaluación de las actividades docentes, investigadoras y de gestión del profesorado universitario ${ }^{6}$ que va a acceder a una plaza, y por tanto, implica a un colectivo bastante reducido. En estos momentos, la evaluación de los méritos investigadores y docentes del candidato se realiza por una agencia de calidad que expide un certificado de acreditación. Los organismos responsables de la evaluación son la Agencia Nacional de Evaluación y Acreditación (ANECA), las agencias de calidad autonómicas y las propias universidades.

Para evaluar la actividad investigadora del profesorado, hace ya bastantes años que el sistema universitario se dotó de unos criterios, que a lo largo del tiempo han ido sufriendo variaciones para ajustarlos a las necesidades del momento, hasta llegar a disponer de un mecanismo sólido y aceptado por una amplia mayoría de los miembros de la comunidad universitaria. Recientemente, el Real Decreto 5337/2012 modula el número de créditos ECTS que debe impartir cada profesor en función de la actividad investigadora reconocida de conformidad con el Real Decreto 1086/1989, es decir, dependiendo de los sexenios de investigación que el profesor acredite, y de cuándo los haya acreditado. Las decisiones fueron tomadas por todas las universidades y de forma conjunta con las administraciones responsables de las enseñanzas universitarias y constituyen un estímulo para fomentar la investigación, la innovación y el desarrollo.

No ha sucedido lo mismo con el sistema de evaluación de la actividad docente, que ha sufrido muchos cambios, no hay criterios bien definidos y existe una gran variedad de modelos para la evaluación de la docencia y su reconocimiento (puede consultarse Murillo 2008, para una revisión exhaustiva). Si bien es cierto que el trabajo realizado en el ámbito de la investigación puede resultar más fácilmente objetivable (número de publicaciones, citaciones, calidad de la publicación, número de patentes, estudios,...) el sistema universitario debería intentar definir criterios aplicables a la docencia. También aquí pueden establecerse indicadores para definir lo que es una docencia de calidad: satisfacción de los alumnos, propuestas de mejora planteadas y ejecutadas por el profesorado, resultados obtenidos por los alumnos (en comparación con otras materias y/o asignaturas del mismo grupo), coordinación entre profesores para facilitar el desarrollo del plan de estudios, valoración por parte de los titulados, formación realizada, proyectos de innovación desarrollados, etc.

La evaluación de la actuación docente del profesorado es competencia de la universidad. En el Real Decreto 1086/1989, sobre retribuciones del profesorado universitario, se introdujo la evaluación de los méritos docentes al establecer, dentro del complemento específico de los funcionarios de carrera de cuerpos docentes universitarios, el componente de méritos docentes. Se reconoce a cada universidad la competencia para evaluar los méritos docentes de su profesorado por períodos mínimos de cinco años a tiempo completo (quinquenio). Los criterios generales para la evaluación de la actividad docente, las actividades objeto de evaluación, así como el procedimiento, fueron propuestos en 1990 por el Consejo de Universidades y a partir de ellos las universidades pusieron en marcha evaluaciones siguiendo sus propios criterios. El año 2001 la LOE otorga a las agencias de calidad las funciones de evaluación, certificación y acreditación, sin embargo, mantiene los complementos retributivos docentes, "quinquenios", que se obtienen casi automáticamente por antigüedad. 
A partir del año 2007, la Ley Orgánica que modifica la LOU, fomenta que las universidades realicen una evaluación de la actividad docente de carácter formativo, que ayude a la mejora de la calidad. En esta línea, la Agencia Nacional de Evaluación y Acreditación (ANECA) ha desarrollado un programa de ayuda a las universidades para crear un sistema de evaluación del profesorado denominado DOCENTIA. El programa se lleva a cabo en estrecha coordinación con las agencias de evaluación autonómicas y como se puede comprobar analizando las guías o procedimientos para la evaluación de la actividad docente, todas ellas establecen cuatro dimensiones en la evaluación del profesorado: planificación de la docencia, desarrollo de la actividad docente, resultados y acciones emprendidas para la mejora e innovación docente. Estos procesos de evaluación se encuentran, para cada universidad, en diferentes fases del proceso de certificación, y pueden diferir mucho de unas universidades a otras. Así, podemos observar que la evaluación puede ser obligatoria en algunas instituciones, mientras que en otras solo se hace a petición del interesado. En otras universidades, como la de Salamanca, se realiza una evaluación obligatoria y otra voluntaria. La periodicidad puede ser distinta (anual, bianual) y su repercusión puede ser muy variada. En la mayoría de universidades la evaluación sólo sirve como información para el docente, el departamento y la universidad, como una medida que le ayuda en la mejora de la calidad del desempeño docente. En unas pocas, la evaluación docente tiene repercusión para la promoción profesional o para la renovación del contrato para profesores no funcionarios, y en algunas comunidades está ligada al complemento retributivo. Por último, los instrumentos de evaluación son variados, desde la encuesta a los estudiantes, el informe de autoevaluación y en algunos casos también se incluye el informe del director de departamento, director de centro o facultad o vicerrectores.

Como se puede ver, la situación es variada, como ejemplo presentamos dos modelos de la situación de las universidades en cuanto a la evaluación docente de su profesorado:

- En las universidades públicas catalanas, que cuentan con modelos ya acreditados por la Agència per a la Qualitat del sistema Universitari de Catalunya (AQU Catalunya): la evaluación de la actividad docente del profesorado está ligada a la concesión del complemento autonómico por méritos docentes. Hay que destacar en este caso que la evaluación es voluntaria, por tanto sólo un porcentaje del total de profesorado es evaluado de una forma completa, es decir, considerando todas las dimensiones de la actividad docente. Por ejemplo, en el año 2009, de los 2.608 profesores de las universidades públicas catalanas que, potencialmente podían someterse a evaluación, sólo se presentaron 1.369, un 52,5\% del total, de ellos 1336 fueron evaluados favorablemente, según datos facilitados por AQU Catalunya.

- En la comunidad de Madrid, el complemento autonómico no se asocia al resultado de la evaluación. Según el modelo DOCENTIA, el complemento no se desagrega, y se evalúa al profesorado de manera conjunta, según el mérito docente, investigador y de gestión. Se observa en estos casos una mayor ponderación de los méritos investigadores sobre los docentes y que la evaluación de estos últimos se relaciona con el reconocimiento de los quinquenios de docencia. 


\section{Retos institucionales en la formación docente del profesorado universitario}

Las unidades de formación de las universidades tienen la responsabilidad de la formación docente del profesorado dentro de su institución, pero su capacidad de decisión puede ser limitada para abordar algunos de los planteamientos de mejora que son necesarios para que dicha formación pueda repercutir en la calidad docente de su profesorado.

Para que la formación pueda incidir en la mejora de la calidad docente, desde el grupo de trabajo (GIFD), queremos dejar constancia de cuáles deberían ser, según nuestro criterio, los retos que cada institución habría de abordar, conscientes de que en algunos casos la toma de decisiones puede requerir el consenso del conjunto de universidades.

- Es necesario abordar la disfunción que representa el hecho de que el profesorado universitario pueda acceder a la función docente sin tener una formación previa. Esta característica, que no se produce en la mayoría de profesiones que componen el sistema laboral del país, puede solventarse tomando decisiones que pueden incluir la exigencia de una formación, previa o inmediatamente posterior al ingreso en la función docente de la universidad. Para ello es necesario tener planificada una formación inicial que el profesorado debe adquirir cuando inicia su carrera profesional o alguna otra solución alternativa que las instituciones consideren correctas para corregir esta disfunción, como por ejemplo, adquirir, o demostrar que se ha adquirido, una formación mínima necesaria, en un plazo máximo de dos años posteriores a la consecución por primera vez de una plaza.

- Cada universidad debería definir y potenciar la formación de su profesorado, integrándola como una característica propia de su razón de ser, imprescindible para conseguir la calidad docente a la que se compromete en su oferta académica. Si la universidad define el perfil docente que desea para su profesorado, podrá transmitirlo con claridad a toda la comunidad docente para que ésta pueda conocer qué se espera de ella.

- Las acciones formativas que la universidad desarrolla deberían dirigirse tanto al profesorado de nuevo ingreso como al profesorado que ya lleva tiempo formando parte de la universidad, ya que en este momento todo el colectivo se enfrenta a retos importantes en su labor docente. La universidad puede transmitir sus objetivos docentes y sus planes de formación del profesorado a través de las unidades académicas (departamentos o centros) y a su vez aprovechar estas unidades para facilitar el contexto de formación del profesorado más adecuado en cada caso. De esta forma se podrían transmitir las necesidades de formación como equipo y se evitaría que la iniciativa en la formación se reduzca solamente a una responsabilidad individual de cada profesor.

- Para conseguir motivar al profesorado deberían establecerse criterios de reconocimiento de una buena actividad docente para que su valoración no se limite a la antigüedad, como ocurre ahora en muchos casos. La valoración de la 
docencia debería incrementarse hasta conseguir niveles comparables a las exigidas en investigación y explicitar que ambas tareas son importantes en el perfil profesional del profesorado universitario para evitar que las diferencias que se producen actualmente vayan en detrimento de la formación docente $y$, por lo tanto, de la calidad docente. Igualmente la calidad docente debería recibir el mismo reconocimiento que se otorga a los méritos de investigación.

Insistimos en que es posible que la responsabilidad en la toma de decisiones, en algunos de los aspectos citados, no se pueda atribuir a una única universidad de forma aislada y por lo tanto, se hace imprescindible una cooperación entre las distintas universidades mediante un trabajo en red, para conseguir cambios legislativos, si éstos son necesarios.

\section{Conclusiones}

Desde el grupo de trabajo GIFD, se han propuesto, de forma consensuada con la comunidad académica de todas las universidades públicas catalanas, las seis competencias que el profesorado universitario debería adquirir, para una adecuada ejecución de su tarea docente en el seno de su ámbito institucional. Partiendo de ese supuesto, se hace necesaria la búsqueda de modelos de formación, dentro de un marco de referencia común, cuyos resultados sean suficientemente positivos para su adopción y aplicación en el contexto actual, y que responda a las exigencias de calidad del EEES, siendo al mismo tiempo adaptable a la idiosincrasia propia de cada institución. Para ello es totalmente necesario definir, previamente, un perfil profesional que sea compatible con las características y sistemas de funcionamiento y evaluación de cada universidad. Todo el esfuerzo dedicado a esa planificación inicial debería partir de una buena definición del perfil docente, en cuanto al momento en el que nos encontramos, para así ajustar la formación a la situación actual: "saber dónde estamos para saber dónde queremos ir".

Por ello se hace necesario, desde la propia institución universitaria, tener claro qué modelo formativo se pretende implementar y concienciarse de que el cambio supone un giro, no sólo en la arquitectura del sistema, sino también en la cultura, teniendo claros cuales son los objetivos que se quieren alcanzar y el contexto en el que deberían realizarse dichas actividades.

La aplicación de estas propuestas también ha de suponer un cambio en la concepción del profesorado que se encuentra actualmente en la universidad. No sólo es necesaria la concienciación de cambio en el profesorado novel, sino que se requiere de una intervención directa sobre las actitudes de los profesores ya consolidados en el tejido universitario. Convendría establecer un cambio generacional graduado sin dejar de lado a las personas que actualmente trabajan en la institución: apostar por una formación continua que permita al profesorado ya asentado replantear sus concepciones sobre la enseñanza.

Entendiendo que no se considera adecuado impartir docencia sin abastecerse de ningún tipo de formación docente previa, la institución debería disponer de un mapa o perfil de competencias básicas y ofrecer un programa de formación al profesorado antes o inmediatamente después de iniciar su docencia universitaria. Así mismo, el 
profesorado debería concienciarse y ser responsable de su propio desarrollo profesional y asumir la gestión de su propia formación permanente.

En educación, el éxito no es sólo fruto de que el docente domine la materia y el estudiante tenga un rendimiento alto. Cuando se contemplan situaciones distintas a ésta, es cuando se abren las posibilidades de cambio. Es necesario poner en cuestión los paradigmas en los que se basa la educación actual. Esto quebrantaría la visión actual de la calidad docente: su evaluación no dependería solamente de su edad o experiencia, sino de su dominio en competencias docentes, de su capacidad de adecuación frente a las demandas y las condiciones de cada contexto formativo.

Dos de los aspectos que se consideran trascendentes en la aplicación de la formación en competencias, no por su directa relación con ellas, sino por su papel como fundamento en la construcción de cualquier plan para la mejora y la innovación docente son:

- Considerar los objetivos de la institución en la planificación de las posibles intervenciones a realizar.

- Promover una continuidad en la formación en competencias.

Hay que tener en cuenta que la capacidad de decisión de las unidades que son responsables de la formación docente del profesorado dentro de las universidades resulta limitada para abordar algunos de los aspectos que se han propuesto aquí como posibles mejoras de la formación, para que ésta pueda repercutir en la calidad docente de la institución. Por ello ya se ha indicado anteriormente que en algunos casos la toma de decisiones puede requerir el consenso del conjunto de las universidades, lo que implica un esfuerzo de coordinación entre todas ellas y de éstas con las agencias y órganos de gobierno autonómico y estatal, que elimine la complejidad y excesiva variabilidad del sistema de evaluación del profesorado universitario en España.

\title{
Notas
}

\begin{abstract}
${ }^{1}$ Mediante los proyectos "Identificación, Desarrollo y Evaluación de competencias docentes en la aplicación de planes de formación dirigidos a profesorado universitario" (EA2010-0099), y "Marc de Referència Competencial pel disseny dels Programes de Formació Docent per al Professorat Universitari" (2010MQD00049).

${ }^{2}$ www.seda.ac.uk

3 www.heacademy.ac.uk

${ }^{4}$ http://www.red-u.org/images/jornadas upv/2/Teaching quality Spain 20120202.pdf

${ }^{5}$ Ley $1 / 2003$ de Universidades de Cataluña, artículo 19, apartados 1 ("El departamento competente en materia de universidades y las universidades públicas deben facilitar que el profesorado, a lo largo de su vida académica y, especialmente, en su primera etapa de actuación docente, goce de las posibilidades de formación adecuadas para ofrecer una docencia de calidad y para actualizar sus conocimientos y sus habilidades ") y 3 (" El departamento competente en materia de universidades y las universidades públicas han de desarrollar programas de formación continua, de incentivos y de reconocimiento de la calidad docente, dirigidos tanto a los profesores y profesoras como a los equipos docentes").
\end{abstract}

${ }^{6}$ Ley Orgánica 6/2001 del 21 de diciembre, de Universidades (LOU), artículo 31, apartado 26. 


\section{Referencias bibliográficas}

AQU Catalunya (2012). Consultado en marzo de 2012 desde www.aqu.cat

Biggs, J.B y Collins, K.F. (1989). Evaluation the quality of learning: the SOLO taxonomy. New York: Academic Press.

GIFD - Grup Interuniversitari de Formació Docent (2011). “Identificación, desarrollo y evaluación de competencias docentes en la aplicación de planes de formación dirigidos a profesorado universitario" (EA2010-0099). Programa estudios y análisis destinado a la mejora de la calidad de la enseñanza superior y de la actividad del profesorado universitario, Ministerio de Educación, Gobierno de España.

JQI - Joint Quality Initiative (2012). Dublín, 18 de octubre de 2004. Consultado en marzo de 2012 des de www.jointquality.nl

Ley de Universidades de Catalunya 1/2003, 19 de febrero de 2003. Artículo 19, apartados 1 y 3.

Ley Orgánica 6/2001, 21 de diciembre de 2001. Boletín Oficial del Estado, n.307, artículo 31, apartado 26.

Ley de Reforma Universitaria, 1983.

Murillo, F.J. (2008). La Evaluación del Profesorado Universitario en España. Revista Iberoamericana de Evaluación Educativa, 1(3), 29-45.

Real Decreto 1086/1989. Palma de Mallorca, 28 de agosto de 1989.

SEDA - Staff \& Educational Development Association (2012). Consultado en marzo de 2012 desde www.seda.ac.uk

Valcárcel, M. (coord.) (2003). “La preparación del profesorado universitario español para la convergencia europea en educación superior" (EA2003-0040). Programa estudios y análisis destinado a la mejora de la calidad de la enseñanza superior y de la actividad del profesorado universitario, Ministerio de Educación, Gobierno de España.

Cita del artículo:

Torra, I.; Màrquez, M.D.; Pagès, T.; Solà, P.; García, R.; Molina, F.; González, A.; Sangrà, A. (2013). Retos institucionales de la formación del profesorado universitario. Revista de Docencia Universitaria. REDU. Vol.11 (1) Enero-Abril. pp. 285-309. Recuperado el (fecha de consulta) en http://www.red-u.net/ 


\section{Acerca de las autoras y autores}

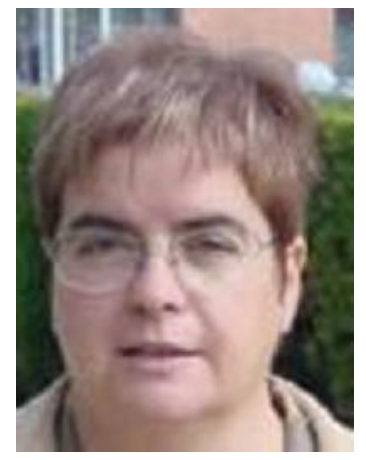

\section{Imma Torra Bitlloch}

Universitat Politècnica de Catalunya

Institut de Ciències de l’Educació

Mail: direccio.ice@upc.edu

Titular de Universidad en el Departamento de Ingeniería Minera y Recursos Naturales de la UPC desde febrero de 1998. Directora del Postgrado de "Gestión y mantenimiento de edificios" organizado por la Fundación Politécnica de Cataluña entre febrero y julio de 2003, directora de la Escuela Universitaria Politécnica de Manresa desde mayo de 1996 hasta mayo de 2002, en la actualidad y desde julio de 2006 es directora del ICE de la UPC. Coordinación de proyecto "Evaluación y seguimiento de las experiencias piloto ECTS en la UPC" financiación del MEC dirección de los proyectos "Análisis de metodologías docentes en la UPC" y "Evaluación del impacto de la formación del profesorado universitario del ICE de la UPC "con financiación del MEC.

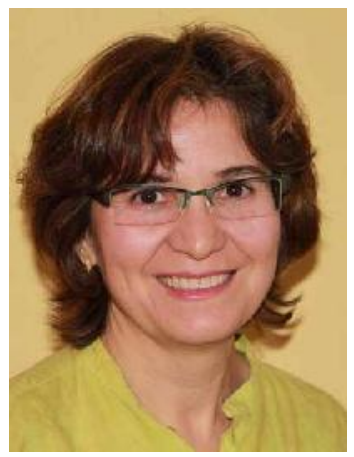

\section{María Dolores Màrquez Cebrián}

Universitat Autònoma de Barcelona

Unitat d'Innovació Docent en Educació Superior

Mail: mariadolores.marquez@uab.cat

Doctora en Ciencias Matemàticas por la Universidad Politècnica de Catalunya, 2002 y Licenciada en Ciencias (Matemàticas) por la UAB, 1989. Titular de Universidad, (Dpt. de Economia y Historia Económica UAB). Coordinadora para la Formación del Personal Docente y Investigador, y la Innovación de la UAB responsable de la Unidad de Innovación Docente en Educación Superior IDES desde Octubre-2009. Videdirectora de Calidad Docente y Ordenación Académica de la Escuela Universitaria de Estudios Empresariales (UAB), 2005-2009 


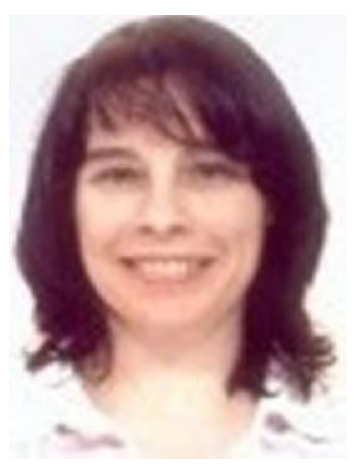

\title{
Teresa Pagès Costas
}

\author{
Universitat de Barcelona
}

Institut de Ciències de l’Educació

Mail: tpages@ub.edu

Profesora Titular de Fisiología de la Universidad de Barcelona (UB). Ha participado en 29 proyectos de investigación y 35 de Innovación Docente de convocatorias públicas. Ha publicado más de 40 artículos en revistas científicas. Es miembro del Grupo de Investigación en Fisiología Adaptativa: Ejercicio e hipoxia, grupo reconocido por la Generalitat de Cataluña, y colaboradora del Servicio de Hipobaria y Fisiología Biomédica del BioPol L'H. En el ámbito académico se coordinadora del Máster oficial de Fisiología Integrativa y Jefe de la Sección de Universidad del Instituto de Ciencias de la educación y responsable de la formación del profesorado de la UB. Autora de 11 capítulos de libro, coautora de 4 manuales docentes y directora revista Colección Cuadernos de Docencia Universitaria, ICE-Octaedro.

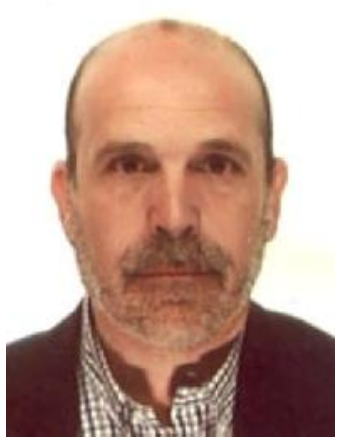

\section{Pau Solà i Ysuar}

\section{Universitat Pompeu Fabra}

Centre per a la Qualitat i la Innovació Docent

Mail: pau.sola@upf.edu

Director Técnico del Centro para la Calidad y la Innovación Docente (Universidad Pompeu Fabra). Miembro del Grupo Interuniversitario de Formación Docente (GIFd). Principales líneas de especialidad e investigación: Implementación de nuevas titulaciones. Medidas de tránsito y adaptación a la universidad de estudiantes de nuevo ingreso (desarrollo de competencias de adaptación a la universidad entre el alumnado de primer curso). 


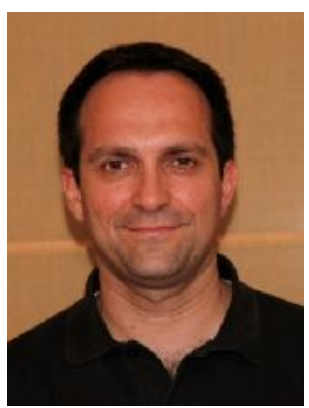

\title{
Rafael García Campos
}

\author{
Universitat de Girona \\ Institut de Ciències de l'Educació \\ Mail: rafael.garcia@udg.edu
}

Doctor en ingeniería y profesor Titular de la Universidad de Girona. Director del Instituto de Ciencias de la Educación Josep Pallach de la Universidad de Girona (UdG). Sus intereses docentes incluyen el diseño de actividades de innovación educativa y la relación entre motivación y aprendizaje. En los últimos años ha centrado su labor docente en el Máster Erasmus-Mundus VIBOT, impartido conjuntamente por la UdG, la Heriot-Watt University (Reino Unido) y la Université de Bourgogne (Francia). Desde 2010 dirige el Grupo de Visión por Computador y Robótica de la UdG, donde está involucrado en diferentes proyectos de investigación tanto a nivel nacional como internacional en el campo de la robótica. Ha realizado estancias de investigación en la Universität der Bundeswehr (Alemania), University College Cork (Irlanda), IRISA-INRIA (Francia), y la Universidad de Miami (EE.UU.).

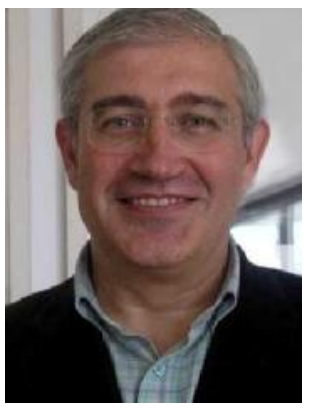

\section{Fidel Molina Luque}

\section{Universitat de Lleida}

Institut de Ciències de l’Educació - Centre de Formació Contínua

Mail: direccio@ice.udl.cat

Dr. Fidel Molina. Catedrático de Sociología de la Universidad de Lleida. Director del Instituto de Ciencias de la Educación- Centro de Formación Continua de la Universidad de Lleida (2011-2012). Decano de la Facultad de Ciencias de la Educación (1998-2001 y 2001-2004). Ha obtenido la Mención de Excelencia (Evaluación Actividad Docente, 2002-2007), así como el Premio (colectivo) "Grundtvig Award - 2005" al mejor proyecto europeo de investigación en Educación de Adultos. Ha publicado diversos libros, artículos y capítulos de libros sobre temas de Sociología de la Educación (Formación continua, Educación Intercultural, Juventud y Socialización), de Sociología del Ocio y del Deporte, Gestión y Formación de Recursos Humanos. Es Postgraduado en Mediación y Resolución de Conflictos y Formador de formadores en Educación Intercultural. 


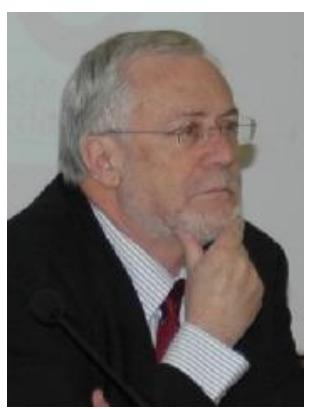

\section{Ángel-Pío González Soto}

\section{Universitat Rovira i Virgili}

Institut de Ciències de l’Educació

Mail: angelpio.gonzalez@urv.cat

Delegado del Rector para la Formación Permanente del PDI y Director ICE Ángel-Pío González Soto es Maestro de Enseñanza Primaria y Licenciado en Pedagogía y Doctor en Ciencias de la Educación. Ejerce profesionalmente como Catedrático de Universidad en el Área de Didáctica y Organización Escolar en el Departamento de Pedagogía, de la Universitat Rovira i Virgili. Como especialista en Didáctica y Organización Escolar ha proyectado su actividad tanto en el ámbito de la educación formal como de la no formal. En la actualidad es Director del ICE de la Universidad Rovira i Virgili y Delegado del Rector para la Formación Permanente del Personal Docente e Investigador.

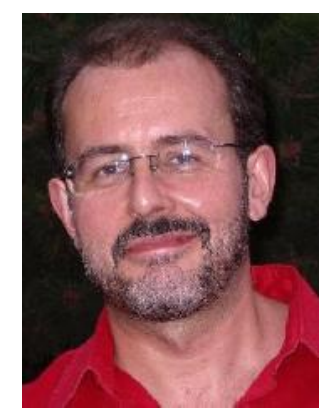

\section{Albert Sangrà Morer}

\section{Universitat Oberta de Catalunya}

eLearn Center

Mail: asangra@uoc.edu

Doctor Europeo en Ciencias de la Educación por la Universitat Rovira i Virgili. Director del eLearn Center, centro de investigación, innovación y formación en e-learning, de la Universitat Oberta Catalunya. Profesor e investigador en dicha universidad. Su campo de investigación es el uso de las TIC en la educación y la formación, especialmente por lo que respecta a las políticas, la organización, la gestión y el liderazgo en su implantación, y el aseguramiento de la calidad del e-learning, ámbitos en los cuales dispone de diversas publicaciones. Ha sido maestro y profesor en los distintos niveles educativos españoles y consultor en diversos proyectos de formación virtual en Europa, América y Asia. Es miembro del grupo de investigación Edulab y Vicepresidente de la European Foundation for Quality in E-Learning (EFQUEL). 


\title{
La enseñanza de competencias para resolver situaciones conflictivas con pacientes agresivos en enfermería mediante el Método del Caso (MdC)
}

\section{Teaching skills to resolve conflicts with aggressive patients in nursing through the} Case Method (MoC)

\author{
Marta Arrue Mauleon \\ Universidad del País Vasco, España
}

\section{Resumen}

Este trabajo trata de diseñar una secuencia de enseñanza que ofrezca a los estudiantes la oportunidad de adquirir los conocimientos, habilidades, actitudes y valores necesarios para enfrentarse a un paciente conflictivo. Para diseñar la docencia se ha optado por una estrategia de enseñanza activa y en particular, por el llamado Método del Caso.

La elaboración del Caso se produjo durante el curso 2010-11 y la implementación del mismo tuvo lugar en el curso 2011-2012, en la asignatura "Relación y Comunicación para el cuidado enfermero", de 20 año de Grado de Enfermería en la Universidad del País Vasco.

Los resultados de la implementación indican que el MdC es una herramienta satisfactoria para la adquisición de las competencias planteadas, además de ser un método de aprendizaje muy bien aceptado por los alumnos en general. Al finalizar el proceso el 97,1\% de los participantes afirma que repetiría con esta metodología y el $100 \%$ opina que "esta metodología me ha ayudado más o mucho más que la metodología tradicional expositiva". Asimismo, todos los alumnos han superado, al menos, el mínimo de los Resultados de aprendizaje exigidos. La experiencia planteada debe servir de incentivo para continuar ampliando el abanico de posibilidades que ofrecen las metodologías activas.

Palabras claves: Enfermería, Método del Caso, Relación y Comunicación, Innovación, Paciente agresivo.

\begin{abstract}
This paper seeks to design a teaching sequence that provides students with an opportunity to acquire knowledge, skills, attitudes and values needed when dealing with an aggressive patient. To design the teaching method, a strategy of active teaching, and in particular the so-called Case Method, has been chosen.
\end{abstract}


The preparation of the case occurred during the 2010-11 academic year and its implementation took place in 2011-2012 during a course called "Relationship and Communication for Nursing Care". The participants were $2^{\text {nd }}$ year nursing students at the Basque Country University.

The results of implementation show that the MoC is a satisfactory tool for the acquisition of the proposed competences, as well as being a learning method that is very well received by students in general. On completion, $97.1 \%$ of the participants said they would repeat this methodology and $100 \%$ said that "this methodology has proven helpful or very helpful to me in comparison with the classic expositive methodology." In addition, all students have passed at least the minimum of the required learning outcome. The proposed experience should be an incentive to continue to expand the range of possibilities offered by active methods.

Key words: Nursing, Case Method, Relationship and Communication, Innovative methodology, Aggressive patient.

\section{Introducción}

La habilidad para saber tratar a la gente es uno de los factores fundamentales en las relaciones profesionales de la sanidad. Si además, nos encontramos ante un paciente agresivo todo se complica mucho más. Saber cuidar de los pacientes agresivos es una habilidad muy valiosa que probablemente toda enfermera necesitará durante su carrera. Tanto las enfermeras como los pacientes estarán más seguros si las enfermeras sabemos cómo manejar estas situaciones. Sin embargo, tal habilidad no se adquiere fácilmente.

Como afirma la Organización Internacional de Trabajo (OIT) y la Organización Mundial de la Salud (OMS) la violencia en el lugar de trabajo se ha ido convirtiendo en un problema mundial. Si bien la violencia afecta prácticamente a todos los sectores y categorías de trabajadores, el sector de la atención de salud corre un riesgo grave. La propia OMS denuncia que casi un $25 \%$ de todos los incidentes de violencia en el trabajo se producen en el sector sanitario. Las agresiones que sufren los trabajadores de la sanidad en el ejercicio de su profesión es, sin duda, un riesgo emergente (Ryan y Maguire, 2006; Organización Colegial de Enfermería, 2007, 2011; Cervantes, Blanch y Hermoso, 2010; Rodríguez et al., 2010).

A pesar de que todas las profesiones del sector salud corren riesgos de sufrir violencia, las enfermeras son las que corren más riesgos (OMS, 2002; Villar y Aranaz, 2005). Ya son muchos los estudios que demuestran que el ataque físico y verbal dirigido hacia los profesionales de enfermería es una lamentable realidad (Consejo General de Enfermería, 2011a; 2011b; 2011c). Tal y como pone de manifiesto el estudio realizado por el Consejo General de Enfermería (2011c), el 33\% de los enfermeros españoles ha sufrido una agresión física o verbal en los últimos 12 meses. Asimismo, de ese total de enfermeros agredidos, un 3,7\% han sufrido un ataque físico. Parece lógico que sea el personal de enfermería el que reciba una mayor proporción de agresiones, ya que su exposición al riesgo es muy superior al de cualquier otro trabajador, dado que su relación directa con el paciente ocupa prácticamente toda la jornada laboral.

Uno de los objetivos de Enseñanza de la asignatura "Relación y Comunicación para el cuidado enfermero" de 2o año del Grado de Enfermería, no sólo es el de mostrar a los estudiantes esta "nueva" problemática, sino hacerles competentes a la 
hora de manejar una situación conflictiva. Para ello resulta necesario que los estudiantes adquieran toda una serie de competencias. En el ámbito sanitario, no son pocos los autores que han demostrado que no es suficiente la utilización del modelo de enseñanza basado en la trasmisión de información, subrayando la necesidad de formar en competencias (Baños y Pérez, 2005; McGloin, 2008; Ruiz, Granados, Gutierrez y Muñoz, 2011).

Debido a la necesidad de adaptación de los nuevos planes de estudio al Marco del Espacio Europeo de Educación Superior, y por ende, a formar en competencias, cada día son más los docentes que, debido a las evidencias de buenos resultados en los procesos de enseñanza-aprendizaje, utilizan las metodologías activas (Arias-Gundín, Fidalgo y García, 2008; Fernández, González, López y Manso, 2010; Castro et al., 2011). Entre las ventajas de utilizar estas metodologías, la investigación educativa menciona los siguientes aspectos (Fernández, Guisasola y Garmendia, 2010):

- Mayor motivación, interés e implicación del estudiante.

- Tiempo de finalización de los estudios más breve y menor tasa de abandono.

- Mayor retención de los conocimientos adquiridos.

- Mayor desarrollo de habilidades y competencias profesionales.

- Mayor conexión entre la teoría y la aplicación, entre conocimiento previo y el que se va aprendiendo y mayor integración de los conocimientos entre diferentes disciplinas.

Sin embargo, en relación con la enseñanza de saber manejar situaciones y pacientes conflictivos en el Grado de Enfermería, no he encontrado publicada ninguna experiencia que haya utilizado el Método del Caso (MdC) para desarrollar las competencias establecidas por la ANECA (Agencia Nacional de Evaluación de la Calidad y la Acreditación).

Este trabajo trata de diseñar una secuencia de enseñanza que ofrezca a los estudiantes la oportunidad de adquirir los conocimientos, habilidades, actitudes y valores necesarios para enfrentarse a un paciente conflictivo. Para diseñar la docencia se ha optado por una estrategia de enseñanza activa y, en particular, por el llamado Método del Caso. Esta herramienta permite a los estudiantes vivir en el aula situaciones que de otra manera no estarían a su alcance y les sirve para conocer problemas similares a los que probablemente se les presentarán en su vida profesional. Es decir, se enfrenta a los alumnos a casos de la vida real impulsándoles a analizar situaciones y buscar soluciones de una manera profesional, dejando a un lado su papel de simples estudiantes y situándose en el lugar de alguien que tiene que tomar decisiones.

Las preguntas que guiaron este trabajo fueron dos: 1) ¿Cómo diseñar una secuencia de enseñanza basada en el Método del Caso?; 2) ¿Cómo adaptar el currículo para implementar el caso diseñado?

Para contestar a estas preguntas la autora participó en el programa ERAGIN de la UPV-EHU que se describe a continuación. Dentro del programa se diseñó el Caso y se implementó. Por último, se obtuvieron los resultados y las conclusiones que se describirán. 


\section{Programa eragin}

Este programa se desarrolla por iniciativa del Vicerrectorado de Calidad e Innovación Docente bajo la dirección y organización del Servicio de Asesoramiento Educativo (SAE) y contribuye de manera sustantiva a la línea estratégica de estimular y apoyar el modelo IKD de enseñanza-aprendizaje cooperativo y dinámico dentro de la UPV/EHU.

El programa ERAGIN (accionar, influir, en euskera) forma al profesorado en el método del caso, del aprendizaje basado en problemas y el aprendizaje basado en proyectos. A través de una formación de larga duración (un año), que incluye formación en el diseño de una asignatura y su implementación, pretende no sólo promover una actitud reflexiva ante la enseñanza y el desarrollo de competencias, sino también para tutorizar, asesorar o formar a otros docentes de la universidad.

El programa sigue las siguientes cuatro fases:

- Talleres de iniciación. Los talleres de iniciación tienen una duración de entre 20 a 25 horas, impartidas a lo largo de 3-4 días de una semana.

- Mentoría colaborativa en la fase de diseño de una propuesta. Los participantes deben ir concretando aspectos de la misma a través de la entrega de varios documentos a lo largo del tiempo de duración de esta fase (5 meses).

- Mentoría colaborativa en la fase de implementación en el aula. Finalizada la fase de diseño, se realiza una evaluación de la propuesta realizada, que si es valorada positivamente se implementa en el aula. Se realiza al menos una observación directa en el aula por parte del mentor.

- Evaluación de resultados y divulgación. Por último, se van recogiendo evidencias de los resultados de la implementación a lo largo de todo el proceso, que se presentan en un informe final y que, junto con la propuesta diseñada, son publicados en un centro de recursos (ikd baliabideak), que queda abierto a la comunidad universitaria.

En resumen, el programa diseñado sigue una estrategia de formación basada en la co-mentoría y en la mentoría educativa (Hargreaves y Fullan, 2000; Mulle, 2000; Feiman-Nemser, 2001) y en la reflexión en la acción (Brockbank y McGill, 2002), combinando la mentorización individual, junto con la colaboración grupal de los participantes. Durante la participación en el programa ERAGIN la autora desarrolló las acciones que se describen a continuación.

\section{Integración del caso en el currículum e implementación}

Ante la necesidad personal y social de mejorar la docencia universitaria y, en particular, la docencia en enfermería, la autora se planteó poner en práctica un estilo docente que facilitara el aprendizaje autónomo de los estudiantes. Este desafío comenzó con el proceso de integración del caso en el currículum de la asignatura y, posteriormente, con el proceso de implementación que a continuación se describen. 


\section{Integración del caso en la asignatura}

El objetivo principal de la implementación del MdC fue, lógicamente, la adquisición de las competencias establecidas para la asignatura de "Relación y Comunicación para el cuidado enfermero". Además, se pretendía incentivar la asistencia a clase, mejorar la motivación y el interés, además de conseguir un mejor rendimiento académico de los estudiantes con respecto a la metodología tradicional expositiva.

Las competencias específicas y transversales seleccionadas para trabajar mediante el Caso fueron las que se recogen en la Tabla 1:

\section{Competencias específicas (CE) y transversales (CT)}

CE.1 Identificar y analizar la influencia de factores internos y externos que influyen en el nivel de salud mental de los individuos, identificando los problemas de salud más relevantes en una comunidad.

CE.2 Reconocer signos normales o cambiantes de la salud psíquica, sufrimiento e incapacidad psicológica de la persona.

CE.3 Establecer una relación terapéutica individualizada a cada situación psiquiátrica.

CE.4 Tomar decisiones respondiendo a dilemas éticos o morales y legales en la práctica diaria.

CE.5 Demostrar autoconocimiento para favorecer la relación terapéutica.

CT.6 Conocer y manejar las fuentes de información y la propia información.

CT.7 Exponer de forma clara y correcta, oralmente y por escrito, las decisiones y el proceso de construcción de las mismas.

CT.8 Trabajar en equipo con los compañeros temas relacionados con los cuidados de enfermería con el fin de desarrollar los hábitos y destrezas propios de un aprendizaje cooperativo basado en la comunicación dentro y fuera del ámbito sanitario.

Tabla n.1. Competencias específicas y transversales trabajadas en el Caso

De ahí que el caso fuera diseñado específicamente para trabajar en la asignatura de "Relación y Comunicación para el cuidado enfermero" del Grado de Enfermería. Se trata de un caso donde se presenta un conflicto de decisiones de una enfermera, ante una situación de violencia verbal de un paciente en tratamiento psiquiátrico, en una consulta de un Centro de Salud. La enfermera tiene que tomar una decisión rápida, activar el protocolo de reducción, con las consecuencias que esto tiene para el resto de pacientes en la sala de espera y para el propio paciente, o utilizar la comunicación persuasiva conteniendo la agresión verbal pero arriesgándose a una agresión física.

El párrafo de inicio del Caso es el siguiente:

"En febrero de 2009, Iñigo López, varón de 45 años, acudió a las 11.00 de la mañana a su cita en la consulta de enfermería, ubicada en el Centro de Salud del barrio de Egia de la ciudad de Donostia-San Sebastián (País Vasco, España). Esa mañana la enfermera Coro se había retrasado con las primeras citas. A las 11.40, Coro salió del despacho y en la sala de espera llamó a Iñigo con un retraso de 40 minutos. El paciente entró en el despacho y cerró la puerta de un portazo, sin sentarse en la silla, con los puños cerrados y en un tono de voz muy alto recriminó a Coro: "iYa es hora, estoy subiéndome por las 
paredes! iNo hay derecho! iEstoy harto de que todo el mundo se me cuele!". Coro ante esta situación inesperada ¿qué podía hacer? activar el protocolo de agresión o intentar calmarle, corriendo riesgo de agresión física."

Mediante este Caso, los estudiantes se enfrentan a una situación real (una agresión verbal por parte de un paciente) en la que tienen que aplicar los nuevos conocimientos para la solución del problema, aprendiendo así de forma autónoma, reflexiva y crítica. Dicho caso, se encuentra publicado en su integridad, junto con sus Notas de Enseñanza correspondientes, en la página web de la Universidad del País Vasco (Ikd Baliabideak: http://cvb.ehu.es/ikd-baliabideak/arrue-03-2012.htm)

Con el objetivo de trabajar el Caso se preparó una estrategia de enseñanza con toda una serie de tareas que ayudaban a los estudiantes a lograr las competencias previamente establecidas (Tabla 2). Como se observa en dicha tabla, se utilizaron diversas modalidades docentes tanto presenciales como no presenciales: clases magistrales, seminarios y prácticas de ordenador. Todas las actividades fueron trabajadas por cada alumno tanto de manera individual como grupal. Además, se valoró el número de horas que supondría cada actividad. En total, se calculó que cada alumno invertiría 36 horas para elaborar el caso (15 horas presenciales y 21 no presenciales).

\begin{tabular}{|c|c|c|c|}
\hline Semana & Actividades & Modalidad & $\begin{array}{l}\text { Horas } \\
\text { estimadas }\end{array}$ \\
\hline 1 & $\begin{array}{l}\text { Presentación de la actividad y formación de grupos } \\
\text { 1a encuesta de evaluación }\end{array}$ & Magistral & $1: 30 \mathrm{~h}$ \\
\hline \multirow[t]{2}{*}{$2-3$} & Búsqueda bibliográfica & $\begin{array}{c}\text { Presencial } \\
\text { (P. de } \\
\text { ordenador) }\end{array}$ & $5 \mathrm{~h}$ \\
\hline & $\begin{array}{l}\text { Trabajo individual } \\
\text { Análisis y síntesis de las lecturas obligatorias } \\
\text { (Completar fichas bibliográficas). Búsqueda de } \\
\text { información complementaria. }\end{array}$ & No presencial & $8 \mathrm{~h}$ \\
\hline \multirow[t]{3}{*}{4} & $\begin{array}{l}\text { Sesión grupal } \\
\text { El grupo de expertos debate sobre la información. } \\
\text { Cada miembro explica y resume el material trabajado } \\
\text { de manera individual en su grupo. Se genera el Dossier } \\
1 \text { con toda la información teórica sobre el caso. } \\
\text { 2a encuesta de evaluación. }\end{array}$ & $\begin{array}{l}\text { Presencial } \\
\text { (Seminario) }\end{array}$ & $2 \mathrm{~h}$ \\
\hline & $\begin{array}{l}\text { Trabajo individual } \\
\text { Cada miembro deberá trabajar (reflexión, análisis y } \\
\text { primera aproximación escrita) las preguntas } \\
\text { detonantes. }\end{array}$ & No presencial & $4 \mathrm{~h}$ \\
\hline & $\begin{array}{l}\text { Sesión grupal } \\
\text { Examen de conceptos }\end{array}$ & Presencial & $1: 30 \mathrm{~h}$ \\
\hline 7 & $\begin{array}{l}\text { Trabajo grupo pequeño } \\
\text { El grupo discutirá las preguntas detonantes y llegará a } \\
\text { un acuerdo sobre la decisión de la enfermera } \\
\text { (Diagrama de flujo). 3ạ encuesta de evaluación. }\end{array}$ & $\begin{array}{l}\text { Presencial } \\
\text { (Seminario) }\end{array}$ & $2 \mathrm{~h}$ \\
\hline
\end{tabular}




\begin{tabular}{llrl}
\hline & $\begin{array}{l}\text { Sesión grupal } \\
\text { Cada grupo elaborará el Dossier 2 con la exposición de } \\
\text { todo el Caso y prepararán la sesión plenaria. }\end{array}$ & No presencial & $6 \mathrm{~h}$ \\
\hline 9 & $\begin{array}{l}\text { Sesión plenaria } \\
\text { Análisis del Caso mediante el pizarrón. Entrega del } \\
\text { Dossier. } \\
\text { 4a encuesta de evaluación. }\end{array}$ & $\begin{array}{c}\text { Presencial } \\
\text { (Seminario) }\end{array}$ & $3 \mathrm{~h}$ \\
\hline 11 & $\begin{array}{l}\text { Trabajo grupo pequeño } \\
\text { Modificaciones del Informe final }\end{array}$ & No presencial & $3 \mathrm{~h}$ \\
\hline & & $\begin{array}{r}\text { Total: } 36 \text { horas } \\
\text { 21 no presenciales }\end{array}$ \\
\hline
\end{tabular}

Tabla n.2. Actividades, modalidades docentes y número de horas estimadas para cada actividad

Una vez elaborado el Caso y determinado cuáles iban a ser las actividades que íbamos a hacer con los estudiantes, se pasó a implementarlo en el aula.

\section{Implementación}

La implementación del Caso se produjo durante los meses de septiembre a noviembre de 2011, dentro de la asignatura "Relación y Comunicación para el cuidado enfermero". Esta asignatura tiene 6 créditos y se imparte en el primer cuatrimestre de 20 curso. En este Caso participaron todos los alumnos matriculados en el grupo de Euskara de la citada asignatura $(n=36)$.

En la primera sesión, una vez expuesto en qué consistía el trabajo, se crearon grupos de 4 alumnos ( 9 grupos en este caso). La creación de estos grupos se realizó de manera aleatoria. Cada grupo cumplimentó una cláusula de compromiso donde figuraban los nombres, e-mail, teléfono y fotografías de cada componente, además de las reglas de comportamiento del grupo. Se les pidió que hicieran 4 fotocopias para que de esta manera, tanto el profesor como cada miembro del grupo, dispusiera de una copia.

Como se ha señalado en la Tabla 2, la implementación del Caso abarcó 6 sesiones presenciales ( 5 horas de prácticas de ordenador, 8 horas y media de seminarios y 1 hora y media de clase magistral) y una estimación de 21 horas de trabajo personal de los estudiantes fuera del aula (búsqueda bibliográfica, lecturas, análisis y elaboración de informes). Asimismo, todos los alumnos trabajaron el Caso de manera individual y grupal (tanto en grupos pequeños como de la clase en su conjunto). Todo el proceso se trabajó a lo largo de 11 semanas.

La retroalimentación con el alumnado intentó que fuera continua e inmediata a cada actividad. Por ejemplo, tras cada Encuesta de Evaluación, se comentaban, de manera anónima, los resultados generales de las mismas, aclarando, puntualizando o recogiendo las opiniones expresadas.

Las primeras horas presenciales y de trabajo individual (semanas 1 a 3 ) se centraron en la búsqueda de bibliográfica y análisis y síntesis de la misma, dado que, 
para que un estudiante pueda aplicar su conocimiento, en primer lugar deberá conocer la información necesaria y luego comprender esta información.

A lo largo de las cinco sesiones presenciales, los grupos tuvieron la oportunidad de expresar sus dudas y la profesora tuvo la ocasión de evaluar el funcionamiento general de cada grupo: las dudas que surgieron, la resolución de conflictos, la participación activa de los miembros, etc.

El Caso se cerró con una sesión plenaria donde todos los alumnos realizaron la exposición conjunta de la problemática, de sus causas, de las soluciones o alternativas, de la evaluación y de la priorización de las mismas. Las mesas se encontraban colocadas en forma de $\mathrm{U}$, con la pizarra y la pantalla del ordenador cerrando el rectángulo. La profesora se colocó en un ángulo de la $\mathrm{U}$, a fin de dinamizar la sesión pero interfiriendo lo estrictamente necesario. Para finalizar la sesión, se resumieron los aspectos teóricos tratados, a los que se incorporó las contribuciones que propiciaron mayor reflexión entre los participantes.

A lo largo de todo este proceso se fue evaluando tanto la metodología del MdC como las competencias logradas por los alumnos que a continuación se describen.

\section{Evaluación de la metodología de enseñanza basada en el MdC y del conocimiento logrado}

En este apartado se aborda el tema de la evaluación. Dicha evaluación abarca dos aspectos: por un lado, la evaluación de la metodología de enseñanza y, por otro, el conocimiento adquirido por los estudiantes.

\section{Evaluación de la metodología de Enseñanza basada en el MdC}

Los instrumentos de evaluación que se han empleado para medir la metodología de enseñanza basada en el MdC son las encuestas de evaluación y la participación. A continuación se describe cada uno de los instrumentos. Asimismo, se presentan los resultados obtenidos.

\section{Encuestas de evaluación}

Con el objetivo de hacer un seguimiento del proceso, tras concluir las sesiones presenciales se administraron varias encuestas de evaluación: la 1a tras la presentación del Caso; la 2a a tras la primera sesión grupal presencial; la $3 \underline{a}$ tras la segunda sesión grupal presencial; y la 4a tras la sesión plenaria. Posteriormente se codificaron todos los datos para su posterior análisis estadístico.

Con respecto a cómo perciben los estudiantes el trabajo con el MdC, las encuestas de evaluación han puesto de manifiesto una evolución positiva en la percepción de los alumnos con respecto a la metodología. Además han servido para poder mejorar aspectos en el futuro. 


\begin{tabular}{|c|c|c|c|c|c|c|c|c|}
\hline \multirow{2}{*}{$\begin{array}{l}\text { Pregunta } \\
n=36\end{array}$} & \multicolumn{2}{|c|}{ No } & \multicolumn{2}{|c|}{ Si } & \multicolumn{2}{|c|}{ No se } & \multicolumn{2}{|c|}{ TOTAL } \\
\hline & $\mathbf{F}$ & $\%$ & $\mathbf{F}$ & $\%$ & $\mathrm{~F}$ & $\%$ & $\mathbf{F}$ & $\%$ \\
\hline $\begin{array}{l}\text { 1) ¿Te ha animado la primera sesión? (1ạ } \\
\text { encuesta de evaluación) }\end{array}$ & 8 & 22,8 & 13 & 37,1 & 14 & 40 & 35 & 100 \\
\hline $\begin{array}{l}\text { 2) ¿La segunda sesión te ha animado a } \\
\text { trabajar con esta metodología? ( } 2 \text { a } \\
\text { encuesta de evaluación) }\end{array}$ & 2 & 5,8 & 27 & 79,4 & 5 & 14,7 & 34 & 100 \\
\hline $\begin{array}{l}\text { 3) ¿Repetirías esta metodología en otras } \\
\text { asignaturas? (4ạ encuesta de evaluación) }\end{array}$ & 1 & 2,8 & 34 & 97,1 & 0 & 0 & 35 & 100 \\
\hline $\begin{array}{l}\text { 4) ¿Conocías la Metodología del Caso? } \\
\text { (1a encuesta de evaluación) }\end{array}$ & 30 & 85,7 & 5 & 14,2 & 0 & 0 & 35 & 100 \\
\hline $\begin{array}{l}\text { 5) ¿La Guía del Estudiante te ha parecido } \\
\text { un instrumento útil? (1a encuesta de } \\
\text { evaluación) }\end{array}$ & 3 & 8,8 & 29 & 82,5 & 4 & 11,1 & 36 & 100 \\
\hline $\begin{array}{l}\text { 6) ¿Crees que con esta metodología estás } \\
\text { aprendiendo más sobre el tema que con } \\
\text { la metodología tradicional? (2a encuesta } \\
\text { de evaluación) }\end{array}$ & 1 & 2,9 & 33 & 97 & 0 & 0 & 34 & 100 \\
\hline $\begin{array}{l}\text { 7) ¿Te encuentras a gusto trabajando en } \\
\text { el grupo? ( } 2 \text { a encuesta de evaluación) }\end{array}$ & 1 & 2,9 & 33 & 97,1 & 0 & 0 & 34 & 100 \\
\hline
\end{tabular}

Tabla n.3. Preguntas sobre la percepción de los estudiantes en torno al MdC

Los resultados obtenidos ponen de manifiesto que, a pesar de haber tenido que invertir más tiempo del estimado, la MdC ha resultado una experiencia positiva para el conjunto de los participantes. En un primer momento, el porcentaje de alumnos satisfechos con la metodología era del 37,1\% (Tabla 3, pregunta n이). Este porcentaje se incrementó al 79,4\% (Tabla 3, pregunta no2) a mitad del proceso y al 97,1\% (Tabla 3, pregunta $\mathrm{n}$-3) tras la sesión plenaria. Únicamente 1 alumno no repetiría con esta metodología.

El porcentaje de descontentos $(22,8 \%$ ) y escépticos $(40 \%)$ de las primeras semanas (Tabla 3, pregunta $\mathrm{n}$-1) quizás se deba a que la mayoría de los alumnos no conocían esta metodología $(85,7 \%$ ) (Tabla 3, pregunta n우). Sin embargo, también hay que plantearse la posibilidad de que la primera sesión de presentación no fuera lo suficientemente atractiva y haya que modificar algunos aspectos de la misma.

Asimismo, quizás haya que plantearse modificar la Guía del Estudiante que se les entregó el primer día, reduciéndola y simplificándola. Sin embargo, queda patente la utilidad de la misma, ya que esto permite que los estudiantes tengan a mano en cualquier momento todo escrito, y no haya discrepancias entre la interpretación de alguno de los estudiantes y lo dicho por el profesor. Más de tres cuartas partes de los participantes (82,5\%) (Tabla 3, pregunta nํ5) consideraron de interés esta Guía. 
Con respecto a la comparación entre la metodología tradicional y la MdC, también se apreció una evolución positiva. A mitad de implementación, un 97\% de los matriculados (Tabla 3, pregunta no6) en la asignatura opinaba que "está aprendiendo más con esta metodología que con la tradicional", incrementándose a un 100\% (Tabla 4) al final de la misma ("esta metodología me ha ayudado más o mucho más").

\begin{tabular}{lccc}
\hline Valoración general & $\boldsymbol{n = 3 5}$ & $\mathbf{F}$ & $\mathbf{\%}$ \\
\hline Mucho más & 12 & 34,2 \\
Más & 23 & 65,7 \\
Igual & 0 & 0 \\
Menos & 0 & 0 \\
\hline Total & $\mathbf{3 5}$ & $\mathbf{1 0 0}$ \\
\hline
\end{tabular}

Tabla n.4. Frecuencias y porcentajes sobre la utilidad de la metodología: ¿Comparando con la metodología tradicional cómo crees que te ha ayudado ésta? (4o Encuesta de evaluación)

\section{Evaluación del trabajo individual y en grupo}

Este apartado fue evaluado tanto por el profesor como por los alumnos al 50\%. Los alumnos cumplimentaron dos matrices de valoración donde se evaluaban la participación individual en el grupo y el funcionamiento del grupo pequeño respectivamente. El profesor, a su vez, evaluó la participación individual en la sesión plenaria y el funcionamiento del grupo pequeño mediante otras dos matrices. Los resultados de aprendizaje evaluados a través de este instrumento fueron los R.7, R.9 y R.10. (ver Tabla 6).

Los criterios utilizados en las matrices de valoración fueron: asistencia, preparación del trabajo, contribuciones personales, autoanálisis, argumentación, crítica y consenso.

En general, el trabajo se desarrolló según lo previsto y hay que destacar el buen ambiente de trabajo que se generó, tanto en las sesiones presenciales como fuera de ellas. La asistencia de los alumnos a las sesiones de trabajo fue casi del $100 \%$.

En relación a la problemática de crear los grupos de forma aleatoria, y a pesar del escepticismo con el que se lo tomaron los alumnos, es necesario señalar sus efectos positivos. Ya en la segunda encuesta del MdC casi la totalidad del alumnado $(97,1 \%)$ (Tabla 3, pregunta no7) refería encontrarse satisfecho en su grupo. Este sistema evitó que se juntasen grupos naturales ya creados, rompiendo subgrupos y provocando el trabajo con otros compañeros del gran grupo, con los que posiblemente, de otra forma, no hubieran tenido relación. En este sentido, según la percepción de todo el profesorado que imparte docencia en este grupo, parece que la implementación de esta metodología ha podido influir en un ambiente relajado y de compañerismo que no se sentía el año pasado.

Un tema que surgió reiteradamente por parte del alumnado fue el del número de horas excesivas no presenciales invertidas. Este problema ya había sido previsto y, 
por lo tanto, el primer día se les pidió que anotaran minuciosamente el número de horas que destinaban a cada tarea. Los autoinformes revelaron que, efectivamente, el número de horas no presenciales invertidas por los alumnos superó las estimadas por el profesor. En el Tabla 5 se presentan las horas reales utilizadas para cada actividad. En el caso de las actividades no presenciales se muestra la media de todos los alumnos que presentaron el cronograma.

Como se observa en dicha tabla, la mayor diferencia entre lo estimado y lo real, se encuentra en el trabajo individual de análisis y síntesis de las lecturas obligatorias. Frente a las 8 horas estimadas, la media de horas invertidas para dicha tarea fue de 11,3 horas. Las diferencias individuales han sido apreciables. Desde una alumna que declaró haber necesitado 23 horas, hasta las 7 horas de otra. La mayoría necesitaron en torno a 10 horas. Con respecto a la reflexión y análisis de las preguntas detonantes, según los registros de los alumnos, habría que aumentar el número de horas de 4 a 6 . Las sesiones de trabajo en grupo se ajustan más a lo estimado y sólo requerirían de una hora más. En este mismo sentido, en la $2^{a}$ encuesta, un número importante de alumnos declararon no haber tenido suficiente tiempo para explicar y resumir el material trabajado en clase.

\begin{tabular}{lcc}
\multicolumn{1}{c}{ Actividad } & $\begin{array}{c}\text { Horas } \\
\text { ESTIMADAS }\end{array}$ & $\begin{array}{c}\text { Horas } \\
\text { REALES }\end{array}$ \\
\hline $\begin{array}{l}\text { Presentación de la actividad y formación } \\
\text { de grupos }\end{array}$ & $1: 30 \mathrm{~h}$ & $1.30 \mathrm{~h}$ \\
\hline $\begin{array}{l}\text { Búsqueda bibliográfica } \\
\text { Trabajo individual } \\
\text { de las lecturas) }\end{array}$ & $5 \mathrm{~h}$ & $5 \mathrm{~h}$ \\
\hline Sesión grupal & $8 \mathrm{~h}$ & $\mathbf{1 1 , 3} \mathbf{~ h}$ (media) \\
\hline $\begin{array}{l}\text { Trabajo intesis } \\
\text { Examen de conceptos }\end{array}$ & $\mathbf{2 h}$ & $\mathbf{2 ~ h}$ \\
\hline Trabajo grupo pequeño & $1.30 \mathrm{~h}$ & $1.30 \mathrm{~h}$ \\
\hline Sesión grupal & $2 \mathrm{~h}$ & $2 \mathrm{~h}$ \\
\hline Sesión plenaria & $6 \mathrm{~h}$ & $7 \mathrm{~h}$ (media) \\
\hline Trabajo grupo pequeño & $3 \mathrm{~h}$ & $\mathbf{4 h}$ \\
\hline
\end{tabular}

Tabla n.5. Número de horas estimadas y reales para cada actividad. 


\section{Evaluación del conocimiento adquirido}

Igualmente se ha valorado si los estudiantes han adquirido las competencias y contenidos específicos establecidos.

La complejidad de la evaluación de competencias hace necesario diseñar estrategias adecuadas que nos permitan tener la certeza de que los estudiantes han adquirido dichas competencias. Los resultados de aprendizaje son más concretos y, por lo tanto, más objetivamente evaluables. De ahí que, como se muestra en la Tabla 6 , para hacer esta evaluación se determinaron diferentes resultados de aprendizaje para cada competencia.

\begin{tabular}{|c|c|c|}
\hline & $\begin{array}{l}\text { Resultados de aprendizaje (R) } \\
\text { Al completar de forma exitosa este módulo, los estudiantes } \\
\text { deben ser capaces de: }\end{array}$ & Competencias (C) \\
\hline R.1. & $\begin{array}{l}\text { Identificar los factores que precipitan la violencia (demora, } \\
\text { hacinamiento, patología...). }\end{array}$ & CE. 1; CE. 2 \\
\hline R.2. & Valorar el riesgo de agresión física. & CE.1; CE. 2 \\
\hline R.3. & $\begin{array}{l}\text { Valorar a los pacientes reconociendo los signos de hostilidad que } \\
\text { indican posibles respuestas conductuales agresivas. }\end{array}$ & CE.1; CE. 2 \\
\hline R.4. & $\begin{array}{l}\text { Identificar los síntomas de la patología y otros datos relevantes que } \\
\text { pueden explicar la agresividad. }\end{array}$ & CE.1; CE. 2 \\
\hline R.5. & $\begin{array}{l}\text { Analizar las diferentes opciones que tiene la enfermera para tratar la } \\
\text { conducta agresiva. }\end{array}$ & CE. 3; CE. 4 \\
\hline R.6. & $\begin{array}{l}\text { Proponer estrategias de mejora para ser capaces de dar respuesta } \\
\text { ante situaciones de violencia. }\end{array}$ & CE. 3; CE. 4 \\
\hline R.7. & $\begin{array}{l}\text { Identificar los miedos, resistencias, inseguridades, sentimientos, ideas } \\
\text { y prejuicios de cada uno ante una situación de violencia. }\end{array}$ & CE. 5 \\
\hline R. 8 & $\begin{array}{l}\text { Encontrar información relevante seleccionando adecuadamente las } \\
\text { fuentes y sintetizarla de forma que sea útil para la toma de decisiones. }\end{array}$ & CT. 6 \\
\hline R.9. & Expone sus propuestas de forma clara, precisa, ordenada y coherente. & CT. 7 \\
\hline R.10. & Desarrollar capacidad de trabajo en equipo. & CT. 8 \\
\hline
\end{tabular}

Tabla n.6. Resultados de aprendizaje trabajados en el Caso

En el marco de este artículo la competencia se define como "el conjunto de conocimientos, actitudes y habilidades necesarias para desempeñar una ocupación dada" (Yániz, 2008), mientras que los resultados de aprendizaje se definen como "enunciados acerca de lo que se espera que el estudiante sea capaz de hacer, comprender y/o sea capaz de demostrar una vez terminado un proceso de aprendizaje" (Kennedy, 2007). Por lo tanto, las actividades de evaluación se han centrado en los resultados de aprendizaje. Para medir dichos resultados de aprendizaje se utilizaron diferentes instrumentos de evaluación: una prueba escrita de conceptos, un informe final y varias rúbricas para valorar la participación.

A continuación se recogen los ejemplos para los siguientes Resultados de Aprendizaje: 
R.4. Identificar los síntomas de la patología y otros datos relevantes que pueden explicar la agresividad.

R.5. Analizar las diferentes opciones que tiene la enfermera para tratar la conducta agresiva.

R. 8 Encontrar información relevante seleccionando adecuadamente las fuentes y sintetizarla de forma que sea útil para la toma de decisiones.

R.10. Desarrollar capacidad de trabajo en equipo.

El Resultado de Aprendizaje número 4 (identificar los síntomas de la patología y otros datos relevantes que pueden explicar la agresividad) se evaluó mediante un examen de conceptos individual y el informe final del grupo.

La prueba escrita individual (20\% respecto a la nota global) se realizó a mitad del proceso de implementación para favorecer que los alumnos asistieran a las sesiones de clase habiendo trabajado el material. En dicha prueba se preguntó sobre las características de la patología que aparecía en el caso y los síntomas de agresividad. Tras esta prueba escrita se comprobó que todos los alumnos cumplían, al menos, con el mínimo requerido. Tras los resultados positivos de la prueba, los alumnos adquirieron confianza en la metodología. En la sesión presencial previa al examen, la presión de tener una prueba al finalizar la misma, hizo que todos los grupos trabajaran activamente. Además, nadie acudió a la sesión plenaria sin haber trabajado el material.

El informe final del grupo (55\% respecto a la nota global) se evaluó mediante una matriz que utilizaba los siguientes criterios: la presentación del trabajo, la utilización adecuada de los conceptos, el uso del razonamiento crítico, la gramática y la ortografía, el uso correcto de la bibliografía, la innovación y la elaboración conjunta del mismo. Este trabajo escrito no fue superado por 1 grupo $(11,1 \%), 5$ grupos $(55,5 \%)$ obtuvieron un aprobado y 3 grupos $(33,3 \%)$ lo superaron con notable. Ningún grupo realizó un trabajo sobresaliente.

El Resultado de Aprendizaje número 5 (analizar las diferentes opciones que tiene la enfermera para tratar la conducta agresiva) se valoró mediante un diagrama de flujo que tuvo que elaborar cada grupo. En dicho diagrama se debían incluir las diferentes opciones que tenía la enfermera para hacer frente al problema. La mayoría de los grupos fueron capaces de señalar la mayoría de las opciones, a pesar de que en un par de casos se detectaron dificultades. En los dos casos los miembros del grupo no sabían elaborar un diagrama de flujo. Tras el feed-back con la profesora, todos los grupos plantearon diagramas donde daban cabida a la mayoría de las opciones.

El Resultado de Aprendizaje número 8 (encontrar información relevante seleccionando adecuadamente las fuentes y sintetizarla de forma que sea útil para la toma de decisiones) se evaluó por medio de la bibliografía y las fichas incluidas como anexos en cada uno de los informes finales. La forma de citar la bibliografía fue uno de los puntos que más problemas supuso para la mayoría de los grupos. A pesar de haberles entregado un resumen de las normas APA (American Psychological Association) e incluso haber puesto unos ejemplos en clase, 7 grupos tuvieron que corregir la bibliografía. Con respecto a los resúmenes de los artículos que debían 
elaborar, la mayoría pecaba de ser excesivamente extensos; por lo que a este aspecto se refiere, 6 grupos también tuvieron que hacer modificaciones en las fichas.

El Resultado de Aprendizaje número 10 (desarrollar capacidad de trabajo en equipo) se valoró mediante dos rúbricas, una completada por el profesor y otra por cada uno de los miembros del grupo. Los criterios de observación fueron los siguientes: 1) Discuten los problemas con pasión y sin prevenciones, 2) Confiesan abiertamente sus debilidades y errores, 3) Han terminado el debate con resoluciones claras y específicas, 4) Se centran en lograr resultados colectivos, 7) Evitan las distracciones, 6) Existe respeto entre los miembros. Únicamente en un caso hubo discrepancias entre lo que opinaba el profesor y lo que expresaban los miembros del grupo.

Al tratarse de una nueva asignatura implantada con el nuevo Grado de Enfermería, no es posible comparar los resultados con años anteriores. Pero con respecto a los resultados obtenidos en el curso 2011-2012, en general, pueden calificar como satisfactorios. A pesar de que únicamente hayan destacado un par de estudiantes por su calificación de sobresaliente en la nota global del Caso ( 2 puntos sobre la nota final de la asignatura), hay que subrayar que todos los alumnos han superado, al menos, el mínimo exigido.

\section{Discusión}

Como hemos comentado en el apartado anterior, a pesar de haber tenido que invertir más tiempo del estimado, la $\mathrm{MdC}$ ha resultado una experiencia positiva para el conjunto de los participantes. Además, se ha comprobado que los Resultados de Aprendizaje establecidos para cada competencia han sido adquiridos de manera satisfactoria.

En cuanto a la percepción acerca del ambiente de trabajo, en conjunto se ha considerado excelente. La mayoría ha participado de manera satisfactoria, cumpliendo las tareas y la sensación es positiva.

Es importante destacar que el alumnado ha tomado conciencia de la complejidad de la valoración e intervención profesional y, sobre todo, de la importancia del tema tratado (palabras de unas alumnas: "cuando leímos el Caso yo pensé, puff, vaya rollo. Pero ahora me he dado cuenta de que es súper importante";"yo nunca imaginé cuando me metí a hacer esta carrera que me podría ver envuelta en una situación asi"). Las prácticas clínicas que los alumnos deberán superar en los próximos dos años, revelarán si, efectivamente, la formación teórica recibida mediante esta metodología permite a los alumnos enfrentarse a este tipo de situaciones satisfactoriamente y son capaces de superar la gran carga emotiva que genera este tipo de circunstancias.

La actividad no presencial dedicada por los alumnos apenas se desvía de forma significativa de las establecidas por la ANECA para la asignatura de "Relación y Comunicación para el cuidado enfermero", por lo que es factible extender la experiencia a otras asignaturas.

Tampoco han sido necesarias modificaciones de los grupos. Los grupos iniciales se han mantenido y sólo en un caso se ha reflejado, de manera anónima, en las 
encuestas la insatisfacción por el grupo al que se pertenecía. Esta persona no acudió en ningún momento a tutorías para expresar su disconformidad y solicitar algún tipo de cambio de manera personal, por lo que no se pudo solventar el problema.

No obstante, es justo reconocer también ciertas limitaciones e inconvenientes con las que nos hemos encontrado a lo largo del proceso. En primer lugar, a pesar de que la gran mayoría de los alumnos que asiste regularmente a clase estuvo interesado en participar en la metodología del Caso, nos encontramos ante un problema con los alumnos que además de estudiar trabajaban. El porcentaje de este tipo de alumnos aumenta año tras año en el Grado de Enfermería. Para los alumnos con este perfil resulta casi imposible asistir a todas las clases presenciales. La solución a este problema no es fácil, ya que este tipo de competencias no se adquieren "haciendo un trabajo extra". Dejarles fuera de la metodología tampoco resultaría satisfactorio para cualquiera de las partes; ellos seguirían sin adquirir las competencias y el profesorado no dispone de horas para proporcionarles un seguimiento individual a cada uno de ellos.

En segundo lugar, no han sido pocos los momentos en los que los alumnos se han sentido desbordados, tanto por las dimensiones del Caso, como por el número tan alto de temas a tratar al mismo tiempo. Quizás esto no habría ocurrido si los alumnos hubieran estado familiarizados con la metodología. Por ello, resultaría interesante familiarizar a los estudiantes con esta metodología desde el primer año académico y hacer el abordaje, sobre todo en los primeros cursos, mediante casos de pequeña dimensión.

Otra limitación es la insuficiencia de recursos humanos que imposibilita el trabajo con grupos tan numerosos como los que tenemos en el Grado de Enfermería. Este tipo de metodologías, indudablemente, requieren de una mayor planificación y trabajo por parte de los profesores que las clases expositivas. Consecuentemente, el seguimiento y la evaluación constante de las competencias con grupos de 70 alumnos (media de alumnos por clase en el Grado de Enfermería de la Universidad del País Vasco), no resulta lo suficientemente individual y exhaustiva. Es necesario añadir que, en muchas ocasiones, la infraestructura de los centros no facilita este tipo de metodología. En muchos casos las aulas no son suficientemente grandes, con las mesas fijadas al suelo, un número insuficiente de ordenadores, etc.

Finalmente, es obligatorio subrayar que se trata de una experiencia aislada. Sólo se ha implementado una vez, por lo que tendremos que esperar a los próximos años para recabar más información sobre la aplicabilidad del Método del Caso en el Grado de Enfermería. No obstante, la propia autora tiene como objetivo la difusión y aplicación de esta metodología en otras asignaturas para que esta experiencia no se resuma a una totalmente aislada práctica del contexto global del Grado de Enfermería.

\section{Conclusiones}

En resumen, experiencia ha aportado evidencias de que el Método del Caso aplicado en la asignatura "Relación y Comunicación para el cuidado enfermero" de 2 o 
año de Grado de Enfermería en la Universidad del País Vasco, presenta las características que resumo a continuación.

La estrategia de enseñanza del "Caso" ha sido bien aceptada por el alumnado. Todos los estudiantes que han realizado el curso opinan que esta forma de trabajar les ha ayudado más o mucho más que la metodología tradicional expositiva. La práctica totalidad del alumnado se ha mostrado satisfecho con su trabajo en grupo. Además, la asistencia de los estudiantes a las sesiones de trabajo fue casi del $100 \%$.

Los resultados de la experiencia muestran que con el MdC los estudiantes tienen que aplicar los nuevos conocimientos para la solución del problema, estimulando así el aprendizaje así autónomo, reflexiva y crítico. Esto ha llevado a que el Método del Caso sea una herramienta satisfactoria para la adquisición de las competencias planteadas, dado que todos los alumnos han superado, al menos, el mínimo exigido. Así mismo, mejora la motivación y el interés por el tema, especialmente, por tratarse de situaciones reales.

Como conclusión general, y a la vista de los resultados, consideramos que el Método del Caso es, por un lado, una herramienta satisfactoria para la adquisición de las competencias planteadas y, por otro, un método de aprendizaje muy bien aceptado por los alumnos en general.

La experiencia planteada debe servir de incentivo para continuar ampliando el abanico de posibilidades que ofrecen las metodologías activas, de forma que el mayor grado de conocimiento que vayamos adquiriendo desde los diferentes trabajos nos sirva de soporte para abrir futuras líneas que traten de seguir profundizando y buscando propuestas de actuación, que traten de corregir aquellos aspectos negativos encontrados, así como el refuerzo de los positivos.

\section{Agradecimientos}

Este trabajo no se habría podido realizar sin la ayuda y sabias sugerencias que me ha brindado Jenaro Guisasola. Por ello, quiero expresarle mi agradecimiento por su tesón y dedicación en el desarrollo de este artículo.

\section{Referencias Bibliográficas}

Arias-Gundín, O., Fidalgo, R. y García, J.N. (2008). El desarrollo de las competencias transversales en magisterio mediante el aprendizaje basado en problemas y el método del caso. Revista de Investigación Educativa, 26(2), 431-444.

Baños, J.E. y Pérez, J. (2005). Cómo fomentar las competencias transversales en los estudios de Ciencias de la Salud: una propuesta de actividades. Educación Médica, 8(4), 216-225.

Brockbank, A. y McGill, I. (2002). Aprendizaje reflexivo en la educación superior. Madrid: Morata. 
Castro, M., Gómez, M.P., Pablo-Romero, M.P., Sanz, M.T. e Yñiguez, R. (2011). Una experiencia en la docencia de introducción a la economía, a través de tecnologías interactivas. Revista de Investigación Educativa, 29(1), 43-57.

Cervantes, G., Blanch, J. M. y Hermoso, D. (2010). Violencia ocupacional contra profesionales sanitarios en Cataluña notificada por Internet (2007-2009). Archivos de Prevención y Riesgos Laborales, 13(3), 135-140.

Consejo General de Enfermería (2011a, Marzo). El 33\% de los enfermeros españoles ha sufrido una agresión física o verbal en los últimos doce meses. Diario Enfermero. Recuperado de http://www.ege.enfermundi.com/

Consejo General de Enfermería (2011b, Noviembre). La Consejería de Sanidad de Castilla y León registra 187 incidentes y 227 profesionales agredidos en el primer semestre, la mayoría de carácter verbal. Diario Enfermero. Recuperado de http://www.ege.enfermundi.com/

Consejo General de Enfermería (2011c, Septiembre). CSIF pide una reunión urgente con la Delegación de Salud de Sevilla por las agresiones a sanitarios. Diario Enfermero. Recuperado de http://www.ege.enfermundi.com/

Feiman-Nemser, S. (2001). Helping novices learn to teach: Lessons from an exemplary support teacher. Journal of Teacher Education, 52(1), 17-30.

Fernández, I., Guisasola, G. y Garmendia, M. (2010). Desarrollo profesional y aprendizaje cooperativo entre docentes universitarios. El programa ERAGIN de formación del profesorado en metodologías activas de enseñanza. Comunicación presentada en el VI Congreso Iberoamericano de Docencia Universitaria, Noviembre, Lima.

Fernández, M.A., González, J.L., López, I. y Manso, M.E. (2010). Evaluación participativa en habilidades para comunicar en 30 año de Grado de Enfermería en el curso 2009/10, la escenificación como método docente y de evaluación. Revista de Docencia Universitaria, 8(2), 73-93.

Hargreaves, A. y Fullan, M. (2000). Mentoring in the New Millennium. Theory into Practice, 39 (1), 50-56.

Kennedy, D. (2007). Writing and Using Learning Outcomes. A practical guide. Irlanda: University College Cork. Disponible en: http://es.scribd.com/doc/54575200/8/Definicion-de-resultados-deaprendizaje\#page $=18$

McGloin, S. (2008). The trustworthiness of case study methodology. Nurse Researcher, $16(1), 45-55$.

Mulle, C. (2000). Constructing co-mentoring partnerships: walkways we must travel. Theory into practice, 39 (1), 3-11.

OMS (2002). Directrices marco para afrontar la violencia laboral en el sector de la salud. Ginebra: Autor.

Organización Colegial de Enfermería (2007). Estudio sobre agresiones a la profesión enfermera. Madrid: Organización Colegial de Enfermería - Consejo General. Disponible en: http://www.index-f.com/lascasas/documentos/lc0446.pdf 
Organización Colegial de Enfermería (2011). Estudio sobre agresiones a la profesión enfermera. Datos actualizados. Madrid: Organización Colegial de Enfermería Consejo General. Disponible en:

http://www.redaccionmedica.com/contenido/images/enfermeria estudio agres iones2011.pdf

Rodríguez, J. J., et al. (2010). Agresiones 2009. Madrid: Organización Médica Colegial de España.

Ruiz, T., Granados, G., Gutiérrez, M.I. y Muñoz, M.J. (2011). Seminario integrado como metodología complementaria en la asignatura de Enfermería Geriátrica. Gerokomos, 22(3), 104-108.

Ryan, D. y Maguire, J. (2006). Aggression and violence - a problema in Irish Accidente and Emergency departments?. Journal of Nursing Management, 14, 106-115.

Villar, M. y Aranaz, J. M. (2005). Violencia en el medio hospitalario por pacientes con enfermerdad mental. Archivos de Prevención y Riesgos Laborales, 9(1), 20-27.

Yániz, C. (2008). Las competencias en el currículo universitario: implicaciones para diseñar el aprendizaje y para la formación del profesorado. Red $\boldsymbol{U}$. Revista de Docencia Universitaria, número monográfico 1. Disponible en http://www.redu.m.es/Red U/m1

Cita del artículo:

Arrue, M. (2013). La enseñanza de competencias para resolver situaciones conflictivas con pacientes agresivos en enfermería mediante el Método del Caso (MdC). Revista de Docencia Universitaria. REDU. Vol.11 (1) Enero-Abril. pp. 311-328 .Recuperado el (fecha de consulta) en http://www.red-u.net/

\section{Acerca de la autora}

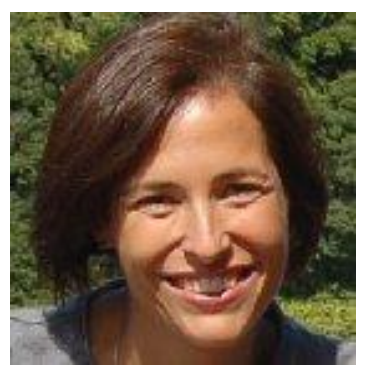

\section{Marta Arrue Mauleon}

\section{Universidad del País Vasco}

Departamento de Enfermería I

Mail: $\underline{\text { marta.arrue@ehu.es }}$

Enfermera Especialista en Salud Mental y Doctora por la Facultad de Psicología (Universidad del País Vasco). Licenciada en Geografía e Historia (Universidad de Salamanca). En la actualidad es profesora de la Escuela Universitaria de Enfermería de de la Universidad del País Vasco. Especialista en Relación y comunicación y Enfermería de Salud Mental. 


\title{
Iniciativa curricular para el desarrollo de competencias transversales en Ciencias de la Salud
}

\section{Curriculum initiative for the development of transversal skills in Health Sciences}

\section{Emilio Gil Martín}

Universidad de Vigo, España

\section{Resumen}

Actividad curricular consistente en la organización, por grupos de alumnos de 5o curso de Biología (de la asignatura Patología Molecular) trabajando en equipo, de una Jornada anual para la detección precoz de la hiperglucemia. La actividad se desarrolla en colaboración con una Asociación de Diabéticos local y precisa de la coordinación con ella y con los responsables universitarios para la disposición de todos los requerimientos técnicos, logísticos y de personal que se necesitan para el evento.

Los objetivos formativos de esta iniciativa son diversos y se extienden a los variados registros de los que se compone una buena formación integral. Entre ellos puede destacarse: i) el fomento y desarrollo de capacidades específicas de la materia (referidas a la capacitación en la obtención, tratamiento y transmisión de datos concernientes a la predisposición a la enfermedad), ii) la familiarización de los estudiantes con el voluntariado social y el asociacionismo de afectados por enfermedades, y iii) el cultivo de competencias transversales referidas a la gestión de recursos, a la mejora de la disciplina, la coordinación y el trabajo en equipo, a la cultura de la calidad y a la conciencia de la necesidad de una formación continuada a lo largo del ejercicio profesional.

El diseño de la actividad es abierto, de manera que primero ejercita respecto al trabajo en el seno de un laboratorio de ideas para, a continuación, canalizar el resultado de éste hasta su materialización práctica mediante un taller preprofesional.

Palabras clave: competencias específicas, competencias transversales, Espacio Europeo de Educación Superior (EEES), formación integral.

\begin{abstract}
The aim of this curricular activity consists in the organization of an annual day for early detection of hyperglycemia. This is accomplished by working groups of fifth-year Biology degree students from the Molecular Pathology course. The activity is developed in collaboration with a local Diabetes Association, which will coordinate with the University and with all the technical, logistical and staff requirements
\end{abstract}


needed for the event.

The educational purposes of this initiative are diverse and extend to the various fields of a good integral training that renders highly skilled students. These include: $i$ ) the promotion and development of specific subject capabilities (referred to training in getting, processing and transmitting data concerning the predisposition to the disease), ii) the familiarization of students with social and voluntary associations of individuals affected by the disease, and iii) the development of transversal skills related to the management of resources, improvement of discipline, coordination and teamwork, quality awareness, as well as the need for ongoing training throughout professional life.

The design of the activity is open, beginning with work within a laboratory of ideas, and finally pre-workshop practice.

Key words: specific skills, transversal competences, European Higher Education Area (EHEA), comprehensive training.

\section{Introducción}

Al abrigo de la reflexión abierta en el colectivo universitario durante la implementación -actualmente en curso- de la reforma de los sistemas de educación superior [cuya meta es la convergencia hacia el Espacio Europeo de Educación Superior (EEES) promulgado en la Declaración de Bolonia de 1999], el profesorado se ha visto en la necesidad de repensar su apuesta formativa en términos de las capacidades (competencias) adquiridas por el alumnado (algo de lo mucho publicado sobre su naturaleza y utilidad puede encontrarse en: Bennet, Dunne \& Carré, 2000; Le Boterf, resalientes de cada uno de ellos. En este sentido, el rendimiento y la calidad de su trabajo fueron más que satisfactorios al brindar su imaginación y talento creativos al servicio de una causa en la que crligados a trabajar de la mano con nuestros alumnos en el fomento de los conocimientos propios de nuestras materias de especialización (competencias específicas), pero también de actitudes y destrezas diferentes de las implícitas en el curriculum tradicional de cada materia (competencias transversales). Estas últimas, no atendidas explícitamente hasta la fecha, han pasado, sin embargo, a desempeñar una función estructurante en los curricula educativos, respondiendo no sólo a la voluntad de fortalecer las competencias técnicas específicas de cada ámbito disciplinar, sino a su materialización en contextos reales o muy cercanos a la realidad, como vía para robustecer las cualidades integrales del futuro profesional.

Nuevas coordenadas del diseño curricular que obligan lógicamente también a resituar los roles de cuantos actores participan o variables inciden en la secuencia enseñanza-aprendizaje. En este sentido, y por lo que a la labor del profesor se refiere, la reformulación de nuestro papel nos emplaza a continuar actuando como vehículos de transmisión de conocimientos (en base a nuestra capacitación experta), mas atendiendo en lo sucesivo a esta nueva -o quizá no tan nueva-, ambiciosa y asimismo discutida (Gimeno Sacristán, 2009a) responsabilidad como agentes facilitadores de entornos de aprendizaje (BOE, 2007). En este recién estrenado papel, nuestra implicación en el aprendizaje de los alumnos cobra utilidad si partiendo de cualquier contenido disciplinar somos capaces de planificar una enseñanza orientada a la ejercitación y desarrollo de competencias. De presidir una docencia meramente 
expositiva/evaluativa, por tanto, se nos insiste en dar el paso de estimular sugestivamente la motivación por aprender de los jóvenes estudiantes, de diseñar programas y actividades formativas que incentiven en ellos los reflejos característicos del futuro profesional, de guiar y supervisar (tutelar) su progresiva capacitación actuando y retroactuando con sagacidad y carisma sobre sus potencialidades, de erigirnos formalmente, en suma, en líderes empáticos con cuyo ejemplo y la ayuda de una atmósfera confidente creada al efecto se surta el efecto de extraer el máximo rendimiento intelectual y la mejor disposición actitudinal.

Comoquiera que sea, de la mano de esta actualización pedagógica y de su particular empeño por hacer aflorar la utilidad práctica (funcional) de los saberes aprendidos, han emergido al debate educativo las aún controvertidas competencias transversales (Bernard, 2000; Gimeno Sacristán, 2009b), según el gusto de cada quien conocidas también por transdisciplinares o genéricas (como, por ejemplo, prefiere el Informe Tuning; ver González \& Wagenaar, 2003). Se trate de un ramillete de cualidades (adaptativas), cuya cotización al alza en la planificación académica arranca de su valor de uso en el contexto de una sociedad que demanda profesionales capaces de desenvolverse en contextos dinámicos, cambiantes (Gil Martín \& González Aguado, 2012). Sin soporte científico en una única área de conocimiento, su naturaleza metadisciplinar las distingue como vitola de la capacidad para el análisis global de la complejidad de los problemas reales, para el tratamiento sistémico y contextual de éstos $y$, en consecuencia, para el alumbramiento de las más acertadas vías de resolución. Incorporarlas, por tanto, al bagaje competencial de los ciclos formativos en ciencias arma a los egresados con una panoplia tan eficaz como necesaria frente a un panorama de crecimiento incesante de la ciencia experimental y de substanciales y aceleradas transformaciones tecnológicas (Esteve, 2003). La adquisición, pues, de los conocimientos, destrezas, habilidades, actitudes y valores que comporta el desarrollo de las competencias transversales no puede sino redundar en egresados mejor formados y más resolutivos. A su vez, la incrementada madurez de éstos habrá de aumentar su potencial de inserción laboral, su empleabilidad, por ceñirse a la peculiar jerga del paradigma dominante (MEC, 2004), y a fin de cuentas el beneficio para la sociedad entera aumentará también merced a la inclusión en sus cuerpos técnicos de profesionales mejor cualificados y capaces de desempeños de mayor eficacia y eficiencia.

\section{Las competencias transversales en la formación en Ciencias de la Salud}

Con el advenimiento de la epistemología constructivista y la fundación de su nuevo paradigma de la enseñanza, se abrió paso en el debate pedagógico la noción de que el conocimiento surge de un proceso de descubrimiento personal del estudiante (Johnson, Johnson \& Smith, 1991). A la luz de este nuevo modo de conceptualización de la enseñanza/aprendizaje, tutores, profesores y conjunto del entorno educativo nos limitaríamos a prestar las condiciones más idóneas para que el alumno pueda dar pie a la "construcción" y "transformación" de su propio conocimiento. Los estudiantes abandonarían así la tradicional pasividad con la que venían aceptando la transmisión meramente expositiva y deductiva del conocimiento heredado y pasarían a 
desempeñar un papel activo en su propio aprendizaje, galvanizando sus estructuras cognitivas y modificándolas conforme se adentrasen en los contenidos del currículo. Enseñar y aprender en este contexto pasaría por diseñar una metodología inductiva en la que, participando en la asunción de los objetivos formativos y trabajando los contenidos sobre situaciones problemáticas simuladas o reales, el alumno alcanzase las competencias pretendidas, lo cual a su vez no resultaría posible si la secuencia enseñanza-aprendizaje no se desenvolviese en un entorno cooperativo y relacional (Fernández March, 2006).

Para un aprendizaje activo y participativo (social) se requieren capacidades múltiples y de naturaleza diversa, pero las conocidas por genéricas o transversales ostentan entre ellas una relevancia significativa. Comunes a todos los estudios de Grado, representan las cualidades que se reconocen en el pensar y actuar de todo profesional competente. Cultivarlas, por lo demás, constituye un aval de la proactividad del egresado para su formación continua, así como para el ejercicio eficaz y productivo de la profesión en cualquier ámbito laboral.

En los laboratorios de discusión intelectual sobre dichas capacidades transversales (Murphy, 2001), los expertos suelen coincidir (en consonancia con la propia taxonomía del Informe Tuning) en distinguir entre las denominadas capacidades instrumentales o metodológicas, volcadas en el fortalecimiento de las habilidades de gestión de la información y organización del trabajo, las capacidades personales (también denominadas sociales o de participación), necesarias para potenciar el trabajo en equipo y la comunicación externa, y las capacidades sistémicas, concebidas como portaestandartes del espíritu crítico y de la voluntad por mejorar que se le reconoce a todo profesional cualificado.

Encontraríamos entre las primeras (instrumentales) la capacidad de aplicación práctica de los conocimientos adquiridos, la resolución de problemas y toma de decisiones, la capacidad de análisis y de síntesis o, entre otras, la capacidad de planificar y gestionar el propio tiempo de trabajo. Una representación significativa de las segundas (personales) estaría integrada por la capacidad de trabajo en equipo (tanto más en equipos multidisciplinares), por el razonamiento (auto)crítico y la asunción de responsabilidades. Entre las últimas (sistémicas) convendría resaltar a título de ejemplo la capacidad de aprender y trabajar autónomamente, el pensamiento creativo $y$, en relación con éste, la capacidad de trabajo en entornos abiertos (cambiantes).

Las Ciencias de la Salud brindan, en mi opinión, uno de los suelos más receptivos para el enraizamiento de una docencia sensible y activamente motivada por el desarrollo de algunas de estas capacidades. A nadie se le escapa, en este sentido, el hondo impacto social que ha distinguido a buena parte de los avances de la Biología Molecular desde mediados del siglo pasado (clonación, transgénesis, etc.). Nadie es ajeno, asimismo, a las inquietudes que algunos desarrollos recientes han elevado de nuevo a la palestra pública (es el caso de las células madre o de la medicina personalizada, por citar algún ejemplo). Así las cosas, cómo negar la pertinencia de pertrechar a los egresados de titulaciones de este ámbito no sólo con un elenco de capacidades de máxima excelencia o con la solvencia técnica requerida en un terreno de tal amplitud y expansión, sino, además y en virtud de lo anterior, con todas las actitudes que los empleadores de dichos titulados universitarios valoran en mayor 
medida (Baños \& Pérez, 2005) y un bagaje de valores labrado conforme a los estrictos acuerdos (bio)éticos vigentes en la comunidad científica y en la sociedad.

En el ámbito de las Ciencias de la Salud la Patología Molecular (PM) representa una referencia obligada y de importancia substancial, ya que sin su aporte de conocimientos y técnicas no se habrían podido obrar los extraordinarios avances de la Biomedicina de las últimas tres décadas. Además, sobre ella pivota el eje de la Medicina Molecular, o lo que es igual, el horizonte soñado de recrear mediante modelos moleculares el normal acontecer de los procesos bioquímicos (normofisiología), así como las más significativas alteraciones de la economía corporal (fisiopatología), pretensión última, por lo demás, de cuantos especialistas trabajamos en dicho ámbito. Se tiene en la PM, por tanto, uno de los más fecundos actores del espectacular desarrollo que ha experimentado la Biología humana durante los últimos años, que ha conducido, entre otros avances significativos, a identificar miles de genes responsables de enfermedad, a esclarecer la base genética de otras tantas patologías humanas y a profundizar en la etiopatogenia molecular de no pocas dolencias que urgen formas de abordaje más eficaces (Alzheimer, mucoviscidosis, corea de Huntington, y tantas más). Y el futuro no hace sino engrandecer sus expectativas, pues, a título de ejemplo, se cuenta con que la carrera actual por obtener los perfiles moleculares de los diferentes tipos de tumores traiga consigo la integración en su seno de la patología oncológica convencional (Cao et al., 2011).

Por igual disciplina básica y aplicada, la PM ha espoleado desde sus inicios buena parte de la evolución de la biología experimental, pues ésta se ha nutrido sistemáticamente del estudio y comprensión de los sistemas disfuncionales, tanto de aquéllos que la naturaleza pone a disposición del científico como de aquéllos otros que éste mismo se provee mediante la cuidadosa e inteligente manipulación de tejidos, órganos, sistemas y organismos modelo. El aforismo "donde hay patología, hay biología" resume el sentido profundo que tiene investigar las "excepciones" para la mejor compresión de la "norma" y, por ello, la imbricación íntima de la PM con la Biología humana en su más genuina acepción. Imbuida, asimismo, de una vocación proyectada a la mejora del diagnóstico y tratamiento de la enfermedad, la PM reconoce como parte integral de su cuerpo de doctrina los objetivos de la terapia médica y acata como propio el compromiso ético de la Declaración de Ginebra (AMM, 1948).

A la vista de esta serie de considerandos, la oferta formativa en PM (y, por extensión, en Ciencias de la Salud) debe incluir, entre sus objetivos específicos, la capacidad de sintetizar los hechos significativos relacionados con la enfermedad (genética) y canalizarlos hacia la evaluación diagnóstica (o preventiva) de los pacientes (y parientes concernidos), así como hacia el escrutinio de las mejores dianas terapéuticas. No son ajenas a estos objetivos de aprendizaje la capacidad de contribuir al avance de la investigación como tampoco, y de modo muy especial, las habilidades relacionadas con el tratamiento de la información sensible obtenida de los pacientes o la correcta comunicación de dicha información a los afectados. No olvidemos, en este sentido, que un foco de empleo potencial para los titulados en Ciencias de la Salud se encuentra, entre otros, en la industria del diagnóstico (clínico y genético) o en los gabinetes de asesoría genética a los pacientes y familiares en riesgo. A estas fuentes de ocupación se verán abocados, por tanto, los futuros graduados en Biología y Grados 
afines o los egresados de los Másteres de especialización en Ciencias de la Salud, razón por la que gana actualidad la acción curricular que aquí se describe.

Partiendo de este análisis de situación sobre los intereses de la formación en Ciencias de la Salud, como responsable de la asignatura Patología Molecular (optativa general de último curso de la Licenciatura de Biología, especialidad de Biología Sanitaria) he coordinado durante los últimos cursos una acción curricular encaminada a potenciar algunas de estas importantes capacidades (tanto específicas como especialmente transversales) en los inminentes titulados. Confío en que en el sucederse de los siguientes apartados se encuentre muestra de la pertinencia y utilidad de una apuesta de esta naturaleza, al igual que de su vigencia una vez extintas las actuales Licenciaturas en favor del nuevo panorama integrado por Grado y Máster, pues la acción a la que me refiero resulta plenamente aquilatable a todos ellos y, en general, a cualquier ciclo formativo en Ciencias de la Salud, a condición de que incorpore créditos de esta materia bajo idéntica denominación u otras equivalentes o afines.

\section{Iniciativa curricular para el desarrollo de competencias transversales en Ciencias de la Salud}

Una de las plataformas más potentes para el desarrollo de competencias (transversales) es el modelo de aprendizaje colaborativo (o Team Based Learning, TBL; Michaelsen, Bauman Knight \& Dee Fink, 2002). En esta aproximación cada discente cuenta con la responsabilidad de construir su propia senda de aprendizaje mientras da respuesta a un reto planteado desde fuera (por el profesor) trabajando coordinadamente en el seno de un equipo de trabajo. En los términos de la excelente definición de Ted Panitz el aprendizaje colaborativo es, antes que una técnica para reproducir en el aula, una filosofía personal que facilita el trato con las personas en cualquier situación y capacita para la formación de grupos de trabajo en los que se respeta y potencia las aportaciones y habilidades de cada quien. EI TBL se basa, por tanto, en la creación, análisis y aplicación sistemática de estructuras o modelos de organización social en el aula (o fuera de ella). Concebido para alumnos de cursos avanzados (Bruffee, 1995), este contexto de aprendizaje responde a un sofisticado diseño en el que los alumnos trabajan (semi)autónomamente organizados en grupos de alto rendimiento. El equipo que aglutinan desarrolla proyectos ambiciosos en entornos de trabajo reales, determinando desde el inicio las necesidades de información (cantidad y tipo) y las herramientas que precisan para la ejecución del proyecto, estableciendo el mejor régimen de trabajo y responsabilidad individual y grupal dentro del equipo y sabiendo articular, asimismo, el protocolo de coordinación y colaboración que asegure el funcionamiento armonioso del conjunto. Por su parte, el profesor "colaborador" ajusta su actuación a la de un -importante- actor secundario responsable del seguimiento del proyecto en marcha; al trabajarse sobre problemas que requieren análisis desde ópticas plurales y a los que hipotéticamente puede darse soluciones diversas, su actuación se ciñe a temporizar y estructurar las condiciones que mejor sirvan al aprendizaje, a monitorizar en régimen de tutoría (ejecutiva y evaluativa) la evolución satisfactoria del proyecto y a la asesoría experta cuando desde el equipo le sea requerida. Se establece así una dinámica de aprendizaje basada en el 
diálogo e interacción entre los estudiantes y de éstos con el profesor y demás agentes del contexto educativo y social (centro, institución, industria, sociedad, etc). Configurado el entorno de enseñanza/aprendizaje bajo tales parámetros, el instructor cede protagonismo y una buena parte de su autoridad al grupo (MacGregor, 1990; Smith \& MacGregor, 1992; Cooper \& Robinson, 1998), siendo ambos (instructor y grupo discente), además del resto de actores capaces de prestar significado (comunidad científica y/o sociedad, por ejemplo), los responsables de juzgar la calidad del proyecto realizado y su adecuación a los fines perseguidos (Rockwood, 1995).

La iniciativa curricular sobre la que versa este artículo responde en sus grandes descriptores a una acción de TBL y se ha promovido, desde luego, con el objeto de alcanzar las prestaciones formativas que se le suponen a esta metodología. La iniciativa ha consistido en la organización de un Jornada anual para la detección precoz de la hiperglucemia (someramente descrita en Gil Martín, 2010), y ha sido llevada a cabo en colaboración con una asociación local de afectados (la Asociación de Diabéticos de Vigo, ADV). El día de la Jornada, en las cuatro ediciones habidas, se eligió cada año en función del calendario escolar y el desarrollo de la misma tuvo lugar en la Facultad de Biología de la Universidad de Vigo. (La edición de 2010 requirió, además del emplazamiento central, de una subsede en otra ubicación dentro del campus para dar cobertura a parte de la demanda que en ediciones anteriores no había sido posible atender.)

El peso organizativo recayó enteramente en estudiantes colaboradores voluntarios de la materia Patología Molecular, como se ha dicho con anterioridad asignatura cuatrimestral de la Licenciatura de Biología, de 5 créditos (3.5 teóricos y 1.5 prácticos). Fundamentalmente cursada por alumnos de 5을 curso de la especialidad en Biología Sanitaria, sin embargo, como optativa general estuvo abierta a todos los alumnos de 2 o ciclo y con frecuencia acogió estudiantes de 40 o 50 curso (de la especialidad en Biotecnología). El tratamiento académico de la iniciativa fue, por lo demás, el correspondiente al de una actividad formativa cualquiera; esto es, se recogió en la programación docente de la materia y se evaluó como una actividad complementaria de acuerdo con los estándares de calidad que se detallan más adelante.

La responsabilidad de asegurar la disposición de los medios de carácter personal, logístico o de comunicación requeridos para la organización y desarrollo de la Jornada descansó por entero en los alumnos organizadores. Ello exigió la formación de diversos grupos de trabajo, que debieron trabajar en estrecha coordinación desde las primeras semanas del cuatrimestre hasta el mismo día de celebración de la Jornada, y más allá de éste durante el tiempo necesario para elaborar las evidencias evaluables del trabajo de cada uno. Aunque según el sello elegido para imprimir a cada edición pudieron necesitarse otros más, de mano se contó siempre con, al menos, un grupo responsable de la realización de los análisis, otro encargado de la logística y de las tareas de publicidad y comunicación, y un tercero dedicado a dar cobertura gráfica a la Jornada.

En el plano estrictamente pedagógico, la iniciativa se concibió como la resolución de un proyecto en un entorno problemático (es decir, real), de manera que la única forma de afrontar su realización fuese el trabajo en equipo y la estratificación acordada de tareas. Se estableció así una disciplina de trabajo en que, partiendo del 
establecimiento de los objetivos estratégicos a cumplir (fase 1), surgiera una distribución de tareas (individuales y por equipos) y su incardinación en el tiempo, tanto durante el tiempo de organización (fase 2) como durante la celebración de la Jornada (fase 3). Este esquema de organización (tabla 1 ) no sólo fue un requisito básico para la coordinación y el trabajo en equipo, sino que constituyó una auténtica secuencia didáctica para los implicados, en la cual, respetándose los acuerdos alcanzados sobre fechas y objetivos, cada participante dispusiera de un margen suficiente para construir su propio aprendizaje a través de la gestión autónoma de los recursos, de la diligencia en la ejecución de tareas y de la puesta en práctica -sin más cortapisas que las limitaciones de presupuesto y la aprobación del resto del comité organizador- de iniciativas propias. Un sistema de distribución del trabajo, asimismo, que, respetando el papel escasamente conductivo del profesor, ha facilitado sin embargo un observatorio desde el que proceder tanto a la evaluación procesual de los equipos como a la de las conductas observables de sus integrantes. Componentes ambas que, sumadas a la evaluación del material acreditativo de los productos obtenidos por cada equipo de trabajo (ver más adelante), ha permitido un juicio de calidad sobre el grado de cumplimiento de los objetivos marcados y, por tanto, de las competencias a desarrollar.

\begin{tabular}{|c|c|l|}
\hline Fase del trabajo & \multicolumn{2}{|l|}{ Acciones a desarrollar } \\
\hline \multirow{2}{*}{ (1) Planificación } & $>$ Establecimiento de los objetivos estratégicos. \\
& $>$ Establecimiento de los grupos de trabajo. \\
\hline (2) Organización & $>$ Distribución de tareas entre los equipos. \\
& $>$ Establecimiento del calendario de actuaciones. \\
\hline (3) Ejecucuión & $>$ Realización de los análisis glucémicos. \\
& $>$ Registro documental de la Jornada. \\
\hline
\end{tabular}

Fuente: Elaboración propia

Tabla n.1. Organigrama del plan de trabajo para la organización de la Jornada

La organización y distribución del trabajo por equipos ha respondido, grosso modo, al esquema que se recoge en la tabla 1 y que se describe con algo más de detalle a continuación. Una vez formado el comité de organización de la Jornada se acometía la primera fase del proceso (1), consistente en la planificación de la Jornada en tutorías consecutivas celebradas con el Profesor responsable, en las que se iban perfilando las acciones estratégicas a poner en marcha. Una vez ultimado el diseño genérico de la Jornada, en semanas posteriores se cerraba una reunión con miembros de la ADV en su sede social para presentarles dicho diseño y recibir sus alegaciones o sugerencias. Concluida la planificación, se establecían los grupos de trabajo y se definían las tareas asignadas a cada uno de ellos, de manera que pudiera iniciarse el trabajo en el seno de cada equipo. En adelante se realizaban tutorías periódicas (abiertas en cuanto a número, calendario, duración y contenido) de cada grupo y del 
conjunto de grupos con el Profesor responsable para supervisar y coordinar las tareas en marcha.

A lo largo de la siguiente fase de organización (2), el equipo encargado de los análisis recibía instrucción de la ADV sobre la toma de muestras y la valoración glucémica. Estudiaba el material didáctico y divulgativo con que cuenta la Asociación, y concretaba los criterios con los que seleccionar el más idóneo para entregar a cada participante de la Jornada y el mejor modo de promover su consulta. Una misión atendida con especial esmero fue la asimilación de las recomendaciones que su personal médico y los propios miembros les hicieron sobre cómo reaccionar en caso de resultados positivos o, en caso contrario, cómo instaurar una buena profilaxis basada en hábitos de conducta saludables.

El equipo de logística y comunicación, por su parte, debió coordinar con la Dirección de los Centros que fueran a albergar algún evento relacionado con la Jornada la disposición de los permisos y espacios requeridos para la celebración. Misión suya fue también diseñar la campaña promocional de la Jornada para darla a conocer a la comunidad universitaria y al entorno social próximo, y en ambos casos hacerlo con la debida antelación para instar la participación en ella. Y, asimismo, fue responsabilidad de este equipo entablar contacto institucional (por medio del Gabinete de Prensa de la Universidad) con los medios de comunicación para comprometer su asistencia y recabar el mayor eco periodístico.

El equipo de cobertura tuvo la encomienda de realizar un amplio registro documental (fotográfico y videográfico) de la Jornada. Mediante entrevistas a cámara, fue responsabilidad suya testar la opinión del público participante, así como del Presidente de la ADV, a quien se le solicitó una valoración de la Jornada y un juicio sobre la atención que merece la enfermedad por parte de las autoridades en materia de salud y la calidad asistencial de los pacientes por parte del sistema público de salud. Aprovechando que la Jornada se clausuraba con una conferencia a cargo de un especialista en diabetes, los integrantes de este equipo debieron asimismo entrevistarlo previamente de acuerdo con un guión elaborado por ellos y supervisado por el profesor responsable. El registro televisivo de esta entrevista y de la conferencia se realizó por el personal del servicio de TV institucional de la Universidad de Vigo (http://tv.uvigo.es/).

Llegado el día de celebración de la Jornada (3), cada grupo tuvo la responsabilidad in toto de su parcela de trabajo. El equipo de análisis realizó las determinaciones glucémicas, trató de ellas directamente con cada participante y atendió eventualmente a los medios de prensa presentes. El equipo de logística y comunicación se preocupó de los requerimientos o incidencias surgidas durante la Jornada y procuró animar la asistencia de la comunidad universitaria. El equipo de cobertura, en fin, realizó las entrevistas pactadas y recogió el material gráfico necesario para el registro documental de la Jornada.

Desde la perspectiva de que esta iniciativa curricular pretendía el desarrollo de competencias transversales, el modo de evaluar el cumplimiento de los objetivos debía apartarse de la mera certificación de conocimientos que se practica en otras partes de la materia. La evaluación, por tanto, tomó como referencia, por un lado, de observaciones procesuales (como la participación activa en las tutorías de 
planificación, la organización del tiempo y del trabajo, la competencia de trabajo en equipo, constancia, iniciativa, autonomía, etc) como también globales, en concreto la calidad de los resultados tangibles. En este sentido, cada equipo debió presentar antes de la finalización del curso una evidencia evaluable del trabajo interno desplegado y de las realizaciones alcanzadas. Así, el equipo de análisis proporcionó una memoria con el registro de todas las determinaciones glucémicas efectuadas, el análisis estadístico y discusión de los resultados y las conclusiones alcanzadas. El equipo de logística y comunicación fue evaluado por la gestión de los recursos puestos a su disposición, por la calidad y despliegue de la campaña de sensibilización y por el resultado organizativo de la Jornada, de todo lo cual quedó registro documental suficiente para efectuar la evaluación. El equipo de cobertura, por su parte, debió acreditar el fruto de su trabajo con una memoria fotográfica etiquetada o un DVD editado con el relato comentado de los aspectos más destacados de la Jornada. Edición tras edición, el alto nivel alcanzado en el conjunto de las tareas asignadas, una vez reflejadas en su correspondiente memoria, mereció el máximo reconocimiento en la calificación final de cada grupo de trabajo (llegando a tener un impacto del 15\% sobre la calificación final).

Como buena prueba de todo ello pueden consultarse algunas de las entrevistas a expertos y conferencias de clausura de la Jornada; grabadas por personal técnico de uvigoTelevisión, una vez editadas por el servicio de realización han sido depositadas en la Mediateca pública de la web institucional de la Universidad de Vigo (uvigo.es), donde actualmente están disponibles las correspondientes a las tres últimas ediciones:

- http://tv.uvigo.es/gl/serial/346.html (año 2008)

- http://tv.uvigo.es/gl/serial/522.html (año 2009)

- http://tv.uvigo.es/gl/serial/759.html (año 2010)

\section{Objetivos formativos}

Han jugado a favor de esta iniciativa curricular la reformulación de los roles de educador y educando que ha traído consigo la Declaración de Bolonia y la creación subsiguiente del EEES, sin obviar, por lo demás, la inexcusable convergencia de las competencias científicas, intelectuales y actitudinales entre los objetivos formativos en Ciencias de la Salud (lógicamente también en PM). Basada en el voluntariado social, el diseño de la actividad descrita pretende valerse de la empatía de nuestros jóvenes estudiantes hacia iniciativas de carácter solidario, acercándoles por esta vía al emergente fenómeno del asociacionismo de afectados por enfermedades (igualmente patologías raras o huérfanas que otras de amplia prevalencia entre la población general, como la diabetes).

La decisión de emprender en el ámbito universitario una tarea de estas características nace también de la necesidad de ofertar modos de aprender más diversos, más acordes con las potencialidades y formas de aprender de cada quien y, por ello, necesariamente más libres en cuanto a formato que las actividades académicas regladas $y$, por ende, en escenarios más abiertos y ricos que los tradicionales. El objetivo, en suma, no ha sido otro que lograr la competencia científica abandonando por un momento el academicismo expositivo y dando pie a un proyecto de aprendizaje investigativo (colaborativo). De dicha reflexión nació el entorno de 
trabajo detallado en los epígrafes precedentes, el cual, con la andadura de los años, supo hacerse mínimamente conductivo y configurarse como una secuencia estructurada de tareas iniciada con un laboratorio de ideas (fase 1 del esquema de trabajo), plataforma de trabajo entre iguales generadora de ideas e iniciativas sobre las que planificar acciones y adoptar estrategias colegiadas, seguido de un taller preprofesional (fases 2 y 3), esto es, un marco de trabajo en el que confrontar lógicas diversas, salvar dificultades, trabar alianzas cooperativas y acercarse cuanto sea posible a la divisoria fronteriza entre la formación universitaria y el real desempeño de la profesión.

Aparte de una vía para fortalecer conocimientos científicos aprendidos en el aula, o para ampliarlos, los participantes en la actividad han tenido la oportunidad de desenvolverse en contextos reales $y$, por lo tanto, relevantes para la práctica profesional, adquiriendo una primera experiencia en la gestión de servicios y recursos en general, así como en la delicada conciliación de intereses diversos. En este sentido, a sabiendas de que el fomento de la autonomía consciente de los alumnos estimula por igual su creatividad y responsabilidad, el marco de aprendizaje informal surgido resulta enteramente propicio al desarrollo de importantes competencias específicas de la Materia, pero especialmente al de otras no menos relevantes referidas a la formación integral de los estudiantes (competencias básicas o transversales).

Como inminentes profesionales, los estudiantes de últimos cursos (tanto de las extintas Licenciaturas como de los actuales Grados o Másteres en Ciencias de la Salud) deben fortalecer competencias específicas muy ligadas a los entornos de inserción laboral que les esperan, lo que para el caso que nos ocupa significa concentrar esfuerzos en el desarrollo, entre otras, de las que se citan a continuación:

1. Comprender la importancia de la (evolución) genética en la comprensión profunda de la biología humana y de la enfermedad.

2. Adquirir dominio de las claves para la evaluación de los avances científicos en el terreno de la PM y de la medicina predictiva.

3. Conocer los medios disponibles para el diagnóstico (clínico o genético) de la enfermedad.

4. Apreciar en su justa importancia el valor de la capacidad de predicción y prevención de la enfermedad.

5. Trabajar en favor de que las personas o pacientes tengan acceso a las pruebas diagnósticas y predictivas apropiadas, e informar acerca tanto de la utilidad y beneficios de éstas como de sus limitaciones y riesgos.

6. Trabajar en pro de que los afectados dispongan de un tratamiento adecuado a su dolencia.

7. Aprender a comunicar de modo apropiado y con rigor la información (clínica y genética), especialmente el significado de las probabilidades en que se expresan los niveles de riesgo (estadístico).

8. Comenzar a comprender las diferentes etapas de maduración psicológica de los individuos, con el fin de facilitar el consejo genético a pacientes de distintas edades y condiciones. 
9. Aprender a comunicar y transmitir la información recibida sobre la enfermedad (genética) de forma clara, no directiva y no sesgada por apreciaciones personales.

10. Conocer las herramientas de uso para mitigar o reducir la ansiedad de los pacientes, sabiendo hacer frente a sus respuestas emocionales.

11. Conocer los más importantes aspectos legales y éticos que conciernen a los desarrollos de la Genética Médica y de la PM.

El trabajo sobre este ramillete de competencias sólo puede actuar en favor de la integración de los titulados en gabinetes de Genética Clínica/Asesoría Reproductiva/Consejo Genético o, por extensión, en los equipos profesionales responsables de la asistencia -en su más amplia acepción- a los afectados por condiciones genético-hereditarias y a sus familiares cercanos. Pero, además, desde este mismo ángulo se tienta la prosecución de competencias transversales referidas al logro de principios de equiparidad universal y de no discriminación por razón de discapacidad, conforme al mandato de nuestro ordenamiento jurídico (Ley 51/2003, 2003) y a las directrices específicas de la Agencia Nacional de Evaluación de la Calidad y Acreditación (ANECA, 2001).

Como primeros interesados en la calidad de su propia capacitación, los estudiantes son conscientes de la importancia de alcanzar también competencias transversales substantivas en su formación integral, en su capacidad ejecutiva y de mejora. Así, por ejemplo, en el ámbito de las Ciencias de la Salud y concretamente en el trabajo especializado sobre diagnóstico y tratamiento de pacientes, no se les escapa la necesidad de resultar empáticos, no directivos, adquirir conciencia clara de las propias limitaciones y solicitar la ayuda especializada y el concurso de otros cuando sea menester. Asimismo, en un terreno tan complejo y dinámico, y sometido a tan acelerada transformación como la Biología Molecular (la PM de modo particular), los alumnos no son ajenos en absoluto a que su salida de la Universidad no interrumpe la necesidad de proseguir con su formación continuada, un requerimiento que se proyectará en el tiempo tanto como lo haga su periplo profesional. En este sentido, cristalizar una voluntad decidida de mejora y actualización de los conocimientos exige a fortiori dotar al futuro profesional de las herramientas precisas para el autoaprendizaje (el manido aprender a aprender o lifelong learning, adoptado en la conferencia de Berlín del año 2003). Teniendo en consideración este conjunto de apreciaciones sobre las necesidades de reciclaje que impone el mercado de trabajo de nuestro tiempo, los estudiantes deberían ser capaces de desarrollar, entre otras, capacidades transversales y actitudes básicas como las que siguen:

1. Apreciar el valor de los equipos humanos numerosos y multidisciplinares y aprender a trabajar en su seno con provecho.

2. Adquirir conciencia de las limitaciones de cada quien y del campo de especialización científico-técnico propio, sabiendo acudir con agilidad al asesoramiento experto de otros especialistas.

3. Comprender y respetar las opiniones y actitudes ajenas (de cualquier índole), sobre todo en el caso de que difieran substancialmente de las propias, 
aprendiendo a conciliarlas cuanto sea preciso con las propias en beneficio de los objetivos comunes.

4. Aprender a colaborar con asociaciones, grupos de apoyo o entidades de servicio público en pro del bienestar de los pacientes.

5. Adquirir el compromiso ético de preservar y defender la confidencialidad de toda información referida a la salud.

6. Desarrollar la capacidad de realizar un análisis crítico de las repercusiones en los sistemas públicos de salud, en su organización y recursos económicos, de los avances en el conocimiento molecular de la enfermedad humana.

7. Aprender a diseñar estrategias eficaces de búsqueda de información significativa y de calidad.

8. Adquirir dominio de las herramientas de documentación científica.

Así las cosas, la acción curricular que aquí se refiere es una plataforma muy potente para acercar al entorno académico la realidad profesional y social tantas veces estudiada y debatida en abstracto en las aulas, y actuar por ello como extraordinario trampolín desde el que cultivar la formación integral de los estudiantes. Acaso por compartir esta visión, la Jornada para la detección precoz de la hiperglucemia contó desde su primera edición con un número creciente de voluntarios y logró concitar el interés del alumnado y personal del Centro organizador y de la comunidad universitaria, hasta erigirse en cita ineludible del calendario escolar del final de cada curso académico.

\section{Algunos de los resultados}

A lo largo de sus primeras cuatro ediciones, la Jornada para la detección precoz de la hiperglucemia ha ido adquiriendo una estructura básica que le ha conferido tanto proyección como consolidación entre los alumnos de PM y demás estudiantes de la Facultad de Biología y Centros próximos. Quizá por tratarse de una actividad protagonizada por un grupo de jóvenes voluntarios, quizá por abordar la problemática de una patología de gran importancia epidemiológica y, por tanto, tan próxima a la realidad personal y/o familiar de muchos de nosotros, quizá por el aval de credibilidad obtenido de la participación de una asociación de pacientes, o quizá tal vez por la sola novedad de su formato, lo cierto es que tanto el estudiantado como el personal de administración y servicios (que se ha volcado en la facilitación logística de la Jornada) han venido participando entusiásticamente en cada cita anual, sometiéndose activamente al análisis glucémico; como dato significativo, las determinaciones analíticas superaron en todas las ocasiones las 200 y rozaron el medio millar (461) en la edición de 2010.

Es de mención obligada la seriedad y aplicación con que se afanaron desde el primer momento los alumnos colaboradores. Gracias a su trabajo ilusionado y de calidad se han logrado mejorar progresivamente múltiples aspectos organizativos de la Jornada. Asimismo, y más importante, es claro que la iniciativa espolea su creatividad y pone a prueba su capacidad de armonizar las capacidades tan diversas como sobresalientes de cada uno de ellos. En este sentido, el rendimiento y la calidad de su 
trabajo fueron más que satisfactorios al brindar su imaginación y talento creativos al servicio de una causa en la que creen y de la que se sienten beneficiados en forma de experiencia (personal, social, profesional) y gratificados por la consideración de sus propios compañeros y de la comunidad educativa a la que pertenecen.

A lo largo de las sucesivas ediciones, del laboratorio de ideas realizado para cada edición surgieron interesantes iniciativas que se fueron implementando en la medida en que lo permitieron los recursos disponibles. Algunas de tales iniciativas han llegado para quedarse, como la creación de un logotipo propio (edición de 2009; figura 1) o la confección de pegatinas (edición de 2010) para la solapa como comprobante de haberse prestado al análisis. El resto de ideas [referidas, por ejemplo, al diseño de la campaña de promoción previa (algo de lo mucho realizado puede verse en la figura 2), a la adecuación del estand, a la animación del público el día del evento para promover la participación, etc, etc] han conferido un perfil particular a cada una de las ediciones. Son fruto, asimismo, de la iniciativa de los alumnos colaboradores algunas de las evidencias presentadas como pruebas de evaluación; el diseño del estudio estadístico de los resultados analíticos (grupo de análisis), el portafolio (del grupo de logística y comunicación) con la memoria de la planificación logística de la Jornada, las diversas actuaciones emprendidas para asegurar la requerida el día de celebración y las evidencias del material publicitario utilizado en la campaña de promoción, o la edición de un video (por parte del grupo de cobertura) como memoria gráfica de la Jornada (que puede limitarse a reflejar el trabajo del equipo de cobertura o cubrir el desarrollo integral de la Jornada; un ejemplo de esta última posibilidad puede consultarse en la dirección: http://tv.uvigo.es/video/43446).

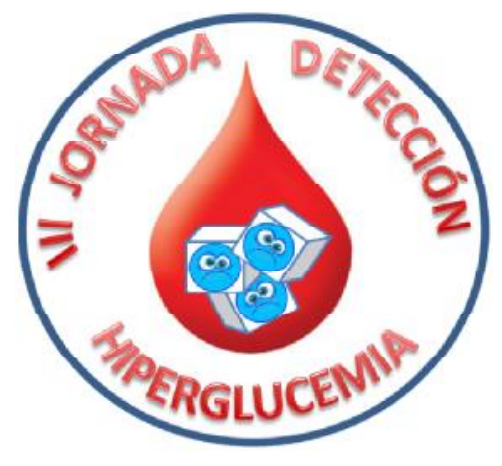

Fuente: Elaboración propia

Figura n.1. Logotipo creado, en 2009, para la III edición de la Jornada para la detección precoz de la hiperglucemia. 

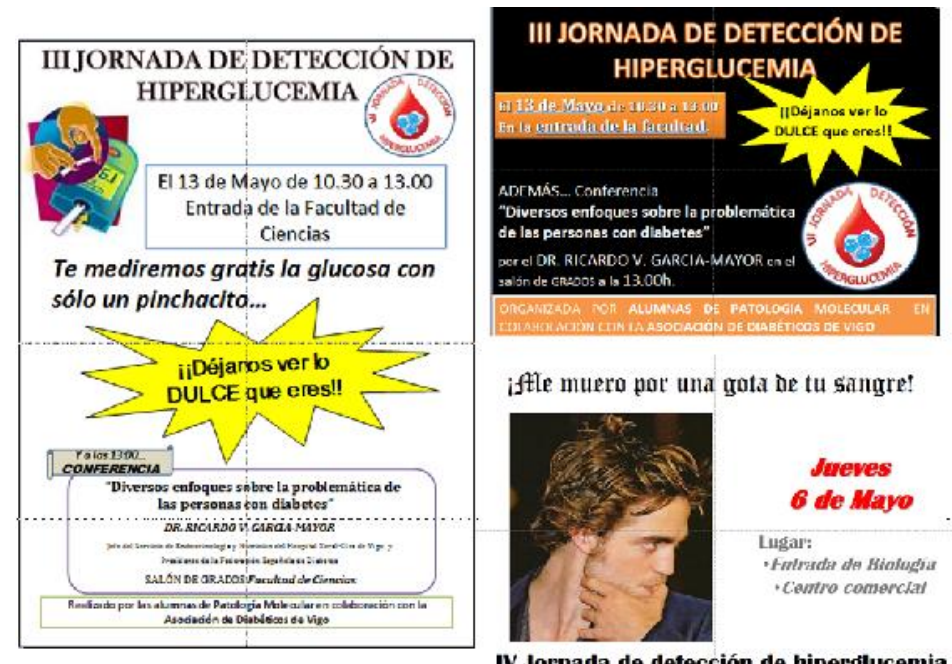

iffle muero pot una gota ion tu sangre!

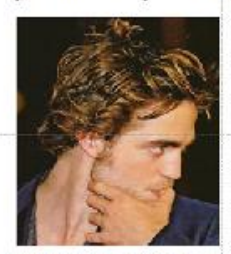

IV Jornada de detección de hiperglucemia

Fuente: Elaboración propia

Figura n.2. Algunos ejemplos de la cartelería digital y en papel utilizada para publicitar la Jornada para la detección precoz de la hiperglucemia.

Por lo que se refiere a la proyección de la Jornada, desde la primera edición de mayo de 2007 las principales cabeceras de prensa de la ciudad de Vigo (Atlántico Diario, Faro de Vigo y La Voz de Galicia, entre otras) se han hecho eco de ella mediante crónicas de diversa extensión, así como el Diario da Universidade de Vigo (DUVI, http://duvi.uvigo.es/). En todas las ocasiones han sido los voluntarios los responsables de atender a estos medios de prensa, de presentarles y explicarles el sentido de la Jornada, así como de responder a todas sus preguntas. En el siguiente enlace ( $L a$ Voz de Galicia, de fecha 31/05/2008) se proporciona una muestra de la repercusión en prensa que han tenido las sucesivas ediciones de la Jornada (la mayoría de las referencias no están disponibles actualmente en la red):

\section{http://www.lavozdegalicia.es/vigo/2008/05/31/0003 6865147.htm}

\section{Conclusiones: la voz de los protagonistas}

Para terminar no encuentro mejor alternativa que ceder todo el protagonismo a quienes ostentan el mérito de haber dado vida, frescura y provecho (para ellos mismos y para la comunidad) a la acción curricular que aquí se desgrana. Procedentes de una encuesta respondida (a finales de 2011) por una quincena de egresados, que en su día participaron en alguna de las sucesivas ediciones de la Jornada, las palabras con las que han expresado su libre parecer, enhebradas a modo de conclusiones, son el mejor instrumento que tengo en mi mano para enjuiciar el sentido (¿por qué?) y la pertinencia (¿para qué?) de la iniciativa.

Sentido. "Yo conozco la enfermedad muy de cerca,..... y considero que es muy importante que la gente se conciencie de que es una enfermedad a tener muy en cuenta y que es muy importante su detección precoz", subraya CCD; idéntica motivación expresa NSO, quien afirma "Realmente, la diabetes es una patología que ha estado presente en mi vida a lo largo de toda ella". Para PPR, sin embargo, la 
motivación es otra: "Yo lo planteé como un reto personal dada mi timidez"; y otra diferente, a su vez, para RGP: "Mi colaboración podía contribuir a ayudar a las personas. Además, se trataba de una actividad diferente", una actividad "...no relacionada con libros o trabajos, sino directamente con las personas", en palabras de PAC. Diferentes motivaciones, como se ve, y tan diversas como nítidas todas ellas aún en la conciencia de los estudiantes.

Pertinencia. La opinión es unánime en cuanto a la ayuda que supuso organizar la Jornada en la mejora de la capacidad de organización, coordinación y trabajo en equipo ("...nos prepara a todos los que terminamos la carrera para ser organizados", estima, por ejemplo, NCS). Pero, además, PPR encuentra que le ayudó "a la hora de organizarme en las tareas diarias y a saber compaginarlas cuando se presenta algún imprevisto; también en relación a la forma de dirigirme a las personas cuando éstas no comprenden tecnicismos"; a CSF le sirvió para darse cuenta de que "es muy fácil difundir la información y asumir papeles que a veces crees que no podrás desempeñar"; GPF aprendió "a ser más práctico" y a "...ser muy riguroso con lo que se hace, y más cuando hay personas (pacientes) de por medio"; para RGP "La organización de la Jornada me ayudó a 'moverme', a buscar la manera de hacer las cosas, de buscar soluciones". Por su parte, MRF vio cómo le "....ayudó, sobre todo, a la hora de confiar en los compañeros de trabajo" y, una vez fuera de la Universidad, trató de animar a la organización de alguna actividad similar a los profesores del Centro en que realizó sus prácticas del Máster para el Profesorado de Educción Secundaria, al tiempo que "desde que participé en la Jornada me di cuenta de que no cuesta tanto colaborar con más gente para poder ayudar a los demás, así que desde entonces, cuando puedo, ayudo en las actividades de ...., una asociación de carácter social".

La iniciativa curricular descrita quiere ser, en fin, una opción plausible para el aprendizaje autónomo, activo y por objetivos, una saludable alternativa que, al menos para las competencias a desarrollar en Ciencias de la Salud, se complementa bien con el aprendizaje transmisivo y más ritualizado que transcurre en el aula. De acuerdo con el pensar de Philippe Perrenoud, cofundador del Laboratoire Innovation-FormationÉducation (LIFE) y experto en innovación pedagógica, "En el mejor de los casos, los jóvenes son 'sabios' cuando salen de la escuela, pero no son necesariamente competentes. Es decir, no aprendieron a movilizar (la cursiva es mía) sus conocimientos fuera de las situaciones de examen" (Brossard, 1999). Considerando que esta necesidad de fomentar el aprendizaje activo y funcional es transversal a todo el sistema educativo, he querido diversificar la experiencia formativa de mis alumnos a través de una iniciativa académica en que, sobre los puntales de la motivación y la responsabilidad (personal y de equipo), se lograse estimular su voluntad de esfuerzo y la formación integral de todos ellos saliera beneficiada. ¿Se ha conseguido? Las palabras de uno de los participantes, que resumen bien el sentir compartido por todos los demás, acaso proporcionen una pista: “...me di cuenta de que no estábamos tan lejos de poder hacer cosas realmente interesantes aunque fuésemos estudiantes", CSF dixit.

"Creo que lo hicimos bien", reflexiona MEZ. Confío en que, después de todo, lo hecho haya sido para el bien de su capacitación profesional. 


\section{Referencias bibliográficas}

Agencia Nacional de Evaluación de la Calidad y Acreditación (ANECA). Guía de Apoyo para la Elaboración de Títulos Oficiales de Grado y Máster. ANECA, 18 de febrero de 2001.

Asociación Médica Mundial (AMM). Declaración de Ginebra. Adoptada por la 2a Asamblea General de la AMM, Ginebra, Suiza, septiembre de 1948. Disponible en la dirección web:

http://www.wma.net/es/30publications/10policies/g1/index.html (consultada en noviembre de 2011).

Baños, J.-E. \& Pérez, J. (2005). Cómo fomentar las competencias trasnversales en los estudios de Ciencias de la Salud: una propuesta de actividades. Edución Médica 8(4), 216-225.

Bennet, N., Dunne, E. \& Carré, C. (2000). Skills development in higher education. The Society for Research into Higher Education \& Open University Press.

Bernard, R. (2000). ¿Existen las competencias transversales? Educar 26, 9-17.

Boletín Oficial del Estado. (2007). Real Decreto 1393/2007, de 29 de octubre, del Ministerio de Educación y Ciencia. BOE no 206, 44037-44048.

Brossard, L. (1999). Construir competencias: todo un programa. Entrevista con Philippe Perrenoud. Vida pedagógica 112, 1-13.

Bruffee, K. (1995). Sharing our toys: Cooperative learning versus collaborative learning. Change 27, 12.18.

Cao, Y., DePinho, R.A., Ernst, M. \& Vousden, K. (2011). Cancer research: past, present and future. Nature Reviews of Cancer 11(10), 749-754. doi: 10.1038/nrc3138.

Cooper, J. \& Robinson, P. (1998). Small-group instruction in Science, Mathematics, Engineering and Technology (SMET). Disciplines: A Status Report and an Agenda for the Future. Journal of College Science Teaching. National Science Teachers Association, Arlington.

Declaración de Berlín (2003). http://www.uco.es/organizacion/eees/documentos/normas-documentos/docbasica/Comunicado\%20de\%20Berlin\%202003.pdf (consultada en noviembre de 2012).

Declaración de Bolonia. Declaración conjunta de los Ministros Europeos de Educación. Disponible en la dirección web:

http://www.educacion.gob.es/boloniaensecundaria/img/Declaracion Bolonia.pd $\underline{f}$ (consultada en noviembre de 2011).

Esteve, J.M. (2003). La tercera Revolución Educativa. La Educación en la Sociedad del Conocimiento. Editorial Paidós, Barcelona.

Fernández March, A. (2006). Metodologías activas para la formación de competencias. Educatio siglo XXI 24, 35-56.

Gil Martín, A. \& Pérez Aguado M.E. (2012). Enseñanza de las ciencias y enseñanza para la vida. Aula de Innovación Educativa (210), 18-22. 
Gil Martín, E. (2010). Organización de la Jornada Anual para la Detección de la Hiperglucemia. En: La docencia en el nuevo escenario del Espacio Europeo de Educación Superior. Ed. por Vicerreitoría de Formación e Innovación Educativa, Universidade de Vigo, 2010, pp. 473-476. ISBN: 978-84-8158-494-3.

Gimeno Sacristán, J. (2009a). Diez tesis sobre la aparente utilidad de las competencias en educación. En: Gimeno Sacristán, J., Comp. Educar por competencias, ¿qué hay de nuevo? Ediciones Morata, S.L., Madrid.

Gimeno Sacristán, J., Comp. (2009b). Educar por competencias, ¿qué hay de nuevo? Ediciones Morata, S.L., Madrid.

González, J. \& Wagenaar, R. (Coord.). Tuning Educational Structures in Europe: Informe Final Fase Uno. Bilbao. Universidad de Desuto, 2003, pp. 27-34. Disponible en la dirección (consultada en noviembre de 2011):

http://www.relint.deusto.es/TUNINGProject/documentos/Tuning phase1/Tunin g phase1 full document.pdf

Johnson, D.W., Johnson, R.T. \& Smith, K.A. (1991). Active Learning: Cooperation in the College Classroom, Edina, MN: Interaction Book Company.

Lasnier, R. (2000). Réussir la formation par compétences. Ed. Guérin, Montreal. ISBN: 9782760156982.

Le Boterf, G. (2001). Ingeniería de las competencias. Editorial Gedisa, Barcelona.

Ley 51/2003, de 2 de diciembre, sobre la igualdad de oportunidades, la no discriminación y la igualdad universal de las personas con discapacidad. B.O.E. no 289, pp. 43187-43195. Disponible en la dirección web (consultada en noviembre de 2011: http://www.boe.es/boe/dias/2003/12/03/pdfs/A43187-43195.pdf

MacGregor, J. (1990). Collaborative learning: Shared inquiry as a process of reform. En: Svinicki, M. D., Ed. The changing face of college teaching, New Directions for Teaching and Learning, no 42. lossey-Bass Publishers, San Francisco.

Michaelsen, L.K., Bauman Knight, A. \& Dee Fink, L., Eds. (2002). Team-Based Learning: A Transformative Use of Small Groups in College Teaching. Praeger Publishers, Wesport, US. ISBN 0-89789-863-X.

Ministerio de Educación y Ciencia (MEC). Programa de Estudios y Análisis de la Dirección General de Universidades del MEC. (2004). Las demandas sociales y su influencia en la planificación de las titulaciones en España en el marco del proceso de convergencia europea en educación superior. Proyecto EA2004-0009, Informe de 2004. Fundación Universidad-Empresa, Ed., pp. 103-104, Madrid. ISBN: 84-7842-194-7. Disponible en la dirección web (consultada en noviembre de 2011): http://www.fue.es/50545212/52932375669.pdf

Murphy, R. (2001). A briefing on key skills in higher education. LTSN Generic Centre: Learning and Teaching Support Network. Assessment Series $\mathrm{n}-5$.

Panitz, T. http://home.capecod.net/ tpanitz/tedsarticles/coopdefinition.htm (consultada en noviembre de 2012).

Rockwood, H.S. III (1995). Cooperative and collaborative learning. The national teaching \& learning forum 4, 8-9. 
Smith, B.L. \& MacGregor, J.T. (1992). What is collaborative learning? En: Goodsell, A.S., Maher, M.R. \& Tinto, V., Eds. Collaborative Learning: A Sourcebook for Higher Education. National Center on Postsecondary Teaching, Learning, \& Assessment, Syracuse University.

Cita del artículo:

Gil Martín, E. (2013). Iniciativa curricular para el desarrollo de competencias transversales en Ciencias de la Salud. Revista de Docencia Universitaria. REDU. Vol.11 (1) Enero-Abril, pp. 329-347. Recuperado el (fecha de consulta) en http://www.redu.net/

\section{Acerca del autor}

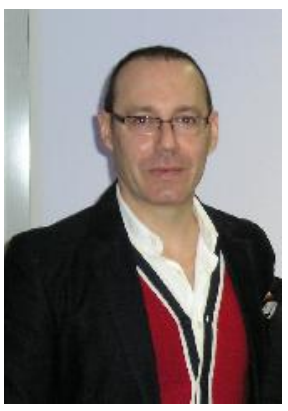

\section{Emilio Gil Martín}

\section{Universidad de Vigo}

Dpto de Bioquímica, Genética e Inmunología

Mail: egil@uvigo.es

Licenciado en Biología y doctorado en Neuroquímica por la Universidad de León, desde 1998 ocupa una plaza como Profesor Titular de Bioquímica y Biología Molecular en el Departamento de Bioquímica, Genética e Inmunología.

Su actividad docente se concentra en la Licenciatura y Grado de Biología, con docencia en Patología Molecular, Bioquímica y Técnicas Instrumentales. Imparte docencia, asimismo, en el Máster en Metodología y Aplicaciones en Biología Molecular y en el Máster interuniversitario en Biotecnología Avanzada.

Integrado en el grupo de investigación de Biomarcadores Moleculares, su área de especialización es la glicobiología, dedicándose concretamente al estudio de las alteraciones moleculares que produce la transformación neoplásica (principalmente el adenocarcinoma colorrectal) sobre enzimas glicosiltransferasas y glicoproteínas. 


\title{
Evaluando el practicum en Educación Social: acciones de mejora ante la puesta en práctica de los nuevos grados
}

\author{
Assessing the practicum in social education: improvement actions to face the \\ implementation of the new undergraduate degrees
}

\author{
Sonia Morales Calvo \\ Universidad de Castilla la Mancha, España
}

\section{Resumen}

Este trabajo, muestra la trayectoria y evolución de nuestro Practicum, unido a los principales resultados de un estudio que, desde la perspectiva de los estudiantes, persigue conocer su grado de satisfacción y la utilidad que les merece el Practicum de la titulación de Educación Social de la Facultad de Ciencias Sociales de Talavera de la Reina (Toledo), con la intención de identificar evidencias que nos ayuden a tomar decisiones para la mejora de estas tareas de formación de gran relevancia en el desarrollo profesional de los estudiantes.

Para ello, y desde una aproximación descriptiva, se ha elaborado un cuestionario ad hoc, validado mediante juicio de expertos, y entrevistas en profundidad destinadas a recoger información de naturaleza cuantitativa y cualitativa a los estudiantes de tercero de Educación Social en el curso académico 2009/2010.

En este sentido, los ejes prioritarios de estudio se han centrado en la organización y secuenciación del Practicum; la acción tutorial ejercida tanto por los tutores del centros de prácticas, como por los profesores tutores de la universidad, la utilidad y sentido de las practicas, para finalizar con las propuestas de mejora que nuestros alumnos nos plantean.

Un avance de los resultados evidencia una serie de necesidades a las cuales hay que dar respuesta, que van desde la mejora de los procesos tutoriales, mediante programas de acogida a los estudiantes en los centros de prácticas, así como la supervisión guiada de los tutores de la universidad, para finalizar en una nueva reconsideración de modalidades del Practicum, en la línea exigida por los presupuestos teóricos que inspiran el Espacio Europeo de Educación Superior.

Palabras Claves: Educación Social, Practicum, organización, acción tutorial, estudiantes, docentes, instituciones prácticas.

\section{Abstract}

This work shows the background and evolution of the Practicum in the Social Education degree together with the main results of a study that, from the students' point of view, tries to find their degree of 
satisfaction and the usefulness of the Practicum of the Degree in Social Education of the Faculty of Social Sciences in Talavera de la Reina (Spain). Our aim is to find evidences which may help us take the appropriate decisions for the improvement of these training tasks which are very important for the professional development of the students.

For that purpose and using a descriptive approach, a questionnaire created ad hoc has been validated by experts' opinions and exhaustive interviews with the aim of collecting qualitative and quantitative information from third-year students of Social Education during the academic year 2009/2010.

In this sense, the main aspects of study have been the organization and sequencing of the Practicum; the tutoring action carried out both by the tutors of the institutions for work experience and by the tutors at University; the usefulness of the work experience; and finally, the improvement proposals made by our students.

An advance of the results shows a series of needs which have to be met and which include the improvement of tutoring processes by means of good welcome programmes for the students in the institutions for work experience, as well as the guided supervision of University tutors. The results also include the reconsideration of the Practicum according to the theoretic guidelines of the European Higher Education Area, which enrich our current model of work experience.

Key Words: Social Education, Practicum, organization, tutoring action, students, teachers, institutions for work experience.

\section{Introducción}

Como pone de manifiesto el Libro Blanco de la Titulación de Grado de Pedagogía y Educación Social (Aneca, 2005) el Practicum se constituye en una materia de gran potencial formativo en estos momentos de reforma de los planes de estudio motivada por la convergencia europea. La importancia de la asignatura de Practicum radica en la fuerte vinculación entre la teoría y la práctica, lo que nos permite la capacidad de construir un pensamiento práctico, es decir, reflexionar la acción, así como adquirir y desarrollar competencias profesionales donde el Practicum se configura (Lobato, 1996:11) " como un espacio de interconexión entre Universidad y Sistema Productivo, a través del cual se contribuye a desarrollar en los futuros profesionales conocimientos habilidades y actitudes propios de un desempeño profesional, por medio de su implicación en actividades profesionales en contextos y condiciones reales para la consecución de un mayor grado profesional" y donde el estudiante debe construir y adquirir el conocimiento profesional necesario para el ejercicio de su profesión. La perspectiva que caracteriza nuestra concepción del Practicum es la que lo concibe como un espacio de reflexión, ya que es posible (González, 2001), ver, hacer, ver hacer y hacer ver.

Desde esta perspectiva, la calidad de nuestras prácticas pasa sin duda alguna por evaluar su proceso, que va desde la organización y estructura curricular y competencial de la asignatura como eje vertebrador de los estudios de nuestro titulados, asegurando el encuentro entre teoría, práctica y experiencia, hasta establecer pautas e indicadores de calidad de las instituciones práctica que recibe a nuestros alumnos. El interesante y detallado análisis del estado de la cuestión del Practicum que realiza Zabalza (2011) que va más allá de los ámbitos de formación docente o de profesiones 
educativas pone de manifiesto algunas cuestiones a tomar en consideración al hablar de la evaluación de tal formación, entre ellas, considerar al Practicum dentro de una visión más curricular e integrada dentro del curriculum de formación, donde la importancia de los aspectos curriculares se deben centran en una mayor atención de los contenidos de aprendizaje definidos de forma concreta. De esta manera, los modelos basados en el aprendizaje de competencias presentan importantes ventajas para el estudiantes, ya que permiten un feedback continuo y claro sobre el logro de las competencias (Bers, 2001). El estudio realizado por el grupo MPI (Modelo de Practicum Integrador, 2011) de la Universidad Autónoma de Barcelona en el marco del proyecto MDQ (Millora de la Qualitat de la Docència) pone de manifiesto la necesidad de mejorar la formación teórica-práctica de los estudiantes de la titulaciones de Educación Social, Pedagogía y Psicopedagogía, presentando las funciones que desde un punto de vista genérico pueden desarrollar los profesionales, los ámbitos de trabajo más frecuentes y las competencias que desarrollan en estos contextos. El mapa competencial extraído (Armegol, et al. 2011) aporta orientaciones claras para la planificación del Practicum, donde cada ámbito concreto de actuación supone adquirir unos conocimientos específicos y que estos deban ser incorporados a los planes de estudio.

Siguiendo con los aspectos curriculares del Practicum, estos deben de incorporar un buen sistema de tutorías y supervisión, donde la acción tutorial se deba de configurar como un espacio didáctico de aprendizaje, y donde la figura del profesortutor sea una de las exigencias para todas las universidades como apoyo y orientación al estudiante en su paso por la Enseñanza Superior (Zabalza, 2003; Gairin, et al. 2004; González, et al. 2010). Además, debe de haber una mayor profundización sobre las funciones de los tutores en las instituciones (Martínez Figueira, 2010), como personas experimentada que proporciona la guía y el apoyo necesario para que el aprendizaje y la inserción sociolaboral resulten lo más enriquecedoras posible, incluyendo estrategias planificadas para desarrollar la identidad del tutor del centro de prácticas. Bajo esta premisa, Zabalza y Cid (1998) formulan dos condiciones necesarias para la realización de una buena tutoría; que la figurara del tutor participe no sólo de la elaboración del plan de prácticas, sino también de las bases conceptuales que sustentan la formación del alumnado de prácticas y la necesidad de un cierto nivel de formalización en todo el proceso para que el programa de formación esté bien estructurado. De esta manera, Martínez y Raposo (2011,169:170) centran su atención en el análisis empírico (a partir de las opiniones de los propios tutores-supervisores) de las funciones más significativas (por su frecuencia de ejecución) a desarrollar con los alumnos tutorizados:

- Establecer con el alumnado una relación de trabajo abierta y de confianza

- Integrar al alumnado en el centro de prácticas durante su estancia en dicho centro

- Estar disponible para el alumnado

- Facilitar al alumnado el conocimiento del contexto y el acceso a la documentación de la institución. 
Burguera y Arias (2011) centran su atención en la figurara del coordinador de Practicum, como eje vertebrador de las acciones entre tutores académicos y centros de prácticas, siendo el agente fundamental en el proceso evaluador, en el control y supervisión del desarrollo, evaluación y mejora del proceso de prácticas.

Otra parte importante de la dimensión curricular del Practicum es la evaluación, donde la aportación de los agentes implicados en este proceso, alumnos, profesores y tutores de la institución prácticas, (Molina, et al.2008; Gónzalez y Hevia 2010) nos ofrecen claves para volver a reformular y repensar el Practicum, ofreciéndonos una vía para analizar la calidad de las prácticas, profundizando en aspectos concretos que permitan un análisis más detallado y posibiliten, tanto el conocimiento de los condicionantes y de las circunstancias en las que se desarrollan los procesos, como la detección de los puntos fuertes y débiles con una clara orientación hacia la mejora de la calidad del programa de prácticas.

\section{EI Practicum en Educación Social en la Universidad de Castilla la Mancha. Contextualización}

El nuevo perfil profesional de Educación Social recogido en el Plan de Estudios de Grado de Educación Social de Castilla La Mancha (2009) corresponde a un profesional que realiza una intervención socioeducativa con personas y colectivos diversos en sus contextos socioeducativos, con el fin de que logren su desarrollo personal y social, su integración y su participación en la comunidad en diferentes espacios sociales. Desempeña, junto a otros profesionales, una función de intervención social y educativa en contextos socio-comunitarios, así en la descripción profesional de la Educación Social, realizada por la Asociación Estatal de Educación Social (ASEDES 2007:12) la define como" derecho a la ciudadanía que se concreta en el reconocimiento de una profesión de carácter pedagógico, generadora de contextos educativos y acciones mediadores y formativas, que son de competencia profesional del educador social".

En relación a la formación que nos ocupa, la de los educadores sociales, podemos mencionar que ésta es una profesión que desarrolla su trabajo educativo en diferentes espacios profesionales. Para ello este profesional tiene que adquirir una serie de conocimientos, destrezas y aptitudes orientadas a desarrollar su trabajo educativo con diferentes colectivos y en diferentes formatos y temáticas tan diversas como el empleo, el ocio, la cultura, la alfabetización o la animación sociocultural. Tanto es así que en el Plan de Estudios de Grado de Educación Social de Castilla La Mancha (2009) se afirma que los tres ejes que definen transversalmente el conjunto de prácticas educativas que el educador desarrolla en contextos y espacios socioeducativos diversos, son los siguientes: creación, coordinación y gestión de espacios educativos, mediación educativa en contextos sociales diversos y formación y transmisión cultural. Ejes que implican el despliegue de una serie de competencias por parte del educador si quiere alcanzar los objetivos que en cada espacio se le demandan, apoyándose siempre en su carácter educativo.

Entre los espacios donde el educador ejerce su labor profesional, (Petrus,1993; Sáez y Molina, 2006) el Libro Blanco del título de grado en Pedagogía y Educación 
Social (2005) hace referencia a: animación sociocultural, inserción socio-ambiental, gestión y difusión cultural, cooperación para el desarrollo, tercera edad, educación de adultos, marginación y delincuencia, menores, discapacidad física-psíquica, inmigrantes y refugiados, turismo sociocultural y ecológico, mediación (social, familiar), acogida y adopción, mujer etc... Estos ámbitos constituyen los lugares habituales de nuestras prácticas en la titulación.

El Practicum se convierte en una materia crucial para completar y enriquecer los aprendizajes de nuestros alumnos, acercando a nuestros estudiantes a la realidad del contexto profesional. De esta manera nuestro Practicum queda estructurado en 24 créditos ECTS, estableciendo como competencias asociadas a esta materia.

\section{COMPETENCIAS BÁSICAS}

Que los estudiantes hayan demostrado poseer y comprender conocimientos en un área de estudio que parte de la base de la educación secundaria general, y se suele encontrar a un nivel que, si bien se apoya en libros de texto avanzados, incluye también algunos aspectos que implican conocimientos procedentes de la vanguardia de su campo de estudio.

Que los estudiantes sepan aplicar sus conocimientos su trabajo o vocación de una forma profesional y posean las competencias que suelen demostrarse por medio de la elaboración y defensa de argumentos y la resolución de problemas dentro de su área de estudio. Que los estudiantes tengan la capacidad de reunir e interpretar datos relevantes (normalmente dentro de su área de estudio) para emitir juicios que incluyan una reflexión sobre temas relevantes de índole social, científica o ética.

Que los estudiantes puedan transmitir información, ideas, problemas y soluciones a un público tanto especializado como no especializado.

Que los estudiantes hayan desarrollado aquellas habilidades de aprendizaje necesarias para emprender estudios posteriores con un alto grado de autonomía.

Capacidad comunicativa y relacional en el trabajo con individuos y grupos.

\section{COMPETENCIAS GENERALES}

Competencia de observación y aprendizaje autónomo en la interacción social y personal con agentes, colectivos y contextos socioculturales diversos.

Competencia para trabajar en equipos plurales e interdisciplinares.

\section{COMPETENCIA TRANSVERSAL}

Capacidad para asumir la necesidad de la formación permanente y el desarrollo profesional, a partir de la reflexión y la evaluación de la propia práctica educativa.

\section{COMPETENCIAS ESPECÍFICAS}

Capacidad para detectar, analizar y valorar de manera reflexiva demandas y necesidades socioeducativas

Habilidad para diseñar, implementar y evaluar estrategias, planes, programas, proyectos y acciones socioeducativas en diversos contextos y ámbitos.

Habilidad para el asesoramiento y acompañamiento educativo de personas y grupos Capacidad de compromiso con el desarrollo de la cultura profesional, a través de la formación, el intercambio profesional, la vinculación con los colegios profesionales y la proyección de la Educación Social

Tabla n. 1. Cuadro de Competencias en el de Educación Social, asociadas al Practicum

A medida que hemos ido avanzando en nuestro proceso curricular, hemos ido planteado diversas modalidades de Practicum quedando estructurado actualmente en 
dos grandes bloques; por un lado el Practicum I, centrado en la formación a través de seminarios prácticos en el aula, por otro la formación en el contexto de la institución propia del escenario profesional de nuestros educadores sociales, constituyendo el Practicum II, donde el alumnado puede optar por las siguientes modalidades de prácticas: investigación, prácticas en la institución o aprendizaje servicio.

En la modalidad de prácticas en la institución, los alumnos analizan críticamente el contexto sociocultural, detectando posibilidades de mejora e intervención. Es un tiempo para la observación y la reflexión mediante los seminarios técnicos que darán paso a la intervención mediante un proyecto social y educativo. Nuestro programa (Morales, 2011) planifica el proceso de manera que los estudiantes conozcan, vivan y actúen en un sólo centro de prácticas de forma que lleguen a integrarse en él y a conocer sus procedimientos de manera plena. Esta modalidad es donde se ubican el mayor porcentaje de alumnos de nuestra titulación.

A partir del 2005, se integra en el Practicum la modalidad de Investigación, motivada por la creación del grupo de investigación en Educación Social de la Facultad de Ciencias Sociales del Campus de Talavera de la Reina "GIES", en ella el alumno debe realizar una fase formativa para poder integrarse en las línea de investigación que en ese momento estén en proceso de realización. Será a partir del 2007 cuando empieza a ponerse en marcha la modalidad de Aprendizaje Servicio, que en un principio denominamos, proyectos de intervención tutorizado (Morales, 2007; Yaniz,et al. 2009) esta modalidad pretende fortalecer el vinculo entre la universidad y el contexto social, pero en la dinámica propia del programa, que permite profundizar indagar y comprender una realidad dando respuestas a las diferentes necesidades sociales en el contexto próximo, teniendo como pilar fundamental el compromiso con la comunidad.

En el curso académico 2009/2010, última promoción de nuestra diplomatura y tras tener criterios internos de evaluación, observamos que dicho programa sigue arrastrando problemas persistentes que queremos identificar, tanto en su estructura como en su supervisión, para ello, los diferentes agentes implicados en la acción formativa se embarcan en el objetivo de "Analizar la posibilidad de enriquecer y mejorar el propio sistema de formación práctica" abordando dos tareas complementarias, una, reflexionar sobre problemas y cuestiones considerados clave en el funcionamiento y organización del Practicum; otra, detectar necesidades y proponer alternativas a los problemas identificados para proceder, finalmente, a "Rediseñar el sistema de Prácticas propio, de acuerdo a las posibles mejoras derivadas del análisis", y que darán lugar al actual Practicum de nuestra titulación que se implantará en el curso académico 2012/2013.

\begin{tabular}{ll}
\hline & \multicolumn{1}{c}{ PRACTICUM GRADO DE EDUCACIÓN SOCIAL } \\
24 créditos ECTS...............4 Curso de Titulación
\end{tabular}

Tabla n.2.Estructura del Practicum en el Grado de Educación Social 
Con lo expuesto anteriormente y teniendo en cuenta que esta investigación forma parte de otra más extensa, nos centraremos en conocer y explicar los niveles de satisfacción de los alumnos con respecto a la asignatura del Practicum II de la Titulación de Educación Social.

Los objetivos marcados se orientan a:

- Analizar el grado de satisfacción con respecto a los aspectos organizativos de las prácticas, y especial de los seminarios que orientan el Practicum.

- Conocer la valoración con respecto a los agentes implicados en el proceso formativo del Practicum: Tutores de la universidad, Tutores de la institución práctica.

- Conocer su percepción sobre la utilidad de las prácticas en su formación profesional.

- Valorar las opiniones que el alumnado tiene sobre los criterios de evaluación, tanto de los seminarios como su participación en las instituciones.

- Conocer las aportaciones de nuestros estudiantes como elemento de mejora del Practicum.

\title{
Método
}

\section{Participantes}

La muestra del estudio responde a un muestreo no probabilístico de tipo casual por accesibilidad (Aliaga, 2000), ya que los participantes son los estudiantes que en el curso 2009/10 estaban matriculados en el Practicum II. La muestra está formada por 65 sujetos, de los 75 matriculados en esta materia, de los cuales 70 alumnos realizan el Practicum I y II, por tanto representan el $92,8 \%$ de la población de referencia, lo que informa suficientemente de su representatividad. La edad de nuestros alumnos se concentra mayoritariamente entre los 20 a 25 años $(64,1 \%)$, existiendo un hecho significativo en nuestra universidad y es el incremento de alumnos mayores de 25 años (30\%). El sexo femenino (84\%) es claramente predominante sobre el masculino $(15,6 \%)$. La titulación de Educación Social ha sido elegido por más del $75 \%$ de nuestros alumnos en primera opción, lo que establece una predisposición inicial positiva hacia los contenidos que se imparten, además más del $50 \%$ de nuestros alumnado continuarán sus estudios una vez finalizado la diplomatura, el cuarto adaptado $(26,6 \%)$ y la realización de máster $(17,2 \%)$ son las opciones elegidas por nuestros alumnos.

\author{
Acceso a los estudios Bachillerato :53,2\% \\ de Educación Social Formación profesional: 32,8\% \\ Prueba de Acceso de mayores de 25 años:(14,1\%). \\ Lugar de Realización Servicios Sociales Básicos:12,5\% \\ de las prácticas Centros de Menores: $21,9 \%$
}




\begin{tabular}{|l|l|} 
& Educación Especial: $20,3 \%$ \\
& Drogodependencias:9,4\% \\
Centros Educativos:7,8\% \\
Educación de Adultos:4,7\% \\
Orientación Sociolaboral:4,7\% \\
Salud mental:3,1\% \\
Animación Sociocultural:1,6\% \\
Otros datos & Modalidad de Aprendizaje Servicio: $9,2 \%$ \\
Investigación: $1,2 \%$ \\
Un 70,3\% recibe becas de estudio \\
Un 28,8\% trabaja, y un $28,1 \%$ lo hace en sectores relacionados con la \\
educación social.
\end{tabular}

Tabla n.3. Descripción de la muestra

\section{Procedimiento de recogida de información y análisis de datos}

Para la recogida de información se ha construido un cuestionario, teniendo en cuenta la revisión documental y bibliográfica, así como las aportaciones y la validación de expertos, que supuso reorganizar algunas de los items planteados. Entre los criterios de elección de expertos se estableció que fueran profesores universitarios con más de cinco años de experiencia en la gestión, coordinación y tutorización de las prácticas, así como investigadores en esta temática.

El instrumento de recogida de datos se organizó en seis dimensiones de análisis con 80 preguntas -codificadas como dicotómicas, politómicas, abiertas y discretasreferidas al Practicum // en Educación Social, a saber: organización y secuenciación, acción tutorial de los tutores de los centros de prácticas y de la titulación, utilidad y sentido de las prácticas y propuestas de mejora. De estas 80 preguntas 60 se utilizaron con formato Likert con 5 categorías para manifestar su grado de satisfacción: (1) nada; (2) poco; (3) normal; (4) bastante; y (5) mucho. El instrumento, una vez realizados los análisis de consistencia interna, arrojó un coeficiente de 0,91 (Alpha de Cronbach) que podemos considar muy buena.

Para su análisis, se ha previsto una estrategia descriptiva y comparativa utilizando como soporte para dicho trabajo el SPSS 19.0 con licencia de la Universidad de Castilla-La Mancha.

Los bloques en los que hemos dividido nuestro cuestionario son:

- Características sociodemográficas, formativas, de participación social y laboral

- Estructura organizativa

- Agentes implicados

- Evaluación

- Utilidad en la formación y el futuro de los profesionales de la educación social

- Propuestas de mejora 


\section{Principales Resultados de la Investigación}

\section{Estructura organizativa del Practicum}

Nuestros alumnos manifiestan en más de un $50 \%$ que la oferta de plazas y la asignación de las mismas, se adecua al perfil profesional y a su sus intereses personales, siendo un proceso adecuado la asignación de los centros, donde se respeta la elección inicial del alumnado y se tiene en cuenta su itinerario formativo para la realización de las mismas. Estos niveles de satisfacción también se manifiestan en el material didáctico $(59,4 \%)$ creado para orientar la estructura y la organización del Practicum (Morales, 2006). Estos niveles de satisfacción decrecen con respecto a la variedad y oferta de plazas existentes, a la información previa que se debe de realizar en la universidad sobre las actividades y programas que realizan las instituciones de prácticas, antes de que el alumno se incorpore, y al tiempo estipulado, donde el nivel de insatisfacción llega al 65,6\% cuando nos referimos al Prácticum II.

Con respecto a los seminarios, un $93,8 \%$, de nuestros alumnos asistieron a los mismos, manifestando una valoración positiva, dirigida a los temas tratados, que ofrecen una visión general del ámbito del educador social, a los ponentes (62\%) y la duración del mismo $(64,3 \%)$, en cambio, estos niveles de satisfacción decrecen en cuanto a las actividades realizadas $(40,6 \%)$ y a los criterios de evaluación $(34,5 \%)$. Debemos de recordar, que estos niveles de satisfacción con respecto a la valoración de los seminarios, son más positivos cuando se introduce el portafolios (Morales, 2010; 2011) como proceso de aprendizaje y de evaluación, con carácter optativo entre nuestros alumnos.

Con respecto a la organización de las instituciones prácticas nuestros alumnos manifiestan su gran satisfacción con respecto a la institución elegida, que se adecúa a las necesidades formativas y expectativas elegidas por el alumnado, unido a la especificación de las funciones por parte de la institución donde, más del $75 \%$ de nuestro alumnado recomendaría dicha institución como lugar de prácticas. 


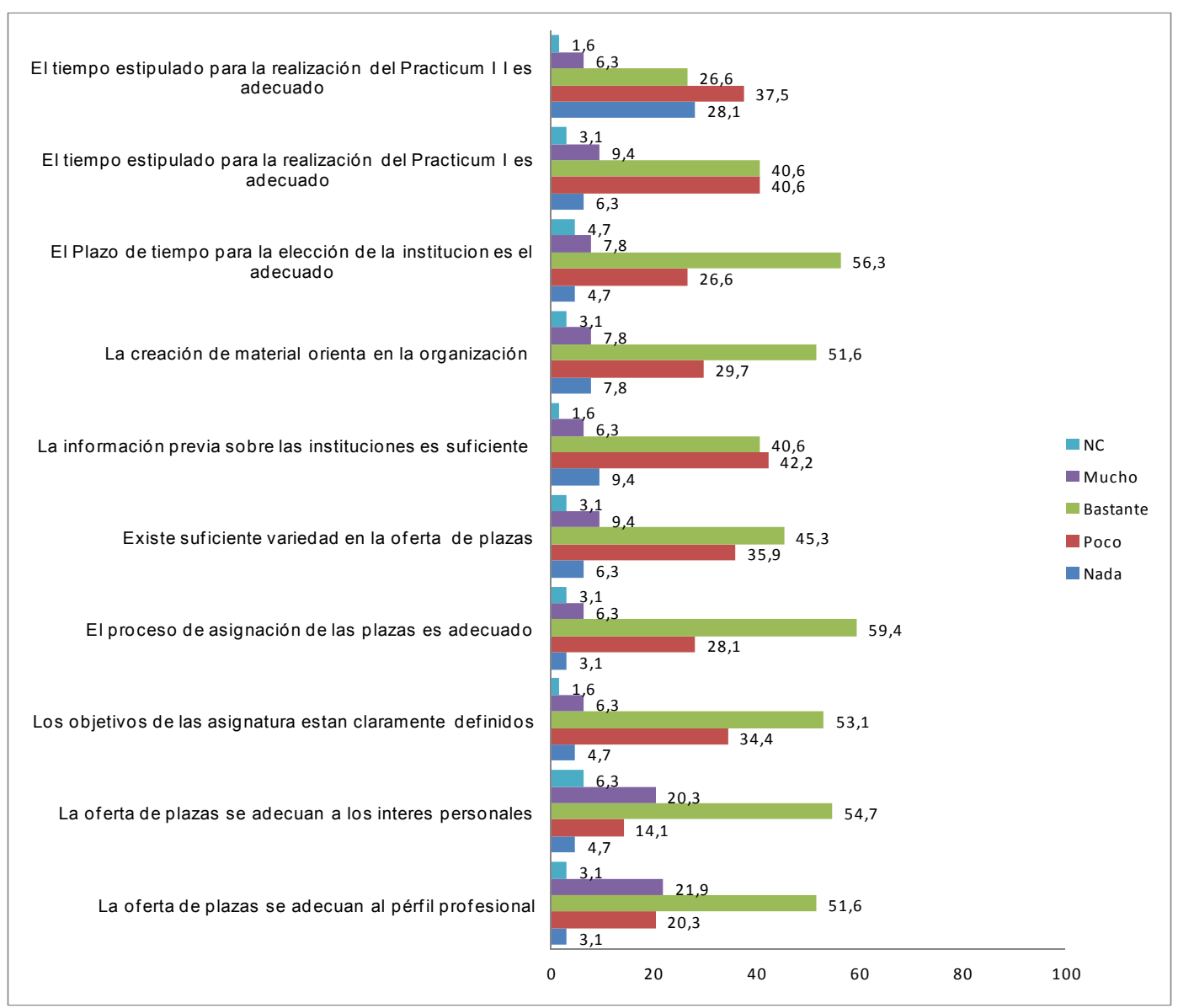

Gráfico n.1. Grado de satisfacción de la organización del Practicum

\section{Agentes implicados en el Practicum.}

Este bloque tiene como dimensión determinar las valoraciones del alumnado respecto a la implicación de los centros/instituciones de prácticas y a la universidad. Con respecto al profesor tutor de la universidad, nuestros alumnos destacan la eficacia de las tutorías individuales, sobre todo, cuando el asesoramiento se centra en la elaboración del informe de prácticas, siendo el seguimiento del alumnado uno de los factores a mejorar, podemos deducir que nuestros alumnos manifiestan niveles de satisfacción superiores cuando son tutorizados por profesores a tiempo completo. Con respecto al tutor de la institución práctica, los niveles de satisfacción se disparan en todas las dimensiones; cercanía hacía el estudiante, descripción de los programas y actividades donde el alumnado se ubicará, en proporcionar material adecuado para la realización de sus prácticas, fomento de la relación del alumno con el resto de profesionales y en el seguimiento y asesoramiento del alumnado. Destacan que a pesar de no existir la figura del educador social como titulado (40\%), sino profesionales habilitados en estas funciones, coinciden en que poseen un alto grado técnico y profesional para ejercer la tutorización en el ámbito de la Educación Social, más del $75 \%$ de nuestros alumnos recomiendan su institución como centro de prácticas. 


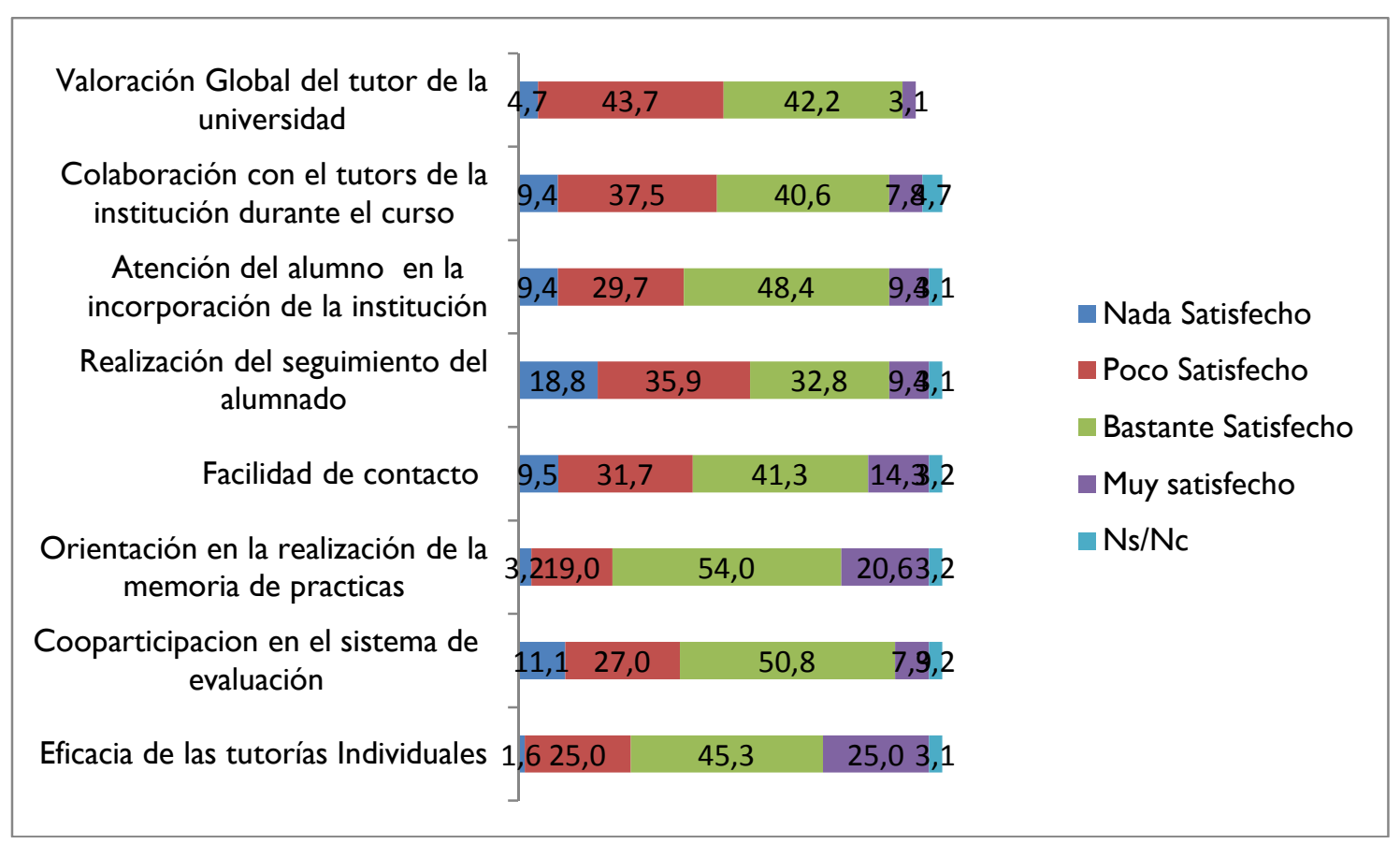

Gráfico n. 2. Niveles de Satisfacción de los alumnos con respecto al tutor de la Universidad

Cualificado técnico profesional para el seguimiento del alumnoen prácticas Proporciona los recursos necesarios para que se lleve a cabo el proyecto

Relación con el tutor de la Universidad

Valoración de la acción tutorial

Seguimiento en la ejecución del proyectos de intervención

Actitud Cercana hacia los alumnos

Fomento de la relación del alumno con la institución

Orientación en la preparación de las actividades

El tutor explica los programas que se desarrollan en el centro

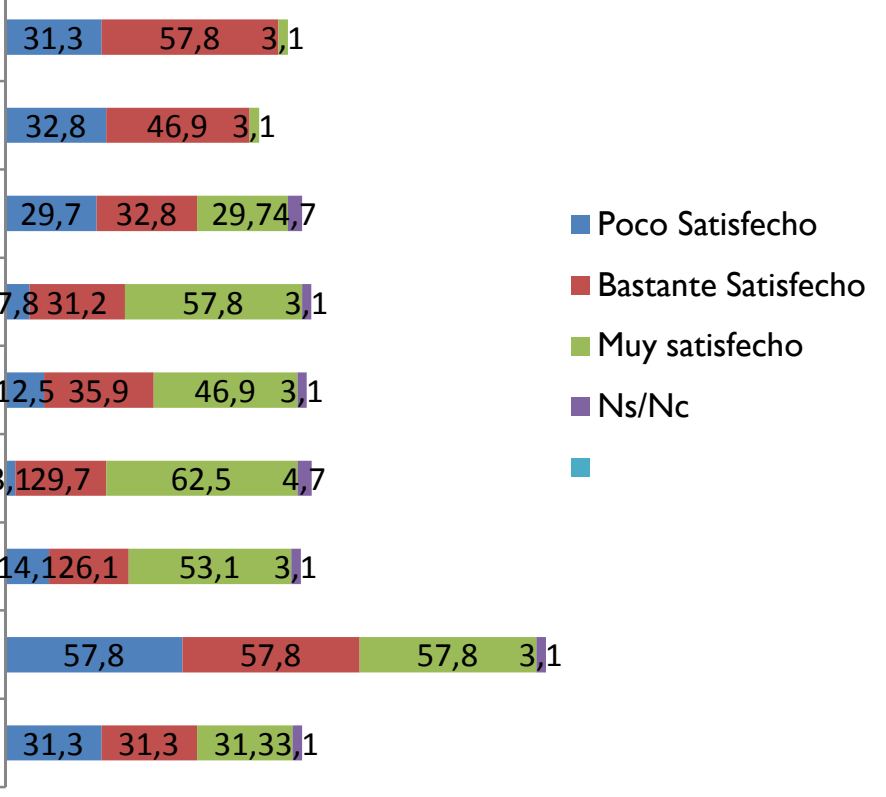

Gráfico n.3. Niveles de Satisfacción del alumnado drigido al tutor de la Institución práctica

\section{Utilidad y Evaluación del Practicum.}

Nuestros alumnos afirman que el grado de utilidad y sentido del Practicum en la titulación, posibilita la cercanía de los estudiantes a los escenarios profesionales reales (67,2\%), completan y amplían los aprendizajes académicos $(56,3 \%)$ y establece 
criterios de reflexión sobre la praxis del educador social, al mismo tiempo facilita salidas profesionales y puede ser facilitador de empleo, un $11,2 \%$ manifiestan que la institución les ha pedido el curriculum una vez finalizadas sus prácticas. Debemos de decir, que estos niveles de utilidad se incrementan en los alumnos que eligen como modalidad de Practicum el aprendizaje-servicio.

Con respecto a la evaluación, nuestros alumnos manifiestan que el diario de campo es fundamental para entender su proceso de aprendizaje dentro de la institución, además manifiestan que el tutor de la universidad actúa como responsable final de la evaluación de los alumnos, sobre todo, en aquellos casos donde se produce una diferencia de valoraciones entre los distintos profesionales. Como aspecto a mejorar destacan una mayor claridad en los criterios de evaluación y una mayor consideración de la nota de del tutor.

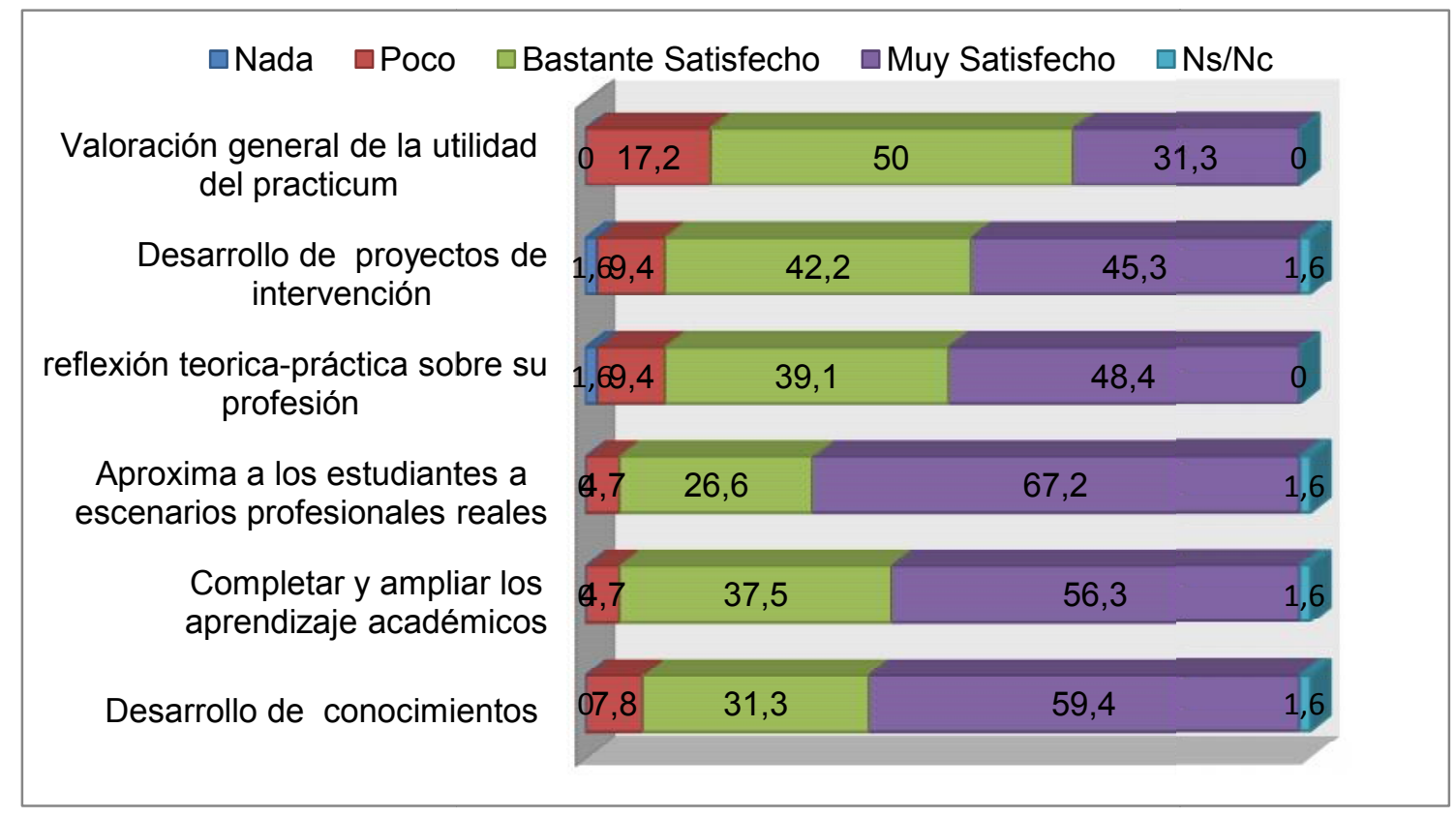

Gráfico n.4. Utilidad Formativa del Prácticum

\section{Conclusiones y Propuestas}

Esta primera fase del estudio, que será completada con las aportaciones de los tutores de las instituciones prácticas, nos permite plantearnos una serie de conclusiones finales que serán plasmadas en el actual Practicum de nuestra titulación. De este análisis se desprende que, aunque existe un alto grado de satisfacción en el Practicum, estos niveles decrecen con respecto a la organización del mismo, manifestando que no hay oferta formativa suficiente en algunos de los ámbitos demandados por nuestros alumnos, como es el caso de los centros de menores o penitenciarios, contextos que en nuestra comunidad autónoma es bastante limitado, de ahí que en algunas ocasiones los convenios de prácticas se realizan con otras comunidades como Madrid o Extremadura, por proximidad geográfica, para dar una alternativa a los alumnos que demandan estos ámbitos profesionales. Además, nuestros alumnos también 
manifiestan que el plazo de tiempo destinado a la elección de la plaza no es suficiente, ya que no hay una información precisa de la institución que precise dónde se realizarán las prácticas, a pesar de que el equipo docente viene realizando un gran esfuerzo para la recopilación de los datos de las entidades (Morales, 2006), aproximadamente unos 110 centros, la información que se les facilita hace referencia aspectos generales de la institución, por lo que sería necesario establecer el programa o servicio concreto en el que quedarán reflejadas las tareas y funciones que debe realizar el alumno.

Como propuesta de mejora estamos elaborando las competencias que deberá realizar el futuro educador social en los diversos contextos profesionales donde realizará su práctica profesional, estableciendo qué debe saber y qué debe saber hacer en el ámbito de prácticas elegido. Además, hemos establecido con algunas instituciones, proyectos de intervención basados en la modalidad de AprendizajeServicio, ampliando el número de alumnos en prácticas con respecto a la modalidad tradicional de prácticas en la institución.

La acción tutorial, ejercida por el tutor de la institución y el profesor de la universidad, se alzará como uno de los pilares básicos para el apoyo y orientación del alumno, si bien con algunas diferencias, otorgando una valoración más positiva en el caso del profesorado universitario cuando éste ejerce su docencia a tiempo completo, frente al profesorado a tiempo parcial, donde el alumnado manifiesta una falta de seguimiento del alumno y coordinación entre instituciones. Al mismo tiempo, valoran en mayor medida la figura del tutor del centro de prácticas, destacando el seguimiento y orientación en la preparación de las actividades prácticas, así como la actitud cercana hacia el estudiante que las realiza, de esta manera, nuestros alumnos ponen de manifiesto que los tutores poseen las competencias propias, habilidades, conocimientos y actitudes que constituyen la base de una efectiva práctica profesional (Field,1994) y donde diversas actuaciones y tareas que el tutor/a realiza responderán (Raposo y Martínez ,2006) a tres grupos de macro-características: humanas (el saber ser), técnicas (el saber hacer) y científicas (el saber saber); siendo las primeras las que se corresponderían con el ámbito personal, y la restante se correspondería con el profesional.

Como propuesta de mejora en este ámbito, hemos intentado que la gestión y el seguimiento del alumno recaiga sobre un sólo profesor para centralizar todas las actuaciones, unido a las entrevistas individuales y grupales para poder dar respuesta a las expectativas personales sobre las prácticas, estableciendo obligatoriamente las tutorías en los momentos claves del Practicum, (inicio, seguimiento y final) y por último se estandarizan todos los protocolos de seguimiento y evaluación de las prácticas para todo el profesorado .

La percepción que tienen nuestros alumnos sobre los criterios y procedimientos de evaluación son positivos, aunque mejorables en varios aspectos, entre ellos destacan la mejora de los criterios de valoración, dirigidos a los procesos de evaluación de los seminarios y a las sesiones formativas que los conforman, así como una mayor representatividad del tutor de la institución. Debemos destacar que estos niveles de satisfacción con respecto a los criterios de evaluación aumentan en aquellos alumnos que optan por la realización del portafolio. 
Como propuesta de mejora se han llevado a cabo tres ejes de actuación en los seminarios; uno dirigido al mundo profesional, otro enfocado a los recursos de la acción práctica y por último la tercera línea de actuación centralizada en la elección de la institución, donde se trabajará el proyecto formativo en colaboración con las institución y el visto bueno de los tutores académicos y de la empresa, dentro del marco establecido en el Real Decreto 1077/2011, del 18 de noviembre que regula las prácticas académicas externas de los estudiantes universitarios.

Por último, para la selección de los centros hemos atendido especialmente a una serie de criterios de calidad como la existencia de la figura profesional del educador social, el grado de motivación o interés en la recepción de nuestros alumnos, que los centros sean ejemplos de buenas praxis educativas y que posean una vinculación objetiva hacía la mejora del proceso de prácticas.

Para finalizar, podemos decir que, aunque la percepción general del Practicum durante estos últimos años ha sido positiva, debemos dotarlo de la flexibilidad necesaria que permita realizar los cambios necesarios para mejorar la calidad de nuestro Practicum, y de esta manera, que ocupe el lugar que le corresponde como eje vertebrados de nuestra titulación.

\section{Referencias Bibliográficas}

ANECA (2005).Libro Blanco del Título de Grado en Pedagogía y Educación Social. Recuperado el 18 de Marzo de 2008, de: http:www.aneca.esactrivin/docs/libroblanco_pedagogia1_035005.pdf.

Aliaga, F. (2000). Bases epistemológicas y proceso de investigación psicoeducativa. Valencia: CSV.

ASEDES (2007). Documentos profesionalizadores. Barcelona: ASEDES y CGCEES.

Armegol, C., Castro, D., Jariot, M., Massot, M y Sala, J. (2011).El Practicum en el Espacio Europeo de Educación Superior (EEES): mapa de competencias del profesional de la educación. Revista de Educación, 354. Enero-Abril 2011, pp.7198.

Bers, T.H. (2001). Measuring and reporting competencies. New Directions for Institutional Resarch, 110, 29-40.

Burguera, J.L y Arias, J.M. (2011). Los coordinadores de prácticas como supervisores del practicum .Revista de Docencia Universitaria, Vol.9 (3), pp. $219-235$.

Cid Sabucedo, A., Pérez Abellás, A y Sarmientos Campos, J.A (2011). La tutoría en el Practicum. Revisión de la literatura. Revista de Educación, 354, 127-154.

Field, B. (1994). The new role of the teachers-mentoriing, en B. Field, et al: Teachers as Mentors: A practical guide. Londres, The Falmer Press.

Martínez Figueira, E. (2010). Ser tutor, ¿cuestión de personalidad o de profesionalidad? Revista Educativa de Orientación y Psicopedagogía, 21(3), pp. 16. 
Gairin,J.; Feixas,M.; Guillamón,C. y Quinquer, D. (2004). La tutoría académica en el escenario europeo de la Eduación Superior. Revista Interuniversitaria de Formación del Profesorado, 18(1),61-77.

Gónzalez, M. (2001). ¿Qué se aprende en el Practicum ? En L. Iglesias, M. Zabalza, A. Cid y M. Raposo (Coords). Desarrollo de competencias profesionales en el Practicum.VI Simposium Internacional sobre el Practicum. Lugo: Unicopia.

González Jiménez, F.E y Equipo (2010). Selección, formación y práctica de los docentes investigadores. La carrera docente. EI EEES. Madrid: Universitas

González, X y Hevia, I. (2010). El Practicum de la Licenciatura de Pedagogía estudio empírico desde la perspectiva del alumnado. Revista de Educación, №354.pp 209-236. Monográfico dedicado a la Formación práctica de estudiantes universitarios: repensando el Practicum.

Lobato, C. (Ed.) (1996). Desarrollo profesional y prácticas: Practicum en la universidad. Bilbao: Universidad del País Vasco

Martínez Figueira, E. y Raposo Rivas, M. (2011). Funciones generales de la tutoría en el Practicum: entre la realidad y el deseo en el desempeño de la acción tutorial. Revista de Educación, 354, 155-181.

Molina Ruiz, E.et al.(2008). Procedimientos de análisis, evaluación y mejora de la formación práctica. Revista de Educación, 346, 335-361.

Morales, S. (2011). Nuevos contextos de enseñanza y aprendizaje en el Espacio Europeo de Enseñanza Superior. Buenos Aires: Miño y Dávila.

Morales, S. (2010). El Practicum en Educación Social: El portafolio como instrumento de seguimiento y evaluación del aprendizaje de los alumnos. Revista Docencia e Investigación, no 20, 33-58.

Morales, S. (2007). La enseñanza Basada en Proyectos de Aprendizaje Tutorizado: una experiencia de coordinación entre la universidad de mayores y el Practicum en Educación Social. En A. Cid, M. Muradas, M.A. Zabalza, M. Sanmamed, M. Raposo y M.L. Iglesias (Coords.). Buenas Prácticas en el Practicum. Santiago de Compostela: Imprenta Universitaria, pp. 910-920.

Morales, S. (2006). El Practicum en Educación Social. Toledo: Universidad de Castilla la Mancha.

Petrus, A. (1993). Educación Social y perfil del Educador Social. En J. Sáez (Coord.). El educador Social. Murcia: Universidad de Murcia.

Real Decreto 1707/2011, de 18 de noviembre, por el que se regulan las prácticas académicas externas de los estudiantes universitarios.BOE,núm.297.

Sáez, J., y García Molina, J. (2006). Pedagogía Social .Pensar la Educación Social como profesión. Madrid: Alianza.

Universidad de Castilla la Mancha. (2009). Plan de Estudios de Grado en Educación Social .Universidad de Castilla la Mancha.

Yaniz, C., Elespuri, I. y Villardón, L. (2009). Aprendizaje Servicio en la Universidad. .En A. Cid, M. Muradas, M.A, Zabalza, M. Sanmamed, M. Raposo y M.L. Iglesias 
(Coords.). Buenas Prácticas en el Practicum. Santiago de Compostela: Imprenta Universitaria, pp.71.78.

Zabalza, M. (2011). El Practicum en la formación universitaria: estado de la cuestión. Revista de Educación , 354, Enero-Abril, 21.43.

Zabalza, M. (2009). Practicum y formación .¿En qué puede formar el Practicum ? En M. Raposo Rivas y otros (Coords). El Practicum más allá del empleo. Formación vs. Training Santiago de Compostela. Pontevedra: Diputación Provincial de Pontevedra, pp. .45-70.

Zabalza, M. (2003).Competencias docentes del profesorado universitario. Calidad y desarrollo profesional. Madrid: Narcea.

Zabalza, M y Cid, A. (1998). El tutor de prácticas: un perfil profesional. En M.A Zabalza (Ed.). Los tutores en el Practicum. Funciones, formación, compromiso institucional. Pontevedra: Diputación de Pontevedra, pp. 17-63.

Cita del artículo:

Morales, S (2013). Evaluando el Practicum en Educación Social: Acciones de Mejora ante la puesta en práctica de los nuevos grados. Revista de Docencia Universitaria. REDU. Vol.11 (1) Enero-Abril, pp. 349-364. Recuperado el (fecha de consulta) en http://www.red-u.net/

\section{Acerca de la autora}

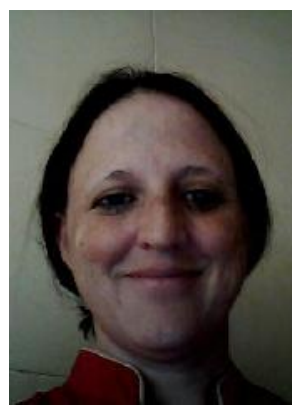

\section{Sonia Morales Calvo}

\section{Universidad de Castilla la Mancha}

Departamento de Pedagogía

Mail: sonia.morales@uclm.es

Doctora en Pedagogía por la Universidad Complutense de Madrid. Es profesora de Educación Social de la Universidad de Castilla La Mancha en la Facultad de Ciencias Sociales, donde realiza tareas de coordinación del Practicum, es docente y coordinadora del área de Tecnología de la Información y de la Comunicación en la Universidad de Mayores "Jóse Saramago" de Talavera de la Reina. Miembro del grupo de investigación en Educación Social (GIES) de la Universidad de Castilla la Mancha participando en varias publicaciones y dirigiendo varias investigaciones orientadas a la acción tutorial del profesor universitario y la mejora de los procesos del practicum , también es miembro del grupo de "Formación y práctica de los docentes investigadores" de la Universidad Complutense de Madrid, donde participa en varias investigaciones orientadas a la calidad de la educación en la enseñanza superior. 


\title{
Reflexiones en torno a la lengua francesa en Castilla y León: secciones bilingües y formación de los profesores de disciplinas no lingüísticas (DNL)
}

\author{
Reflections concerning the French language in Castilla y León: bilingual sections \\ and trainig of teachers of not linguistic disciplines (NLD)
}

\author{
Ana $M^{a}$ Elisabeth Cuervo Vázquez \\ Rosa María Alonso Díaz \\ Montserrat Sabadell González \\ Universidad de Valladolid, España
}

\section{Resumen}

Este trabajo presenta un recorrido a través del panorama de la enseñanza de las lenguas extranjeras y sus resultados de aprendizaje, centrado especialmente en la lengua francesa. Iniciaremos este estudio partiendo de un plano general, analizando la situación en la que se encuentra nuestro país, España, para finalmente abordar, de manera más concreta, el caso particular que presenta nuestra Comunidad autónoma, Castilla y León. Durante este proceso, iremos desvelando diversos datos que nos servirán de apoyo, correspondientes a diferentes estudios realizados en Europa, que ponen en relieve cuáles son las lenguas extranjeras predominantes en el ámbito educativo y el grado de consecución de los objetivos planteados.

Como profesionales dedicados a la formación de futuros profesores de francés lengua extranjera, hemos sentido la necesidad de elaborar este trabajo en el que nos planteamos una revisión de la oferta educativa en lenguas extranjeras en Castilla y León y de las condiciones en que ésta se desarrolla, centrando nuestra atención en la lengua francesa y más concretamente en las secciones bilingües de francés, considerando sus ventajas y objetivos, y en las necesidades de formación de su profesorado. A partir del análisis de la situación actual, llevaremos a cabo una reflexión para intentar mejorar la cuestión planteada al inicio, partiendo de la idea de que una oferta amplia de lenguas extranjeras no sólo es factible, sino imprescindible, y que la implantación de dos lenguas extranjeras en el curriculum escolar debiera tener carácter obligatorio, y no optativo.

Palabras clave: enseñanza, lenguas extranjeras, francés, secciones bilingües, formación, profesorado, resultados de aprendizaje, oferta educativa. 


\begin{abstract}
This study explores the panorama of foreign language teaching of and its learning outcomes, with a special emphasis on the French language. We will start by analyzing the situation in our country, Spain, before moving on to tackle, more specifically, the particular case presented by our autonomous Community, Castilla y León. During this process, we will be divulging diverse supporting data and information gleaned from studies conducted in Europe that highlight the predominant foreign languages in the educational area and the degree of attainment of specific objectives.

As professionals in the education of future teachers of French as a foreign language, we have felt the need to conduct this study in which we present a review of the educational offer in foreign languages in Castilla y León and of the conditions in which this develops, focussing on the French language and more concretely on the French bilingual sections, considering their advantages and aims, and the training needs of teachers. From the analysis of the current situation, we will reflect upon the need to try to improve the question raised at the beginning, starting from the idea that a wide offer of foreign languages is not only feasible, but indispensable, and that the incorporation of two foreign languages in the school curriculum should be mandatory, not optional.
\end{abstract}

Key words: education, foreign languages, French, bilingual sections, formation, teachers, learning outcomes, educational offer.

\title{
Introducción: Panorama general de las lenguas extranjeras
}

Nadie cuestiona hoy en día la necesidad de saber comunicarse en una lengua extranjera. Ya sea por motivos profesionales, laborales, culturales o de ocio, la demanda social para que se favorezca la adquisición de esta competencia desde la escolaridad más temprana va en aumento. Además, existen otras motivaciones puramente educativas. Los aparentes beneficios cognitivos que se derivan del aprendizaje de lenguas extranjeras, y su contribución a la adquisición de los objetivos educativos generales, han impulsado que no sólo se haya adelantado la edad de aprendizaje, sino que además se reivindique la inclusión de una segunda lengua extranjera durante la etapa de escolaridad obligatoria. En paralelo, los programas de enseñanza bilingüe también han conseguido el apoyo de las autoridades educativas, extendiéndose, en nuestro país, a todas las Comunidades Autónomas. Si bien queda por saber si siempre se ha hecho en las condiciones idóneas, cuestión que pretendemos desvelar a través de este trabajo en el que nos planteamos una revisión de la oferta educativa en lenguas extranjeras en Castilla y León, centrando nuestra atención en la lengua francesa y más concretamente en las secciones bilingües de francés, así como de las condiciones en que ésta se desarrolla.

El aprendizaje de las lenguas extranjeras es un tema que ha suscitado grandes debates desde hace tiempo. Pero hemos comenzado un nuevo siglo y, si bien la metodología ha evolucionado adaptándose a las necesidades y los contextos educativos, el problema en cuanto a resultados de aprendizaje parece persistir, a tenor de los estudios que se han ido publicando, alguno de ellos muy reciente.

Partimos del hecho de que el multilingüismo es algo común a la sociedad. Por una parte se da un multilingüismo endógeno, propio de cada país, donde conviven diferentes lenguas minoritarias junto a la lengua llamada "nacional"; y por otro no podemos obviar el multilingüismo exógeno que afecta al conjunto de países, propio de 
una creciente movilidad económica y profesional. De modo que, hoy en día, sólo una minoría de países de la UE es monolingüe y España no está entre ellos.

En los últimos 50 años, el Consejo de Europa, desde su Departamento de Política Lingüística, ha propiciado una política activa de promoción de la diversidad lingüística y del aprendizaje de lenguas en el ámbito de la educación, siendo su contribución más relevante el establecimiento del Marco común europeo de referencia para las lenguas: aprendizaje, enseñanza, evaluación (2001), así como su herramienta complementaria: el Portfolio europeo de las lenguas. Las acciones ulteriores que ha venido llevando a cabo la Comisión Europea para favorecer el aprendizaje de lenguas se han centrado fundamentalmente en:

- El Programa de Aprendizaje Permanente (PAP), dentro del cual el objetivo de intercambio, cooperación y movilidad entre los sistemas de educación y formación de los países europeos se ve apoyado por diferentes acciones orientadas al aprendizaje de lenguas.

- Dos grupos de trabajo que desarrollan o han desarrollado su actividad bajo el Método Abierto de Coordinación: Grupo Temático de Aprendizaje Temprano de lenguas extranjeras (que finalizó su mandato en marzo de 2011); Grupo temático sobre idiomas y empleo (con mandato hasta diciembre de 2012).

- El Estudio Europeo de Competencia Lingüística (EECL), pensado para ofrecer datos comparables sobre la enseñanza y el aprendizaje de idiomas en la UE que permitan revisar los planteamientos pedagógicos y metodológicos al respecto, así como facilitar la toma de decisiones en materia de política educativa (sus resultados se han hecho públicos en junio de 2012).

Podemos afirmar que desde hace varias décadas la mayoría de los europeos tienen garantizado el aprendizaje de al menos una lengua extranjera durante la etapa de escolaridad obligatoria. $Y$ a partir del Consejo Europeo celebrado en Barcelona en $2002^{1}$ se recomienda incluso la ampliación a dos lenguas extranjeras; recomendación que se reitera en las Conclusiones del Consejo de Educación de 12 de mayo de 2009 sobre un marco estratégico para la cooperación europea en el ámbito de la educación y la formación (ET 2020) ${ }^{2}$, invitando a la Comisión a que, a finales de 2012, presente al Consejo una propuesta de posible punto de referencia en este ámbito, basada en los trabajos en curso llevados a cabo por los lingüistas que participan en el Programa de Aprendizaje Permanente.

Por su parte, España tiene ya una tradición en el aprendizaje temprano de lenguas desde los niveles preescolares, aunque con un claro dominio del inglés (95\%). Además, el Plan de Acción 2010-11 incorporó como objetivo específico el plurilingüismo o impulso al aprendizaje de idiomas, para lo cual se diseñó el Programa integral de Aprendizaje de Lenguas Extranjeras (2010-2020), acordado con las Administraciones educativas y dirigido tanto a los estudiantes de todos los niveles, como a los profesores, a los centros educativos y a las familias.

Siguiendo esta misma línea, podemos señalar que en todas las Comunidades Autónomas se ha desarrollado la implantación de la enseñanza bilingüe en la modalidad AICLE (Aprendizaje Integrado de Contenidos y Lengua), un enfoque que trata de potenciar simultáneamente el conocimiento específico y lingüístico, 
contribuyendo así al desarrollo de una competencia comunicativa significativa que optimiza y facilita los aprendizajes posteriores.

No obstante, el Informe español 2010-2011 sobre Objetivos Educativos Europeos y Españoles Estrategia Educación y Formación 2020 reconoce abiertamente que uno de los grandes obstáculos a la movilidad de la población española continúa siendo el escaso conocimiento de lenguas extranjeras. De hecho, según datos de Eurostat publicados en 2010, España es el cuarto país de la UE en el que menos adultos hablan idiomas, superada únicamente por Rumanía y Hungría con un $75 \%$ cada una y Portugal (51\%). Casi la mitad de los adultos españoles $(46,6 \%)$ reconoce no hablar ningún idioma extranjero, mientras que la media de la Unión Europea se sitúa en el $38 \%$. Según estas mismas fuentes, Luxemburgo es el único país de la UE donde el $100 \%$ de sus estudiantes aprenden 2 o más lenguas extranjeras en los niveles ISCED 2 y $3^{3}$ (un $83,6 \%$ lo hace incluso en el nivel ISCED 1 ), datos que contrastan enormemente con el $5,9 \%$ (nivel ISCED 1), o el 39,4\% y 23,4\% (ISCED 2 y 3 respectivamente) en España ${ }^{4}$. Podemos observar que, aunque la recomendación del estudio de al menos dos lenguas extranjeras se va implantando tímidamente en la secundaria, nuestro país se sitúa aún, en el entorno europeo, en uno de los últimos lugares en cuanto a dominio de lenguas extranjeras se refiere por parte de nuestros estudiantes.

En un intento de solucionar el problema, el Ministerio de Educación ha elaborado el ya citado Programa Integral de Aprendizaje de Lenguas Extranjeras, cuyo objetivo es mejorar la competencia lingüística en todas las edades y los niveles educativos. Lo que, sin embargo, no se ha traducido en una política educativa que desarrolle y potencie realmente el aprendizaje de varias lenguas extranjeras, pues, si bien existe la posibilidad de cursar una segunda lengua extranjera (LE2) en el período de la enseñanza secundaria, ésta tiene un carácter optativo y no goza de un apoyo institucional explicito, de modo que sólo el inglés constituye una apuesta clara (ofrecido como LE1). También es importante reseñar que la oferta de esta segunda lengua se limita a la lengua francesa, quedando excluidas otras, como es el caso del portugués, que por su proximidad geográfica a Castilla y León y por sus relaciones comerciales e institucionales podría constituir una oferta interesante.

Vemos entonces que el prototipo creciente de sujeto es aquel que se maneja en una segunda lengua, o al menos se declara conocedor de ella, aunque sea a un nivel básico (Snow, 1999), sin embargo, las políticas educativas no consiguen aún impulsar de un modo efectivo el plurilingüismo.

\section{La situación del francés en el panorama general de las lenguas extranjeras}

Abordaremos este punto a partir de los resultados que arrojan los análisis realizados a nivel europeo. Desde el análisis de la situación actual, llevaremos a cabo una reflexión para intentar mejorar la cuestión planteada, partiendo de la idea de que una oferta amplia de lenguas extranjeras no sólo es factible, sino imprescindible, y que la implantación de dos lenguas extranjeras en el curriculum escolar debería tener carácter obligatorio y no optativo. 
Los datos obtenidos del estudio Eurostat corroboran la primacía del inglés sobre el resto de las lenguas extranjeras elegidas por los ciudadanos de estos estados, siendo obligatorio en buen número de países donde lo cursan casi el $100 \%$ de los alumnos en la educación primaria (figura n.1).

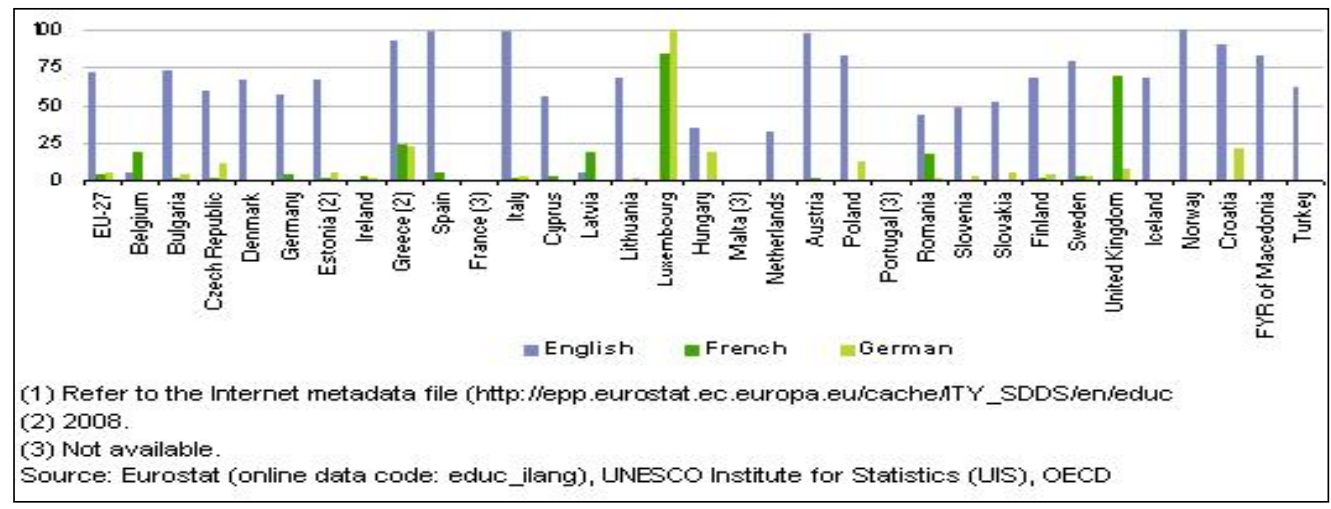

Figura n.1

En cuanto a la educación secundaria, ese mismo estudio (Tabla n.1) muestra que, en 2009 , un $94,6 \%$ de los estudiantes de toda la UE en el nivel ISCED 3 estaban estudiando inglés como lengua extranjera, en comparación con aproximadamente una cuarta parte que estudiaba alemán $(26,5 \%)$ o francés $(25,7 \%)$.

\begin{tabular}{|c|c|c|c|c|c|c|c|c|}
\hline & \multirow{2}{*}{\multicolumn{2}{|c|}{$\begin{array}{l}\text { Proportion of } \\
\text { students learning } 2 \\
\text { or more languages } \\
\text { at ISCED level } 3 \mathrm{GEII}\end{array}$}} & \multicolumn{6}{|c|}{ Upper secondary education (ISCED 3) } \\
\hline & & & \multicolumn{2}{|c|}{$\begin{array}{c}\text { Pupils learning } \\
\text { English in general } \\
\text { programmes }\end{array}$} & \multicolumn{2}{|c|}{$\begin{array}{c}\text { Pupils learning } \\
\text { French in general } \\
\text { programmes } \\
\end{array}$} & \multicolumn{2}{|c|}{$\begin{array}{l}\text { Pupils learning } \\
\text { German in general } \\
\text { programmes }\end{array}$} \\
\hline & 2005 & 2009 & 2005 & 2009 & 2005 & 2009 & 2005 & 2009 \\
\hline EU-27 & & 64.7 & 79.6 & 94.6 & 27.7 & 25.7 & 25.4 & 26.5 \\
\hline Belgium & 88.7 & 89.3 & 94.4 & 95.0 & 47.8 & 49.3 & 28.4 & 29.3 \\
\hline Bulgaria & 76.9 & 74.5 & 83.1 & 87.5 & 15.4 & 14.5 & 40.3 & 35.9 \\
\hline Czech Republic & 100.0 & 100.0 & 98.1 & 100.0 & 22.4 & 24.8 & 72.2 & 60.6 \\
\hline Denmark & 67.3 & 60.5 & 82.6 & 91.6 & 16.8 & 10.7 & 49.7 & 35.1 \\
\hline Germany & & & 93.8 & 91.8 & 30.0 & 28.1 & - & - \\
\hline Estonia (2) & 80.9 & 97.7 & 92.6 & 96.2 & 6.1 & 6.9 & 44.1 & 39.2 \\
\hline Ireland & 8.9 & 7.8 & - & - & 61.7 & 58.2 & 19.1 & 16.4 \\
\hline Greece (2) & 6.7 & 7.9 & 94.5 & 95.0 & 8.6 & 8.2 & 2.4 & 3.3 \\
\hline Spain & 28.1 & 27.7 & 95.3 & 94.4 & 28.0 & 27.0 & 1.3 & 1.1 \\
\hline France & 89.6 & 90.6 & & 99.5 & - & - & $:$ & 21.3 \\
\hline Italy & 208 & 25.8 & 85.1 & 96.5 & 18.1 & 20.1 & 6.5 & 6.9 \\
\hline Cyprus & & 84.4 & 89.1 & 91.4 & 34.5 & 40.6 & 3.4 & 2.2 \\
\hline Latvia & 74.1 & 773 & 93.7 & 97.2 & 3.6 & 39 & 38.8 & 28.8 \\
\hline Lithuania & 55.0 & 47.6 & 80.2 & 90.8 & 5.9 & 3.8 & 28.4 & 18.8 \\
\hline Luxembourg & 1000 & 100.0 & 96.7 & 97.7 & 96.7 & 100.0 & 96.7 & 100.0 \\
\hline Hungary & & 44.9 & 73.0 & 79.4 & 6.0 & 6.6 & 51.4 & 48.7 \\
\hline Malta & 14.0 & & 65.6 & & 6.6 & & 1.7 & \\
\hline Iletherlands & 100.0 & 77.8 & 100.0 & 100.0 & 69.5 & 64.2 & 86.2 & 69.8 \\
\hline Austria & 76.2 & 68.2 & 96.9 & 98.5 & 54.1 & 42.5 & - & - \\
\hline Poland & & 73.6 & 96.3 & 92.2 & 12.1 & 87 & 72.5 & 54.4 \\
\hline Portugal & 17.1 & & 49.9 & & 19.1 & & 2.5 & \\
\hline Romania & 91.8 & 96.4 & 94.2 & 98.1 & 84.2 & 85.3 & 11.9 & 12.1 \\
\hline Slovenia & 95.0 & 92.2 & 98.8 & 92.8 & 10.9 & 9.8 & 78.2 & 66.4 \\
\hline Slovakia & 99.3 & 99.8 & 97.3 & 98.3 & 14.4 & 16.4 & 75.2 & 67.9 \\
\hline Finland & 99.7 & 99.7 & 99.7 & 99.7 & 19.3 & 17.9 & 37.9 & 27.8 \\
\hline Sweden & 92.6 & 91.8 & 100.0 & 99.9 & 24.2 & 200 & 34.5 & 27.0 \\
\hline United Kingdom & 6.6 & 7.4 & - & - & 40.0 & 28.9 & 15.2 & 10.7 \\
\hline Iceland & 67.8 & 63.3 & 77.2 & 73.2 & 16.4 & 13.7 & 32.4 & 25.4 \\
\hline llorway & & & & 44.8 & & 12.8 & $:$ & 20.3 \\
\hline Croatia & 90.6 & 90.1 & 98.4 & 99.0 & 3.8 & 3.7 & 66.2 & 62.5 \\
\hline Turkey & $:$ & $:$ & : & 81.8 & $:$ & 1.4 & $:$ & 14.8 \\
\hline \multicolumn{9}{|c|}{$\begin{array}{l}\text { (1) Refer to the Internet metadata file (http:liepp.eurostat.ec.europa.euicachelITY_SDDSienieduc_esms.htm). } \\
\text { (2) } 2008 \text { instead of } 2009 \text {. } \\
\text { Source: Eurostat (online data codes: educ_thfrlan, tps00057, tps00058 and tps00059), Unesco Institute for } \\
\text { Statistics (UIS), OECD }\end{array}$} \\
\hline
\end{tabular}

Tabla n.1 
En lo que se refiere a España, esas estadísticas marcan un 94,4\% de alumnos que estudian inglés en ese mismo nivel, frente a un $27 \%$ que eligen el francés y un $1,1 \%$ el alemán.

Esta situación se confirma en Castilla y León, donde la enseñanza obligatoria del inglés se ha ido generalizando en los primeros cursos de la Educación Primaria e incluso en la Educación Infantil, dejando escaso margen a la elección del francés y una presencia casi insignificante del alemán.

Retrospectivamente, vemos que el contacto de los alumnos con una primera lengua extranjera evoluciona progresivamente hacia edades más tempranas. Hasta 1990 el aprendizaje de la lengua extranjera comenzaba a los 12 años (en principio inglés o francés), pero a partir de esta fecha el aprendizaje del inglés se adelanta a los 8 años y el francés va desapareciendo progresivamente de la enseñanza primaria. En 2006, no sólo el inglés se convierte en materia obligatoria a partir de los 6 años, sino que además se propone su aprendizaje desde los 3 años, en la segunda etapa de Educación Infantil, en la mayoría de la Comunidades Autónomas ${ }^{5}$, quedando el francés relegado a LE2 en el tercer ciclo de la Educación Primaria (a partir de los 10 años), como proyecto piloto, en las Comunidades Autónomas de Aragón, Castilla y León y Extremadura ${ }^{6}$.

Pero es en 2009 cuando esta incorporación como LE2 se hace oficial en Primaria. Si bien también se puede impartir como tal el alemán, portugués o italiano, la realidad es que apenas hay centros que ofrecen alemán (actualmente sólo existen dos centros con sección bilingüe en alemán en nuestra Comunidad, uno en Valladolid y otro en León), y ninguno que imparta la lengua portuguesa e italiana.

En cuanto a la enseñanza secundaria, España se encuentra todavía entre los países donde el aprendizaje de la segunda lengua extranjera se ha convertido en un derecho, pero no en una obligación; es decir, es imperativo ofrecer una segunda lengua extranjera entre las materias de opción obligatoria, lo que no garantiza que todos los alumnos la elijan. Según esto, el currículo de Castilla y León ofrece una única lengua extranjera obligatoria, garantizando el poder cursar una segunda en el módulo de optatividad. Así, en aplicación de la Orden EDU/1046/2007 de 12 de junio de 2007, el francés se incorpora como materia optativa a lo largo de toda la etapa de la Secundaria obligatoria, con una carga horaria de dos horas a la semana en los tres primeros cursos. No obstante, tiene un tratamiento particular, ya que en los dos primeros años esta materia tiene carácter de "optativa de oferta obligada", excepto para los alumnos que necesitan refuerzo en Lengua Española y Matemáticas. Y en 4은 de ESO el francés pasa a ser optativa en todas las opciones curriculares con una dotación horaria de tres horas a la semana, concurriendo en todas ellas con Informática y la optativa específica de cada opción. Como Segunda Lengua extranjera no precisa un número mínimo de alumnos para ser impartida, pero en 3 을 de ESO, en el caso de querer establecer una diferencia de nivel para atender las incorporaciones tardías, es decir aquellos alumnos que no la cursaron en 1으 y 2ํㅡ, el número mínimo de alumnos debe ser superior a 15 y debe de existir profesorado disponible.

Durante los dos años de Bachillerato, la segunda lengua extranjera continua siendo optativa para todas las modalidades del mismo, con una dotación horaria de tres horas a la semana en 10 y de cuatro en 2‥ Mientras que en la Formación 
Profesional el módulo de Lengua extranjera sólo existe en algunos ciclos, como es el caso de las familias profesionales de Administración y de Hostelería y Turismo.

Pero, aunque el aprendizaje temprano de una lengua extranjera como asignatura obligatoria ha evolucionado positivamente de un modo apreciable con respecto a otros países europeos, no podemos decir lo mismo del tiempo dedicado a su enseñanza. Centrándonos en este aspecto, para los alumnos de entre 9 y 11 años (tabla n.2), comparando el porcentaje de carga horaria obligatoria dedicadas a las distintas áreas de conocimiento con las medias de los países de la OCDE y de la UE, vemos que en España éste es ligeramente inferior en Artes, idéntico en Lengua, similar en Ciencias de la Naturaleza, y superior en el resto de las asignaturas, destacando las horas dedicadas a Lengua extranjera cuyo porcentaje es claramente superior.

\begin{tabular}{|c|c|c|c|c|c|c|c|c|c|}
\hline & Lengua & Matemáticas & CCNN & CCSS & $\begin{array}{c}\text { Lengua } \\
\text { extranjera }\end{array}$ & Artes & $\begin{array}{c}\text { E. } \\
\text { Física }\end{array}$ & $\begin{array}{c}\text { Horario } \\
\text { flexible }\end{array}$ & Total \\
\hline ES & 23 & 17 & 9 & 9 & $\mathbf{1 3}$ & 10 & 10 & 8 & 100 \\
\hline OCDE & 23 & 16 & 9 & 8 & 9 & 11 & 9 & 4 & 100 \\
\hline UE-21 & 23 & 16 & 8 & 8 & 9 & 12 & 9 & 4 & 100 \\
\hline
\end{tabular}

Tabla n.2. Porcentaje de horas lectivas por área para alumnos de 9 a 11 años (2009).

El horario escolar mínimo obligatorio que establecía el R.D. 1513/2006 (LOGSE) para la lengua extranjera era de 340 horas anuales. Mientras que la LOE lo incrementa en 45 horas, es decir, un total de 385 horas anuales. Sin embargo, Castilla y León aplica la flexibilidad que le permite la ley, según la cual los centros pueden adecuar sus horarios en función de sus características particulares. Así, en la práctica, las horas dedicadas a la enseñanza de la Lengua extranjera en Educación Primaria eran 420 con la LOGSE y 560 con la LOE.

En cuanto a los alumnos de entre 12 y 14 años (tabla n.3), el porcentaje horario es también similar al de la OCDE y de la UE, siendo igual en Matemáticas, superior en Lengua y en Artes, y algo inferior en el resto de las áreas, pero muy inferior sobre todo en Lengua extranjera.

\begin{tabular}{|c|c|c|c|c|c|c|c|c|c|}
\hline & Lengua & Matemáticas & CCNN & CCSS & $\begin{array}{c}\text { Lengua } \\
\text { extranjera }\end{array}$ & Artes & $\begin{array}{c}\text { E. } \\
\text { Física }\end{array}$ & $\begin{array}{c}\text { Horario } \\
\text { flexible }\end{array}$ & Total \\
\hline ES & 17 & 13 & 11 & 10 & $\mathbf{1 0}$ & 10 & 7 & 5 & 100 \\
\hline OCDE & 16 & 13 & 12 & 12 & 13 & 8 & 8 & 6 & 100 \\
\hline UE-21 & 16 & 13 & 12 & 12 & 13 & 8 & 8 & 5 & 100 \\
\hline
\end{tabular}

Tabla n.3. Porcentaje del número de horas lectivas por área para alumnos de 12 a 14 años (2009).

Según esto, en Secundaria el número de horas dedicadas a la enseñanza de idiomas es un $3 \%$ inferior al que se dedica en Primaria, factor que nos diferencia de la mayoría de los países de la OCDE y de la UE. Como rasgo general podemos afirmar que la política de estos países es ir dedicando mayor tiempo al aprendizaje de las lenguas extranjeras a medida que se avanza en el nivel de los estudios, de modo que en la enseñanza Secundaria superior el tiempo consagrado a las lenguas extranjeras es equivalente al de otras materias consideradas principales, como la Lengua materna 0 las Matemáticas, o incluso está por encima de él. Sin embargo, en nuestro país no se 
produce esta progresión y, además, por lo que se refiere a LE2, en secundaria se convierte en una materia optativa que, bajo el criterio prioritario de adquirir las destrezas básicas en Lengua castellana y literatura y/o Matemáticas, puede incluso, en la práctica, ver imposibilitada su elección en primero y segundo de ESO.

Por lo que se refiere al Bachillerato, aunque el informe de la OCDE no establece esta comparativa, el currículo de Castilla y León reserva tan sólo un $10 \%$ de las horas lectivas a la Primera Lengua extranjera, la única que es obligatoria.

Y ya en el ámbito de la enseñanza universitaria, en 2001, en la "Resolución sobre las Universidades y la enseñanza superior en el Espacio Europeo del conocimiento", el Parlamento Europeo solicitó a los Estados miembros y a las Universidades el reconocimiento de la importancia del aprendizaje de lenguas extranjeras a este nivel educativo, incluyéndolas también en los planes de estudios diferentes a las humanidades o estudios lingüísticos resultantes del proceso de Bolonia, lo que facilitaría la movilidad y la integración europea de los estudiantes. Esta recomendación quedó recogida en las primeras directrices generales de planes de estudio y hay que decir que, aunque por lo general se ha tratado de dar respuesta a esta demanda, los compromisos no siempre se han respetado y, en los casos en que ha sido atendida, la lengua ofrecida mayoritariamente vuelve a ser el inglés.

Vemos por lo tanto que la preponderancia del inglés se extiende desde la educación infantil hasta la universitaria, dejando en todos los niveles al francés en segundo lugar, pero con una presencia muy moderada.

\section{La enseñanza bilingüe en francés en Castilla y León}

Junto al adelanto antes citado en cuanto a la edad de iniciación en el aprendizaje de lenguas extranjeras, también hemos de anotar que se da un gran avance en la implantación de los programas de bilingüismo en la modalidad AICLE, abiertos a todos los alumnos, es decir, sin criterios de admisión para seleccionar a los participantes. En Castilla y León los programas bilingües en francés datan del curso 2001-2002 ${ }^{7}$. Fruto de un acuerdo bilateral firmado entre España y Francia en $1969^{8}$, se pusieron entonces en marcha, de forma experimental, las primeras 10 secciones bilingües que hoy conocemos como "Proyecto de clases bilingües Español-Francés». En estas secciones, la lengua de enseñanza es principalmente el español, impartiéndose en francés algún área o materia distinta a la lengua extranjera. Los objetivos de aprendizaje, los programas y los contenidos son los oficiales en España, aunque se produce un incremento de dos períodos lectivos semanales asignados a la lengua francesa, cuyo currículo deberá incorporar el conocimiento de Francia y su cultura. A todos los alumnos se les garantiza el continuar con el estudio de la lengua inglesa como segunda lengua extranjera.

En 2005, este proyecto se prolonga y materializa en un acuerdo marco ${ }^{9}$ que pretende reconocer y regularizar las acciones ya existentes con anterioridad al establecimiento de nuevas disposiciones por parte de la Comunidad Autónoma. Así, en el curso académico 2006-2007, el "Proyecto de clases bilingües Español-Francés» se transforma en "Secciones bilingües Español-Francés», ajustándose al marco 
normativo ${ }^{10}$ fijado para los establecimientos públicos de castilla y León, con el fin de garantizar la calidad de este tipo de enseñanza. Este marco establece las condiciones en cuanto a la estructura y a la organización de las secciones así como los documentos que deben de acompañar su petición de aprobación; y en una segunda orden reguladora $^{11}$ se autoriza la creación de programas bilingües en varios idiomas en el mismo establecimiento. La Segunda Lengua extranjera puede ser el inglés, el francés, el alemán, el italiano o el portugués.

Por lo que se refiere al francés, nuestra Comunidad, a través de la Consejería de Educación, ha suscrito recientemente un acuerdo con la Embajada de Francia en España $^{12}$ cuyo objetivo es impulsar el bilingüismo en francés. En dicho acuerdo se contempla la formación específica para los docentes, intercambios de alumnos y profesores, programas de movilidad estudiantil y hermanamientos entre centros de ambos países; en definitiva, un conjunto de acciones que tenderán a favorecer la convivencia de las lenguas y culturas española y francesa.

Ya en el curso 2010-2011 se pusieron en marcha los programas Erasmus de movilidad de estudiantes para realizar prácticas en empresas 'EURO-FP-CYL' y el intercambio escolar con la región de Poitou-Charentes, pues es un hecho demostrado el interés que el español despierta en los alumnos franceses en esta región. Esta movilidad Erasmus en FP se verá favorecida sensiblemente por el apoyo que desde los programas bilingües se dará a los alumnos que la soliciten, facilitando su acceso a los cursos de Lengua francesa ofrecidos por las universidades públicas de la Comunidad y fomentando el acercamiento a las empresas españolas que tengan sedes o subsedes en Francia o aquéllas francesas que tienen especial presencia en nuestra Comunidad (Michelin y Renault entre otras).

El acuerdo anteriormente citado recoge el compromiso de potenciar la oferta cualificada de centros escolares que ofrecen enseñanza bilingüe en lengua francesa, así como la del francés como Segunda Lengua extranjera en el tercer ciclo de Educación Primaria, en Secundaria y en Bachillerato. Al mismo tiempo, se pretende estimular al alumnado a obtener certificados de nivel de dicho idioma (Delf).

Con la incorporación en el curso actual de una sección bilingüe en el Instituto de Enseñanza Secundaria Parquesol de Valladolid son ya 19 los centros de Castilla y León que cuentan con sección bilingüe en francés: 10 en colegios de Educación Infantil y Primaria (con inicio todos ellos en el curso 2007/2008) y 9 en la etapa de Educación Secundaria Obligatoria (destacando las provincias de Valladolid con 3, donde la más antigua data del curso 2006-2007, y Segovia con 2$)^{13}$. Como dato positivo hemos de comentar la existencia de dos CEIP donde se ofrece un programa bilingüe en alemán: uno en la provincia de León, desde el curso 2009-2010 y otro en la provincia de Valladolid, desde el curso 2010-2011. El dato negativo, sin embargo, lo constituye la provincia de Palencia, donde no existe más bilingüismo que el ofrecido en lengua inglesa. Por otro lado, las provincias de Soria y Zamora no ofrecen, al menos hasta la fecha, la posibilidad de continuidad de los programas bilingües en Secundaria, lo cual supone, a nuestro entender, un freno a su crecimiento.

La elección de las disciplinas enseñadas total o parcialmente en francés está abierta, pues cualquier materia puede entrar a formar parte del currículo integrado -la música, la historia, la biología, etc. -siempre que existan profesores competentes 
desde el punto de vista lingüístico para garantizar su enseñanza. Ciertas materias musicales, artísticas o deportivas entre otras se prestan mucho a este tipo de aprendizajes.

Otro aspecto positivo lo presenta el hecho de que Castilla y León es una de las primeras Comunidades españolas donde se ha regulado la sección binacional francés/español "Bachibac", conducente a una doble certificación de fin de estudios secundarios. Son cuatro los institutos donde se ha autorizado el currículo mixto vinculado a esta doble titulación que permitirá la integración de nuestros alumnos en el sistema de enseñanza superior del país implicado: el IES Eras de Renueva de León, el IES La Albuera de Segovia, el IES Condesa Eylo y el IES Leopoldo Cano, estos dos últimos de Valladolid.

\section{Objetivos}

Los currículos oficiales de lenguas extranjeras tanto en Primaria como en Secundaria, establecen explícitamente, en lo que respecta a los objetivos, una equiparación de todas las destrezas comunicativas, es decir, de las cuatro competencias principales. Por lo tanto, el objetivo principal es el desarrollo de la capacidad para comunicarse en la lengua enseñada en situaciones cotidianas.

Por lo que respecta al objetivo europeo de alcanzar un plurilingüismo sigue siendo algo deseable, aunque no se encuentra recogido como tal en los programas oficiales, salvo en aquellas regiones que parten de un bilingüismo propio. Sin embargo, teniendo en cuenta la inclusión en el currículo de una segunda extranjera, en la mayoría de los casos el francés, si se lleva a cabo con rigor, este hecho posibilita el desarrollo de la competencia plurilingüe y pluricultural incluso en regiones monolingües, como es el caso de Castilla y León.

En cuanto a la enseñanza bilingüe, hoy en día, parece claro que el objetivo comúnmente aceptado es de orden lingüístico y cultural: la enseñanza bilingüe Español-Francés debe procurar un buen manejo de ambas lenguas y un conocimiento y mejor percepción de las culturas que representan, favoreciendo en los alumnos las competencias orales y escritas en dichas lenguas con el objetivo de hacer de ellos mejores ciudadanos europeos.

Evidentemente, el aprendizaje de una lengua es siempre más efectivo cuando contribuye a enriquecer otras adquisiciones, sin embargo, esto no significa que la llamada enseñanza bilingüe pretenda formar alumnos bilingües en el sentido literal del término, sino que más bien persigue optimizar las habilidades cognitivas de los alumnos. Hagège (1996), claro defensor del plurilingüismo, afirma que el bilingüismo precoz favorece el aprendizaje de otras lenguas, opinión que también es compartida por Jasone Cenoz y Durk Gorter, catedráticos de la Universidad del País Vasco que investigan el valor añadido del multilingüismo y la adquisición de segundas o terceras lenguas. Además, tanto desde la sociolingüística como de la psicolingüística, investigadores como Bain (1974), Cummins (1976), Tunmer y Myhill (1984), Deshays (1990), Duverger (1995) Siguan (1996), De Houwer (1999) o Baker (2001) resaltan los beneficios que el bilingüismo ofrece a nivel sociopolítico y cognitivo y opinan que las 
personas bilingües tienen más desarrollada la conciencia metalingüística, además de poseer una flexibilidad cognitiva superior a la de las monolingües e incluso mayor creatividad. Esta flexibilidad comunicativa puede favorecer y potenciar el plurilingüismo en los individuos, un plurilingüismo que es cada vez más común en Europa.

Pero las modalidades de aprendizaje de una sección bilingüe también dan respuesta a los objetivos pedagógicos y didácticos específicos. Incluso si los objetivos de las disciplinas no lingüísticas que se imparten en francés coinciden con los programas nacionales, estos conocimientos no se presentan sólo en los términos de estos programas, sino que frecuentemente están interrelacionados con los de otras disciplinas.

Además, no cabe duda alguna sobre la influencia que estos programas ejercen en los intercambios internacionales, tanto por lo que se refiere a los alumnos como a los profesores o los responsables educativos, y su capacidad para implementar proyectos conjuntos o promover y desarrollar el trabajo en equipo, a modo de las "tríadas reflexivas" propuestas por Canabal García, C y García Campos, M.D. (2012), que propiciarán el intercambio de métodos, experiencias y materiales didácticos. Igualmente, estos programas innovadores pretenden fomentar la obtención de certificados de estudios de los dos sistemas educativos (español y francés).

Y por último, las secciones bilingües tienen que lograr cumplir las metas educativas y profesionales que la sociedad les demanda, contribuyendo a la formación de ciudadanos capaces de participar en una sociedad europea cada vez más multicultural y multilingüística, y así poder comprender mejor la realidad que les rodea $y$, sobre todo, desarrollar actitudes de tolerancia y respeto hacia la diferencia cultural y étnica.

\section{Ventajas}

En nuestra opinión, a la vista de estos planteamientos, de este enfoque educativo podemos inferir una serie de ventajas entre las que destacamos las siguientes:

- Los estudiantes adquieren un mejor nivel lingüístico en la lengua extranjera a la vez que obtienen beneficios en otras materias.

- Disfrutan de una nueva perspectiva que pone a su alcance una visión plural de la sociedad que les acerca al ciudadano europeo del siglo XXI, al desenvolverse en un contexto que integra múltiples culturas y lenguas. Este nuevo sistema, si se lleva a cabo en condiciones apropiadas, presenta ventajas que en el momento actual nadie discute, ya que favorece el desarrollo de diversas capacidades integrando al mismo tiempo los valores de respeto y de tolerancia, muy necesarios en una sociedad cada día más abierta a nuevas realidades culturales.

- Estos alumnos tendrán más facilidad para integrarse en una realidad cada vez más internacionalizada con la que conviven de modo natural a lo largo de sus etapas formativas y profesionales gracias a los intercambios escolares y a los periodos prácticos realizados en el extranjero. 
No es exagerado decir que la generalización del aprendizaje de idiomas es fruto del amplio desarrollo y de la proliferación de las secciones bilingües en la escuela primaria. En los últimos años el aprendizaje de una lengua extranjera se ha erigido en algo absolutamente esencial para que los estudiantes dispongan de la posibilidad de emplear sus habilidades lingüísticas a la hora de acceder a nuevos conocimientos. Pero sólo quienes contemplan la posibilidad de hacerse especialistas en el campo del lenguaje -como profesor investigador, traductor, intérprete- están altamente motivados para continuar el aprendizaje de la lengua extranjera en la enseñanza secundaria. Para el resto de los alumnos la motivación inicial decae y sería necesario generar un objetivo funcional de carácter totalmente inmediato (FOS) para impedir la regresión o fosilización de las adquisiciones realizadas en lengua extranjera e incentivar la continuidad de su estudio.

\section{Características y modelo pedagógico}

En nuestra Comunidad la enseñanza bilingüe se basa en los parámetros que define el modelo de inmersión lingüística de Snow (2001), su objetivo es el de potenciar el contacto con una segunda lengua extranjera amoldándose a la realidad monolingüe de nuestra realidad escolar. Generalmente es en los centros de enseñanza primaria y secundaria donde se imparten estos estudios, que consisten en elegir un número concreto de materias no lingüísticas del currículo -máximo tres- que son enseñadas de forma coordinada con el profesor de la materia lingüística.

Las secciones bilingües españolas son semejantes a las existentes en Francia en el marco del Programme de Sections Internationales para el español. Disfrutan del apoyo que ofrece el Servicio Cultural de la Embajada de Francia, que brinda su colaboración tanto a través de cursos de formación como con la aportación de material especializado. Este modelo de bilingüismo en francés pretende ser diferente al asociado a otras lenguas, y está inspirado en la situación del bilingüismo en Francia, donde existe un gran interés por consolidar los intercambios entre estudiantes, profesores, materiales y experiencias.

La característica de estos programas es que funcionan tanto en centros públicos como concertados y formarán parte de la oferta educativa del centro, debiendo iniciarse en el primer curso de la etapa educativa correspondiente, aunque rara vez comienzan antes de la Primaria. Es un proceso voluntario por parte del centro, que elabora el proyecto de enseñanza bilingüe garantizando la participación de los profesores y una continuidad mínima. Por lo general, se trata de secciones bilingües que a veces coexisten con otras que no lo son.

Podrán impartirse, como vimos anteriormente, en el idioma específico de la sección bilingüe contenidos correspondientes a un mínimo de dos disciplinas no lingüísticas y un máximo de tres. Pero el total de las horas impartidas en el idioma específico no podrá suponer más de un $50 \%$ del horario total de los alumnos. Con el fin de incrementar las horas destinadas a la enseñanza del idioma específico de la sección, los centros podrán modificar su horario semanal, ampliándolo hasta las 27 horas en el caso de centros que impartan Educación Primaria, o hasta las 32, en el caso de centros que impartan Educación Secundaria Obligatoria. 
La LOE, en su Art. 105, descarga en las Administraciones educativas la adopción de medidas para el "reconocimiento del trabajo de los profesores que impartan clases de su materia en una lengua extranjera en los centros bilingües". Por su parte, la Junta de Castilla y León, con el fin de dotar a los profesores de herramientas, recursos y medidas eficientes para su actualización pedagógica y profesional en relación con la competencia lingüística en lengua francesa, se ha comprometido a potenciar el Programa de Colaboradores Extranjeros de Lengua Francesa vinculados a los centros bilingües (futuros maestros en prácticas, maestros extranjeros colaboradores 0 alumnado universitario participante en programas de movilidad internacional) y a desarrollar actuaciones de formación específica para el profesorado participante en centros bilingües de Educación Primaria, Secundaria y Formación Profesional. Se favorecerá además el trabajo en común entre centros educativos franceses y castellano-leoneses para difundir e intercambiar materiales de aprendizaje específicos y ejemplos de buenas prácticas.

El principal personal de apoyo lo constituyen los auxiliares de conversación. Durante un tiempo se contó además con la colaboración de estudiantes extranjeros que participaban en programas de movilidad, nombrándoles Colaboradores Internacionales de Lenguas Extranjeras, con una dedicación de diez horas mensuales de contacto directo con los alumnos y profesores de la sección bilingüe con la que estén vinculados a cambio de una beca mensual. El objetivo de este programa era doble: por una parte se pretendía que los alumnos extranjeros se beneficiaran educativa, lingüística y culturalmente de la experiencia del aprendizaje en otros países europeos $y$, por otro lado, se buscaba que los alumnos de los programas bilingües en nuestra región dispusieran de otro medio más para mejorar su capacidad de comprensión y expresión en lengua extranjera. Pero, en el curso 2011-2012 este plan de colaboradores extranjeros no se ha hecho efectivo en algunas provincias.

\section{Competencias exigidas al profesorado de DNL}

Hasta el momento actual, el profesorado de Primaria que impartía una DNL en una sección bilingüe debía acreditar un nivel B1 del MCERL en la lengua extranjera correspondiente. Pero, como consecuencia de la aplicación del R.D. 1549/2011 de 4 de noviembre, BOE de 9 de noviembre de 2011, para impartir en una lengua extranjera las enseñanzas de Educación Infantil, o materias de un área distinta a la de dicha lengua en Educación Primaria, en centros cuyos proyectos educativos comporten un régimen de enseñanza plurilingüe, a partir del curso académico 2013/2014 será necesario acreditar, al menos, competencias de un nivel B2 del MCERL en la lengua extranjera correspondiente, en nuestro caso el francés.

En cuanto a los profesores de DNL que imparten enseñanza AICLE en francés en Secundaria, lo primero a tener en cuenta es que el primer ciclo puede estar impartido también por maestros. Además de los condicionantes propios para la docencia de la disciplina en sí, los requisitos exigidos en nuestra comunidad a estos docentes son los que a continuación pasamos a citar:

- Licenciado en Filología Francesa o en Traducción e Interpretación Lingüística en Francés. 
- Certificado de Aptitud (cinco cursos completos) o Certificado del Nivel Avanzado en Lengua Francesa, expedido por la Escuela Oficial de Idiomas.

- Diplôme Approfondi de Langue Française (D.A.L.F.)

En cualquier caso, al docente se le ofrece la posibilidad de acogerse a la autoevaluación de sus competencias conforme al MERC, en cuyo caso se le podrá someter a una prueba de comprobación evaluada por profesores de la EOI.

La Consejería de Educación de la Junta de Castilla y León lanza una convocatoria anual de acreditación lingüística para los profesores que deseen impartir DNL en lengua extranjera. Precisamente uno de los obstáculos para el desarrollo de la enseñanza AICLE en francés es la carencia de profesores cualificados, dado el escaso número de estudiantes que se forman en esta lengua en los niveles superiores.

\section{Nuevos retos para la formación de los docentes}

El evidente interés que manifiestan las instituciones, tanto locales como nacionales o europeas, por la puesta en marcha de las secciones bilingües -llamadas también secciones europeas, secciones internacionales, con metodología CLIL, EMILE o AICLE-, nos lleva a cuestionarnos si la actual formación del profesorado cubre los requisitos de este tipo de enseñanza, pues nos encontramos, sin duda, frente a un nuevo perfil profesional cuya formación no debe ser infravalorada, ya que es elemento clave de la enseñanza bilingüe. Para afrontar este análisis, debemos empezar por definir unos principios básicos en los que apoyar nuestros planteamientos y que se convertirán en el eje vertebrador del plan de formación.

Partiremos, en primer lugar, del concepto de la enseñanza bilingüe o plurilingüe que plantea Duverger (2005) y que entiende como una situación que no es nunca la suma de dos o más enseñanzas monolingües, pues no se trata de remplazar un sistema monolingüe por otro, sino que una enseñanza bilingüe o plurilingüe se define por el hecho fundamental de utilizar dos o más lenguas para la explicación de los posibles conceptos en las materias implicadas. Este hecho genera la necesidad de aplicar nuevas prácticas pedagógicas que deben ser definidas, exploradas y trabajadas tanto en la formación inicial del profesor como en la formación continua.

En segundo lugar, pero no menos importante, no hay que olvidar que el objetivo final de este tipo de enseñanza es una mejora tanto en las competencias lingüísticas como en la elaboración de los conceptos disciplinares de las materias implicadas. Hay que recordar que en las secciones bilingües se cursan dos o tres materias en la lengua extranjera correspondiente, en nuestro caso el francés, lo cual implica que los contenidos han de ser transmitidos y trabajados teniendo en cuenta el nivel de lengua extranjera de los alumnos. La consideración de este objetivo nos lleva a establecer las pautas que hemos de seguir para nuestro plan de formación del profesorado, que analizaremos teniendo en cuenta la formación del mismo.

En cuanto a los docentes que imparten francés en los niveles de Infantil y Primaria, habría que hablar de un antes y un después de la reforma de Bolonia. Si hablamos de los profesionales formados en las antiguas Diplomaturas de Maestro (3 
años de formación), en la etapa de Educación Infantil no existían maestros especialistas en lenguas extranjeras, por lo que son los titulados de Educación Infantil los que asumen su enseñanza, apoyados, en la medida de lo posible, por nativos o profesores especialistas. Mientras que en la enseñanza Primaria, hasta el momento actual, son los maestros especialistas en lenguas extranjeras los cualificados para esta tarea. A estos especialistas se les reconocía incluso el derecho a impartir esta asignatura en el primer ciclo de la Secundaria Obligatoria. En estos niveles, los profesores especialistas de lenguas extranjeras pueden además encargarse de la docencia de las asignaturas que forman parte del proyecto bilingüe y que se imparten parcialmente en lengua extranjera. Su formación inicial incluye materias pedagógicas (didáctica y contenidos específicos de la etapa, psicología de la edad escolar, organización escolar, etc.) y materias específicas relacionadas con la enseñanza de las lenguas extranjeras (de contenido lingüístico, literario y didáctico-metodológico), además de períodos de prácticas en centros educativos.

Sin embargo, desde el curso 2009-10, con la implantación de los nuevos programas nacidos de la reforma de Bolonia, se han registrado cambios importantes en la formación inicial del profesorado de todos los niveles, tanto de Infantil como de Primaria o de Secundaria. Todos ellos tendrán que seguir estudios de Grado (Bachillerato +4 años de formación universitaria) y, en el caso de Secundaria, el Máster de carácter profesional en un campo disciplinar específico sigue siendo obligatorio para aquellos que quieran desarrollar la docencia. A los futuros profesores se les aconseja una estancia de un curso académico en el extranjero $y$, aunque no es obligatoria, sí se promueve la movilidad a través de los programas ERASMUS, y también existe la opción de solicitar una estancia anual como Auxiliar de Conversación en un país de habla francesa.

Fruto de esta nueva situación en la formación inicial se pueden detectar cambios significativos que afectan al aprendizaje de la lengua extranjera y que pasamos a exponer a continuación.

La formación específica de los futuros maestros supuestamente "especializados" en la enseñanza de lenguas extranjeras se ha visto reducida a la aportada por la mención correspondiente. Quiere esto decir que, del total de 60 ECTS que corresponden al itinerario especializado, sólo 30 ECTS van destinados a materias de carácter lingüístico, literario, didáctico y metodológico, reservándose los otros 30 ECTS para el prácticum en colegios públicos o concertados y el Trabajo Fin de Grado (TFG).

Si bien los graduados reciben en su etapa de formación una base lingüística y didáctica, es importante reseñar que ésta no tiene como fin preferente el dar respuesta a las necesidades del "nuevo profesional", como lo denomina Duverger, que desempeña su función en las secciones bilingües, ya que no se les prepara para entender que el desarrollo de su tarea docente está estrechamente relacionado con la del profesor cuya disciplina se enseña en lengua extranjera, y que la lengua que enseñan es, además de lengua de comunicación, una lengua escolar que, junto con la materna, permite acceder a conocimientos de otras disciplinas, a construir nuevos conceptos disciplinares.

Los licenciados en Secundaria, por su parte, así como los nuevos graduados de las titulaciones filológicas, poseen una formación inicial más centrada en la adquisición 
de contenidos. Sólo los que se dediquen a la docencia deberán superar un Máster con materias ligadas a las disciplinas pedagógicas y con una estancia práctica en centros de secundaria. Dichas prácticas docentes permiten a los futuros profesores de cualquiera de las etapas educativas obtener una experiencia inicial en un ambiente real de trabajo.

En cuanto a los docentes de las DNL, partimos del hecho de que generalmente han recibido una formación inicial polivalente para primaria y especializada para secundaria, pero carecen también de una formación específica que pueda responder a las nuevas necesidades que plantea la enseñanza bilingüe, pues ésta no constituye objeto específico de estudio en los planes vigentes. En cuanto al nivel lingüístico requerido, cabe recordar que deben acreditar un nivel B2 del MERCL, nivel que no adquieren con las materias de lenguas extranjeras incluidas, en el mejor de los casos, en sus currículos. Hemos de reseñar que actualmente las Universidades de nuestra región están incorporando a sus Másteres programas de especialización en enseñanza bilingüe, si bien, al igual que ocurre en los anteriores niveles con la oferta de lenguas extranjeras, los programas de estos estudios se centran principalmente en la enseñanza bilingüe en inglés.

Es evidente que estamos ante un nuevo reto en la formación inicial de los docentes que no parece haber sido satisfecho con las reformas de planes de estudios recientes y el análisis de las carencias nos ayuda a establecer las pautas que hemos de seguir para nuestro plan de formación del profesorado, que analizaremos teniendo en cuenta sus necesidades formativas. Si lo que pretendemos es el desarrollo de una conciencia plurilingüe y multicultural de los castellano-leoneses, la situación de partida a la que nos enfrentamos, con una ausencia de formación inicial plurilingüe de nuestros titulados universitarios, nos obliga a tomar decisiones respecto al perfil formativo de los colectivos implicados en la docencia de las secciones bilingües de nuestra Comunidad. Actualmente investigadores de diferentes áreas intentan ofrecer soluciones y encontrar respuestas y parece que la modalidad de la formación a lo largo de la vida es la que puede brindar soluciones más duraderas y eficaces. A este respecto, cabe preguntarse qué papel juega la Universidad en esta fase de la formación.

Coincidimos absolutamente con Delors (1998) en que la Universidad ha de desempeñar cuatro funciones esenciales:

- Preparar para la investigación y para la enseñanza, como lugar de ciencia y fuente de conocimiento que lleva a la investigación teórica o aplicada, o a la formación de profesores.

- Ofrecer formación muy especializada, que permita adquirir calificaciones profesionales conforme a unos estudios universitarios y unos contenidos adaptados a las necesidades de la vida económica y social, en los que se aúnan conocimientos teóricos y prácticos a un alto nivel.

- Abrirse a la sociedad para responder a los múltiples aspectos de lo que llamamos educación permanente en el sentido más amplio del término, como plataforma privilegiada de la educación a lo largo de la vida. 
- Cooperar en el ámbito internacional, actuando como interlocutor privilegiado en el intercambio de profesores y estudiantes.

Sólo mediante la observancia escrupulosa de estas funciones conseguiremos que la Universidad tienda lazos con el mercado de trabajo y prepare eficazmente a los distintos profesionales.

Volviendo a nuestras secciones bilingües, no conviene olvidar que en situaciones de bilingüismo consideramos esencial la formación lingüística de los diferentes profesionales implicados en el manejo de la lengua extranjera de la sección. El objetivo primordial de dicha formación consistiría en mejorar el conocimiento de la lengua y de la cultura del país, en nuestro caso la lengua y la cultura francesas, ya que es innegable que el hecho de enseñar y aprender una lengua extranjera no sólo radica en desarrollar habilidades lingüísticas como la fonología, la morfología o la sintaxis; en el uso de una lengua lo intelectual y lo afectivo son difícilmente separables. La palabra, según Bally (1965), es sobre todo sentimental. Aprender una lengua extranjera implica entonces la apropiación de aspectos conformados por diferentes competencias de tipo lingüístico y sociocultural, es decir desvelar una nueva realidad intercultural. Por otro lado, Snow (1989) identifica dos tipos de competencias diferentes que afectan al profesor de DNL: las competencias lingüísticas obligatorias y las competencias lingüísticas compatibles. Las primeras son los contenidos lingüísticos indispensables de la disciplina, que garantizan la adquisición de los conceptos disciplinares; las segundas afectan a competencias estrictamente lingüísticas, que no son indispensables para la apropiación de conceptos disciplinares.

Aunque la cuestión lingüística se nos presenta como la más evidente y necesaria, no hay que ignorar que cuando enseñamos una materia en dos lenguas entran en juego otras competencias, recogidas por Nunan y Lam (1998), citado por Cavalli (2005: 259), y entre las que cabría destacar:

- Un conocimiento de la lingüística y del bilingüismo.

- La capacidad de elaborar materiales.

- La capacidad de crear nuevos instrumentos de evaluación, para el alumno y para el profesor.

- La capacidad de comprender y manejar las relaciones con la comunidad educativa.

- Competencias para una gestión eficaz de las relaciones en la comunidad escolar.

- Desarrollar competencias sobre el aprendizaje cooperativo.

- Una capacidad de valorar la cultura del alumno y la posibilidad de responder positivamente a la diversidad de comportamientos en circunstancias interculturales.

Un sistema de enseñanza bilingüe de calidad debe contar con un buen plan de formación y perfeccionamiento profesional adaptado a esta realidad y establecer además unos parámetros adecuados a este perfil en la contratación de profesores. 
En los programas de secciones bilingües de francés de Primaria, en los que escasean docentes de DNL bien preparados para llevar a cabo la docencia en esta lengua, por lo general son los propios maestros especializados en lenguas extranjeras los que asumen la responsabilidad de estas secciones bilingües $y$, en muchos casos, también de las asignaturas implicadas (matemáticas, conocimiento del medio...). Sin embargo, en la ESO la situación difiere, puesto que las diversas asignaturas son impartidas por profesores especializados en la materia. Pero, para que esta situación idónea funcione y la integración de los docentes de las DNL sea eficaz, es sumamente importante que éstos posean una formación complementaria en lengua extranjera que asegure la viabilidad de estos proyectos, sin olvidar la importancia de la figura del auxiliar de conversación.

Junto a estas competencias, podemos también señalar otras que son determinantes en el contexto en el que nos movemos y que implican el desarrollo de capacidades para relacionar dos currículos diferentes, contenidos, materiales, escalas y modalidades de evaluación, en una palabra, hay que crear un programa que integre dos perspectivas diferentes para un mismo tema; y esto no puede improvisarse, ya que el alumno no puede perder de vista su currículo nacional si queremos que llegue a la Universidad sin desfases o lagunas en su formación previa. Para ello el profesor debe aprender a elaborar sus programaciones en función de los límites de su programa nacional pero integrando documentos, materiales y manuales de la L2. Estos nuevos profesionales se enfrentan también a la necesidad de manejar dos lenguas en el aula, y de su acierto depende el no cortar la comunicación en clase, pues la lengua extranjera se convierte en un instrumento más de aprendizaje, lo que nos permite elaborar síntesis o resúmenes en las dos lenguas.

Para llevar a cabo esta nueva tarea es muy aconsejable la creación de equipos pedagógicos, cuyo funcionamiento se concibe como un todo que responderá a las necesidades de cada uno de los participantes como miembros autónomos y diferentes. El diálogo entre los profesores implicados y el asistente de lenguas ha de ser constante, puesto que los problemas habrán de ser solucionados mediante un enfoque cruzado entre la lengua de la sección, la lengua en situación y la disciplina no lingüística enseñada. Por este motivo se hace también imprescindible la adaptación de los horarios, de modo que se facilite la concertación que requiere un trabajo en equipo.

Como decíamos anteriormente, la solución a las carencias formativas parece venir de la mano de la formación continua o permanente. En nuestra Comunidad, la Dirección General de Calidad e Innovación y la formación de profesores de Castilla y León, a través del Centro de Formación de los profesores de idiomas (CFPI), propone una oferta de formación continua para la adquisición y el perfeccionamiento de la competencia lingüístico-comunicativa para todas las lenguas. No obstante, pese a los esfuerzos realizados y a la amplia variedad de programas, éstos no siempre están en sintonía con las recomendaciones teóricas, ni con las necesidades reales de los profesores de las secciones bilingües. En nuestra opinión, pese a los esfuerzos realizados, la propuesta formativa está excesivamente vinculada a la lengua inglesa que, como ya hemos dicho, se constituye en lengua prioritaria frente a otras que pasan a un plano inferior; así mismo, se presentan una diversidad de opciones formativas que no responden a una estructura definida, de ahí su carácter errático; y por último, 
muchas de ellas tienen un diseño muy general y no están debidamente enfocadas a cada una de las etapas educativas.

La tarea no es sencilla; el aprendizaje de lenguas para el profesor de DNL debe centrarse en las disciplinas implicadas y, para ello, lo más recomendable sería que cualquier tipo de formación que se le ofrezca incluya, además de una formación lingüística de carácter específico de acuerdo con su especialidad, una estancia en el ámbito escolar del país de referencia, lo que le proporcionaría ejemplos de prácticas pedagógicas eficaces. Pero esto no siempre es posible y depende, en la mayoría de los casos, de las disposiciones presupuestarias que hoy en día no nos permiten albergar muchas esperanzas.

\section{Conclusiones}

En España, y por supuesto en Castilla y León, se ha experimentado en las últimas décadas un aumento del número de años de enseñanza obligatoria de al menos una lengua extranjera. En paralelo, se ha ido avanzando en el descenso en la edad de comienzo de esta enseñanza. Sin embargo, las estadísticas europeas siguen situándonos en una posición delicada en cuanto al dominio de las lenguas extranjeras por parte de la población, lo cual debería llevarnos a centrar el problema en los diferentes agentes o factores que intervienen en la enseñanza de las lenguas y, consecuentemente, en los resultados de aprendizaje.

Creemos que para que la diversidad lingüística europea se convierta en una ventaja competitiva real, desde las instituciones con competencias en materia educativa se debe impulsar activamente el plurilingüismo, fomentando el aprendizaje de un amplio abanico de lenguas en todos los niveles educativos. Es una tarea que nos compete especialmente, pues facilitar la adquisición de otras lenguas distintas de la nativa y el dominio de las destrezas comunicativas básicas es una responsabilidad de las Universidades y de las Administraciones.

A partir de la etapa de secundaria y durante la educación superior (formación profesional y universitaria), se deben introducir módulos de aprendizaje de lenguas menos teóricos y con una mayor orientación práctica. En este sentido, se ha de insistir en ampliar la gama de lenguas extranjeras en todos los itinerarios formativos, incluyéndolas al menos como materias optativas y con objetivos específicos.

Descuidar la oferta de lenguas extranjeras va contra los objetivos europeos de facilitar la movilidad de estudiantes, profesores, trabajadores y ciudadanos en general. Aunque parezca difícil en momentos de crisis como el actual, y hasta económicamente poco práctico, desde la Universidad deberíamos tender a potenciar el aprendizaje de lenguas a lo largo de toda la vida, dado el valor añadido en relación con las futuras oportunidades laborales. Lamentablemente, haciendo nuestras las palabras de la Comisaria europea de educación, a tenor de la escasa presencia y variedad que las lenguas extranjeras tienen en los nuevos grados, sentimos que "el aprendizaje de una lengua extranjera no es una prioridad para los estudiantes universitarios, y debería serlo para todos". En este sentido, quizá la Universidad debiera plantearse una revisión de los actuales planes de estudio para ofrecer una completa y necesaria formación inicial en idiomas conforme a los niveles demandados en Europa para que nuestros 
alumnos adquieran, desde la propia Universidad y no desde centros privados, una sólida y adecuada preparación y puedan alcanzar el nivel lingüístico oral y escrito exigido, ejerciendo así su papel como garante de una formación muy especializada adaptada a las necesidades de la vida económica y social y en la que se aúnen conocimientos teóricos y prácticos a un alto nivel.

Además, al igual que en la mayoría de los países europeos, los títulos o certificados que se otorgan al término de las distintas etapas educativas deberían incluir el reconocimiento del nivel de competencia en lengua extranjera.

Castilla y León, que parte de una realidad monolingüe, ha emprendido varios proyectos acertados con el fin de afianzar el plurilingüismo. Aun así, en nuestra opinión este esfuerzo no es suficiente si se mantienen las prioridades entre unos idiomas y otros, puesto que ninguna lengua debería posicionarse por delante de las demás, como ocurre con el inglés en nuestra comunidad, donde se sitúa por delante del estudio de todas las demás lenguas, convirtiendo el francés en una materia de carácter optativo y minimizando la presencia del alemán, portugués u otras posibles opciones. Parece pues ineludible una mayor diversificación en el número de idiomas ofertados en la enseñanza primaria, la secundaria y la universidad, de modo que cursar el alemán, el italiano u otros idiomas resulte una posibilidad real. Esta aspiración, que hasta el momento actual no es más que una utopía, nos aleja claramente a los castellanoleoneses del ciudadano plurilingüe que preconiza Europa.

Por ello, consideramos que la situación de nuestra comunidad y otras de nuestro país cuya característica es el monolingüismo, provoca a nuestro entender una desventaja frente a otras que disfrutan de un bilingüismo de partida. En el contexto actual, el alumno sólo puede aspirar a dominar una lengua extranjera (aquella que es impartida como primer idioma extranjero) y a adquirir conocimientos muy básicos que a menudo son interrumpidos en la etapa de secundaria- de una segunda. Por este motivo, consideramos que una política de incentivación del plurilingüismo es aún más necesaria en nuestro caso.

En cuanto a las secciones bilingües de francés, siguen abriéndose camino en Castilla y León. Existe actualmente un importante número de centros que las ofertan, 19 en su totalidad, y no dudamos en destacar los beneficios que éstas pueden aportar a nuestros alumnos ya que favorecen las estrategias orales y escritas de la lengua de estudio, incrementan su conocimiento cultural y optimizan sus habilidades cognitivas. Esta enseñanza favorece igualmente el conocimiento y manejo de ambas lenguas e integra valores de respeto y tolerancia muy apreciados en nuestra sociedad, cada vez más abierta al exterior. Además, no olvidemos que las modalidades de aprendizaje de una sección bilingüe también responden a objetivos pedagógicos y didácticos específicos y favorecen los programas de intercambio tanto de los alumnos como de los profesores, facilitando de este modo la posibilidad de trabajar en equipo.

No obstante, advertimos que no nos encontramos en el mejor momento para recibir el tan esperado y necesario apoyo financiero por parte de nuestro gobierno y que todo ello nos puede llevar a prescindir de becas y programas de movilidad que hasta ahora favorecían la formación continua de nuestros alumnos mediante los intercambios culturales que se realizaban entre los diferentes países. Y, por otra parte, no parece existir una relación directa entre los esfuerzos por conseguir ser bilingües y 
las horas dedicadas a la enseñanza de idiomas, y en consecuencia, por mucho que se alardee de la proliferación de centros escolares bilingües, los resultados distan aún mucho de ser los deseables.

Para concluir, cada vez es más evidente que la reforma llevada a cabo en los planes de estudio de las lenguas extranjeras no ha satisfecho las demandas previstas inicialmente y vemos cómo el nivel lingüístico de nuestros alumnos no es, en la mayoría de los casos, el adecuado. Pensamos que de este modo será casi imposible alcanzar la tan deseada realidad plurilingüe si continuamos pensando que lo que cuenta es la formación continua y no la inicial. Creemos que lo más importante es poseer una buena formación inicial respaldada por la universidad para después seguir con la formación continua de apoyo. Para ello, la Universidad debería de preparar para la investigación y enseñanza, ofrecer los niveles de formación requeridos según la normativa y facilitar el intercambio en el ámbito internacional con otros centros formativos contando con el apoyo gubernamental.

\section{Notas:}

${ }^{1}$ Sus conclusiones pueden consultarse en: http://www.consilium.europa.eu/uedocs/cms data/docs/pressData/es/ec/70829.pdf

${ }^{2}$ Diario Oficial de la Unión Europea C 119/2 de 28.5.2009.

${ }^{3}$ ISCED 0 corresponde a la Educación Infantil, ISCED 1 a la Educación Primaria, ISCED 2 a la Secundaria Obligatoria, e ISCED3 a la Secundaria postobligatoria (Bachillerato) y Formación Profesional de Grado Medio.

${ }^{4}$ Fuente: Eurostat yearbook 2011: http://epp.eurostat.ec.europa.eu/portal/page/portal/eurostat/home

${ }^{5}$ Aunque la Ley Orgánica de Educación (LOE, 2006) introduce el estudio obligatorio de la lengua extranjera en la Educación Infantil a partir de los tres años, su desarrollo se hace efectivo en Castilla y León durante el curso 2008/2009 (Real Decreto 122/2007, de 27 de diciembre).

${ }^{6}$ Conviene aclarar que esta experiencia no ha de confundirse con los programas bilingües en francés.

7 No fuimos pioneros en este tipo de enseñanza, pues esta experiencia ya funciona en Andalucía en 1998 y en Aragón y Galicia en 1999.

${ }^{8}$ Convenio de cooperación cultural, científica y técnica entre la República Francesa y España. Firmado en Madrid, el 7 de febrero de 1969. (BOE de 23 de diciembre de 1969).

${ }^{9}$ Acuerdo Marco entre el Gobierno del Reino de España y el Gobierno de la República Francesa sobre los programas educativos, lingüísticos y culturales en los Centros Escolares de los dos Estados, hecho en Madrid el 16 de Mayo de 2005.

${ }^{10}$ Orden EDU/6/2006, del 4 de enero (BOCYL 12 de enero de 2006).

${ }^{11}$ Orden EDU/1847/2007, del 19 de noviembre (BOCYL 23 de noviembre de 2007).

${ }^{12}$ Ver la nota de prensa del Gobierno de la Comunidad autónoma de Castilla y León en http://ambafrance-es.org/france espagne/IMG/pdf/Convenio Embajada Francesa 8-0311 .pdf

${ }^{13}$ Fuente: http://www.educa.jcyl.es/es 


\section{Referencias Bibliográficas}

Bain, B. (1974). Bilingualism and cognition: Towards a general theory. En S.T. Carey (Ed.). Bilingualism, biculturalism and education (pp. 119-128). Edmonton, University of Alberta Press.

Baker, C. (2001). Foundations of Bilingual Education and Bilingualism. (3a. ed.) Clevedon: Multilingual Matters.

Bally, CH. (1965). Le langage et la vie. Génève: Droz.

Canabal García, C.; García Campos, M.D. (2012). Profesorado universitario que indaga colaborativamente: una propuesta formativa para mejorar la práctica docente. Revista de Docencia Universitaria. REDU. Monográfico: Buenas prácticas docente en la enseñanza universitaria. 10 (1), 215-35. Recuperado el (25-062012) en http://redaberta.usc.es/redu

Cavalli, M. (2005). Éducation bilingue et plurilinguisme. Le cas du Val d'Aoste. Paris: Didier.

Cummins, J. (1976). The influence of bilingualism on cognitive growth: A synthesis of research findings and explanatory hypotheses. Working Papers on Bilingualism, 9, 1-43.

Decreto 40/2007, de 3 de mayo, por el que se establece el Currículo de Educación Primaria en la Comunidad de Castilla y León. (BOCyL de 9 de mayo de 2007)

Decreto 52/2007, de 17 de mayo, por el que se establece el currículo de la Educación Secundaria Obligatoria en la Comunidad de Castilla y León. (BOCyL de 23 de mayo de 2007)

De Houwer, A. (1995). Bilingual Language Acquisition. En P. Fletcher, y B. MacWhinney (Eds.). The handbook of child language, (pp. 219-250). Londres: Blackwell.

Delors, J. (1998). La educación encierra un tesoro. Informe a la UNESCO de la Comisión Internacional sobre la educación para el siglo XXI. UNESCO: Santillana, S.A.

Deshays, E. (1990). L'enfant bilingue. Paris: Robert Laffont.

Duverger , J. (1995). Repères et enjeux. Revue Internationale d'Éducation de Sèvres, 7, 29-44. Enseignements bilingues.

Duverger , J. (2005). L'enseignement en classe bilingue. Paris: Hachette.

Hagège, C. (1996). L'enfant aux deux langues. Paris: Éditions Jacob.

Junta de Castilla y León. Normativa reguladora sobre secciones bilingües. [En Línea: http://www.educa.jcyl.es/educacyl/cm/educacyl/tkContent?pgseed=126527584 9065\&idContent $=76049$ \&locale $=e s \quad$ ES\&textOnly=false]

Ministerio de Educación. Secretaría de Estado de Educación y Formación Profesional (2011). Objetivos Educativos Europeos y Españoles Estrategia Educación y Formación 2020. Informe español 2010-2011. Secretaría General Técnica. Subdirección General de Documentación y Publicaciones.

Ministerio de Educación (2011). Programa integral de Aprendizaje de Lenguas Extranjeras. Conferencia de Educación, 23 de marzo de 2011. 
Nunan, D.y Lam, A. (1998): Teacher education for multilingual contexts: models and Issus. En J. Cenoz y F. Genessee (Eds). Beyond Bilingualism, Multilinguallism and multilingual educacion, (pp. 117-140). Clevedon: Multilingual Matters.

Orden $\mathrm{ECl} / 1128 / 2006$, de 6 de abril, por la que se desarrolla el Real Decreto 717/2005, de 20 de junio, por el que se regula la ordenación de la enseñanzas en los centros docentes acogidos al convenio entre el Ministerio de Educación y Ciencia y The British Council. (BOE de 19 de abril de 2006)

Orden EDU/2157/2010, de 30 de julio, por la que se regula el currículo mixto de las enseñanzas acogidas al Acuerdo entre el Gobierno de España y el Gobierno de Francia relativo a la doble titulación de Bachiller y de Baccalauréat en centros docentes españoles, así como los requisitos para su obtención. (BOE de 7 de agosto de 2010)

Siguan, M. (Comp.) (1996). La enseñanza precoz de una segunda lengua en la escuela. Barcelona: Horsori.

Snow, M., Met, M. y Genesee, F. (1989). A Conceptual Framework for the Integration of language and Content in Second/Foreign Language Instruction. En TESOL Quaterly 23/2, 201-217.

Snow, C. (2001). Bilingüismo y Adquisición de una segunda lengua. En J. Berko. y N. Berstein (Eds.). Psicolingüística (pp.477-508). (2aed.). Madrid: McGraw Hill.

Tunmer, E.E. y Myhill, M.E. (1984). Metalinguistic awereness and bilingualism. En W.E. Tunmer, C. Pratt y M. L. Herriman (Eds.). Metalinguistic awereness in children (pp. 169-87). Berlín: Springer Verlag.

Cita del artículo:

Cuervo Vázquez, A.M.E.; Alonso Díaz, R.M; Sabadell González, M. (2013). Reflexiones en torno a la lengua francesa en Castilla y León: secciones bilingües y formación de los profesores de disciplinas no lingüísticas (DNL). Revista de Docencia Universitaria. REDU. Vol.11 (1) Enero-Abril, pp. 365-388. Recuperado el (fecha de consulta) en http://www.red-u.net/

\section{Acerca de las autoras}

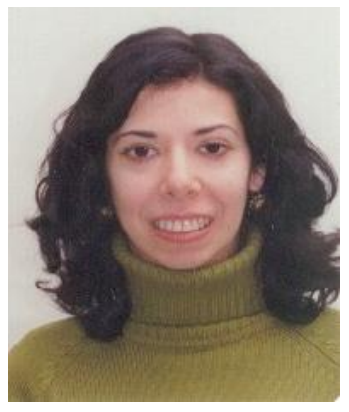

\section{Ana $M^{a}$ Elisabeth Cuervo Vázquez}

\section{Universidad de Valladolid}

Departamento de Filología Francesa y Alemana

Mail: anaelisa@dlyl.uva.es 
Es profesora doctora del Departamento de Filología Francesa y Alemana de la Universidad de Valladolid. Su experiencia docente abarca la enseñanza de la Lengua y la Literatura Francesa y formación en profesorado bilingüe en la Facultad de Educación de la Universidad de Valladolid. Dicha experiencia, al igual que sus trabajos de investigación, le han llevado a explorar diferentes campos relacionados con la enseñanza y aprendizaje de las lenguas, el plurilinguismo y el bilingüismo. Actualmente es miembro del Centro Transdisciplinar de Investigación en Educación (CETIE-UVa) y de la Asociación de Profesores de Francés de la Universidad Española (APFUE).

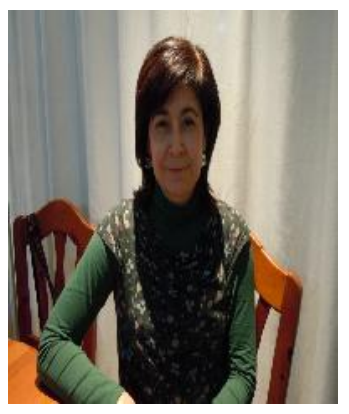

\section{Rosa María Alonso Díaz}

\section{Universidad de Valladolid}

Departamento de Filología Francesa y Alemana

Mail: rmalonso@ffr.uva.es

Es profesora del Departamento de Filología Francesa y Alemana de la Universidad de Valladolid. Su experiencia docente abarca la enseñanza de la Lengua y la Literatura Francesa y su Didáctica en la Facultad de Educación de la Universidad de Valladolid. Dicha experiencia, al igual que sus trabajos de investigación, le han llevado a explorar diferentes campos relacionados con la enseñanza y aprendizaje de las lenguas, la literatura y el bilingüismo. Actualmente es miembro del Centro Transdisciplinar de Investigación en Educación (CETIE-UVa).

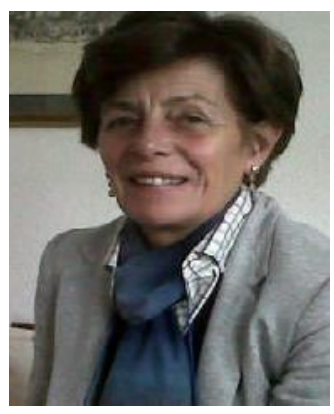

\section{Montserrat Sabadell González}

\section{Universidad de Valladolid}

Departamento de Filología Francesa y Alemana

Mail: montsesg@ffr.uva.es

Es profesora del Departamento de Filología Francesa y Alemana de la Universidad de Valladolid. Su experiencia docente abarca la enseñanza de la Lengua y la Literatura Francesa y su Didáctica en la Facultad de Educación de la Universidad de Valladolid. Dicha experiencia, al igual que sus trabajos de investigación, le han llevado a explorar diferentes campos relacionados con la enseñanza y aprendizaje de las lenguas y el bilingüismo. Actualmente es miembro del Centro Transdisciplinar de Investigación en Educación (CETIE-UVa). 


\title{
La responsabilidad social de las universidades a través de la memoria cinematográfica
}

The social responsibility of universities through the prism of cinematic memory

\author{
María José Chisvert Tarazona
}

Universidad de Valencia, España

\section{Resumen}

Este artículo rescata una interesante película británica filmada en 1983 por Lewis Gilbert, "Educando a Rita", referencia fiel a la memoria de los inicios de la Open University, a su espíritu de innovación educativa, pionera en la educación universitaria a distancia en Europa. La cinta explora temas relevantes relativos a la educación superior y susceptibles de análisis en al menos cuatro dimensiones: la cultura de la institución universitaria, la profesionalidad docente, la metodología de enseñanza y el papel del alumnado en los estudios superiores. Las dimensiones consideradas requieren de un elemento posibilitador, de reflexión curricular: la orientación de los planes de estudio. El interés por cuestionar el sentido del espacio universitario recupera la noción de misión académica vinculada a la idea de excelencia intelectual y al desarrollo científico que subyace a tres actividades principales dirigidas a cumplir su función de generación y difusión del conocimiento y la cultura: la investigación, la enseñanza y la extensión. Esta última, la extensión universitaria, nace con miras a fortalecer la vinculación universidad-entorno social bajo el enfoque de la responsabilidad social universitaria, si bien su incorporación al quehacer de las instituciones de educación superior adolece de escaso reconocimiento como función sustantiva. Se convierte así en la gran olvidada en los planes de estudio actuales inspirados en el EEES al limitarse a generar pruebas de acceso específicas dirigidas a personas adultas. Las transiciones tardías a la esfera universitaria requieren nuevas propuestas integradoras en los planes de estudio para estos colectivos.

Palabras clave: transiciones educativas, planes de estudio, universidad, cine, personas adultas.

\begin{abstract}
This article reviews 'Educating Rita', an interesting British film shot in 1983 by Lewis Gilbert. The film offers a faithful depiction of the beginnings of the Open University, a pioneer in university-level distance learning in Europe thanks to its innovative educational spirit. The film explores relevant issues at the core of higher education, analyzing them in at least four dimensions: the culture of the educational institution, teachers' professionalism, teaching methodology and the role of students in higher education. A facilitating element and theme for curricular reflection underlying these four dimensions is
\end{abstract}


the orientation of university curricula. An interest in questioning the meaning of universities is linked to the recovery of the notion of academic mission. These are closely connected to the notion of intellectual excellence and to the scientific development underlying three main activities: research, teaching and outreach. All three addressed the university mission to generate and disseminate knowledge and culture. University outreach aims to strengthen the links between higher education institutions and their social surroundings, as an expression of social responsibility within the university. However, outreach lacks sufficient acknowledgment as a privileged function. For this reason, it is often left aside in recent curricula developed by the European Higher Education Area, where only specific entry tests for adult learners are offered. Late transitions into university requires new curriculum proposals if they are intended to integrate adult learners.

Key words: educational transitions, curricula, university, cinema, adult learners

\section{Introducción: contexto y texto en la universidad}

El lenguaje fílmico es un instrumento de comunicación, de educación colectiva, un repositorio cultural que a través de narrativas artísticas y documentales nos aproxima a representaciones de la realidad. En palabras de Clarembeaux (2010) el cine se define como el arte de la memoria colectiva e individual y requiere de la convergencia entre una pedagogía de educación para el cine y la voluntad de conservar la memoria y el patrimonio cinematográfico.

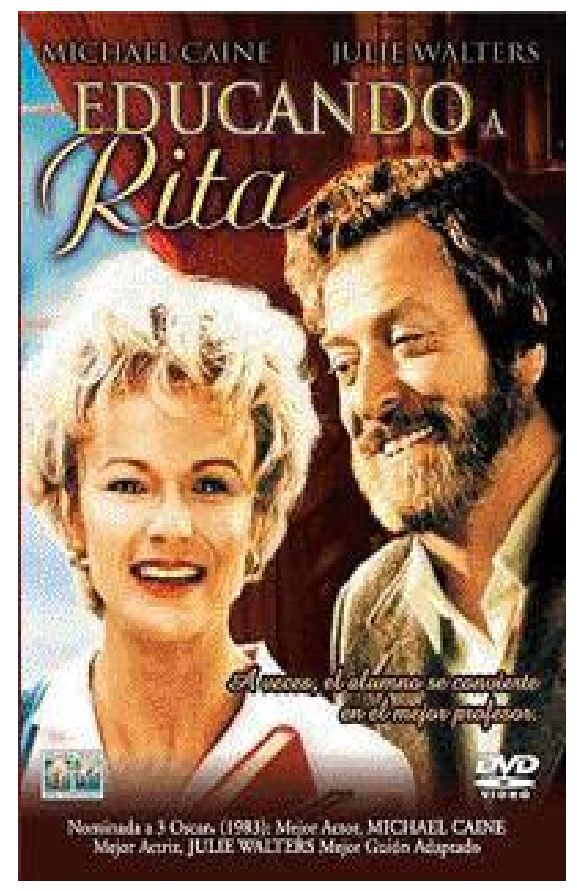

Esta es la razón de rescatar un interesante celuloide británico filmado en 1983 por Lewis Gilbert, "Educando a Rita". En esta proyección podemos encontrar una referencia fiel a la memoria de los inicios de la Open University, a su espíritu de innovación educativa, pionera en la educación universitaria a distancia en Europa. Esta recomendable película cuenta la historia de Susan, de Rita, como gustaba llamarse al inicio, una mujer adulta que desea ser alguien diferente y para ello inicia sus estudios en la universidad.

La cinta explora temas relevantes relativos a la educación superior y susceptibles de análisis en al menos cuatro dimensiones: la cultura de la institución universitaria, la profesionalidad docente, la metodología de enseñanza y el papel del alumnado en los estudios superiores.

Ilustración I: Cartel de "Educando a Rita"(1983).

El contexto socioeducativo en el que nace la Open University busca fórmulas de superación de la segregación, de recuperación de colectivos que crecieron en las fronteras del sistema educativo británico. La Ley Butler, promulgada en la Inglaterra de 1944 por el partido conservador hizo obligatoria la formación hasta los quince años, estableciendo el denominado "sistema tripartito", que contemplaba una formación básica general hasta los once años y cuatro años más de estudios en alguno de los tres 
modelos de escuela que se crearon: las Grammar Schools, para el mejor alumnado; las Technical Schools, para quienes deseaban recibir una formación profesional; y las Modern Schools, que ofrecían una enseñanza básica al alcance de la gran mayoría de la población escolar. La admisión en uno u otro modelo se realizaba atendiendo a los resultados escolares. Este sistema fue considerado por el partido laborista socialmente injusto y segregador. En 1965, Anthony Crosland, ministro laborista de educación, obligó a unificar los tres sistemas en uno sólo, las Comprehensive Schools, prohibiendo que las admisiones en los centros de secundaria se hicieran en función de méritos académicos o intereses personales. Otro cambio importante se produce en la educación superior, al introducir un sistema binario en el que se unieron a las universidades tradicionales las instituciones politécnicas (Woolwich Politécnica). También la Open University responde a estos principios políticos basados en la inclusión. Sus raíces académicas se remontan a 1926, año en el que el pedagogo e historiador J.C. Stobart escribió un memorándum relativo a la "Universidad del aire". Antecedentes más próximos, en los años sesenta del pasado siglo, alimentaban el debate de propuestas como la "teleuniversity", que combinaba conferencias de difusión de los textos de correspondencia y visitas a universidades convencionales. Si bien no es hasta la victoria electoral del partido Laborista, en 1964, cuando el proyecto de la Open University se hace realidad impulsado por la Ministra de Educación Jennie Lee.

El contexto que inspira el nacimiento de esta universidad se traslada al texto universitario tal y como se representa a través del film. La cultura de este espacio recreado a través del cine impregna cada fotograma mostrando el sentido y significado de la educación a distancia y de la educación a lo largo de la vida en la universidad. Las estrategias de inclusión propias de la institución, así como el valor otorgado a la socialización, favorecen el establecimiento de alianzas duraderas de este espacio universitario con el entorno, transformándolo. La profesionalidad docente se construye en las relaciones mantenidas entre el profesor y la estudiante, en el requisito de dominio del conocimiento y de la cultura, así como en las expectativas y prejuicios respecto del rendimiento académico. La metodología de enseñanza, inserta en un ambiente híbrido de aprendizaje, se evidencia en la mentorización, en el uso de medios y recursos formativos audiovisuales, en los criterios incorporados para la evaluación de los desempeños. Si bien, es quizá el alumnado, a través de Rita, el que se reivindica con mayor clarividencia, mostrando su disposición hacia el aprendizaje, el peso de los antecedentes educativos, el contexto familiar que apoya o dificulta el proceso, las transformaciones personales provocadas por el acceso a nuevo conocimiento. Las dimensiones ya consideradas requieren de un elemento posibilitador, de reflexión curricular: la orientación de los planes de estudio. Currículum como proceso que invita a la consideración sobre qué enseñar, pero que no puede obviar respuestas al para qué enseñar, cómo enseñar, qué, cómo y cuándo evaluar. El currículum, parafraseando a Kemmis (1993), se constituye en puente que fomenta la interactividad entre la universidad y el contexto, entre la teoría y la práctica.

La riqueza de esta producción para el análisis del entorno universitario radica en que los procesos educativos son dimensiones significativas en la narración de la película. Invita a revisar la misión de la universidad en tiempos de reforma, a valorar la 


\section{La Open University: oportunidad para la inclusión}

El complejo proceso de reforma universitaria que se está acometiendo en la actualidad con objeto de favorecer la Convergencia Europea en el acceso al conocimiento incorpora importantes cambios en el ámbito formal o estructural. Las fuentes de financiación, los sistemas de garantía de la calidad, entre otros, son revisados con esta finalidad. La creación del Espacio Europeo de Educación Superior (EEES) supone un desafío en la articulación de criterios convergentes relativos al modo de aprender del alumnado universitario, a su papel activo en su propio proyecto formativo, al nuevo rol del profesorado, a las metodologías de enseñanza; con la intención de posibilitar la homologación y equiparación de los títulos superiores. El debate avanza, constatándose a través de las declaraciones y conferencias de La Sorbona (1998), Bolonia (1999), Praga (2001), Berlín (2003), Bergen (2005), Londres (2007) y Lovaina (2009). A pesar de las controversias y críticas al proceso de construcción del EEES (Fueyo, 2004; Bolívar, 2009), la construcción de espacios de intercambio académico marcha con firmeza. El proceso de reforma de la enseñanza superior tendrá que hacer frente a dos retos importantes: a la internacionalización del currículum y la apuesta clara por su virtualización (Gijón y Crisol, 2012). Todo ello implica su traslación a los planes de estudio.

Rescatar lo que significó la Open University en sus orígenes podría abrir nuevas miradas al debate sobre el sentido del espacio universitario. Mostrarlo a través del cine, de la proyección "Educando a Rita", proporciona la proximidad tangible del ejemplo, a pesar de tratarse de una recreación.

El interés por cuestionar el sentido del espacio universitario recupera la noción de misión académica vinculada a la idea de excelencia intelectual y al desarrollo científico que subyace a tres actividades principales dirigidas a cumplir su función de generación y difusión del conocimiento y la cultura: la investigación, la enseñanza y la extensión. Esta última, la extensión universitaria, nace con miras a fortalecer la vinculación universidad-entorno social bajo el enfoque de la responsabilidad social universitaria y ha sido incorporada al quehacer de las instituciones de educación superior. Sin embargo su reconocimiento como función sustantiva apenas ha ido cobrando espacio. Quizás una de las dificultades más importantes ha sido la falta de claridad sobre su significado o conceptualización, tanto desde el profesorado universitario, como desde la dirección institucional y demás miembros de la comunidad universitaria.

Sin embargo existen experiencias alentadoras en el ámbito universitario europeo que podrían acompañar a la reflexión sobre la significación de la extensión universitaria, tal y como se pone de relieve en "Educando a Rita".

La narración fílmica protagonizada por Rita se sitúa en una institución de estudios superiores real, la Open University británica, universidad que abrió sus puertas en abril de 1969 en respuesta al gran aumento de la población estudiantil universitaria y a su diversidad. Este centro de estudios superiores inicia actividades de extensión universitaria cubriendo la actualización profesional, temas sociales y comunitarios y temas de interés personal, que posteriormente expandió a sus programas de grado y más tarde de postgrado universitario. Fue pionera en la introducción de tecnologías de la información y la comunicación al servicio del 
aprendizaje, posibilitando el acceso a personas sin oportunidad de asistir a universidades convencionales. Wiltshire (1973) atribuía a la combinación de diversos medios educativos -cursos por correspondencia, emisiones por radio y televisión, entrevistas y controles personales, etc.-, su innovación en la enseñanza y aprendizaje. A partir de la década de los setenta del pasado siglo, otras instituciones y programas de nivel superior adoptaron y/o adaptaron su modelo con éxito.

La Open University británica desde su apertura comenzó a acoger gran número de estudiantes "no diplomados". La oferta del plan de estudios remitía en sus inicios a cuatro cursos básicos como introducción general y multidisciplinaria a las materias que se enseñaban en las Facultades de Letras, Matemáticas, Ciencias Sociales y Ciencias. Cada curso comprendía lecciones por correspondencia, semanales o quincenales, una emisión por televisión y otra radial cada semana. Los envíos postales comprendían, de ordinario, un folleto impreso de 60 a 80 páginas, con notas explicativas y comentarios sobre las emisiones por radio y televisión, ejercicios de autoevaluación y prácticas orientadas por el profesorado responsable del estudiante. En ciertas materias, al alumnado se le proporcionaba los elementos necesarios para el trabajo práctico, de carácter experimental, que realizaba en su domicilio (Wiltshire, 1973). La rigidez en los planes de estudio era mínima. Los estudiantes tenían la facultad de combinar los cursos, según su voluntad, dentro de ciertos límites lógicos determinados por el propósito de evitar la especialización extrema. Los cursos se complementaban con el envío de libros, editados para satisfacer las necesidades y objetivos planteados.

El aparato administrativo académico de la Open University incorporó en sus inicios una red de oficinas regionales y centros de estudio locales distribuidos en todo el país, que ofrecían la posibilidad de que el alumnado estableciera contactos entre sí y asistiera a las tutorías, rompiendo el aislamiento de la mayoría de los estudiantes en el sistema de enseñanza a distancia. Los grandes centros disponían de una red informática a la que también podían acceder haciendo uso de ordenadores disponibles en estos centros y tenían la obligación de entrevistarse con una figura docente consejera cada quince días.

Por último, y como complemento de los métodos de enseñanza indicados, la Open University ya contaba con cursos de verano. Su característica específica era que el alumnado debía permanecer interno durante una semana en las diversas universidades del país. Tales cursos constituían un estímulo, a la vez que permitían la realización de estudios en equipo intensivos, trabajos de laboratorio, experiencias y el establecimiento de contactos con el profesorado de los cursos, a los que sólo habían conocido con antelación por sus textos y emisiones.

La evaluación se basaba en un control continuo sobre el trabajo del estudiante y en un examen escrito. Se ponía especial cuidado en que los títulos fueran equivalentes a los otorgados por las otras universidades británicas. Los estudios se acreditaban con la obtención del correspondiente diploma. Seis "unidades de valor", traducibles en créditos, permitían obtener el diploma Bachelor of Arts, y ocho, el Honours Degree. Los exámenes $y$, en general, las evaluaciones, se realizaban bajo el control externo de otras universidades.

Esta institución universitaria experimental, al tiempo que preparaba para la obtención de grados universitarios, ofrecía respuestas a alumnado que iniciaba su 
tránsito a la cultura y al conocimiento en la edad adulta. Su cariz innovador no reside exclusivamente en los medios y tecnologías puestos al servicio de la educación a distancia, sino en la apertura de su acceso a estudiantes sin requisitos de formación previa. También era innovadora la tutorización y mentorización ejercida por el profesorado, así como la disposición institucional de espacios físicos de proximidad que acercaban el entorno universitario a las vidas de personas adultas ya incorporadas al mercado laboral. El aprendizaje a lo largo de la vida toma sentido bajo esta fórmula flexible de acceso al espacio universitario.

En la actualidad la Open University, considerada la mayor organización dedicada a la educación a distancia, desarrolla un entorno de aprendizaje virtual que ofrece lugares de encuentro entre profesorado y estudiantes para labores de apoyo, intercambio de documentos y de recursos educativos con base a los permisos de entrada de cada estudiante, foros de discusión, anuncios online o acceso remoto al entorno de aprendizaje desde cualquier ordenador y a cualquier hora. Ello no significa que abandone en los medios y tecnologías la referencia de calidad, de hecho cuenta con programas específicos de formación del profesorado. El Post Graduate Certificate in Education de la Open University ya forma a un $10 \%$ del profesorado de Reino Unido y ha ganado premios por su calidad. Se caracteriza por ser llevado a cabo durante el desempeño profesional ("in-service") y en el seno de las instituciones ("in-house").

Si regresamos a la narración del film, exquisita en la reproducción de la estructura y cultura de esta universidad, encontramos una mirada avanzada sobre lo que representa la incorporación de estudiantes diversos en el espacio universitario, sobrelos requisitos que la institución debe asumir para favorecerlo. La película muestra en algunos fotogramas el acceso de Rita a los documentos recibidos a través de envíos postales, los cursos en la televisión o la radio, sin embargo se incide especialmente en la tutorización, en las relaciones establecidas con el profesor, también en los cursos de verano. Efectivamente el rol docente requería de una adaptación a esta nueva realidad. En la actualidad una pregunta recurrente alude al significado del nuevo rol del profesorado definido en la reforma universitaria, rol que afecta también a la definición de los planes de estudio. Tal vez sería de interés iniciar desde un análisis relativo a lo que la sociedad espera de esta figura y para ello pudiera ser de utilidad revisar referentes cinematográficos.

\section{La Cinematografía y la profesión docente universitaria: el caso del Dr. Frank Bryant}

La filmografía rodada en el espacio universitario europeo y norteamericano da prueba del imaginario construido en las sociedades postmodernas sobre el rol del profesorado universitario. Si revisamos algunas de las películas más significativas de este género confirmaremos que el dominio del conocimiento y la conexión con el alumnado son las claves que la sociedad reconoce en la excelencia de la docencia universitaria. También la investigación se introduce en algunas películas, pero su representación es más limitada y en la mayoría de las ocasiones aparece desligada de la docencia. 
No es el caso de "Ágora" (Amenábar, España, 2009). En esta producción que nos remonta al s. IV, Hipatia enseña matemáticas, astronomía y filosofía a los hijos de la élite en la escuela de Alejandría sin alejarse de su trayectoria como investigadora.

Esta profesora conecta con el conocimiento, se conmueve ante los textos de los Elementos de Euclides, ante el cono de Apolonio, el sistema geocéntrico de Ptolomeo o el heliocéntrico de Aristarco de Samos.

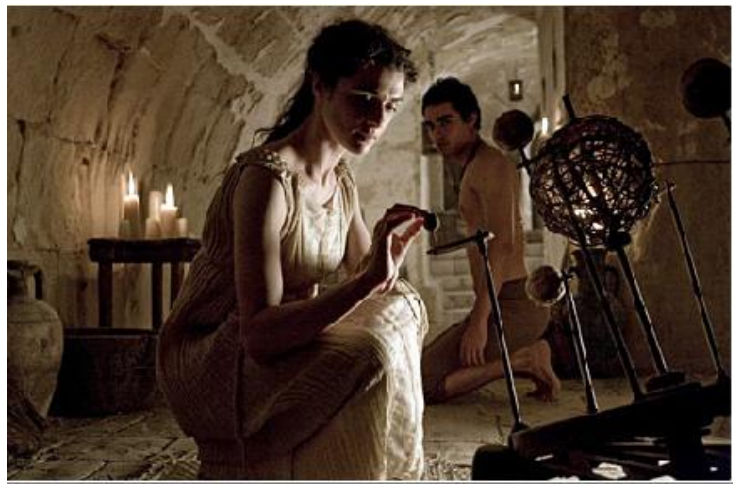

Ilustración II: Fotograma de "Ágora"(España, 2009).

Su interés hacia la investigación se constata a través de la búsqueda y resolución del enigma astronómico que plantean los planetas errantes, vislumbrando en la elipse la solución que hallarán más de mil años después, en el siglo XVI, Copérnico y Kepler en su reformulación, hoy vigente, de la teoría heliocéntrica de órbitas elípticas.

La película muestra así la búsqueda de respuestas desde la ciencia. Hipatia a través de la docencia deja entrever este espíritu investigador, quedando patente no sólo el conocimiento científico de la escuela neoplatónica, sino también sus dudas e incertidumbres, preguntas y supuestos que buscará verificar desde la investigación, desde el acto mismo de pensar, a solas y en colaboración con los estudiantes a los que invita a reflexionar sobre las incertidumbres que busca resolver.

En "El amor tiene dos caras" (EEUU, 1997), dirigida y protagonizada por Barbra Streisand, encontramos fotogramas de interés hacia el tema tratado que relaciona

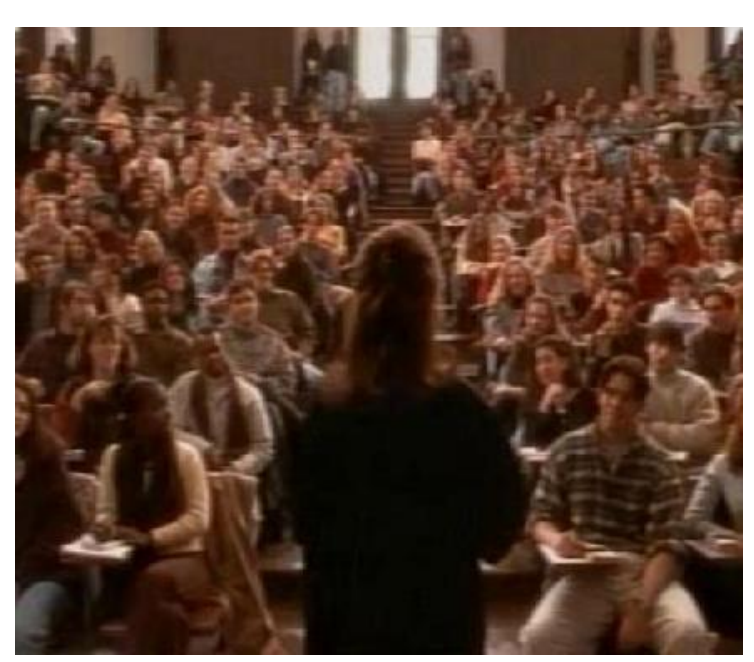
conocimientos y capacidad docente para compartirlos. Rose Morgan es una brillante profesora de literatura en la Universidad de Columbia, y conoce a Gregory Larkin que enseña matemáticas en la misma universidad. Esta comedia romántica de enredo nos deja ver a cada uno de ellos en sus aulas. El aula de Rose está repleta de alumnado. La siguen con la mirada, la escuchan, están hechizados por sus conocimientos, por su conexión al mundo real, cargada de ejemplos tangibles, por su sentido del humor...

Ilustración III: Fotograma de "El amor tiene dos caras" (EEUU, 1997)

En otra aula, el profesor Gregory Larkin deja a su espalda a su escaso alumnado, mirando hacia la pizarra en la que traza símbolos incomprensibles, incapaz de trasmitir su propia pasión por las matemáticas, de posibilitar el aprendizaje.

Es el método lo que está en tela de juicio y es el alumnado el que lo evalúa. El paso que pretende el proceso de Bolonia del instruction paradigm al learning paradigm, conceptos planteados por Barr y Tagg ya en 1995. 
En la misma línea se representa la figura del profesor en el film de Alan Parker "La vida de David Gale" (EEUU, 2003). Un universitario de Texas increpa a su nutrido alumnado, les atrapa en su oratoria, les invita a la reflexión: "venga, pensad, quiero que me digáis, que nos digáis a todos con qué fantaseáis, ¿con la paz mundial? (silencio). Lo imaginaba (ríen). Fantaseáis con la fama internacional (aplausos), fantaseáis con poder ganar un premio pulitzer o el premio nobel de la paz o un premio musical de la MTV, fantaseáis con poder conocer algún genio...".Y cita a Lacan para mostrar como la permanente ausencia de los objetos deseados es requisito del deseo: "No es eso lo que deseas, sino la fantasía de eso.". De nuevo conocimiento y conexión con el alumnado aparecen como pruebas fehacientes de la calidad docente.

El cine parece encontrar unanimidad sobre qué es un buen profesor o profesora en el espacio universitario. Seduce a través del discurso, con elocuencia, lucidez y una cierta dosis de humor, a un alumnado que abarrota sus aulas, responde a preguntas, argumenta, ríe, toma notas, se entrega, al fin, a su autoridad en un conocimiento inédito. Si bien es indiscutible que sin formalizar los problemas referidos al conocimiento no existe discurso riguroso sobre la enseñanza, y mucho menos en los niveles de especialización que implica la enseñanza universitaria. Una visión integral de los procesos de enseñanza-aprendizaje requiere una revisión profunda de la actividad, de los procesos, de los métodos, de los agentes.

Por otra parte, se alude también a profesorado que investiga, en su búsqueda del saber, de respuestas a viejos o nuevos enigmas, aunque mayoritariamente se tiende a disociar la investigación de la docencia en el cine. En el film "El club de los poetas muertos" (Weir, EEUU, 1989) vemos el rol del profesor que conjuga la transmisión de lecciones de vida con la pasión por la poesía. Del otro lado, la película dirigida por Ron Howard "Una mente maravillosa" (EEUU, 2001) nos aproxima a la investigación, a las ciencias matemáticas. Narrativas cinematográficas que no distan de algunas de las propuestas actualmente sometidas a debate relativas a la separación de roles académicos que diferencien profesionales de la docencia y de la investigación.

Sin embargo nuestro profesor en "Educando a Rita", interpretado por Michael Caine, no nos recuerda a ninguna de las películas citadas. La cinta se inicia en una clase de literatura. El Dr. Bryant, ajeno a las demandas del alumnado, observa el exterior del aula a través de su ventana. Se detecta fácilmente su inteligencia, e incluso su honestidad, pero también el desinterés hacia su alumnado. El alcohol ha debilitado los vínculos con su práctica y se traduce en aburrimiento e ironía. Sirve de ejemplo una de las escenas iniciales de la película en la que se dirigía a Rita en su primera entrevista en la universidad y exponía su versión sobre su propia profesionalidad con el objetivo de que la alumna escogiera otro tutor: "Entre Ud. y yo y las paredes..., de hecho soy un profesor atroz. Eso es casi todo el tiempo...Mi atroz enseñanza combina con mis atroces alumnos.".

En ocasiones recuerda a Walter Vale, un profesor gris interpretado por Richard Jenkins en "The visitor" (McCarthy, EEUU, 2007). Este film muestra a través de las aulas universitarias la tristeza de la cotidianidad, el hastío que le produce al viejo profesor la enseñanza, la investigación. Film inquietante que se aleja de las aulas para mostrar dónde late la vida. 
A diferencia de esta película, "Educando a Rita" posibilita el reencuentro del Dr. Bryant con su motivación por la enseñanza. Seducido por la frescura de esta estudiante, Rita, comienza a realizar esfuerzos de conexión, a posibilitarle el acceso a saberes literarios, a convertirse en un comunicador, en un divulgador de la sabiduría que se le presupone. El académico se reencuentra a través de Rita con su propio conocimiento. Es ajeno a la mirada habitual del profesorado universitario, que tiende más a lamentar la precariedad de la preparación cultural con la que llega el alumnado que acude a la institución que a tomar conciencia de la parte de responsabilidad indirecta que le compete. Sin embargo, no llega a convertirse en un intelectual comprometido con la comunidad a la que pertenece, tal vez por sus dudas sobre el calado de ese conocimiento, sobre el beneficio que podría acarrear poner dicho conocimiento a disposición de la sociedad.

El profesor desdeña el marco institucional que propone la Open University, lo cuestiona, transgrede sus normas y es, en varias ocasiones, gravemente amonestado. Sin embargo traslada a su alumna todas las oportunidades formativas y evaluativas que esta institución ofrece, posibilitando la acreditación de sus nuevos aprendizajes y la apertura a espacios profesionales y de estudio. Pero será su alumna la que le obligue a reflexionar sobre el calado del trabajo que desarrolla como mentor, sobre el efecto que ha tenido en sus avances, como posibilitador de una toma de decisiones crítica sobre su proyecto profesional y de vida. Se produce un proceso en el que la autoridad que ejerce el docente se debilita en el tiempo en la medida en que será la alumna la que va ampliando el control sobre su aprendizaje.

El Espacio Europeo de Educación Superior (EEES) propone una construcción del conocimiento próxima a esta idea en la que la mediación se dirige hacia un aprendizaje autónomo y profundo que tiende a reducir paulatinamente la directividad del profesorado en beneficio del alumnado (Zabalza, 2011). Si bien la nueva Ley Orgánica 4/2007 de Universidades no ha redefinido las funciones dispuestas en el artículo primero de la LOU para el profesorado, que siguen siendo: (a) La creación, desarrollo, transmisión de la ciencia, la técnica y la cultura; y (b) La preparación para el ejercicio de actividades profesionales que exijan la aplicación de conocimientos y métodos científicos y para la creación artística. La masificación, el excesivo número de créditos por curso y la falta de formación son, entre otros, motivos de peso que obstaculizan los objetivos de Bolonia.

Este rol docente en revisión nos lleva a preguntas inevitables sobre los métodos en la educación a distancia y en el aprendizaje de personas adultas. Regresando al film, se analizan ambientes educativos híbridos.

\section{La mentorización y los medios}

Los ambientes híbridos de aprendizaje son aquellos que combinan la formación presencial con formación mediada por las tecnologías de la información y de la comunicación (Reay, 2001; Young, 2002). Las secuencias de aprendizaje que se generan responden a realidades espacio-temporales diversas: presencial, virtual y autónoma (Osorio y Duart, 2011). Estos "lugares" de aprendizaje responden a una necesidad creciente de flexibilidad en la esfera universitaria, especialmente cuando 
ésta asume entre sus objetivos la extensión a la comunidad. Si cualquier estudiante universitario se encuentra en la mayoría de los casos con un nuevo escenario educativo: nuevos compañeros y compañeras, nuevas formas de aprender, nuevo profesorado; la situación en el caso de las transiciones tardías se agrava debido a que las referencias institucionales educativas previas están muy alejadas en el tiempo y en muchos casos son referentes de fracaso o de abandono temprano; así como por los conocimientos previos disponibles, que distan de las enseñanzas comúnmente consideradas universitarias.

La tutoría universitaria cobra por ello un papel especial al proporcionar la orientación en el desarrollo personal, académico y profesional del alumnado desde un proceso de enseñanza-aprendizaje personalizado (González, 2006; Herrera, 2011). EI afiche de la película permite apreciar la idea del tutor como sostén y como guía para el crecimiento. El Dr. Bryant propone lecturas, evalúa los trabajos, sugiere actividades, lanza preguntas...

Las relaciones personales que se establecen entre el profesorado y el alumnado, así como con la institución, son sin duda elementos cruciales para la mejora de la calidad que ofrece el sistema universitario (Guardia, 2000; Rico, Defior, Sánchez y Coriat, 2001; Rué, 2004; Herrera, 2011). Las tutorías implican un rol profesional capaz de crear entornos de aprendizaje complejos en los que el alumnado participa activamente en su proceso de enseñanza aprendizaje a través de estrategias y actividades (Zabalza, 2003, Herrera, 2011) puestas al servicio del acceso a un conocimiento sustancial y valioso.

Pero podríamos aportar un paso más allá de la tutorización, aludiendo a la mentorización. La mentoría, consistente en interacciones establecidas entre una persona con amplia experiencia (mentora) y otra con menor experiencia (mentorizada) tiene el objetivo de desarrollar competencias y capacidades de difícil acceso si no se dispone de la ayuda adecuada (Single y Muller, 1999; Sánchez, Manzano, Rísquez y Suárez, 2011). En el ámbito anglosajón desde los años setenta la mentoría (mentoring) y la mentoría electrónica (e-mentoring) son prácticas habituales utilizadas dentro y fuera de las universidades. En España tiende a identificarse la mentoría con el apoyo y relación entre el alumnado, diferenciándose de este modo del concepto de tutoría que se establece básicamente entre el profesorado y el alumnado (García-Nieto, Oliveros, García-García, Ruíz y Valverde, 2005). Sin embargo recupero el término de mentorización, más liberado de la carga de sumisión de la persona de menor experiencia, que finalmente decide si llevar a término o no los consejos recibidos, de ahí su idoneidad en los estudios superiores.

Encontramos escasos ejemplos de mentorización en la filmografía dedicada a la enseñanza universitaria. Podríamos recordar la cinta "Leones por corderos" dirigida y protagonizada por Robert Reford (EEUU, 2007), en la que interpreta a un profesor universitario de Ciencias Políticas, Stephen Maller. Escenas en su despacho reunido con un alumno brillante que ha dejado de asistir a sus clase, se dirigen a inspirarle, a proponerle dejar de lado la apatía, al compromiso, no sólo político, sino también de vida. Otra película que merece ser citada es "El indomable Will Hunting"(VanSant, EEUU, 1997). Cuenta como un muchacho de los suburbios con un don especial para resolver problemas matemáticos es arrastrado a una encrucijada que le obliga a 
decidir hacia dónde orientar su proyecto de vida. La mentorización es de nuevo la clave.

En "Educando a Rita" se produce una mentorización entre pares cuando la propia Rita participa en el Campus de espacios de reflexión dialógica informales transmitiendo a los estudiantes más jóvenes su propio análisis crítico sobre autores literarios. Pero la más sustancial es la que mantiene el Dr. Bryant con su alumna mostrándole su conocimiento, su pensamiento, pero sin determinismos. La escena en la que el Dr. Bryant alaba una de las producciones de Rita a pesar de distar de los criterios evaluativos universitarios, muestra la disidencia de una alumna adulta, consciente de sus propios objetivos y de cómo acceder a ellos, enfrentando a las valoraciones del profesor su tenacidad y su entrega al estudio. La clarividencia de estas relaciones adultas ofrece oportunidades de aprendizaje bidireccionales. Profesor y alumna se benefician del proceso.

Los films citados y otros que pudiéramos rescatar de la cinematografía tienen una gran influencia en el establecimiento de la memoria colectiva, en la visión crítica que aporta a la pedagogía y la didáctica (Martínez-Salanova, 2010). La producción fílmica en la esfera universitaria introduce narrativas que operan en la apropiación que el público espectador hace del objeto cinematográfico, del espacio textual articulado (Carmona, 2005). En este caso, el cine genera un conocimiento sobre el sentido y significación de la mentorización en el imaginario colectivo.

La educación mediática se erige como otra de las claves en la educación a distancia, complemento posibilitador del proceso que requiere de un aprendizaje sistematizado de sus códigos, lenguajes, discursos y procesos de enseñanza aprendizaje (Aguaded-Gómez, 2011). Sin embargo en esta película el estímulo del aprendizaje reside en la tutorización, o mejor, en la mentorización, en las relaciones establecidas, y no en los medios. La idea de red se promueve en "Educando a Rita" desde la presencialidad, en su entorno, con los desplazamientos físicos a las universidades, con los contactos generados entre iguales y con el profesorado. Las redes de aprendizaje no se limitan a lo que actualmente se consideran entornos de aprendizaje en línea dirigidos a facilitar a los participantes el desarrollo de sus competencias colaborando y compartiendo información. Parten de una red física, de contacto permeable con el entorno, que podría, y sin duda debería, complementarse con un entramado digital.

En palabras de Benkler (2009) se evidencia que las instituciones de educación superior deben concentrarse en convertir en permeables las fronteras entre las universidades, así como entre las universidades y el mundo más allá de ellas. La política de la Unión Europea en el ámbito de la enseñanza superior tiene como objetivo principal elevar al $40 \%$ la proporción de personas de entre treinta y cuarenta años con estudios superiores en 2020, tal y como se expresa por la Comisión Europea (2010) en la Estrategia Europa 2020. Esta ampliación de la formación requiere de la creación de entornos abiertos de aprendizaje favorecidos por redes de comunicación. En esta línea, se ha puesto en marcha el sistema europeo de transferencia de créditos (ECTS), con objeto de reconocer los estudios realizados y facilitar la movilidad de los estudiantes y el profesorado a través de programas como el Erasmus o el Leonardo. "e-Europe" es también una iniciativa que ha potenciado desde 1999 el desarrollo de un campus virtual entre estudiantes e investigadores en las universidades europeas. 
En el marco español encontramos desde 1972 la Universidad Nacional de Educación a Distancia (UNED). Uno de sus objetivos básicos desde sus inicios ha sido favorecerla igualdad de oportunidades en el acceso a la formación universitaria tanto inicial como permanente (García-Aretio, 2006). Para ello se crearon centros regionales desde los que el profesorado tutoriza y asesora al alumnado. Al uso de la radio y la televisión como sistemas de emisión de contenidos se añaden, en los años noventa del pasado siglo las nuevas tecnologías. La incorporación de sistemas multimedia, tanto en la elaboración de materiales como en su distribución, se hace extensiva a todas las disciplinas.

La Universitat Oberta de Catalunya (UOC) apostó por las posibilidades emergentes que ofrecían las tecnologías de la información y la comunicación, por romper las barreras espacio-temporales que aproximan a la ciudadanía a un modelo educativo constructivista basado en la personalización y el acompañamiento integral (Santacana, 2006). Tecnologías que se utilizan como medio que complementa la enseñanza presencial, manteniendo el contacto directo entre estudiantes y profesorado.

Estas redes físicas y virtuales del aprendizaje ubican al alumnado en una posición más activa y participativa, si bien la revisión de su rol en las universidades requiere reparar en conceptos como inclusión y ciudadanía.

\section{El alumnado adulto universitario}

Carecer de educación implica la exclusión de la participación social. Estar o no educado, ser o no instruida, se convierte en la actualidad en la llave que hace posible el ejercicio de una ciudadanía democrática efectiva. La democratización del acceso a las universidades implica la diversificación de la procedencia de sus estudiantes: culturas, expectativas, ideologías, creencias diversas cohabitan en la esfera universitaria. La escasa vertebración del alumnado evidencia identidades y subjetividades claramente diferenciadas y el profesorado ha de responder a éstas.

Si nos situamos en los inicios de la película "Educando a Rita", una mujer adulta, carente de los modales y del registro lingüístico que habitualmente se atribuye a la buena educación, acude por primera vez a la universidad para conocer a su tutor. Se presenta como Rita, en honor a Rita Mae Brown ${ }^{1}$, aunque en su solicitud la inicial corresponde al nombre de Susan. Es curioso, los nombres propios, por su naturaleza designativa, tienen una referencia singular, pero carecen de significado lingüísticamente construido. La visión del yo, lejos de ser homogénea y estable se define por su carácter distribuido y dialógico. Esta película ilustra la narrativa del yo (autobiográfica) asociada a la experiencia educativa. Nos invita a reflexionar sobre el papel de la educación superior en el proceso de construcción cultural de la identidad personal. Se trata de una construcción discursiva que está mediada, como todos los relatos, por instrumentos semióticos (Santamaría y Martínez, 2005) de los que Rita se apropia a lo largo de su proceso de transformación, evidenciándose no sólo en las acciones que argumentan la acción, también en el uso que hace del lenguaje y da cuenta de su conciencia, de la transformación simbólica. Rita le pregunta a su profesor en su primera entrevista: "¿Cuándo, ya sabe, cuándo empezará a enseñarme algo?". 
Frente a lo que el profesor Bryant le manifiesta dudas: "¿Qué puedo enseñarle?". Su respuesta fue sencilla, o tal vez no tanto: "Todo".

Resulta inevitable recordar "Pygmalion" (Reino Unido: 1938), film dirigido por Anthony Asquith y Leslie Howard e inspirado en la obra de teatro homónima de Bernard Shaw "My Fair Lady" en el que el profesor Higgins, experto en fonética, realiza un experimento con Elisa, una florista callejera. La convertirá a través de la educación de la voz y de la revisión de sus modales en una aparente dama de la alta sociedad, su dicción ya no identificará su origen social. Al tratarse de un experimento, una vez finalizado la vendedora de flores debe regresar al lugar de origen. Pero Elisa encarna una mujer fuerte, autónoma y tenaz que no acepta el servilismo y desea aprender.

El cambio que se produce es comparable a la transformación de Rita en una mujer culta, con capacidad crítica para la toma de decisiones. Esta evolución queda expresada en las escenas finales de la película en la que Rita reconoce el mérito de su profesor: "Gracias a lo que tú me has dado pude elegir". Sin embargo, recupera su nombre original, Susan, dotando de valor sus experiencias previas, en un constante proceso de resignificación de la identidad. Atribución de significado como acto social, sujeto al poder que le ha aportado la educación, pero también sus propias experiencias de vida.

Ya es una evidencia que estudiantes de las universidades actuales no tienen garantizada su proyección laboral. El desempleo tiende a despertar un clima competitivo y a situar sus intereses más allá del debate, de la colaboración, del aprendizaje; dirigiendo sus esfuerzos a nutrir su expediente con títulos atractivos para el mercado de trabajo antes que hacia la adquisición de conocimientos sustanciales. La educación integral de y para la formación de una ciudadanía que desarrolle identidades autónomas y reflexivas con su propio sentido de lo social, como misión de las universidades, resulta menos evidente, repercutiendo en el papel que traza el alumnado en ellas.

Narrativas cercanas a la visionada en la película podemos encontrarlas entre el alumnado que accede a través de pruebas destinadas a mayores de 25 o de 45 años, o en las universidades de mayores, también en los cursos de extensión universitaria. Una aportación significativa en esta línea es la investigación que presenta GonzálezMonteagudo (2010). Desde un enfoque biográfico y narrativo se entrevista a estudiantes universitarios con dificultades para desarrollar y concluir sus estudios ${ }^{2}$.La lectura del caso de Laura permite analizar la transformación de su identidad como consecuencia de los estudios universitarios. El aumento de su confianza, de su capacidad de relación con personas que poseen un nivel académico alto.

Podemos preguntarnos si debería preocupar a la universidad las condiciones en las que se producen las transiciones de colectivos vulnerables. ¿Es la universidad un espacio accesible a estos colectivos? ¿Qué requieren los planes de estudio para posibilitar estas transiciones? 


\section{La extensión universitaria: gran olvidada en los planes de estudio}

En los años setenta del pasado siglo se reclamaba una universidad de masas, es decir, aquella que permite el acceso de gran parte de la población, superando la imagen de universidad restringida a las élites sociales y económicas. Este proceso de evolución de las universidades se hace posible gracias a la Ley General de Educación (1970) que estructuró la educación universitaria en tres ciclos, incluyendo a las Escuelas Universitarias, lo cual representó un paso adelante hacia la ampliación y democratización del acceso al espacio universitario. Supuso un considerable aumento de la diversidad de la oferta universitaria y la adaptación a las nuevas necesidades de la sociedad y economía españolas (ICED, 1987).

En la actualidad las universidades españolas dirigen su mirada a Europa. Los espacios de convergencia en el ámbito educativo que se están propiciando en los Estados miembros de la Unión Europea y Estados asociados a ella, favorecen la mejora del proceso de reconocimiento de titulaciones con objeto de facilitar la movilidad del alumnado universitario, así como la integración de los títulos en un mercado laboral único. Entre los objetivos más relevantes de la Declaración de Bolonia se encuentra: (1) la armonización de los sistemas nacionales de titulaciones basada en los ciclos de grado y postgrado; (2) el establecimiento de un sistema de créditos europeo, el ECTS; y (3) la implantación de un suplemento europeo ${ }^{3}$ a los títulos emitidos por las instituciones educativas de enseñanza superior. El profundo esfuerzo que representa propiciar la convergencia de los planes de estudio podría hacernos olvidar la extensión universitaria, espacio de compromiso con la ciudadanía, de vínculo con la sociedad, mostrado con claridad en la Open University a través de la narrativa fílmica de "Educando a Rita".

Los planes de estudio en el ámbito nacional responden a dos preceptos constitucionales: la autonomía universitaria, recogida en el artículo 27, y la competencia estatal reguladora de las condiciones de obtención, expedición y homologación de los títulos universitarios, presente en el artículo 149. Se trata por tanto de un autogobierno limitado, de una autonomía tutelada que participa en la elaboración de planes de estudio en los que el Gobierno de la Nación determina qué se enseña y las universidades deciden cómo se organiza ese conocimiento dentro de las directrices generales comunes a los planes de estudio (Infante, 2010). Siguiendo a Zabalza (2011), los procesos de cambio en la educación superior responden a cuatro fases en la toma de decisiones con un preciso y diferenciado orden jerárquico: (1) los poderes políticos a través de la norma; (2) las Universidades, supeditadas en el proceso seguido con Bolonia a los recursos disponibles; (3) los centros académicos (Facultades, Escuelas Técnicas, etc.) en los que se configuran los Planes de Estudios, los horarios, los periodos de prácticas, las tutorías, la evaluación, etc.; y (4) el profesorado, que tiene que reconocer por primera vez niveles de decisión superiores al individual. La toma de decisiones se jerarquiza, se hace más compleja, se amplifican los espacios de reflexión en la esfera universitaria, pero también de crítica y desacuerdo con decisiones asumidas en otro plano jerárquico.

La sociedad demanda de las instituciones de Enseñanza Superior fórmulas para abrir la Universidad a grupos de personas que solicitan sus servicios sin la formación previa requerida (Pérez, 2006). Sirva de ejemplo la enseñanza universitaria de las 
personas mayores, hecho relativamente reciente, como lo demuestran las investigaciones sobre el tema y el gradual aumento de Programas Universitarios de Mayores en España desde 1990 (Martín, 2007; Orte, 2006; Requejo, 2009).

Las condiciones de acceso a los estudios universitarios oficiales se regulan a través del Real Decreto 1892/2008 que, en su capítulo V, articula criterios para las personas que hubieran cumplido 25 años de edad, las que hubieran cumplido 40 años y acrediten una determinada experiencia profesional o laboral, así como las personas que hubieran cumplido 45 años. Pero en ningún caso se incorpora en los planes de estudio universitarios procesos que favorezcan estas transiciones. La propuesta queda limitada a pruebas de acceso que conllevarán, de ser superadas, la participación en igualdad de condiciones que el resto del alumnado en el plan de estudios. Las políticas educativas a través de la norma restringen cualquier posibilidad favorecedora del acceso en las propias universidades. Por tanto es ya la primera fase citada por Zabalza la que aleja a las universidades españolas de una experiencia como la propuesta por la Open University. El impacto de la norma es tradicionalmente escaso en la práctica pero sí tiene un profundo efecto limitador en términos de exclusión: qué cosas no se pueden hacer, no son objeto de financiación. El relato de este artículo queda de este modo excluido de las opciones curriculares de los actuales planes de estudio en las universidades españolas.

\section{Conclusiones}

La narrativa cinematográfica, el lenguaje audiovisual, pueden convertirse en instrumentos de estudio en la representación de la memoria colectiva. Patrimonio cultural que recoge, interpreta, recrea espacios educativos. Este artículo se ha situado en la esfera universitaria tomando como eje un film paradigmático. Se dice de "Educando a Rita" que es un viaje hacia el autodescubrimiento, un cuento de hadas con final abierto, un documental social. Este film entiende la educación superior como algo más que una preparación propedéutica dirigida a satisfacer las demandas del mundo laboral, valora la vida humana por encima de la ciencia. Contrasta con la orientación de las universidades españolas del siglo XXI en las que se cuestionan las funciones básicas que definiera Ortega y Gasset (1975): formar para profesiones intelectuales, cultivar y preparar para la investigación, sin olvidar la transmisión de la cultura general. Esta visión humanista de las universidades, volcadas hacia el saber desinteresado, hacia un conocimiento valioso por sí mismo, se reconduce en la actualidad bajo orientaciones más pragmáticas, hacia la transmisión de un conocimiento al servicio de profesiones cualificadas, enfatizando en la experiencia, en el control sobre los procesos y en los resultados obtenidos. Ortega y Gasset (1975) alerta sobre este pragmatismo identificando al profesional formado en la universidad con el nuevo bárbaro, más sabio que nunca, pero más inculto también. Sin embargo ese profesorado universitario erigido tradicionalmente como fuente de transmisión y de saber ya es caduco. La acción, la actividad, los procesos, la didáctica entendida como técnica de enseñanza, las tecnologías educativas, dejan de estar relegadas a un segundo plano. Sin embargo, en esta convivencia en la que todavía se busca el equilibrio, la universidad de masas no puede obviar la significación del efecto "civilizador" del saber universitario. Algunas universidades españolas ya introducen en 
sus programas una contradicción al mostrar una oferta de titulaciones de grado y postgrado profundamente especializadas que no responden a las cambiantes actividades laborales de la sociedad actual, cada vez más compleja, priorizando hacia el estudio de profesiones minoritarias, cursadas por "mayorías". Una menor especialización en pro de un modelo curricular más abierto, dirigido a fomentar en el estudiantado conocimientos más generales, aproximaría a la idea de formación a lo largo de la vida, a la inclusión, al compromiso con la comunidad, con la sociedad democrática. Este papel de las universidades se trasladaría en sus ofertas, en su funcionamiento y en la delimitación de la profesionalidad docente (Gimeno, 1996).

Los requerimientos de la sociedad del conocimiento solicitan una importante reforma de nuestro sistema de formación superior con objeto de fomentar el desarrollo del aprendizaje a lo largo de la vida. La flexibilidad se vería favorecida por elementos ya disponibles en esta película: mentorización; currículum más generalista, coincidiendo con la propuesta del EEES al definir cinco ramas de conocimiento con materias básicas comunes a todos los grados adscritos a ella; construcción e intercambio de conocimiento en entornos potenciados por la tecnología y por el contexto físico; o la apertura de espacios en los planes de estudio favorecedores de transiciones tardías. El análisis de esta producción fílmica aporta sin duda elementos de interés en el debate abierto de la reforma universitaria. Elementos que podrían transformarse en acciones desde la introducción de estas reflexiones en el debate relativo al diseño y revisión de los planes de estudios.

\section{Notas}

1 Prolífica escritora estadounidense, conocida por sus novelas de misterio y de otros géneros. Adicionalmente, como guionista ha sido nominada al premio Emmy. También se la conoce por haber sido una comprometida activista feminista y a favor de los derechos LGBT.

${ }^{2}$ Se trata de un Proyecto Leonardo, Acces and Retention: Experiences of Non-traditional Learners in Higher Education, enmarcado en el tópico "Promoción de la excelencia, la eficiencia y la equidad en la enseñanza superior".

${ }^{3}$ Documento que añade información al título obtenido mediante una descripción de su naturaleza, nivel, contexto y contenido.

\section{Referencias Bibliográficas}

Aguaded-Gómez, J.I. (2011). La educación mediática, un movimiento internacional imparable. La ONU, Europa, España apuestan por la educomunicación. Comunicar, 37, XIX, 7-8. DOI. Recuperado de http://dx.doi.org/10.3916/C372011-01-01

Barr, R. y Tagg, J. (1995). From Teaching to Learning. A new paradigm for undergraduate education. Change (November-December, 1995), 13-25. Recuperado de http://ilte.ius.edu/pdf/BarrTagg.pdf

Benkler, Y. (2009). The Tower and the Cloud: Higher Education in the Age of Cloud Computing. In R. KATZ, (Ed.). The University in the Networked Economy and Society: Challenges and Opportunities. Educause; 51-61. 
Bolívar, A. (2009). La planificación por competencias en la reforma de Bolonia de la educación superior: un análisis crítico. Educaçao Temática Digital, Campinas, 9, n. esp., 68-94. Recuperado de http://www.fe.unicamp.br/revista/index.php/etd/article/viewArticle/1702

Bruner, J. (2003). Self-making narratives, en R. Fivush y C.A. Haden (Eds.), Autobiographical memory and the construction of a narrative self. Developmental and cultural perspectives. Mahwah, NJ: Lawrence Erlbaum Associates.

Carmona, R. (2005). Cómo se comenta un texto fílmico. Madrid: Cátedra.

Clarembeaux, M. (2010). Educación en cine: memoria y patrimonio. Comunicar, no 35, v. XVIII; 25-32. Recuperado de: www.revistacomunicar.com/verpdf.php?numero=35\&articulo $=35$. .

Comisión Europea (2010). Comunicación de la Comisión Europa 2020. Una estrategia para el crecimiento inteligente, sostenible e integrador. Bruselas 3-3-2010. COM 2020.

Declaración de Bergen (2005). El espacio europeo de educación superior. Alcanzando las metas. Comunicado de la Conferencia de ministros europeos responsables de educación superior. Bergen, 19-20 de mayo de 2005. Recuperado de http://www.eees.es/pdf/Bergen ES.pdf

Declaración de Berlín (2003). Educación Superior Europea. Comunicado de la Conferencia de Ministros responsables de la educación superior. Berlín, 19 de septiembre de 2003. Recuperado de http://www.eees.es/pdf/Berlin ES.pdf

Declaración de Bolonia (1999). El espacio Europeo de la enseñanza superior. Declaración conjunta de los ministros europeos de educación reunidos en Bolonia el 19 de junio de 1999. Recuperado de http://www.eees.es/pdf/Bolonia ES.pdf

Declaración de La Sorbona (1998). Declaración conjunta para la armonización del diseño del Sistema de educación Superior europeo (a cargo de los cuatro ministros representantes de Francia, Alemania, Italia y el Reino Unido). La Sorbona, París, 25 de mayo de 1998. Recuperado de http://www.eees.es/pdf/Sorbona ES.pdf

Declaración de Praga (2001). Hacia el área de la Educación Superior Europea. Declaración del encuentro de los ministros europeos en funciones de la educación superior en Praga, 19 de mayo de 2001. Recuperado de http://www.eees.es/pdf/Praga ES.pdf

Education Act,(1944). 7\&8 GEO. 6. CH. 31. Recuperado de http://www.educationengland.org.uk/documents/pdfs/1944-education-act.pdf

FilmAffinity. Recuperado de http://www.filmaffinity.com/es/film741389.html

Fueyo, A. (2004). Evaluación de titulaciones, centros y profesorado en el proceso de Convergencia Europea ¿de qué calidad y de qué evaluación hablamos? Revista interuniversitaria de formación del profesorado, nำ51, 207-220. 
García-Aretio, L. (2006). La Universidad Nacional de Educación a Distancia (UNED) de España. AIESAD, 9, 1 y 2, 17-51.

García-Nieto, N.; Oliveros, L.; García-García, M.; Ruíz, C.; Valverde, A. (2005). La Mentoría. Una experiencia con estudiantes de la Universidad complutense. Madrid: ICE, Universidad Complutense.

Gijón, J. y Crisol, E. (2012). La internacionalización de la Educación Superior. El caso del Espacio Europeo de Educación Superior. Revista de Docencia Universitaria. REDU. Monográfico: Buenas prácticas docentes en la enseñanza universitaria. 10 (1), 389-414. Recuperado de http://redaberta.usc.es/redu

Gimeno-Sacristán, J. (1996). La profesionalidad escindida de los profesores en la universidad, en G. Quintás, Reforma y evaluación de la universidad. Valencia: Guada.

González, I. (2006). Dimensiones de evaluación de la calidad universitaria en el Espacio Europeo de Educación Superior. Electronic Journal of Research in Educational Psicology, 4, 3, 445-468.

González-Monteagudo, J. (2010). Biografía, identidad y aprendizaje en estudiantes universitarios no tradicionales. Estudio de caso de una mujer trabajadora. Revista de currículum y formación del profesorado, 14, 3, 131-147.

Guardia, J. (2000). La gestión de las acciones de orientación universitaria: Una cuestión de estructura, en H. Salmerón; V.L. López (Coord.), Orientación educativa en las Universidades, 99-106. Granada: Grupo Editorial Universitario.

Herrera Torres, L. (2011). Orientación, tutoría y mentorización en educación Superior: una labor destinada tanto al alumnado como al profesorado universitario. DEDICA. Revista de Educaçao e Humanidades, 1, 425-452.

Hurtado, J. y Jardón, P. (2011). El cinema a l'educació. Alzira: Germanía.

ICED (1987). La reforma universitaria española. Evaluación e informe. Madrid: Consejo de universidades.

Infante, J. (2010). La reforma de los planes de estudio universitarios de la España democrática (1977-2000). Revista de Educación, 351, 259-282.

International Council for Educational Development (1987). La reforma universitaria española. Evaluación e informe. Madrid: Consejo de Universidades. Secretaría General.

Kemmis, S. (1993). El currículum, más allá de la teoría de la reproducción. Madrid: Morata.

Ledesma, N.; García-Raffi, X. y Hernández, F.J. (Ed.) (2010). Per a una pedagogia de la ficció audiovisual. Alzira: Germanía.

Leuven and Louvain-la-Neuve Communiqué (2009). The Bologna Process 2020. The European Higher education area in the new decade. Communique of the conference of European ministers responsible for Higher Education. Leuven and Louvain-la-Neuve, 28-29 April 2009. Recuperado de http://www.eees.es/pdf/Leuven Louvain-laNeuve Communique April 2009.pdf 
Ley 14/1970, de 4 de agosto, General de Educación y Financiamiento de la Reforma Educativa (BOE 6-8-1970).

Ley Orgánica 6/2001, de 21 de diciembre, de Universidades (BOE 24-12-2001).

Ley Orgánica 4/2007, de 12 de abril, por la que se modifica la Ley Orgánica 6/2001, de 12 de diciembre, de Universidades (BOE 13-04-2007).

London Communiqué (2007). Towards the European Higher education area: responding to challenges in a globalised world. London, 18 May 2007. Recuperado de http://www.eees.es/pdf/London Communique18May2007.pdf

Martín, A.V. (2007). Gerontología educativa: enquadramento disciplinar para o estudo e intervençao socioeducativo com idosos. En A. Requejo y F. Cabral, As pessoas idosas: contexto social e intervençao educativa, 47-73. Lisboa: Editorial Horizontes Pedagógicos.

Martínez-Salanova, E. (2010). Los sistemas educativos en la memoria heterodoxa del cine europeo. Comunicar, 35, XVIII, 53-60.

Orte, C. (2006). El aprendizaje a lo largo de toda la vida. Los programas universitarios de mayores (pp.263-311). Madrid: Dykinson.

Ortega y Gasset, J. (1975). Misión de la universidad y otros ensayos afines. Madrid: El Arquero.

Osorio, L.A.; Duart, J.M. (2011). Análisis de la interacción en ambientes híbridos de aprendizaje. Comunicar, 37, XIX, 65-72.

Pérez, G. (2006). Estereotipos, vejez y bienestar social. En G. Pérez (Coord.), Calidad de vida en personas mayores, 51-75. Madrid: Dykinson.

Real Decreto 1393/2007, de 29 de octubre, por el que se establece la ordenación de las enseñanzas universitarias oficiales (BOE 30-10-07).

Real Decreto 1892/2008, de 14 de noviembre, por el que se regulan las condiciones para el acceso a las enseñanzas universitarias oficiales de grado y los procedimientos de admisión a las universidades públicas españolas (BOE 24-112008).

Reay, J. (2001). Blended Learning a fusion for the future. Knowledge Management Review, 4, 3, 6 .

Requejo, A. (2009). La Educación de "Personas Mayores" en el Contexto Europeo. Revista Electrónica de Educación y Formación, 3, 45-63.

Rico, L.; Defior, S.; Sánchez, A.; Coriat, M. (2001). Calidad de la enseñanza en la Universidad de Granada. Revista de Currículum y formación del profesorado, 5, 2, 1-20. Recuperado en http://www.ugr.es/ recfpro/rev52ART1.pdf

Rué, J. (2004). La convergencia europea: entre decir e intentar hacer. Revista interuniversitaria de Formación del Profesorado, 18, 1; p. 39-59.

Sánchez, M.; Manzano, N.; Rísquez, A. y Suárez, M. (2011). Evaluación de un modelo de orientación tutorial y mentoría en la educación superior a distancia. Revista de Educación, 356, 4, 719-732. 
Santacana, T. (2006). El model pedagògic de la universitat oberta de Catalunya (UOC): una visió des de l'aula. Coneixement i Societat: Revista d'Universitats, Reserca $i$ Societat de la Informació, 10, 56-71.

Santamaría, A. y Martínez, M. (2005). La construcción de significados en un marco de la Psicología Cultura del pensamiento narrativo, en M. Cubero y Ramírez, Vygotsky en la Psicología Contemporánea. Buenos Aires: Miño y Dávila.

Single, P.B., Muller, C.B. (1999). Electronic mentoring: Issues to Advance Research and Practice. Annual meeting of the International Mentoring Association, Atlanta, 1517 de abril.

Wiltshire, H.C. (1973). La Universidad Abierta. Boletín del Centro de Documentación Patronato de Obras Docentes del Movimiento, no 46. Madrid.

Yung, J.R. (2002). Hybrid Teaching Seeks to End the Divide between Traditional and Online Instruction. Chronicle of Higher Education; 48 (28); A33.

Zabalza, M.A. (2003). Competencias docentes del profesorado universitario. Calidad y desarrollo profesional. Madrid: Narcea.

Zabalza, M.A. (2011). Metodología docente. Revista de Docencia universitaria. REDU. Monográfico: El espacio europeo de educación superior. ¿Hacia dónde va la Universidad Europea? 9, 3, 75-98. Recuperado en http://redaberta.usc.es/redu

\section{Filmografía}

Amenábar, A. (Director). (2009). Ágora [Película]. Estados Unidos, España: Himenóptero, Telecinco, Telecinco Cinema, Cinebiss, Mod Producciones.

Asquith, A.; Howard, L. (Directores). (1938). Pigmalion (Pygmalion) [Película]. Reino Unido: Pascal Film Productions.

Gilbert, L. (Director). (1983). Educando a Rita (Educating Rita) [Película]. Reino Unido: Acorn Pictures.

Howard, R. (Director). (2001). Una mente maravillosa (A Beautiful Mind) [Película]. Estados Unidos: Dreamworks / Universal Pictures / Imagine Entertainment.

McCarthy, T. (Director). (2007). The Visitor [Película]. Estados Unidos: Overture Films / Groundswell Productions / Participant Productions.

Parker, A. (Director). (2003). La vida de David Gale (The Life of David Gale) [Película]. Estados Unidos, Alemania: Universal Pictures, Intermedia Films, Saturn Films, Dirty Hands Productions, Mikona Productions GmbH \& Co. KG.

Redford, R. (Director). (2007). Leones por corderos (Lions for Lambs) [Película]. Estados Unidos: United Artists.

Streisand, B. (Director). (1997). El amor tiene dos caras (The Mirror Has Two Faces) [Película]. Estados Unidos: Columbia Pictures Corporation, Barwood Films, Phoenix Pictures.

Van Sant, G. (Director). (1997). El indomable Will Hunting (Good Will Hunting) [Película]. Estados Unidos: Miramax Films, Lawrence Bender Productions. 
Weir, P. (Director). (1989). El club de los poetas muertos (Dead Poets Society) [Película]. Estados Unidos: Touchstone Pictures / Silver Screen Partners IV.

Cita del artículo:

Chisvert Tarazona, M.J. (2013). La responsabilidad social de las universidades a través de la memoria cinematográfica. Revista de Docencia Universitaria. REDU. Vol.11 (1) Enero-Abril, pp. 389-410. Recuperado el (fecha de consulta) en http://www.redu.net/

\section{Acerca de la autora}

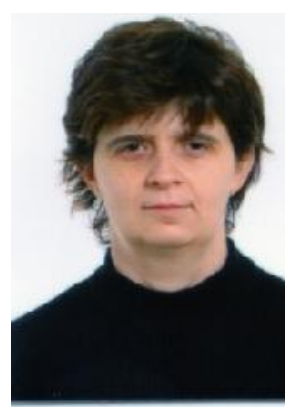

\section{María José Chisvert Tarazona}

Universidad de Valencia

Departamento de Didáctica y Organización Escolar

Mail: Maria.Jose.Chisvert@uv.es

Profesora doctora de la Universidad de Valencia perteneciente al departamento de Didáctica y Organización Escolar. Ha sido docente en la Licenciatura de Pedagogía, en el Grado de Magisterio y en el Máster de Educación Secundaria, dónde enseña Organización y dirección de centros, Didáctica e Innovación Educativa. Participa en el equipo de investigación sobre formación y transiciones entre educación y empleo con colectivos vulnerables dirigido por Fernando Marhuenda. 


\title{
Entorno virtual de aprendizaje compartido en Educación Superior
}

Shared virtual learning environments in higher education

\author{
Margarita R. Rodríguez Gallego \\ Antonia López Martínez \\ Universidad de Sevilla (España)
}

\section{Resumen}

Cada vez son más demandadas las plataformas virtuales apoyadas en el concepto web 2.0 y en las Redes Personales de Aprendizaje (PLN) al garantizar la conversación entre agentes educativos y la colaboración orientada a la producción conjunta de conocimiento.

Desde este punto de vista, surgieron los Entornos Virtuales de Aprendizaje (VLE) dónde se configuran, integran y combinan nuevas aplicaciones adaptadas a las necesidades de los usuarios.

El propósito final de esta experiencia es la creación de un entorno virtual compartido para lograr que el aprendizaje no se vincule exclusivamente a una actividad memorística y que a través del uso combinado de la plataforma virtual, herramientas de la web 2.0 y la red social los estudiantes puedan crear su propio espacio de trabajo en el que quede reflejado la búsqueda y transformación de la información, creación de recursos para el aprendizaje y tareas de colaboración.

Nuestro entorno virtual de aprendizaje compartido ha sido puesto a prueba, implementado y evaluado durante el segundo cuatrimestre, en el marco de la asignatura Didáctica General en la Facultad de Ciencias de la Educación de la Universidad de Sevilla (España) durante el curso académico 2009-2010.

Una encuesta efectuada revela hechos interesantes sobre el éxito de este entorno virtual compartido en relación con la motivación y los resultados de aprendizaje de los estudiantes.

Palabras clave: Entorno Virtual de Aprendizaje (VLE), Plataforma virtual WebCt, Constructivismo, Conectivismo, Redes sociales, Educación Superior, Entorno Personal de Aprendizaje (PLE).

\section{Abstract}

There is an increasing demand for virtual platforms based on the concept of Web 2.0 and on Personal Learning Networks (PLN) as these guarantee conversations between educational agents and cooperation aimed at the joint knowledge production. 
From this point of view, Virtual Learning Environments (VLE) have emerged in which new applications adapted to users' needs are configured, integrated and combined.

The ultimate purpose of this experience is the creation of a shared virtual environment to ensure that learning is not linked exclusively to rote activity. Through the combined use of the virtual platform, Web 2.0 tools and social networking, students can create their own workspace in which the search and processing of information, as well as the creation of learning resources and collaboration tasks are reflected.

Our shared virtual learning environment has been tested, implemented and evaluated in the second semester, as part of the General Teaching Methodology course in the Faculty of Education at the University of Seville (Spain) during the 2009 - 2010 academic year.

A survey reveals interesting facts about the success of the shared virtual environment in relation to the motivation and learning outcomes of students.

Key words: Virtual Learning Environment (VLE), WebCT virtual platform, Constructivism, Connectivity, Social Networks, Higher Education, Personal Learning Environment (PLE).

\section{Marco teórico}

La situación en la que se encuentran actualmente los estudios de Grado en las distintas titulaciones y los continuos avances tecnológicos en el ámbito educativo nos ha llevado a investigar entornos de aprendizaje facilitadores del proceso de formación de nuestros estudiantes. Esta investigación ha sido aprobada en la convocatoria de Innovación y Mejora Docente del Vicerrectorado de Docencia de la Universidad de Sevilla, en el curso académico 2009-2010.

Diferentes estudios realizados sobre e-learning recomiendan que no se deben utilizar plataformas de enseñanza únicamente como depositarias de diferentes objetos de aprendizaje, sino que además de la estructuración de los materiales, de la aplicación de una serie de metodologías y estrategias específicas por parte del profesorado, es conveniente emplear herramientas de comunicación propias de la web 2.0 y redes personales de aprendizaje -PLN- (Jolliffe et al., 2001; Salmon, 2002; Simpson, 2002; Hanna, 2002; Palloff y Pratt, 2003; Coppola et. al., 2003).

En este sentido, siguiendo a Downes (2005), hablamos de la aplicación e-learning 2.0 si los usuarios de la red emplean la web 2.0 multimedia, por ejemplo software social como wikis, weblogs o actividades colaborativas de aprendizaje para producir autónomamente sus propios contenidos de aprendizaje. La característica central del escenario e-learning 2.0 es que los aprendices sean autónomos en la producción de contenidos y en la adquisición de aprendizaje (Fitzgerald, 2006). Un entorno e-learning 2.0 es un indicio de contenidos de aprendizaje que además de contener metadatos y referencias a fuentes online puede también derivar en contenidos realizados por uno mismo o en herramientas en línea aptas para aprender. Para Kerres $(2007$, p.6) "el entorno de aprendizaje debe ofrecer un "mecanismo" para recoger e integrar contenidos y herramientas de una forma orientada a objetivos".

En cuanto al uso de plataformas virtuales, los sistemas LMS (Learning Management System) que vienen utilizándose mayoritariamente en las universidades corresponden a software comercial o a desarrollos propios. Hay que señalar que las universidades han realizado, en muchos casos, grandes inversiones económicas para 
desarrollar software propietario o comprar plataformas de software comercial. Sin embargo, hoy en día esta infraestructura podría convertirse, más que en un activo, en una barrera, pues la mayoría de estas herramientas se han quedado anticuadas, y necesitan configurarse, integrarse y combinarse con nuevas herramientas que están disponibles para crear aplicaciones adaptadas a las necesidades personales de los usuarios. Cada vez son más las plataformas virtuales apoyadas en el concepto de web 2.0 y en las aplicaciones que garantizan la conversación entre agentes educativos y precisan de la colaboración en la gestión del proceso por parte de la institución y de los docentes para la producción conjunta de conocimiento. Atendiendo a los principios de la web 2.0, algunos autores (Downes, 2007; Wilson, 2005) han decidido diferenciar los clásicos Entornos Virtuales de Enseñanza-Aprendizaje (EVEA) de los nuevos espacios de comunicación e interacción para el aprendizaje desarrollados a partir de las nuevas aplicaciones, enfatizando la dimensión personal y social de ésta frente al valor tecnológico de las clásicas plataformas de enseñanza. Este nuevo espacio se conoce como Entorno de Aprendizaje Personal -PLE-.

El uso de estos entornos virtuales de aprendizaje (VLE) y entornos personales de aprendizaje (PLE) se está extendiendo cada vez más en el ámbito universitario. En el Reino Unido, un equipo de la Universidad de Bolton ha desarrollado una aplicación para proporcionar a los estudiantes una plataforma que facilite el acceso a las redes de personas y recursos. En la Universidad de Mary Washington en Virginia, los estudiantes y profesores usan UMW Blogs, una plataforma de publicaciones multiusuario de WordPress personalizada por la Universidad para ofrecer espacios flexibles donde los estudiantes presenten sus trabajos, compartan sus ideas y colaboren en proyectos. Enfoques similares se han utilizado en Baylor University, Penn State y la Universidad de British Columbia. En España, hay muchas Universidades que están apostando por nuevas herramientas tecnólogicas, la Universidad de Sevilla, Córdoba, País Vasco, Universidad Jaume I de Castellón, entre otras. En estos entornos de aprendizaje se ofrece un sitio donde explorar herramientas y servicios libremente, como StumbleUpon, Flickr, YouTube y otros lugares que permitan almacenar información, conectar con sus compañeros y acceder a colecciones de recursos.

Desde este punto de vista, el valor de la web 2.0, plataformas virtuales y redes sociales pueden llegar a una alianza, al poner el énfasis en la creación más que en el consumo, en la descentralización del contenido, proceso de enseñanza-aprendizaje y adquisición de competencias. La idea es proporcionar al estudiante un espacio en la red que le permita desarrollar y compartir sus ideas.

Bajo esta premisa, hemos integrado herramientas de la web 2.0 y la red social en un entorno virtual de aprendizaje compartido. La creación de un entorno personal de aprendizaje (PLE) requiere más preparación tecnológica por los estudiantes y dejar a un lado la plataforma institucional. Los PLE proporcionan una base para el establecimiento de una forma de educación que va más allá de los modelos centrados en el currículo y los cursos, y proponen un modelo de aprendizaje continuo centrado en el estudiante y controlado por el propio usuario. Pues como plantea Lubensky (2006), el PLE requiere algún tipo de instalación o lugar para que un usuario tenga acceso, agregue, configure y manipule los recursos y referencias digitales provenientes de sus experiencias de aprendizaje. Un entorno de aprendizaje es, por lo tanto, algo que nosotros hacemos por nosotros mismos, utilizando nuestras aplicaciones favoritas: 
nuestro blog, wikis, e-porfolio, marcadores sociales, correo web, etc. Más que herramientas o plataformas específicas, los PLE son concebidos como entornos de enseñanza que se sitúan en la intersección entre los VLE, la web 2.0 y el portafolio electrónico como estrategia evaluativa de enseñanza-aprendizaje (Lubensky, 2006). En definitiva, nuestro VLE describe las herramientas, las comunidades y los servicios que constituyen profesores y estudiantes en la plataforma educativa y que se emplea para dirigir el propio aprendizaje y conseguir las metas educativas.

Un VLE típico, por ejemplo, podría incorporar, en la plataforma institucional, los blogs dónde los estudiantes comentan sobre lo que están aprendiendo, y sus comentarios pueden aparecer reflejados en la web, en sitios como YouTube o en los canales RSS de noticias. En los campus que apoyan los VLE, los estudiantes son alentados a recurrir a estas redes y colecciones de recursos externos, para que los utilicen como herramientas y hagan un esfuerzo por ampliar sus experiencias de aprendizaje, más allá de los límites del campus. Conforme se van creando ideas surgen discusiones y el contenido legitimado en este ambiente alimenta la ayuda combinada de compañeros, colegas, amigos, así como expertos y críticos.

La novedad es que en el VLE incorporamos elementos de todas las teorías del aprendizaje. De los enfoques conductista, el estudiante debe encontrar una respuesta dada a uno o varios estímulos presentados en pantalla. A este uso de ordenador se le denomina "Enseñanza Asistida por Ordenador" (EAO), se centra en programas de ejercitación y práctica muy precisos basados en la repetición. De la teoría cognitivista, no solo dar una respuesta, sino resolver problemas, tomar decisiones para conseguir un determinado objetivo y realizar tareas para desarrollar estrategias y capacidades cognitivas. De la teoría constructivista, las ideas subyacentes de la web 2.0, la idea de una web social donde el conocimiento se construye y reconstruye en colaboración con los demás. De la tendencia conectivista, la utilización combinada de distintas herramientas que facilitan el acceso a las fuentes (nodos) del conocimiento.

Así pues, hay una tendencia en aprendizaje contemporánea hacia una mayor actividad, autoproductividad y autonomía, que pretende cambiar el acento en el carácter del aprendizaje del producto frente al proceso. Estos desarrollos están expresados fundamentalmente, en las teorías de aprendizaje constructivistas y en las prácticas conectivistas. Desde una perspectiva constructivista, el aprendizaje es un proceso constructivo, activo, emocional, autónomo, social y situacional al que Siemens (2004) introduce una nueva faceta, denominada conectivismo. De acuerdo, con este autor, los resultados satisfactorios del aprendizaje dependen de la puesta en marcha de redes apropiadas que contengan bases de distribución de conocimiento. Aprender en un sentido conectivista requiere entornos de aprendizaje virtuales abiertos que permitan conexiones e intercambios con otros participantes de redes, los cuales construirán comunidades de aprendizaje productivas.

Los principios del conectivismo, propuestos por Siemens (2004), como tendencia emergente del aprendizaje en redes son:

1. El aprendizaje y el conocimiento se basa en la diversidad de opiniones.

2. El aprendizaje es un proceso de conectar nodos especializados o fuentes de información.

3. El aprendizaje puede residir en dispositivos no humanos. 
4. La capacidad de aprender es más importante que lo que se conoce hoy en día.

5. Es necesario cultivar y mantener conexiones para facilitar el aprendizaje continuo.

6. La capacidad de ver conexiones entre campos, ideas y conceptos es una capacidad fundamental.

7. La actualización del conocimiento es la intención de todas las actividades de aprendizaje conectivista.

8. La toma de decisiones es en sí un proceso de aprendizaje.

Consideramos que los entornos virtuales de aprendizaje compartido permiten al estudiante la creación de una zona de construcción del conocimiento propia y de trabajo con otras personas, y la regulación cognitiva al aprender el manejo de nuevas herramientas tecnológicas (autoaprendizaje, aprendizaje regulado y aprendizaje colaborativo). El estudiante al permanecer en continuo aprendizaje genera nuevas zonas de construcción del conocimiento que pasan a formar parte de su estructura cognitiva al generar conexiones para facilitar el aprendizaje.

El estudio y reflexión de las experiencias sobre VLE y PLE llevadas a cabo por distintos autores (Attwell, 2008; Lubensky, 2006; Cabero, Barroso y Llorente, 2010; y Adell y Castañeda, 2010) y la aportación de las teorías y tendencias emergentes sobre el aprendizaje refuerzan la idoneidad, relevancia y pertinencia de nuestro entorno virtual.

\section{Proceso metodológico}

Nuestro modelo didáctico ha sido diseñado e implementado para 160 estudiantes del Grado de Pedagogía de la Facultad de Ciencias de la Educación de la Universidad de Sevilla, durante el curso académico 2009-2010, en la asignatura Didáctica General, sin embargo, han participado 147 estudiantes.

Este trabajo se centra fundamentalmente, en la creación de un entorno de aprendizaje en la educación superior con la finalidad de alcanzar los siguientes objetivos:

- Presentar un entorno efectivo para orientar al estudiante en el aprendizaje de la asignatura.

- Diseñar tareas motivadoras que faciliten la asimilación de los contenidos de la materia.

- Promover el empleo significativo de la información (búsqueda y transformación de la misma, creación de recursos para el aprendizaje y tareas de colaboración).

Para la consecución del primer propósito, se han organizado los contenidos de la asignatura en la plataforma virtual WebCt de la Universidad de Sevilla. Ésta va a ser el eje del curso y el contexto para organizar los contenidos. A través de ella el estudiante tiene acceso en todo momento a los objetivos, metodología y sistema de evaluación que se plantean a lo largo del curso, desarrollo de las sesiones y progreso de los contenidos, recursos complementarios (bibliografía, apuntes, material audiovisual), noticias, formularios, etc. Con este fin, se publica en la plataforma virtual una bitácora que pretende ser el espacio principal donde se muestra la información anteriormente aludida creada para la asignatura, y donde también se ofrece al estudiante un conjunto 
de herramientas como apoyo del aprendizaje (vídeos, material complementario, presentaciones en power-point, etc.).

De la aplicación WebCt hemos utilizado diversas herramientas configurables:

- Herramientas de gestión y seguimiento de los estudiantes. Nos permite tener información de todos los matriculados en nuestra asignatura, del uso que hacen de los recursos y de las calificaciones obtenidas en las prácticas y prueba de autoevaluación.

- Grupos de trabajo. Hemos configurado grupos de trabajo con sus propios administradores de archivos y herramientas de comunicación internas al grupo.

- Gestión de archivos. Cada estudiante o grupo de trabajo posee un espacio web en el que puede dejar y consultar archivos.

- Herramientas de comunicación. El curso dispone de sus propios sistemas de correo electrónico, foros, chats, pizarras compartidas y consejos para los estudiantes.

- Herramientas de organización y publicación de contenidos. En este bloque hemos utilizado los módulos de contenidos para colgar los temas de la asignatura, un glosario de términos por orden alfabético, biblioteca de imágenes, calendario y el índice del curso

- Herramientas de evaluación. En la plataforma virtual hemos utilizado únicamente los exámenes de autoevaluación pero con notas explicativas para aclarar las respuestas dadas.

La plataforma virtual ha sido esencial en esta investigación pues se ha constituido como un punto de referencia estable para los estudiantes. Además, ha superado la curva de aprendizaje inicial al no ser expertos en el uso de la web 2.0.

Como cada entorno de aprendizaje puede ser implementado de varias formas, la decisión de nuestras implementaciones, a menudo, ha dependido de experiencias de software, objetivos de aprendizaje y competencia multimedia de los estudiantes.

En este sentido, hemos combinado aplicaciones complementarias como el modelo de contenidos basado en objetos de aprendizaje (WebCt, RODAS y OpenCourseWare) y uso de las herramientas surgidas de los servicios 2.0.

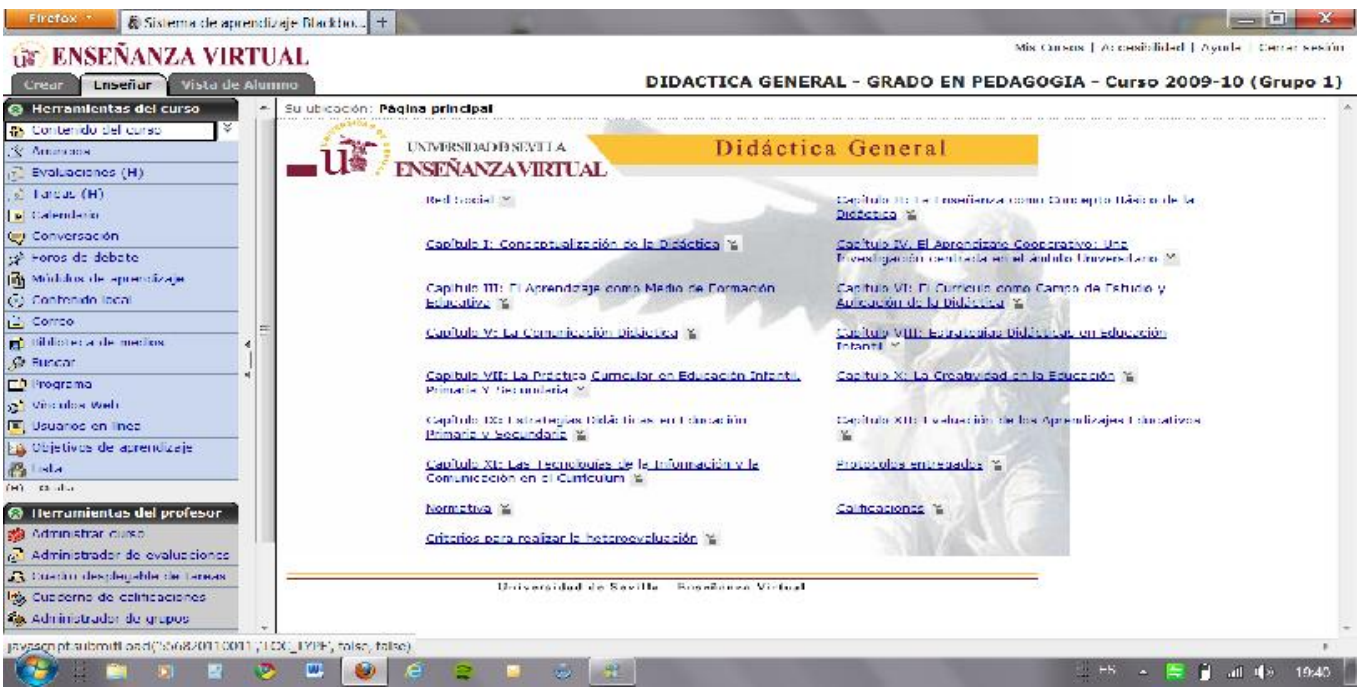

Imagen n.1. Plataforma WebCt de la asignatura Didáctica General. 
En lo concerniente al segundo objetivo de la investigación se han organizado los créditos prácticos de la asignatura través de la publicación de las producciones de cada grupo en un blog. Para este fin se ha creado una red de aprendizaje denominada Red Didáctica General. El software público Wordpress es muy atractivo para los estudiantes pues cuentan con un reproductor media fácil de usar, que ha permitido la integración de audios o vídeos en formatos comprimidos; la generación de archivos de sonido y todo el software y hardware requeridos, que han podido ser escuchados o bajados del blog y que han sido utilizados como fuente de aprendizaje. La decisión de crear una red social ha sido atendiendo a que Twitter, Facebook, Myspace, Tuenti etc. no son redes profesionales y podían dispersar más que formar a los estudiantes.

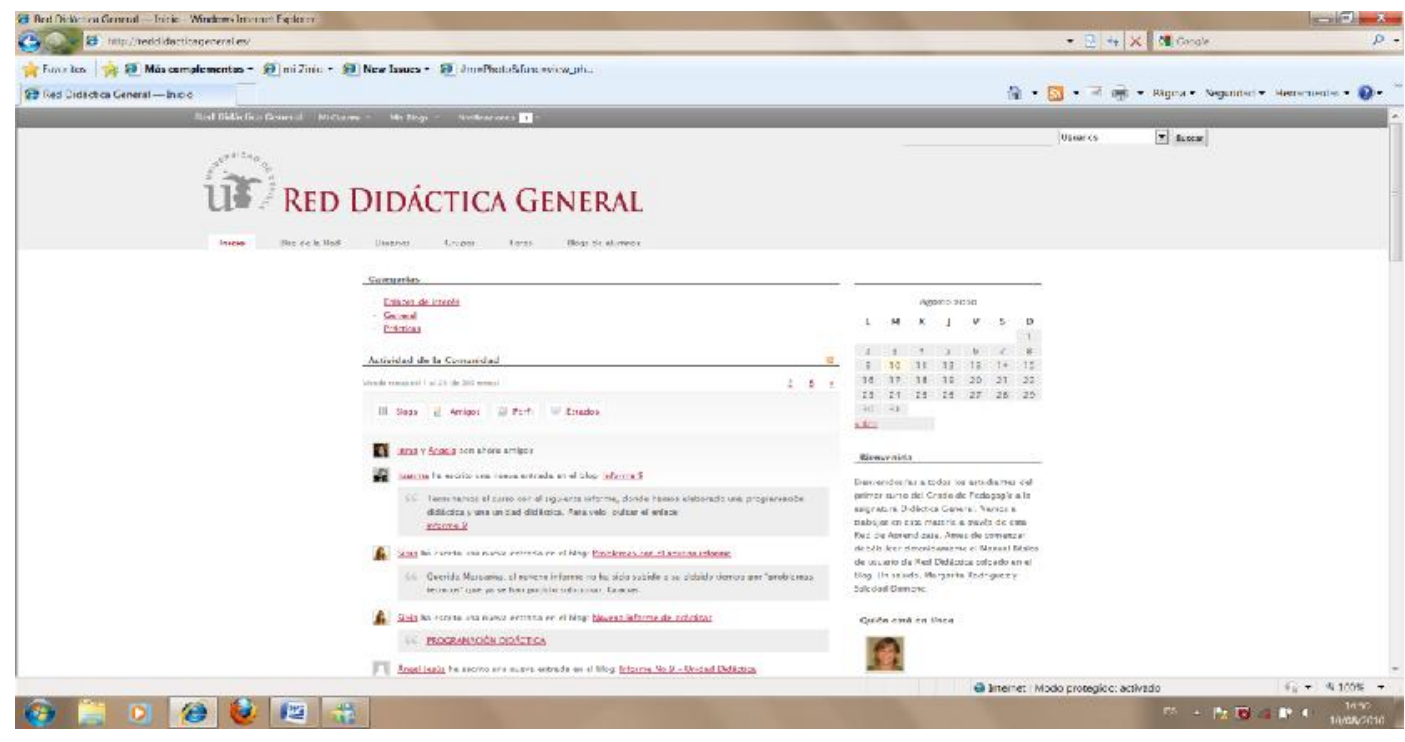

Imagen n.2. Red Social Didáctica General

En la red encontramos herramientas de apoyo al aprendizaje como acceso a enciclopedias online, traductores, diccionarios, directorio de fotos, etc. Los estudiantes crean materiales de aprendizaje mientras trabajan problemas actuales, tales como textos o archivos audiovisuales que han servido a otros compañeros; cada uno ha aprendido gracias a las contribuciones de otros, de esa forma la reciprocidad de aprendizaje, que es aprendizaje social, ha sido muy elevada (Schaffert et. al., 2006).

Para desarrollar mapas conceptuales se han empleado herramientas online, Gliffy y CmapTools, aplicaciones gratuitas de extraordinario valor didáctico.

A través de las formas de alimentación RSS (Really simple Syndication) los estudiantes han podido suscribirse a muchos contenidos de páginas web sin la necesidad de visitar cada uno de los lugares en los que está interesado y chequear por actualizaciones. De esta forma, han creado un servicio personal de alertas de distintos canales de información, distinto tipo de fuentes, imágenes y otros multimedia (D'Souza, 2006).

Los informes de prácticas se han presentado en el blog y han sido evaluados considerando la personalización de la información (se ha primado la presentación de mapas conceptuales, esquemas, resolución de casos prácticos, simulaciones, etc, y se 
ha penalizado la copia), presentación elaborada de los informes (incluyendo fotografías, animaciones, vídeo y distintas grafías) y facilidad de navegación (links, referencias externas y menú de navegación).

Cada estudiante ha participado, por defecto, del blog principal como suscriptor y del blog asociado a su grupo. Para administrar esta participación hay que entrar en el "Panel de Control" del blog en cuestión. Para ello debemos ir a la barra de menú superior y pinchar en el ítem "Mis Blogs - "Nombre del blog" - Panel de Control" (como se ve en la imagen 3 ).

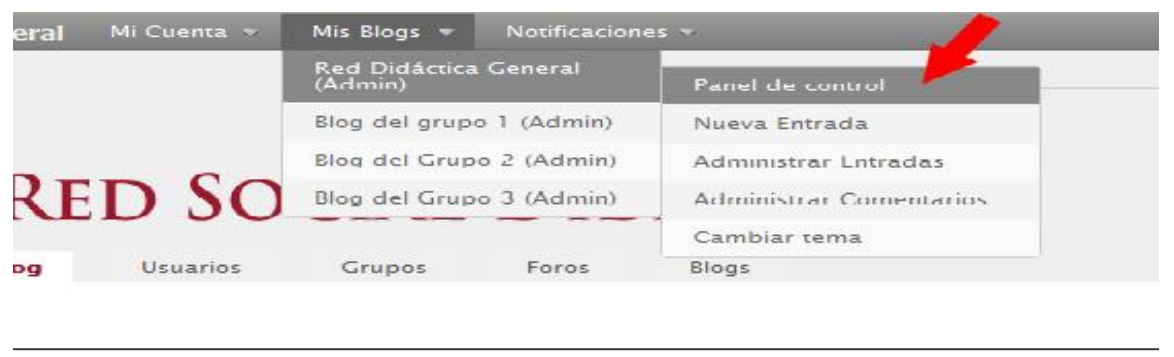

Imagen n.3. Cómo crear un blog en la Red Social Didáctica General

El propósito final ha sido lograr que el aprendizaje no se vincule exclusivamente a una actividad memorística, y que a través del uso combinado de la plataforma virtual, herramientas de la web 2.0 y la red social los estudiantes puedan integrar el conocimiento de la asignatura a través de la búsqueda y transformación de la información, creación de recursos para el aprendizaje y tareas de colaboración.

En la figura 1 mostramos una descripción de nuestro entorno virtual compartido con los recursos disponibles en la red. El acceso a la plataforma WebCt y base de datos ERIC sólo es posible a través de un intermediario, en este caso la Universidad. Para el acceso a Wordpress hemos creado la Red Social alojada en un servidor particular y todos los estudiantes han tenido que registrarse para poder participar.

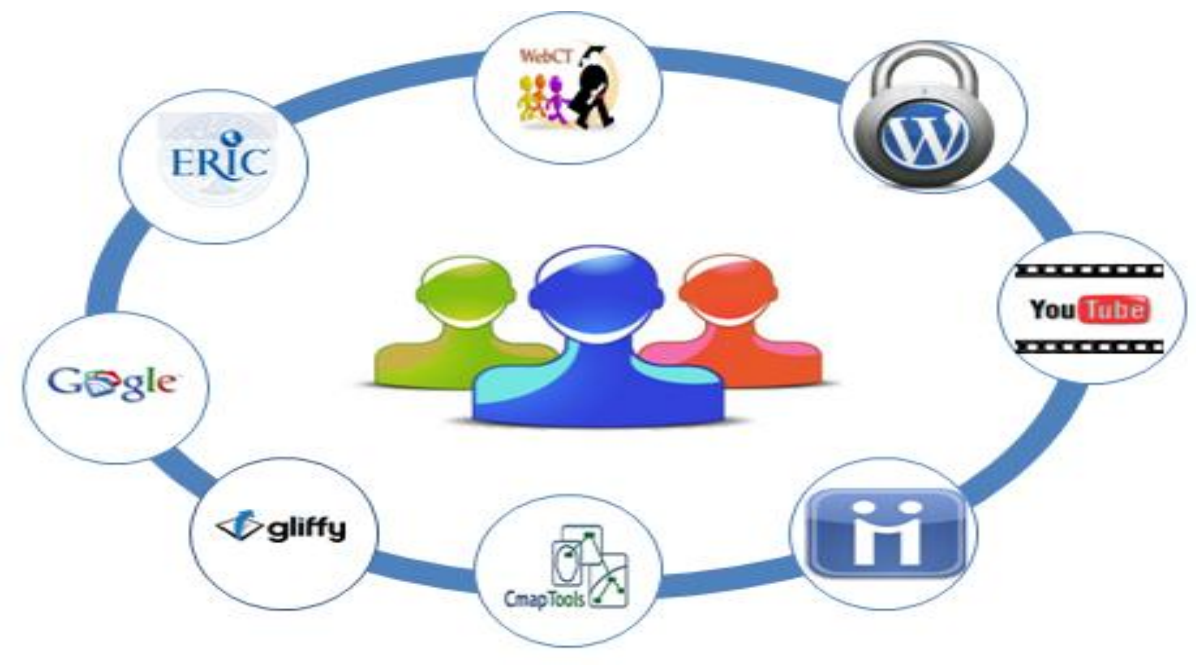

Fuente: elaboración propia

Figura n.1. Entorno Personal de Aprendizaje (PLE) de la asignatura Didáctica General 
Por ejemplo, en el caso que se muestra en la figura anterior, el estudiante comienza la asignatura en la plataforma WebCt, pincha en el icono Red Social se da de alta y cada grupo crea su blog a través de Wordpress. Además, han recogido información sobre los temas que le interesan a través de distintos espacios y recursos, se han suscrito a otros blogs sindicándose a través de RSS (Google Reader), utilizan marcadores sociales para almacenar y compartir enlaces, tienen un canal en Youtube y realizan mapas conceptuales para elaborar los resúmenes de la asignatura con la herramienta CmapTools. Finalmente, estos conocimientos son volcados nuevamente en el blog.

Para evaluar nuestro entorno virtual compartido fue necesario crear un instrumento que nos ayudara a determinar el grado de satisfacción en el uso de la plataforma WebCt, herramientas Web 2.0 y la Red Didáctica General. Para lograr nuestro objetivo elaboramos un inventario con 40 ítems, cinco alternativas de respuesta y dos preguntas abiertas. Las respuestas de los ítems se valoran de 1 a 5 que van desde nada satisfactorio (valor 1) a muy satisfactorio (valor 5) y distribuidos en cuatro categorías (accesibilidad, diseño, información y creación de recursos de aprendizaje) de la siguiente forma:

- Categoría Accesibilidad: Comprende los ítems (1, 2, 4, 10, 28, 29, 30 y 31) para determinar el nivel de satisfacción acerca del acceso a la plataforma WebCt y Red Didáctica General (blog), sistema de navegación, caminos de avances y retrocesos, funcionalidades y modos de comunicación de la red (amigos, hilos, grupos etc.).

- Categoría Diseño: A través de los ítems (3, 5, 8, 9, 38, 39 y 40) que forman esta categoría conoceremos el nivel de satisfacción de los estudiantes sobre el diseño de la plataforma en relación a los medios auxiliares (correos, biblioteca, buscadores, enlaces a páginas web,...) que facilitan el aprendizaje, la interacción con las tutoras y la combinación de entornos utilizados.

- Categoría Información: Los ítems asociados a esta categoría $(6,11,12,13,14$, $15,16,17,18,19,20,24,25,26$ y 32 ) nos van a permitir analizar el nivel de satisfacción de los estudiantes en relación a la información que le ofrece la plataforma y la red en su proceso formativo en cuanto a las noticias que aparecen, el programa de la asignatura y los contenidos. Consideramos necesario averiguar si la información proporcionada responde a sus expectativas, es decir, si los conocimientos son aplicables a la práctica, las simulaciones refuerzan la adquisición de conocimientos, los contenidos permiten acceder a una formación más especializada, los documentos complementarios son de gran ayuda y las tutoras orientan correctamente y contestan a las dudas planteadas.

- Categoría Creación de recursos de aprendizaje: Comprende los ítems (7, 21, 22, $23,27,33,34,35,36$ y 37 ) a través de los cuales tratamos de analizar el nivel de satisfacción de los participantes en relación a los foros de discusión, vídeos, presentaciones proporcionadas, actividades, recursos y blog. 


\section{Análisis de los datos}

Para validar el contenido del inventario elaboramos un protocolo en el que se pedía a los expertos que asignaran una puntuación de 1 a 5 , en la medida que los ítems recogidos en las cuatro categorías, se adecuaran o no a los objetivos propuestos. En este sentido, hemos consultado los estudios realizados por López y Díaz 2007; González y Raposo 2008; Outoni y Suárez 2010 y Cabero y Batanero 2008. Para la validación solicitamos el juicio de expertos formada por un total de 28 profesores de las Facultades de Ciencias de la Educación de Sevilla, Huelva y Granada. De los resultados obtenidos podemos deducir la validación del inventario con puntuaciones entre 4.2 y 5 .

Una vez administrado el inventario entre los 147 participantes procedimos al vaciado de los resultados en una matriz de datos para su posterior análisis a través del programa estadístico. En primer lugar, transformamos los datos brutos del inventario en códigos que pudieran ser analizados adecuadamente. A continuación, asignamos un número a cada uno de los sujetos (estos números abarcan desde el 1 hasta el número máximo de sujetos que respondieron al inventario, en nuestro caso 147). Los datos de las preguntas efectuadas a través del inventario se codificaron en función de las cinco alternativas de respuesta: Nada satisfactorio, Poco satisfactorio, Indiferente, Satisfactorio, Muy satisfactorio. A cada una de estas categorías se les otorgó un número del 1 al 5 con objeto que las respuestas pudieran ser identificadas.

Finalizado el proceso descrito procedimos a realizar el análisis de los datos a través del programa SPSS. En concreto, la información obtenida en el inventario se sometió a un análisis de distribuciones, donde se realizan unas pruebas estadísticas en función de los objetivos perseguidos. En el estudio descriptivo se calculan las puntuaciones medias en las cuatro categorías contempladas en esta investigación, con el fin de adquirir una visión general de todos los resultados y cómo se han distribuido durante el proceso. Se estudia individualmente cada categoría a nivel de medias por ítems y por dimensión, para obtener una visión global a través de la correspondiente representación gráfica.

\section{Resultados}

En este apartado se presentan los resultados obtenidos tras el análisis de los datos. Para la realización de este proceso tomamos como base las cuatro categorías del inventario, éstas son: accesibilidad, diseño, información y creación de recursos de aprendizaje y llevamos a cabo un estudio descriptivo a nivel global. La tabla 1 recoge los resultados de las puntuaciones medias y las desviaciones típicas de las cuatro categorías analizadas. 


\begin{tabular}{|c|c|c|c|c|}
\hline & Accesibilidad & Diseño & Información & $\begin{array}{c}\text { Creación } \\
\text { Recursos de } \\
\text { aprendizaje }\end{array}$ \\
\hline Validos & 147 & 147 & 147 & 147 \\
\hline Perdidos & 0 & 0 & 0 & 0 \\
\hline Media & 4,8089 & 4,0375 & 4,5571 & 4,8000 \\
\hline D. Típica & ,40966 & ,42163 & ,44361 & ,49142 \\
\hline
\end{tabular}

Tabla n.1. Resultados de las categorías del inventario

En relación con la tabla anterior, y atendiendo a las puntuaciones medias, podemos decir que la categoría Diseño ha obtenido la puntuación más baja con un resultado de $(4,03)$ que está por encima de la media. Sin embargo las categorías Accesibilidad, Información y Recursos de aprendizaje se sitúan en la opción de respuesta "muy satisfactorio" al estar muy por encima de la media con las puntuaciones 4,80, 4,55 y 4,80 respectivamente. A continuación vemos en la tabla 1 la representación gráfica de los resultados que se han comentado.

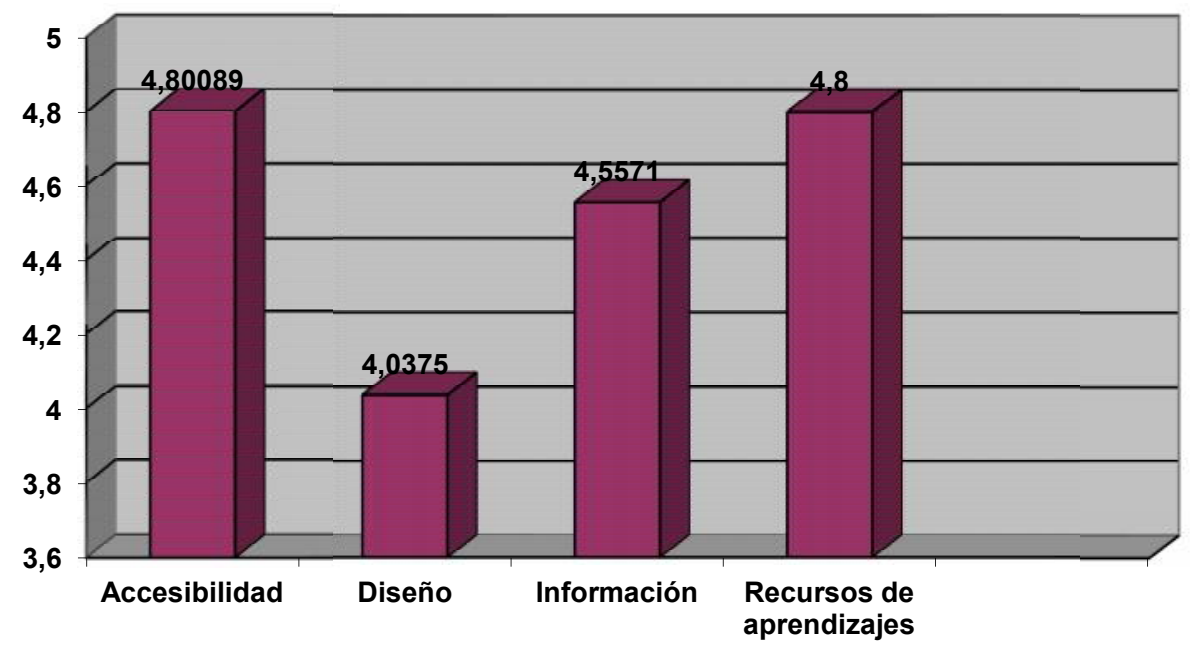

Gráfica n.1. Puntuaciones medias de las categorías Accesibilidad, Diseño, Información y Creación de Recursos de aprendizaje.

\section{Resultados de las Categorías}

Una vez analizadas las puntuaciones medias y las desviaciones típicas de las cuatro categorías pasaremos a analizar las respuestas obtenidas en los ítems más relevantes de cada categoría:

En la categoría accesibilidad destacamos el ítem 1 "El acceso al entorno de aprendizaje es sencillo" ha sido valorado satisfactoriamente por el 85,23\%. El ítem 2 "El sistema de navegación es de fácil manejo" ha obtenido la máxima puntuación (76,01\%). El ítem 4 "En el entorno de aprendizaje es fácil orientarse, encontrar los caminos de avances y retrocesos" el 62,12\% lo considera muy satisfactorio. El ítem 29 
"Las funcionalidades y modos de comunicación (amigos, hilos, grupos etc.) son accesibles" ha sido respondida, con el valor 5, por el 73,26\% de los estudiantes.

En la categoría diseño, el ítem 3 "El entorno de aprendizaje cuenta con medios auxiliares que facilitan el aprendizaje (correo, biblioteca, buscadores, enlaces a páginas web,.." ha sido contestado, con el valor 5 , por el $68,78 \%$ de los estudiantes. El ítem 8 "La interacción con las tutoras a través de las herramientas de comunicación disponibles en el entorno de aprendizaje está bien diseñada", ha sido respondido satisfactoriamente por el 60,64\% de los estudiantes. Y el ítem 38 "La combinación de la plataforma WebCt, Red Social y herramientas web 2.0 ha facilitado el aprendizaje de la asignatura" ha sido puntuado con el máximo valor por el $80,44 \%$ de los participantes. Esto significa que el diseño de nuestro entorno virtual compartido para la adquisición de conocimiento y la utilización de los servicios y herramientas ofrecidos ha tenido una acogida muy positiva.

En la categoría información, destacamos un buen nivel de satisfacción de los estudiantes en relación a la información que se le ofrece. Así el $70 \%$ ha contestado satisfactoriamente en el ítem 6 "Las noticias y el tablón de anuncios sirven de ayuda en el proceso formativo" y el ítem 12 "Los contenidos del curso se han expresado de forma clara y comprensible", el $68,85 \%$ de los estudiantes han respondido muy satisfactorio. El ítem 20 "Los documentos complementarios de estudio ayudan en la adquisición de los conocimientos de la asignatura" ha sido contestado, con el valor 5, por el 76,92\% de los estudiantes.

En la categoría creación de recursos de aprendizaje, el ítem 34 "El poder crear nuestro propio blog ha motivado al grupo en su aprendizaje" ha sido respondido, con el máximo valor, por el 92,01\%. Los estudiantes manifiestan que ha sido positivo trabajar, por ellos mismos, aunque es necesario tener todos los contenidos de la materia y presentaciones en power point para su estudio. Enfatizaron que los contenidos expuestos en el blog dan una panorámica muy útil de la asignatura y producen una reposición buena de conocimientos. El feedback ha sido positivo en referencia al desarrollo de competencias en el entorno; los estudiantes han planteado que han sido capaces de incrementar sus competencias didácticas así como las técnicas y multimedia mediante el uso de la plataforma, blog y de otras herramientas web 2.0 integradas en el entorno de aprendizaje. Además, la elaboración del blog junto con un feedback regular de las profesoras ha mejorado sus capacidades de escritura. A continuación se muestran dos opiniones que lo avalan.

"El blog me parece una muy buena forma de mostrar los conocimientos adquiridos y para aplicar la teoría a la práctica. Además adquirimos conocimientos informáticos, aprendemos a escribir y podemos mantener el contacto con los compañeros, de manera que es posible intercambiar opiniones y dudas entre nosotros" (Alumno 25).

"Al trabajar con nuevas herramientas estamos más motivados en el grupo y nos lleva a realizar los informes de prácticas más minuciosamente ya que serán vistas y comentadas por otros compañeros" (Alumno 8).

En el ítem 39 "¿Qué ventajas consideras que tiene trabajar con este entorno virtual de aprendizaje?, el $82,06 \%$ de los encuestados ha destacado el efecto de atracción social y motivación y el $68,27 \%$ la sencillez para comunicarse profesores y alumnos. La mayoría de los estudiantes $(80,75 \%)$ consideran que ha favorecido la 
recopilación de todos los materiales de la asignatura para su estudio y el $(72,41 \%)$ que el sistema de evaluación es bastante acertado al adaptarse las pruebas a cada tipo de contenido. Otra de las ventajas planteadas es que el correo electrónico ofrece un intercambio privado de mensajes, mientras que el tablón de anuncios lo realiza de forma pública (65,51\%). El chat permite establecer una comunicación sincrónica y en tiempo real entre los usuarios y no hay que desplazarse al despacho de las profesoras (42,06\%).

"Este entorno ha dado un cambio en la vida de los estudiantes ya que en los últimos años los alumnos tenían que acudir a tutoría o a otros compañeros para solucionar las dudas y ahora gracias a este entorno lo podemos realizar más rápido y fácilmente" (Alumno77).

"Es muy útil para organizar y estudiar los materiales de la asignatura" (Alumno 55).

"Es de gran ayuda tener en el entorno todo lo relativo a la asignatura porque desde cualquier sitio podemos tener acceso a ella y verlo de forma más clara" (Alumno 142).

Por último, el 40 "¿Qué inconvenientes consideras que tiene trabajar con este entorno de aprendizaje?", el 24,56\% plantean que tienen acceso a demasiada información que en algunos casos no son capaces de procesar y organizar.

"En determinados momentos que me encontrado saturado de información y he tenido que recurrir a la profesora" (Alumno 91).

\section{Conclusiones}

Con el entorno presentado hemos utilizado aplicaciones más activas e interactivas para favorecer una mayor participación de los estudiantes $y$, de este modo, potenciar su interés hacia el aprendizaje y hacia la actividad de la asignatura. En este sentido, podemos afirmar que los objetivos propuestos se han conseguido.

Destacamos que se han diseñado técnicas y herramientas vinculadas al concepto de web 2.0 buscando siempre introducir en el aula momentos para la propia producción y para posibles experiencias creativas a través del uso de blogs, mapas conceptuales, RSS, etc.

Además, hemos integrado las herramientas que ofrece la web 2.0, Red social y la plataforma WebCt en las clases de Didáctica General del Grado de Pedagogía de la Facultad de Ciencias de la Educación. El motivo es tanto didáctico como técnico. Desde un punto de vista didáctico, consideramos que los entornos virtuales de aprendizaje compartido permiten a los estudiantes el autoaprendizaje al crear conocimiento, el aprendizaje colaborativo a través del trabajo con otras personas y el aprendizaje regulado al aprender el manejo de nuevas herramientas tecnológicas. Desde un punto de vista técnico, diseñamos este entorno virtual de aprendizaje porque las plataformas virtuales no nos permiten abrir los materiales de nuestra materia a otros estudiantes y profesionales que cursan o imparten la misma asignatura pero con distinto profesor, o de alumnos matriculados en diferentes asignaturas que puedan tener relación con la nuestra. Además de no disponer de herramientas como blog, wikis, mapas conceptuales, etc. Si bien es cierto, que las plataformas de enseñanza virtual ofrecen servicios, como foros y chats, que fomentan la participación y motivación del alumno, 
las posibilidades de la web 2.0 nos llevan a un nuevo nivel basado en la colaboración de profesores y alumnos. En este sentido, la respuesta del alumnado se ha correspondido, de una forma claramente mayoritaria, con las expectativas creadas. Los estudiantes han respondido al desafío de utilizar la plataforma virtual, web 2.0 y la red con interés y motivación y, en general, se han esforzado por alcanzar los objetivos de la asignatura y por elaborar publicaciones interesantes sobre las cuestiones planteadas.

El servicio que ofrecen los entornos virtuales de aprendizaje compartido es la preparación para ser utilizados por un mayor número de personas lo que los hace ideales para actuar como centro de partida para las actividades de toda una institución educativa. Entre las ventajas, que hemos ido descubriendo en esta investigación, destacamos:

- El efecto de atracción social para los alumnos que implica un acercamiento del aprendizaje informal con el formal, así como el acercamiento de su vida privada a la vida docente.

- La sencillez y fomento de la comunicación con los estudiantes, en ambas direcciones, al estar todos en un mismo espacio. Hemos comprobado que, desde la existencia de este entorno virtual en nuestra asignatura, el medio habitual para la comunicación a través de Internet se ha desplazado en parte hacia la red social. Esto es debido a que es mucho más fácil localizar las personas dentro de la red que mediante otros medios, ya que no es necesario mantener una lista con todos nuestros contactos. Además, algunos profesores son reacios a dar su dirección de correo electrónico a los estudiantes y en las comunicaciones a través de la red social éste nunca es desvelado, aunque los mensajes se reciban en él.

- Cuando un curso virtual acaba, los estudiantes pierden la opción de seguir participando en el curso, y los mensajes se eliminan normalmente de un año a otro, lo que impide aprovechar el aprendizaje de sus predecesores. Es probable, incluso, que el profesor tenga que explicar algo que ya fue respondido en el pasado. La ubicuidad y perdurabilidad de la red social arroja, sin embargo, nueva luz sobre una posible enseñanza continuada de la que todos se puedan beneficiar y que puedan mejorar con el paso del tiempo.

También podemos afirmar que el uso de nuestro entorno virtual no sólo ha contribuido a la creación necesaria de conocimiento autónomo en temas de contenido, sino que les ha permitido desarrollar habilidades multimedia $y$ de información requeridas para trabajar con estas herramientas. Esto incluye búsqueda, selección y filtro, al igual que presentar y estructurar el conocimiento, todas estas aptitudes son importantes para una formación continuada y exitosa en futuros trabajos y proyectos profesionales. Además de un incremento considerable de aprobados en la asignatura.

La mayoría de nuestros estudiantes consideran que ha habido feedback continuado, por parte de las profesoras, que han acudido en su ayuda para la realización de las actividades de aprendizaje. 
Los trabajos escritos que los participantes han publicado son de buena a muy buena calidad en referencia a criterio y contenido formal. Por ello, hemos derivado todo este trabajo a una publicación en $C D$ con una introducción y una panorámica de los informes prácticos sobre la asignatura que va a ser usada posteriormente como fuente de aprendizaje.

Consideramos que el entorno de aprendizaje presentado puede ser adaptado para cualquier curso, asignatura y aprendizaje. Por ejemplo: el modelo introducido puede transferirse a otros contextos como cualquier asignatura de Grado, la continuación vocacional de los estudios o el aprendizaje en el puesto de trabajo.

Por tanto, podemos afirmar que la creación de nuestro VLE ha proporcionado un entorno educativo flexible donde los estudiantes han podido, además de aprender, compartir experiencias y conocimientos con otras comunidades virtuales. Los estudiantes no sólo han consumido información a través de canales independientes, tales como la biblioteca o los libros, sino que además han establecido conexiones con una matriz cada vez mayor de recursos. Los estudiantes y profesoras hemos podido interactuar mejorando aspectos como la comunicación y la optimización de los tiempos de aprendizaje $y$, sobre todo, posibilitando la puesta en práctica de una metodología más activa.

En definitiva podemos concluir que la enseñanza no ha sido tanto una cuestión de transmisión de datos, sino un cambio metodológico debido al ejercicio de colaboración en la recogida, organización e integración de datos para la construcción del conocimiento. El objetivo de nuestros estudiantes ha sido recopilar información y establecer conexiones para conseguirla, difundirla y colaborar en su uso.

En esta asignatura hemos comenzado una nueva andadura, creando un conjunto de objetos de aprendizaje para poder construir un verdadero repositorio de materiales que puedan ser puestos a disposición de estudiantes y profesores que quieran recrearlos y reutilizarlos.

Queremos dar un paso más e ir avanzando en la investigación sobre PLE para obtener importantes consecuencias en nuestra práctica docente pero sabiendo que lo importante es el punto de vista personal y que la tecnología es un instrumento para conseguir el aprendizaje y para facilitar nuevos modelos y estrategias de formación.

\section{Referencias bibliográficas}

Adell, J. y Castañeda, L. (2010). Los entornos personales de aprendizaje (ples): Una nueva manera de entender el aprendizaje. En R. Roig Vila \& M. Fiorucci (Eds.), Claves para la investigación en innovación y calidad educativas. Alcoy: MarfilRoma TRE Universitadeglistudi.

Attwell, G. (2007). Personal Learning Environments - the future of eLearning? ElearningPapers, 2(1), 1-8.

Attwell, G. (2008). Personal Learning Environments - the future of eLearning? [presentación visual] Recuperado el 9 de mayo de 2012 http://www.slideshare.net/GrahamAttwell/personal-learning-environment-thefutur-of-education-presentation 
Cabero, J., Barroso, J. y Llorente, M.C. (2010). El diseño de Entornos Personales de Aprendizaje y la formación de profesores en TIC. Digital Education Review, 18, 27-37. Recuperado el 25 de mayo de 2012 en http://greav.ub.edu/der

Cabero, J. y Batanero, J.M. (2008). Servicios universitarios de producción de nuevas tecnologías de la información y comunicación. Construcción y validación de un instrumento para su evaluación y autoevaluación. Bordón, 60 (2), 7-24.

Castaño, C. y otros (2008). Prácticas educativas en entornos web 2.0., Madrid: Editorial Síntesis.

Coppola, N. et. Al. (2002). Becoming a virtual profesor: pedagogical roles and asynchronous learning networks. Journal of Management Information Systems, $18,4,169-189$.

Downes, S. (2005). E-Learning 2.0. Recuperado el 15 de mayo de 2012 http://www.elearnmag.org/subpage.cfm?section=articles\&article=29-1

Downes, S. (2007). Learning networks in practice. Emerging Technologies for Learning Recuperado el 18 de mayo de 2012 http://nparc.cisti-icist.nrccnrc.gc.ca/npsi/ctrl?action=rtdoc\&an $=8913424$

Downes, S. (2010). New Technology supporting informal learning. Journal of Emerging Technologies In Web Intelligence, Vol. 2 N. 1. Recuperado el 10 de abril de 2012 http://www.academypublisher.com/ojs/index.php/jetwi/article/viewArticle/020 $\underline{12733}$

D'Souza, Q. (2006). RSS Ideas for Educators. Recuperado el 12 de mayo de 2012 http://www.teachinghacks.com/wpcontent/uploads/2006/01/RSS\%20Ideas\%20for\%20Educators111.pdf

Esteve, F.M. y Gisbert, M. (2011). El nuevo paradigma de aprendizaje y las nuevas tecnologías. Revista de Docencia Universitaria. REDU. Monográfico: El espacio europeo de educación superior. Hacia dónde va la Universidad Europea?. 9 (3), 55-73. Recuperado el 26 de abril de 2012 en http://redaberta.usc.es/redu

Fitzgerald, S. (2006) Creating your Personal Learning Environment. A workshop presented for the August 3rd LearnScope Workshop. Australian Technology Park $\begin{array}{llllll}\text { Recuperado el } & 30 & \text { de } & \text { abril de }\end{array}$ http://seanfitz.wikispaces.com/creatingyourple

González, M. y Raposo, M. (2008). Necesidades formativas del profesorado universitario en el contexto de la convergencia europea. Revista de Investigación Educativa, 26, (2), 285-306.

Hamburg, I. \& Hall, T. 2008. Informal learning and the use of Web 2.0 within SME training strategies. elearningPapers. Recuperado el 2 de mayo de 2012 http://www.elearningeuropa.info/files/media/media17541.pdf

Hanna, D. (ed.) (2002). La enseñanza universitaria en la era digital, Barcelona: Octaedro-EUB.

Jolliffe, A. et. al. (2001). The online learning handbook, London: Kogan Page. 
Kerres, M. (2007). Microlearning as a challenge to instructional design. In: Hug, T. \& Lindner, M. (Eds) (2007) Didactics of Microlearning. Münster: Waxmann. Recuperado el 10 de mayo de 2012.

López, A. y Díaz, L. (2007). Validación de una escala observacional para el análisis de los documentos de planificación. Revista de Investigación Educativa, 25(2), 267-285.

Lubensky, R. (2006). The present and future of Personal Learning Environments (PLE). $\begin{array}{lllll}\text { Recuperado el } & 20 & \text { de } & \end{array}$ http://www.deliberations.com.au/2006/12/present-and-future-of-personallearning.html

http://mediendidaktik.uni-duisburg-essen.de/system/files/Microlearningkerres.pdf

Outoni, P. y Suárez, A. (2010). Adaptación y validación del test de dislexia Bangor. Revista de Investigación Educativa, 28, (2), 445-457.

Palloff, R. y Pratt, K. (2003). The Virtual student, San Francisco: Jossy-Bass.

Salmon, G. (2002). E-tivities. The key to active online learning, Londres: Kogan Page.

Schaffert, S. et. al.(2006). Learning with Semantic Wikis. First Workshop "SemWiki2006 - From Wiki to Semantics", co-located with the 3rd Annual European Semantic Web Conference (ESWC), Budva, Montenegro. Recuperado el 18 de abril de 2012 http://www.salzburgresearch.at/research/gfx/semantic wiki elearning.pdf

Siemens, G. (2004). Connectivism: A Learning Theory for the Digital Age. Recuperado el 10 de mayo de 2012 http://www.elearnspace.org/Articles/connectivism.htm

Simpson, O. (2002). Supporting students in online, open and distance learning, Londres: Kogan Page, $2^{\mathrm{a}}$ ed.

Waters, S. (2008). Here Are Resultats From My PLN Survey! Recuperado 23 de abril de 2012 http://suewaters.com/2008/12/04/here-are-the-resultats-from-my-plnsurvey/

Wilson (2005). Future VLE - the Visual Version. Recuperado el 2 de abril de 2012 http://zope.cetis.ac.uk/members/scott/blogview?entry=2005012517020

Cita del artículo:

Rodríguez Gallego, M. y López Martínez, A. (2013). Entorno virtual de aprendizaje compartido en Educación Superior. Revista de Docencia Universitaria. REDU. Vol.11 (1) Enero-Abril, pp. 411-428. Recuperado el (fecha de consulta) en http://www.redu.net/ 


\section{Acerca de las autoras}

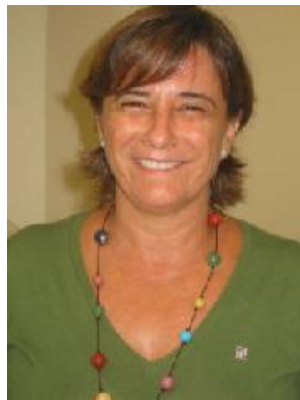

\section{Margarita R. Rodríguez Gallego}

\section{Universidad de Sevilla}

Departamento de Didáctica y Organización Educativa

Mail: margaguez@us.es

Licenciada en Ciencias de la Educación. Trabaja como profesora Titular del Departamento de Didáctica y Organización Educativa, de la Facultad de Ciencias de la Educación de la Universidad de Sevilla. Su actividad docente y sus líneas de investigación se centran, fundamentalmente, en la didáctica general, tecnologías de la información y la comunicación en los distintos niveles educativos y la mejora del prácticum en la Licenciatura y Grado de Pedagogía.

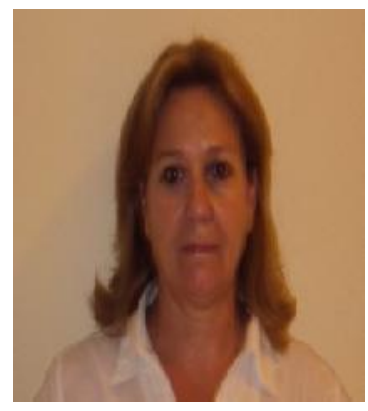

\section{Antonia López Martínez}

\section{Universidad de Sevilla}

Departamento de Didáctica y Organización Educativa

Mail: anlomar@us.es

Profesora Titular del Departamento de Didáctica y Organización Educativa de la Facultad de Ciencias de la Educación de la Universidad de Sevilla. Su actividad docente y sus líneas de investigación se centran, fundamentalmente, en la organización y dirección de los centros educativos para atender las necesidades e intereses de los alumnos. 


\title{
Puntos fuertes y débiles en la formación por competencias según los graduados universitarios españoles
}

\author{
Strong and weak points in competence - based training according to Spanish \\ Higher Education graduates
}

\author{
Andrea Conchado Peiró \\ José Miguel Carot Sierra
}

Universidad Politécnica de Valencia, España

\section{Resumen}

El objetivo del trabajo es el análisis exploratorio descriptivo de las competencias que los titulados universitarios consideran puntos fuertes o débiles en sus estudios. Este objetivo se plantea a raíz de la creciente importancia que las competencias han cobrado en los últimos años debido al proceso de adaptación de las titulaciones universitarias al Espacio Europeo de Educación Superior. Para ello se describen los resultados obtenidos en base a una muestra de 5.474 graduados universitarios españoles y trece titulaciones universitarias. Dichos datos proceden del proyecto de investigación REFLEX «El Profesional Flexible en la Sociedad del Conocimiento: Nuevas exigencias en la Educación Superior en Europa» en el que participaron universidades de catorce países europeos y Japón, entre cuyos objetivos se encontraba el estudio de las competencias en el ámbito universitario. Los resultados muestran que los graduados universitarios señalan como puntos fuertes de sus estudios las competencias relacionadas con las metodologías docentes tradicionales como los conocimientos, la capacidad de análisis y aprendizaje o el trabajo en equipo. Por el contrario, las competencias relacionadas con la gestión de las tecnologías de la información y las comunicaciones así como las habilidades de liderazgo y comunicación en idiomas extranjeros se revelan como puntos débiles de los estudios universitarios. Asimismo se han identificado determinadas particularidades de la formación en competencias en función de las siguientes áreas de estudio: Humanidades, Ciencias, Ingeniería, Arquitectura y Economía y finalmente Bellas Artes.

Palabras clave: Competencias, Titulaciones universitarias, Aprendizaje, Enfoque del aprendizaje, Contexto de aprendizaje, Puntos fuertes y débiles. 


\begin{abstract}
The objective of this study is to analyze from a descriptive point of view the competences considered by higher Education graduates as strengths or weaknesses in their studies. This objective arises from the growing importance that competences have acquired during the preceding years due to the adaptation process of university degrees to the European Higher Education Area. With this aim in mind some specific results, based on a sample of 5,474 Spanish university graduates and thirteen study programs, are described. Data were obtained from the research project REFLEX «The Flexible Professional in the Knowledge Society: New Demands on Higher Education in Europe». Universities from fourteen European countries and Japan participated in the project, and one of the aims was the analysis of competencies in Higher Education. Results describe how graduates point out to competences related to traditional teaching methods as strengths of their studies, such as knowledge of the field of study, ability to analyze and learn and teamwork, whereas competences related to information and communication technology, and also leadership and ability to speak foreign languages, are revealed as weaknesses of university studies. Besides, several peculiarities have been identified according to the following fields of study: Humanities, Sciences, Engineering, Architecture and Economy and finally Fine Arts.
\end{abstract}

Key words: Competences, University degrees, Learning, Learning approach, Learning environment, Strong and weak points

\title{
Introducción
}

Durante los últimos años las competencias han cobrado cada vez más importancia a consecuencia del proceso de adaptación de las titulaciones universitarias al Espacio Europeo de Educación Superior. Específicamente, durante las conferencias de Ministros responsables de la Educación Superior mantenidas en Berlín (2003) y Bergen (2005) se enfatizó en su importancia para el desarrollo sostenible y la cohesión social y se resaltó que engloban tanto el conocimiento y las destrezas como las actitudes y los valores. A pesar de que no se trata de un concepto nuevo en el área de la Educación Superior, en los últimos años se ha producido un incremento muy notable del número de estudios en torno a la definición y selección de competencias clave, sobre todo en lo referente a la inserción laboral de los titulados universitarios.

La cuestión del empleo se ha convertido en los últimos años en un tema controvertido que genera cierta sensación de frustración entre los jóvenes titulados. El sondeo sobre la juventud publicado por el Centro de Investigaciones Sociológicas (2009), y dirigido a los jóvenes residentes en España con edades comprendidas entre los 15 y 29 años, pone de manifiesto que éstos perciben el desempleo como su mayor problema. Como causa principal de esta dificultad apuntan a los obstáculos del mercado laboral español para obtener experiencia laboral, requerida por la mayoría de los empresarios. Así, los jóvenes titulados afirman que existe cierta incongruencia en los perfiles profesionales que demandan los puestos de trabajo, pues son excesivamente específicos y exigen una combinación de experiencia laboral, elevado nivel de estudios y baja edad prácticamente inalcanzable. Igualmente observan mayor competencia en el acceso al trabajo debido al elevado número de titulados 
universitarios, que inevitablemente conlleva un aumento de la precariedad del mercado laboral (ANECA, 2009).

En cuanto a la educación, los jóvenes parecen atribuir menor responsabilidad a la universidad en cuanto a la adecuación de su formación para encontrar trabajo. Como aspectos positivos de su experiencia en la universidad subrayan la potenciación de la capacidad de aprendizaje, la adquisición de "gusto por el conocimiento», la amplitud de perspectivas en relación al entorno donde desarrollar la actividad laboral y el aprendizaje de competencias de cooperación mediante trabajos en grupo. No obstante, describen la formación universitaria como excesivamente teórica, generalista y alejada del mundo del trabajo y apuntan especialmente hacia los idiomas como una carencia generalizada. Por esta razón, en los últimos años las universidades han incorporado a sus informes de empleabilidad análisis específicos sobre las competencias que pueden adquirirse por medio de la formación universitaria (Alonso, Fernández y Nyssen, 2009).

De hecho, un estudio elaborado por Accenture y Universia (2007) apunta que esta percepción de papel de la universidad en relación a la formación en competencias coincide con la visión de los empleadores. Los entrevistados procedentes de este grupo de interés opinan que los estudios universitarios deberían tener mayor orientación práctica e igualmente señalan las carencias en idiomas. Sin embargo, especifican que el conocimiento de herramientas informáticas no es una debilidad de los titulados universitarios. Finalmente comentan que lo que realmente se valora en el mundo de la empresa son ciertas actitudes relacionadas con el trabajo, como la responsabilidad y disciplina, la iniciativa, la disponibilidad, la capacidad de adaptación al cambio y movilidad geográfica, la predisposición para la inclusión en grupos y desenvolvimiento en situaciones sociales e incluso la presencia física. Asimismo reconocen que, aunque los estudios universitarios no contribuyen al desarrollo de estas actitudes, tampoco lo hace el sistema educativo en conjunto, la familia o la sociedad actual, que en realidad serían los entornos más propicios para su aprendizaje.

A raíz de estas iniciativas el término "Competencia» ha enriquecido su acepción inicial predominantemente enfocada al ámbito laboral, introduciendo un nuevo enfoque a nivel multidimensional. Específicamente Hartog (1992) define las competencias como las aptitudes, destrezas y capacidades de los graduados en Educación Superior que contribuyen a aumentar su productividad con un enfoque multidimensional y De Miguel et al. (2005) inciden especialmente en las características subyacentes de las competencias como son la motivación, los rasgos de la personalidad, el autoconcepto, los conocimientos y las habilidades.

En este sentido, la existencia de las componentes actitudinal y relativa a las destrezas, conlleva la imposibilidad de realizar una medición mediante los procedimientos tradicionales de evaluación de conocimientos. Si bien es cierto que en los últimos años se han desarrollado nuevos instrumentos de evaluación por competencias, como la rúbrica y el portafolio, la aplicación de ambos instrumentos no resulta pertinente en estudios sobre titulados universitarios, sino de forma continuada durante la realización de los estudios. En este caso la autoevaluación surge como un método adecuado para obtener información sobre la percepción de las personas sobre sí mismas, y especialmente en el caso de las competencias, que se ha empleado con anterioridad de forma generalizada. Así, la metodología de obtención de datos 
mediante autoevaluación puede ser administrada a un gran número de encuestados con independencia de su localización geográfica y la forma de contacto (online, telefónica, correo postal o entrevista personal). En cuanto a las competencias universitarias, este método de recogida de datos posibilita obtener información cuantitativa sobre un amplio espectro de conocimientos, habilidades y actitudes, difícilmente accesibles mediante otras formas de observación externa. Por el contrario, es necesario ser consciente de las dificultades inherentes al uso de la autoevaluación como método de obtención de información. El principal inconveniente de esta metodología una mayor incidencia del error de medida. Este error puede deberse a distintas razones, como la presencia de preguntas redactadas de forma ambigua o incomprensible, las limitaciones en la capacidad del encuestado para valorar ítems de forma abstracta, una definición de escalas de medida poco precisas e incluso la influencia de la deseabilidad social en las respuestas de los encuestados (Allen y Van der Velden, 2005).

En este contexto, resulta totalmente imprescindible conocer los puntos fuertes y débiles de las titulaciones universitarias españolas en cuanto a la formación por competencias, con el fin de determinar en qué medida el esfuerzo dedicado por los profesionales académicos están produciendo resultados en las competencias adquiridas por los estudiantes. En estas circunstancias, la presente investigación profundiza en las valoraciones de los titulados universitarios sobre las competencias que adquirieron gracias a sus estudios mediante un estudio descriptivo detallado.

\section{La importancia del enfoque del aprendizaje adoptado por el estudiante}

El término «Enfoque del aprendizaje» se define como los procesos de aprendizaje que emergen de la percepción que el estudiante tiene de la tarea académica influida por sus propias características personales (Marton, Hounsell y Entwistle, 1984). Estos enfoques del aprendizaje están influidos tanto por las características personales del estudiante y el contexto de enseñanza, como por la interacción entre ambos elementos. Concretamente, el aprendizaje académico se define como la integración de tres dimensiones: la conciencia que el estudiante tiene de su propio aprendizaje, el contenido de la materia y el contexto de aprendizaje. Esta conciencia del estudiante sobre el acto del aprendizaje es una de las claves para el éxito académico y se relaciona con el desarrollo de competencias metacognitivas. Por otro lado, estas estrategias están condicionadas por el contenido de la materia, segunda dimensión del concepto de aprendizaje académico. Al mismo tiempo, el contexto de aprendizaje abarca tanto las experiencias de aprendizaje previas, como las propias expectativas y valores del estudiante, el estilo de enseñanza del profesorado e incluso la institución, la metodología docente y los recursos y materiales docentes empleados en el aula así como el sistema de evaluación seleccionado por el profesor. Estos enfoques de aprendizaje no son características psicológicas estables, sino que dependen de la naturaleza de la tarea, el sistema de evaluación, el método de enseñanza, la percepción que el estudiante, la relevancia del curso, el interés del estudiante o el estilo de aprendizaje.

A grandes rasgos existe un consenso generalizado sobre la existencia de dos componentes fundamentales en su definición: las motivaciones que revelan las 
intenciones que mueven al estudiante a aprender y unas estrategias coherentes con dichas intenciones. Atendiendo a ambos elementos, es posible identificar hasta tres enfoques diferentes de aprendizaje, de modo que todas constituyen formas transculturales o universales de aproximarse al aprendizaje, independientemente del sistema educativo nacional. En primer lugar el «Enfoque Superficial» se basa en una motivación extrínseca. En este caso el aprendizaje en el contexto académico se considera un medio para lograr un objetivo concreto, como conseguir trabajo o aprobar un examen, y las estrategias para lograrlo se basan en minimizar el esfuerzo y reproducir lo aprendido a través de un aprendizaje memorístico. Este enfoque superficial es adoptado como consecuencia de una percepción insatisfactoria del estudiante en cuanto al ambiente de aprendizaje, de forma que considera que las tareas y procesos de aprendizaje son algo impuesto externamente. Por ello, el estudiante se limita a completar los requisitos mínimos exigido en cada tarea y memorizar el contenido de la materia con el fin de superar la evaluación sin relacionar los contenidos nuevos con los adquiridos previamente ni reflexionar sobre su posible transferencia a otros ámbitos. Según Valle et al. (2000), en este caso el esfuerzo de los estudiantes no está guiado por ninguna de las metas relacionadas con la motivación (aprendizaje y rendimiento), sino por la meta de evitación del trabajo. El «Enfoque Profundo» se basa en una motivación propia del estudiante por las materias, de forma que el estudiante se interesa por el trabajo académico y tiene la intención de comprender e interaccionar con los contenidos previos. Las estrategias surgen de esa motivación y se utilizan para maximizar la comprensión, de forma que la curiosidad del individuo sea satisfecha. Por último, el «Enfoque de Alto Rendimiento» o «Enfoque de Logro" se basa en la valoración que el individuo hace de sí mismo a través de la obtención de un alto rendimiento académico. Esta motivación constituye una forma particular de motivación extrínseca características de estudiantes competitivos, autodisciplinados, esmerados y sistemáticos y cumplen todos los requisitos que demanda la educación superior. En estas circunstancias las estrategias se relacionan con la organización del tiempo, la organización del espacio y el dominio de las materias de la forma más eficiente posible. En uno de los escasos estudios sobre la cuantificación del porcentaje de estudiantes con mayor tendencia a adoptar uno u otro enfoque del aprendizaje Hernández y Hervás (2005) estiman que, en una muestra de 360 estudiantes universitarios, aproximadamente la mitad de los encuestados adopta un enfoque profundo $(47,5 \%)$ o un enfoque superficial $(42,2 \%)$ mientras que los estudiantes que adoptan el enfoque de alto rendimiento constituyen una minoría $(10,2 \%)$.

\section{La influencia de los factores personales en la adopción del enfoque del aprendizaje}

Respecto a la relación entre el uso del tiempo y los factores personales y los enfoques del aprendizaje se han planteado numerosos estudios descriptivos desde muy diversas perspectivas, aunque es posible encontrar elementos comunes entre ellos. En muchos de ellos se plantea el análisis de los enfoques de aprendizaje de los estudiantes en función de su género, concluyendo que en la gran mayoría de ellos no existen diferencias significativas. Otros factores relevantes considerados en diversos estudios son la edad, la etapa educativa y el curso, factores que parecen fomentar la adopción 
de un enfoque más profundo a medida que se incrementan en el tiempo. Sobre la relación entre el área de estudio y el enfoque de aprendizaje adoptado se han realizado muy diversos estudios pero no se han alcanzado un consenso en base a conclusiones coherentes debido a las limitaciones de recursos en la fase de recogida de datos así como por la heterogeneidad en los planteamientos de investigación (Biggs 1982; Biggs, Kember y Leung, 2005; Kember y Wong 2000). En cualquier caso, la adopción de uno u otro enfoque del aprendizaje sólo muestra influencia en el rendimiento académico si se contextualizan en el contexto de enseñanza aprendizaje.

\section{Los contextos activos de enseñanza - aprendizaje}

Tradicionalmente la enseñanza proporcionada por las Instituciones de Educación Superior se ha fundamentado en que los estudiantes estudien los contenidos, asistan a clase para comprender mejor dichos conceptos y finalmente demuestren sus conocimientos en los exámenes finales. Así, estas clases teóricas se estructuran fundamentalmente en torno a la presentación de los contenidos de la materia de forma sistemática por parte del profesor.

Sin embargo, en este contexto de aprendizaje se corre el riesgo de que los estudiantes adquieran conocimientos sin ningún significado para ellos, con la consiguiente probabilidad de ser olvidados rápidamente después de la evaluación. Asimismo, este contexto de aprendizaje apenas fomenta la implicación de los estudiantes, lo cual es habitual que se manifieste en una motivación muy baja en el aprendizaje de la materia. Por último, se ha señalado que la subdivisión de la materia de estudio en diversas asignaturas y temarios resulta un tanto artificial y alejada de la realidad laboral, generalmente organizada en torno a problemas, cuya resolución habitualmente requiere la aplicación de conocimientos procedentes de diversas materias. En otras palabras, se considera que la estructuración de contenidos realizada en la lección magistral no refleja la interdisciplinariedad de las situaciones que posteriormente deberán afrontar los titulados universitarios. Al mismo tiempo presenta algunos inconvenientes que la convierten en una opción poco recomendable para la formación en determinadas competencias. Por su propia naturaleza su aporte en la adquisición de las capacidades de liderazgo, innovación y cooperación resulta prácticamente nulo. Asimismo, en otras competencias como organización personal y comunicación, se considera que tiene un efecto parcial, ya que aunque existe comunicación, ésta se produce en un único sentido, desde el profesor hacia el estudiante. Del mismo modo la capacidad de organización la adquiere posteriormente el alumno, fundamentalmente gracias a su trabajo autónomo y no a consecuencia del discurso recibido en el aula.

Debido a estas críticas a los contextos tradicionales de aprendizaje, y concretamente al empleo de la lección magistral como método docente, se ha fomentado la introducción de nuevos contextos de aprendizaje que demandan una participación más activa por parte del alumnado. En estos contextos de aprendizaje activos es habitual aplicar determinados métodos de enseñanza - aprendizaje como trabajos en grupo, prácticas en empresa, trabajos escritos o aprendizaje basado en problemas u orientado a proyectos. De este modo las instituciones tratan de introducir 
cambios en las metodologías docentes con el fin de garantizar que los titulados universitarios posean las competencias genéricas que posteriormente les permitirán desenvolverse con soltura en el ámbito laboral. Concretamente tanto profesores universitarios como empleadores coinciden en que determinadas competencias son especialmente relevantes en el entorno laboral, como son la resolución de problemas, la comunicación y el trabajo en equipo.

Una de las principales aportaciones de los contextos activos de aprendizaje es el incremento de la implicación de los estudiantes en el proceso de aprendizaje. Otra característica de los contextos de aprendizaje activos es que los estudiantes aprenden a través de la aplicación de los conocimientos teóricos a problemas reales. Esta experiencia obtenida mediante ejemplos prácticos y/o reales o incluso mediante simulación de situaciones prácticas, resulta una condición muy relevante para una adecuada preparación profesional, y adicionalmente facilita la adquisición de experiencia profesional. Schön (1987) incide en que los profesores envíen a los estudiantes a que tomen contacto con la realidad de su entorno laboral desde el inicio de los estudios, de modo que el aprendizaje se lleve a cabo mediante la ejecución de tareas. De hecho, las estancias en entornos laborales a través de prácticas en empresa son una metodología muy valorada por los empleadores, y en los últimos años también por las Instituciones de Educación Superior así como por los titulados universitarios, como un método muy eficiente para la adquisición de las competencias demandadas en el puesto de trabajo.

\section{Metodología}

Los datos fueron obtenidos en el marco de trabajo del Proyecto REFLEX «El Profesional Flexible en la Sociedad del Conocimiento: Nuevas exigencias en la Educación Superior en Europa». En este proyecto participaron universidades de Alemania, Austria, España, Finlandia, Francia, Italia, Noruega, Países Bajos, Reino Unido, Bélgica, República Checa, Portugal, Suiza, Japón y Estonia, y en él se entrevistó a más de 40.000 graduados sobre su experiencia en la Universidad y la transición al mercado laboral, así como las competencias que adquirieron durante el período académico. Los entrevistados fueron seleccionados mediante muestreo aleatorio estratificado en función de la región geográfica y el área de estudio, atendiendo en este caso a la siguiente agrupación realizada a partir de la clasificación propuesta por la UNESCO: Educación, Humanidades, Ciencias Sociales, Economía y empresa, Derecho, Técnicas, Salud y Ciencias. El trabajo de campo se desarrolló durante el curso académico 2005/2006 y las entrevistas se realizaron por medio de tres vías: online, por correo postal y a través de llamadas telefónicas.

La encuesta se dirigió a graduados universitarios que hubieran finalizado sus estudios cinco años antes del momento de la entrevista. Esta selección fue realizada por los expertos que participaron en el Proyecto REFLEX, considerando que en esta fase vital los graduados tienden a la reflexión sobre sus experiencias pasadas y sus posibilidades futuras en el mercado laboral. En el contexto español la encuesta se aplicó a graduados en 33 universidades españolas, públicas y privadas. El porcentaje de mujeres en esta muestra alcanzó el $65.7 \%$ de estos graduados, y la edad promedio fue 30.53 años (DT = 3.32). Asimismo la muestra incluyó graduados en distintas áreas de 
estudio y concretamente en este estudio se presentan resultados relativos a distintas titulaciones universitarias, sin profundizar en diferencias a nivel de especialidad: Diplomatura en Magisterio ( $N=585)$, Licenciaturas en Filología $(N=201)$, Historia $(N=$ 133), Bellas Artes ( $N=69)$, Economía ( $N=147)$, Administración de Empresas ( $N=380)$, Derecho ( $N=379)$, Medicina o Medicina y Cirugía $(N=91)$, Biología y Química $(N=$ 161), Matemáticas y Física $(N=56)$, Ingenierías Técnicas y Superiores $(N=963)$, Arquitectura Técnica y Superior $(N=148)$. Específicamente 5474 titulados universitarios españoles seleccionaron, entre un listado de diecinueve competencias, tres competencias como puntos fuertes de sus estudios, y otras tres como puntos débiles. El estudio de cada bloque de valoraciones - puntos fuertes y débiles - se realizó mediante análisis de tablas de contingencia obtenidas en base a conjuntos de respuestas múltiples por medio del programa PASW Statistics 18.

\section{Las competencias más valoradas por los graduados: Puntos fuertes de las titulaciones universitarias}

A continuación se muestran los resultados más significativos en cuanto a las competencias mejor valoradas por los graduados universitarios en relación a la importancia que tuvieron en sus estudios universitarios. Como se observa en la Figura 1 , un alto porcentaje de graduados seleccionó la competencia trabajar en equipo (34.7\%), seguido del dominio de tu área o disciplina (32.6\%), la capacidad de adquirir con rapidez nuevos conocimientos (31.4\%), el pensamiento analítico (27.8\%), la capacidad de redactar informes o documentos (21.1\%) y la capacidad de rendir bajo presión (20.8\%). Estos porcentajes corresponden a las posiciones que ocupan estas competencias en función del número de veces que han sido seleccionadas por los graduados, independientemente de si fueron escogidas en primera, segunda o tercera opción. El listado ordenado de competencias que se muestra en la Figura 1 se ha segmentado en cuartiles, facilitando así la selección de aquellas competencias cuyos porcentajes superan al cuartil como las más relevantes según las valoraciones de los graduados universitarios. En la parte superior de la figura se muestran aquellas competencias cuyo porcentaje de graduados que las considera puntos fuertes de la carrera supera el tercer cuartil, mientras que en la parte inferior se ubican las competencias cuyos porcentajes se encuentran por debajo del primer cuartil. Por consiguiente, aquellas competencias que se encuentran entre el primer y el tercer cuartil parecen tener una importancia media para los titulados. 


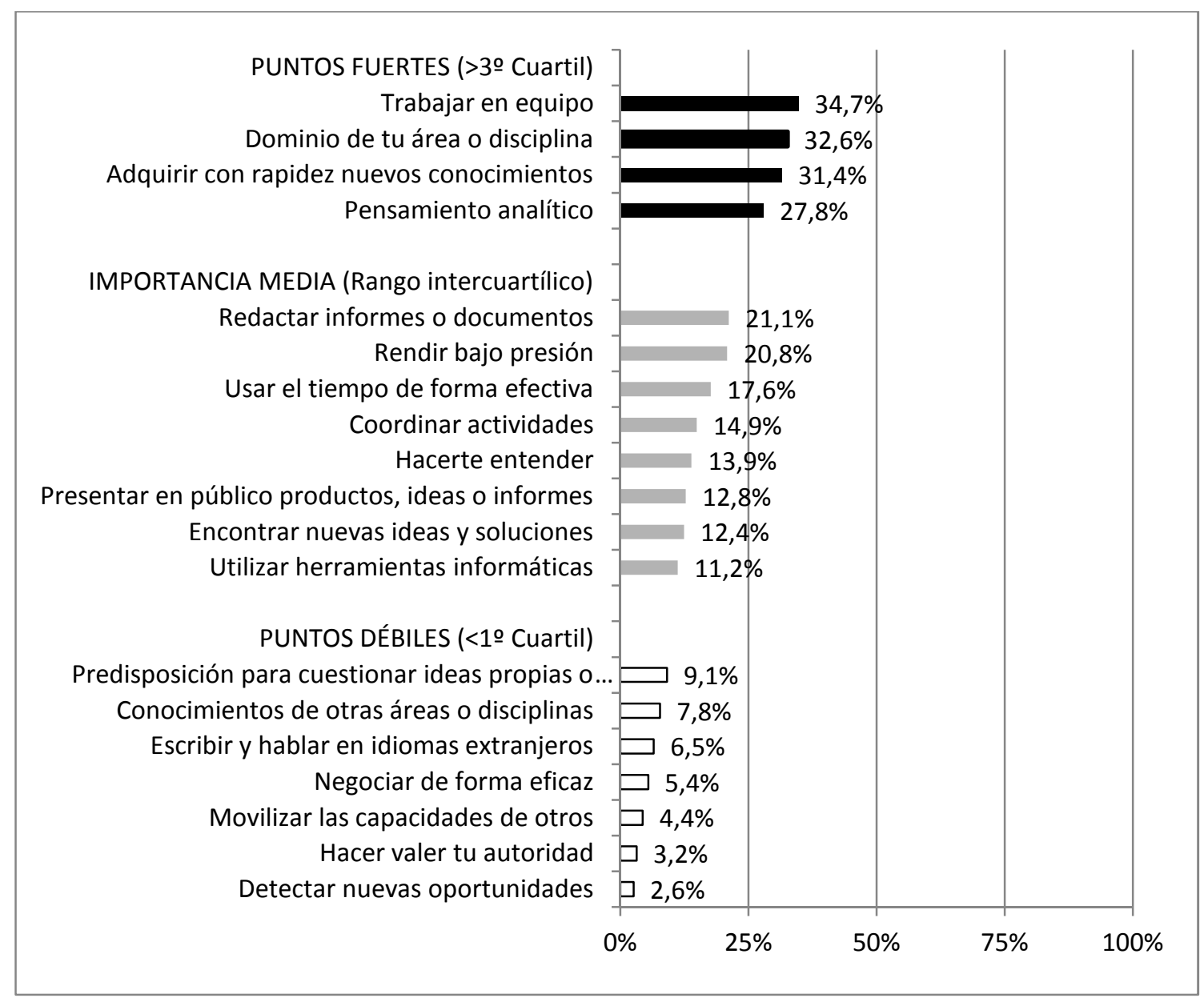

Fuente: Elaboración propia

Figura n.1. Porcentaje de graduados que seleccionaron cada competencia como punto fuerte de su titulación

El trabajo en equipo es la competencia que ocupa el primer puesto en esta lista (34.7\%), y en este aspecto cabe destacar la importancia de no confundir esta capacidad con determinadas metodologías docentes como el estudio y trabajo en grupo. En efecto, es habitual que los estudiantes universitarios deban realizar trabajos en grupo como parte de las actividades propuestas en determinadas asignaturas. No obstante, en la mayoría de los casos esta tarea se resuelve asignando las distintas subtareas a cada miembro del grupo, sin llegar a establecer una sinergia con los compañeros. A pesar de ello, muchos estudiantes finalizan sus estudios con la percepción de que tuvieron que realizar muchos trabajos en grupo durante la carrera, y por ello valoran este aspecto de forma tan notable. En cualquier caso, aún siendo consciente del riesgo de confusión entre los términos «trabajo en grupo» y «trabajo en equipo», resulta sorprendente el gran número de graduados que han seleccionado esta competencia como punto fuerte de sus titulaciones.

Profundizando a nivel de titulación universitaria, son los graduados en Magisterio los que más seleccionan esta competencia como fortaleza de sus estudios (54.4\%), donde frecuentemente se enfatiza en el desarrollo de competencias sociales que faciliten a los futuros maestros la interacción con sus alumnos. Asimismo ocurre con los titulados en Administración y Dirección de Empresas (38.7\%) y Biología o Química 
(36.0\%), quienes habitualmente deben colaborar con sus compañeros en la gestión de grupos de trabajo a nivel empresarial en el primer caso, y para la realización de análisis y ensayos de laboratorio en el segundo. Por el contrario, otros graduados opinan que en sus carreras no se desarrolló suficientemente esta competencia, y concretamente un porcentaje considerable de los graduados en Derecho (17.1\%) e Historia (17.6\%) seleccionaron esta competencia como punto débil de sus estudios.

Esta competencia se encuentra muy relacionada con otras de índole social, como la capacidad de coordinar actividades. De hecho, a pesar de que sólo el $14.9 \%$ de los graduados españoles la señala como punto fuerte de sus estudios, este porcentaje se incrementa hasta el $25.5 \%$ de los diplomados en Magisterio, e incluso hasta el $27.2 \%$ de los Arquitectos (Técnicos y Superiores), quienes trabajan frecuentemente en equipos de trabajo con el fin de elaborar proyectos multidisciplinares. Por otro lado, se plantea la competencia movilizar las capacidades de otros, únicamente seleccionada por el $13.6 \%$ de los graduados. No obstante, este porcentaje aumenta hasta alcanzar el 20.4\% para los licenciados en Filología, más conscientes que el resto de graduados de sus carencias a la hora de movilizar a las personas para formar equipos de trabajo o lograr una mayor motivación para consecución de objetivos comunes.

El segundo puesto en el ranking de las competencias mejor valoradas por los graduados universitarios lo ocupa el dominio de la propia área de conocimiento (32.6\%). En este aspecto, el porcentaje de titulados que coinciden con esta afirmación se incrementa a medida que aumenta el nivel de especialización de los estudios. Concretamente este porcentaje se incrementa de forma muy notable para los licenciados en Medicina o Medicina y Cirugía (55.9\%) y Filología (40.8\%). Evidentemente la adquisición de un gran número de conocimientos, teóricos o procedimentales, pero muy especializados sobre la propia disciplina de la titulación, conlleva una reducción de los conocimientos relativos a otras áreas. Es por ello que el porcentaje de titulados que considera esta última competencia como un punto débil de sus carreras se incrementa para ambas titulaciones respecto al global $(17.0 \%)$, alcanzando el $23.3 \%$ para los licenciados en Medicina o Medicina y Cirugía y el $20.9 \%$ para los licenciados en Filología.

Seguidamente ocupan el tercer y cuarto puesto respectivamente, la capacidad de adquirir conocimientos con rapidez (31.4\%) y el pensamiento analítico (27.8\%). Ambas competencias hacen referencia al llamado metaconocimiento o la capacidad de análisis y aprendizaje del estudiante y forman parte, junto con los conocimientos analizados anteriormente, del conjunto de capacidades que tradicionalmente se han transmitido a los estudiantes en las universidades. A nivel de titulación, ambas competencias adquieren una especial relevancia en carreras relacionadas con las ciencias. Específicamente la adquisición de conocimientos con rapidez es muy valorado por un alto porcentaje de licenciados en Matemáticas y Física (62.1\%), Economía (45.1\%), Ingenierías Técnicas y Superiores (44.6\%) y Biología y Química (35.2\%), e igualmente ocurre con el pensamiento analítico donde se alcanzan porcentajes similares e incluso superiores: licenciados en Matemáticas y Física (70.3\%), Economía (50.5\%), Ingenierías Técnicas y Superiores (42.8\%) y Biología y Química (36.8\%).

A continuación la capacidad de redactar informes o documentos ocupa el quinto puesto de la lista (21.1\%). Esta competencia parece ser característica de determinadas titulaciones del área de humanidades, donde el porcentaje de graduados que la han 
seleccionado como punto fuerte de sus estudios resulta especialmente elevado. Éste es el caso de los licenciados en Historia (44.4\%), Derecho (43.4\%) y Filología (36.7\%). En contraposición existe un alto porcentaje de graduados en otras titulaciones que señalan esta misma competencia como punto débil de sus estudios, como ocurre con los licenciados en Matemáticas y Física (22.0\%), Bellas Artes (17.0\%) y los diplomados en Magisterio (16.7\%). No obstante, este resultado no implica necesariamente una carencia en la capacidad de comunicación de los graduados en estas titulaciones. De hecho, un elevado porcentaje de los diplomados en Magisterio afirma que es capaz de hacerse entender (25.7\%). Del mismo modo los licenciados en Filología (26.8\%) y Derecho (23.3\%) coinciden con esta afirmación, quienes inevitablemente desarrollan en mayor medida sus habilidades comunicativas.

Por último aparecen en los últimos puestos del conjunto de competencias con porcentajes de selección superiores al tercer cuartil, las capacidades de rendir bajo presión (20.8\%) y usar el tiempo de forma efectiva (17.6\%). Ambas competencias han sido escogidas por una parte considerable de los licenciados en Medicina, llegando a duplicar su valor para la capacidad de rendir bajo presión (40.6\%) y con un incremento moderado en el uso efectivo del tiempo (25.2\%). Sin embargo, la capacidad de rendir bajo presión parece ser característica de titulaciones universitarias con métodos de evaluación muy concretos, donde los estudiantes deben cumplir con rigurosidad ciertos plazos para la entrega de trabajos o proyectos o evalúan los conocimientos adquiridos en exámenes globales, que implican una gran carga de trabajo y riesgo durante su realización. Este es el caso de un porcentaje considerable de los Arquitectos Técnicos y Superiores e Ingenieros Técnicos y Superiores (29.3\%). Por otro lado, otros graduados que coinciden con los licenciados en Medicina y Medicina y Cirugía en cuanto a la importancia de sus estudios en el aprendizaje de la gestión del tiempo son los licenciados en Bellas Artes (23.5\%), quienes consideran esta competencia un punto fuerte de sus estudios.

\section{Las competencias menos valoradas por los graduados: Puntos débiles de las titulaciones universitarias}

Respecto a las competencias valoradas como puntos débiles destaca en gran medida la capacidad de escribir y hablar en idiomas extranjeros, seleccionada por más de la mitad de los universitarios (62.0\%). Posteriormente, la capacidad de utilizar herramientas informáticas ocupa el segundo lugar (32.7\%), seguida de la capacidad de presentar en público productos, ideas o informes (26.0\%). A continuación es posible encontrar otras competencias, asociadas a habilidades de liderazgo, como la capacidad de negociar de forma eficaz (25.5\%) y la capacidad de hacer valer tu autoridad (19.4\%). Finalmente los conocimientos de otras áreas o disciplinas (17.0\%) emergen como una posible área de mejora, en contraste al dominio de la propia área o disciplina, uno de los puntos fuertes de las titulaciones universitarias, tal y como se muestra en la sección anterior. Estos seis ítems constituyen el conjunto de capacidades situadas por encima del tercer cuartil, calculado en base al porcentaje de graduados que señalan cada competencia como un punto débil de su carrera. En la Figura 2 se han señalado en la parte superior dichas capacidades, mientras que aquellas competencias con porcentajes inferiores al primer cuartil se encuentran en la parte inferior. Por ende, se 
encuentran entre ambas aquellas competencias cuyos porcentajes se encuentran entre el primer y el tercer cuartil.

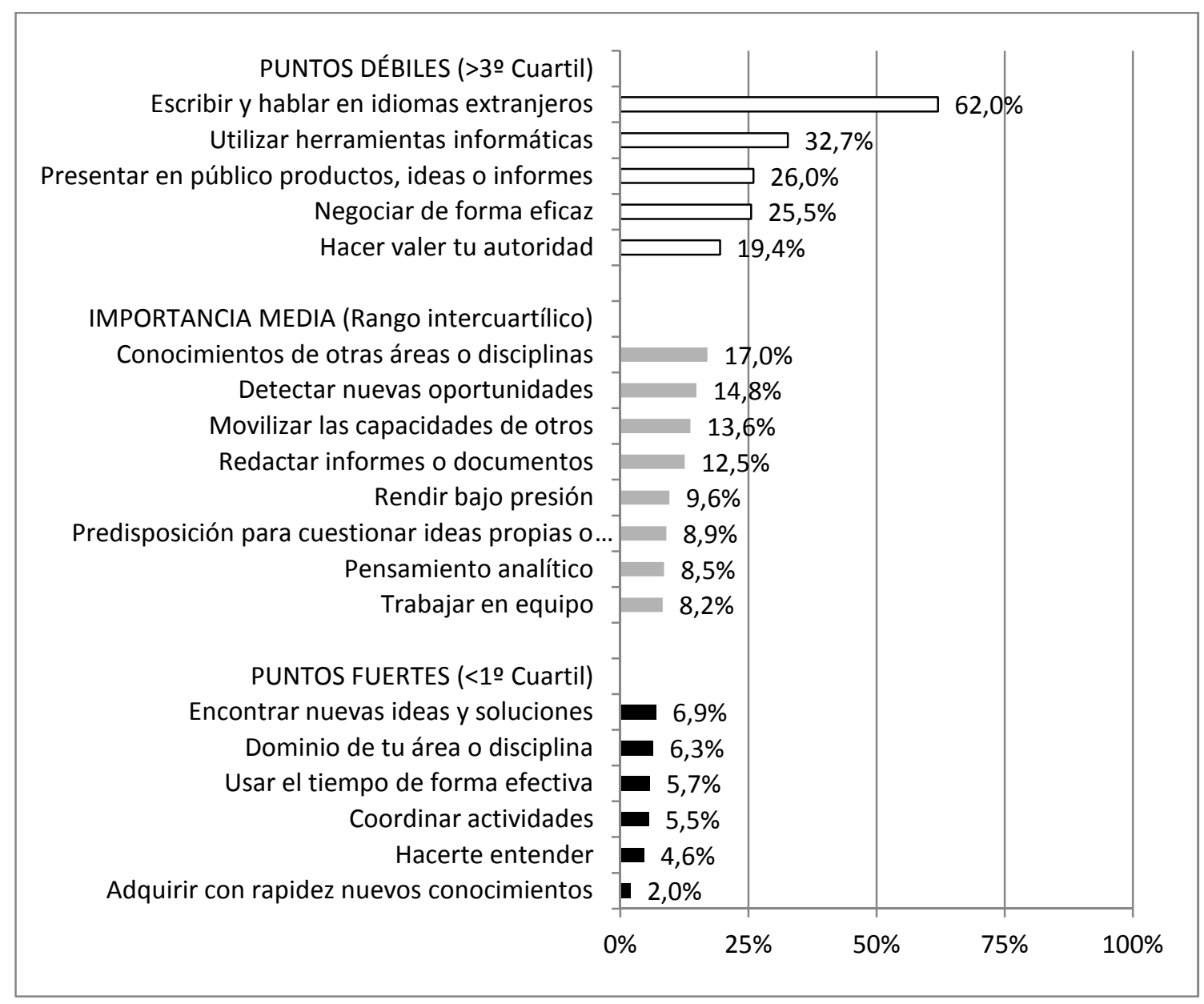

Fuente: Elaboración propia

Figura n.2. Porcentaje de graduados que seleccionaron cada competencia como punto débil de su titulación

La percepción de cierta insuficiencia formativa en idiomas extranjeros es un elemento común entre los graduados de todas las titulaciones $(62.0 \%)$, con la única excepción de los licenciados en Filología, entre los que el porcentaje de los que señalan esta competencia como punto débil disminuye hasta el $19.9 \%$. Los idiomas extranjeros constituyen sin duda uno de los aspectos que las universidades españolas deben mejorar, con el fin de lograr una mayor adaptación de los titulados universitarios a la llamada sociedad del conocimiento, basada en el uso intensivo de las tecnologías de la información y las comunicaciones y en la actualidad, son muchas las instituciones de Educación Superior que ya se encuentran trabajando para mejorar esta laguna.

También ligado al desarrollo de la sociedad del conocimiento, los graduados señalan en segundo lugar a la capacidad de utilizar herramientas informáticas (32.7\%). Esta competencia constituye una carencia más acentuada en determinadas titulaciones, en las que el porcentaje de los graduados que las considera puntos débiles asciende notablemente. Así ocurre con los licenciados en Filología (51.1\%), 
Medicina o Medicina y Cirugía (49.2\%), Derecho (46.6\%) e Historia (42.8\%), cuyos estudios no requieren, en principio, el aprendizaje de complejos conceptos informáticos para el desempeño de la profesión.

En relación a las competencias comunicativas, si bien la capacidad de redactar informes o documentos se mostraba en la sección anterior como un punto fuerte de las titulaciones españolas, la capacidad de presentar en público productos, ideas o informes aparece ahora como un punto débil de los estudios universitarios. Concretamente experimentan cierta falta de preparación los titulados en determinadas carreras del área de ciencias, como los licenciados en Matemáticas y Física (39.0\%), Biología y Química (35.7\%), Ingenierías Técnicas y Superiores (33.9\%) y licenciados en Economía (33.5\%). Todas estas titulaciones tienen en común el énfasis en el desarrollo del pensamiento analítico y adquirir conocimientos con rapidez, tal como se ha mostrado anteriormente. No obstante, parece que una mayor formación en la mejora de las habilidades comunicativas fomentaría mayor confianza entre los titulados en estas carreras en cuanto a su capacidad para realizar presentaciones.

Otro aspecto que los titulados señalan que podría mejorarse son las competencias relacionadas con el liderazgo, como son la capacidad de negociar de forma eficaz (25.5\%) y la capacidad de imponer tu autoridad (19.4\%), que ocupan el cuarto y quinto puesto del ranking de puntos débiles, respectivamente. Esta insuficiencia formativa la perciben especialmente los titulados en carreras del área de ciencias y tecnología. Así, un alto porcentaje de licenciados en Matemáticas y Física opinan de esta forma en cuanto a sus capacidades de negociación (32.0\%), al igual que los titulados en Ingenierías Técnicas y Superiores (35.9\%) y Administración y Dirección de Empresas (30.6\%). Del mismo modo, un alto porcentaje de licenciados en Matemáticas y Física señalan que en sus carreras no se enfatizó en cómo imponer tu autoridad (27.8\%), e igualmente piensan los titulados en Ingenierías Técnicas y Superiores (19.5\%) y Economía (22.6\%). En este aspecto cabe señalar que habitualmente estos estudios conducen al desempeño de puestos que implican funciones relacionadas con la dirección y/o coordinación de equipos de trabajo, lo que puede justificar una mayor concienciación de la importancia de dichas competencias.

Finalmente, los conocimientos relativos a otras áreas o disciplinas surge como un punto débil (17.0\%), en coherencia con el hecho de que el dominio de la propia área o disciplina se reveló en la sección anterior como un punto fuerte de la mayoría de los estudios universitarios. Como es de esperar, esta opinión se encuentra generalizada entre los licenciados en Medicina (23.3\%), quienes anteriormente seleccionaron el dominio de la propia área o disciplina como una de las competencias características de sus estudios, debido al elevado nivel de especialización.

\section{La innovación: una competencia difícil de catalogar}

Con independencia de las competencias descritas hasta el momento, que ocupan posiciones muy específicas en los rankings construidos anteriormente, tres competencias muy relacionadas entre sí adoptan posiciones intermedias en ambos listados: la capacidad de encontrar nuevas ideas y soluciones, la predisposición para cuestionar ideas propias o ajenas y la capacidad de detectar oportunidades. Este conjunto de competencias hacen referencia a las capacidades de innovación y 
creatividad y cómo el titulado universitario es capaz de modificar y renovar el entorno en el que desarrolla sus tareas con el fin de lograr una mejora. Estas competencias son claramente minoritarias, y aunque no apuntan hacia ellas como posibles áreas de mejora, sólo una pequeña parte de los titulados las selecciona como puntos fuertes de su carrera. Concretamente, tan sólo el $12.4 \%$ tiene esta opinión de la capacidad de encontrar nuevas ideas y soluciones, el $9.1 \%$ respecto a la predisposición para cuestionar ideas propias o ajenas y el $14.8 \%$ en cuanto a la capacidad de detectar oportunidades.

No obstante, la percepción de estas competencias se modifica notablemente para los licenciados en Bellas Artes. Entre estos graduados existen mayores porcentajes de los que consideran que en su carrera se aprende a encontrar nuevas ideas y soluciones (46.8\%) e igualmente ocurre con los licenciados en Matemáticas y Física (40.1\%). Asimismo existe un elevado porcentaje de graduados en Bellas Artes e Historia que valoran el desarrollo de la predisposición para cuestionar ideas propias o ajenas como un punto fuerte de sus carreras ( $24.0 \%$ y $26.0 \%$, respectivamente), así como la capacidad de detectar oportunidades ( $19.7 \%$ y $21.9 \%$, respectivamente).

Por tanto, el desarrollo de habilidades relacionadas con la innovación y la creatividad parece estar muy vinculado a determinadas carreras universitarias. Considerando el entorno en que hoy en día los titulados universitarios deben desarrollar su trabajo, mucho más dinámico y cambiante que hace una década, cabría plantearse la necesidad de incluir en el resto de titulaciones nuevas competencias transversales relativas a la innovación y la creatividad.

\section{Conclusiones}

En este trabajo se han analizado las competencias que los titulados universitarios consideran puntos fuertes y débiles de sus estudios. Los resultados muestran que las competencias relacionadas con las metodologías docentes tradicionales son las más señaladas de forma generalizada como puntos fuertes de sus estudios. Concretamente los titulados apuntan al trabajo en equipo, el dominio de la propia área o disciplina, la capacidad de adquirir conocimientos con rapidez, el pensamiento analítico, la capacidad de redactar informes o documentos y la capacidad de rendir bajo presión. Por el contrario, las competencias que los graduados subrayan como puntos débiles de sus estudios se encuentran más relacionadas con la gestión de las tecnologías de la información y las comunicaciones así como las habilidades de liderazgo. Específicamente los titulados apuntan a la capacidad de escribir y hablar en idiomas extranjeros, la capacidad de utilizar herramientas informáticas, la capacidad de presentar en público productos, ideas o informes, la capacidad de negociar de forma eficaz, la capacidad de imponer tu autoridad y los conocimientos de otras áreas o disciplinas. Cabe destacar que esta percepción de la realidad en cuanto a la formación en competencias de los titulados universitarios coincide con la de los empleadores, excepto en la necesidad de ampliar el conocimiento de herramientas informáticas. Como se ha expuesto anteriormente, las empresas valoran la capacidad de aprendizaje de los titulados y los conocimientos que poseen gracias a la universidad. Sin embargo, consideran que la formación universitaria debería estar más orientada al mundo laboral y tener mayor enfoque práctico. 
A nivel de titulación se han detectado diferencias entre las valoraciones de los titulados respecto a las competencias que se adquieren en sus estudios. En función de estas apreciaciones es posible distinguir entre tres grupos de titulaciones. En primer lugar las carreras del área de humanidades: diplomatura en Magisterio y Licenciaturas en Filología, Historia y Derecho, cuyos graduados se sienten seguros en su capacidad de redactar informes o documentos o en cualquier caso de hacerse entender. La mayoría de los graduados en estas titulaciones coinciden en la falta de preparación en herramientas informáticas y trabajo en equipo, con la excepción de los Diplomados en Magisterio, quienes afirman destacar en este aspecto e incluso en la coordinación de actividades. Esta competencia es un punto fuerte especialmente característico de estos estudios, así como lo es la capacidad de escribir y hablar en idiomas extranjeros en la Licenciatura en Filología y una mayor predisposición para cuestionar ideas propias o ajenas en Historia.

Por otro lado, existen igualmente elementos comunes entre las carreras del área de Ciencias: Ingenierías Técnicas y Superiores, Arquitectura Técnica y Superior, Medicina y Medicina y Cirugía, Biología y Química, Matemáticas y Física, Economía y Administración de Empresas. En primer lugar una mayor confianza en el dominio de la propia área o disciplina, así como en la capacidad para adquirir conocimientos con rapidez y el pensamiento analítico. Adicionalmente los ingenieros y médicos se encuentran suficientemente preparados en su capacidad para rendir bajo presión, al igual que los arquitectos, quienes también dicen estar bien formados para la coordinación de actividades. Como contrapartida, tanto los ingenieros como los licenciados en Matemáticas y Física, Economía y Administración de Empresas manifiestan cierta insuficiencia en la formación que recibieron en cuanto a su capacidad para realizar presentaciones en público, negociar de forma eficaz e imponer su autoridad. Finalmente se presenta el caso de los licenciados en Bellas Artes, quienes afirman haber recibido formación en competencias relacionadas con la innovación y la creatividad.

A partir de este trabajo es posible extraer determinadas implicaciones a nivel metodológico de gran utilidad para las instituciones académicas, como la necesidad de enfatizar en mayor medida en la formación en idiomas en todas las titulaciones universitarias. Asimismo se plantea la posibilidad de aplicar los trabajos en grupo no sólo para el desarrollo de competencias colaborativas, sino para fomentar el aprendizaje de las habilidades de liderazgo, competencia que en la actualidad se percibe como poco impulsada por las universidades españolas. Del mismo modo se propone un mayor uso de las exposiciones orales como método para la mejora de las competencias de comunicación de los titulados, y concretamente para el aprendizaje de técnicas de presentación y hablar en público. No obstante, en cuanto a los conocimientos teóricos aportados por los estudios, se propone que prevalezca la actualización de los mismos frente a su ampliación, pues la formación teórica de los titulados parece ser más que suficiente para abordar las tareas de sus puestos de trabajo.

Finalmente es necesario considerar las limitaciones de este estudio, no sólo en lo referente a los problemas asociados al uso de la autoevaluación como método de recogida de datos, sino también por la realización de una única medida. Esta encuesta se dirigió a titulados universitarios durante el curso académico 2005/2006, por lo que 
es necesario considerar que la edad promedio de los titulados se sitúa en torno a los treinta años y el momento de la realización de las entrevistas es anterior al inicio de recesión económica en España. Por consiguiente, sería recomendable disponer de estudios longitudinales que permitiesen evaluar estos resultados a lo largo de la trayectoria profesional de los titulados, información que podría obtenerse a través de los observatorios de inserción laboral de las universidades españolas.

\section{Referencias bibliográficas}

Accenture y Universia. (2007). Las competencias profesionales en los titulados. Contraste y diálogo Universidad - Empresa. http://www.accenture.com/eses/industry/Pages/carrera-prof.aspx. [Consulta: 15/11/2012].

ANECA. (2009). Los procesos de inserción laboral de los titulados universitarios en España. Factores de facilitación y de obstaculización. http://www.aneca.es/Documentos-y-publicaciones/Otros-documentos-deinteres/Insercion-laboral. [Consulta: 13/11/2012].

Allen, J. y Van der Velden, R. (2005). The role of self - assessment in measuring skills. Artículo presentado en Transition in Youth Workshop, celebrado en Research Centre for Education and the Labour Market, Maastricht University.

Alonso, L.E., Fernández, C.J. y Nyssen, J.M. (2009). El debate sobre las competencias. Una investigación cualitativa en torno a la educación superior y el mercado de trabajo en España. http://www.aneca.es/Documentos-y-publicaciones/Otrosdocumentos-de-interes/Insercion-laboral. [Consulta: 13/11/2012].

Biggs, J. (1982). Student motivation and study strategies in University and CAE populations. Higher Education Research and Development, 1(1):33-55.

Biggs, J., Kember, D. y Leung, D. (2005). The influence of active learning experiences on the development of graduate capabilities. Studies in Higher Education, 30(2):155-170.

Centro de Investigaciones Sociológicas. (2009). Sondeo sobre la Juventud. Estudio no 2822.

http://www.cis.es/cis/opencm/ES/1 encuestas/estudios/ver.jsp?estudio=10724 [Consulta: 12/11/2012].

De Miguel, M., Alfaro, I., Apodaca, P., Arias, J.M., García, E., Lobato, C. y Pérez, A. (2005). Modalidades de enseñanza centradas en el desarrollo de competencias. Orientaciones para promover el cambio metodológico en el Espacio Europeo de Educación Superior. Madrid: Programa de Estudios y Análisis. Dirección General de Universidades. Ministerio de Educación y Ciencia

Fernández, F., García, P. y Maquillón, J. (2005). Análisis del cuestionario de procesos de estudio - 2 factores de Biggs en estudiantes universitarios españoles. Revista Fuentes, 6.

Hartog, J. (1992). Capabilities, Allocation and Earnings. Boston: Kluwer.

Hernández, F. y Hervás, R.M. (2005). Enfoques y estilos de aprendizaje en Educación Superior. Revista española de orientación y psicopedagogía, 16(2):283-299. 
Kember, D. y Wong, A. (2000). Implications for evaluation from a study of students' perceptions of good and poor teaching. Higher Education, 40(1):69-97.

Marton, F., Hounsell, D. y Entwistle, N. (1984). The experience of learning. Edinburgh: Scottish Academia Press.

Schön, D. (1987). Educating the reflective practitioner: towards a new design for teaching and learning in the professions. San Francisco: Jossey - Bass Publishers.

Valle, A., González, R., Núñez, J. C., Suárez, J. M., Piñeiro, I. y Rodríguez, I. (2000). Enfoques de aprendizaje en estudiantes universitarios. Psicothema, 12(3):368375.

Cita del artículo:

Conchado, A. y Carot, J.M. (2013). Puntos fuertes y débiles en la formación por competencias según los graduados universitarios españoles. Revista de Docencia Universitaria. REDU. Vol.11 (1) Enero-Abril, pp. 429-446. Recuperado el (fecha de consulta) en http://www.red-u.net/

\section{Acerca de la autora y el autor}

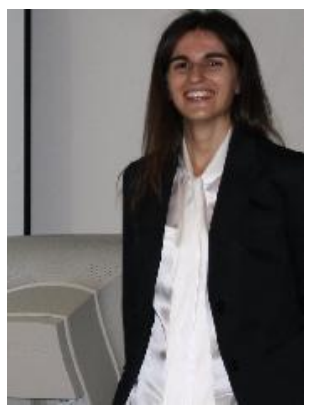

\section{Andrea Conchado Peiró}

\section{Universidad Politécnica de Valencia}

Departamento de Estadística e Investigación Operativa Aplicadas y

Calidad / Centro de Gestión de la Calidad y del Cambio

Mail: anconpei@upvnet.upv.es

Doctora Ingeniera Industrial, Profesora Asociada en el Departamento de Estadística e Investigación Operativa Aplicadas y Calidad de la Universidad Politécnica de Valencia. Trabaja como investigadora en el Centro de Gestión de la Calidad y del Cambio de la misma universidad, donde ha participado en diversos proyectos de investigación a nivel nacional e internacional. Sus áreas de investigación son la estadística y la metodología de la investigación, y específicamente el empleo de ecuaciones estructurales para la evaluación de la fiabilidad y validez de escalas de medida y modelos causales. Asimismo ha colaborado en varios artículos de investigación y capítulo de libro en el campo de la estadística aplicada a la educación. 


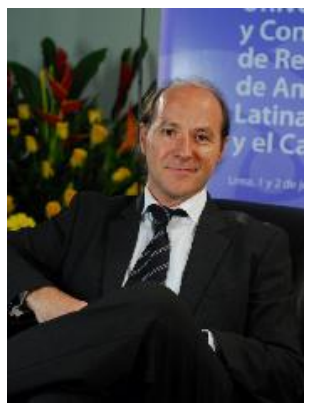

\section{José Miguel Carot Sierra}

\section{Universidad Politécnica de Valencia}

Departamento de Estadística e Investigación Operativa Aplicadas y Calidad / Centro de Gestión de la Calidad y del Cambio Mail: jcarot@eio.upv.es

Doctor en Ciencias Matemáticas, Profesor Titular en el Departamento de Estadística e Investigación Operativa Aplicadas y Calidad de la Universidad Politécnica de Valencia donde actualmente es director del Área de Evaluación Académica y subdirector del Centro de Gestión de la Calidad y del Cambio. Ha sido investigador principal en cinco proyectos europeos y ha participado otros 23 proyectos de investigación. Las áreas en las que actualmente desarrolla su trabajo son: análisis multivariante, diseño de experimentos, métodos estadísticos para la calidad, diseño y análisis de encuestas y gestión de la educación superior. 


\section{REDU}

Revista de Docencia Universitaria

Vol $11 \mathrm{~N}^{\mathrm{O} 1}$ 



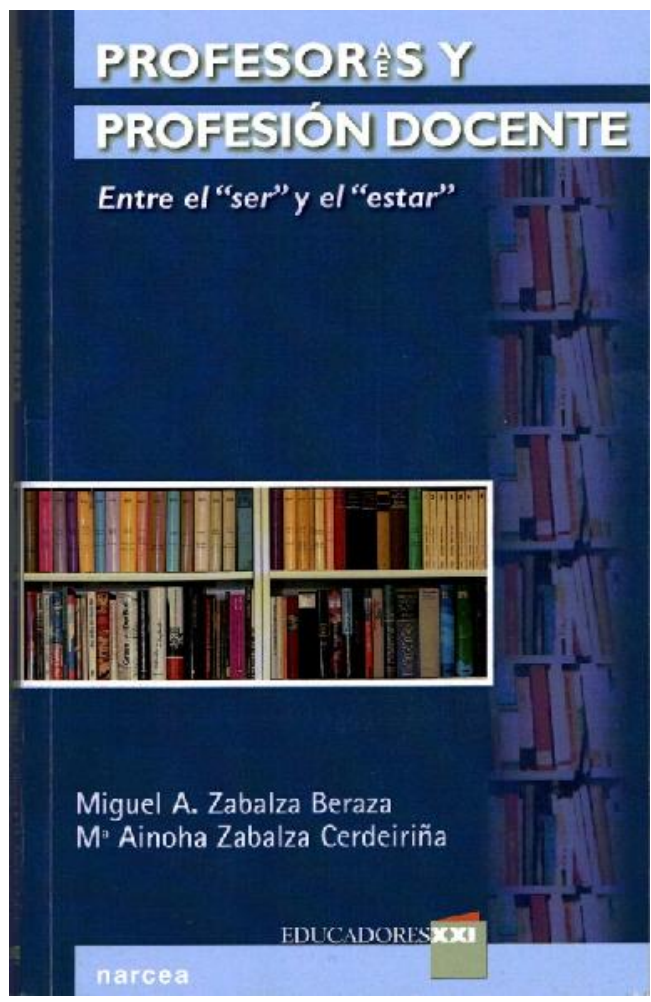

\section{Profesores y profesión docente.} Entre el "ser" y el "estar"

Autores: Miguel A. Zabalza Beraza Mạ Ainoha Zabalza Cerdeiriña

ISBN: 978-84-277-1809-8

Depósito Legal: M-45.623-2011

Editorial: Narcea

Año de edición: 2012

№ Edición: 1a

Lugar edición: Madrid

№ páginas: 165

Idioma: Español

El libro que, nos ofrece de la Editorial Narcea en su colección EDUCADORES XXI, con tres bloques o módulos constituidos por cinco capítulos y 165 páginas, nace - según sus autores- de la preocupación por lo que significa ser "maestro" en los tiempos actuales. Para ello, parten de un primer reconocimiento como es el de que hoy no es fácil se "profesor/a" en ninguna etapa del sistema educativo debido a le exigencia de combinar tradición y posmodernidad en su ejercicio profesional. Esto implica la necesidad de acomodarse a un "nuevo perfil" docente. Este "nuevo perfil" implicará que el docente sea una persona "culta" que domine su materia; además, han de ser profesionales "técnicos" que dominen las competencias básicas de su trabajo como educadores; también, buenos "artesanos", capaces de elaborar materiales didácticos propios, de generar recursos, de emplear efectivamente las tecnologías; por supuesto, que sean profesionales "reflexivos capaces" de documentar y revisar sus prácticas y, finalmente, "actores sociales" relevantes y comprometidos.

Por si lo anterior no fuese suficiente, se plantean la disyuntiva, como subtítulo, entre el "ser" y el "estar" docente. Esto es, entre lo que implica la idea de una "vocación docente" (eso sí, laica) y el desempeño del "rol" docente.

Para responder a estas cuestiones surge este libro. Escrito por padre e hija reflexionando desde sus experiencias como profesores y formadores de profesores. $\mathrm{Si}$ bien, cada uno de ellos con experiencias muy distintas, uno terminando su carrera y la 
otra iniciándola. Experiencias y visiones que le da a la obra una extraordinaria riqueza, frescura y actualidad. Además, al estar escrita con rigor, actualidad, claridad y anécdotas hacen muy fácil y atractiva su lectura.

Como hemos indicado, el libro, se estructura en tres módulos. En el primero de ellos, con un solo capítulo, titulado LA DOCENCIA EN UN MUNDO EN CAMBIO, los autores intentan provocar un debate e intercambio de ideas sobre el trabajo de los profesores en un mundo de cambio. Así, se plantean algunos dilemas importantes que los profesores han de afrontar en su trabajo, como son:

- El dilema entre lo natural y social: el alumno como sujeto. El decir, si lo que se pretende es dejar fluir y, como mucho, supervisar su desarrollo natural o, por el contrario imbuirles pautas culturales y sociales.

- El dilema entre la vida y la academia, al resultar difícil moverse adecuadamente entre el complejo mundo de las necesidades y demandas que los niños, familias y sociedad plantean a la educación.

En el módulo segundo titulado EL PROFESORADO EN EL CALEIDOSCOPIO, los autores, dentro de las muchas formas de abordar la figura de los docentes (como en un caleidoscopio), optan por destacar tres perfiles básicos de todo profesor: lo que es como persona, lo que es como profesional y lo que es como trabajador. Entendiendo que los tres aspectos influyen de una manera clara en el desarrollo de su función y condicionan en que su trabajo resulte de mayor o menor calidad.

En el primero de los capítulos de este módulo se aborda el aspecto de "LOS PROFESORES Y PROFESORAS COMO PERSONAS". Es decir, su perfil personal. Para ello partiendo de cómo la vieja sentencia de que "los profesores enseñan tanto por lo que saben cómo por lo que son", ha recibido escasa atención en el ámbito de la formación docente. $Y$ sin embargo, los autores ponen de manifiesto cómo buena parte de la influencia que los profesores ejercen en los estudiantes se deriva de lo que somos como personas, de nuestra forma de presentarnos y de nuestras modalidades de relación con ellos. Bajo esta consideración, seguidamente se analizan aquellos aspectos que constituyen al docente como persona. Estos hacen referencia a la persona que enseña, los ciclos de vida del profesorado, los núcleos de satisfacción e insatisfacción personal y profesional, la carrera profesional docente, el narcisismo como fuente de energía personal y el docente como persona que enseña a ser personas.

El segundo de los capítulos se aborda el aspecto de "LOS PROFESORES $Y$ PROFESORAS COMO PROFESIONALES". Es decir, su perfil profesional. Este es el punto clave para entender la función docente del profesorado. Para lo cual los autores parten de la pregunta siguiente ¿Qué es enseñar, una profesión o un trabajo? Para contestarla, los autores nos indican que la profesionalidad de los docentes parece una cuestión clara, pero que no se trata de algo que haya madurado sin controversias. Pero como nunca lleve a gusto de todos, también existen movimientos. Vinculados en general a organizaciones sindicales, que no quieren oír hablar de profesionalidad docente y prefieren defender una visión estrictamente laboral. Será por tanto la dimensión profesional de la docencia a la que pretenden prestar mayor atención al considerar que constituye la esencia lo que un docente es y debe hacer. No obstante, el ejercicio de la profesión plantea requisitos que, sin duda, han fortalecido la 
identidad de la tarea docente: una formación específica y de alto nivel; unos sistemas de selección; el reconocimiento de la autonomía en su trabajo; la formación permanente, la carrera profesional y un largo etc. Todos estos aspectos son analizados en este capítulo.

En el tercer capítulo de este módulo, bajo el título de "LOS PROFESORES $Y$ PROFESORES COMO TRABAJADORES", los autores abordan la tercera de las perspectivas desde la que se puede analizar la función docente: la dimensión laboral, la condición de trabajadores de quienes la ejercemos. Consideran, asimismo, que en una consideración puramente teórica de la figura de los profesores, esta dimensión pudiera pasar desapercibida y de hecho así suele suceder, pero parece claro que sin ella cualquier cosa que se diga resulta incompleta y probablemente, incorrecta. Para abordarla nos indican que los profesores somos trabajadores que desempeñamos tareas específicas en instituciones educativas bajo unas ciertas cláusulas laborales, y que estas no pueden de dejar de ejercer influencia en nuestro compromiso y dedicación. Seguidamente, analizan a través de un documento de la OCDE, los modelos actualmente vigentes referidos al profesorado: la funcionarización o el contrato, con sus ventajas y desventajas y desarrollan los aspectos referidos al "mundo laboral" como el acceso a la docencia, las relaciones interpersonales y el clima laboral y la satisfacción y la motivación en el trabajo.

En el tercero y último módulo titulado "LOS BUENOS PROFESORES", los autores hacen un pequeño recuento de cuáles podrían ser las características o cualidades de un buen docente. Para ello se plantean las preguntas de sí ¿Existen los buenos profesores? ¿Cómo son? y ¿dónde están? Pretenden con sus respuestas cerrar el círculo de los análisis anteriores. Consideran, sin pretender ser exhaustivos ni excluyentes, que los docentes han de poseer seis cualidades. Cualidades que explican a través de los seis compromisos siguientes: compromiso consigo mismo y con el propio desarrollo profesional, compromiso con los conocimientos, compromiso con la cultura profesional, compromiso con los estudiantes: tacto pedagógico, compromiso con los colegas: trabajo en equipo y compromiso con la comunidad: compromiso social.

Nos hallamos, por tanto, ante una obra que, además de servir como una magnifica guía para los estudiantes y personas que quieran iniciarse o profundizar en el mudo de lo que significa ser "maestro", constituye un excelente recurso para los profesionales docente de las distintas etapas educativas a quienes llevará a profundizar sobre los diferentes aspectos que constituyen el "perfil docente" a partir de las reflexiones suscitadas por los autores. Reflexiones que, sin duda, van a servir no sólo para el mejor conocimiento del quehacer docente sino también para mejorar la calidad de su actuación.

Alfonso Cid Sabucedo

Universidad de Vigo

acid@uvigo.es 


\section{REDU}

Revista de Docencia Universitaria

Vol $11 \mathrm{~N}^{\mathrm{O} 1}$ 



\section{Eventos relacionados con docencia universitaria}

\section{JORNADAS SOBRE RELACIÓN PEDAGÓGICA EN LA UNIVERSIDAD. Desdibujando fronteras, buscando puntos de encuentro.}

La Universidad Autónoma de Madrid acoge durante los días 20 y 21 de junio de 2013 estas jornadas que apuestan por "lo transdisciplinar y por valorar el trabajo de los estudiantes que acompañan los docentes universitarios. Seguir explorando el valor de la innovación, la reflexión colectiva de los docentes, la desburocratización del cambio y la búsqueda de nuevos escenarios para nuestro trabajo". Las temáticas abordadas en esta convocatoria son:

- Transdisciplinariedad y nuevas formas de construir conocimiento.

- Docencia compartida.

- Resolución de problemas. Las resistencias de los estudiantes.

- El error, como forma de aprendizaje. Favorecer en el estudiante un reproductor o a un autor de conocimiento.

- Si dedicamos tiempo a otro tipo de actividades, qué pasa con los contenidos. Papel del estudiante.

- Estudiantes y profesorado ya acostumbrados a investigar y aprender haciendo.

- Tiempo de clase, ¿para qué? El papel del estudiante en el diseño del curso.

- Evaluar, ¿̇para qué? Aprender sin contexto. Papel del estudiante.

- La perversión de una supuesta evaluación continua. Papel del estudiante.

Información completa en http://ijdesdibujandofronteras.blogspot.com.es/

\section{SYMPOSIUM INTERNACIONAL SOBRE EL PRACTICUM Y LAS PRÁCTICAS EN EMPRESAS EN LA FORMACIÓN UNIVERSITARIA. Un Practicum para la formación integral de los estudiantes}

Durante los próximos 26, 27 y 28 de junio de 2013 se celebra en Poio (Pontevedra) la duodécima convocatoria del Symposium Internacional sobre el Practicum, centrada en la convicción de que el PRACTICUM juega un papel fundamental en la formación integral de los estudiantes, tal y como lo van reconociendo paulatinamente las políticas curriculares universitarias. Se trata de destacar que el buen PRACTICUM es aquel que cumple adecuadamente con su función formativa, es decir, que se trata de 
una experiencia que mejora a los estudiantes como futuros profesionales pero también como personas, que completa sus aprendizajes académicos pero que les ayuda también a ampliar su experiencia vital e ir construyendo un proyecto de vida más estimulante. Es algo a tomar muy en cuenta cuando se planifica el Practicum y cuando se plantean las condiciones para mejorarlo.

Entre las actividades académicas programadas se celebrarán las siguientes conferencias:

- Aprendizaje activo y formación integral de los estudiantes universitarios. Dr. Günter Huber (Univ. de Tübingen, Alemania)

- La supervisión clínica del practicum. Dr. Enrique Correa (Univ. de Sherbrooke, Canadá)

- Formarse en la Universidad del siglo XXI. Dra. Agueda Benito (Rectora de la Universidad Europea de Madrid)

- El practicum como contexto de aprendizaje. Dr. Miguel Angel Zabalza Beraza (Univ. de Santiago, AIDU).

También se presentan dos mesas redondas, una centrada en los Avances en la gestión institucional del Practicum y otra sobre Datos recientes obtenidos en la investigación sobre el tema, con participación del profesado de distintas universidades (A Coruña, Castilla La Mancha, País Vasco, Minho, Navarra, Valencia) y campos de conocimiento (Económicas, Educación, Medicina, Trabajo Social).

Además de comunicaciones, pósters y simposiums autoorganizados, el programa académico se completa con la oferta de talleres sobre la Evaluación de competencias, la Práctica reflexiva, el Estudio de casos, la Guía de prácticas, la Tutoría y supervisión del practicum.

Información más detallada en http://redaberta.usc.es/poio/ 